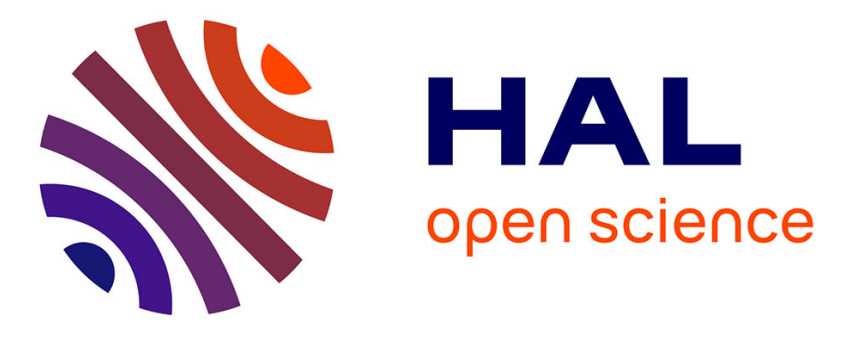

\title{
Reference Value Advisor: a new freeware set of macroinstructions to calculate reference intervals with Microsoft Excel
}

Anne Geffre, Didier Concordet, Jean-Pierre Braun, Catherine Trumel

\section{To cite this version:}

Anne Geffre, Didier Concordet, Jean-Pierre Braun, Catherine Trumel. Reference Value Advisor: a new freeware set of macroinstructions to calculate reference intervals with Microsoft Excel. Veterinary Clinical Pathology, 2011, 40 (1), pp.107-112. 10.1111/j.1939-165X.2011.00287.x . hal-02649142

\section{HAL Id: hal-02649142 \\ https://hal.inrae.fr/hal-02649142}

Submitted on 29 May 2020

HAL is a multi-disciplinary open access archive for the deposit and dissemination of scientific research documents, whether they are published or not. The documents may come from teaching and research institutions in France or abroad, or from public or private research centers.
L'archive ouverte pluridisciplinaire HAL, est destinée au dépôt et à la diffusion de documents scientifiques de niveau recherche, publiés ou non, émanant des établissements d'enseignement et de recherche français ou étrangers, des laboratoires publics ou privés. 
\%PDF - 1.3

\%äãÏò

$44 \odot$ obj

$<<$

/Linearized 1.0

/0 46

$/ \mathrm{H}\left[\begin{array}{ll}1474 & 407\end{array}\right]$

/L 328678

/E 43085

$/ \mathrm{N} 6$

/T 327754

$>>$

endobj

4453

$000000001500000 \mathrm{n}$ $000000138900000 \mathrm{n}$ $00000018810000 \odot \mathrm{n}$ $0000002147 \quad 0000 \odot \mathrm{n}$ $\odot 0000023380000 \odot \mathrm{n}$ $\odot \odot \odot \odot \odot \odot 256 \odot$ $\odot \odot \odot \odot \mathrm{n}$ $000000280500000 \mathrm{n}$ $000000343500000 \mathrm{n}$ $000000378200000 \mathrm{n}$ $000000406700000 \mathrm{n}$

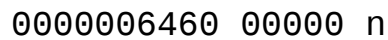
0000006950 $00000 \mathrm{n}$ $00000072410000 \odot \mathrm{n}$

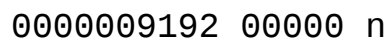
$\odot \odot \odot \odot \odot \odot 979 \odot \quad \odot \odot \odot \odot \odot ~ n$

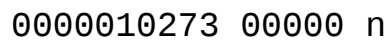
$000001487700000 \mathrm{n}$ $000001506500000 \mathrm{n}$ $000001546600000 \mathrm{n}$ $000001581300000 \mathrm{n}$ $000001918300000 \mathrm{n}$ $\odot \odot \odot \odot ० 19467$ $\odot \odot \odot \odot \odot \mathrm{n}$ $\odot \odot \odot \odot \odot 19713$ $\odot \odot \odot \odot ~ n$ $\odot \odot \odot \odot \odot 20846$ ๑๑९९९ n $\odot \odot \odot \odot \odot 21468$ ๑०९०९ $\mathrm{n}$

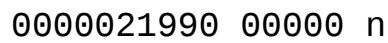
$0 \odot \odot \odot \odot 2871600 \odot \odot \odot \mathrm{n}$ $000002920200000 \mathrm{n}$ $000002955500000 \mathrm{n}$ $000003421100000 \mathrm{n}$ $\odot \odot \odot \odot \odot 34377$ ๑०००९ n $000003459400000 \mathrm{n}$ $\odot \odot \odot \odot \odot 3480 \odot \quad 0 \odot \odot \odot \odot \mathrm{n}$

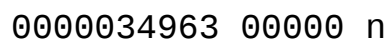
$\odot \odot \odot \odot \odot 35197$ ๑०९०९ n

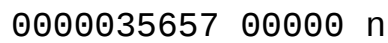
$00000357250000 \odot \mathrm{n}$ $000003581900000 \mathrm{n}$ $000003591500000 \mathrm{n}$ $000003606100000 \mathrm{n}$ $000003623500000 \mathrm{n}$ $\odot 0000364090000 \odot \mathrm{n}$ $000003655700000 \mathrm{n}$ 0000036705 $\odot \odot \odot \odot \odot \mathrm{n}$ $\odot \odot \odot \odot \odot 37504$ ๑๑९९९ n $\odot \odot \odot \odot \odot 38256$ ๑०९०९ n $000003908700000 \mathrm{n}$

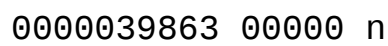
$\odot \odot \odot \odot \odot 4 \odot 68 \odot \odot \odot \odot \odot \odot \mathrm{n}$ 


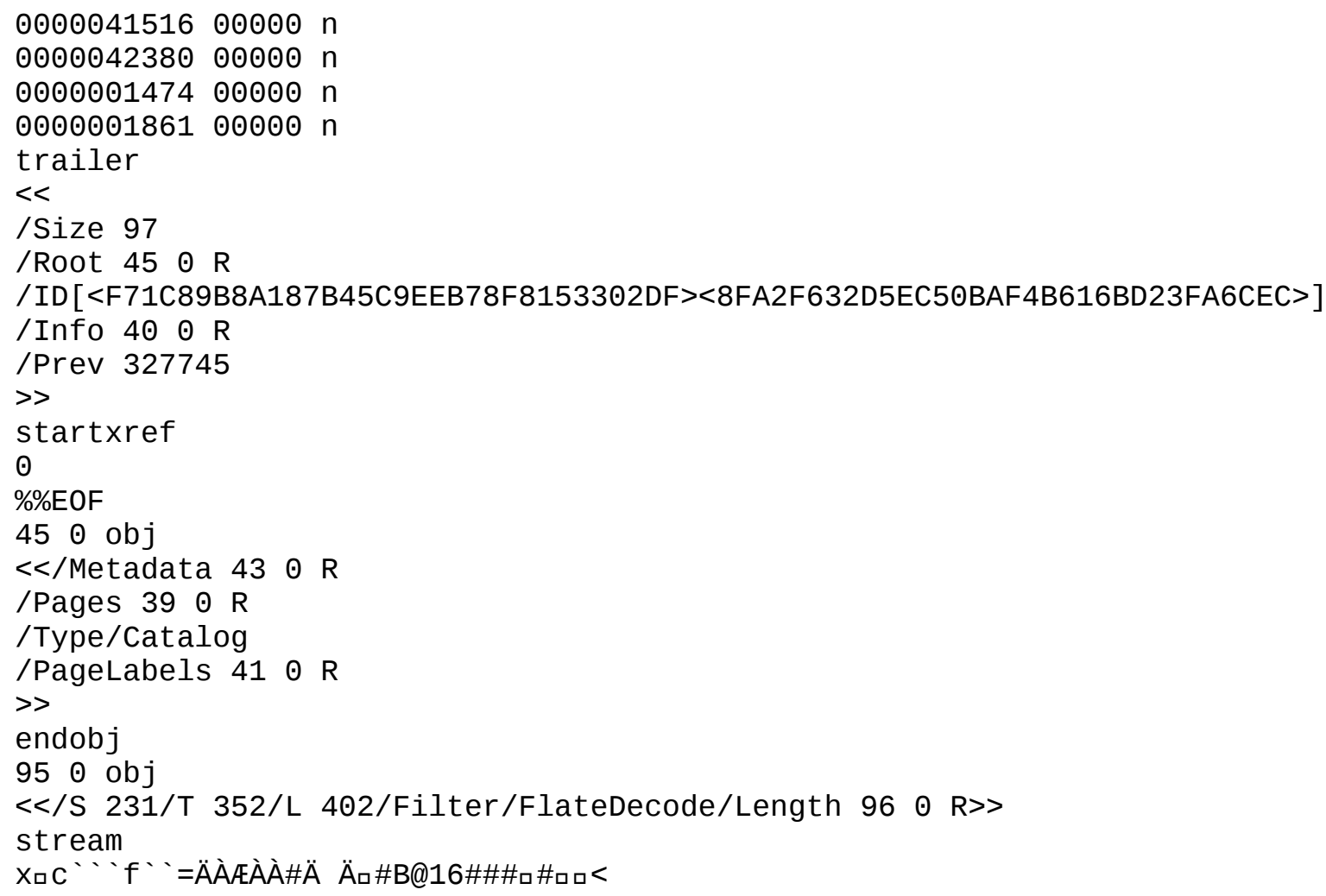




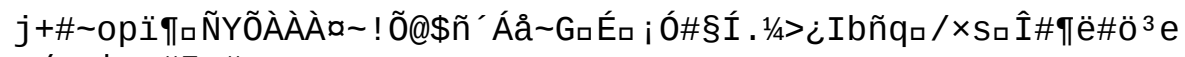
òuqma ${ }^{a} \ddot{i}$ i $X 9 v Q \ddot{I} f \wedge$

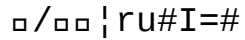

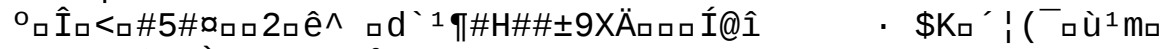

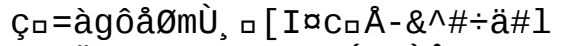

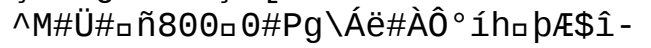

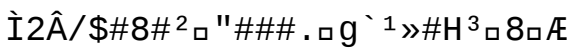




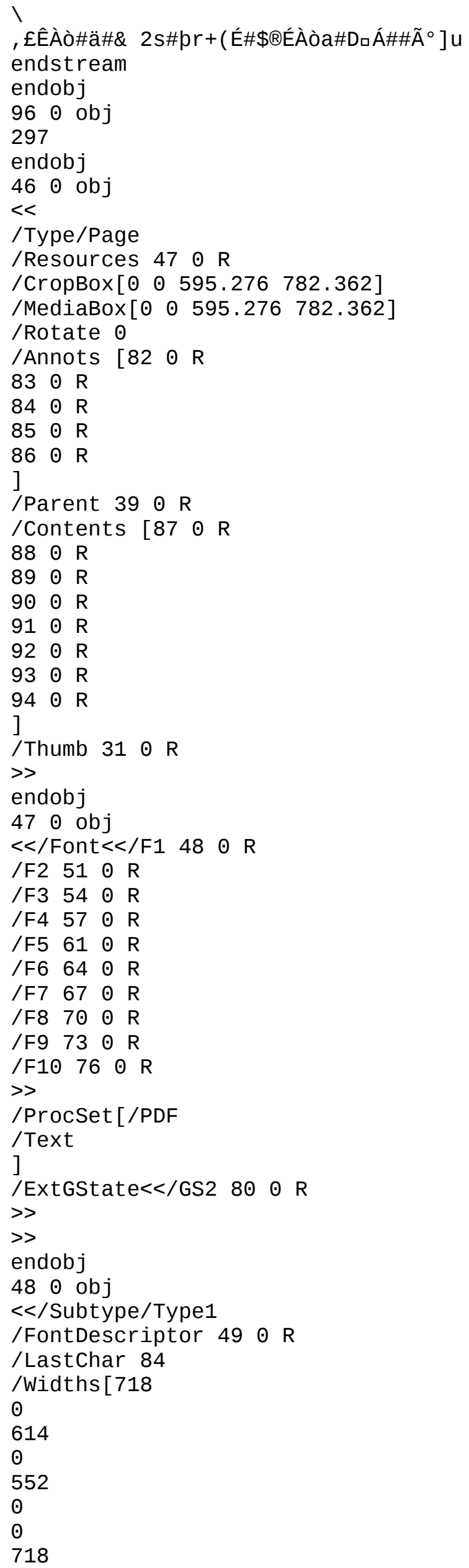




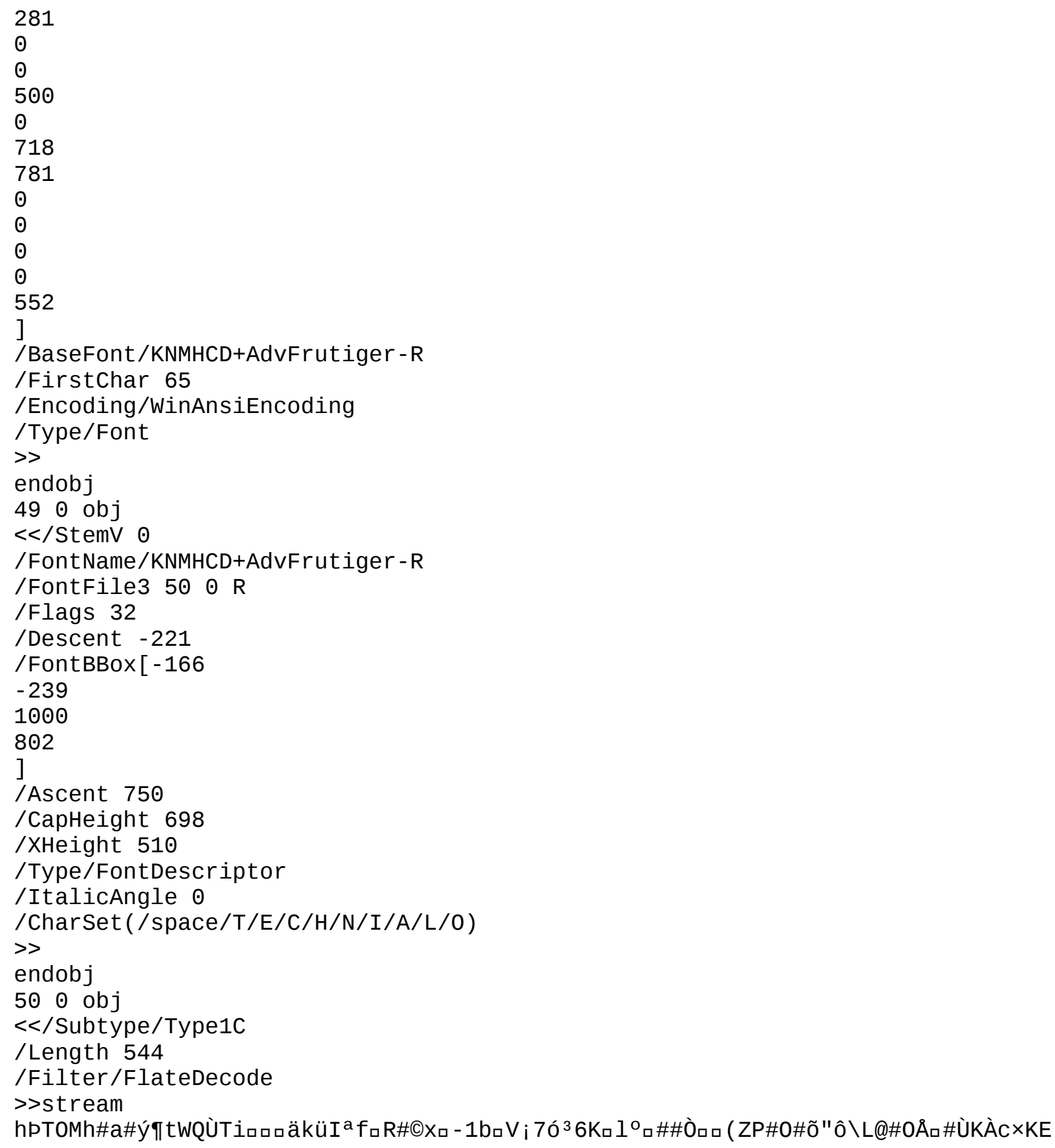




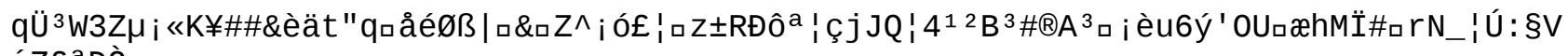
'ZE $\mathrm{D}$ DÒ

\#\&/Đ\¥\#0̃Ø\%ã\#kyc - a 9$] \cup$ 


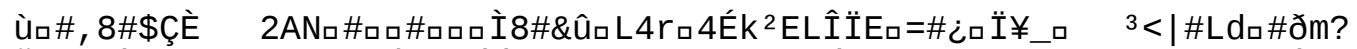

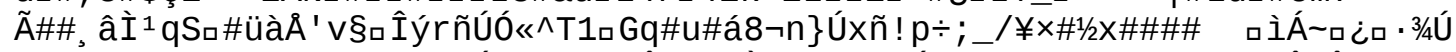

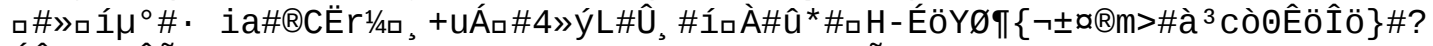

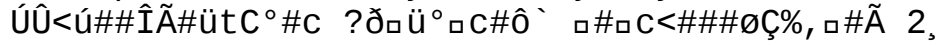


\%(à\#ĖXb\#A\#\# q\#n2\#a-gs

ÊธØİ॰Ú\{1̂?\# 
\#å\#\#x

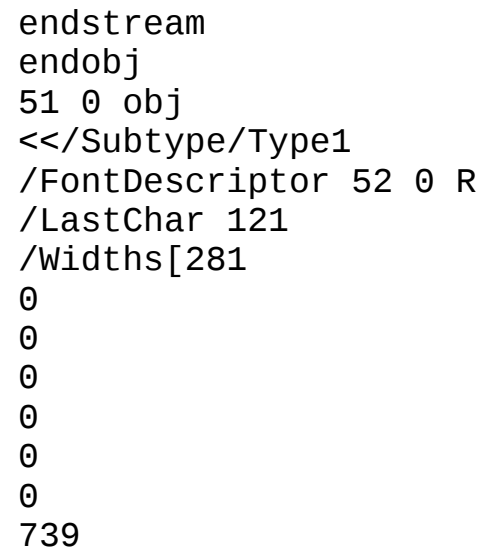


]

/BaseFont/KNMHCE+AdvCgse

/FirstChar 58

/Encoding/WinAnsiEncoding

/Type/Font

$>$

endobj

$52 \odot$ obj

$<</$ StemV 0

/FontName/KNMHCE+AdvCgse

/FontFile3 $53 \odot \mathrm{R}$

/Flags 32

/Descent - 228

/FontBBox [-197

$-218$

1000

791

]

/Ascent 734

/CapHeight 667

/XHeight 521

/Type/FontDescriptor

/ItalicAngle $\odot$

/CharSet (/space/R/e/f/r/n/c/V/a/l/u/A/d/v/i/s/o/colon/w/t/m/h/M/E/x/p/U/D/c/b/y)

$>$

endobj

$53 \odot$ obj

$<</$ Subtype/Type1C

/Length 2306

/Filter/FlateDecode

$>$ stream

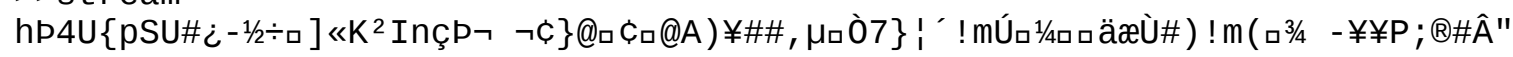




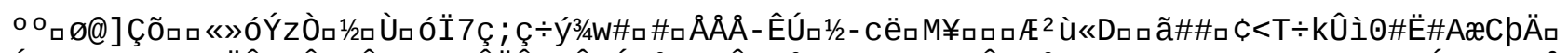

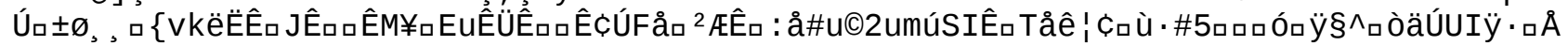
\#£९ì\# 
[\#ロ\%aØ* 


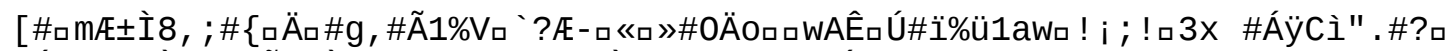

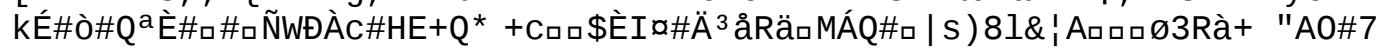

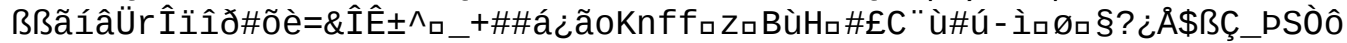

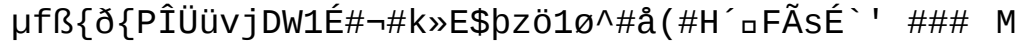




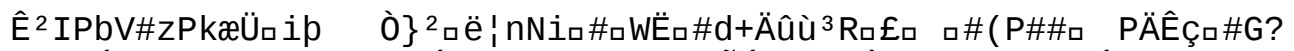

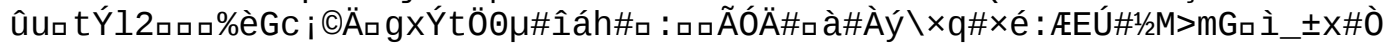

Àx ${ }^{21 / 2 ß \# n ̃ n}$

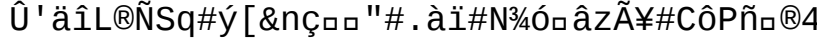

E\#War á 


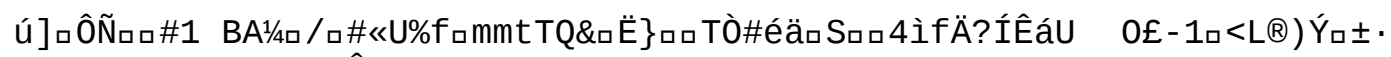

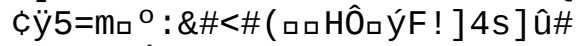

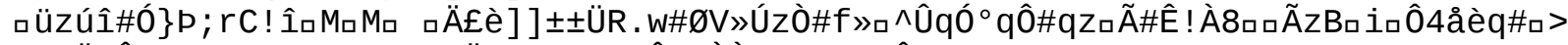

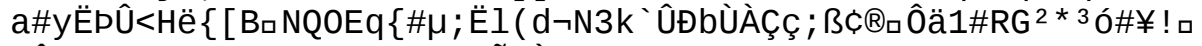

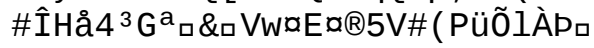




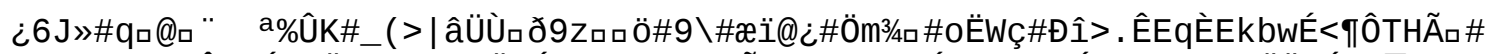

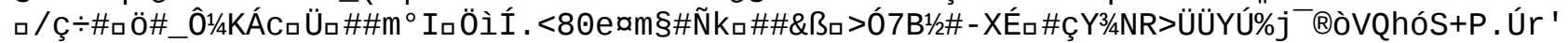

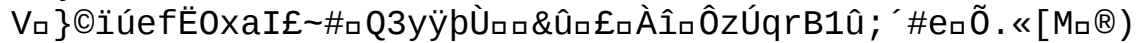




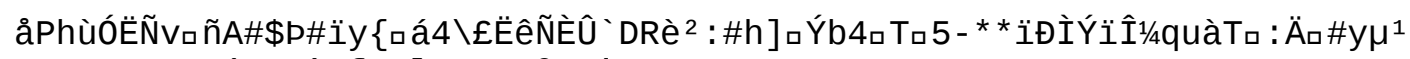

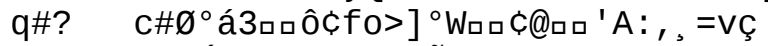

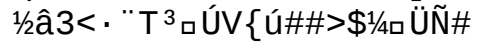

$\square Z^{\wedge} \square 0 ̈ \# \times u ̈ y ́ C o P o F e ̈ \uparrow\{\# 1$

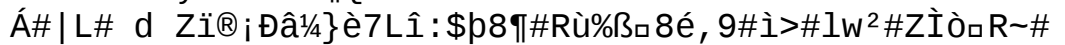

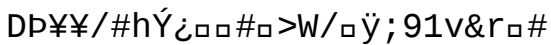

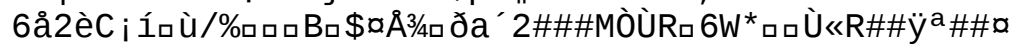

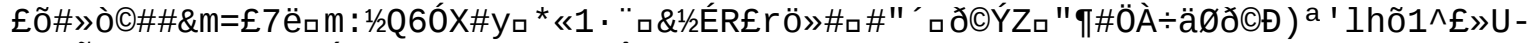

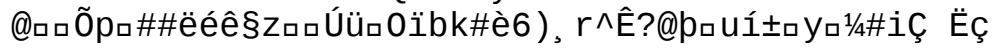




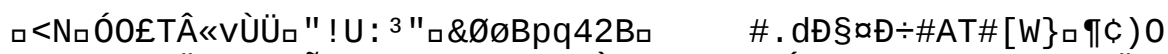

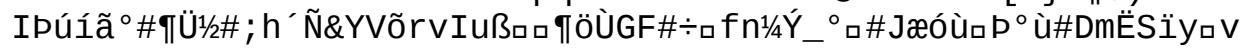




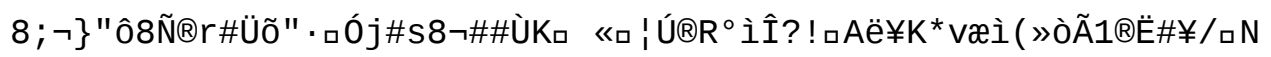




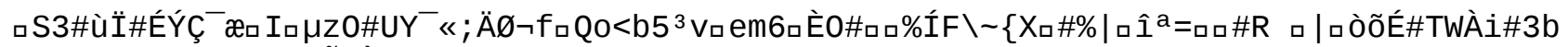

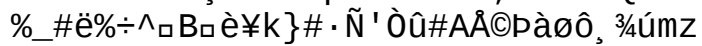




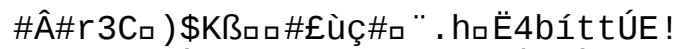

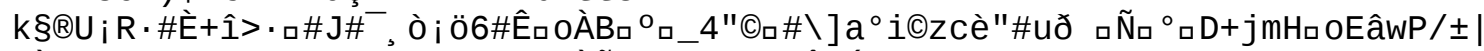

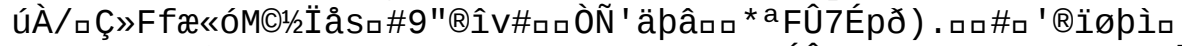

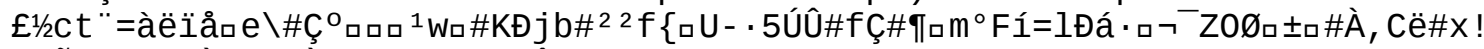

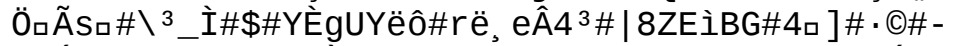

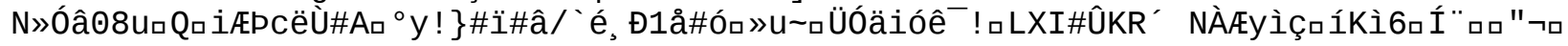

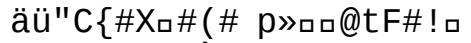

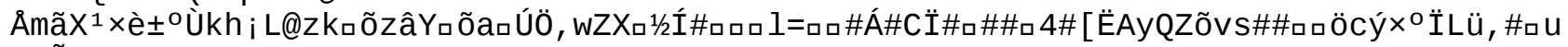
ôûÃ"1L-æç\$ÿ\#` $\# Z^{2} \square S$

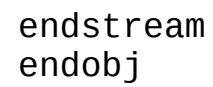



/BaseFont/KNMHDE+AdvCgs

/Firstchar 44

/Encoding/WinAnsiEncoding

/Type/Font

$>$

endobj

$55 \odot$ obj

$<</$ StemV 0

/FontName/KNMHDE+AdvCgs

/FontFile3 $56 \odot \mathrm{R}$

/Flags 32

/Descent - 225

/FontBBox [-166

$-218$

1000

760

]

/Ascent 732

/CapHeight 667

/XHeight 512

/Type/FontDescriptor

/ItalicAngle $\odot$

/

CharSet (/space/A/n/e/G/f/r/acute/one/comma/D/i/d/c/o/c/t/two/J/a/hyphen/P/B/u/h/ $\mathrm{T} / \mathrm{m} / \mathrm{l})$

$>>$

endobj

$56 \odot$ obj

$<$ /Subtype/Type1C

/Length 1864

/Filter/FlateDecode

>sstream

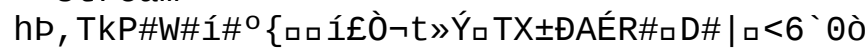

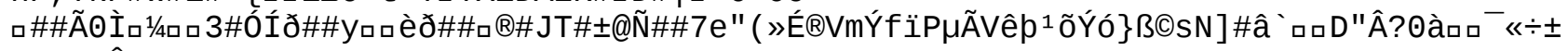

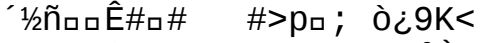

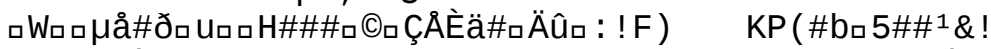

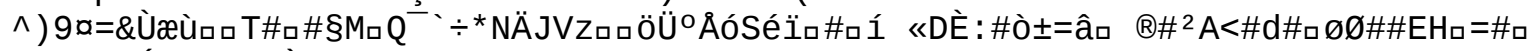
口EH. . ÜÉÆ\#q@P\#Ò\#Q $x_{\square W}$ 


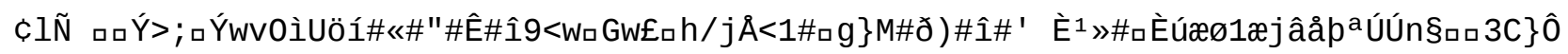
口\#\#ù 


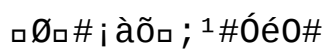




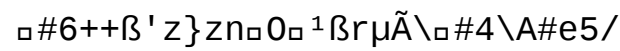

İð6plI3C口

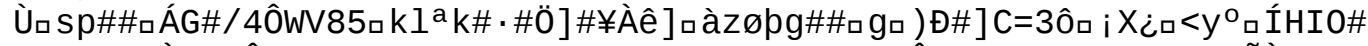

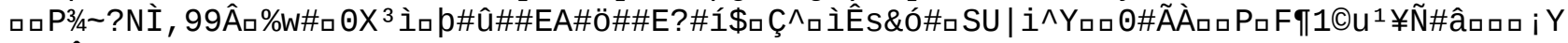
ØûıÂM 


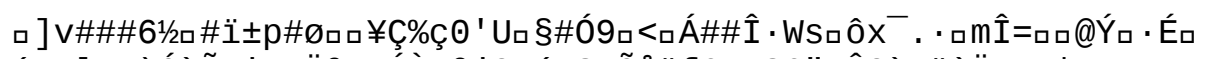

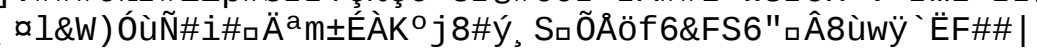




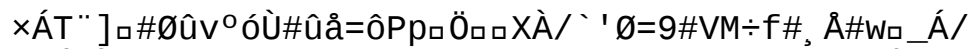

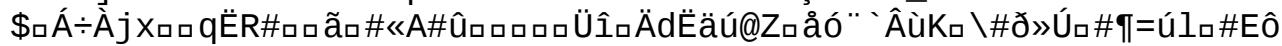

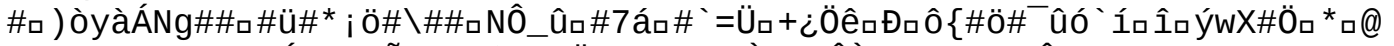

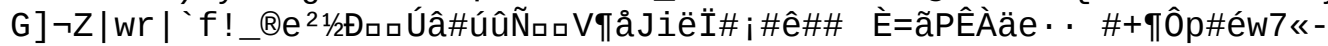

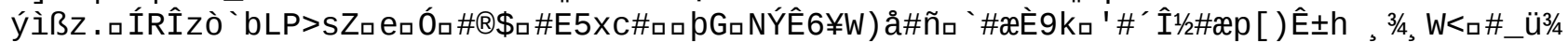

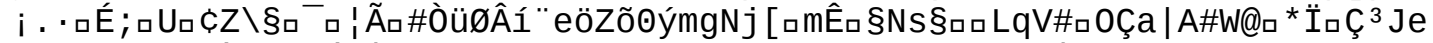

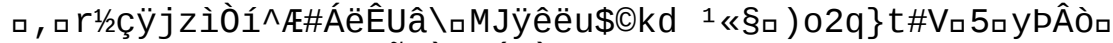

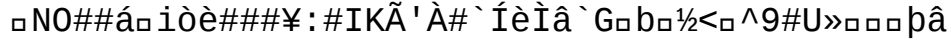




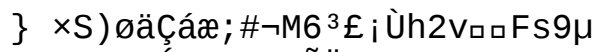

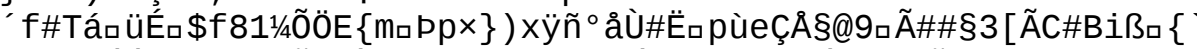

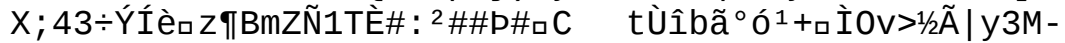

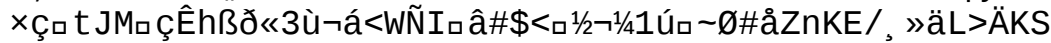

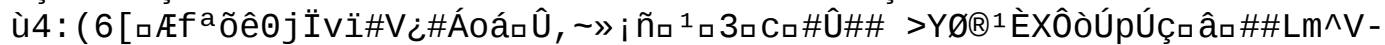

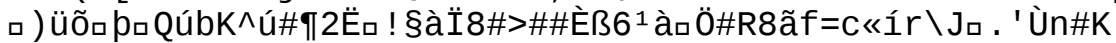

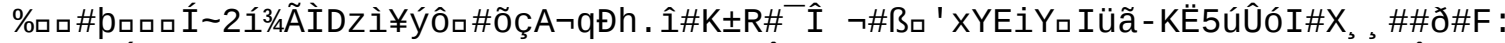

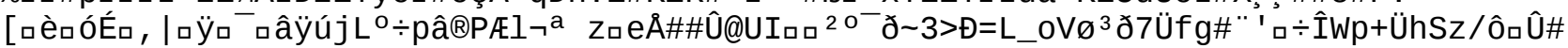
p 


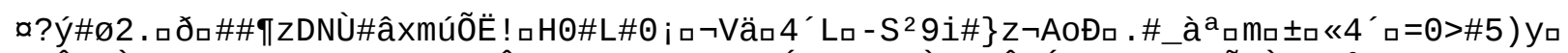

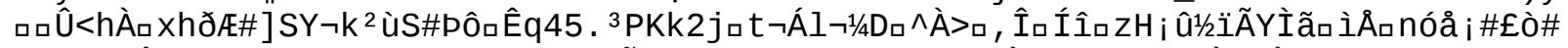

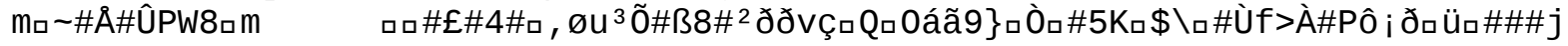

IL

endstream

endobj

$57 \odot$ obj

$<</$ Subtype/Type1

/FontDescriptor $58 \odot \mathrm{R}$

/LastChar 180

/Widths [458

0

218

$\odot$

$\odot$

0

$\odot$

$\odot$

$\odot$

0

260

260

0

$\odot$

260

260

260

260

520

520

520

520

520

520

520

520

520

520

260

260

○

520

0

$\odot$

833

625

572

583

625

489

479

604

635

239

0

0

458

812

645

666

552

0

552 
510

510
625
614

614

○

593

$\odot$

$\odot$

260

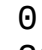

260

$\odot$

$\odot$

$\odot$

489

552

468

552

510

291

531

531

218

218

458
218

812

531

552

0

354

437

322

531

479

729

479

479

$\odot$

$\odot$

$\odot$

0

$\odot$

$\odot$

$\odot$

$\odot$

$\odot$

$\odot$

$\odot$

$\odot$

$\odot$

$\odot$

$\odot$

0

$\odot$

$\odot$

$\odot$

$\odot$

239 


\section{$\odot$}

0

0

500

0

$\odot$

$\odot$

$\odot$

$\odot$

$\odot$

$\odot$

$\odot$

0

218

0

$\odot$

$\odot$

0

$\odot$

$\odot$

$\odot$

$\odot$

$\odot$

$\odot$

$\odot$

$\odot$

260

0

$\odot$

0

$\odot$

$\odot$

218

]

/BaseFont/KNMHDF+AdvCgsl

/Firstchar 30

/Encoding $60 \odot \mathrm{R}$

/Type/Font

$>>$

endobj

580 obj

$<</$ StemV 0

/FontName/KNMHDF+AdvCgsl

/FontFile3 $59 \odot \mathrm{R}$

/Flags 32

/Descent - 224

/FontBBox [-156

$-218$

1000

739

]

/Ascent 731

/CapHeight 667

/XHeight 508

/Type/FontDescriptor

/ItalicAngle 0

/

CharSet (/space/one/D/e/p/a/r/t/m/n/o/f/c/l/i/c/s/s/d/two/U/M/R/eight/P/h/y/g/T/x /E/acute/I/N/A/comma/V/u/F/B/endash/fi/v/G/b/period/three/zero/seven/six/hyphen/ colon/at/0/slash/j/nine/five/X/four/parenleft/parenright/H/L/w/bracketleft/brack etright/equal/k/quoteright/semicolon)

$>$

endobj 
$59 \odot$ obj

<</Subtype/Type1C

/Length 4517

/Filter/FlateDecode

>sstream

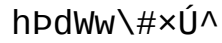




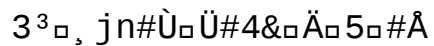

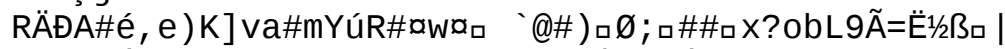

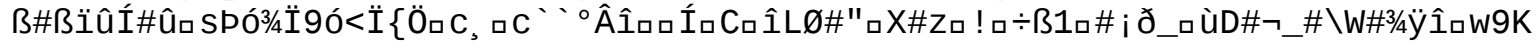





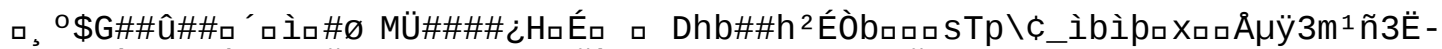

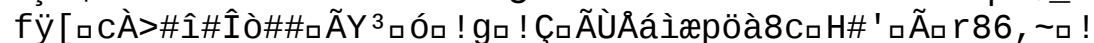

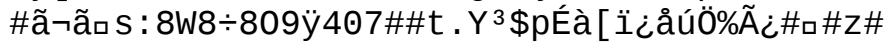

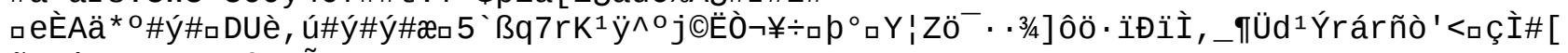

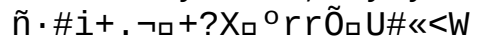

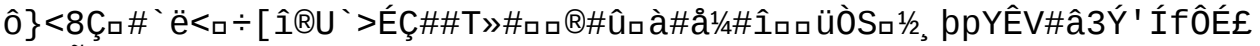

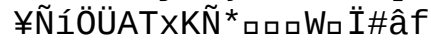




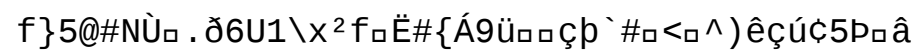

On\#|»l\#ä\#\#dı\$lc\$\#

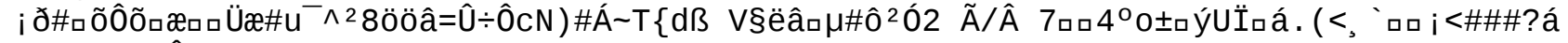
l\#X\# \#×ZÂwa 


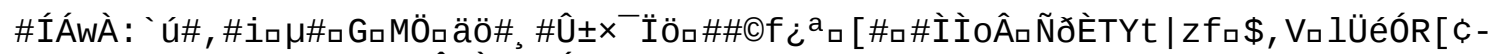

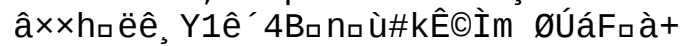




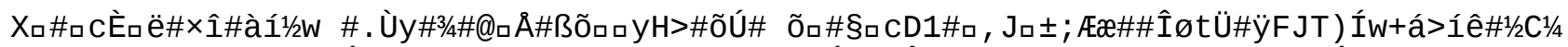

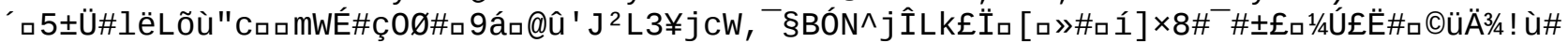

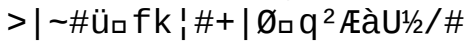


$\mathrm{nB} \ll+\grave{A}$

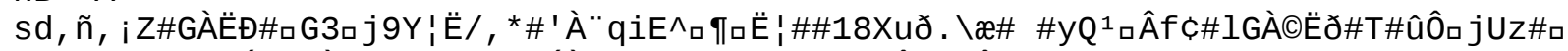

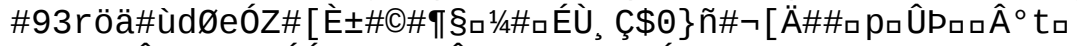

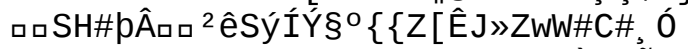

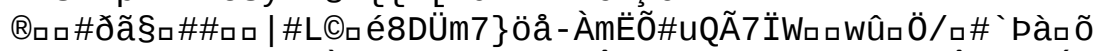

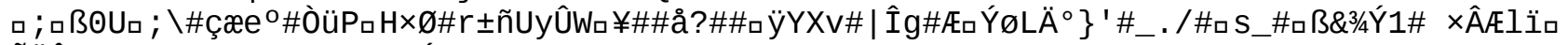
$\tilde{A} \ddot{I} \hat{A}\left(\square \square X^{1} m^{\prime} L \sim \emptyset \# P \# \emptyset W \cdot \# \square U ́\right.$ 


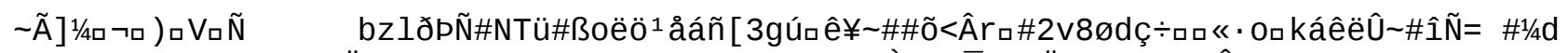

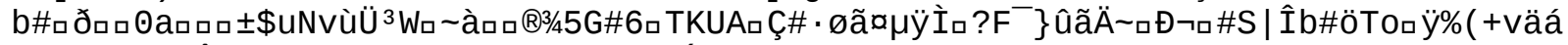

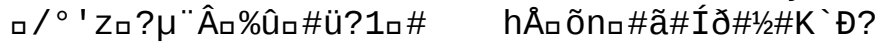

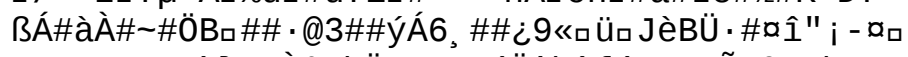
$\left.{ }^{\circ} \# v a ̀\right\}$ ö ${ }^{3} \#$ bäJv×Zïýö ' bê $\left\{e ́<M \wedge \# \tilde{N} \#{ }^{a}\right.$ ChV<R\#

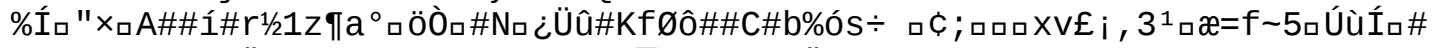

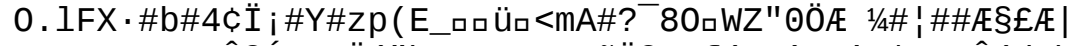

Z“" JI

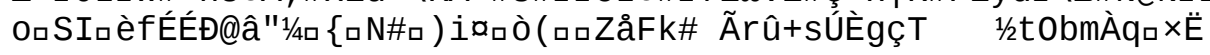




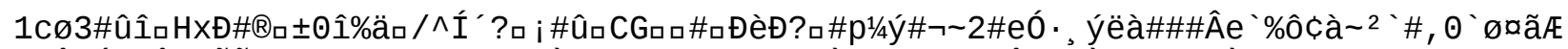

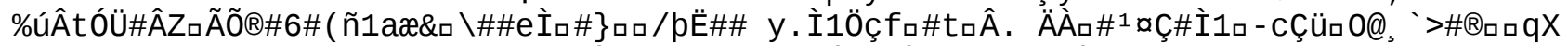

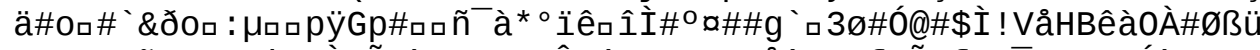

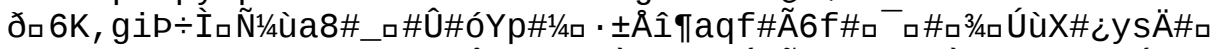

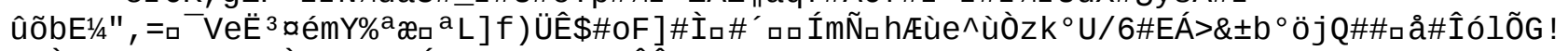

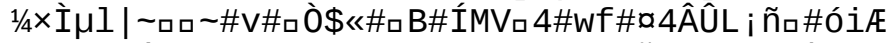

Í\#QqgõVı\#Itqü:\#ロĐK\#\#à1\#<ÃAä\#ロp\#áÎ\# 1+ød_á\{À

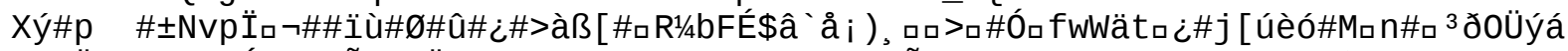

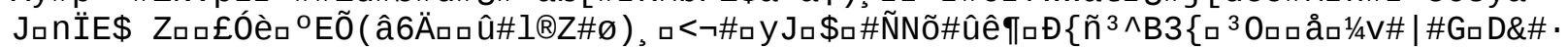

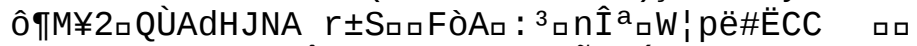
口

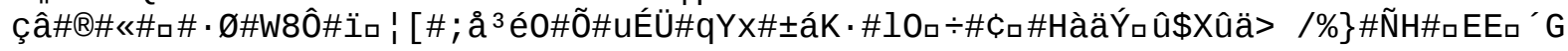
£\#SJ ${ }^{1} \mathrm{C}$

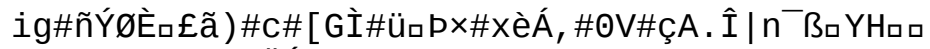

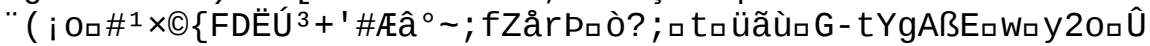

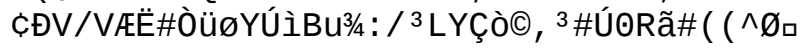




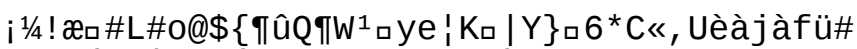

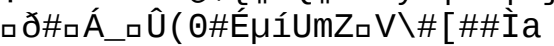

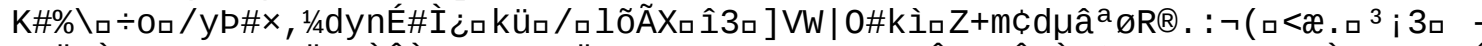

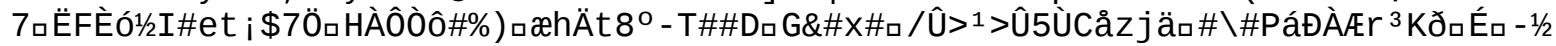

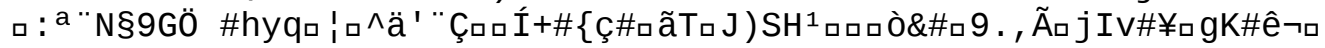


$\mu$

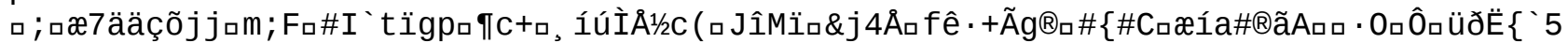

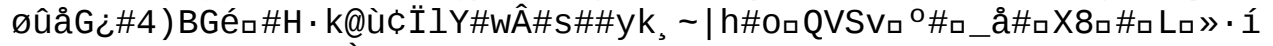

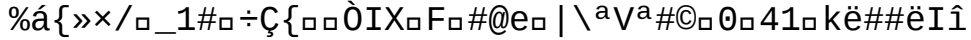

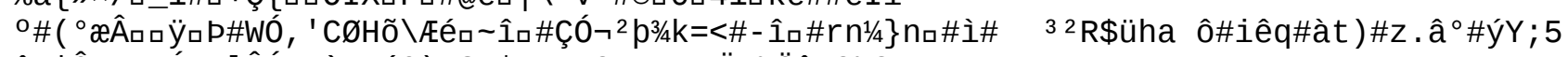

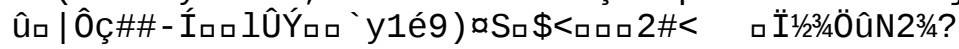




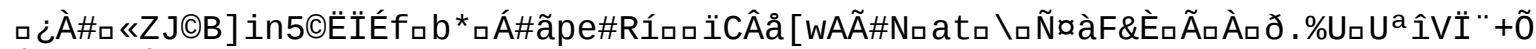
Êôr ${ }^{1} \bigvee \hat{A}-\square C ̧ \S ! \# G \backslash \# \#, a^{\circ} F_{\square}$

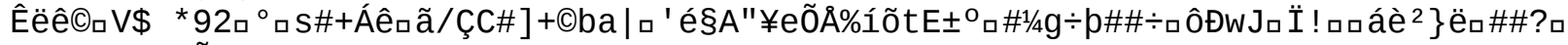

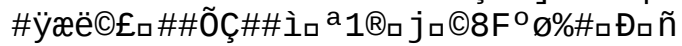

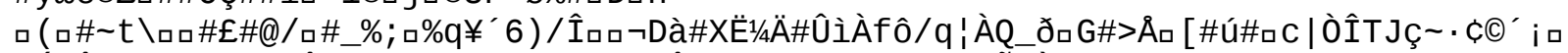

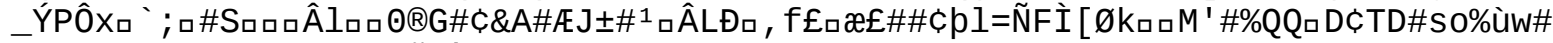

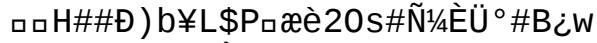

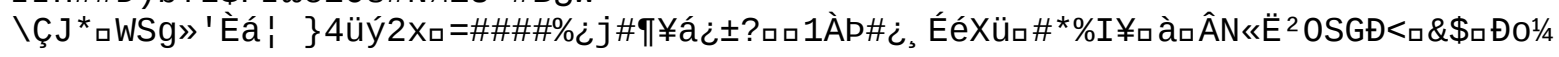




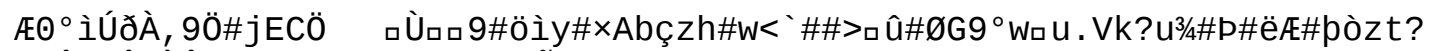

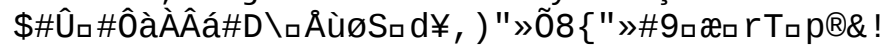

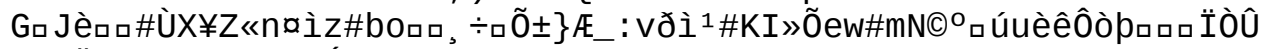

üÄäâ

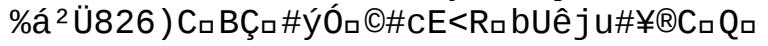




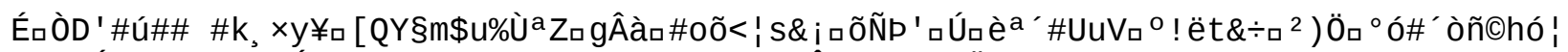

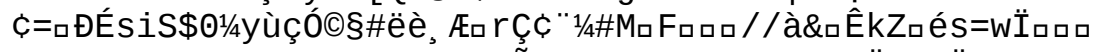

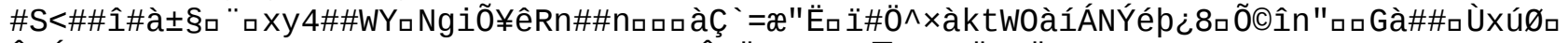

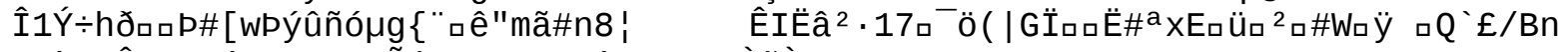

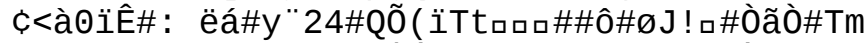

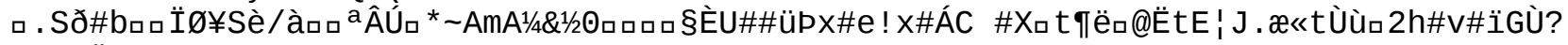
\#éĐ\#Ö $\cdot P^{\circ}$

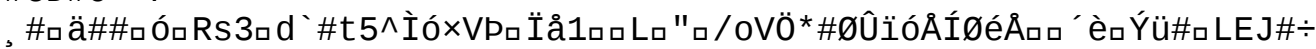

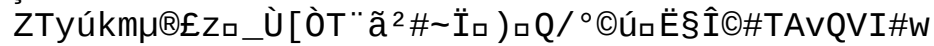




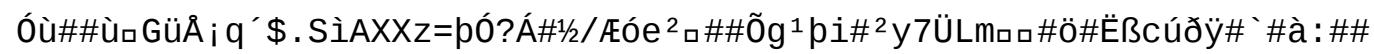

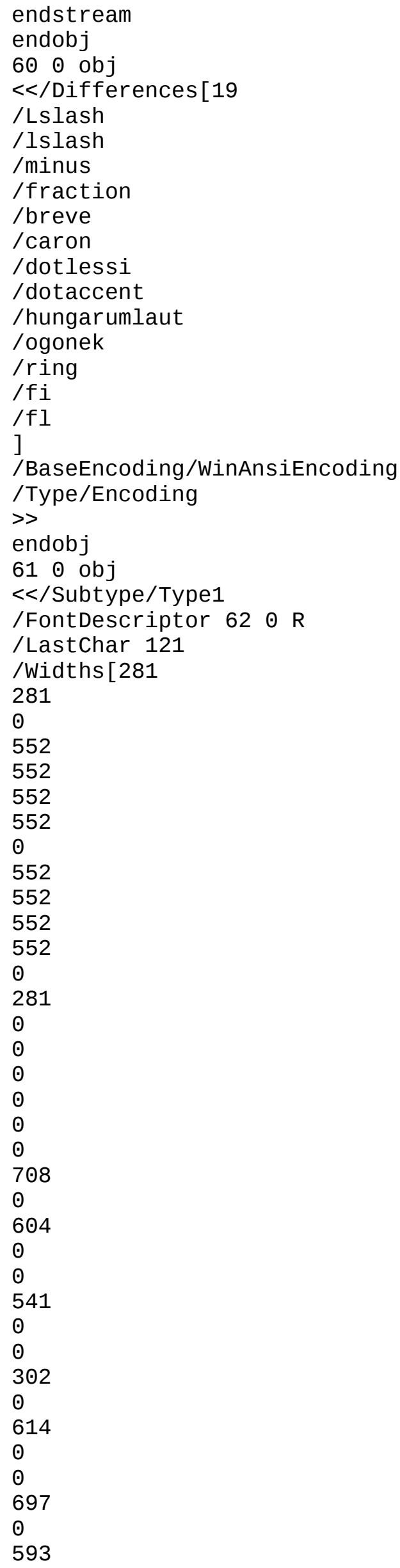


]

/BaseFont/KNMHDG+AdvCgsb

/Firstchar 45

/Encoding/WinAnsiEncoding

/Type/Font

$>$

endobj

$62 \odot$ obj

$<</$ StemV $\odot$

/FontName/KNMHDG+AdvCgsb

/FontFile3 $63 \odot \mathrm{R}$

/Flags 32

/Descent - 226

/FontBBox [-177

$-218$

1000

781

]

/Ascent 733

/CapHeight 667

/XHeight 517

/Type/FontDescriptor

/ItalicAngle $\odot$ 
CharSet (/space/K/e/y/W/o/r/d/s/C/p/n/c/A/b/t/a/colon/V/i/l/P/h/g/I/S/N/zero/two/ seven/five/hyphen/six/three/eight/T/one/period/F/u/k/w/m/R/f)

$>$

endobj

$63 \odot$ obj

$<</$ Subtype/Type1C

/Length 3283

/Filter/FlateDecode

>sstream

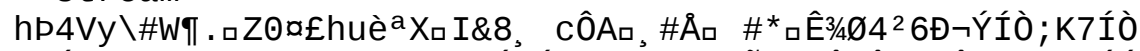

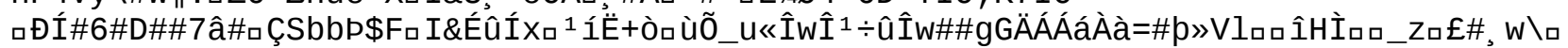

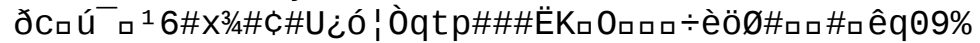

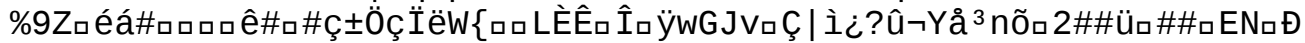

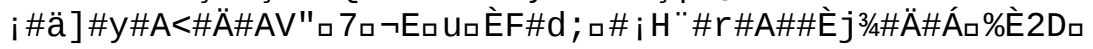




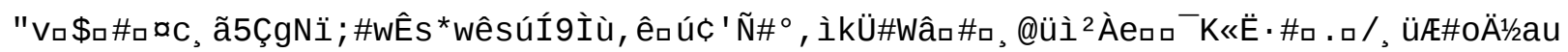
$\% \backslash \# \varnothing$

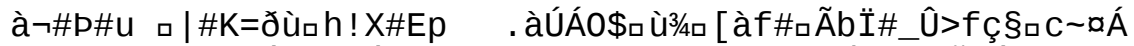

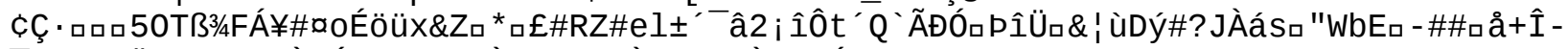

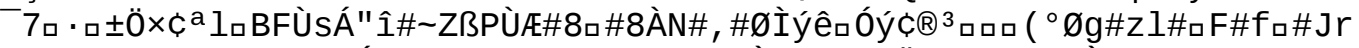

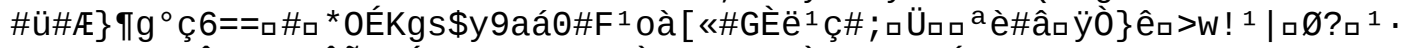

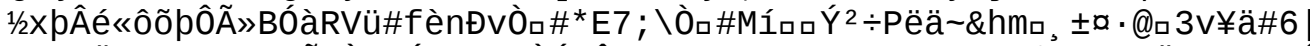

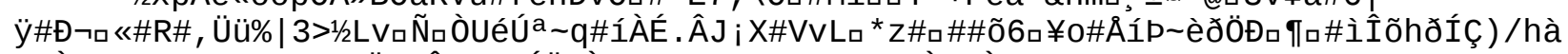

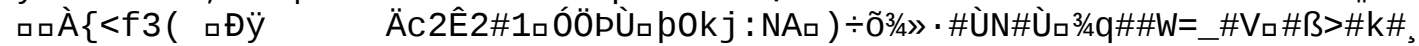

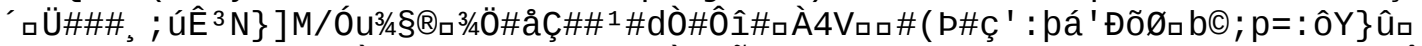

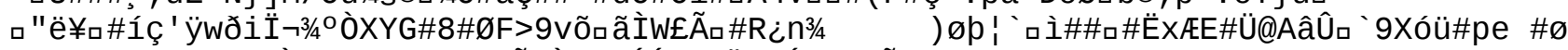

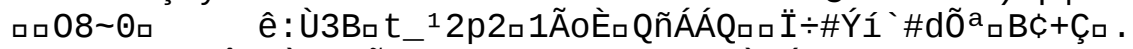

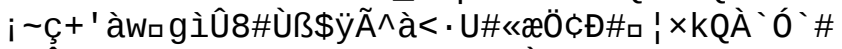

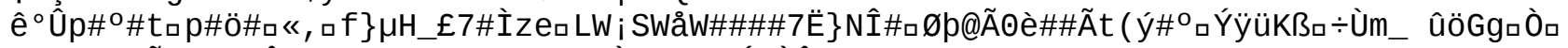

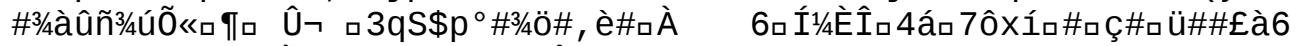

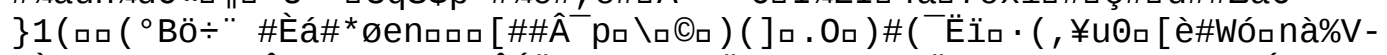

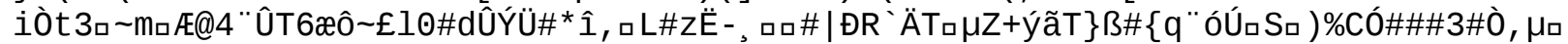

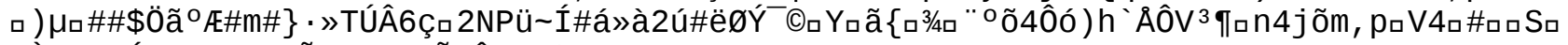

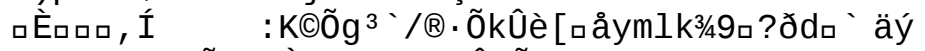

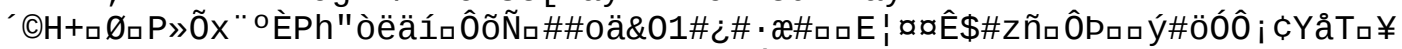

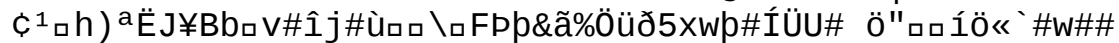




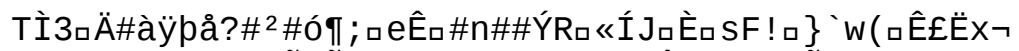

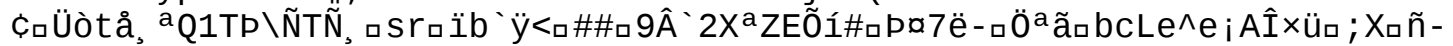

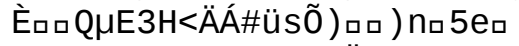

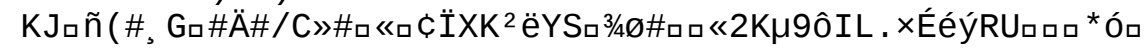
$\mathrm{dD}^{\circ} \mathrm{a}\left(>_{\square Z} \mathrm{Z} \mathrm{I} \ddot{\mathrm{I}} \# . \mathrm{I}_{\text {_\# }}\right.$ 


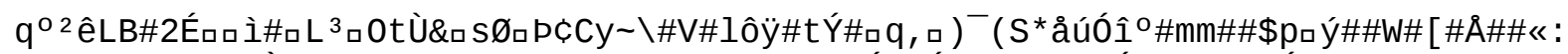

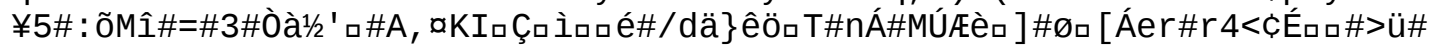




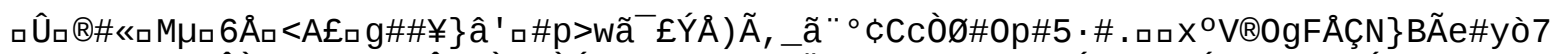

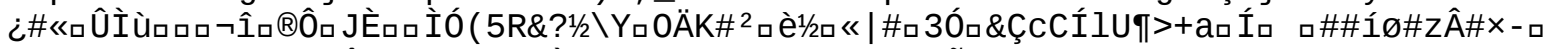

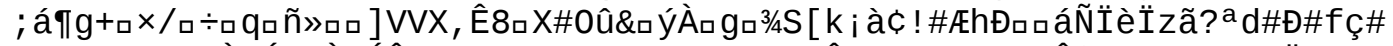

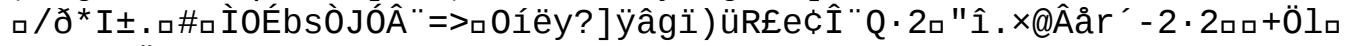

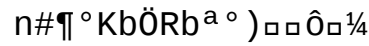




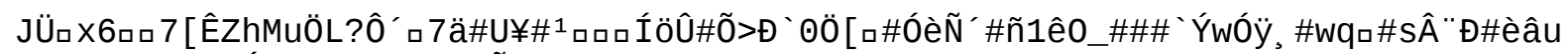

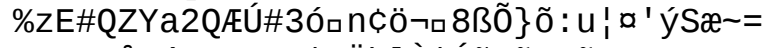

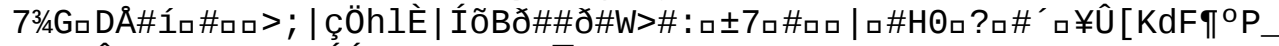

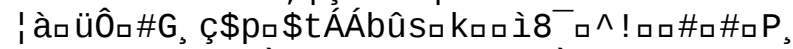

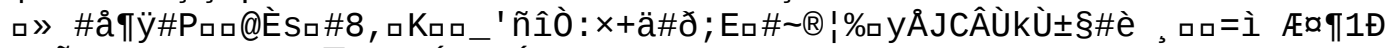

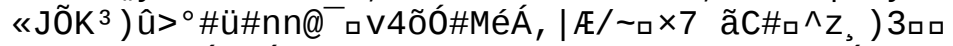

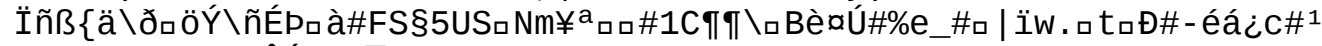

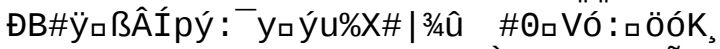

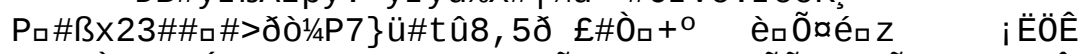

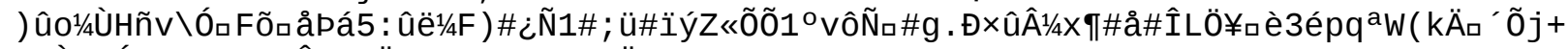

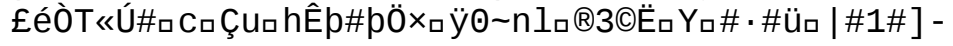




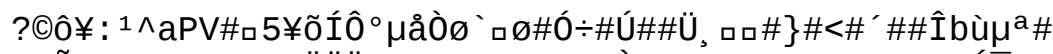

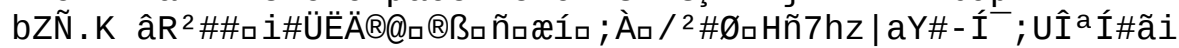

èıðg (ÄuÀ\#

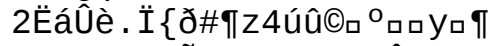

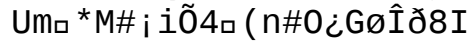




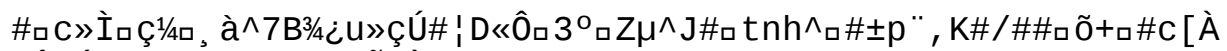

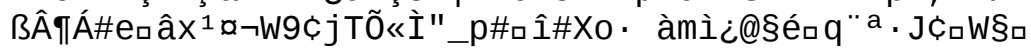




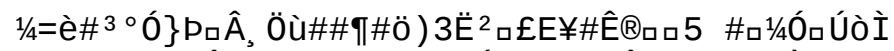

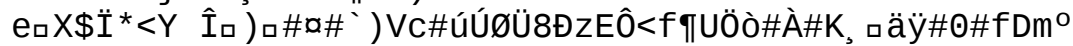

endstream

$64 \odot$ obj

$<</$ Subtype/Type1

/FontDescriptor $65 \odot \mathrm{R}$

/LastChar 116

/Widths [552

0

$\odot$

$\odot$

$\odot$

$\odot$

$\odot$

$\odot$

0

$\odot$

$\odot$

$\odot$

552

666

0

$\odot$

$\odot$

○

614

0

$\odot$

$\odot$

$\odot$

$\odot$

0

0

$\odot$

$\odot$

0

541

0

0

0

500

0

0

531

218

0

0

218

○

531

552

0

$\odot$

0

$\odot$

322

]

/BaseFont/KNMHDH+AdvCgsli

/FirstChar 67

/Encoding/WinAnsiEncoding

/Type/Font 
$>$

endobj

$65 \odot$ obj

$<</$ StemV 0

/FontName/KNMHDH+AdvCgsli

/FontFile3 $66 \odot \mathrm{R}$

/Flags 32

/Descent - 224

/FontBBox [-208

$-218$

1000

739

]

/Ascent 731

/CapHeight 667

/XHeight 508

/Type/FontDescriptor

ItalicAngle 0

/CharSet (/space/Q/V/e/t/c/l/i/n/P/a/h/o)

$>$

endobj

$66 \odot$ obj

$<</$ Subtype/Type1C

/Length 1046

/Filter/FlateDecode

>sstream

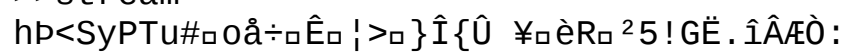




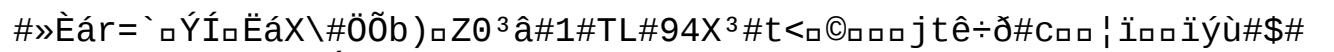

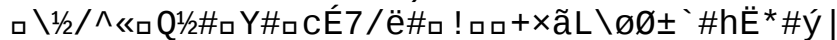

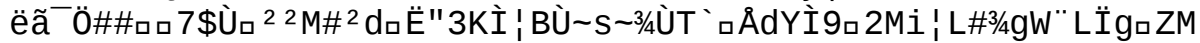

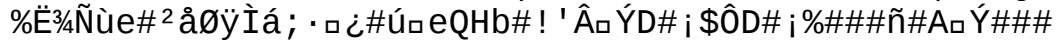

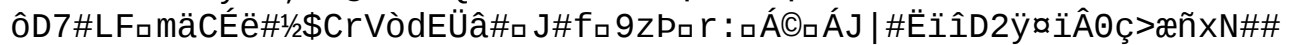


R\%\#KãıMZ?ZW1/2mp7Åyû?í9\}\&ðëp1ßUfî\#« ${ }^{\circ}{ } \#^{3}$ u\#núoÝ

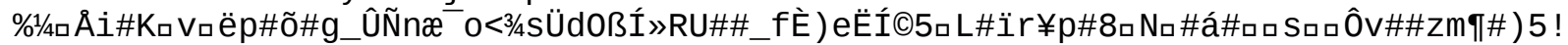

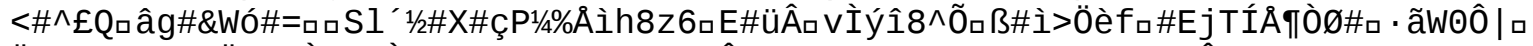

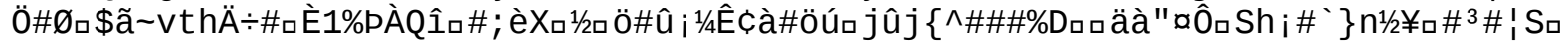




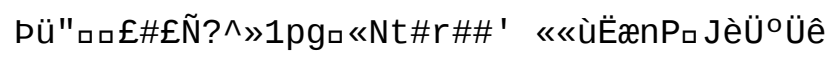




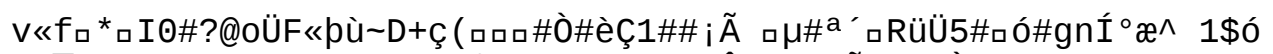

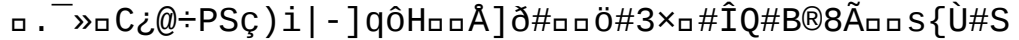

[RVYQ13/4ם

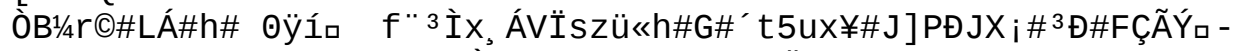

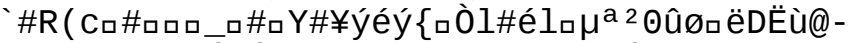

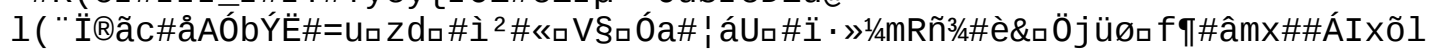




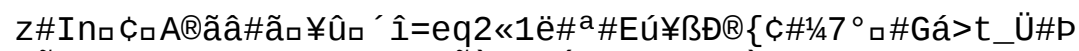

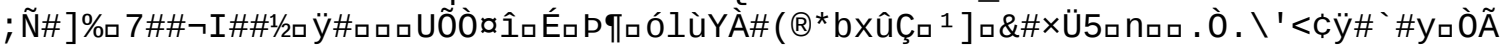

endstream
endobj

670 obj

$<</$ Subtype/Type1

/FontDescriptor $68 \odot \mathrm{R}$

/LastChar 180

/Widths [614

614

281

0

0

0

0

1000

0

0

385

385

0

$\odot$

281

385

281

385

552

552

552

552

552

552

552

552

552

552

281

281

0

0

0

500

$\odot$

718

666

666

718

614

552

718

718

333

447

718

552

947

718

760

552

0

666

552

552 

/BaseFont/KNMHDI+AdvMeridien-R

/Firstchar 30

/Encoding $60 \odot \mathrm{R}$

/Type/Font

$>>$

endobj

$68 \odot$ obj

$<</$ StemV $\odot$

/FontName/KNMHDI+AdVMeridien-R

/FontFile3 $69 \odot \mathrm{R}$

/Flags 34

/Descent - 217

/FontBBox [-145

$-208$

1000

729

]

/Ascent 698

/CapHeight 634

/XHeight 460

/Type/FontDescriptor

ItalicAngle $\odot$

I

CharSet (/space/I/n/t/e/r/a/i/o/l/c/m/d/s/f/v/h/b/y/u/p/comma/g/B/x/endash/c/w/pe $\mathrm{riod} / \mathrm{U} / \mathrm{W} / \mathrm{R} / \mathrm{V} / \mathrm{A} / \mathrm{M} / \mathrm{E} / \mathrm{F} / \mathrm{parenleft/nine/zero/percent/fi/bracketleft/bracketright/par}$ enright/four/semicolon/hyphen/D/T/k/fl/z/H/colon/slash/question/six/three/j/one/ $\mathrm{L} / \mathrm{S} /$ seven/q/two/eight/G/P/N/quoteleft/quoteright/five/K/X/acute/J/Y/O)

$>$

endobj

$69 \odot$ obj

$<<$ Subtype/Type1C 


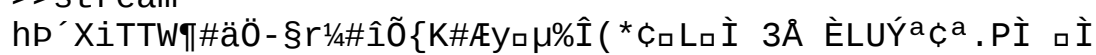

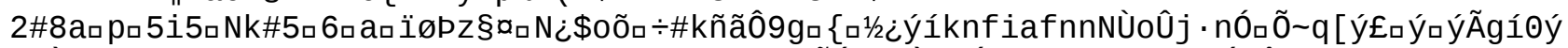

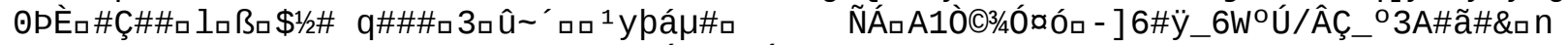

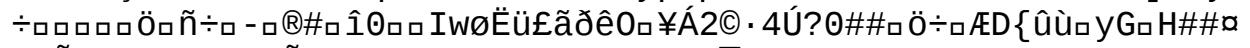

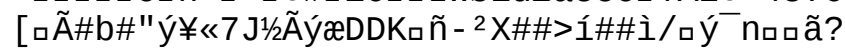

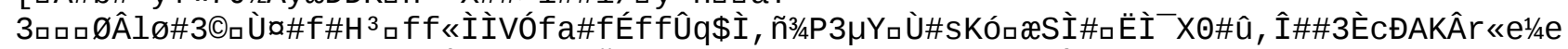

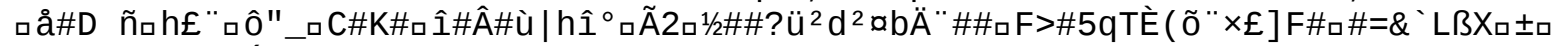

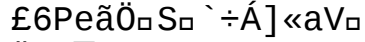

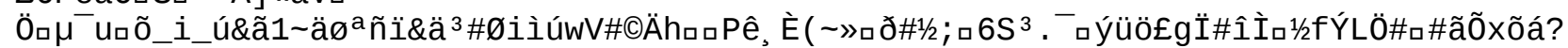

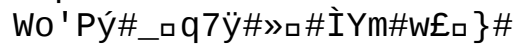

$\square \square \frac{1}{2}{ }^{*}$ $\wedge 6 . ¥ J E \odot$ 
æ\#EeÙロ\#०* ) '" *> - *ロ “” 
2

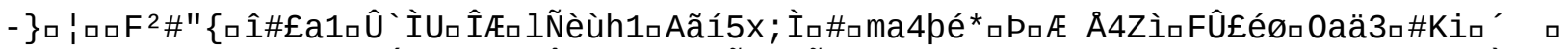

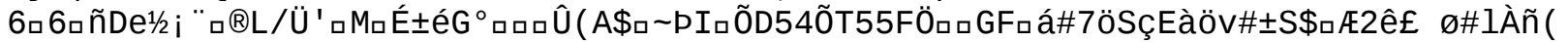


zýäÜB\#ロ\#1̂CEl8\#\#\#\#ú“\#\} 
\#Àö"8Á4ロê 
$+\oplus$

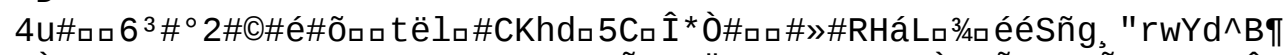

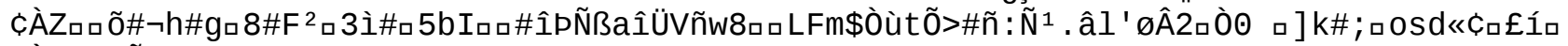
$1 / 2$ Iே »1ิSÑpD 1

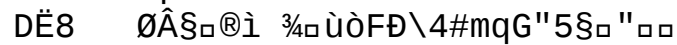

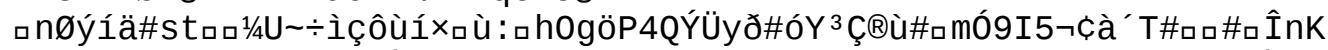

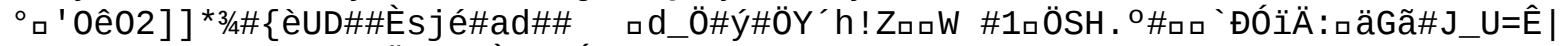

\#\#Ph\#ロロlW i ) \>Aùa\#Ä_\#\#\#İ§>Fóc\#5ロ\#ף 


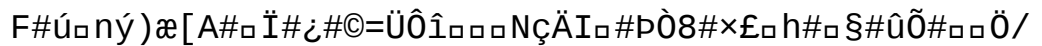

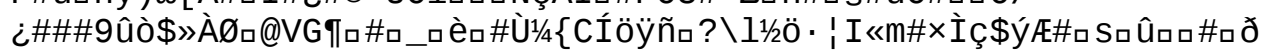
$\#$ a

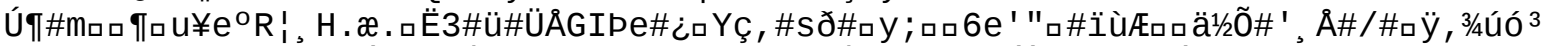

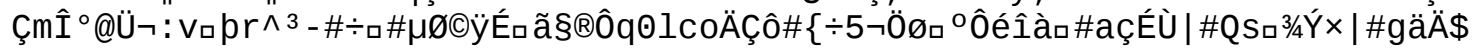
$\square \operatorname{Pa} \odot$

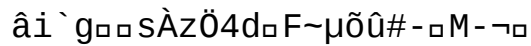




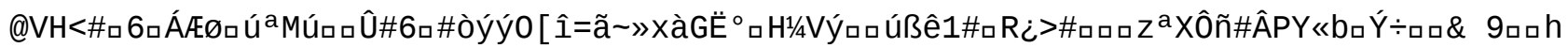
:WDT\# 


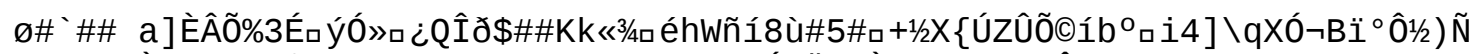

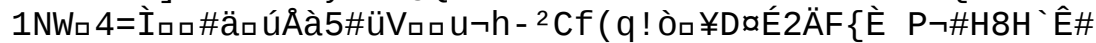

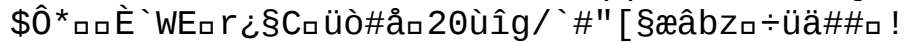

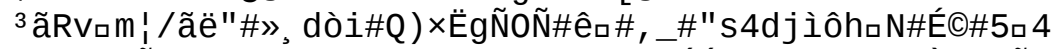

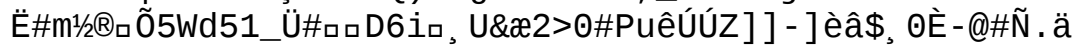

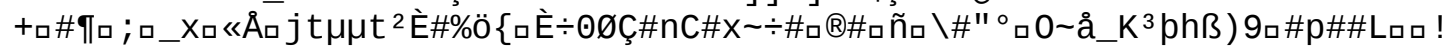


Ùýa $r+i ̀ 2 \_\# ? \_A \varnothing u ̂ / N E ́$ "n=\#@+TT 


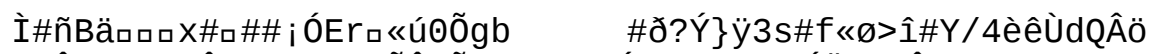

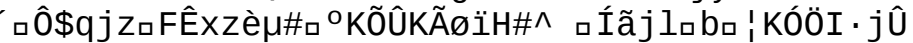

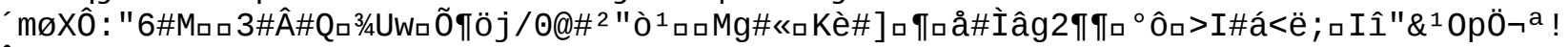

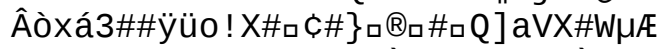

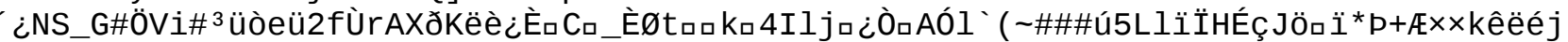

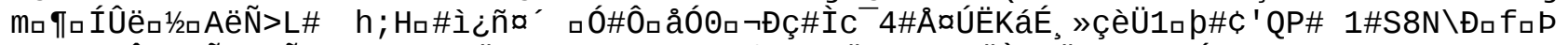

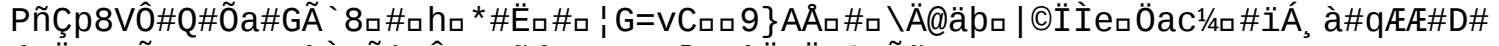

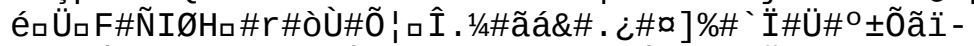

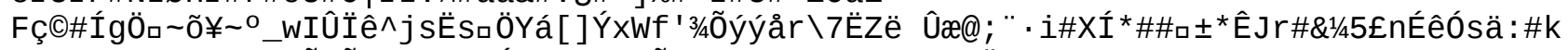

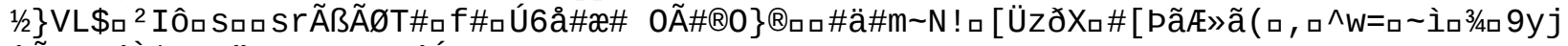
“Ñ9a\#éÙ*mRa"\#\#ロëVa\#3/4íÍç jsñก̃̃\#>

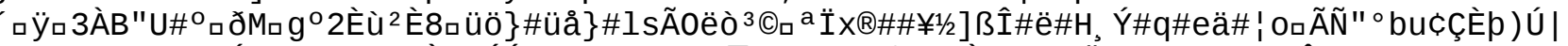

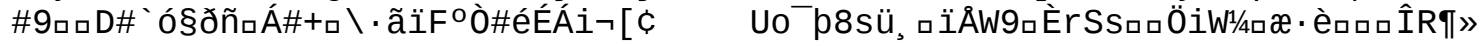




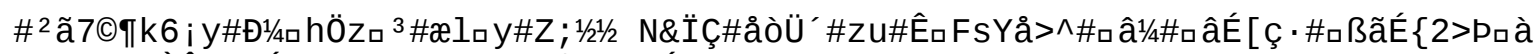

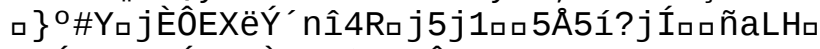

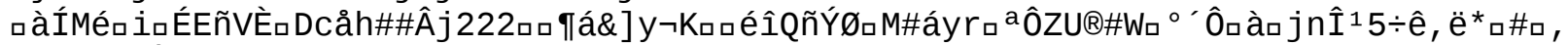

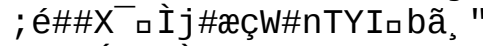

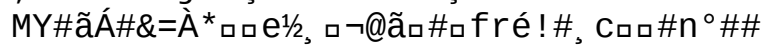

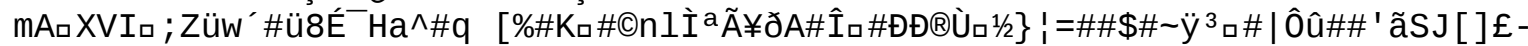

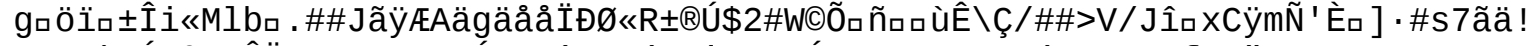

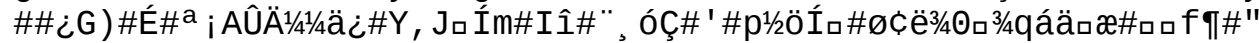

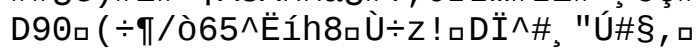

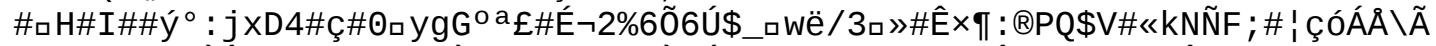

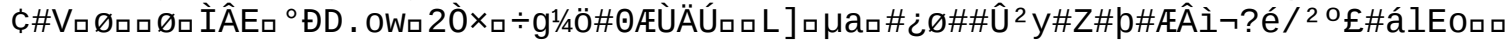




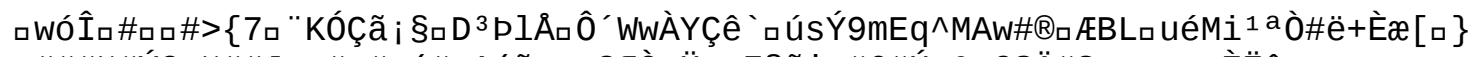

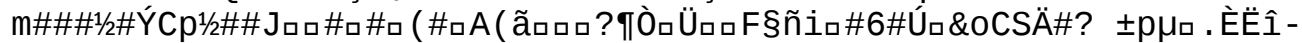

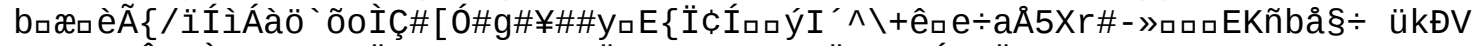

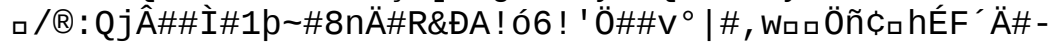

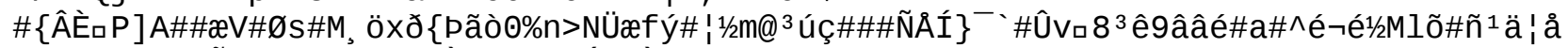

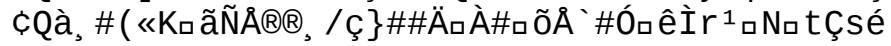




\section{ÖñËÍß\#aKZ}

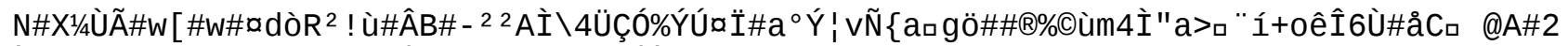

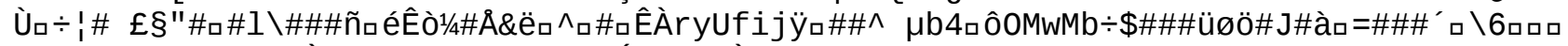

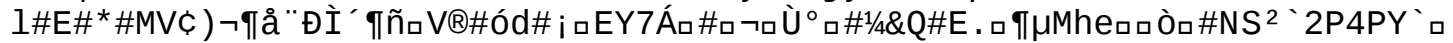

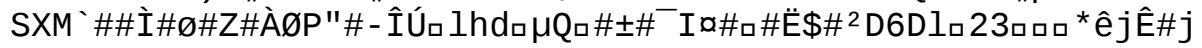




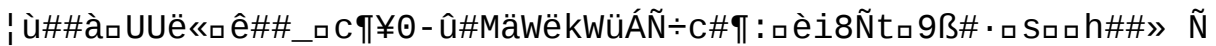

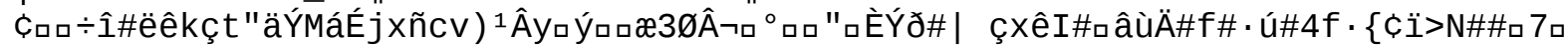

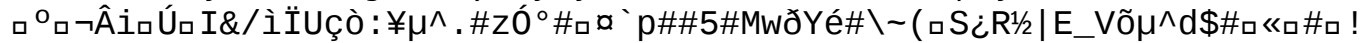

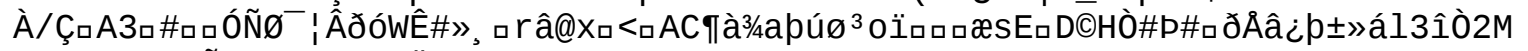

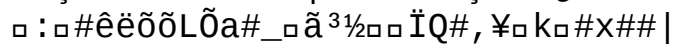

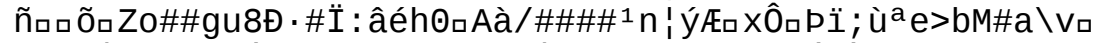

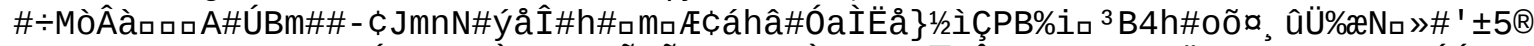

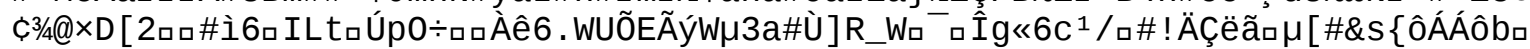

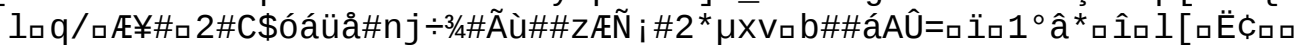

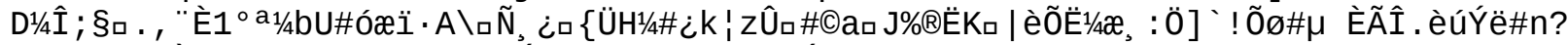

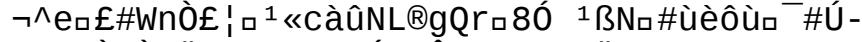

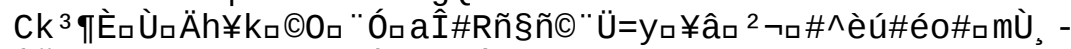

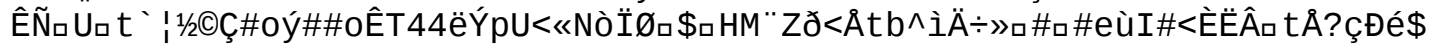

c\&3[ıüi40॰\#+gw\$à \x;

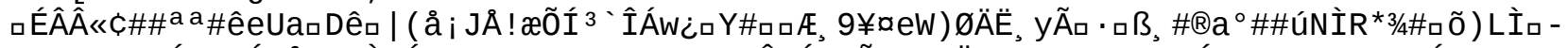

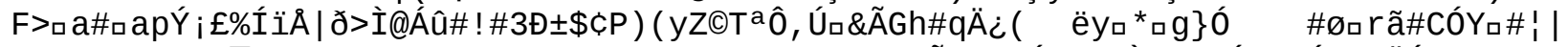

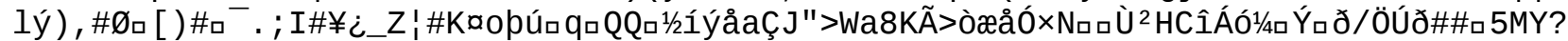

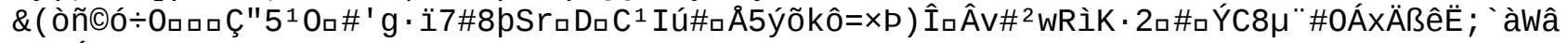
口R\#ÝM4j\$ãıé

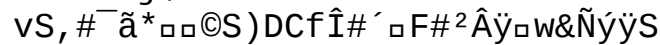

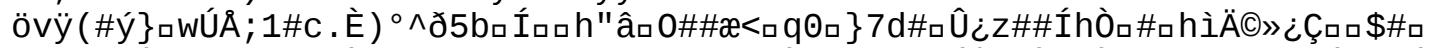

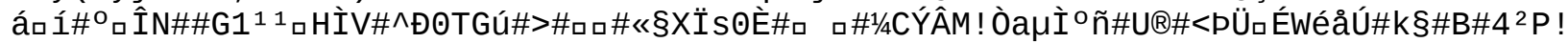

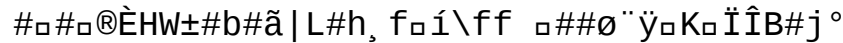

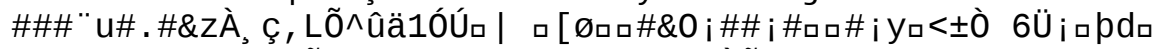

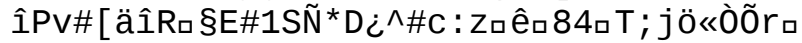




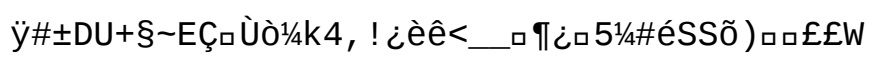




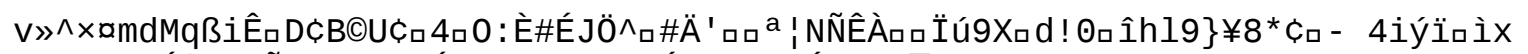

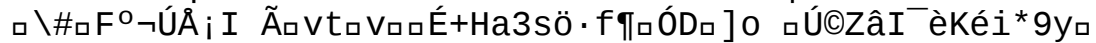

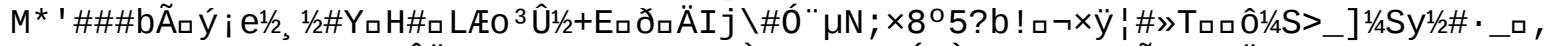

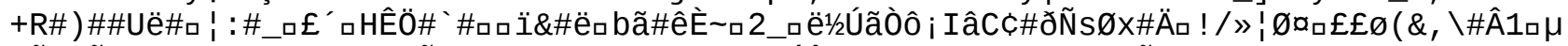

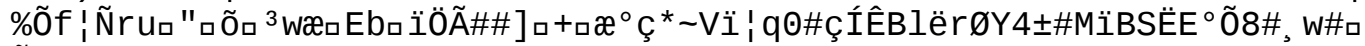

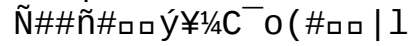




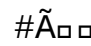

B\#ףWfםฉロ§U 


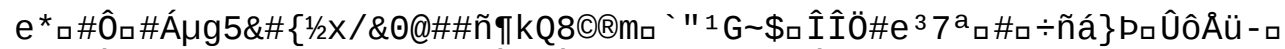

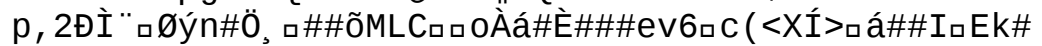

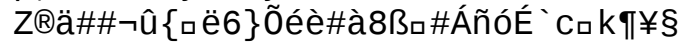

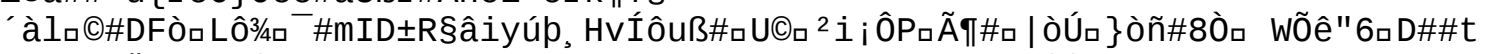

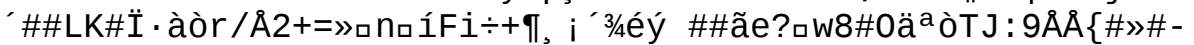

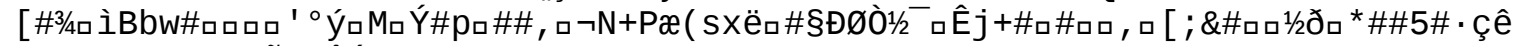

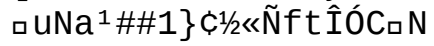

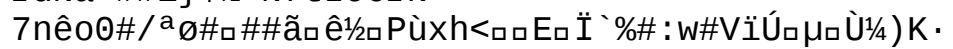

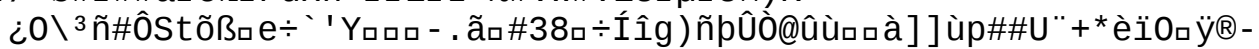

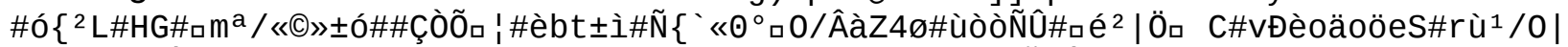

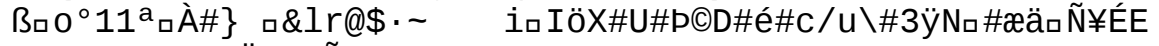

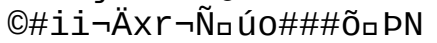

\section{endstream}

endobj

70 ○ obj

$<</$ Subtype/Type1

/FontDescriptor $71 \odot \mathrm{R}$

/LastChar 146

/Widths [500

$\odot$

250

0

0

0

0

$\odot$

0

0

0

$\odot$

0

0

$\odot$

$\odot$

0

0

$\odot$

0

0

$\odot$

○

0

0

0

$\odot$

○ 



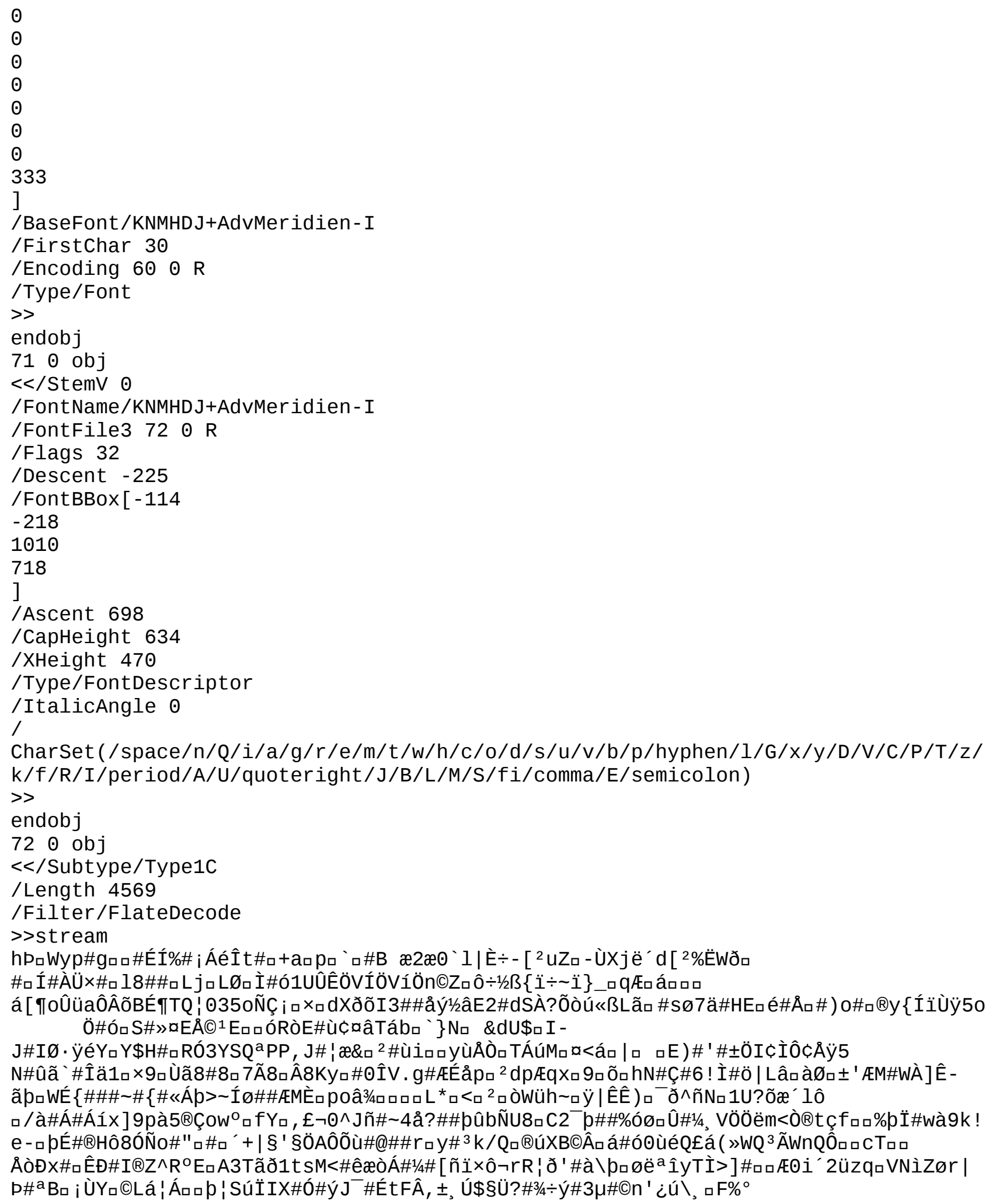




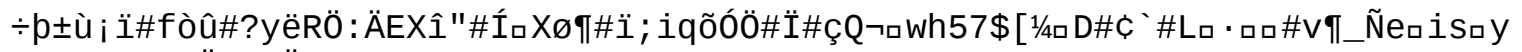
口) \#óðìW=\#Üß゚०Ä8K 


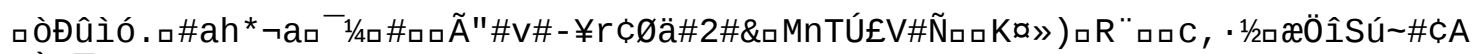
口ÀN ${ }^{-}$. ÇQ* Ú\#Zロ\#

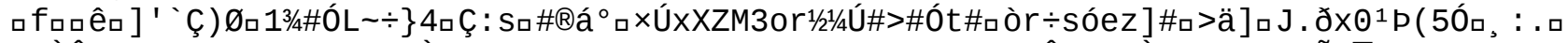

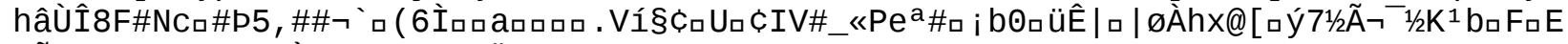

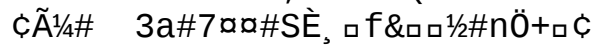




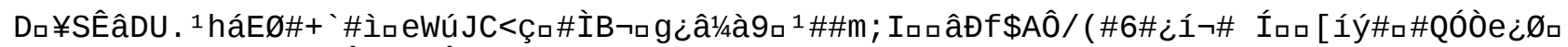

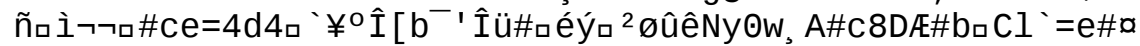

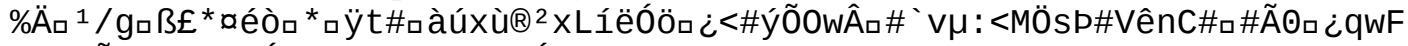
\%\#D $\left\{\tilde{N}{ }^{\prime \prime} x \# \mu \mu 40 A ́ J 2 G a ́\right.$ \#im. ñ|\#Á

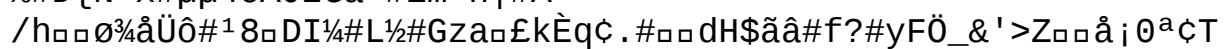

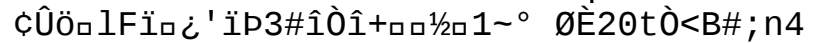




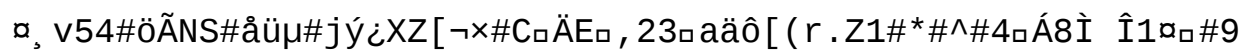




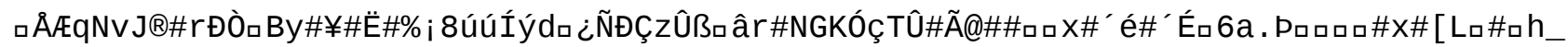

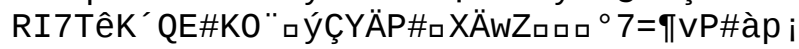




\section{Ấ\#DO\#̈̈\#ѐÝB]}

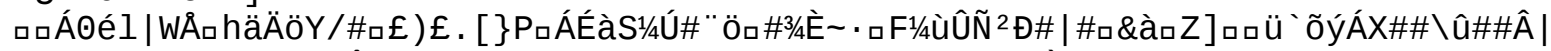

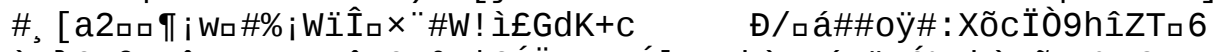

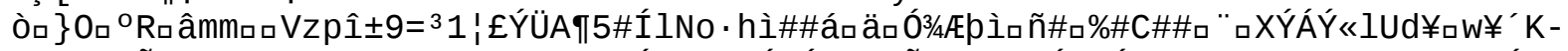

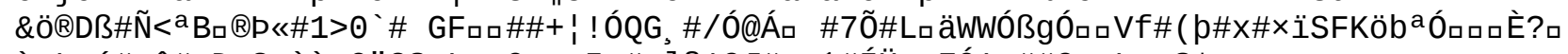

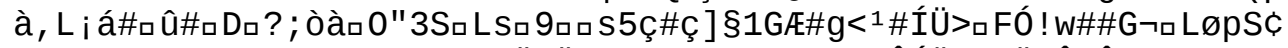

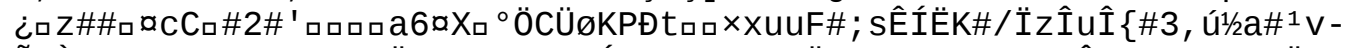

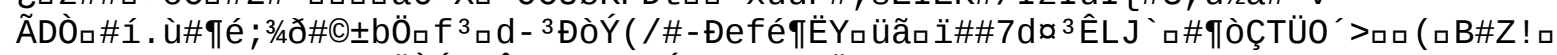

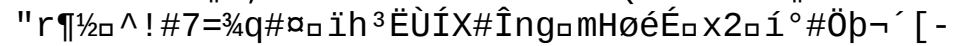

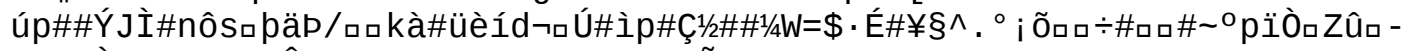

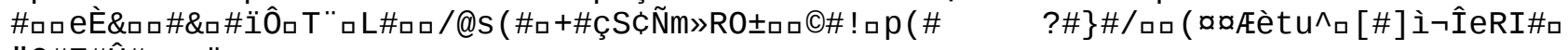

"Q\#Z\#Û\#Wæ_ÿ

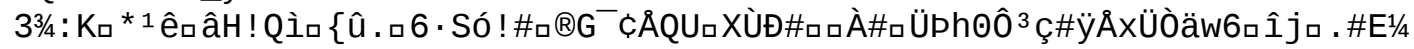




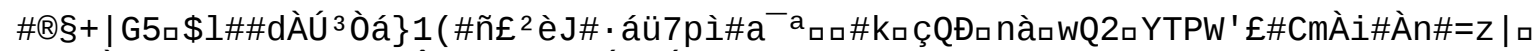

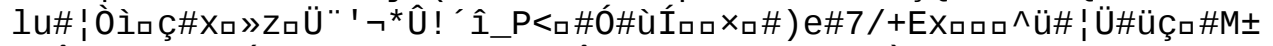

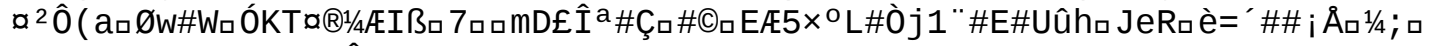
$\pm S \# \mathrm{D}^{\circ} \emptyset \# \hat{\mathrm{a}} \odot$, ${ }^{\mathrm{p} p a \# ! ~ v E ̂ e ̈ Z ® ~}$ 


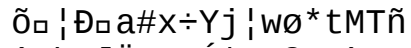

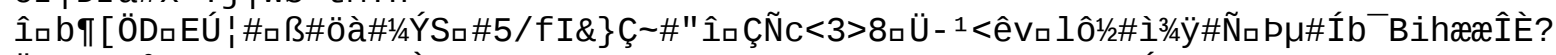

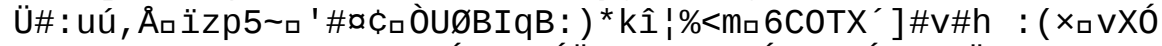

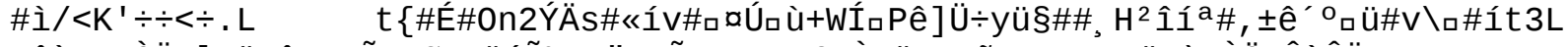

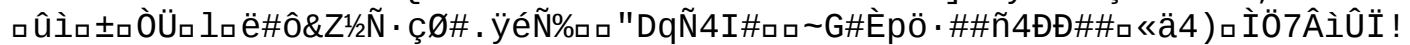

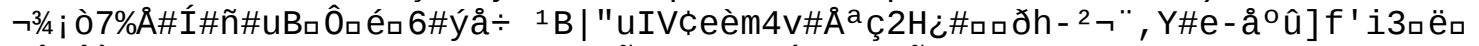

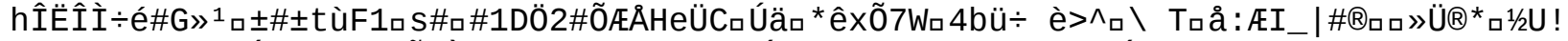

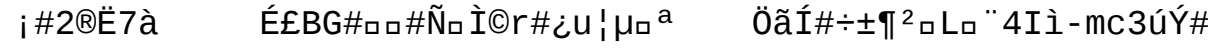




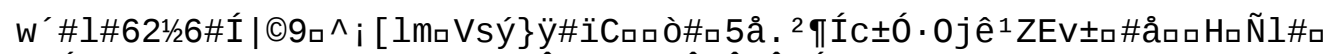

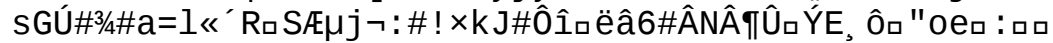

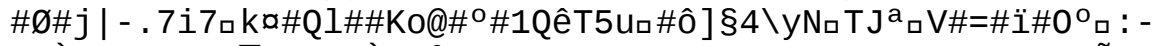

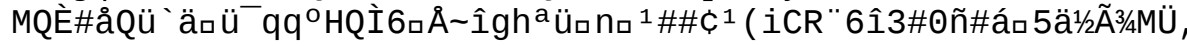




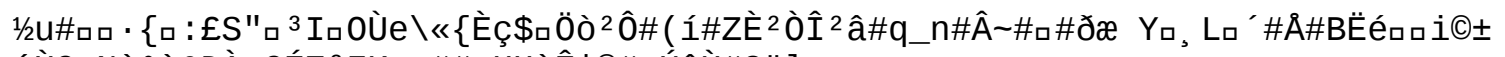

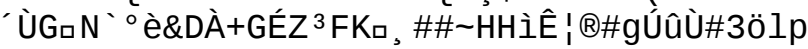




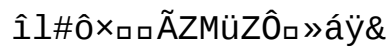

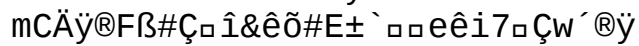


òâ̂Íì̃̃̃

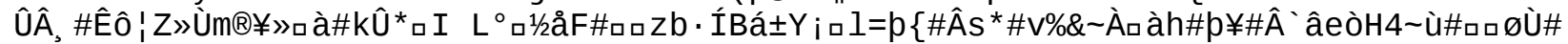

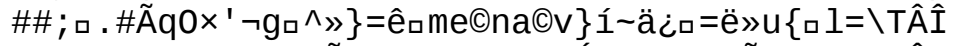

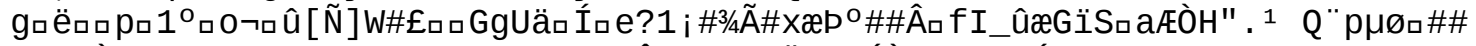

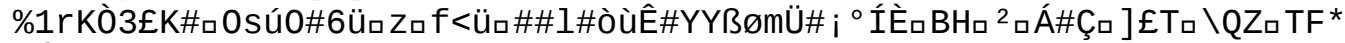

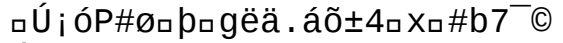

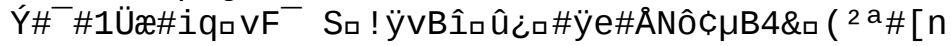




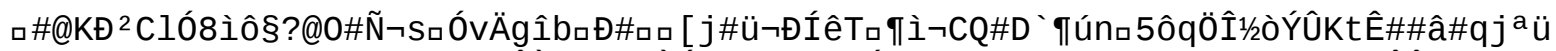

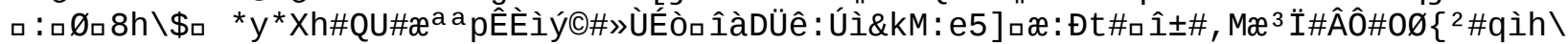

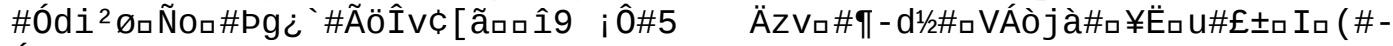

Úı\& \#40M\#ロ+\#ÇZ« 


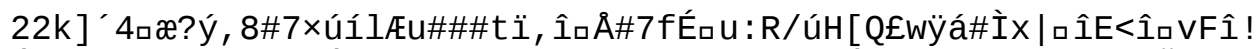

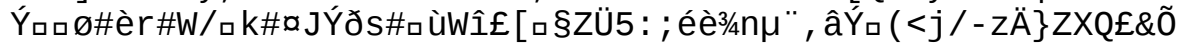

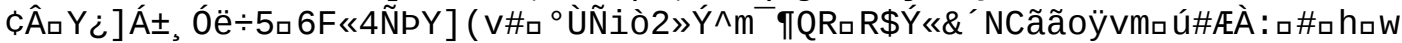

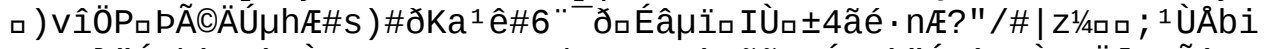

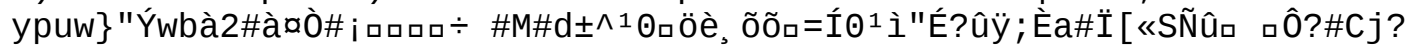

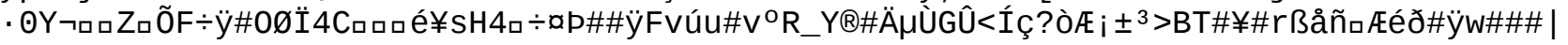
$\# \mathrm{r} "$

endstream

endobj

$73 \odot$ obj

$<</$ Subtype/Type1

/FontDescriptor $74 \odot \mathrm{R}$

/LastChar 90

/Widths [979

]

/BaseFont/KNMHDK+AdvEls-ent5

/Firstchar 90

/Encoding/WinAnsiEncoding

/Type/Font

$>>$

endobj

$74 \odot$ obj

$<$ < StemV $\odot$

/FontName/KNMHDK+AdVEls-ent5

/FontFile3 $75 \odot \mathrm{R}$

/Flags 34

/Descent - 98

/FontBBox $[9$

$-343$

1166

791

]

/Ascent 528

/CapHeight 714

/XHeight 634

/Type/FontDescriptor

It talicAngle $\odot$

/CharSet (/Z)

$>>$

endobj

$75 \odot$ obj

$<$ </Subtype/Type1C

/Length 120

/Filter/FlateDecode

>stream

hpbd`ad `dd\#ööóõpñövL)sÍ)ÖMÍ+1\#

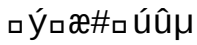




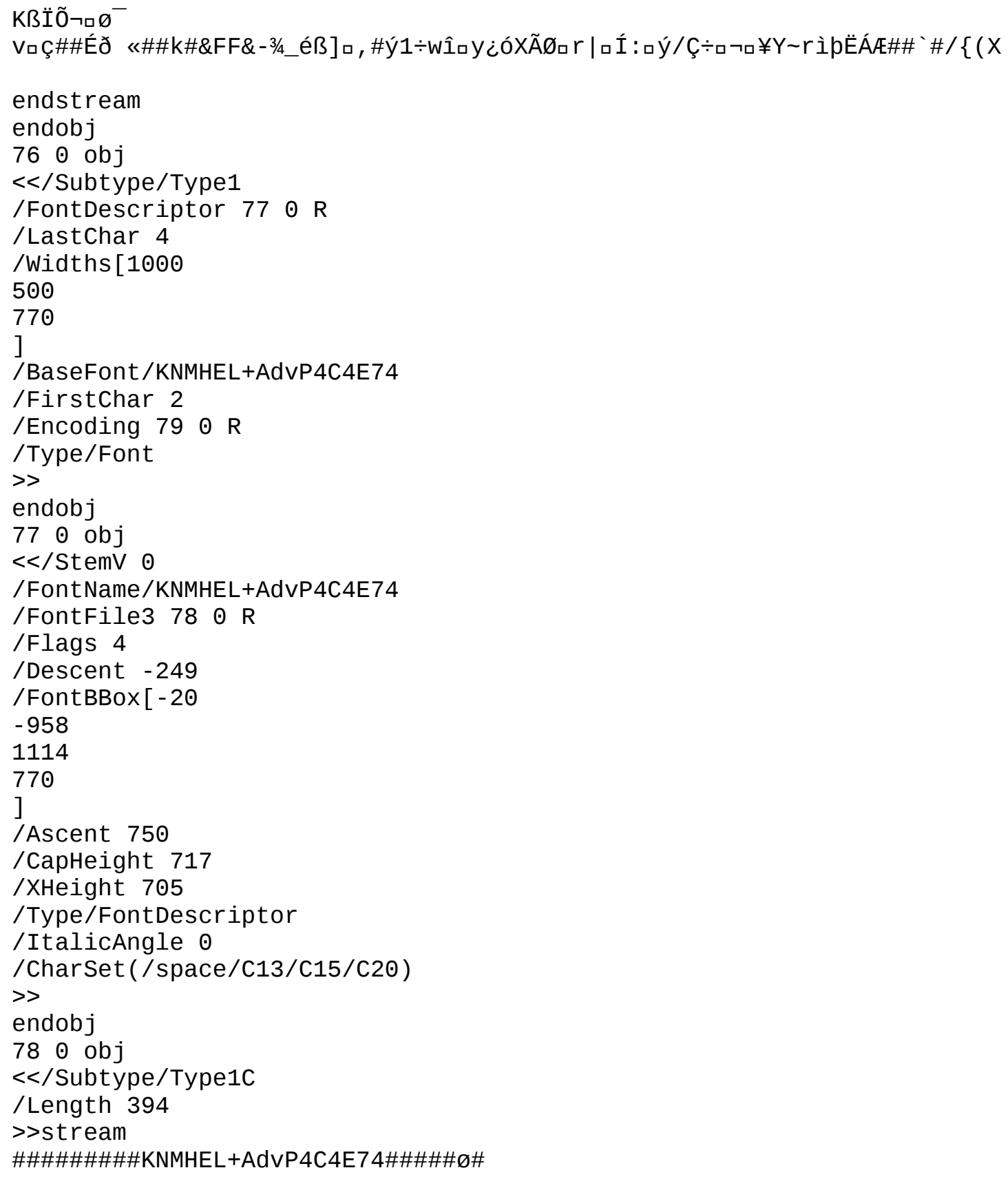




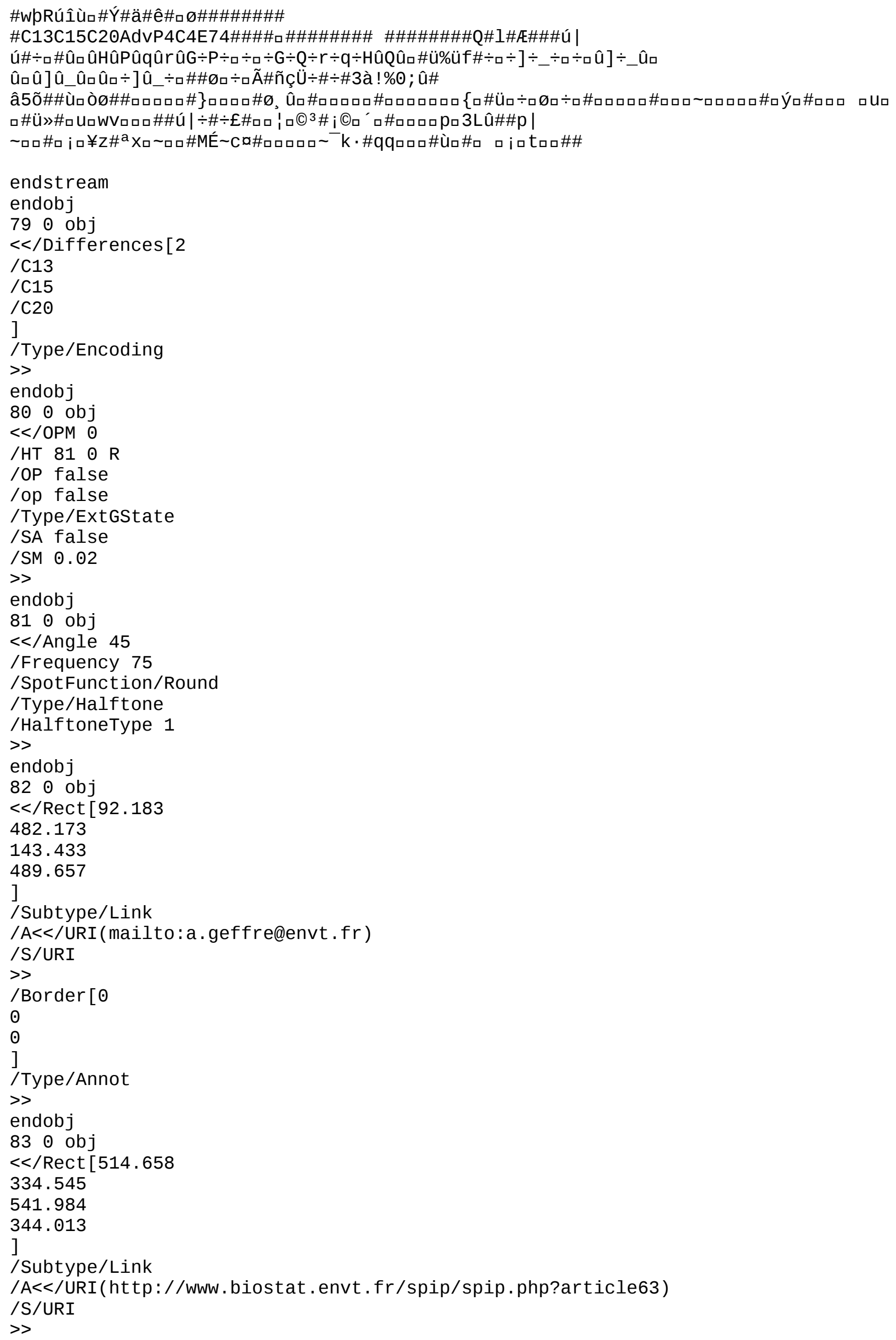




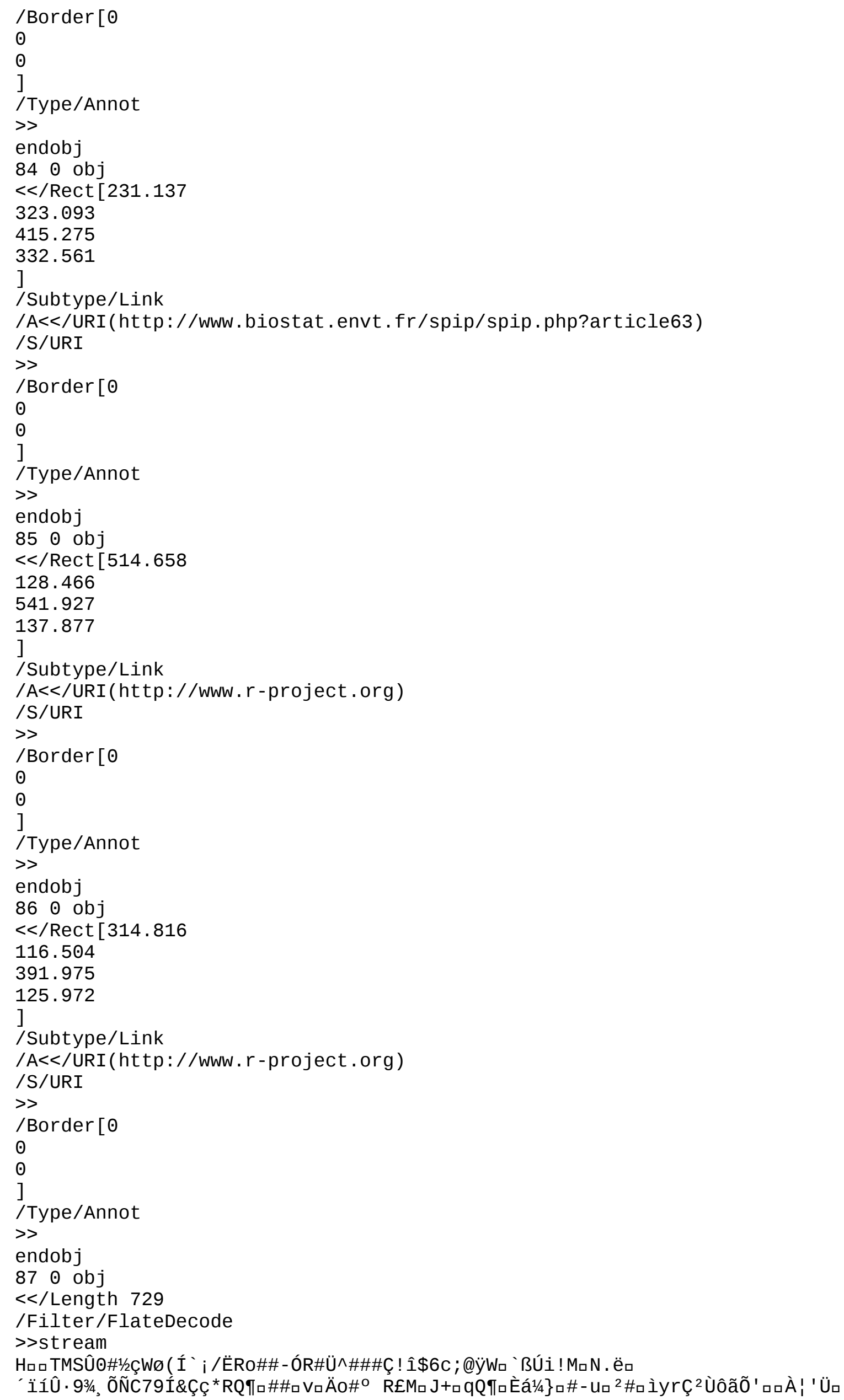




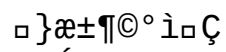

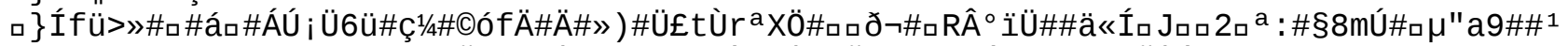

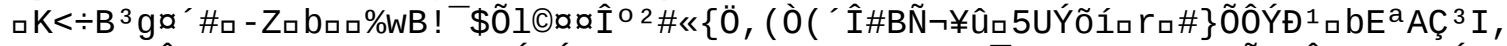

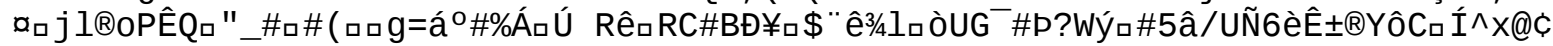

(WÛ́㇒

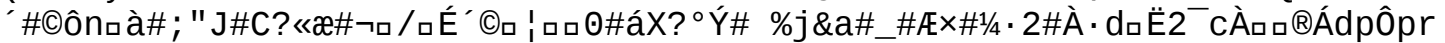

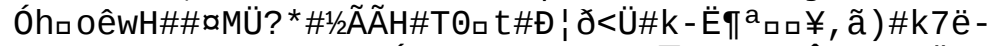

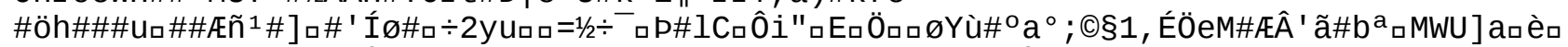

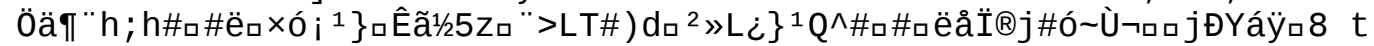

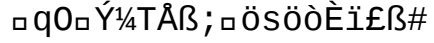


\#ÉロI»

endstream

endobj

$88 \odot$ obj

$<</$ Length 682

/Filter/FlateDecode

>stream

H口ฉSËnÛ@\#1/4û\#

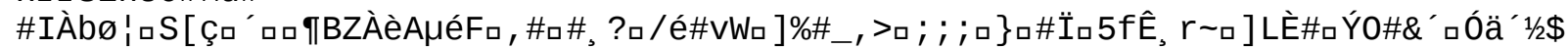

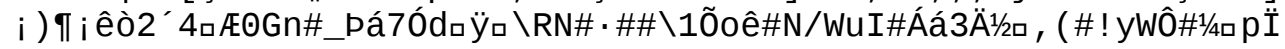




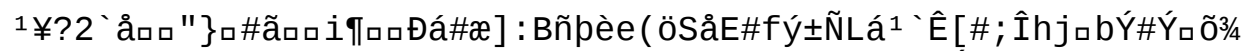

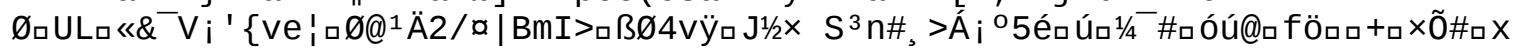

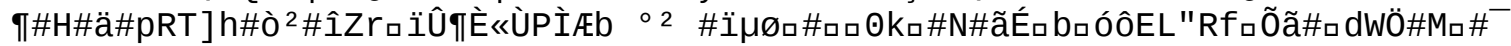




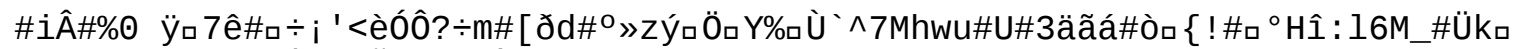

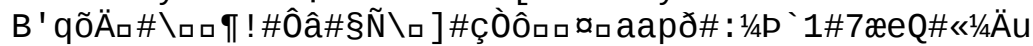

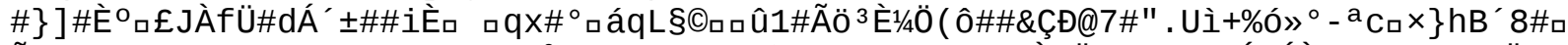

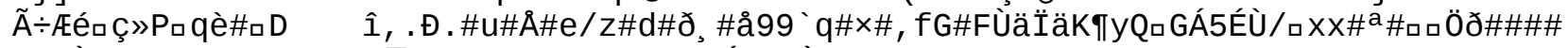

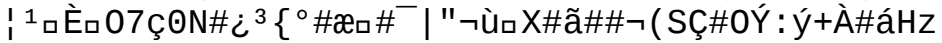

endstream

endobj

$89 \odot$ obj

$<</$ Length 761

/Filter/FlateDecode

>sstream

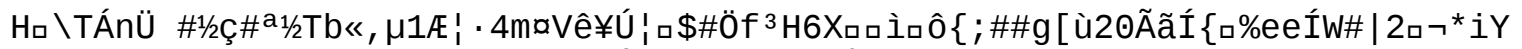

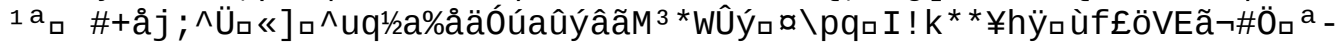

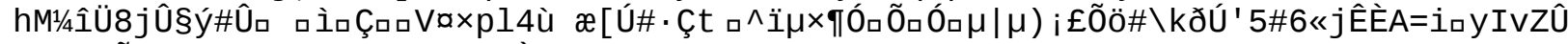

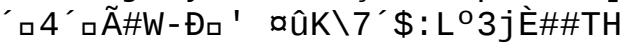




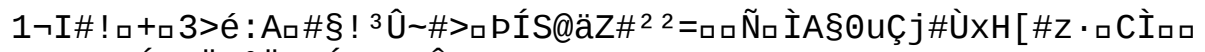

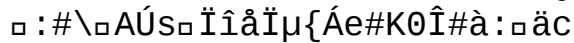

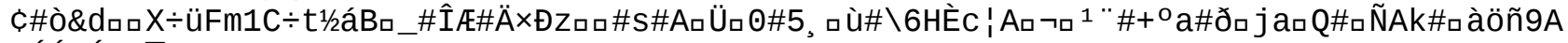
○ÉÁtÉ» $\mathrm{G}^{-} \not \mathrm{E} \square \times 5$

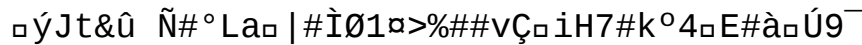

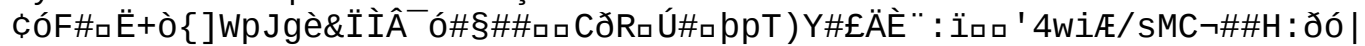

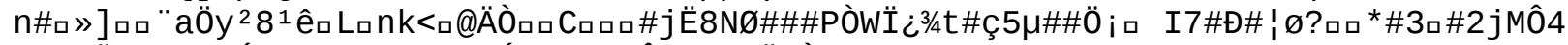

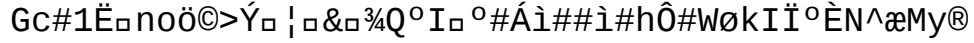




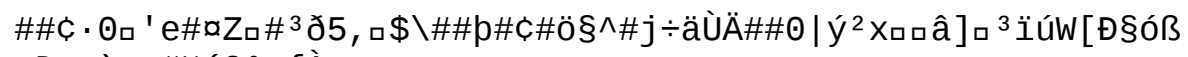
OĐ : ; ) ᄆ_\#1/2é $2^{\circ} \times\{$ À

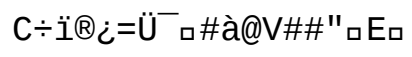


)?\#àoô ${ }^{\circ}+\hat{A}^{2} a^{2}{ }^{2} \mathrm{~B}$

endstream

endobj

$9 \odot \odot$ obj

$</$ Length 706

/Filter/FlateDecode

>sstream

HalS»ロÛ@\#ìý\#. é\#V\$a\#E\ロäロ"]\#U RĐ\#\}ÇDa 


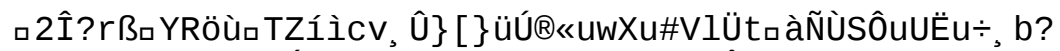

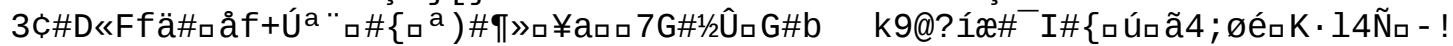

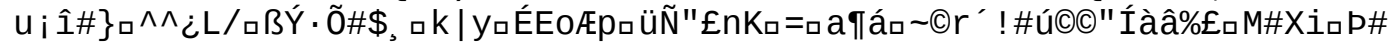




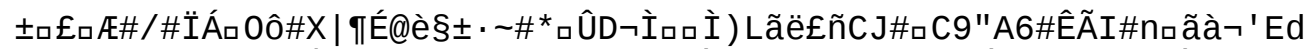

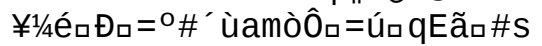

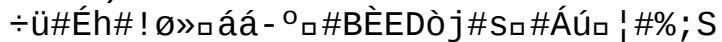

hi'a(\$ë6rqï \%- 


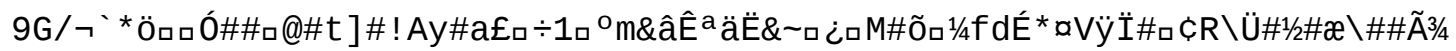
口) ñ@g\#f3à\#\$8æ 


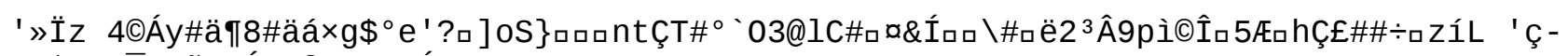

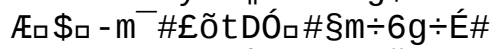

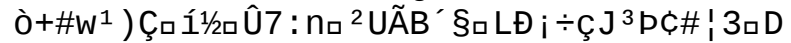

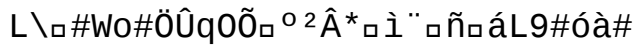

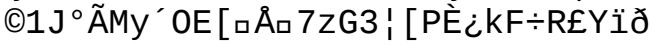


sjロCLÈ\#"Ùi

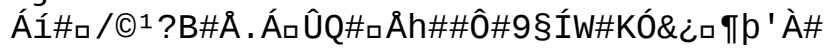


3уг

endstream

endobj

$91 \odot$ obj

$<</$ Length 747

/Filter/FlateDecode

>stream

Ha \TÁnÛO 


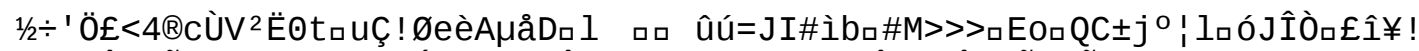

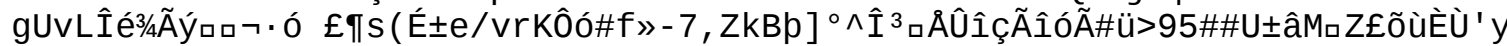

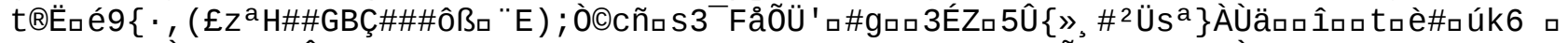

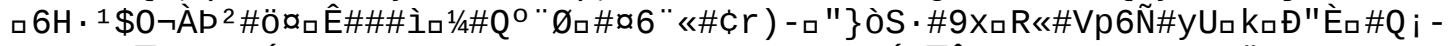

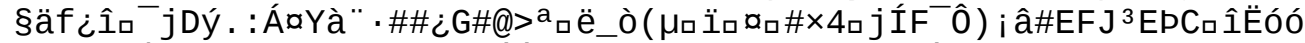

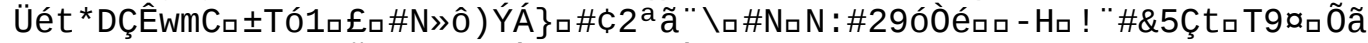

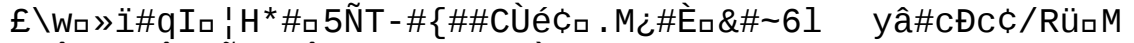

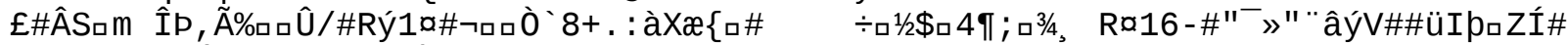

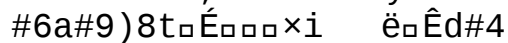

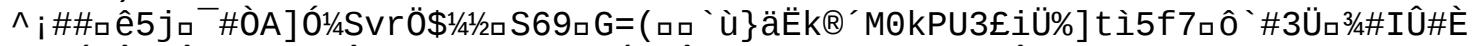

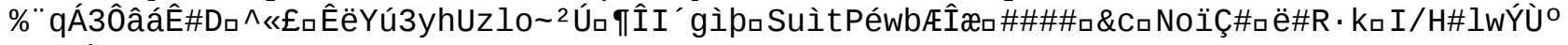

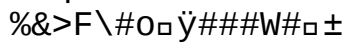

endstream

endobj

$92 \odot$ obj

$<$ /Length 766

/Filter/FlateDecode

>stream

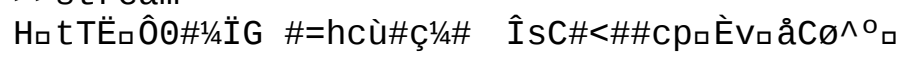




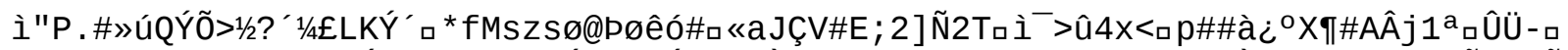

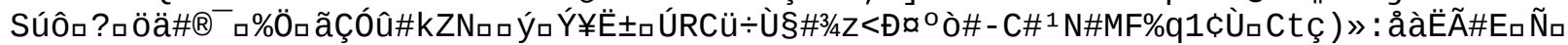

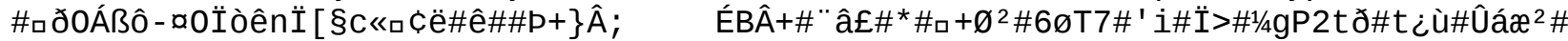

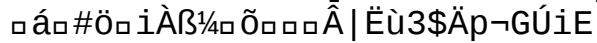

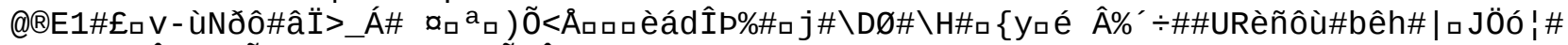
ç\}×ðロQÛ

Õ॰Æ\#е

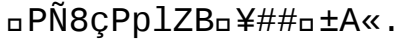

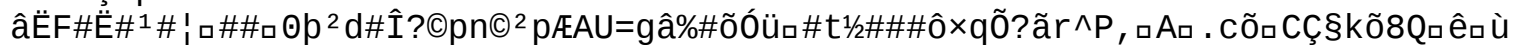
\#Và ${ }^{2} \# \#$ a! çùU

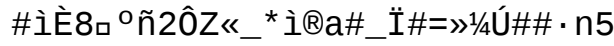




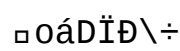




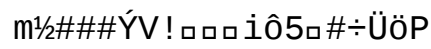




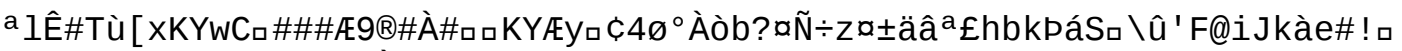

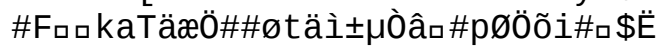




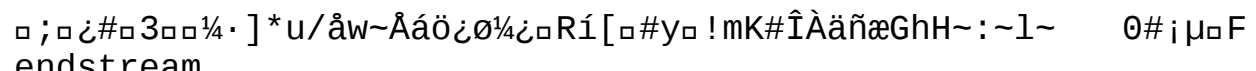
endstream

endobj

$93 \odot$ obj

$<</$ Length 794

/Filter/FlateDecode

>stream

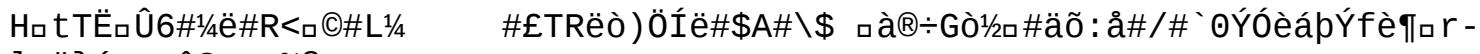

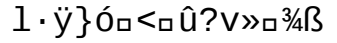

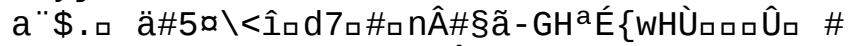

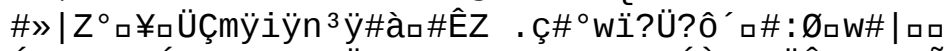

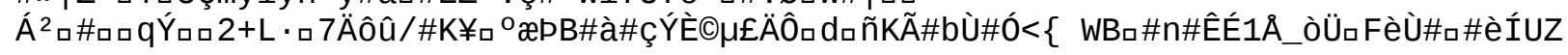




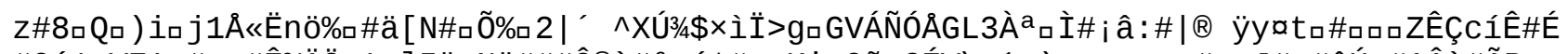

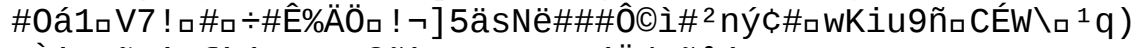

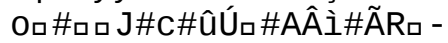

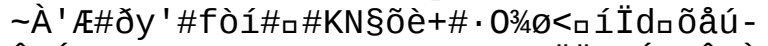

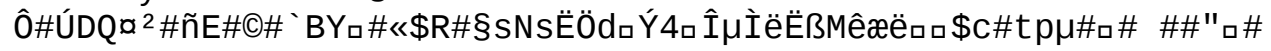




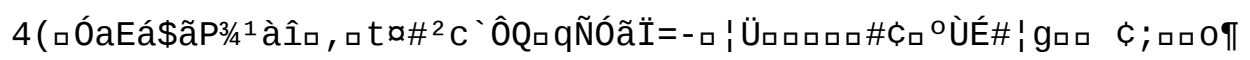




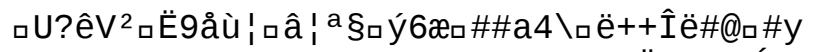

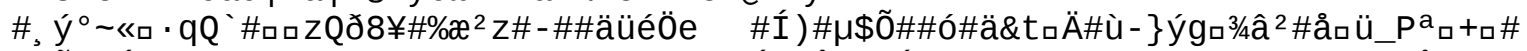

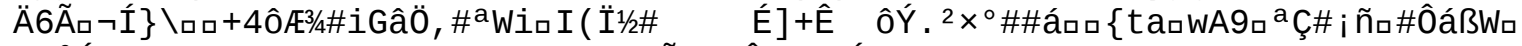

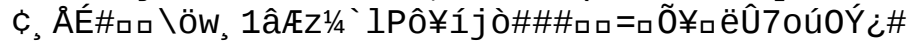


$\# \div$ Ç $\mathrm{C}$

endstream

endobj

$94 \odot$ obj

$<</$ Length 635

/Filter/FlateDecode

>stream

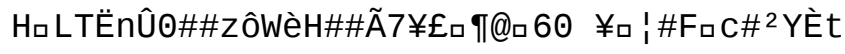




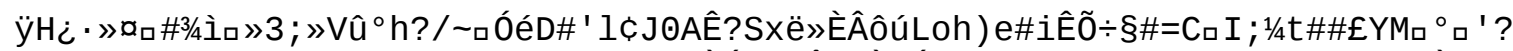

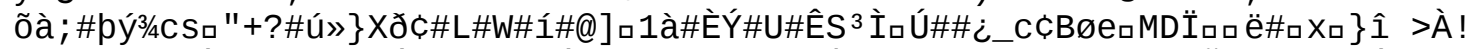

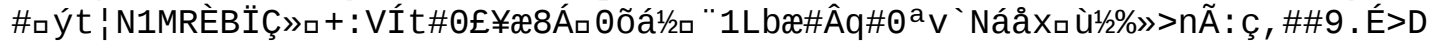




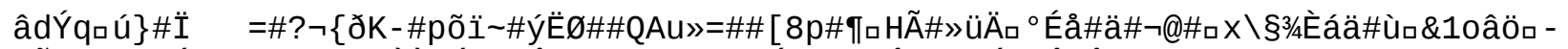

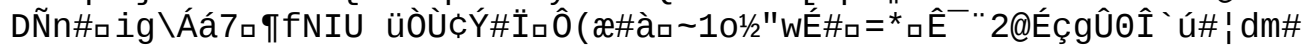

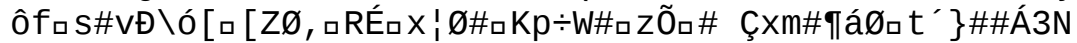

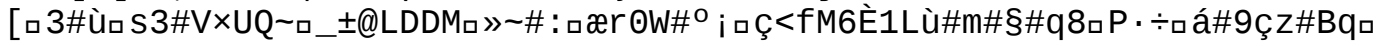
Zı 8“" ò\$ם\%\#U

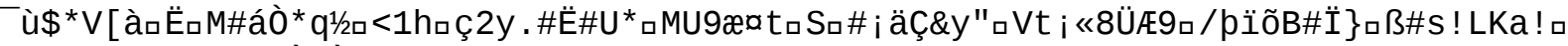

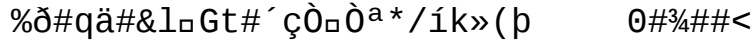

endstream

endobj

$1 \odot$ obj

$<<$

/Type/Page

/Resources $2 \odot \mathrm{R}$

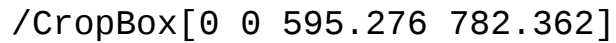

/MediaBox $\left[\begin{array}{llll}\odot & \odot & 595.276 & 782.362\end{array}\right]$

/Rotate $\odot$

/Parent $39 \odot \mathrm{R}$

/Contents $3 \odot \mathrm{R}$

/Thumb $32 \odot \mathrm{R}$

$>>$

endobj

$2 \odot$ obj

$<</$ Font $<</ F 1225 \odot \mathrm{R}$

$/ \mathrm{F} 2510 \mathrm{R}$

/F4 57 ○ R

/F5 $6100 \mathrm{R}$

/F6 $640 \mathrm{R}$

/F7 $67 \quad 0 \mathrm{R}$

/F8 $70 \quad \odot \quad \mathrm{R}$

/F9 $73 \odot \mathrm{R}$

/F10 $76 \odot \mathrm{R}$

/F11 $28 \odot \mathrm{R}$

$>$

/ProcSet [/PDF

/Text

]

/ExtGState $<</ G S 280 \odot \mathrm{R}$

$>>$

$>>$

endobj

$3 \odot$ obj

$<</$ Length 5218

/Filter/FlateDecode

>stream

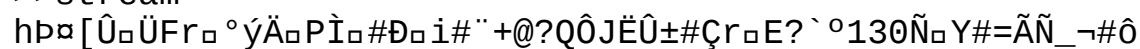

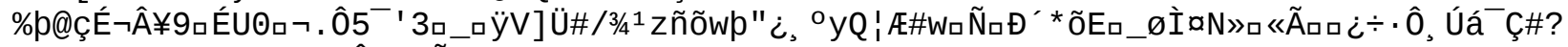

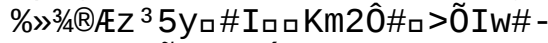

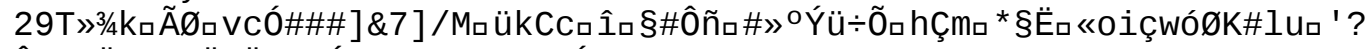

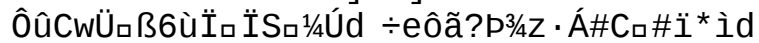

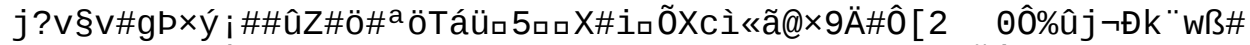

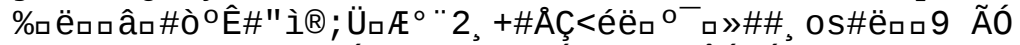

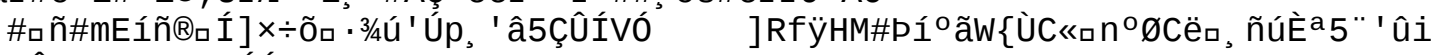

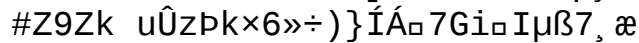

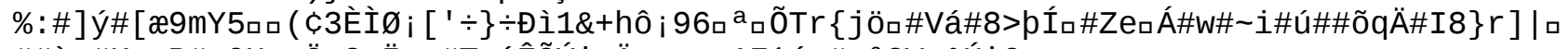

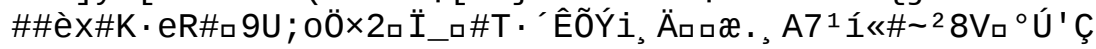

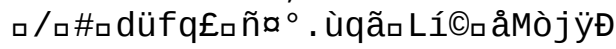




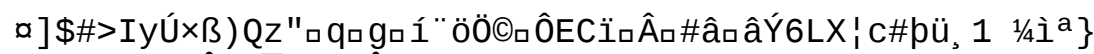

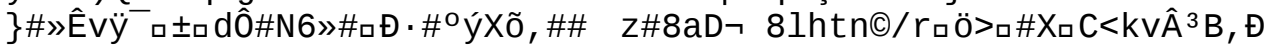
$\mathrm{sU}^{2}{ }^{-}$\#êđ|»' ÒNä口X( çáÂ 
)W\#0̃̊̊ 


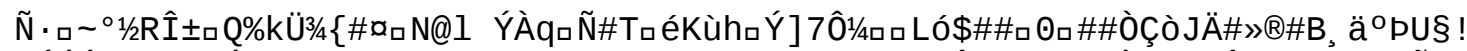

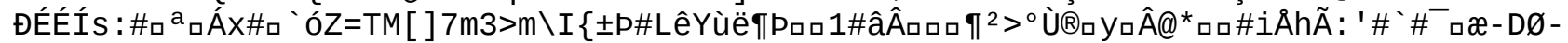

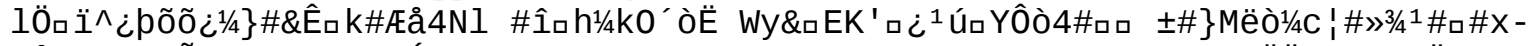

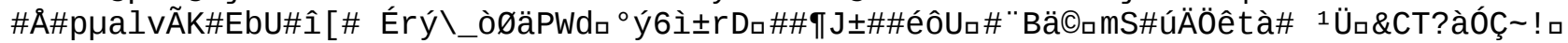
ÿ@\#1 ðçòàjã i «õ@âd3\#Ü口ÍrX\#, +qêঃÜV; r 


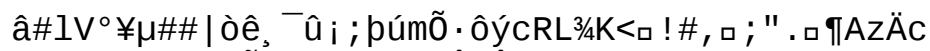

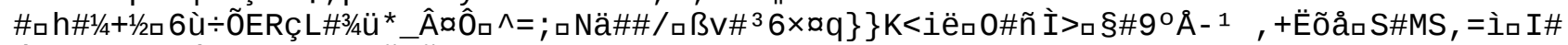

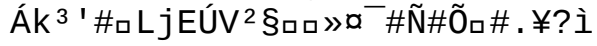




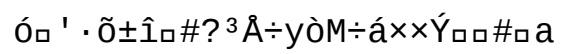




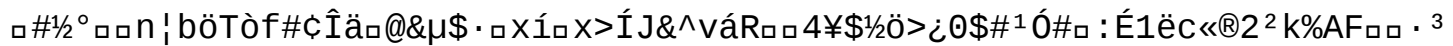

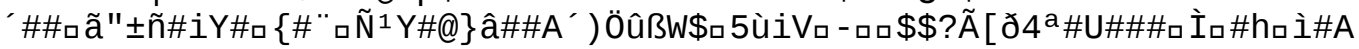




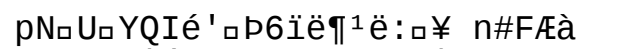

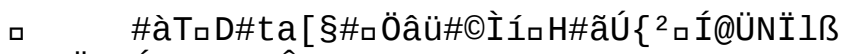

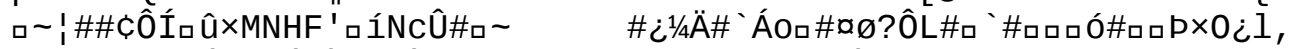

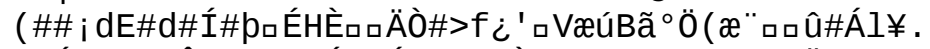

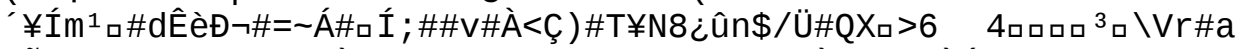

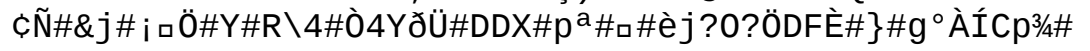


6ロ Ö\#1/4

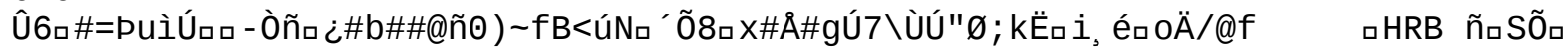

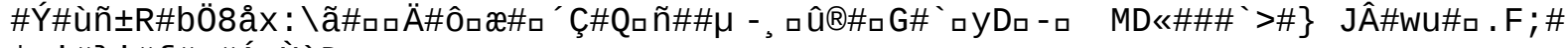
*ai\#\}i\#f\#»\#Ágù̀̀ 


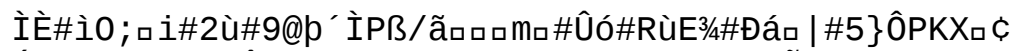

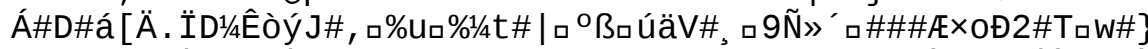

òMïykË\#ロロ!

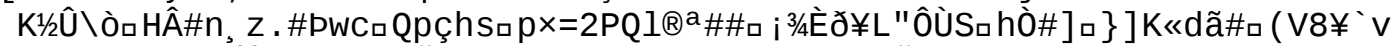

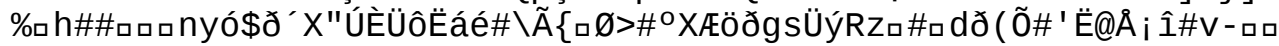

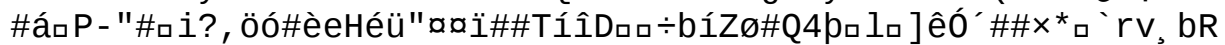


$\left.\mathrm{Gq}_{\square} ß 3 \mathrm{H}\right\} 5$ 


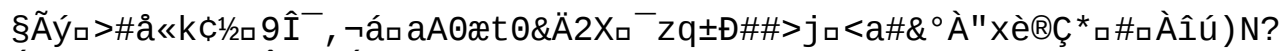

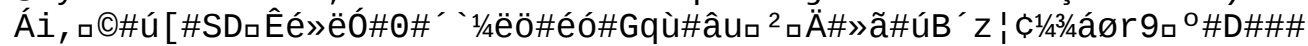

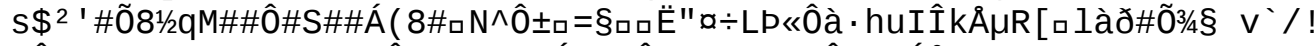

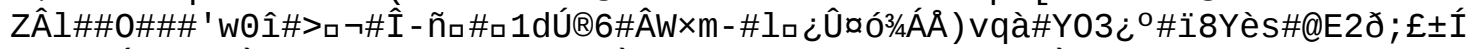

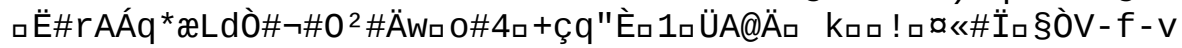




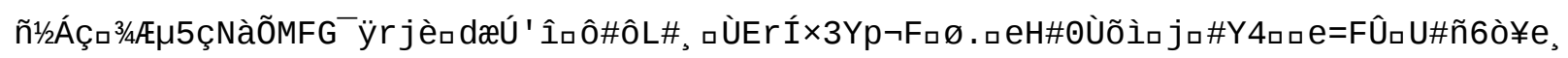




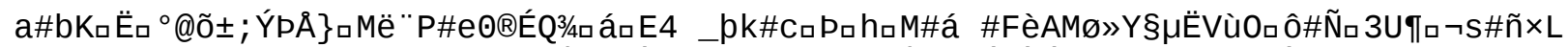

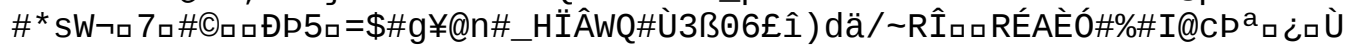

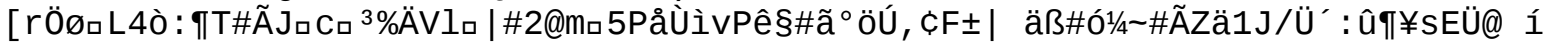

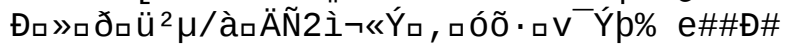
$Đ \mathrm{t} \AA \mathrm{J} \mathrm{a} \backslash$

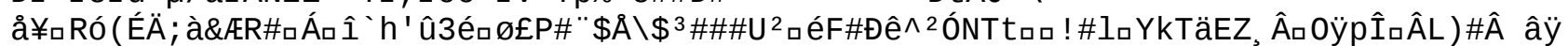
4§úï̈口0̃ 


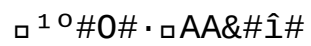




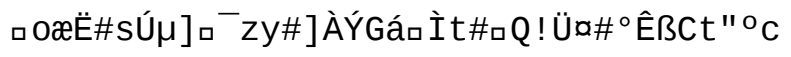

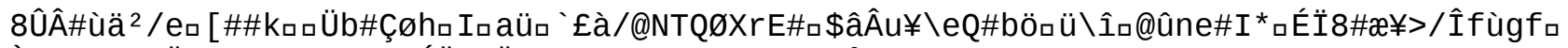

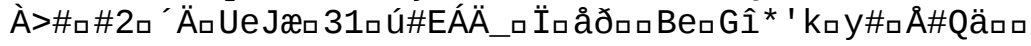




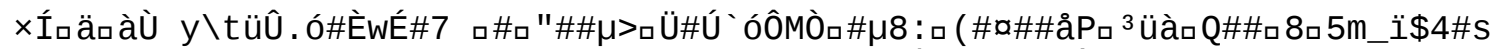

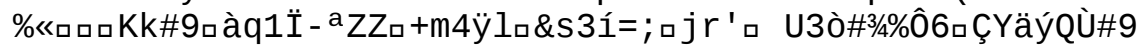
\#canCÇi , ûquF?TÇñ 9 s oW JÂ\#3ロ ë\#’ å(

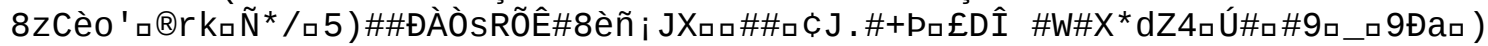

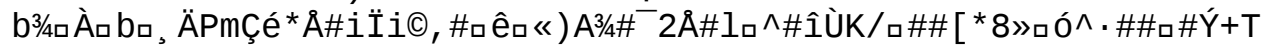

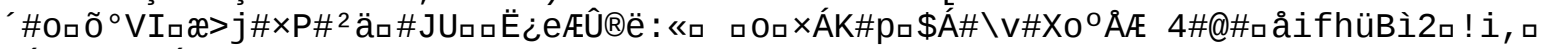
Túó\# ' OWêÉ\#qñ 


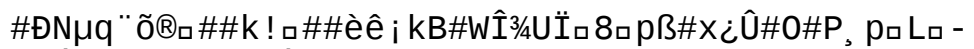

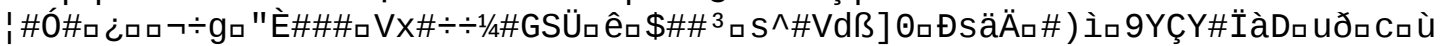

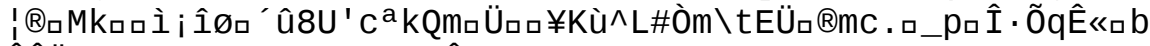

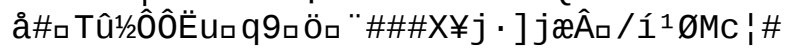


ò¿! hâ\#\#é£óp0?× 


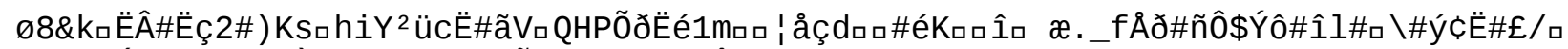

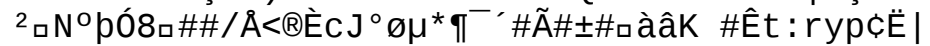

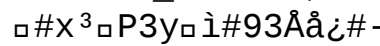

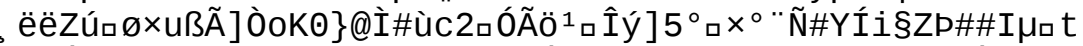

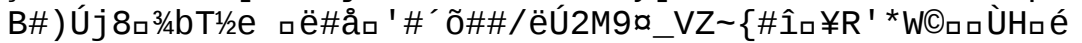

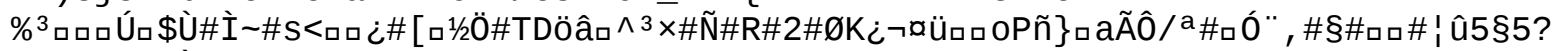

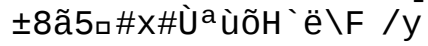

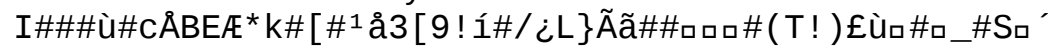




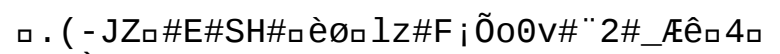

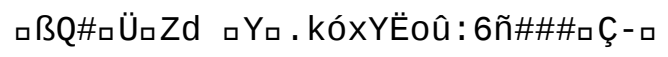
ë»äİ $\mu$ ê $1 / 4 \mathrm{~d} \tilde{n}\left\{\square / I A q^{\wedge}\right)>$ t $3 \# \#$

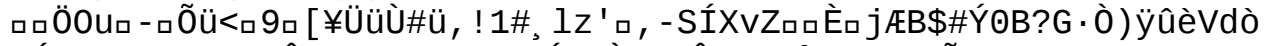

'óó\{ )у 
ù $(71$ ! p\$á"ם \#|

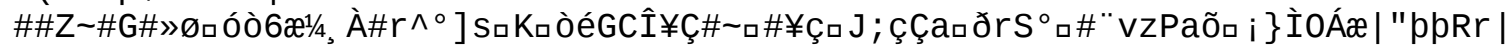

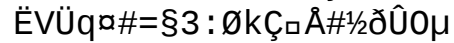

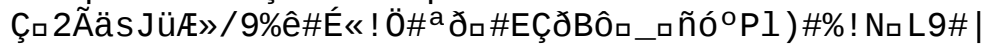

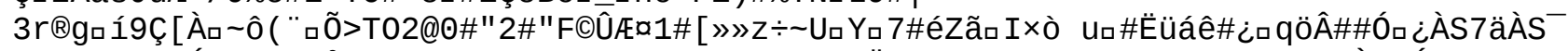

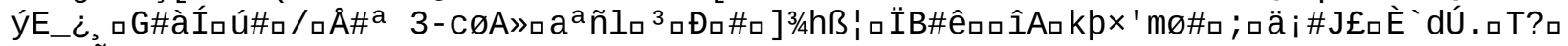
$\# H_{\text {HegÂ }} i$

$\mathrm{Y}$,

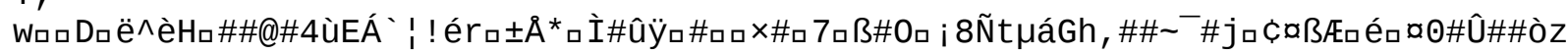




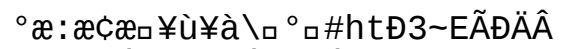

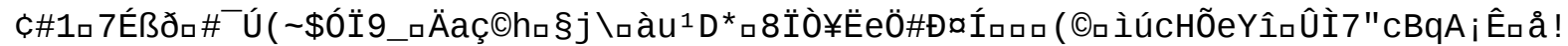

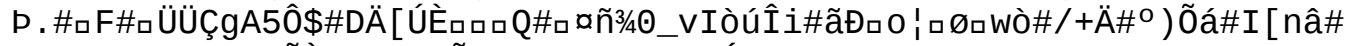

c7x\#70û ) ùqc\#ロÕì\#\&\#áhpÑò ; h\#?ýçaâıG\#ÝuíZú

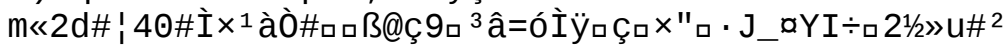

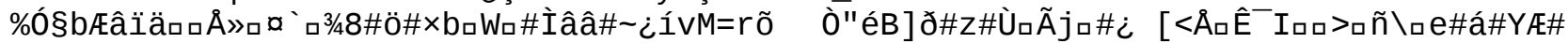

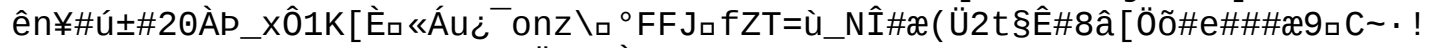

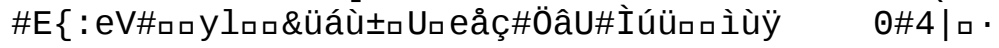

endstream

endobj

$4 \odot$ obj

$<<$

/Type/Page

/Resources $5 \odot \mathrm{R}$

/CropBox[0 0 ○ $595.276 \quad 782.362]$

/MediaBox[ $\left[\begin{array}{llll}\odot & \odot & 595.276 & 782.362\end{array}\right]$

/Rotate 0

/Parent $39 \odot \mathrm{R}$

/Contents $11 \odot \mathrm{R}$

/Thumb $33 \odot \mathrm{R}$

$>$

endobj

$5 \odot$ obj

$<</$ Font $<</ F 1225 \odot \mathrm{R}$

/F13 $6 \odot \mathrm{R}$

/F2 $51 \odot \mathrm{R}$

/F4 57 ○ R

/F5 $6100 \mathrm{R}$

/F6 $6400 \mathrm{R}$

/F7 67 ○ R

/F8 $70 \quad 0 \mathrm{R}$

/F10 76 ○ R

/F11 $28 \odot \mathrm{R}$

$>$

/Procset [/PDF

/Text

]

/ExtGState $<</ G S 280 \odot \mathrm{R}$

$>>$

$>>$

endobj

$6 \odot$ obj

$<</$ Subtype/Type1

/FontDescriptor $7 \odot \mathrm{R}$

/LastChar 49

/Widths [833

]

/BaseFont/KNMHJM+AdVMPi- one

/Firstchar 49

/Tounicode $9 \odot \mathrm{R}$

/Encoding $10 \odot \mathrm{R}$

/Type/Font

$>$

endobj

$7 \odot$ obj

$<</$ StemV $\odot$

/FontName/KNMHJM+AdVMPi-One

/FontFile3 $8 \odot \mathrm{R}$

/Flags 6

/Descent - 205

/FontBBox [-20

$-208$

1000 
770

]

/Ascent 696

/CapHeight 712

/XHeight 442

/Type/FontDescriptor

/ItalicAngle $\odot$

/CharSet (/space/one)

$>$

endobj

$8 \odot$ obj

$<</$ Subtype/Type1C

/Length 108

/Filter/FlateDecode

>stream

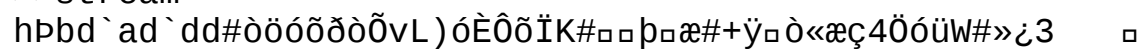

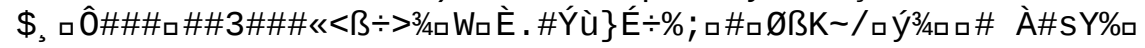

endstream

endobj

$9 \odot$ obj

$<</$ Length 214

/Filter/FlateDecode

>sstream

hPTP1nÃ○ 
Üõ

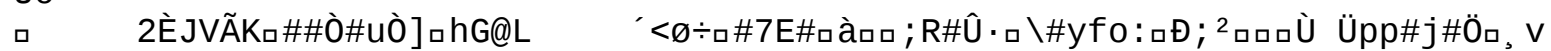

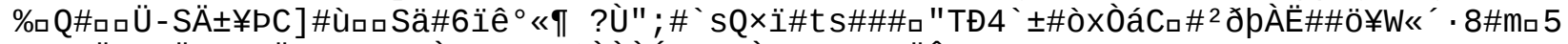

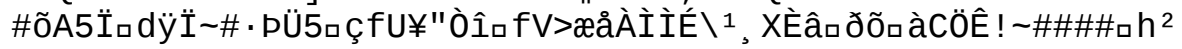

endstream

endobj

$10 \odot$ obj

$<</$ Differences [49

/one

]

/Type/Encoding

$>$

endobj

$11 \odot$ obj

$<</$ Length 4837

/Filter/FlateDecode

>sstream

hP॰ZÛräFr ${ }^{\circ} ß æ \# 13 / 48 \# t$ 


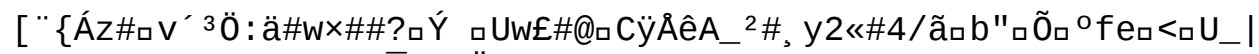

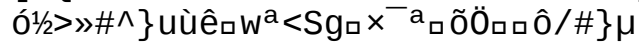

Fa3Wuituv ${ }^{1}\left\{\mathrm{Ur}_{\square} \mathrm{p}_{\square}\right.$

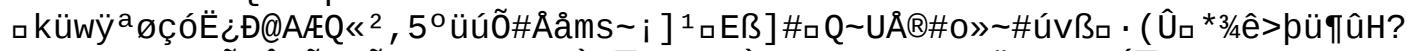

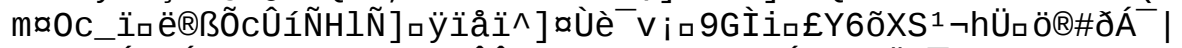

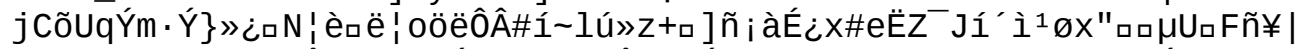

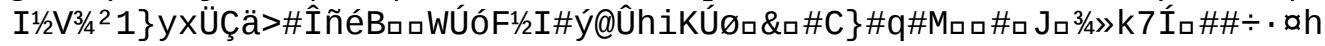




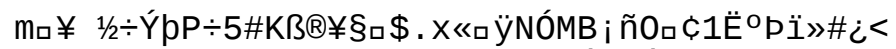

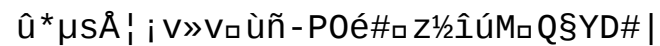

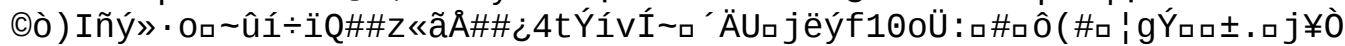

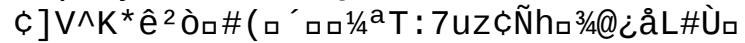

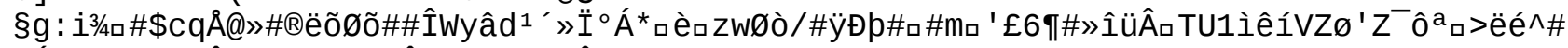

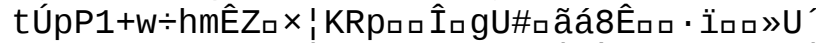

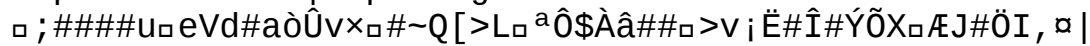

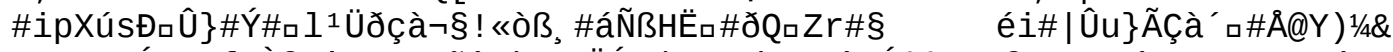

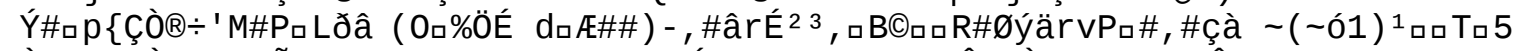

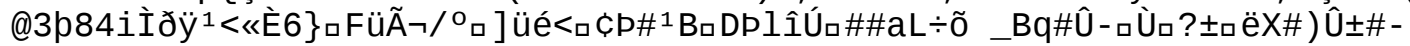

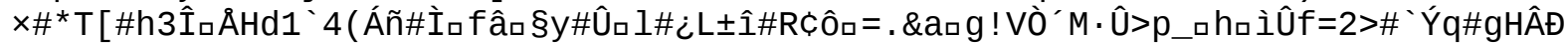

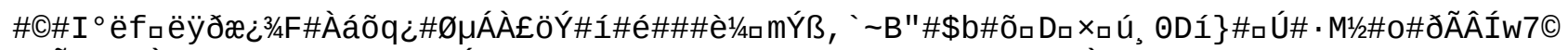

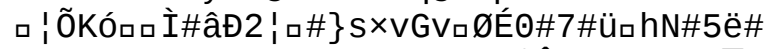

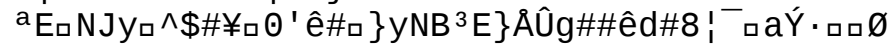

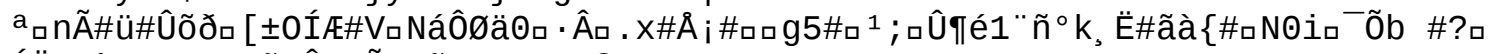

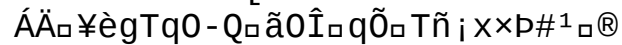

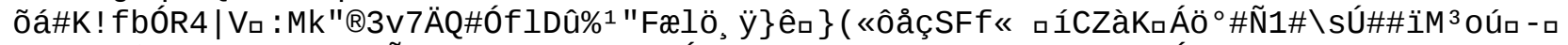

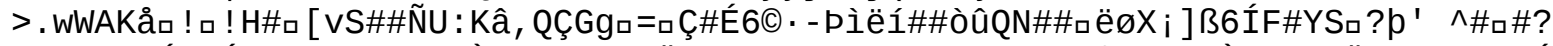

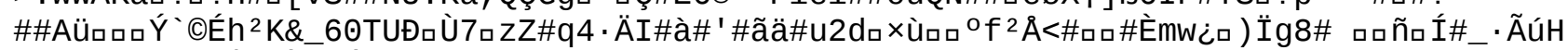

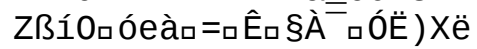




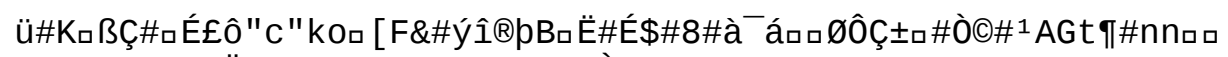

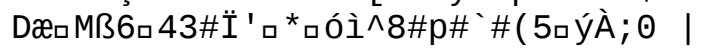


$\hat{E} \# \# H \backsim c \backslash \Re L T C ̧ \# X$ 


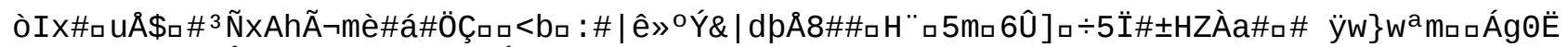

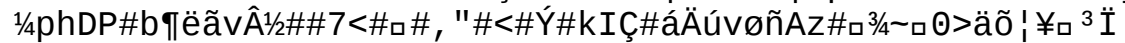

\#1

ロ

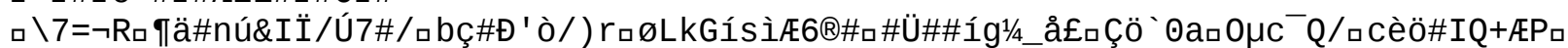

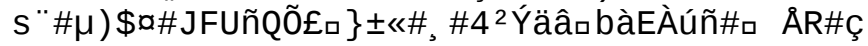




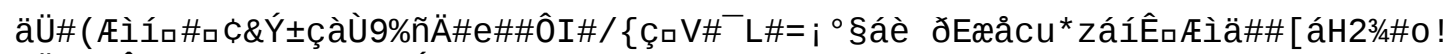

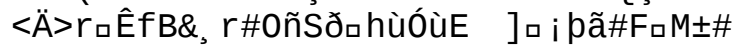




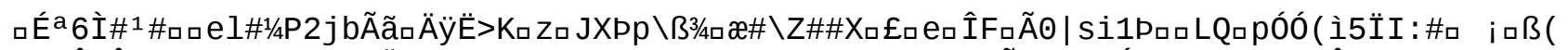

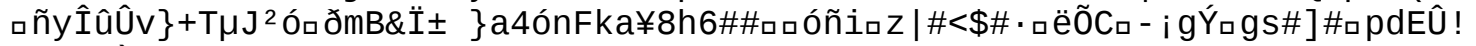

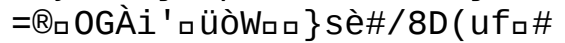




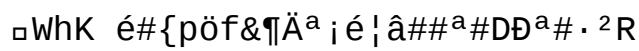




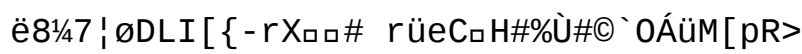




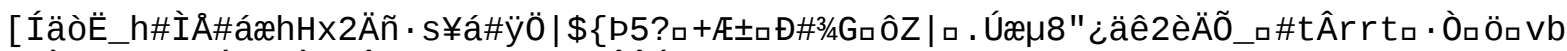

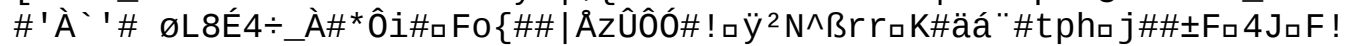

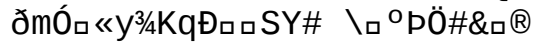

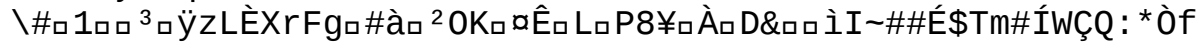

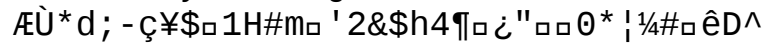


ðM॰ Î ! \#VÜ 
a

- >Íø\#\#8\#\#1D

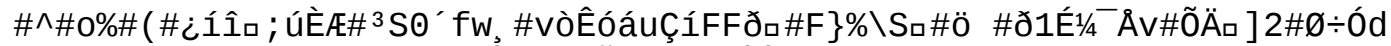

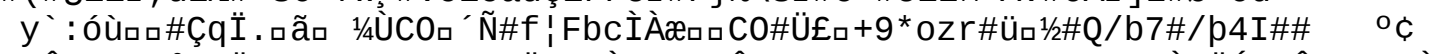

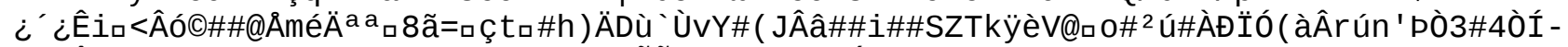

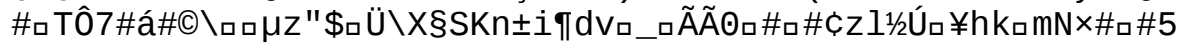

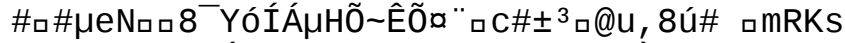

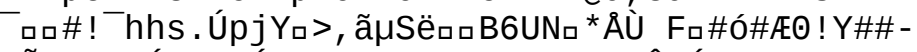

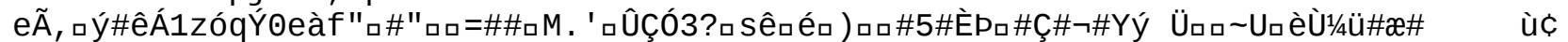

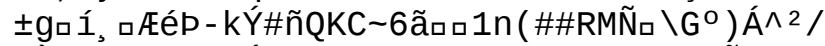

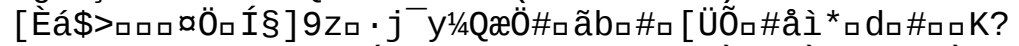

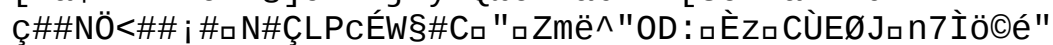

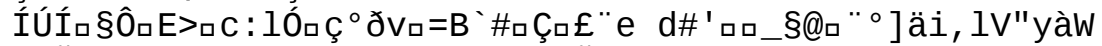

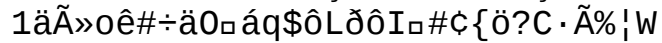




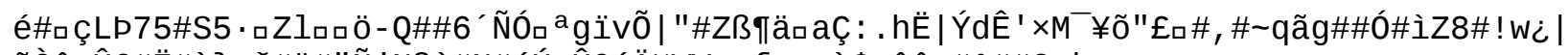

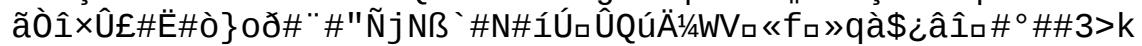




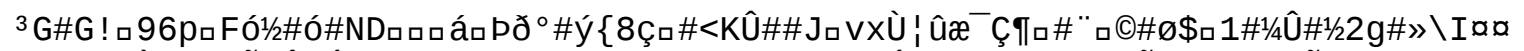

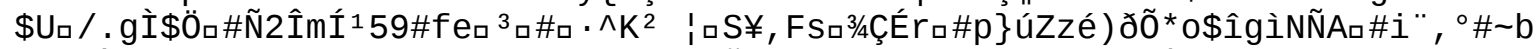

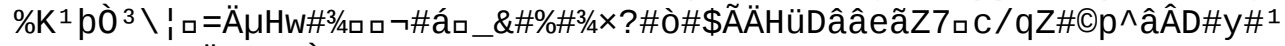

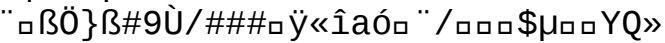

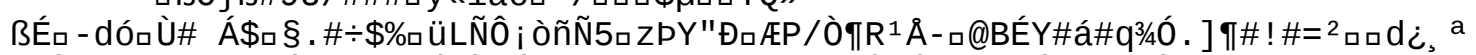

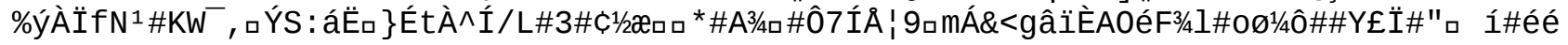
\#aGú \:\#JQ 


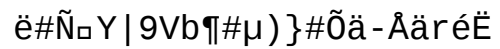

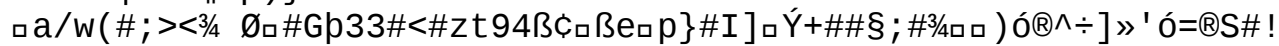

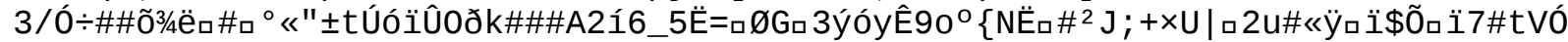

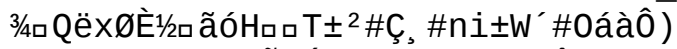

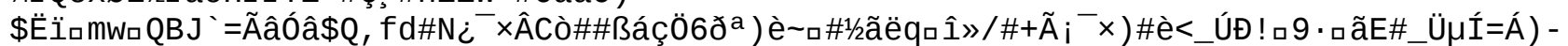

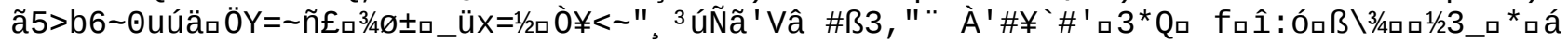




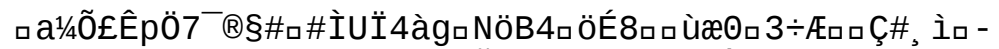

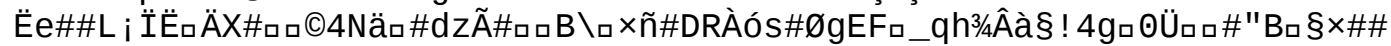

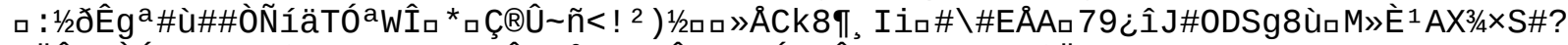

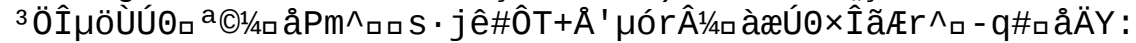

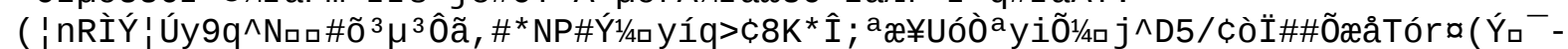

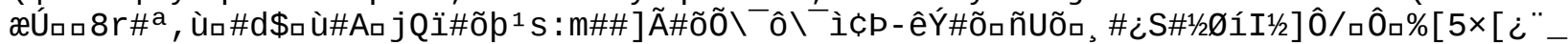

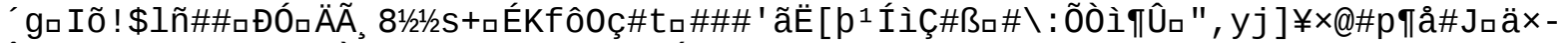

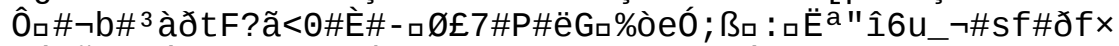

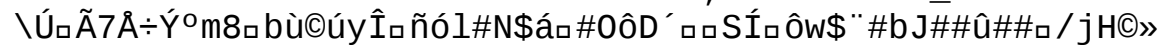

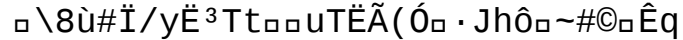




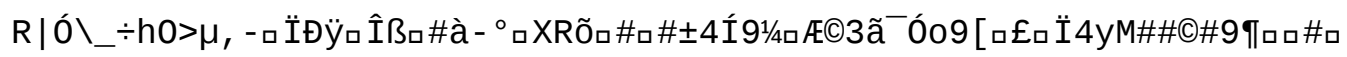




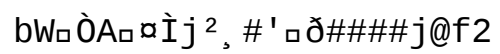

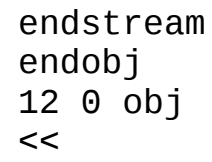

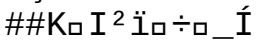




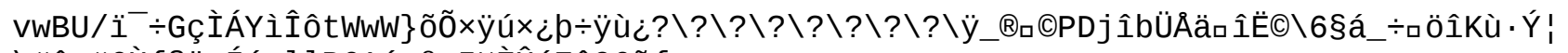

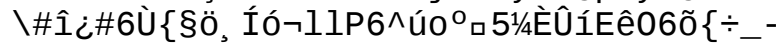

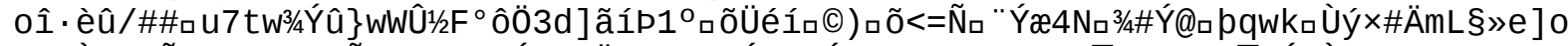

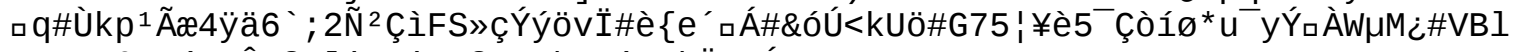

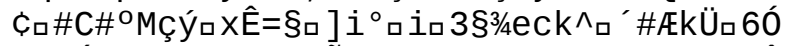

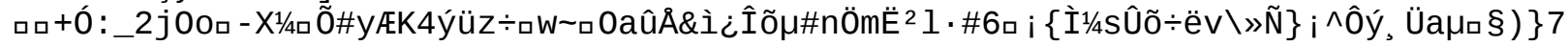




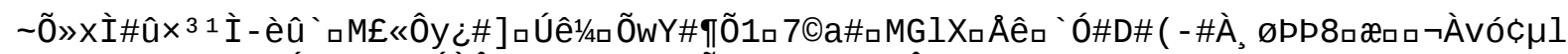

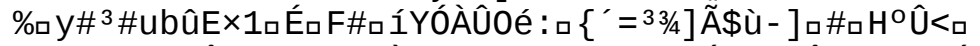

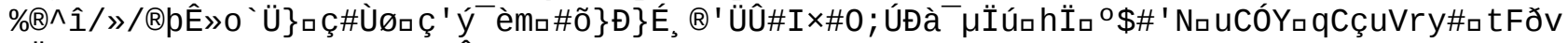

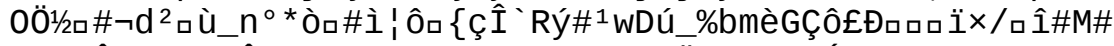

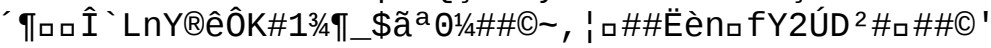




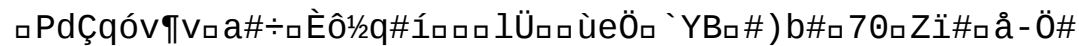

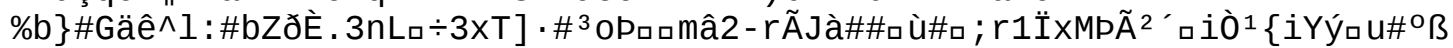

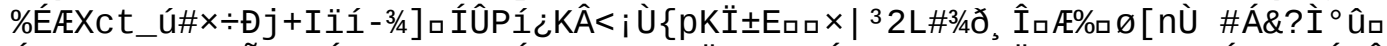

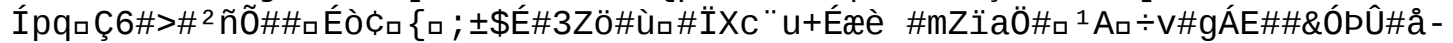

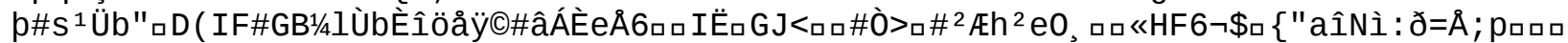

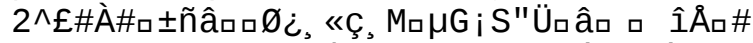

DaP; \#2 $2^{\prime} \# 23 / 4++\ddot{A}^{\circ}$ Í $q$ nE

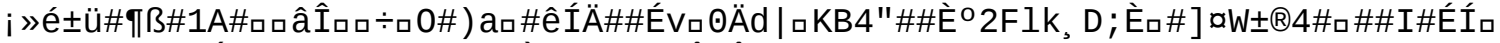

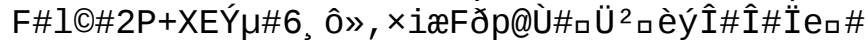

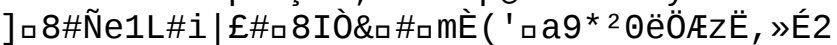

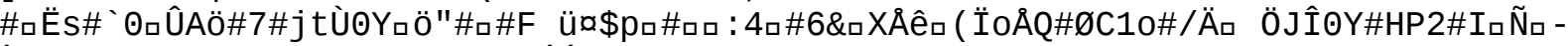

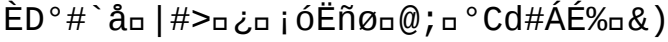


$m \hat{I}^{\circ}$ đ]

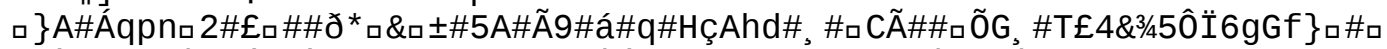

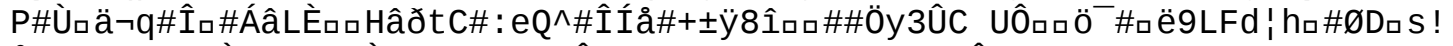

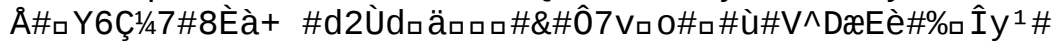

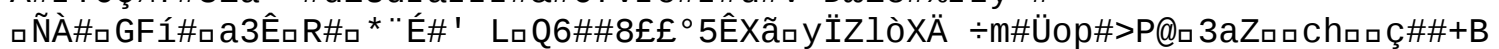

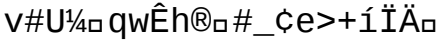




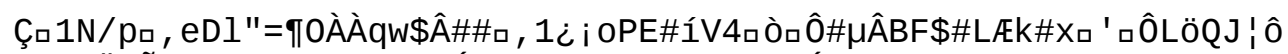




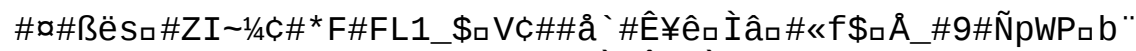

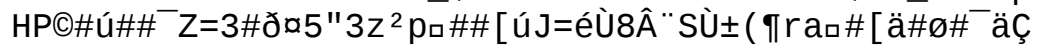




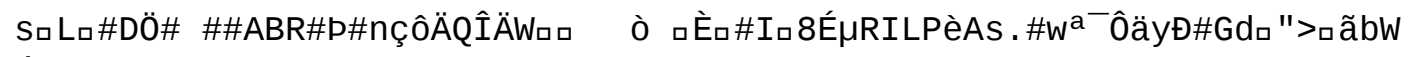
4

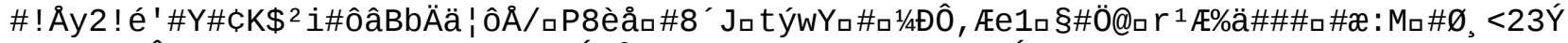

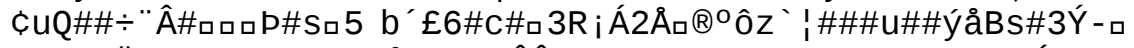

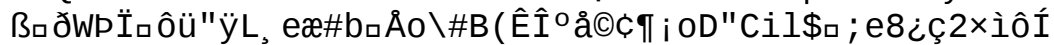

\#2Âôqs 
$e_{a} a ̀ \# e_{,} \# \# \square K^{\prime} d Q$

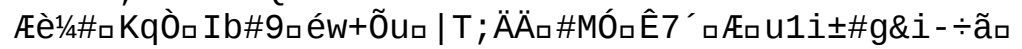

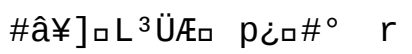

\%ý76gGÖ\#ロ\# i \$C 


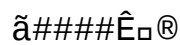




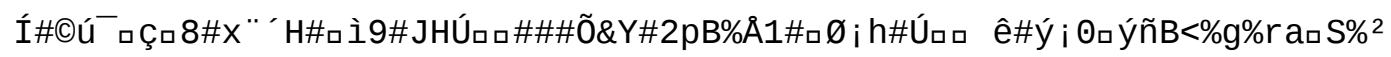




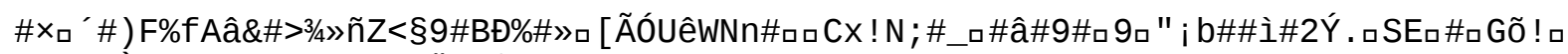

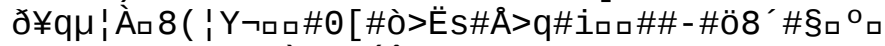

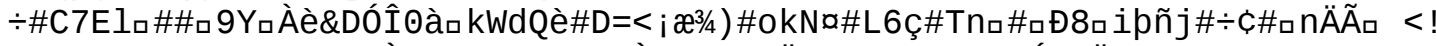

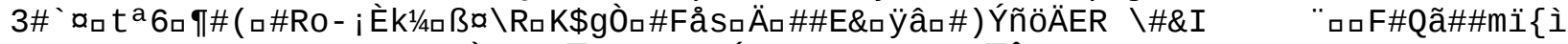

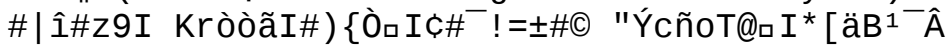

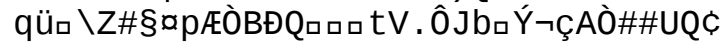

$+$ 


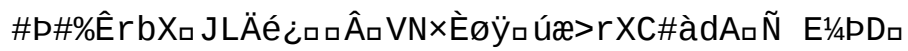


'õaöá : Ij [qaqa $\left.{ }^{a}\right\} \# T a q u ́$

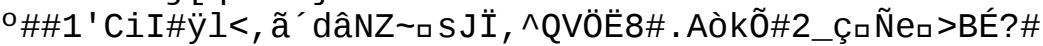

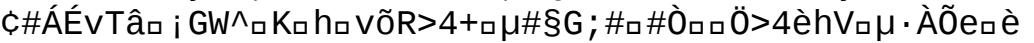

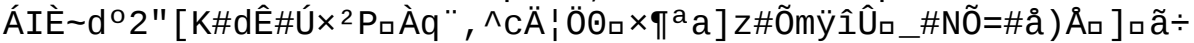

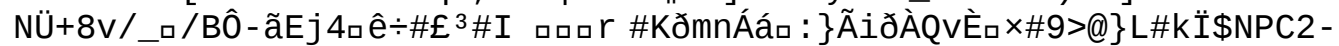

Lã \#É \pm , $\square E ́ X \# \square \& \llbracket \#^{3} A a \tilde{A}$ 


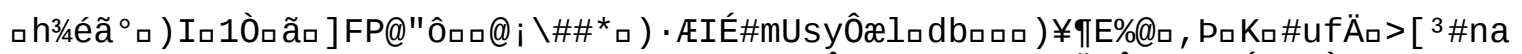

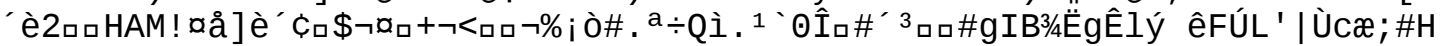


AÍÑòUtò> \Å§̊ロ ¿ ' ÎÁ¥|\#\#ロ\#rèô" 


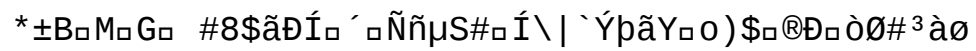
$\mathrm{Eb}^{\circ}$ 
\#ÉÓe|\#๑Úロ1 


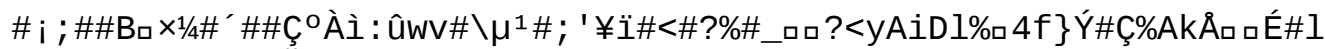

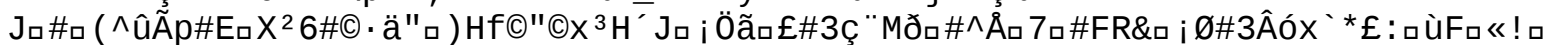

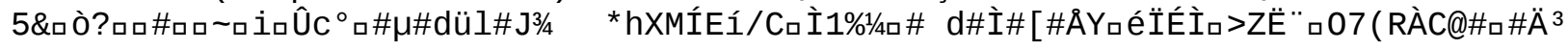




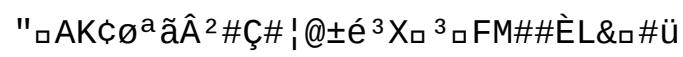


\#

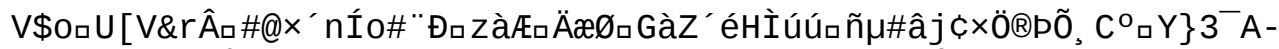

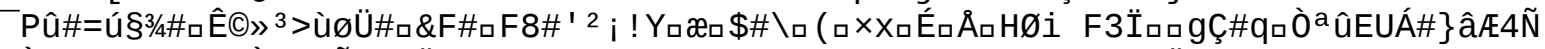

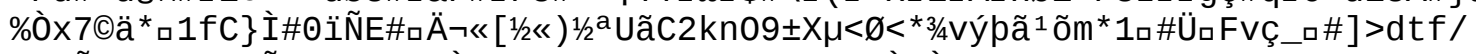

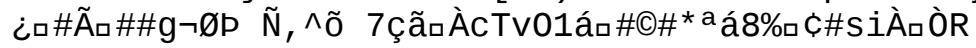




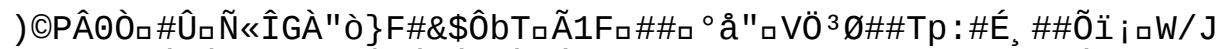

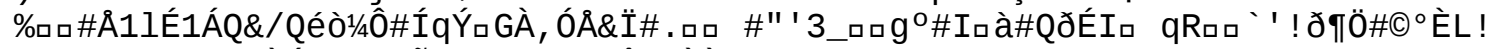

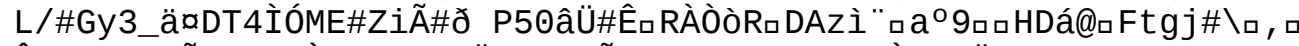

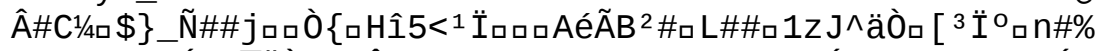

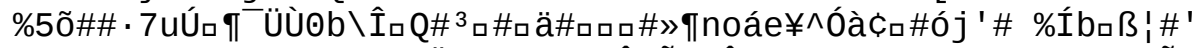

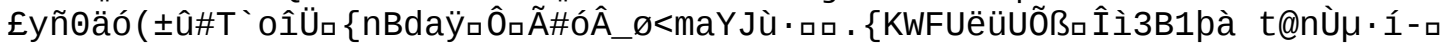

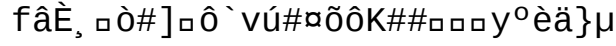


DC/ロ:\#ËEX 


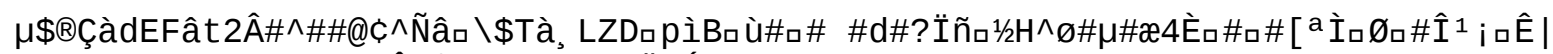

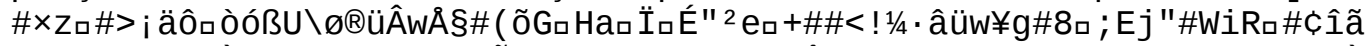

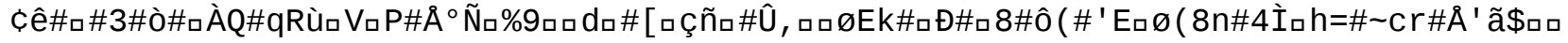


\#èóe0ñ \pm Ãt Âa âEE< $<^{\circ}$ Üè + \pm ðòôa

\#! \}ÜS\#AÊ̂VíjL, , 'd ${ }^{3} S \emptyset_{\square} l_{\square}$ (ðåÄÙE \{

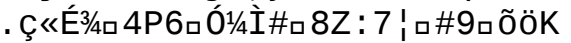

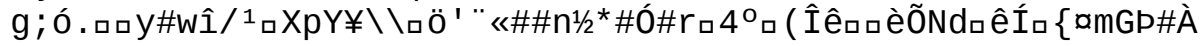




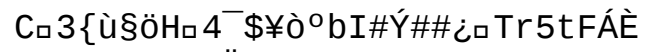

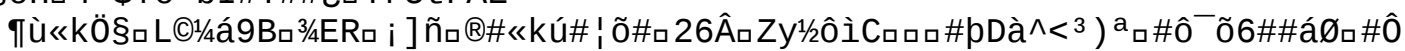
\%! Ü\#åsVí\#ß\#5À\#LÅ] 8WÇ1

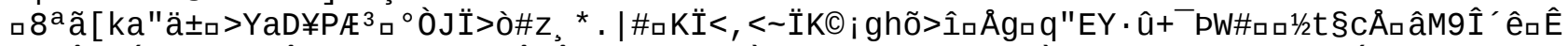

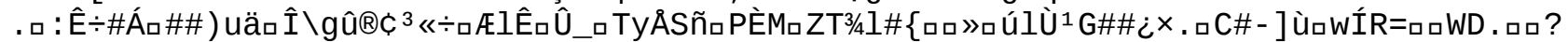

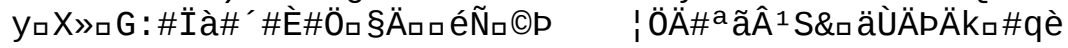




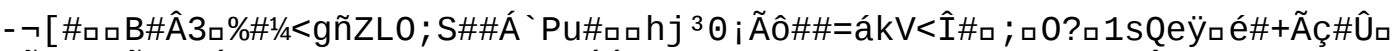

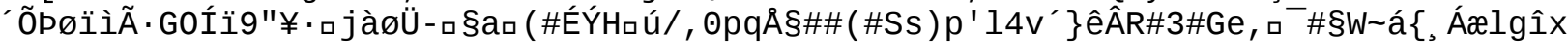
- व \}hxÜCô

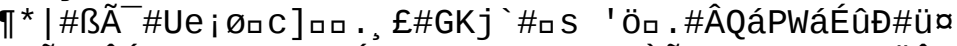

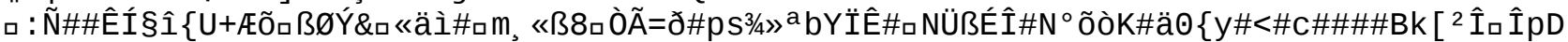

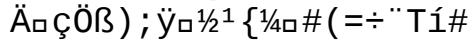




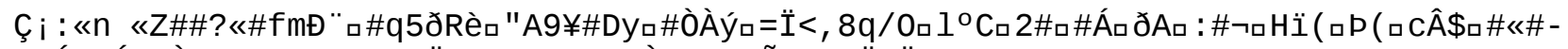

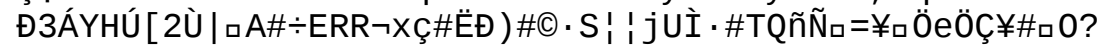

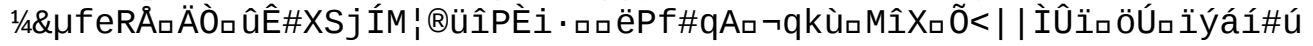




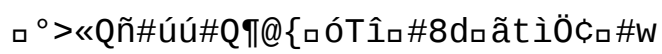




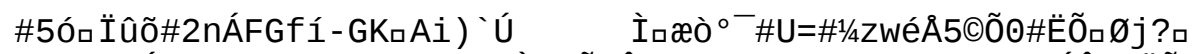

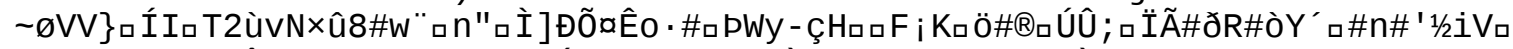

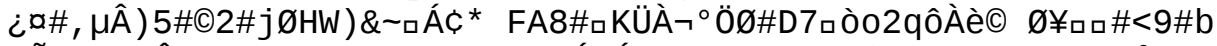

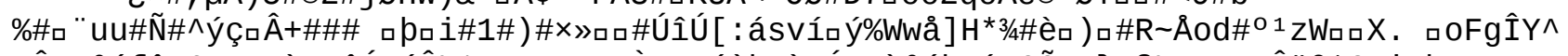

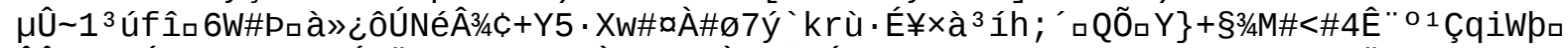
ÔÊë1 êNÉSaquú; ;

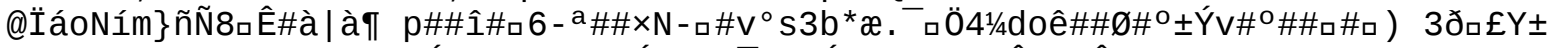

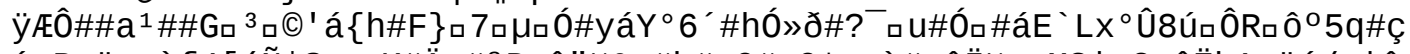

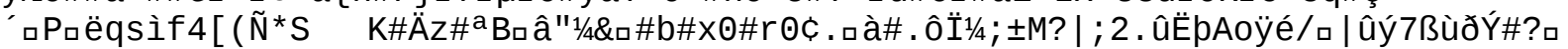

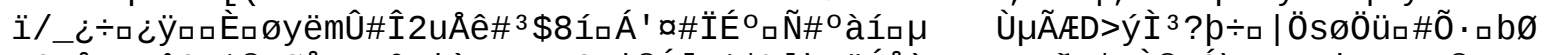

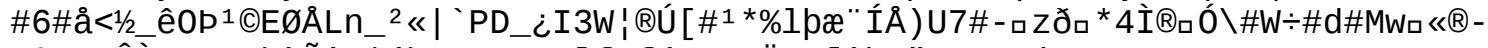

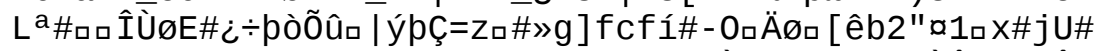

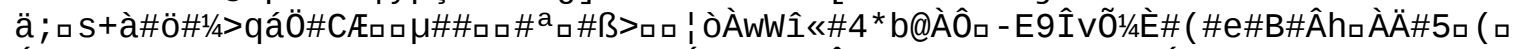

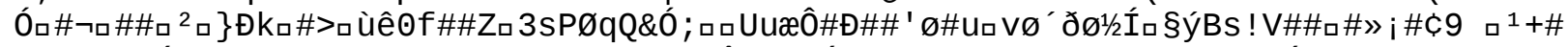

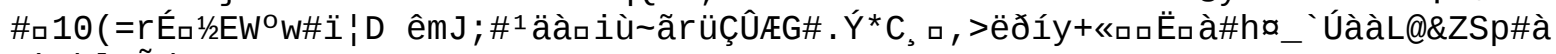
$\mathrm{X})^{1 / 4 \mathrm{~b}} \mathrm{~b} \sim \tilde{\mathrm{A}} / 4 \# \sim$

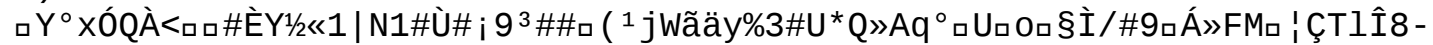

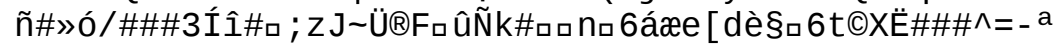




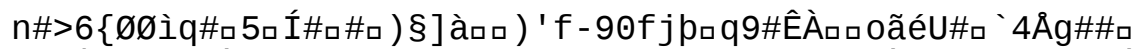

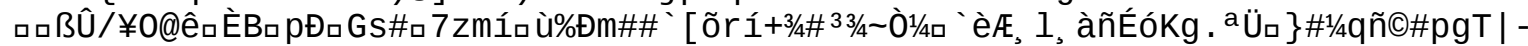
L\#4\#6£\#\#]fí, ÝMĐZàD ${ }^{\circ}[. \varnothing$

D\#Ó\#ÝP口\}\#ì | \#? «\#, £\&ם

$\emptyset_{\square \square{ }^{3}}{ }^{\# p} \times_{\square}, \tilde{A} a^{3}$

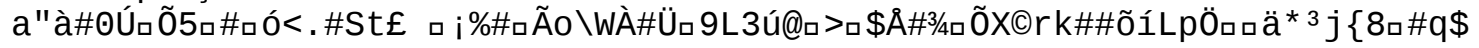




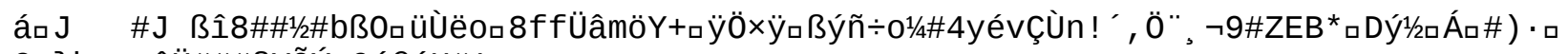

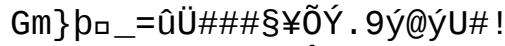

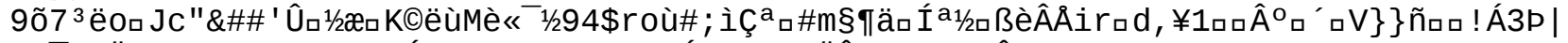

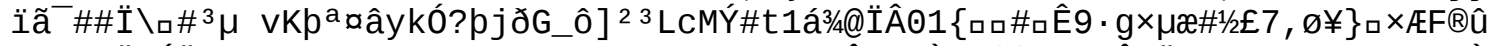

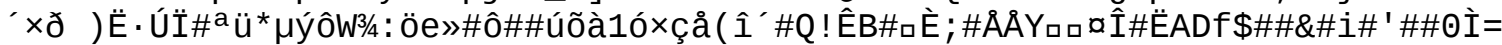

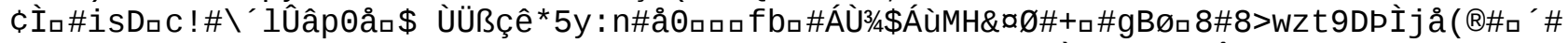

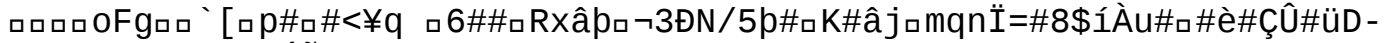

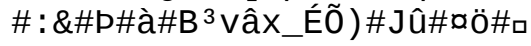




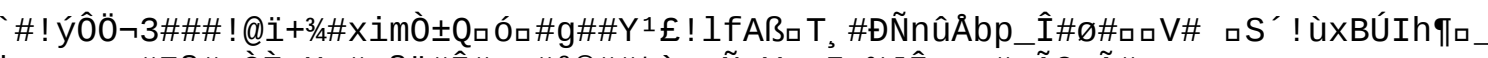

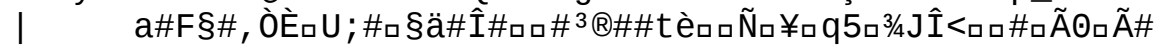




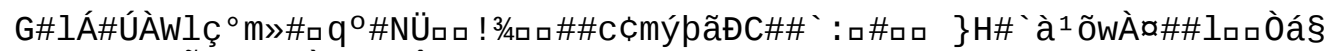

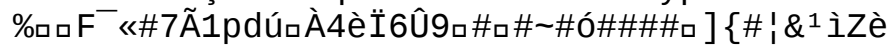




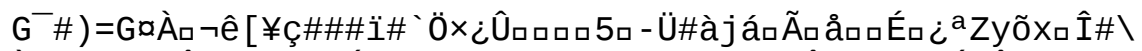

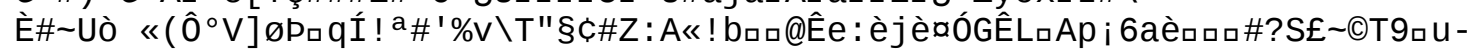

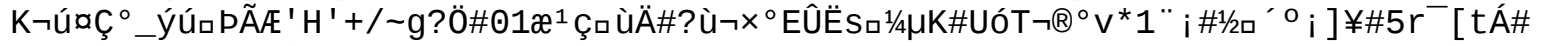
CR9\!®7]ฉí Jãå<

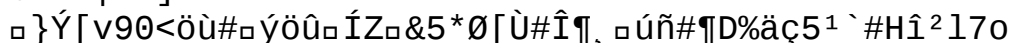

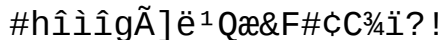

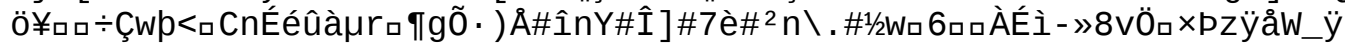

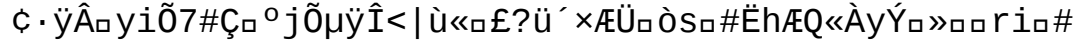




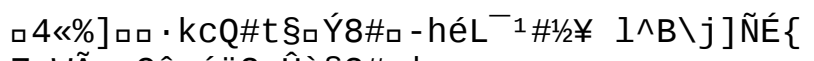
ZpWÃmrÇîaóë2ロÛì2\#ロ! 


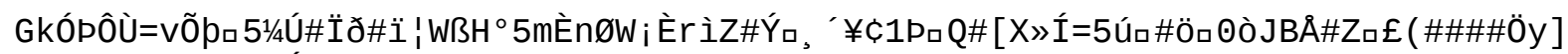

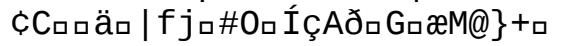




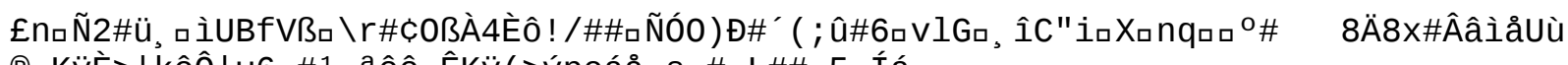

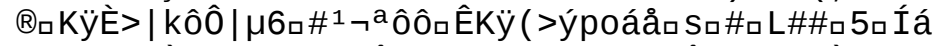

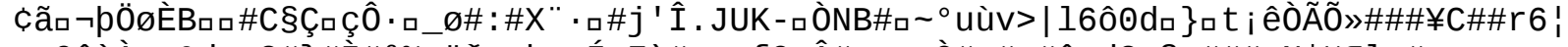

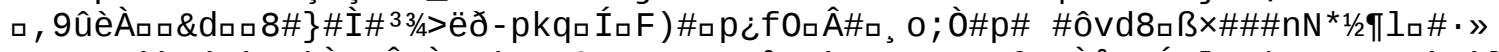

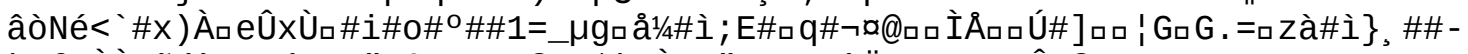

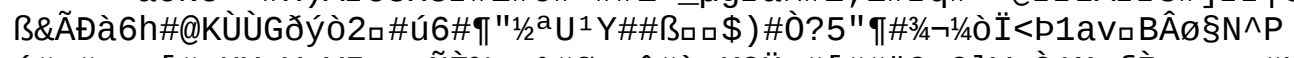

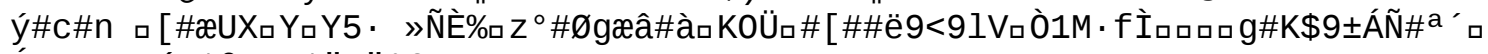

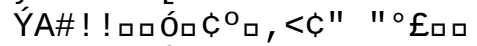

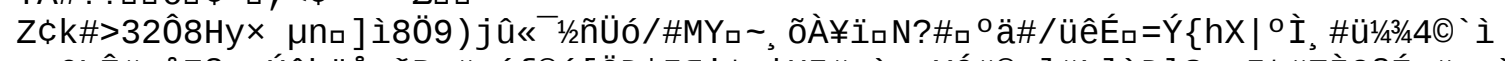

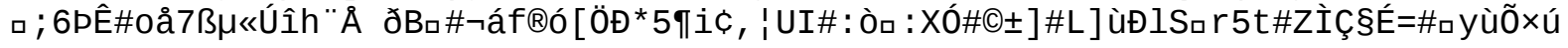




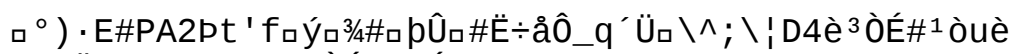

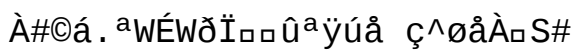

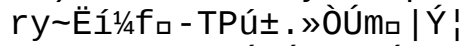

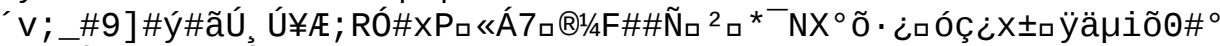

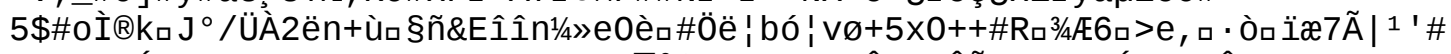

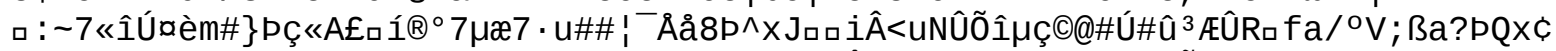

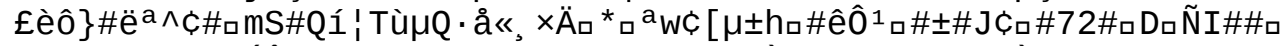

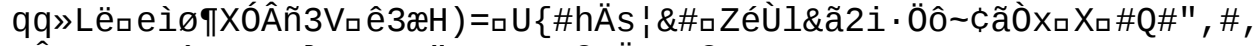

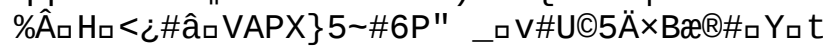




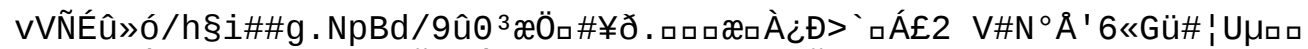

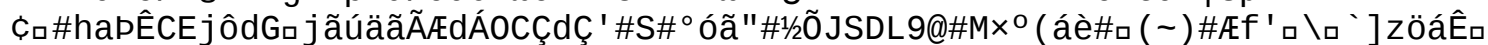

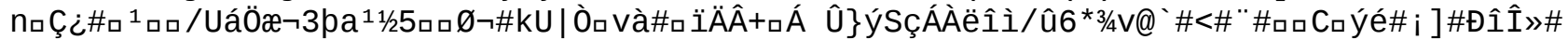

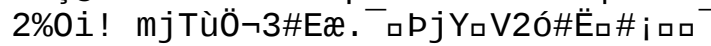
aWU^pÇ3/4â+'XE 


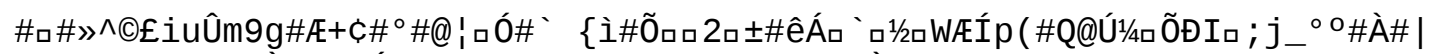

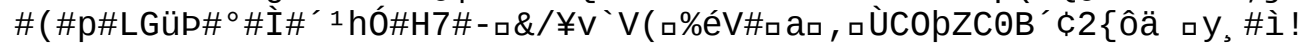

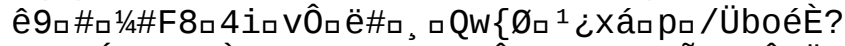

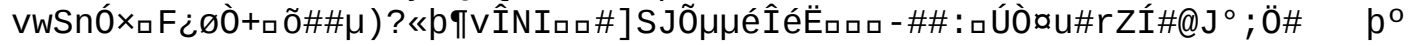




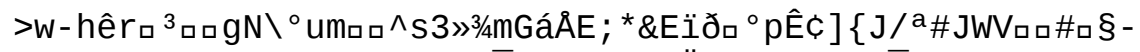

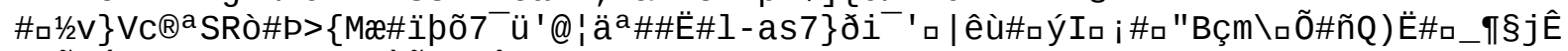

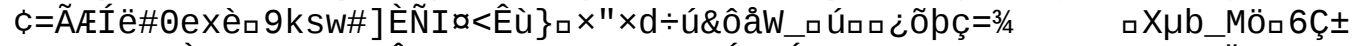

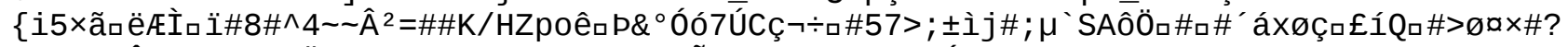

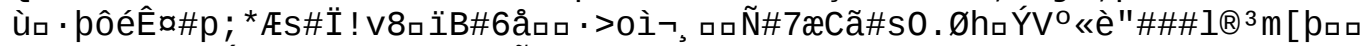
$\# £$ "V

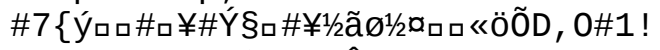

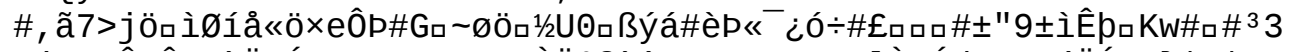

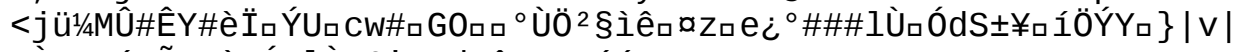

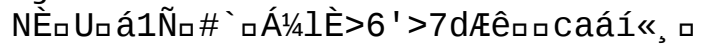

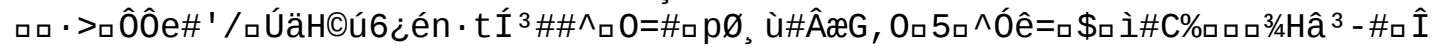




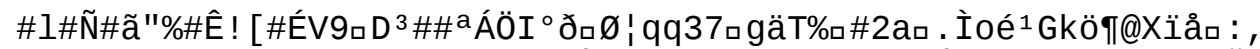

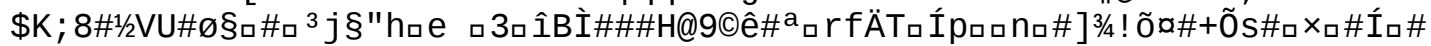

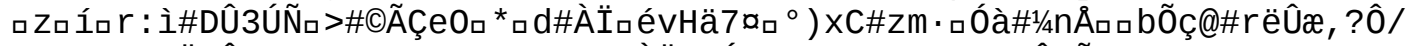

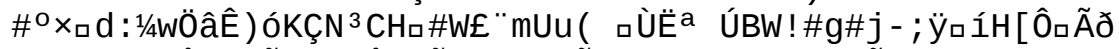

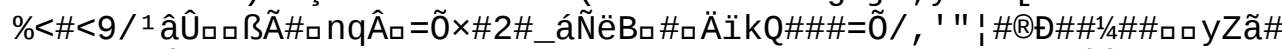

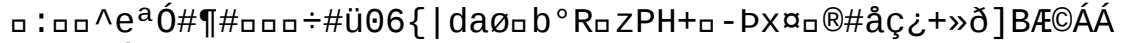
口ÓMHÖÚýÇ $O$

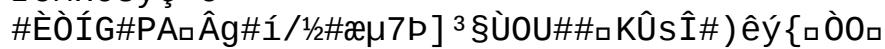




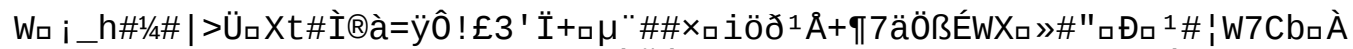

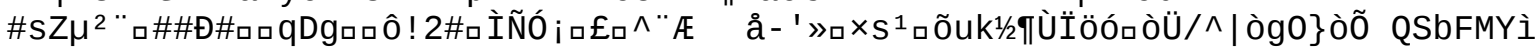

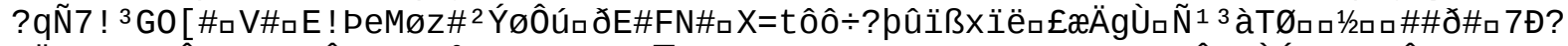

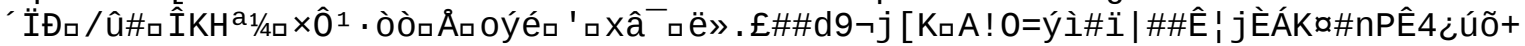

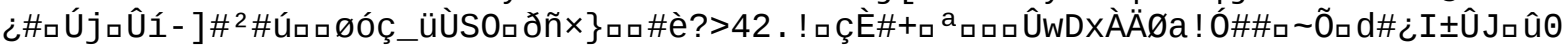

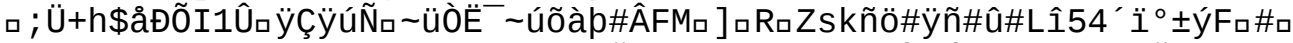

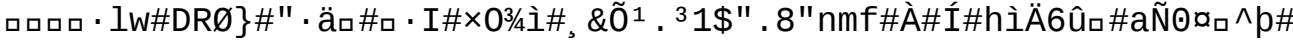

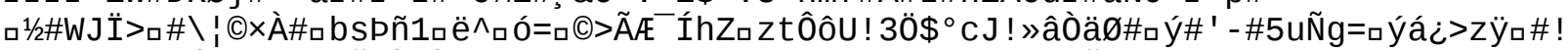

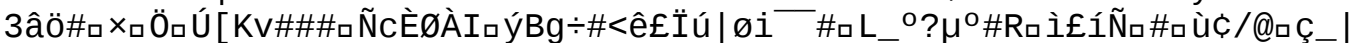

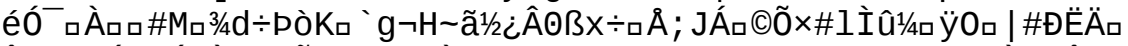

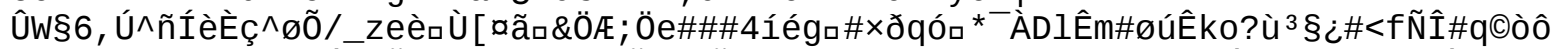

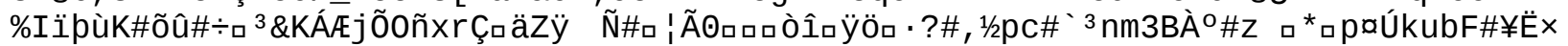




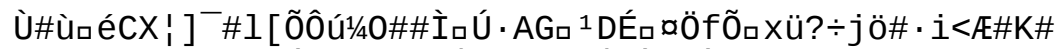

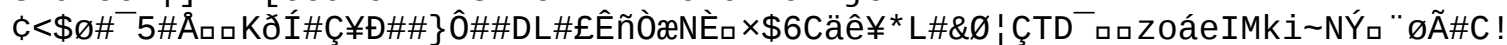

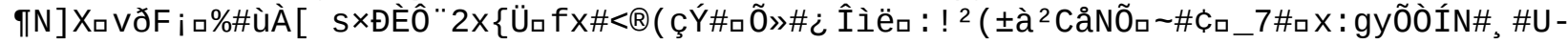

\#- ]

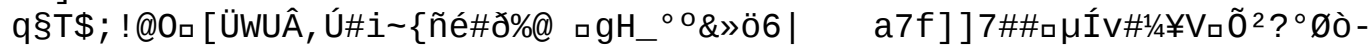

òà eàĐ $i: p \times D \#,{ }^{*} C 5 \# \square 5$

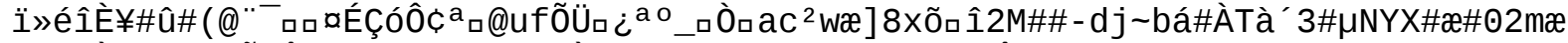

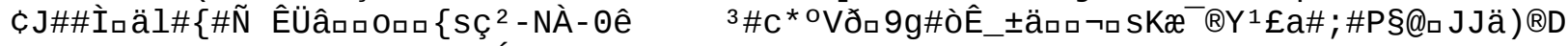

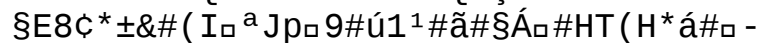

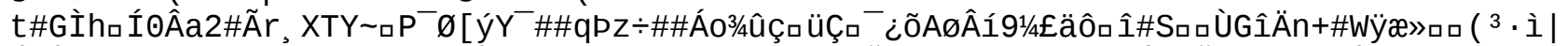

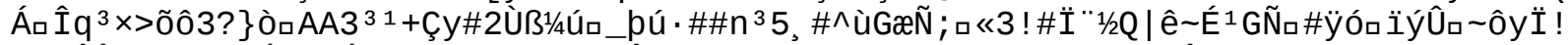

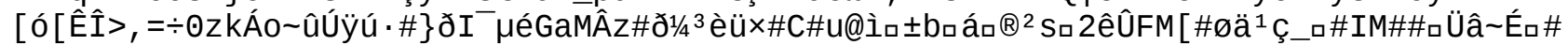

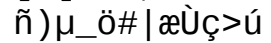

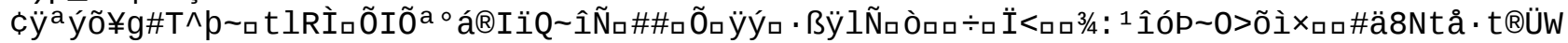

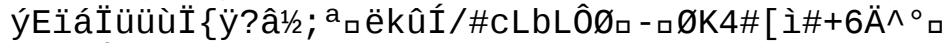

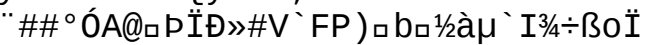




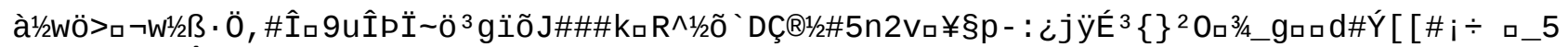
ù $\ddot{y}^{\circ} \tilde{n} 6 \circledast I 1 \hat{E} u ̀ 2$. $u$

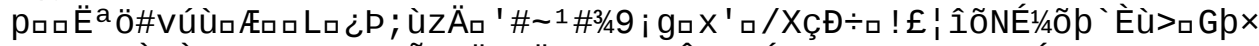

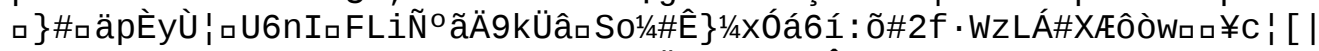

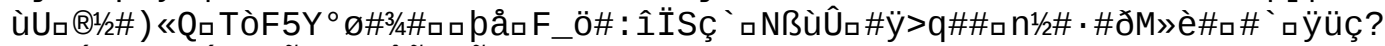

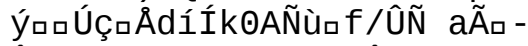

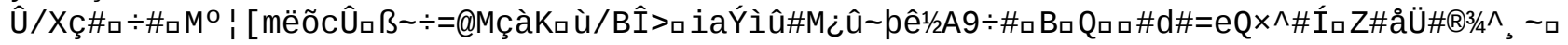
EF_5løhör ${ }^{\circ}{ }^{3} \#$ j

\$\ÈMÚ2L/ÁD\<ÆØúX 


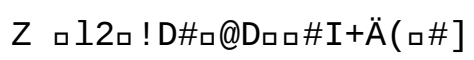

Mь 


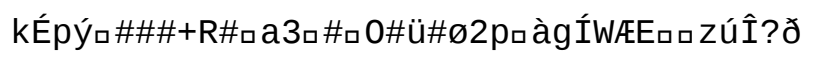




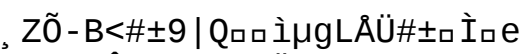

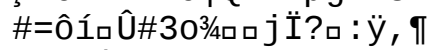

$\wedge_{\square}<\div 0 \hat{0} \ddot{I}<{ }^{3} Đ \#$

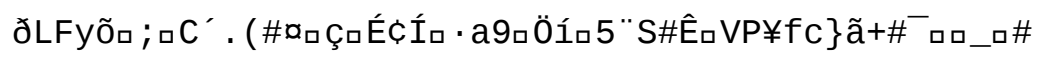




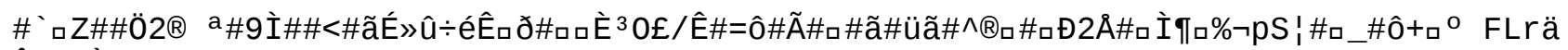
0ิ58nÙ ·̈̈口

İ\} 


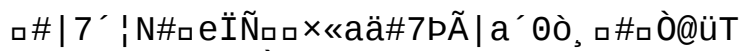

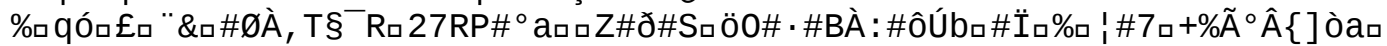

$\neg \mathrm{Z \# \square} \mathrm{g}^{2} \mathrm{y} 8$ 


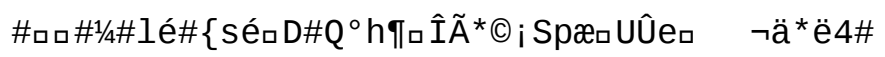




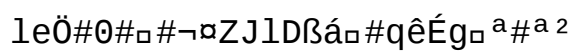




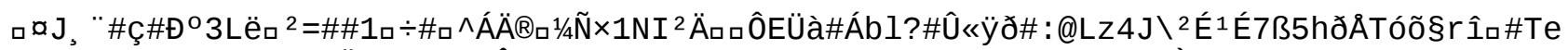

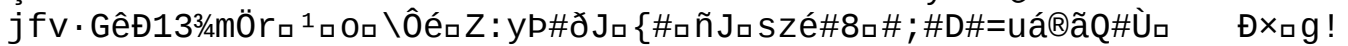

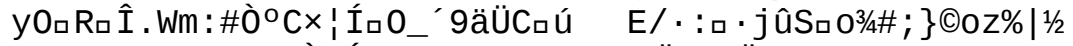

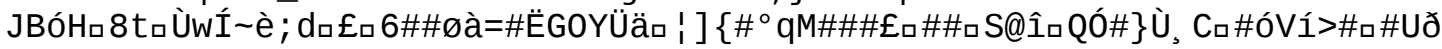




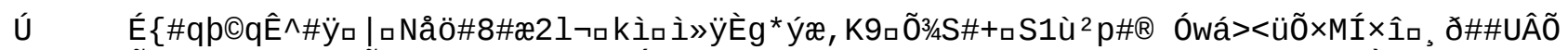

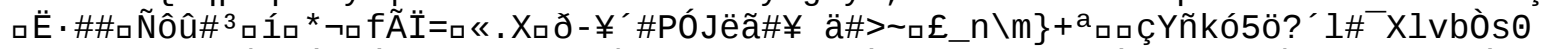

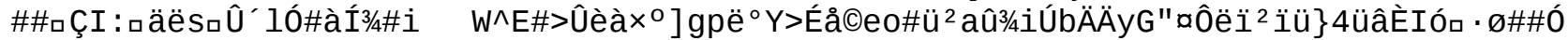

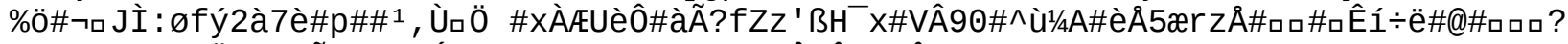

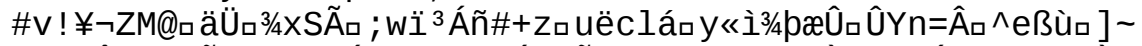

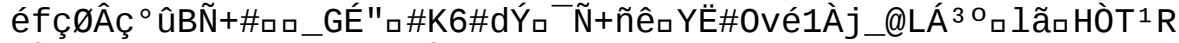
\%Àt\#: : «EU\#hBY"ם"ם\#ロ4ÒXı \-\#f ฉý\#-ロ\}ß| 


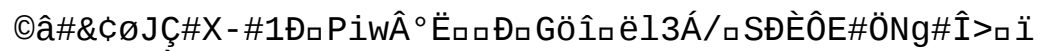

\#sËロ\#\#*ǍbCâ

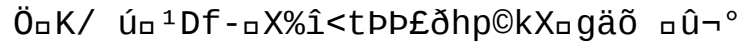

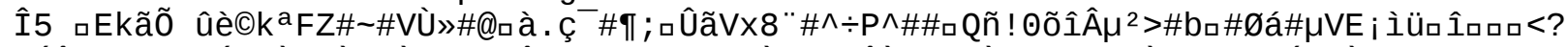

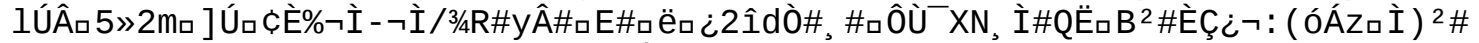

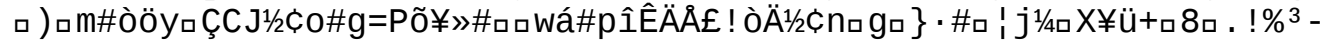

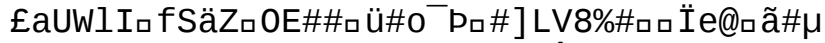

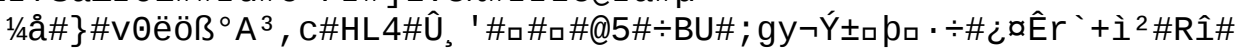

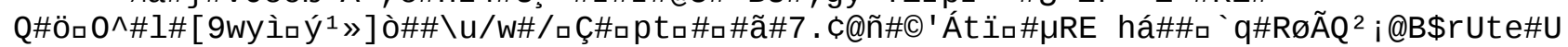
ä $\left(\Theta^{\circ}>\right.$ 


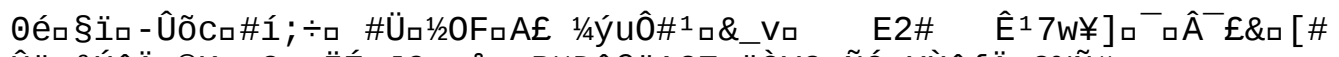

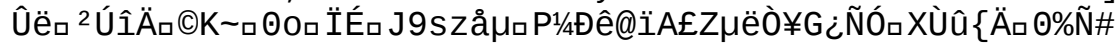

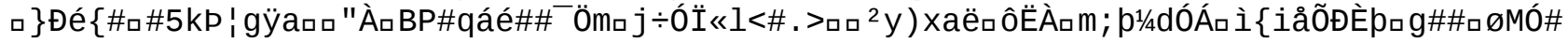
: ØàÆJä

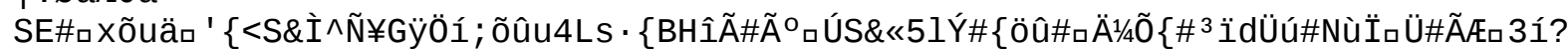

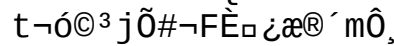

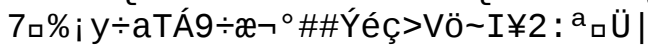

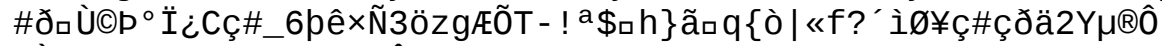

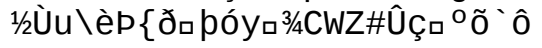




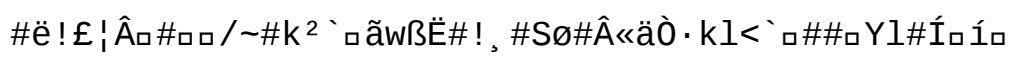




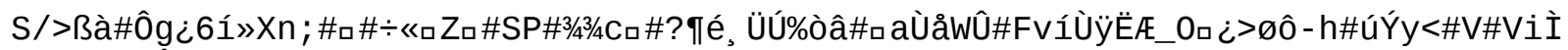

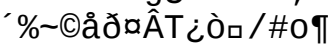




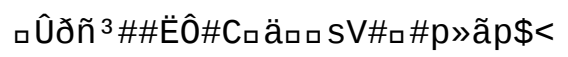




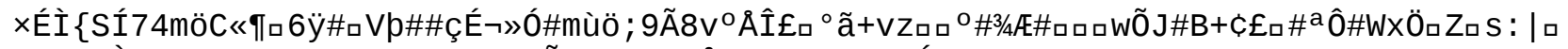

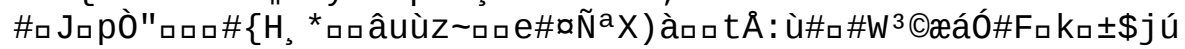

C口ù+âN\&ß9ûà¿SËß\#qp ${ }^{3} ; z q a ́ \# a \_R E+; \hat{o}^{2} \mathrm{j} \# \varnothing \#$ 


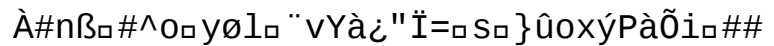

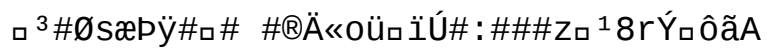

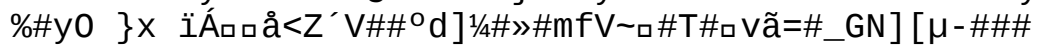

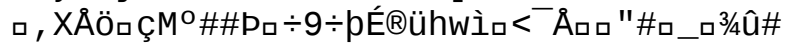




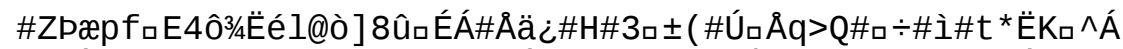

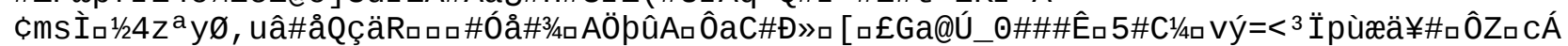

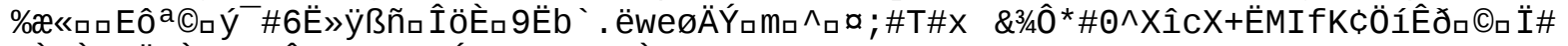

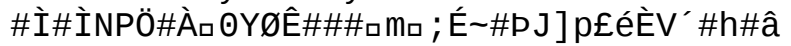

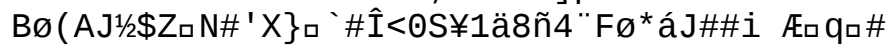

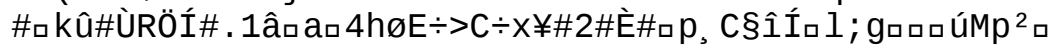

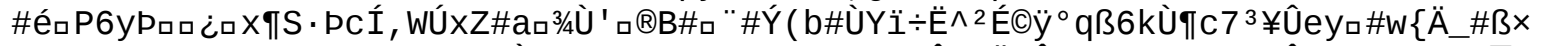

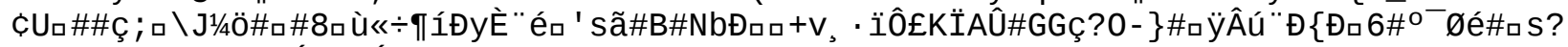

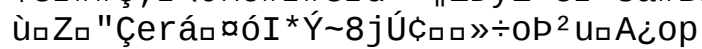




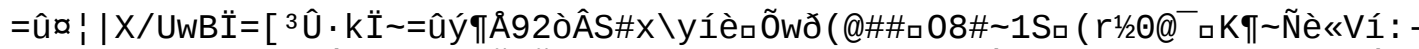

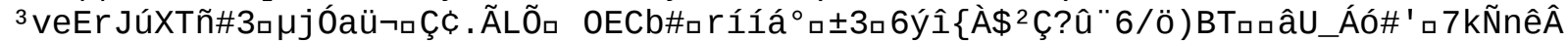

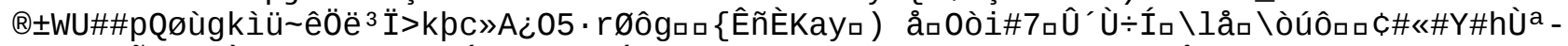

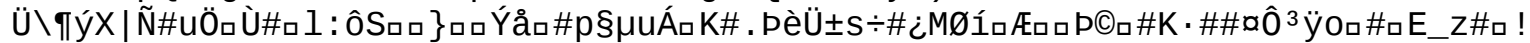

$\left.4 \ddot{i} \tilde{N}{ }^{*} b \ll G a \hat{I}\right\} V \hat{i} o ̀$

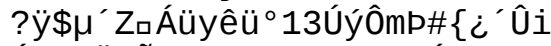

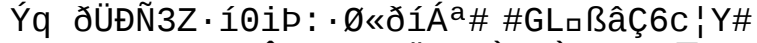

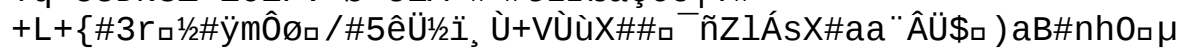




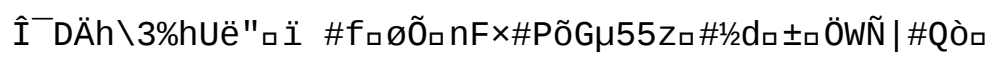




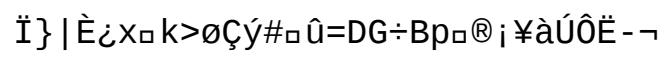




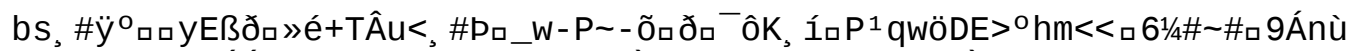

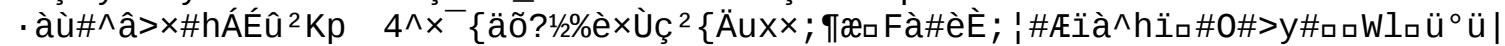

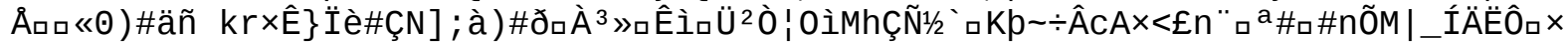




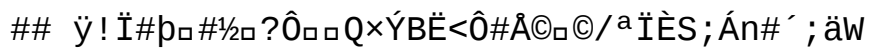


口\#Hı_a 
ð7ロ\#Đ\# 


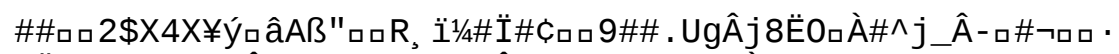

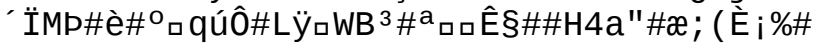

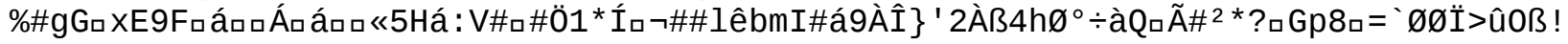

£\#| ${ }^{3}$ !

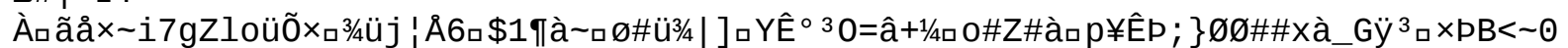

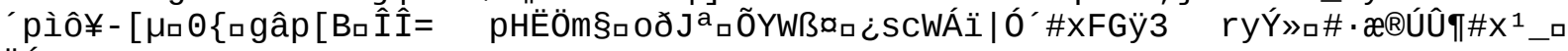
ÄÉj\#¥! 


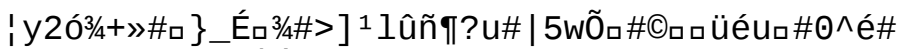

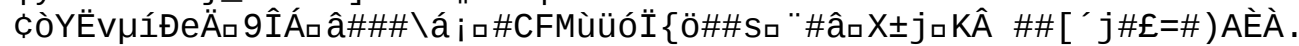

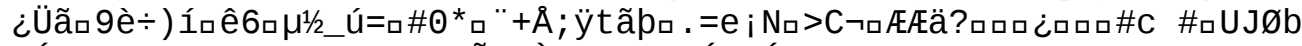

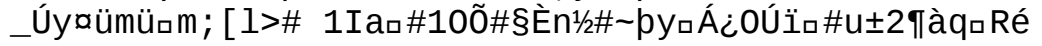




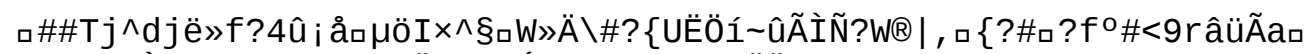

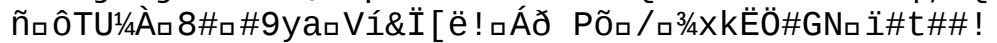

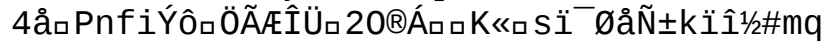

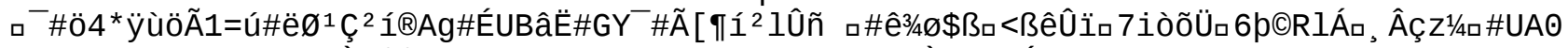

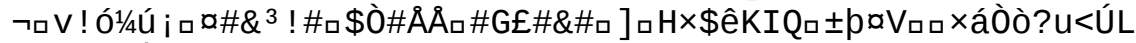

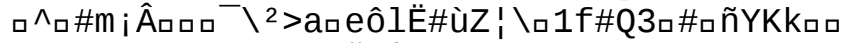

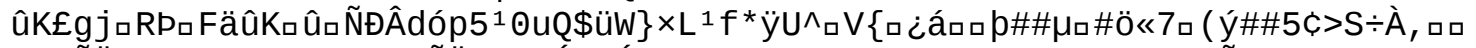

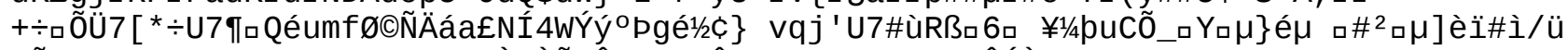

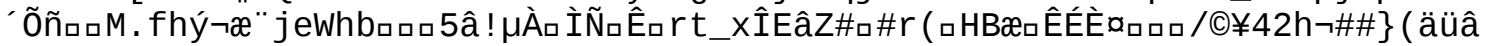

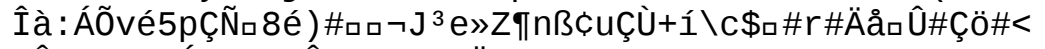

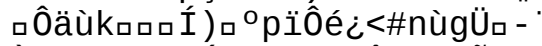

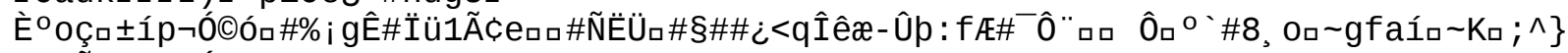

口] ùÑ $)(\cdot\{$ aÁıgú 


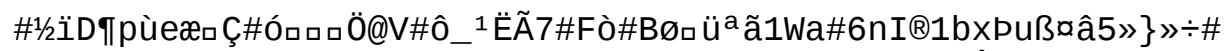

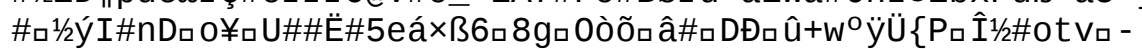




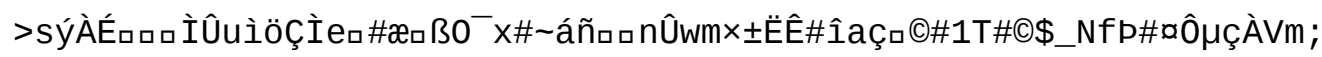




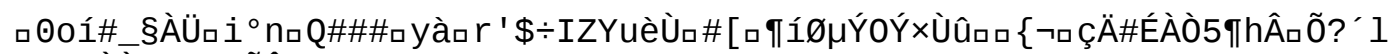

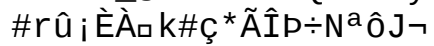




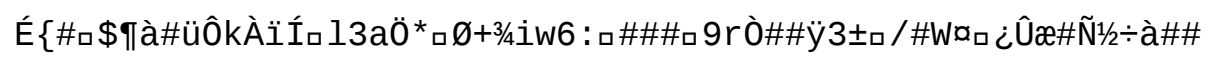




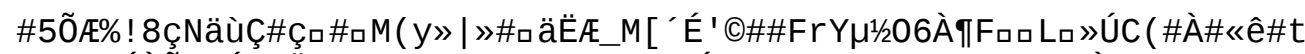

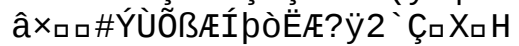

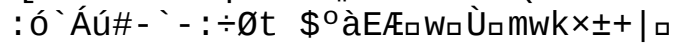

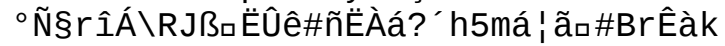




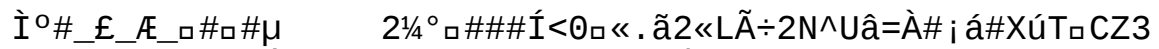

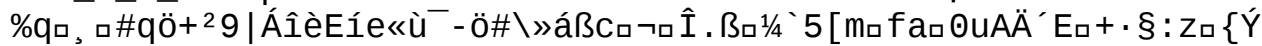

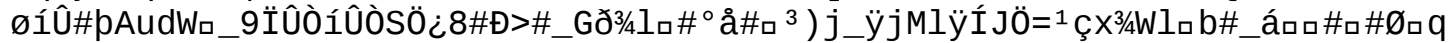

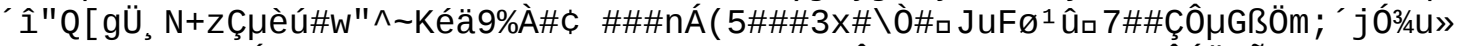

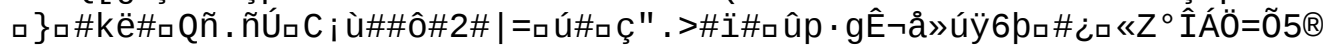




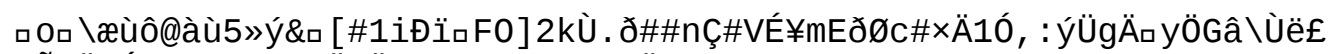

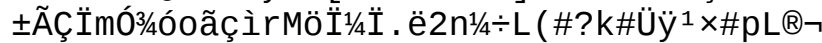




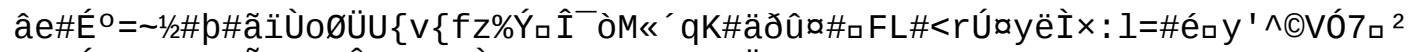

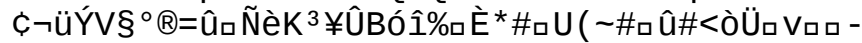

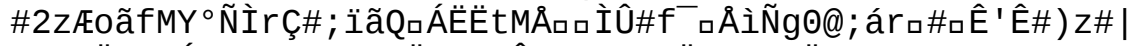

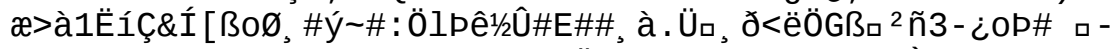

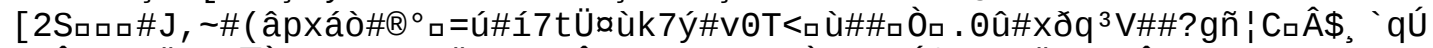

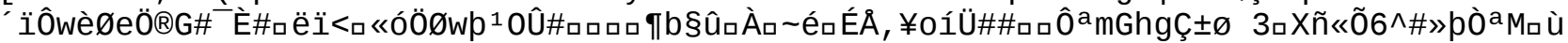

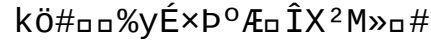




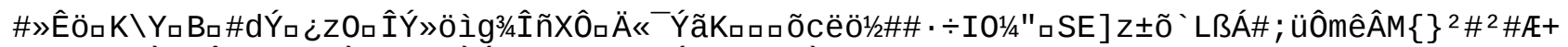

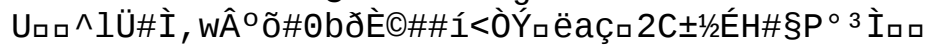

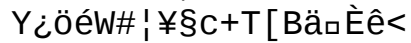




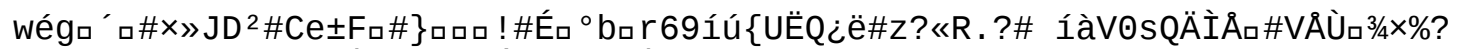

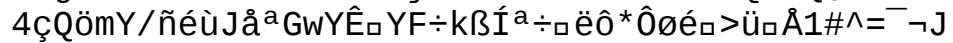

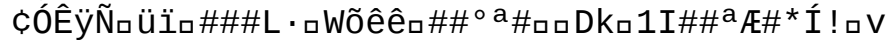

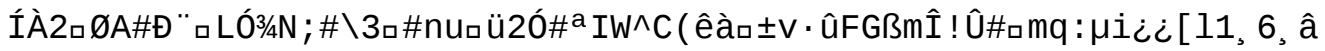

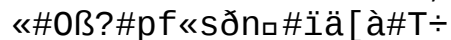

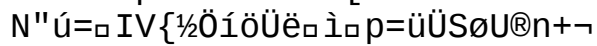

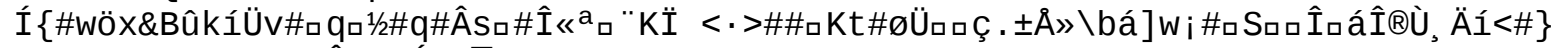

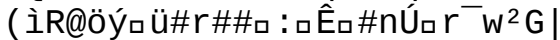

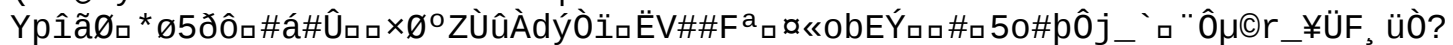

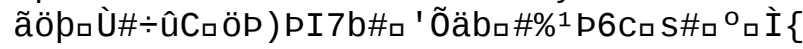

E ${ }^{\circ}{ }^{\circ} \mathrm{GÂUAA} K \#$ 


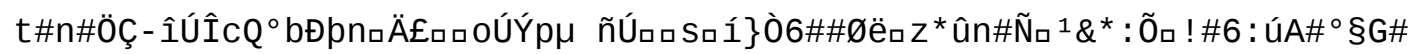
ar\#a_"\#ם 


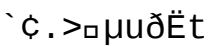

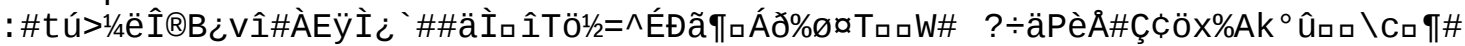

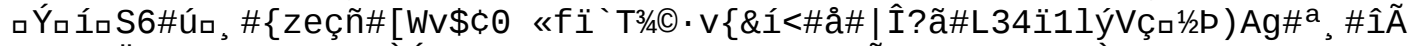

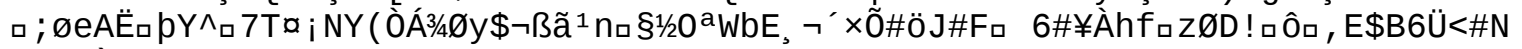
\#æ\#Rİ

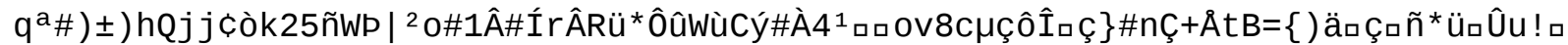

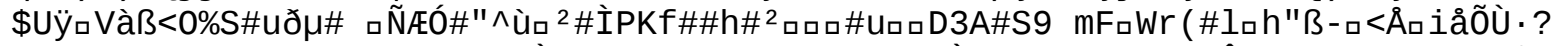

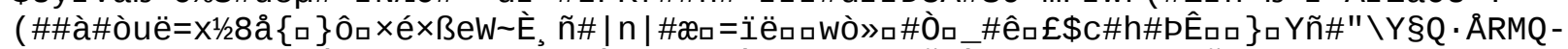

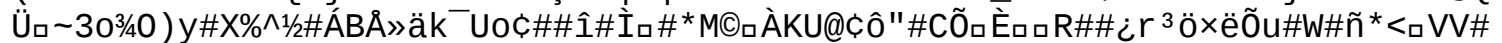

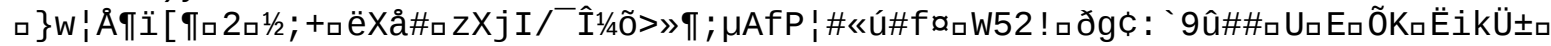

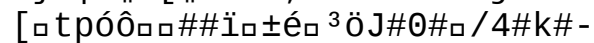

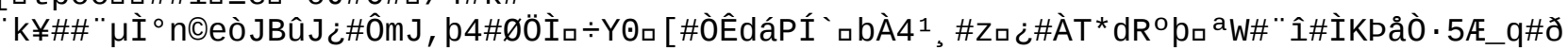

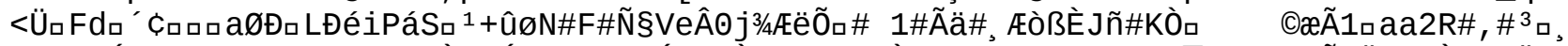

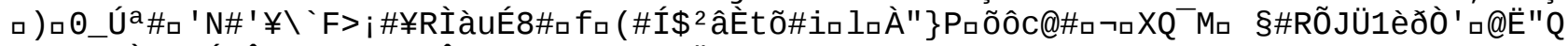

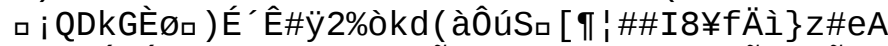

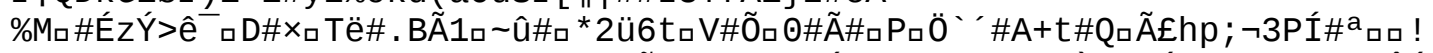

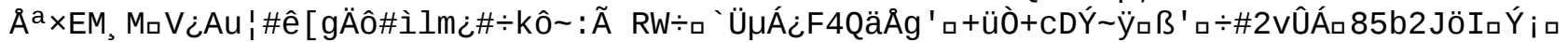

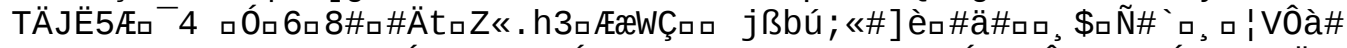

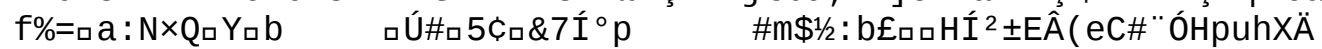

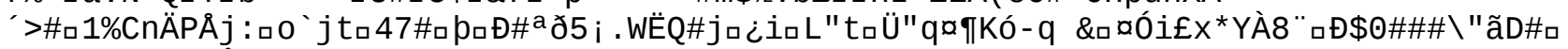
qu\#口£\#A\#â\#SÂ?\#\&ם/f

kiec\#AĖ\#"ם]\%Ôaúロ_;\#\&ם 


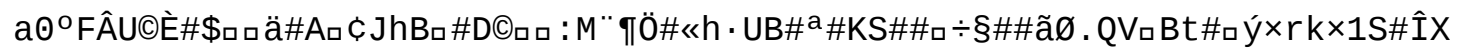

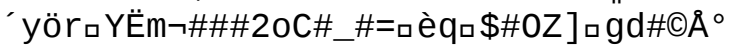

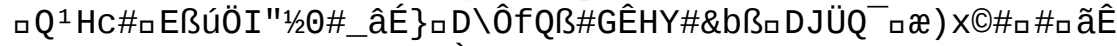

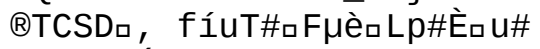

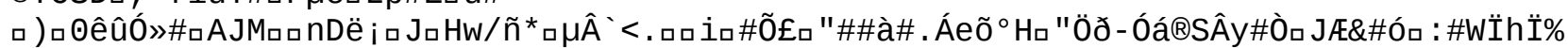

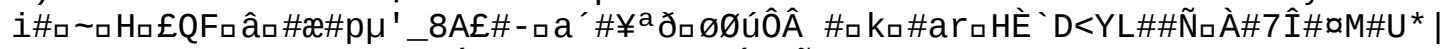

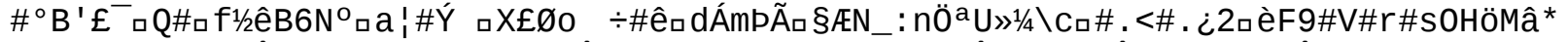

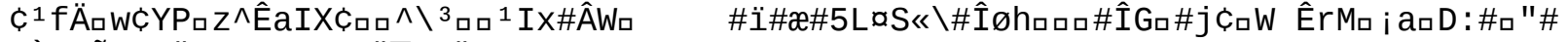

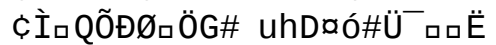

口l\#\#\#1n' ÎD\#Êdıâ^"

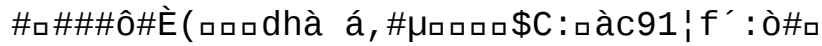

åptDC\#*?«0'c\#"8 


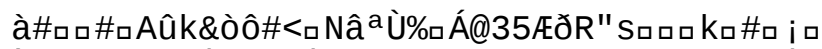

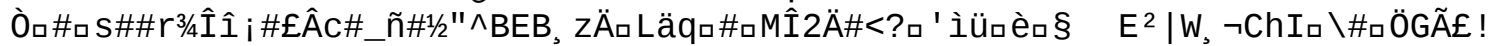

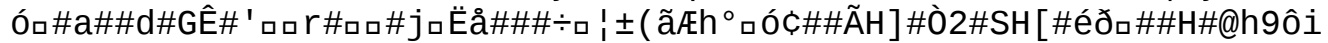

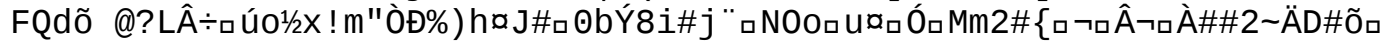




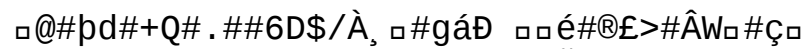

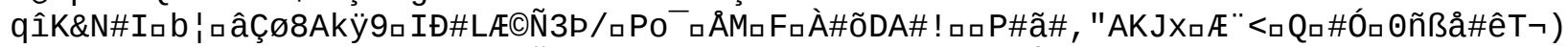

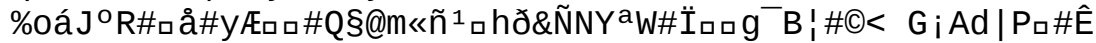

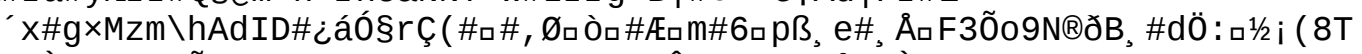

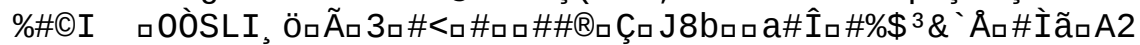

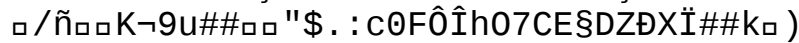

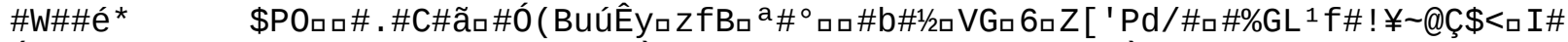

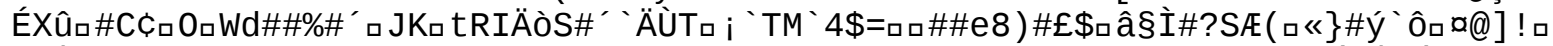

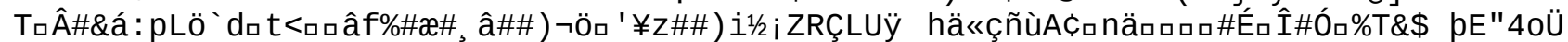
P口\#\#3^)RK - -

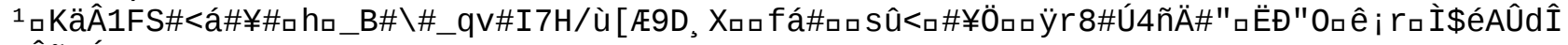

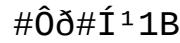




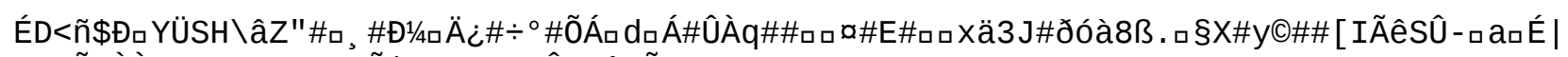

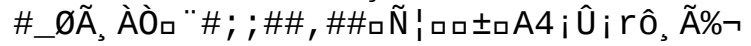

i

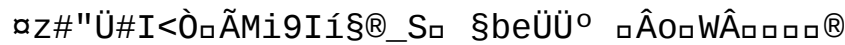




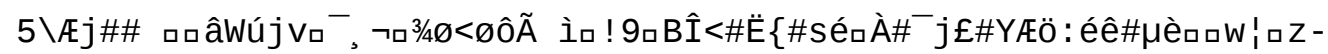

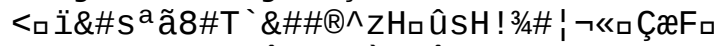

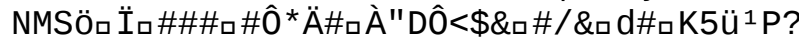

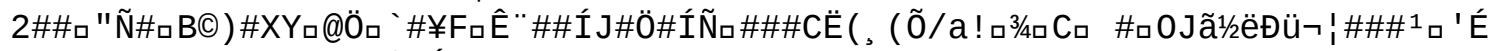

\$口 J\#S<○ß\#ロTNr\#ü"òÅń2

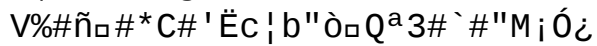

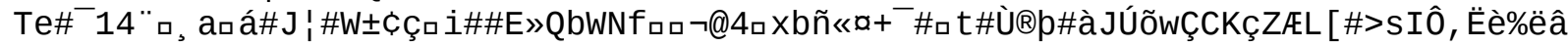

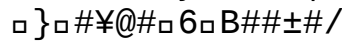


b1C̣ı\#ua, ÖN"U\#Úロ 3ÚĐ1"\#ÆcUSa

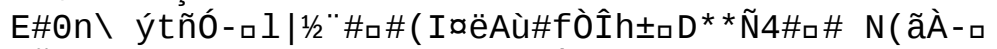

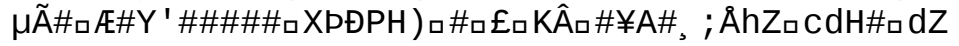




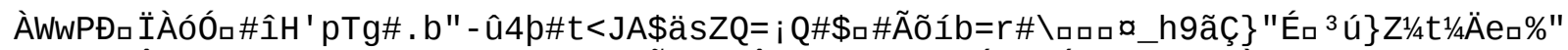

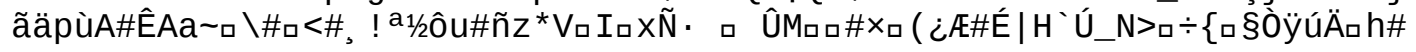




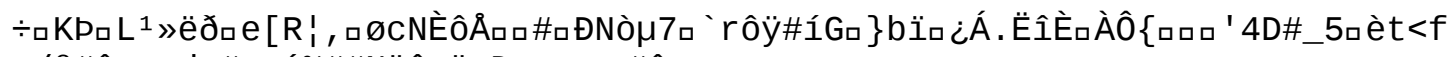

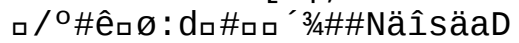
\#â

$4 \hat{\mathrm{A}}, \mathrm{v} \# \% \mathrm{r}$ 口

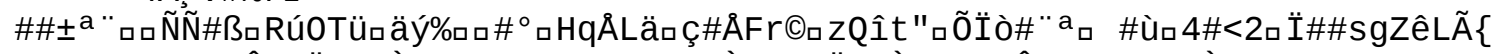

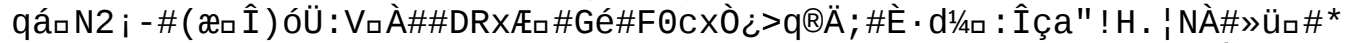

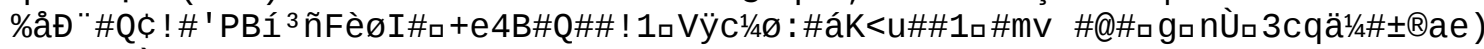

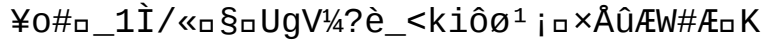

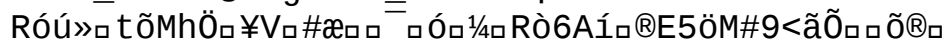

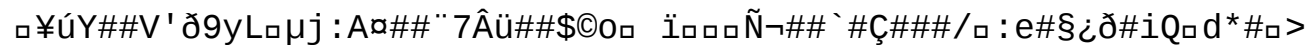

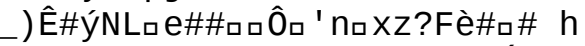

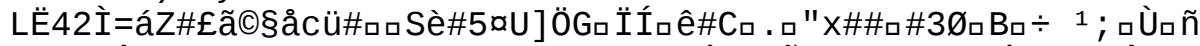

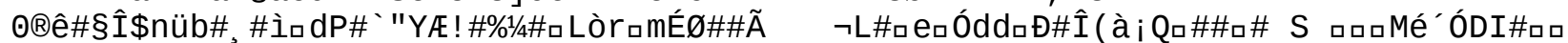

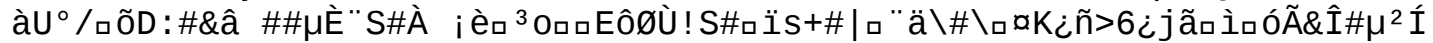

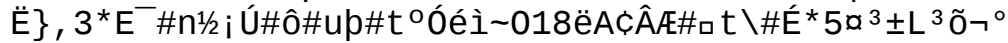

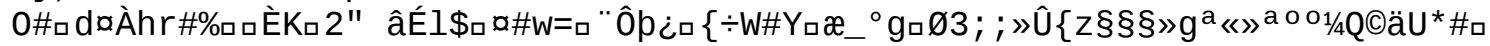




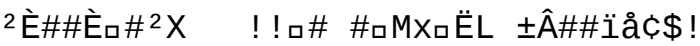

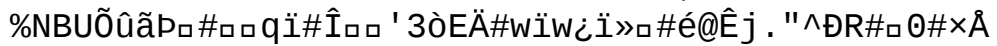


ÈXÃEWÝàÌs«\#qa\#aÉ"lIUaLò6b¿1っËq10̂6

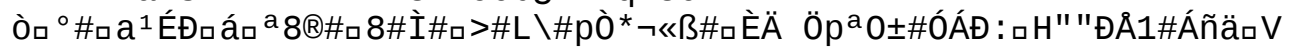
\#]WK2Q\#ѐJ 


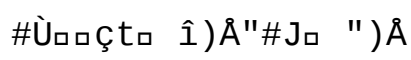




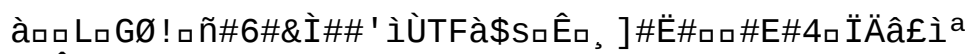
quÊE.\#

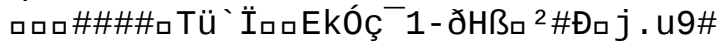




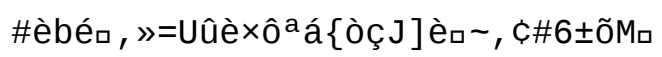




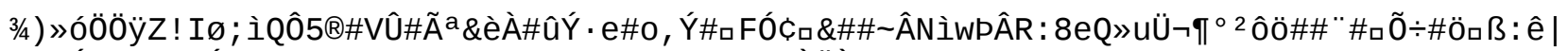

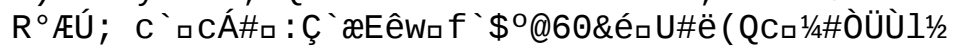


Í\#ê@\#Ò\# ( õ !\#n 


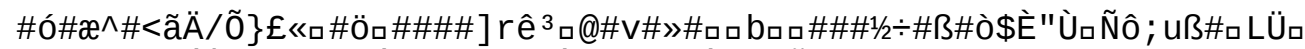

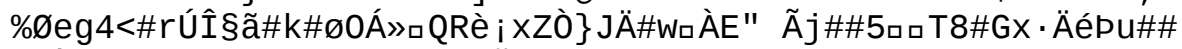

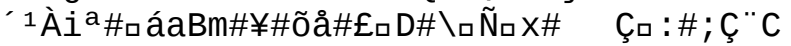


${ }^{1} \mathrm{n} \# \#$ \#\#84ra\}\#\#ãî 


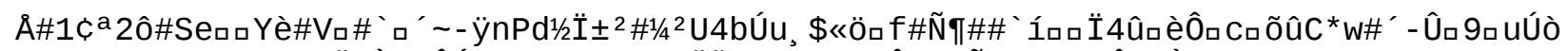

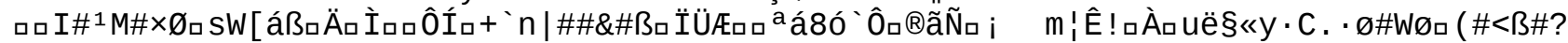

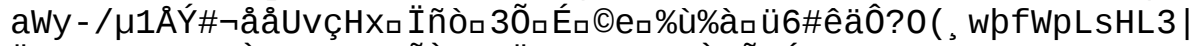

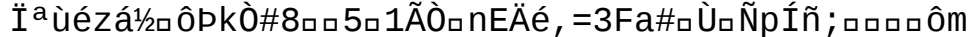

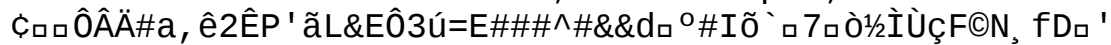




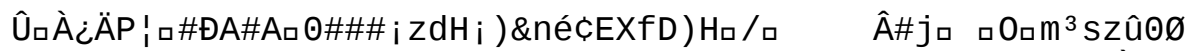

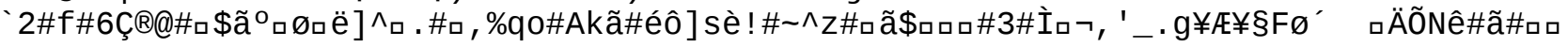
£。ë_\#\#

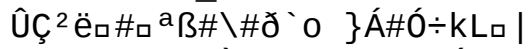

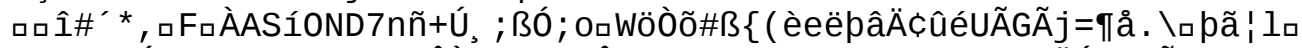

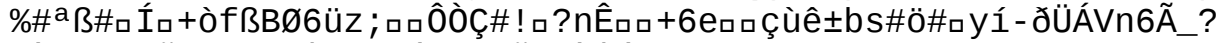

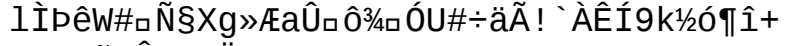

${ }^{1} \emptyset_{\square} \mathrm{Q} \tilde{\mathrm{I}} \#$ \# $\mathrm{eÖ}$ 


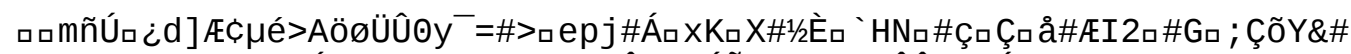

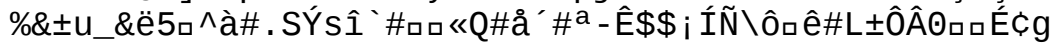




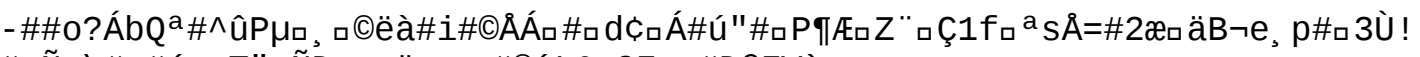

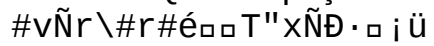
\#`L\& CFWa\#D@FWè 


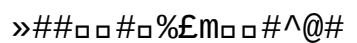

JffdtWÑ $\square$ $0 \tilde{0} 51 \tilde{n} \sim{ }^{\circ} \notin 1 \square \mu$ 


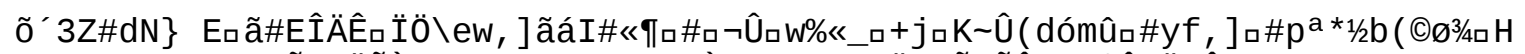

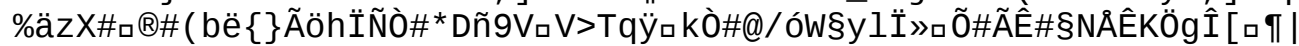

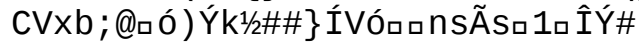




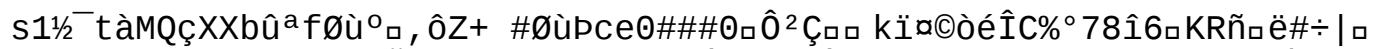

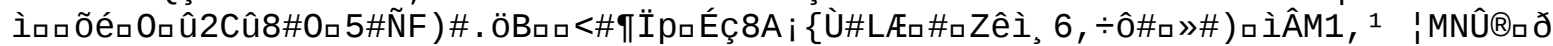

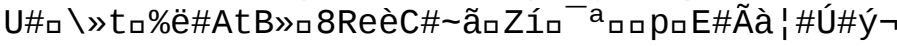




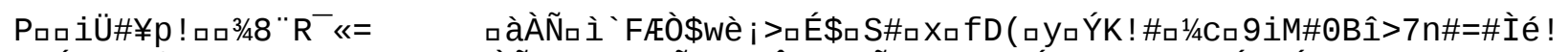

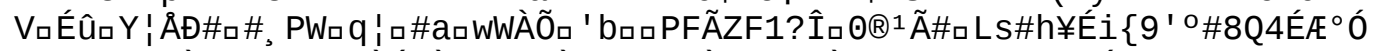

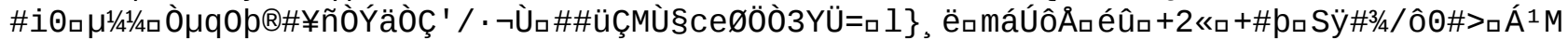

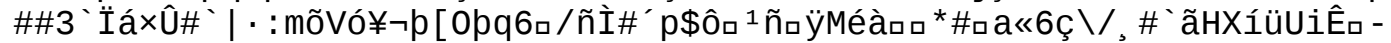

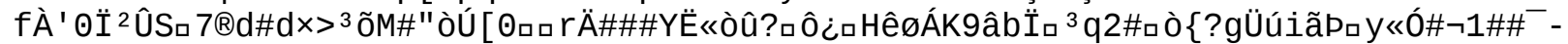

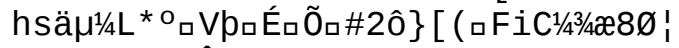

( $F \quad \#$ pî́ma \#P

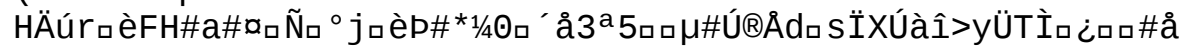




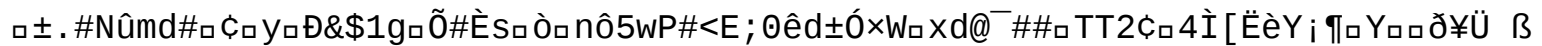

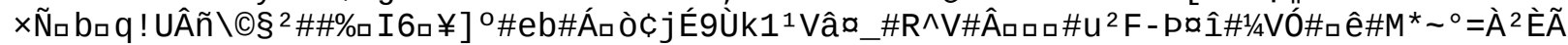

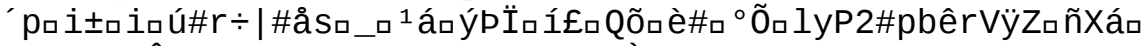

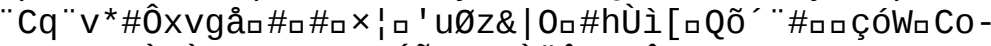

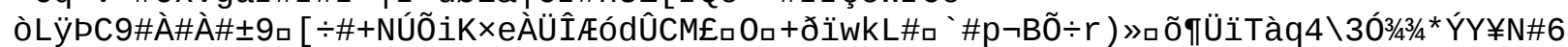

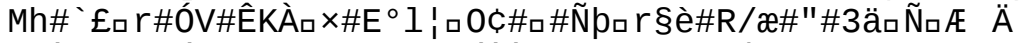

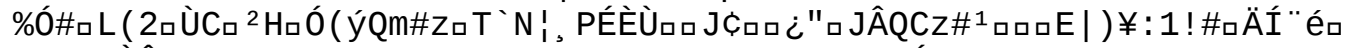

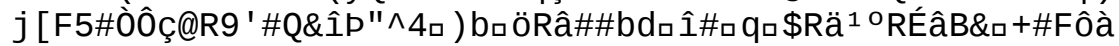

${ }_{1}^{1} \mathrm{MN}$ 


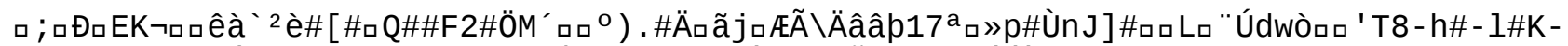

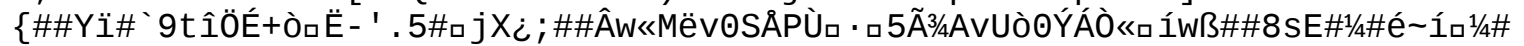

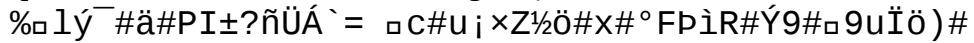

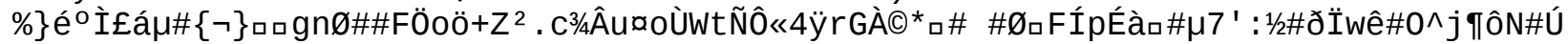

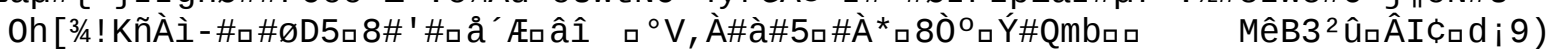

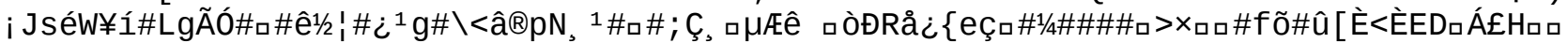

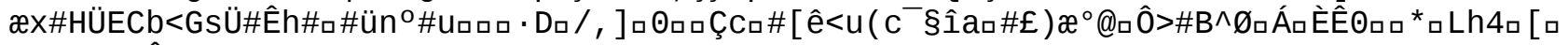

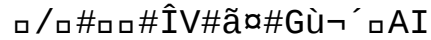

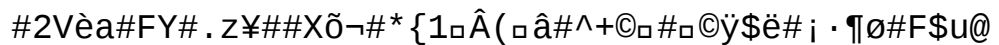

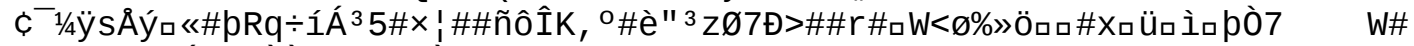

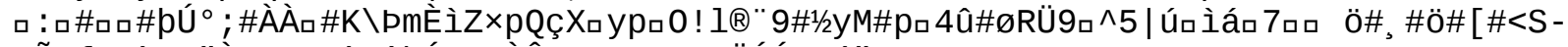

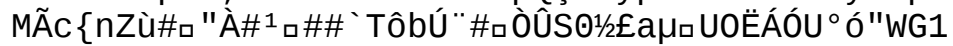

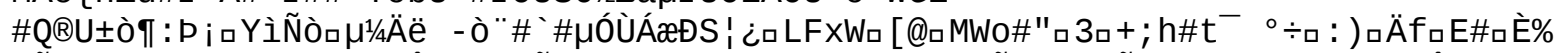

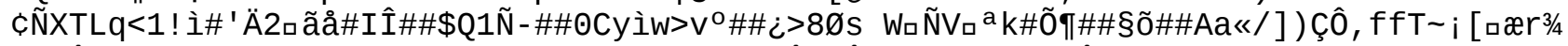

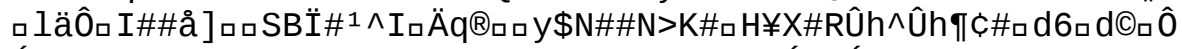

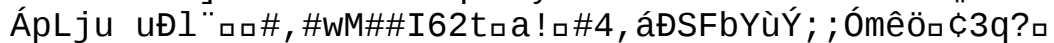

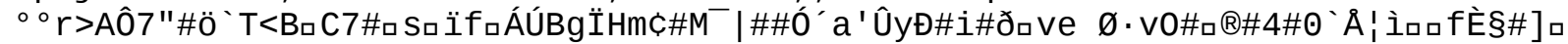

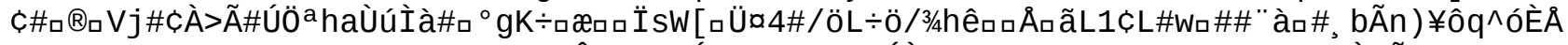

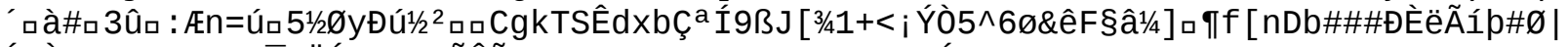

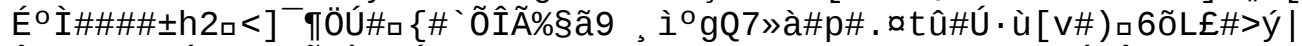

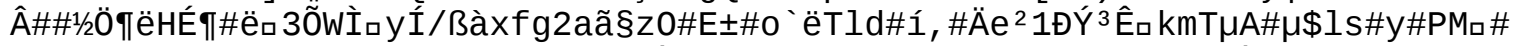

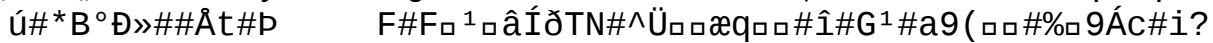

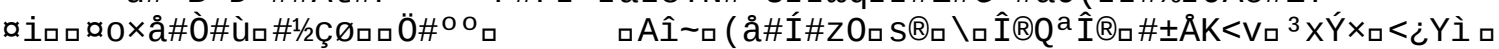

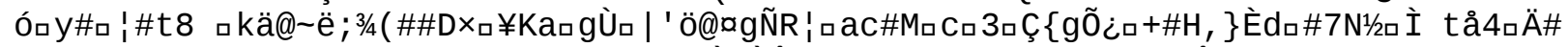

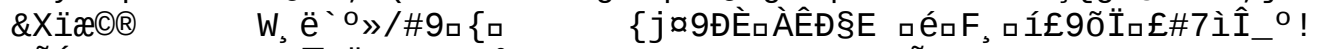

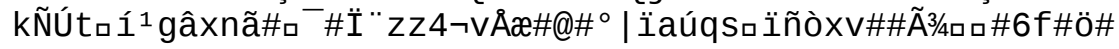




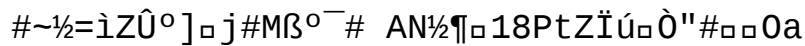

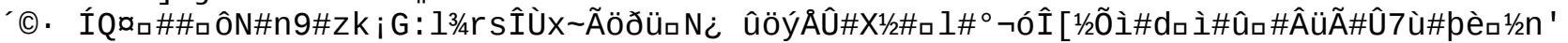
口 \\# Àkop

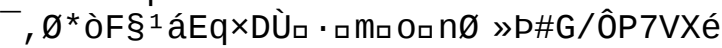

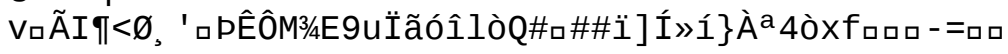




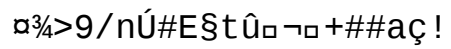

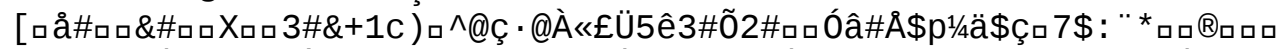

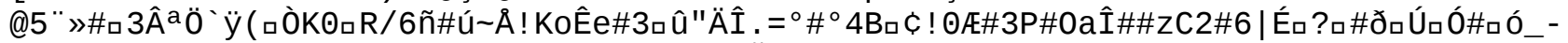

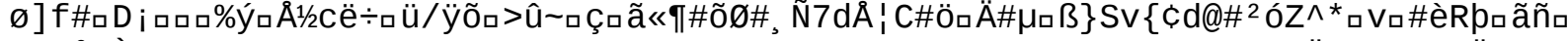

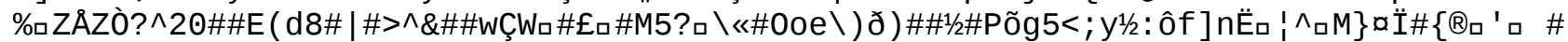
口fı\#ýas ýı<'

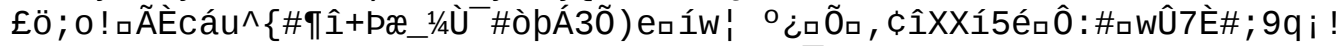

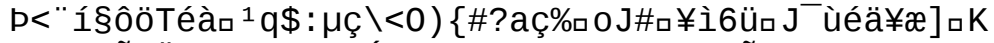

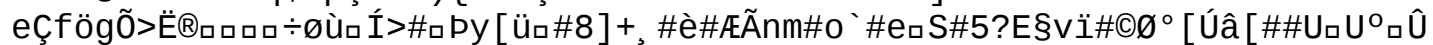

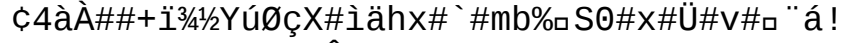

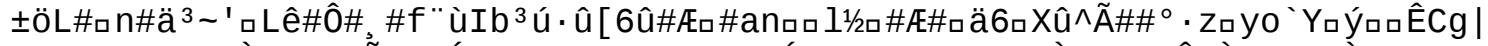

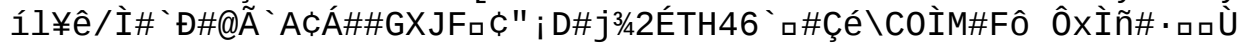

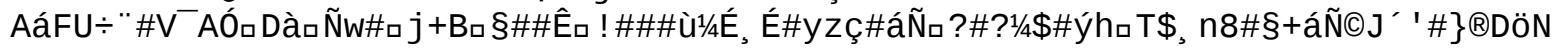




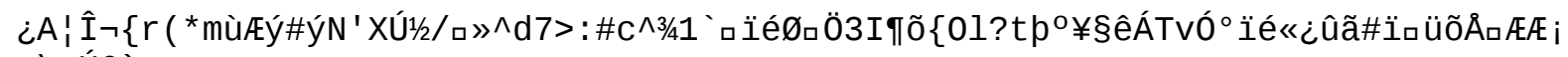
व`口Ú6ù

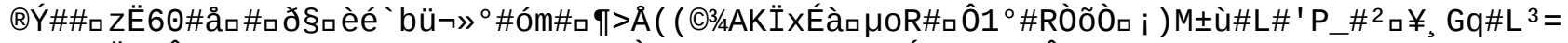

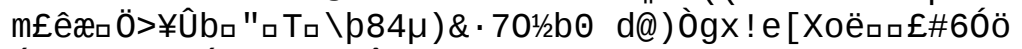

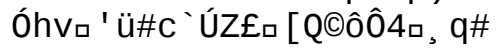




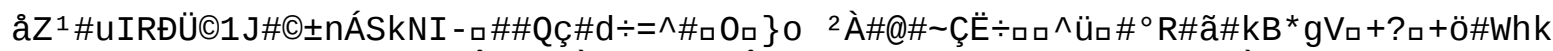

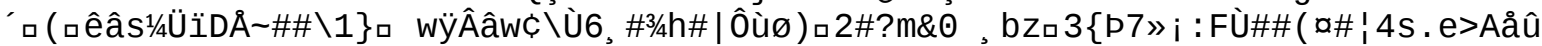

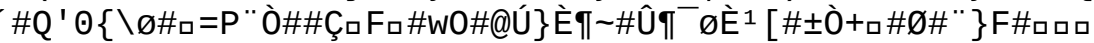

$\times f T \# C ̧ \cdot a \hat{A} E \#) Y ́ 7 K \# \tilde{A} \# \square j a \# X^{3} / 4 a C ̧ A U \hat{\&} \& \mid G J^{-} a ̈$ 


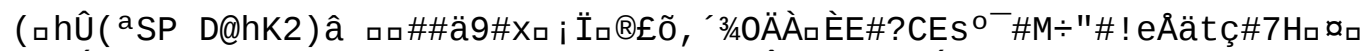

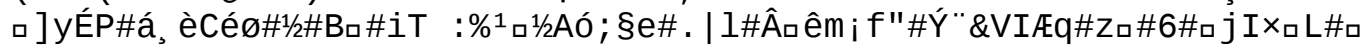

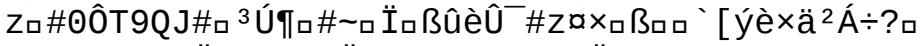

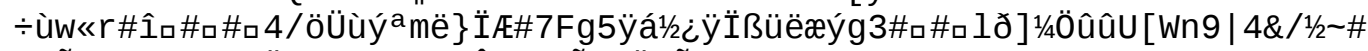

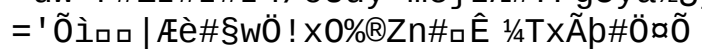




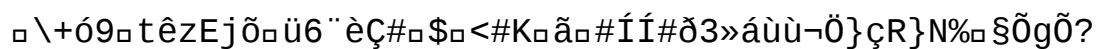

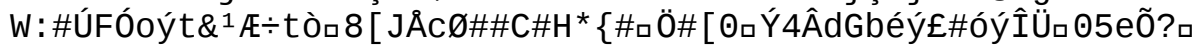

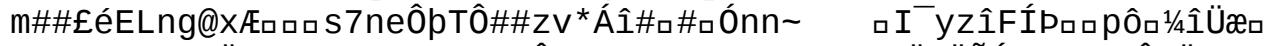

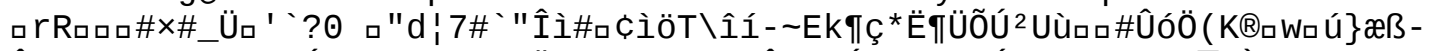

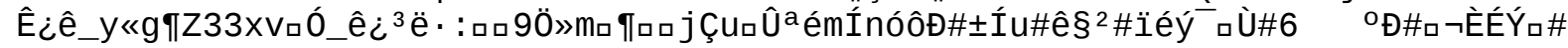
口Mê\#3U 


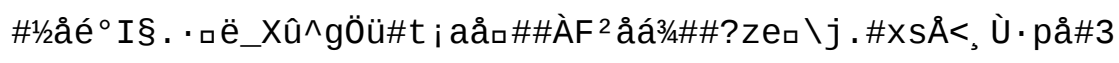




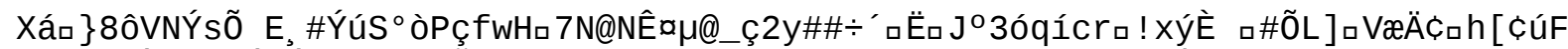

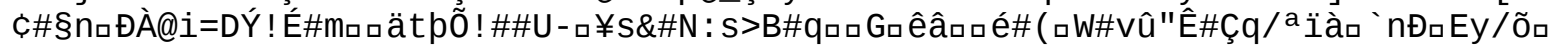

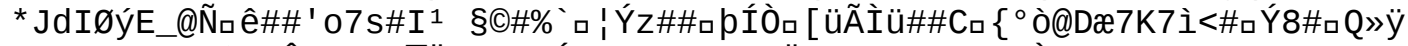

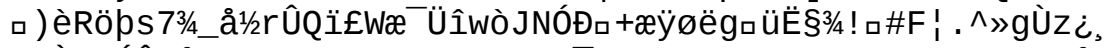

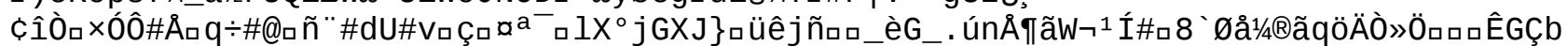

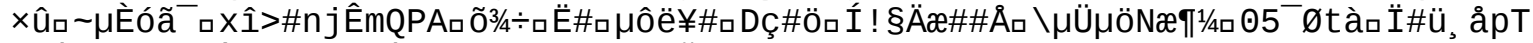

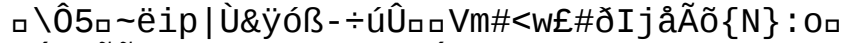

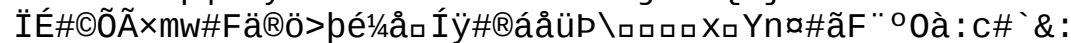

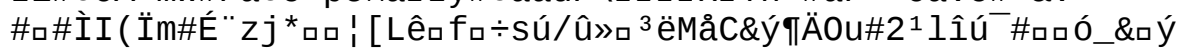




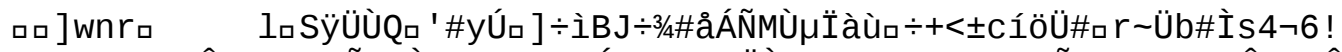

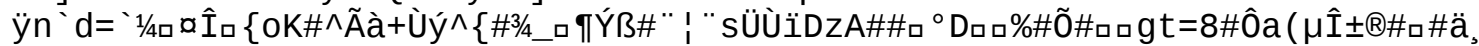

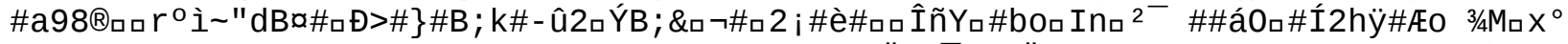

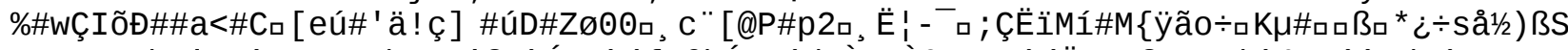

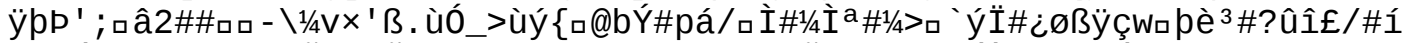

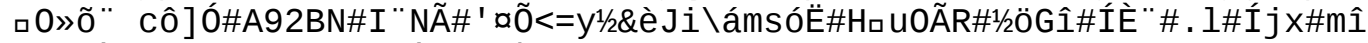
MmJzİøËロ å\#»N\#DdÝ $\neg$, \#WÔ $\left\{\Theta^{*} \ddot{I}\right.$ ?

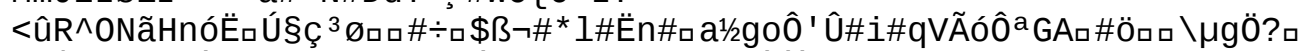

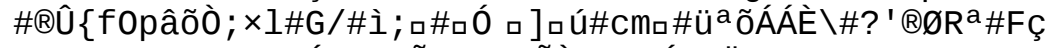

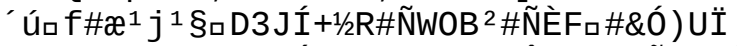

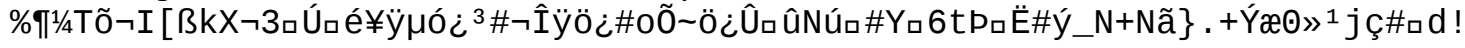


p $1 / 2 \pi æ Æ \# \#-r \# G 4 \mu \%$

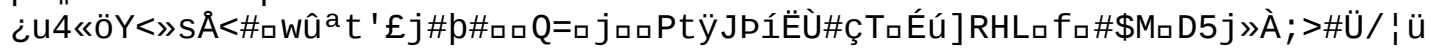

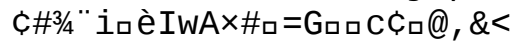

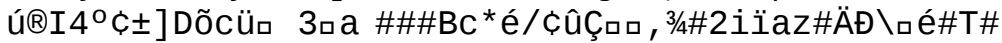

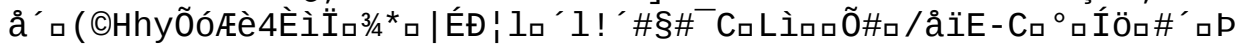

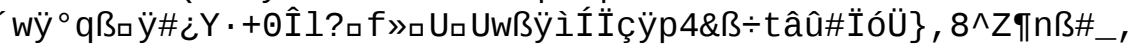


○\苂?òÿpûaCâ

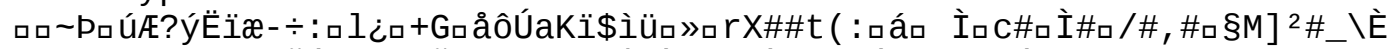

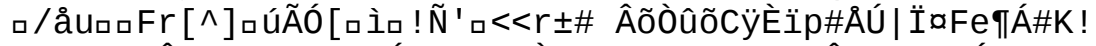

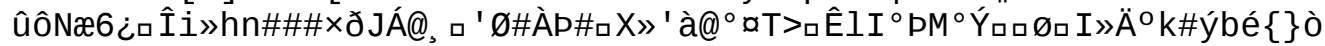

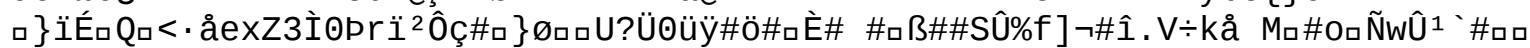
\#HFC ${ }^{3}$ Úý pau?Ýa\#×ôÿI 


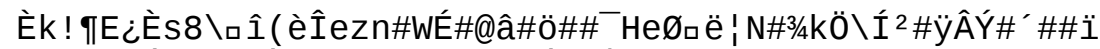

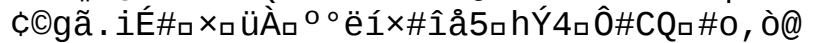

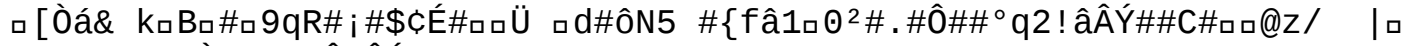

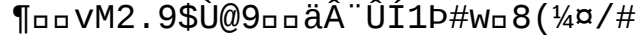




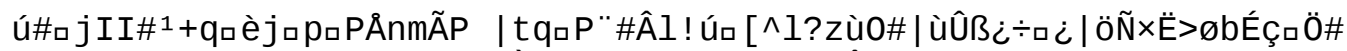

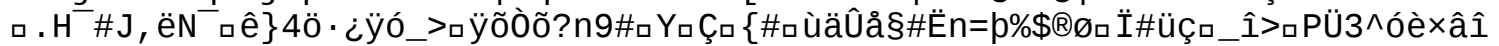
$\$ a s \$ 6 c / \square+\# q$,

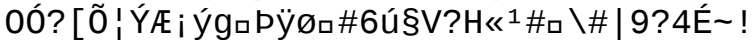

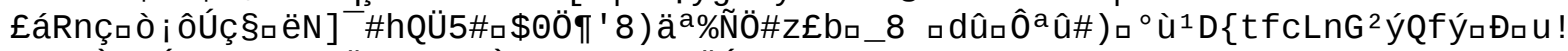

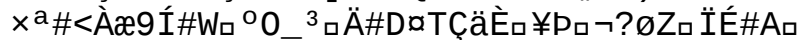

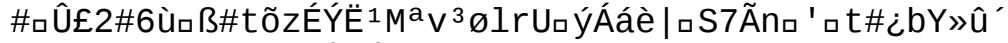

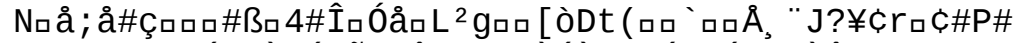

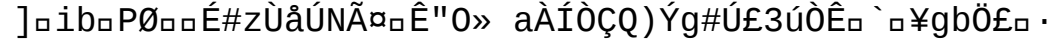




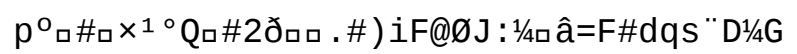




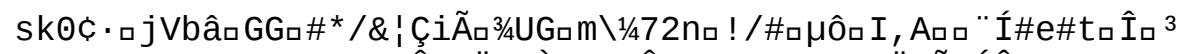

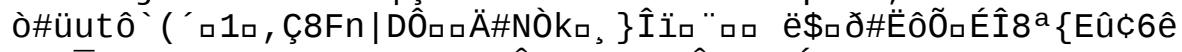
$\square \backslash H=\# \square \# \#$ \#\#ם ( , épXRPtFaO
口cüÎि $\left[\varnothing L^{\prime} Y\right.$ Y $\left.>\right\} \mu a ̀ X T e a^{3}$

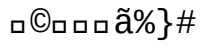
व०

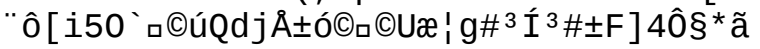




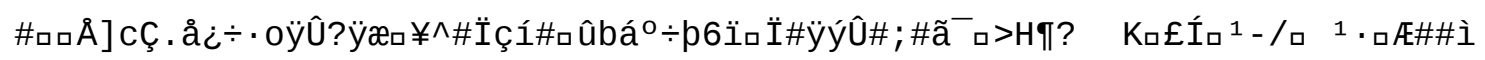




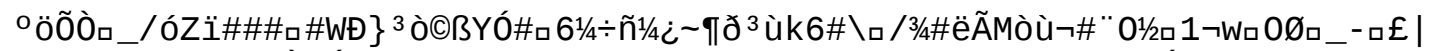

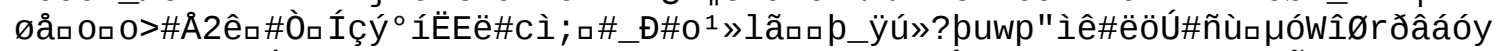
\#\#ACËDÚ

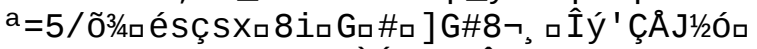

\%å-P3a3"3ÈJâ $\div$ NÀ» I i åMïap\#

oeIßëÒÁ7ð゚îิjI` 口S

C $\tilde{A} \wedge$ ฉ 


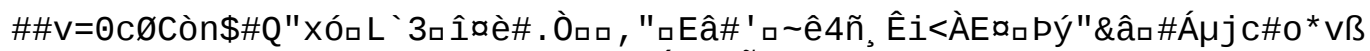

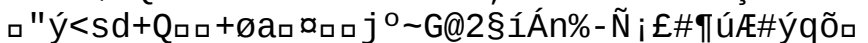

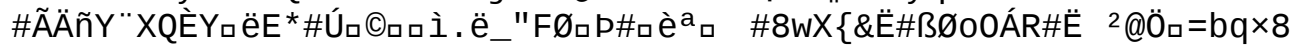




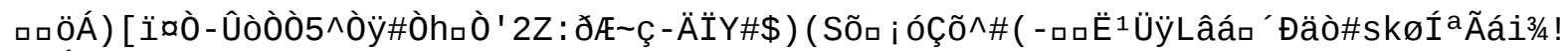
$\tilde{n}>A ́ q^{3 / 4}$ !

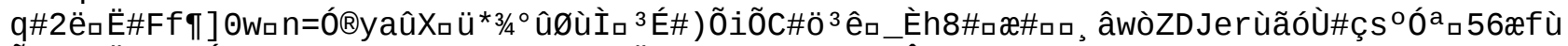

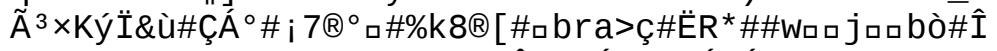

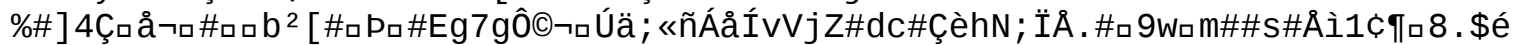

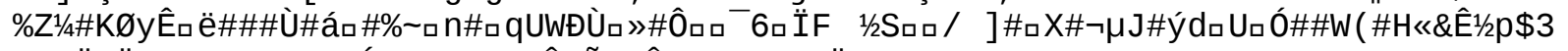

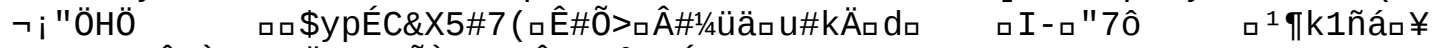

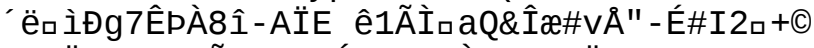

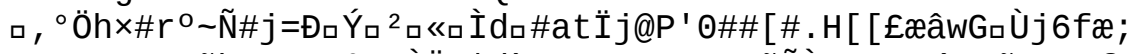

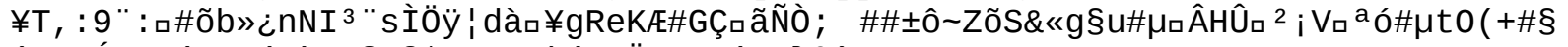

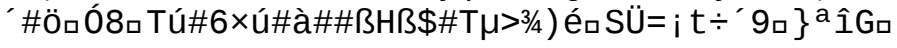




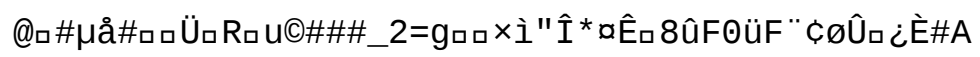




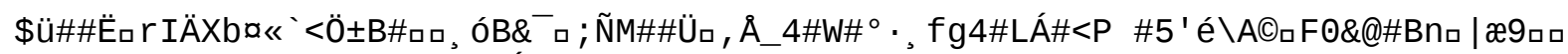

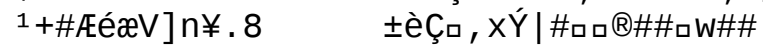

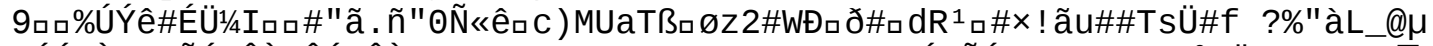

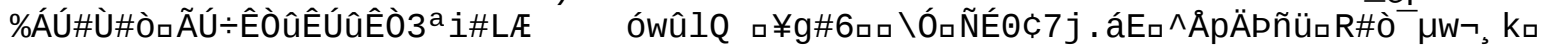
ýBıqóêb

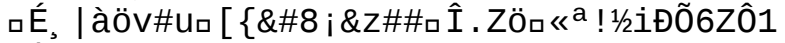

口ÎÖ?Z2\#ロCıí

Ä*ìquã¥ロC\%ý\#e\#ロ1

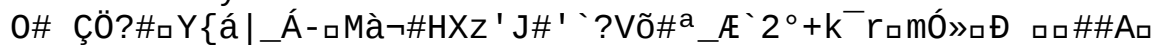




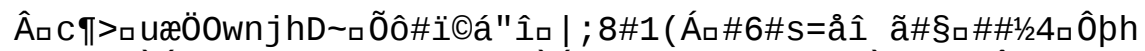

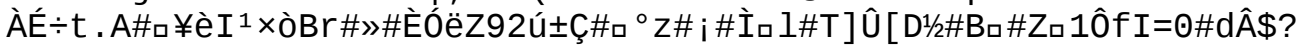

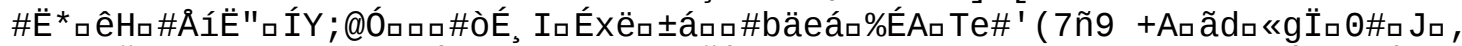

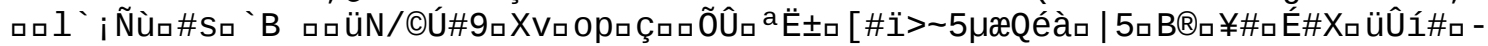

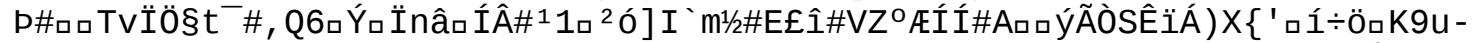

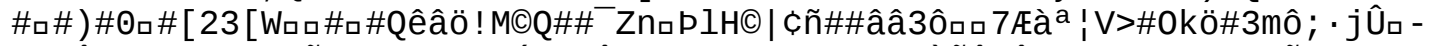

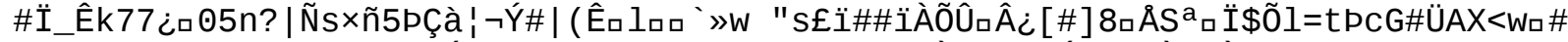

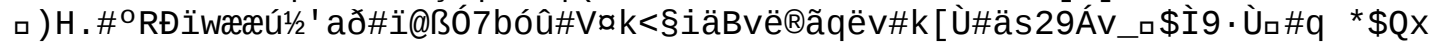
cVVıNú . ฉ\#Õ=\#åHß口Lzü 


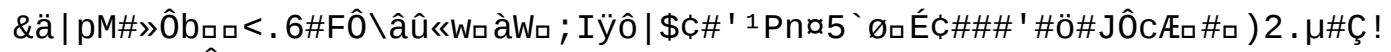
4\#Z9nHCè; Ô' l' lhøäd\#aロì

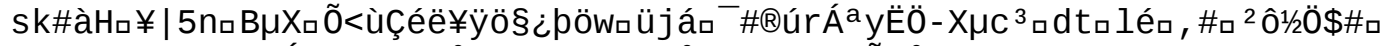

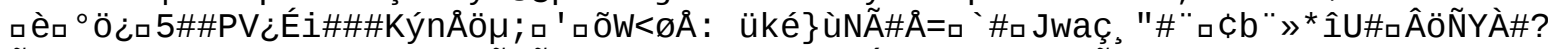
õõ ${ }^{\circ}$ lveqÏ\#

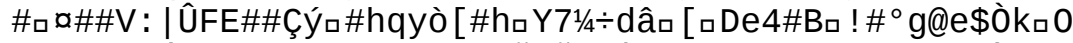

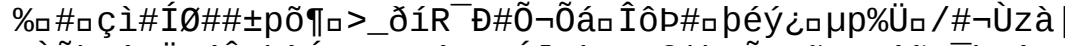

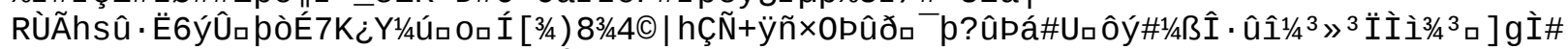

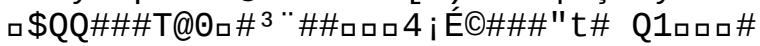


8ュ3ÚàWÝí

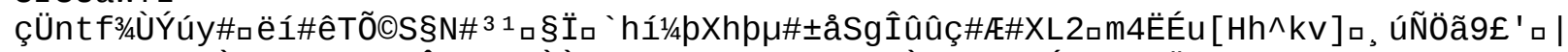

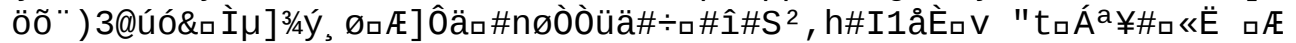




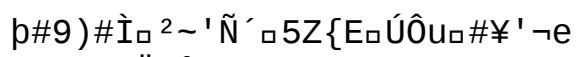

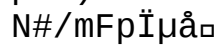




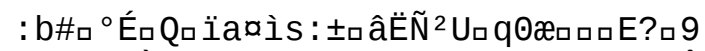

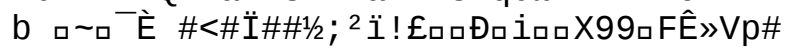

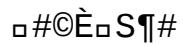




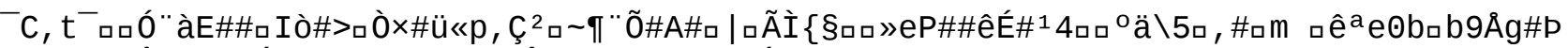

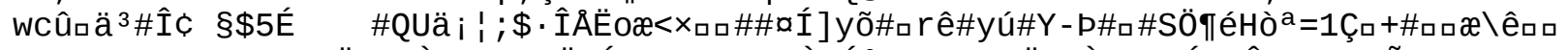

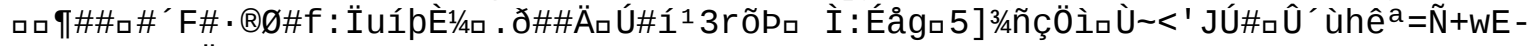

Ó口ó: tríp8Ï॰\#»B

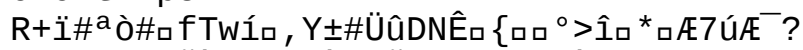

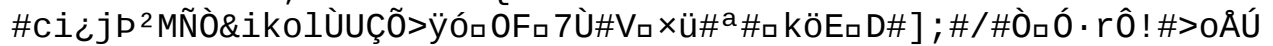

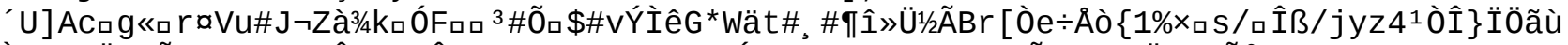

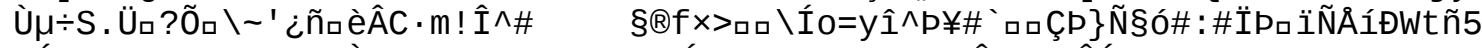

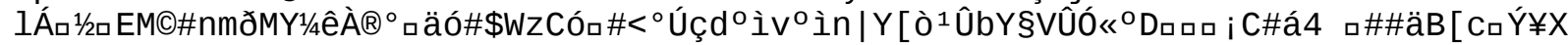

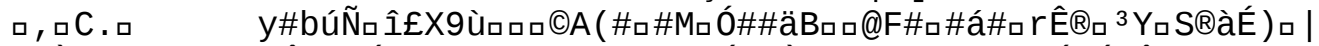

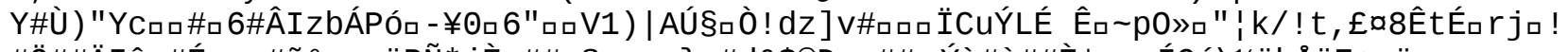

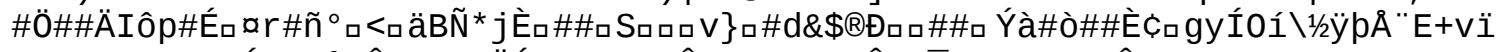

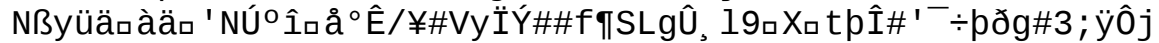

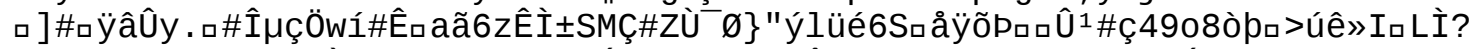

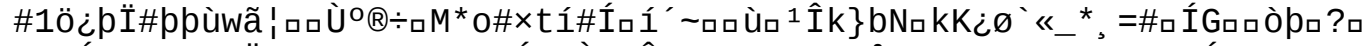

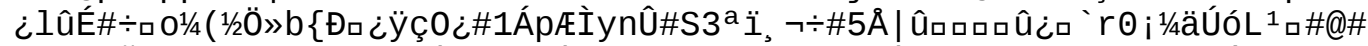

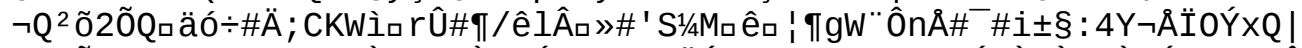

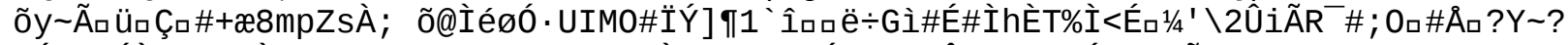

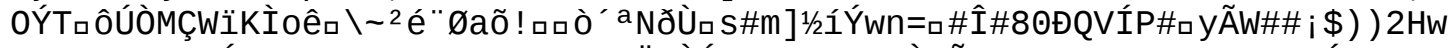

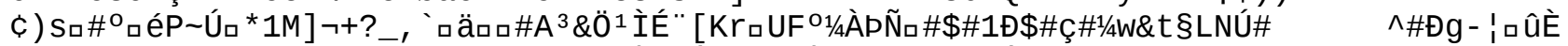

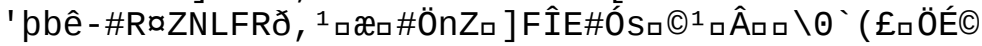

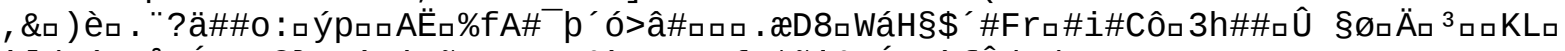

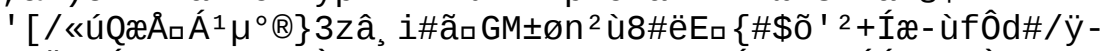

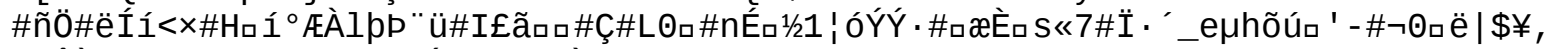

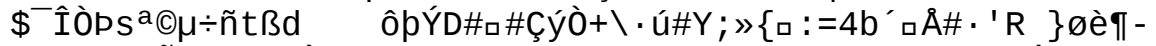

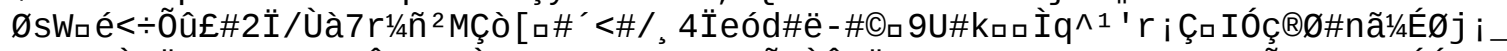

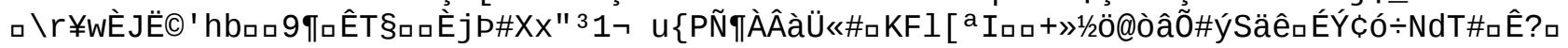
$\mathrm{t}$

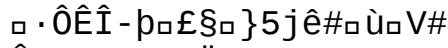

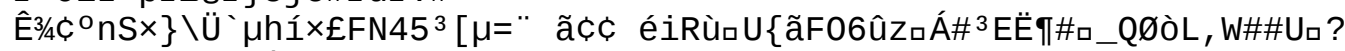

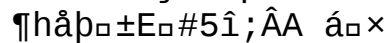

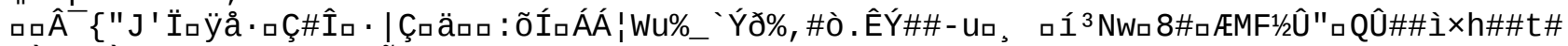

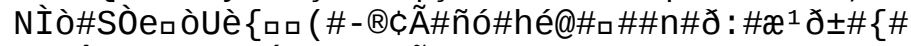

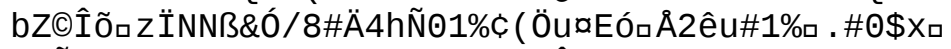

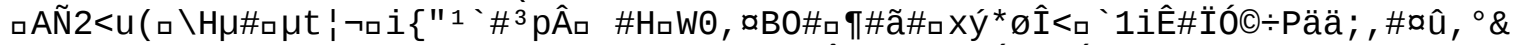

口 \û>,

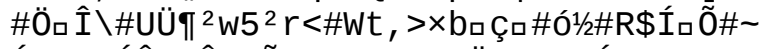

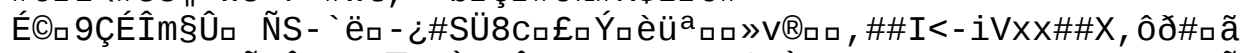

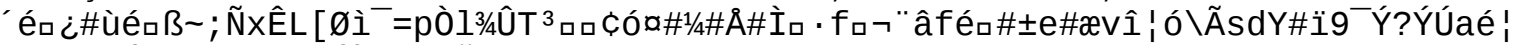

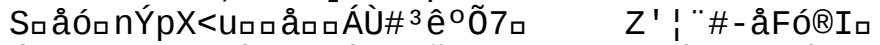

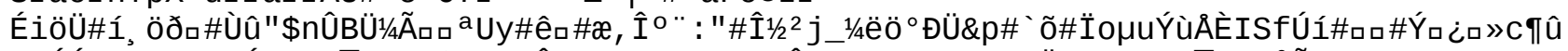

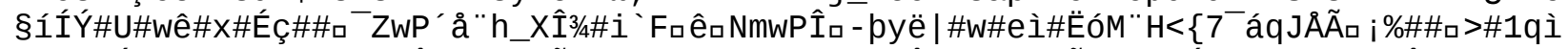

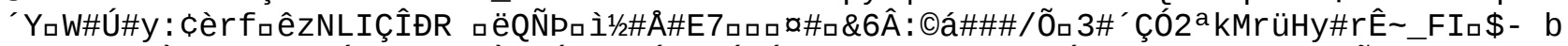

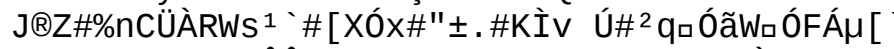

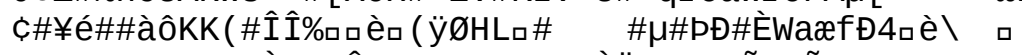

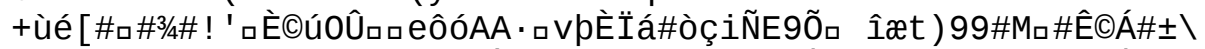

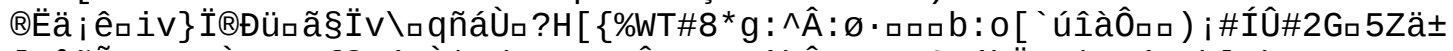

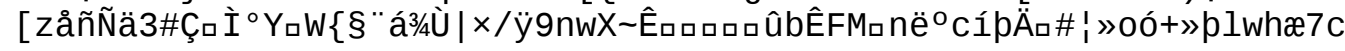




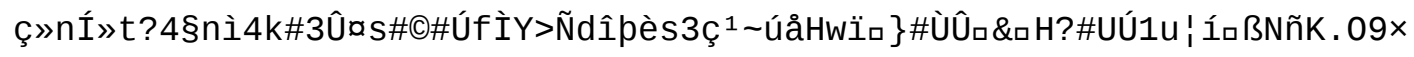




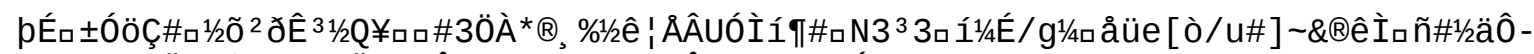

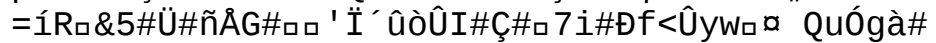

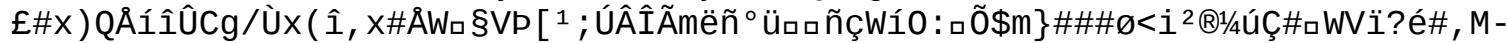
ì\#IåôõaUv ${ }^{2}$ Røk\#í 


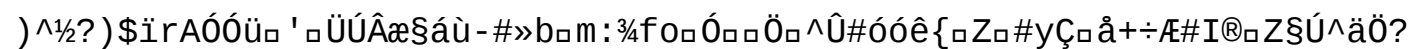

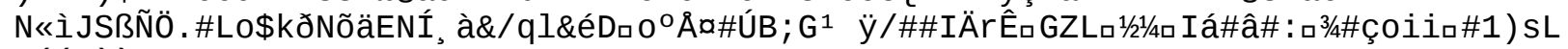
âÁú§̇̀̀̀! , â)\#Wםr 


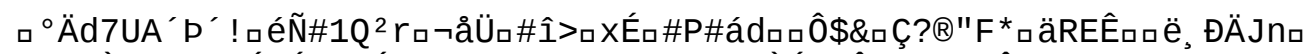

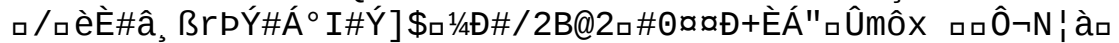

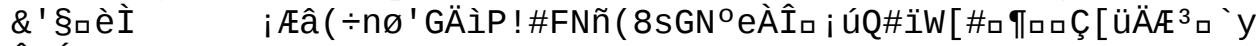

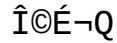




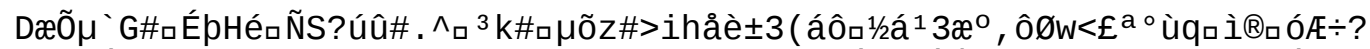

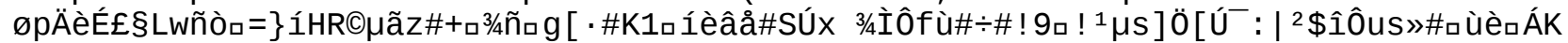

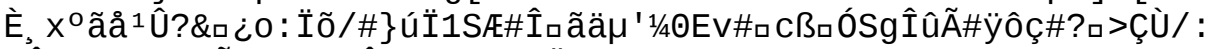

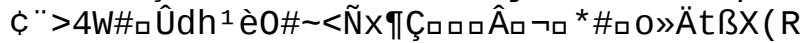

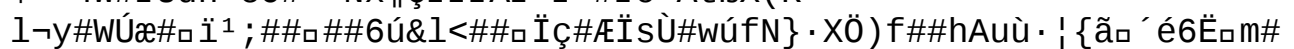




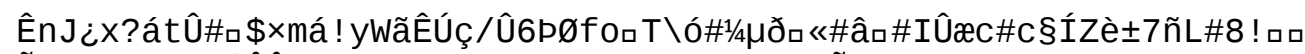

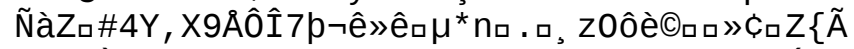

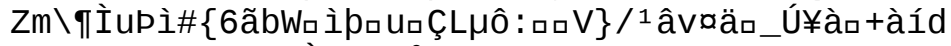

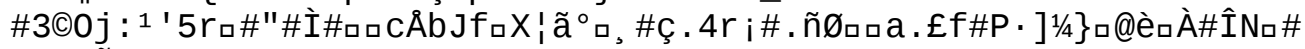
\%_\#口Ñ̃féç\#ä\#grê\#M, x 


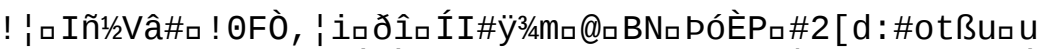

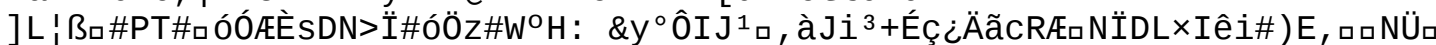

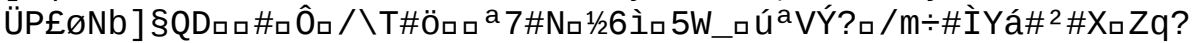

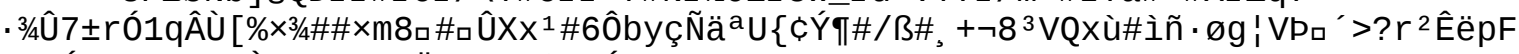

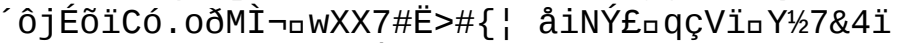

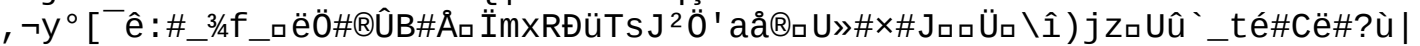

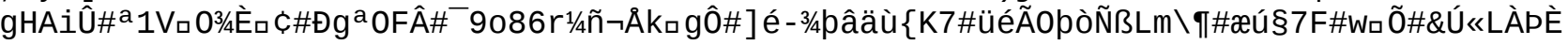
ck [\#ran

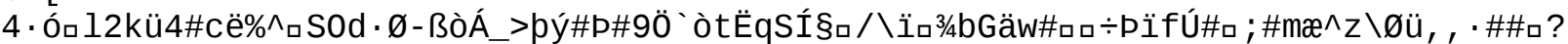

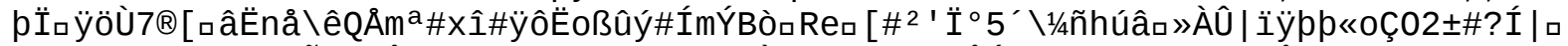

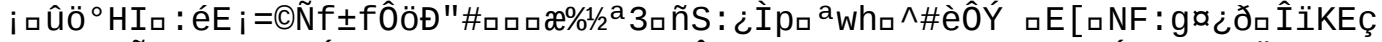

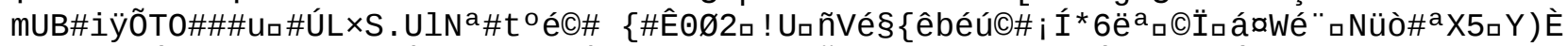

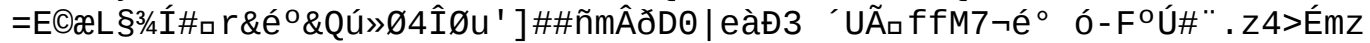

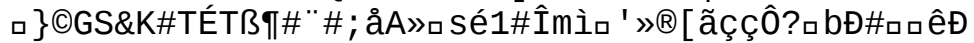

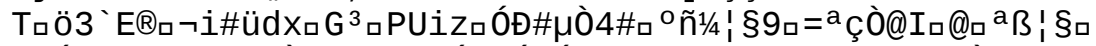

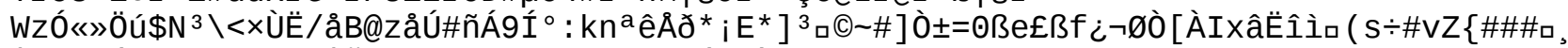

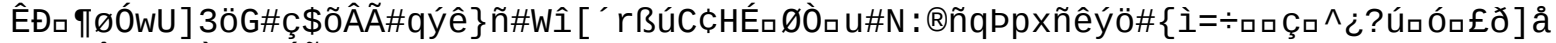

' $\mathrm{d} \mu$ "ÂÂिÜ 


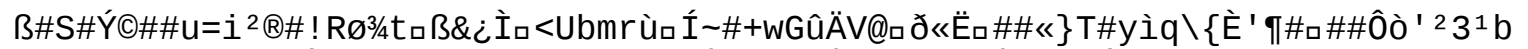

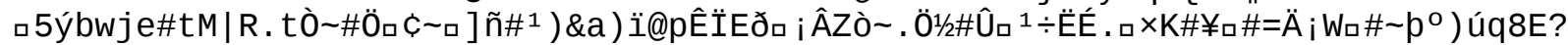

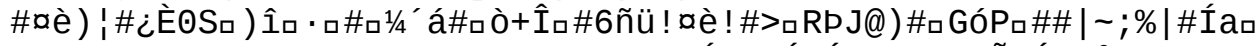

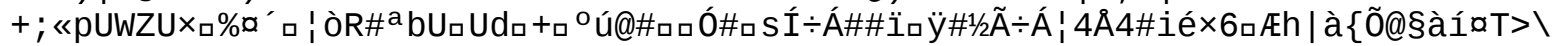

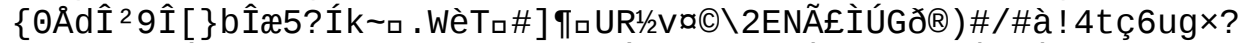
\#_Th॰ÆaAÓ\#ð\#1/42 ì

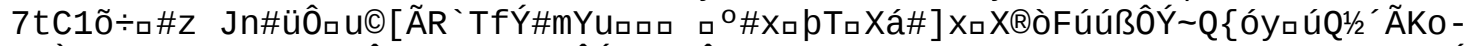

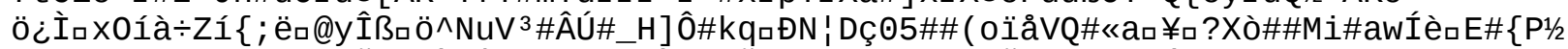

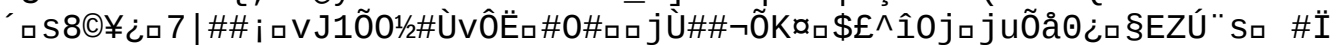

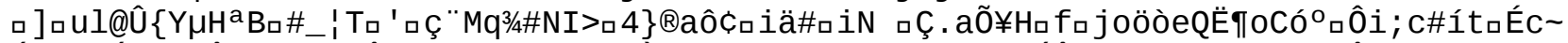

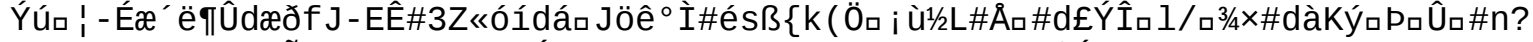

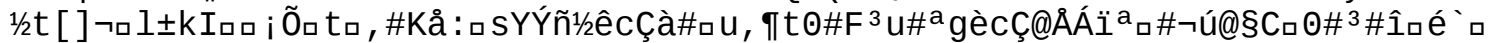

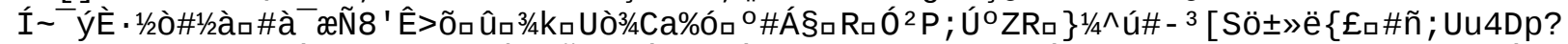

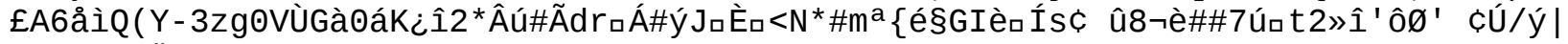

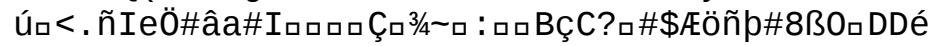




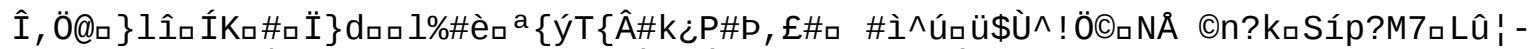

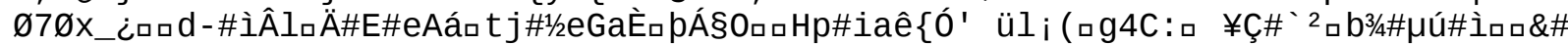

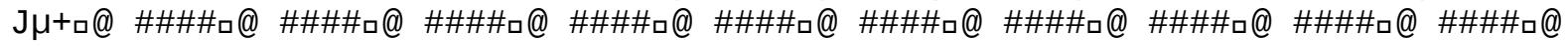

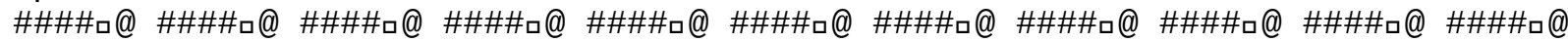

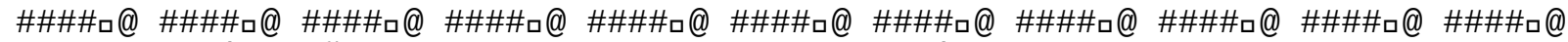

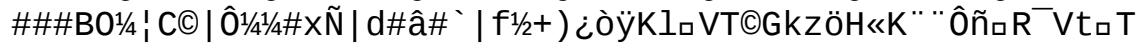

${ }^{\circ} \mathrm{B} \times \mathrm{XPn}$ úpL, ) व 


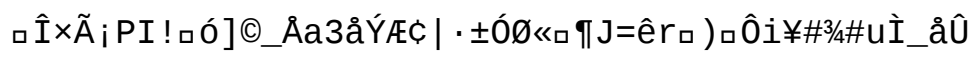




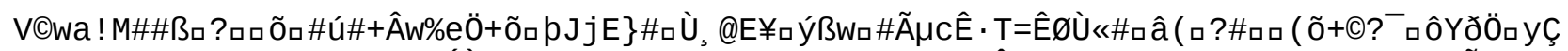

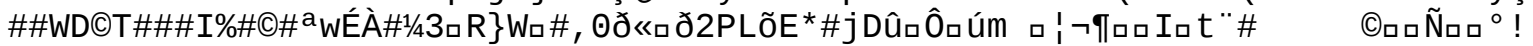

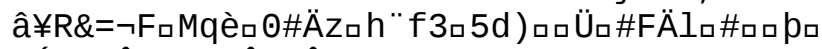

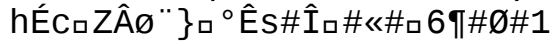

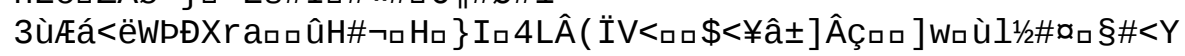




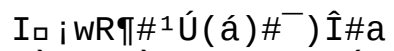

EÊæa ÉmıdParÒçX

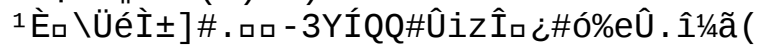

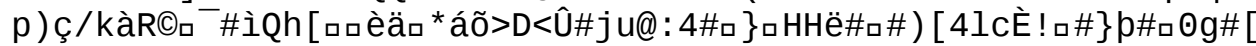

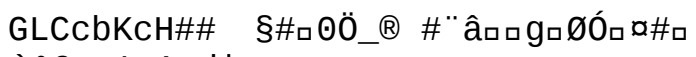

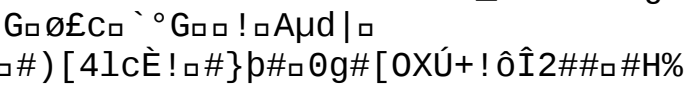




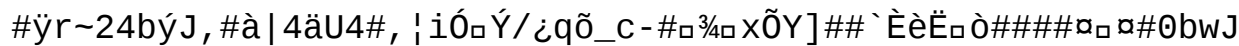

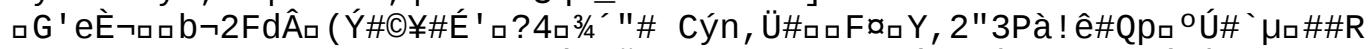

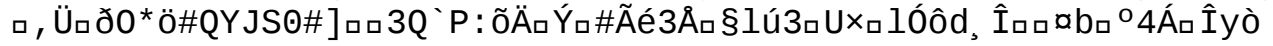




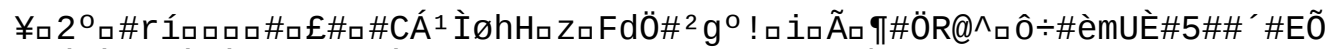

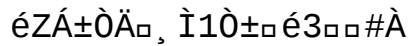

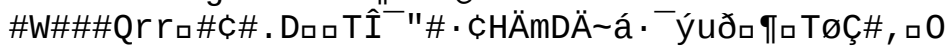




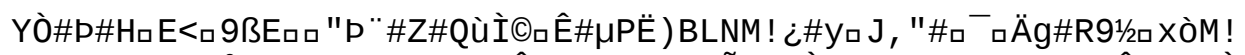

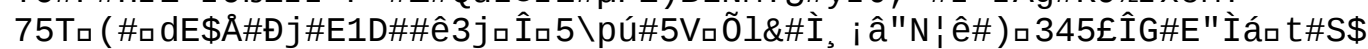




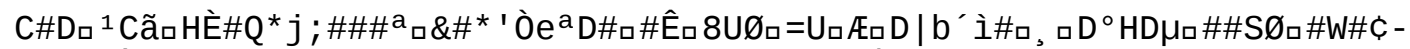

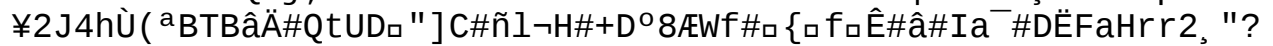

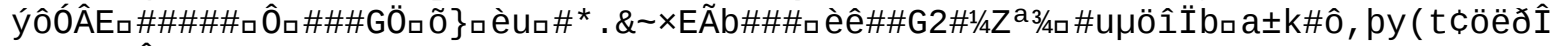

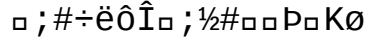




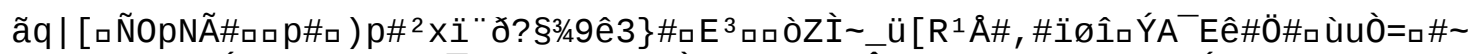

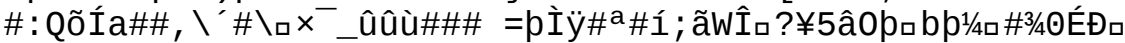

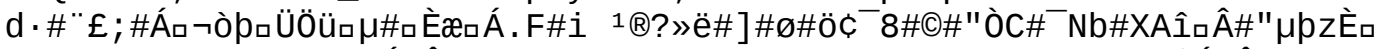

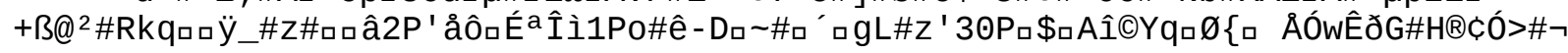
๑.\&\#ѐgロ 8 


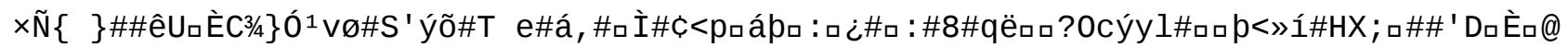

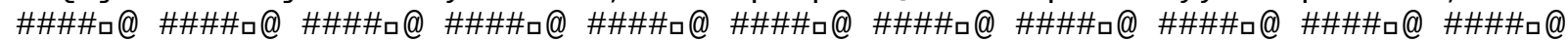

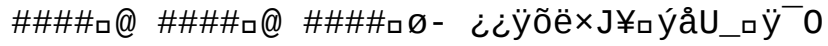




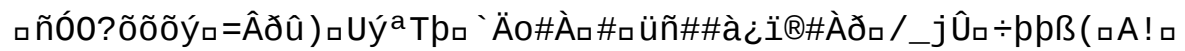
¿Zw\}êùHı\#ð]@]\#\#\#â_\#===¥¥

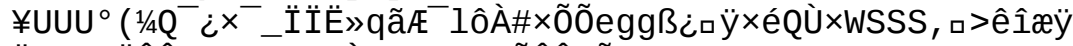

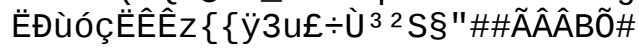

IJJzøðá; đ 
Ú\#\#\#\#\#\# 


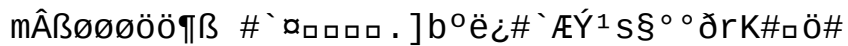

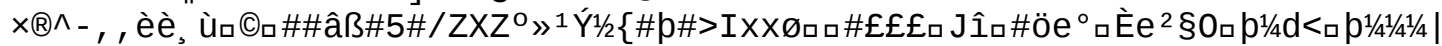

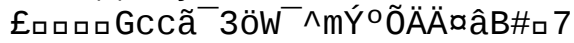

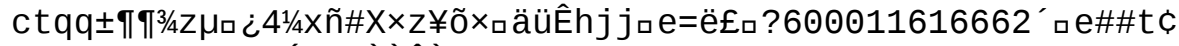

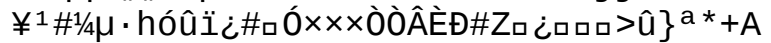

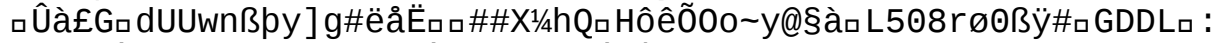

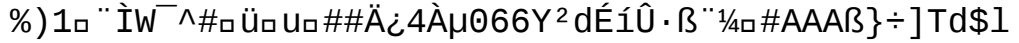




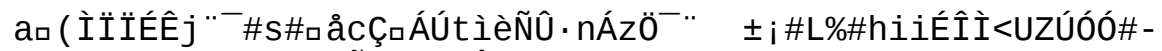

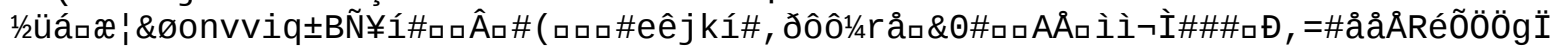
口] ni. aHòrr

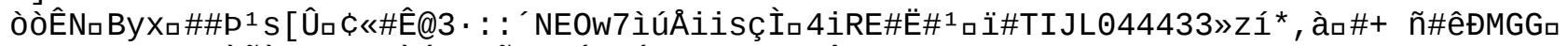

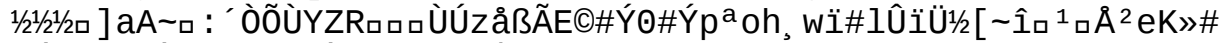

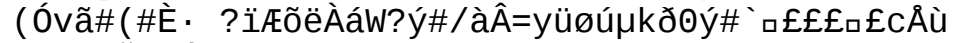

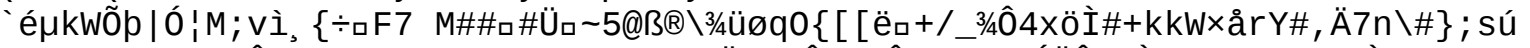

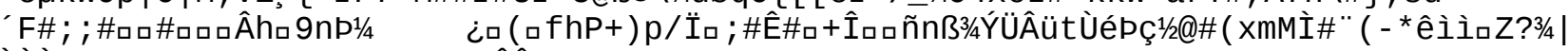

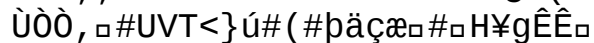

\# $\mathrm{i}$

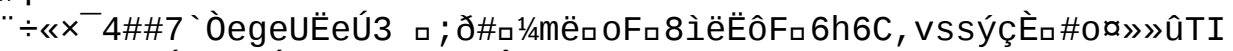

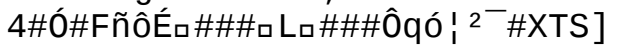

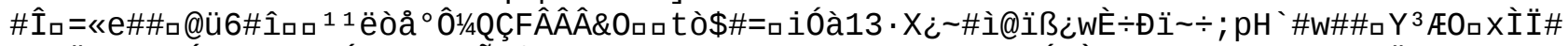

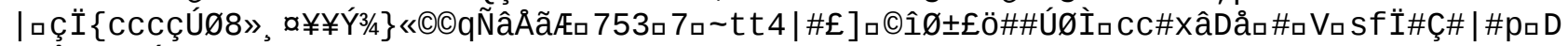
c\#Û\#\# kÓıf.KauUW(\#yyyº\#abbbjjja]ם

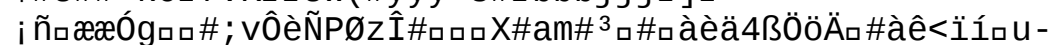

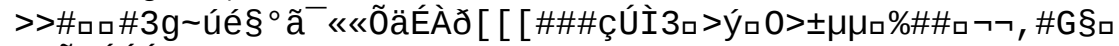

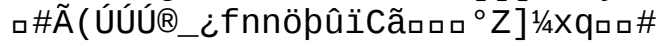




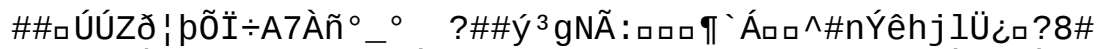

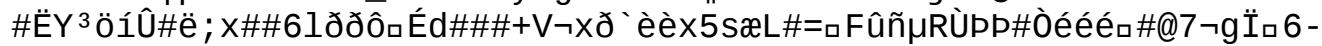

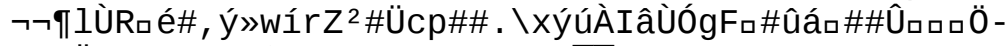

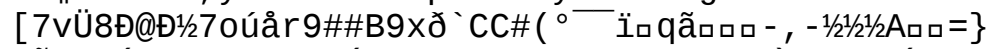

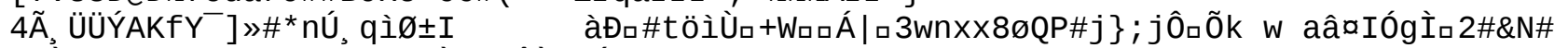

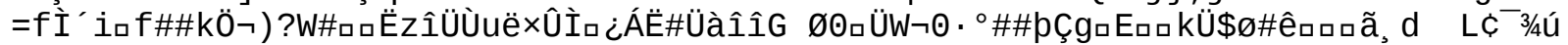

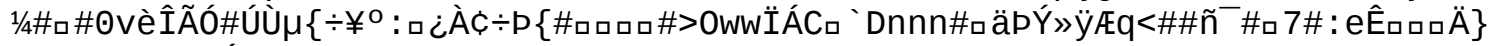

$\{\div$ ¿\#9\#pæçåÁßEロ\#ロU 


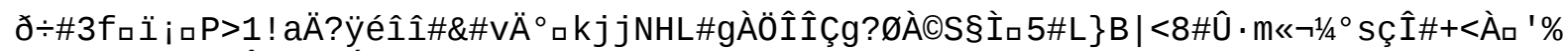

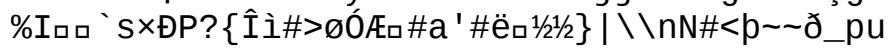

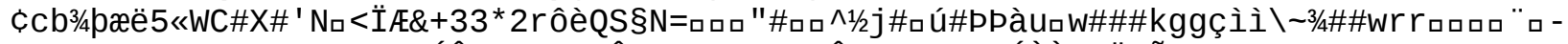

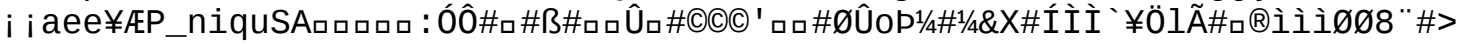




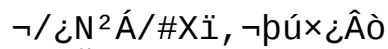

$n^{\prime} 1 / 4 \tilde{A}$

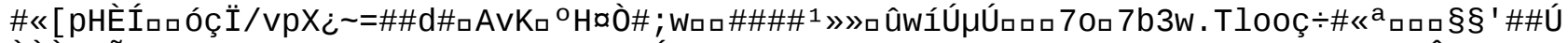

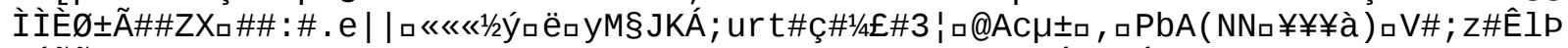

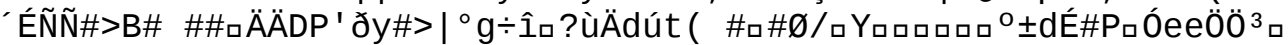




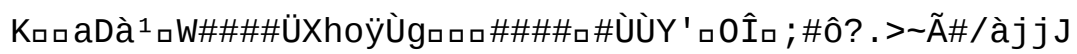


Önฉฉ¿¿\# 


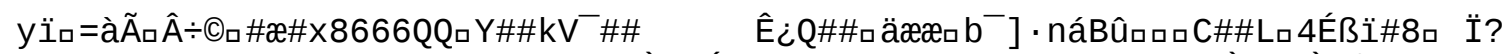

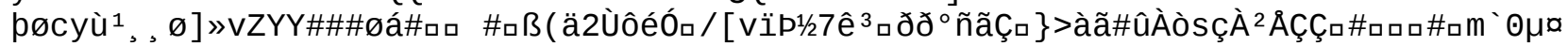
a\#

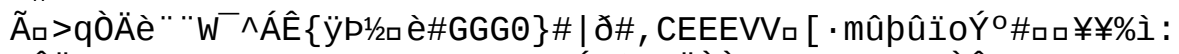

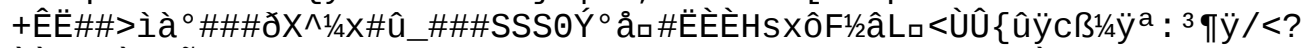

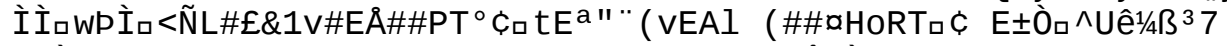

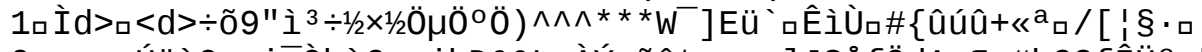

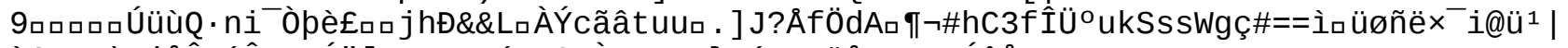

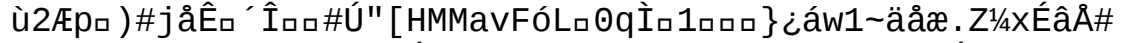

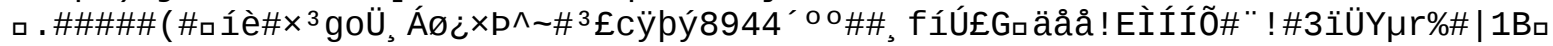



ô·w(ם

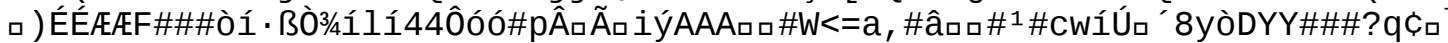

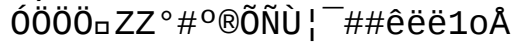

$M \| \notin \wedge$ ロ \#A-ä

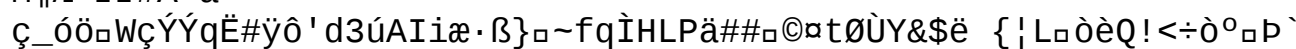

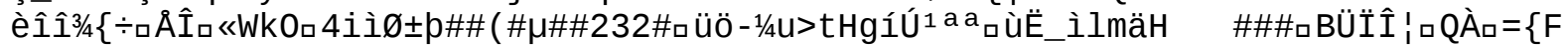

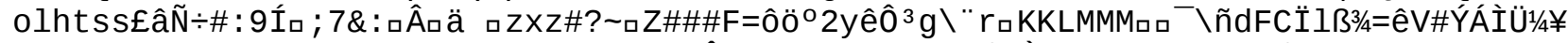

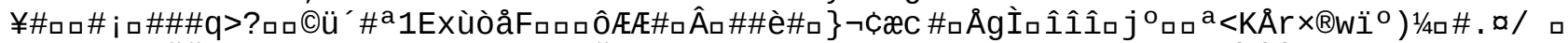

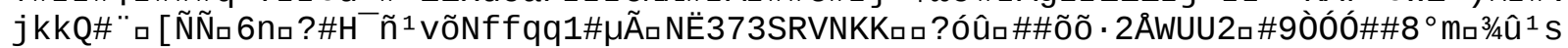




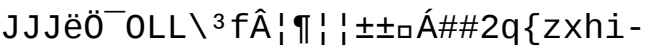

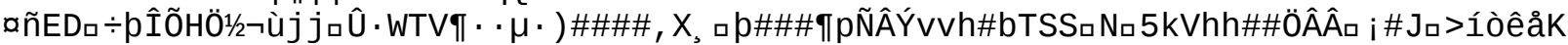

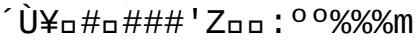

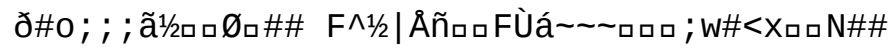




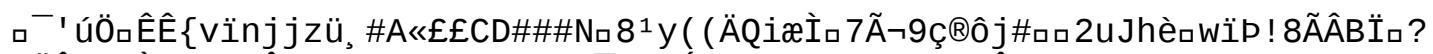

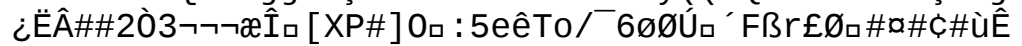

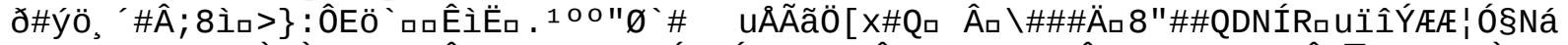

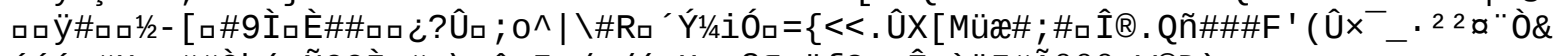
“ 


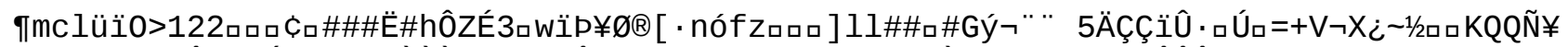

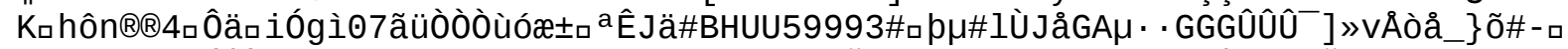

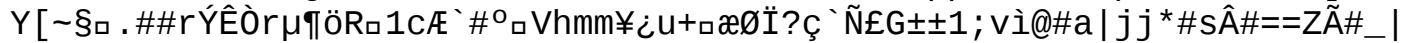

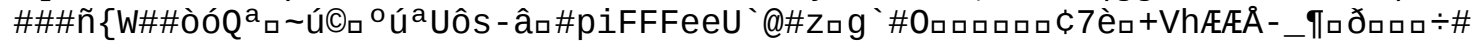




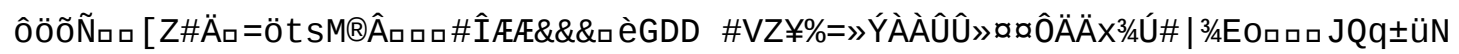
\#GEêëëCKTå\#õ

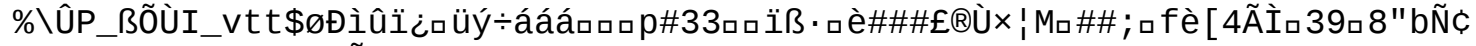

$[\llbracket 1 A 3 c 2 \# I \bar{D} 1 / 2 \# \square$ fggÃ $[\square$

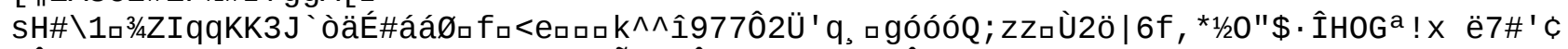

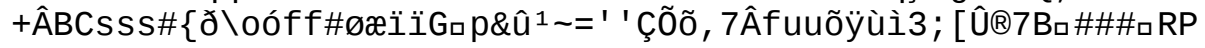




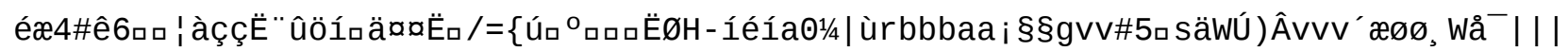
èp 


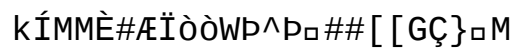




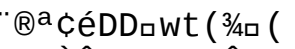

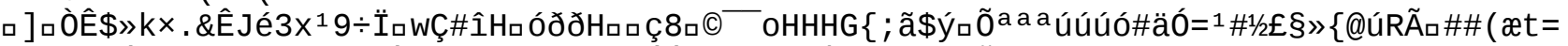

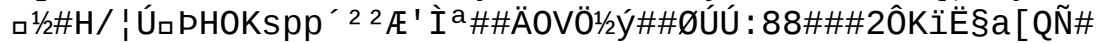




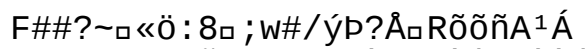

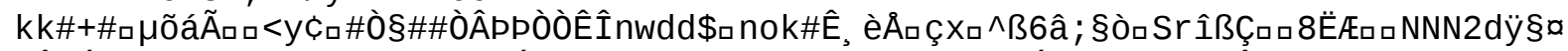

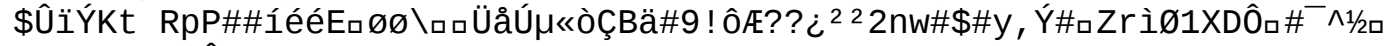

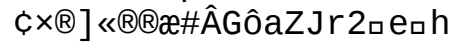




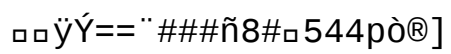

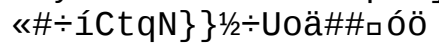

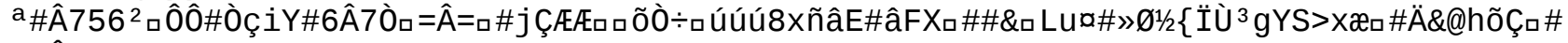

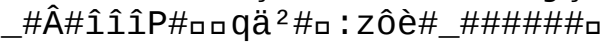

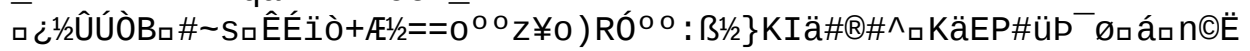




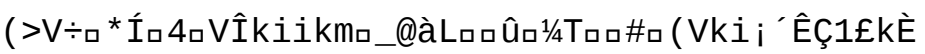




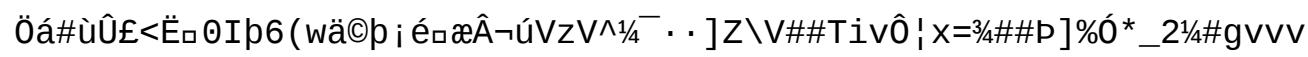




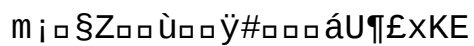

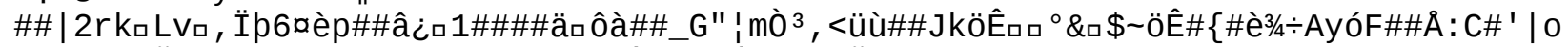

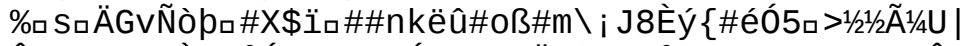

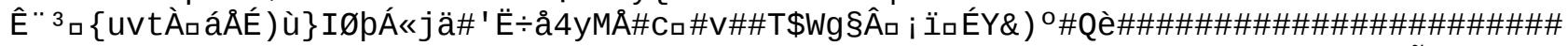

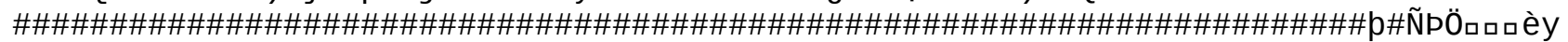

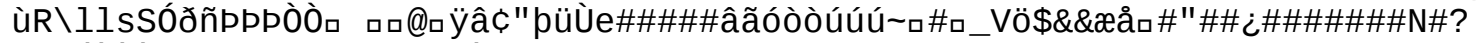

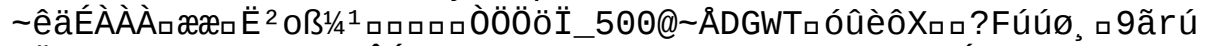

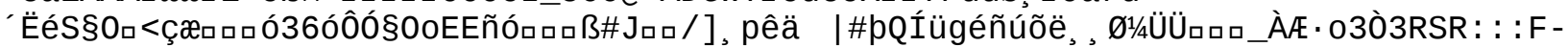

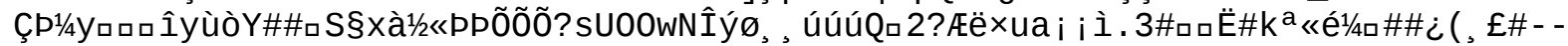

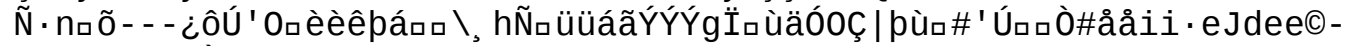

$X^{\circ} \mathrm{k}_{\square} \AA_{\square}{ }^{\circledR} 7 \dot{i} \mathrm{E}_{\square} \mathrm{D} 3 / 4>$ ???

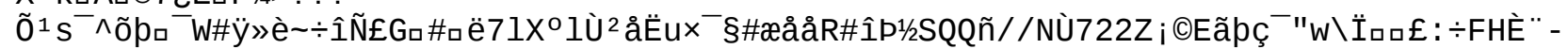

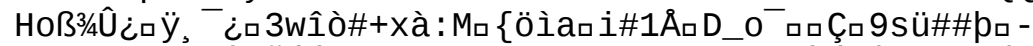

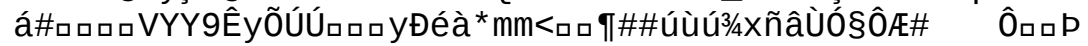

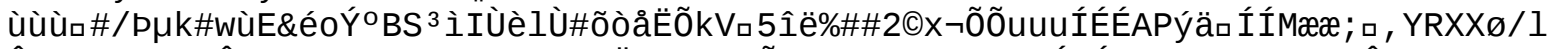

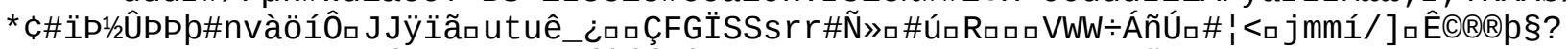

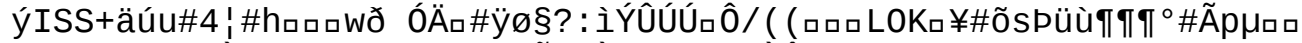

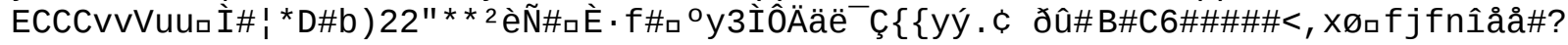
CùP177\#p\# 


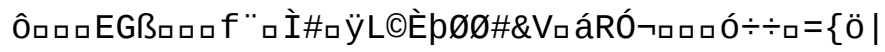

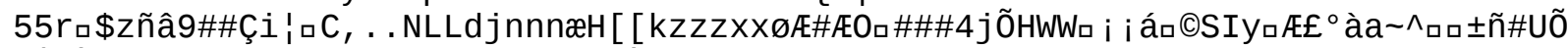

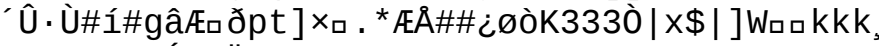

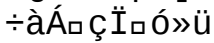

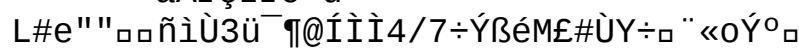

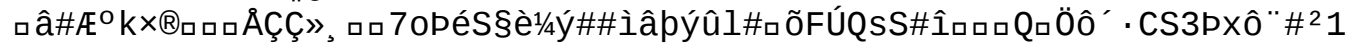




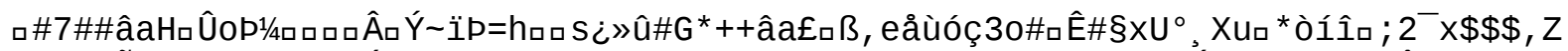

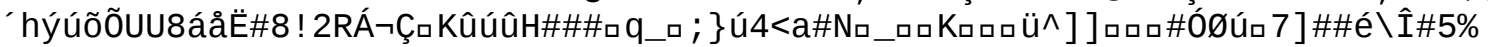

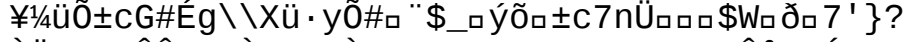

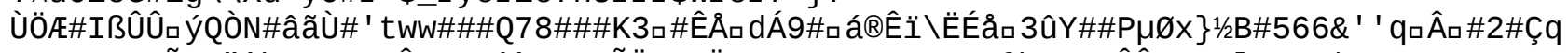

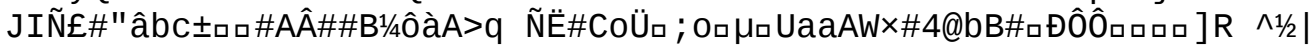

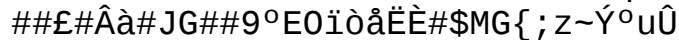




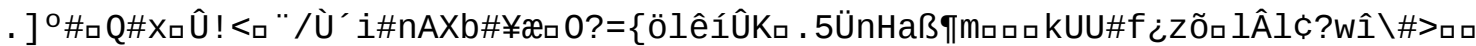

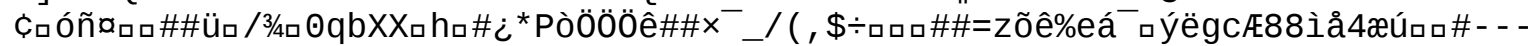
eËロyzzÖÖÕ⿻ruêéé \#?

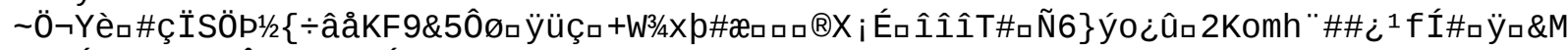
c\#ロÚ · 3P\#\#; WÎ̃ 9300àÁna\#

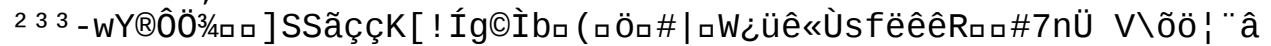

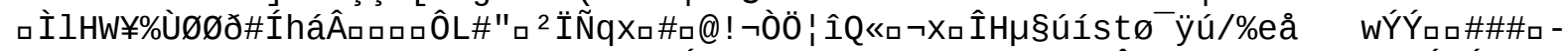

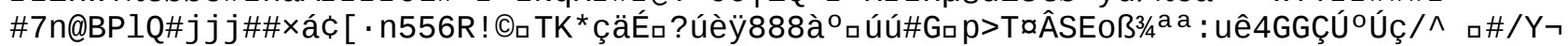

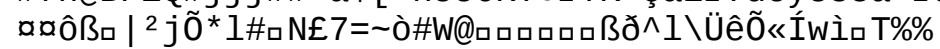

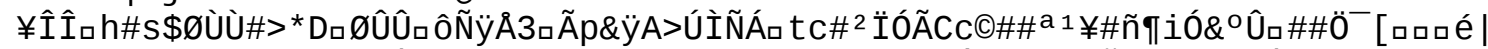

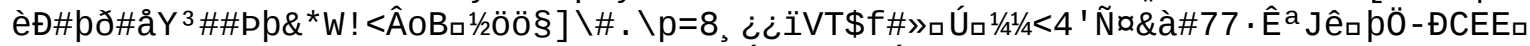

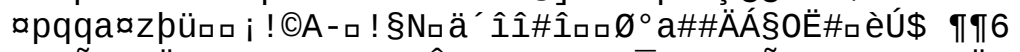

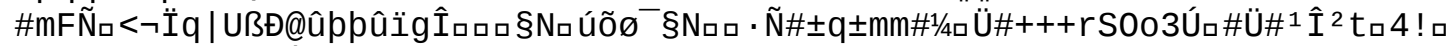

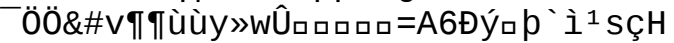

Ràg1/4aロu»tÙ2\#Ntt4.Ú1/2\{7\#áaİ 


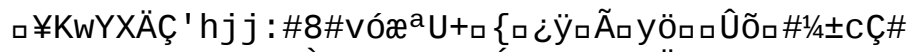

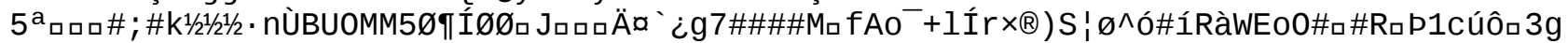

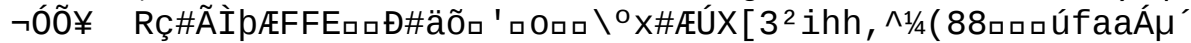


8ם '\#\#\#] ${ }^{2} \times 1 R \S^{\prime} a D \_e^{\wedge} \wedge$ 


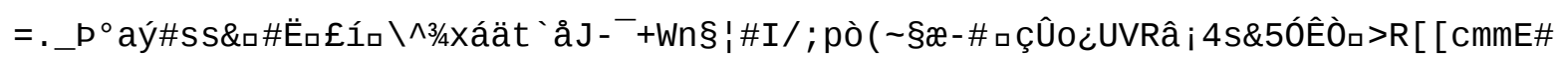




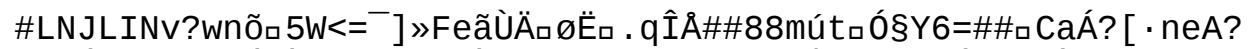

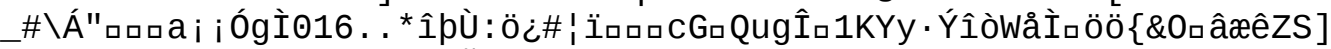

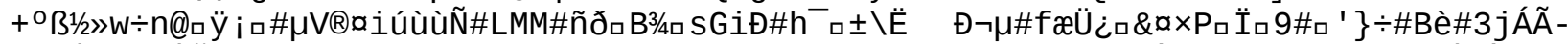

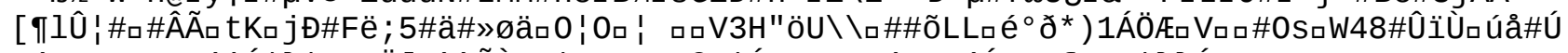

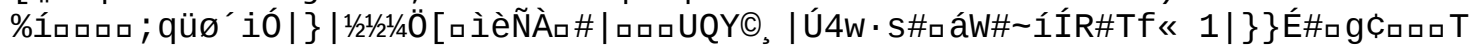

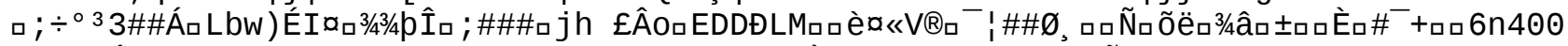

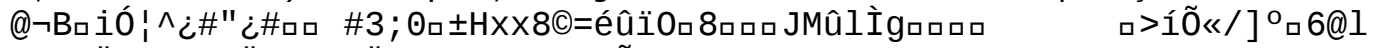

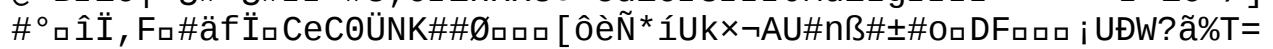

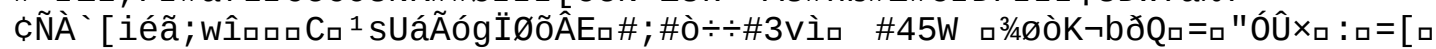

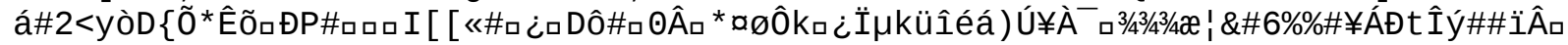

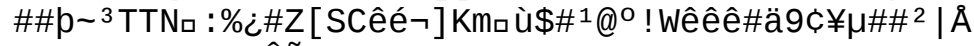

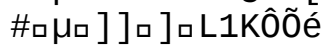

ëê(/ 


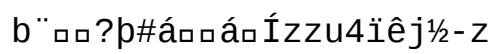


\#YYYÜ1̂̂ôó́ó§̈Ï\# 


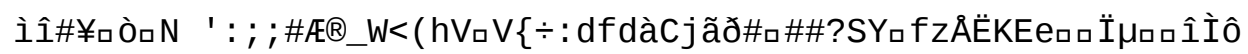




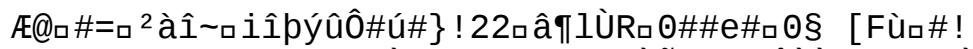

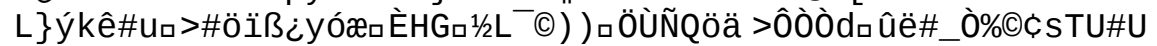

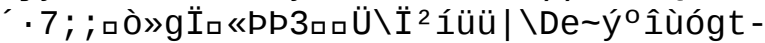

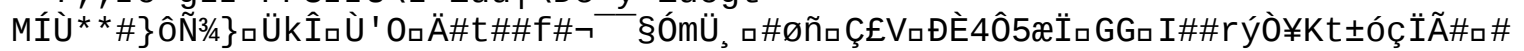

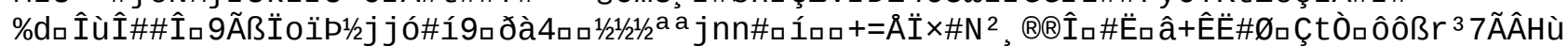

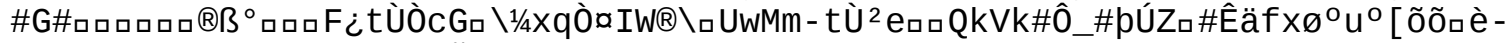

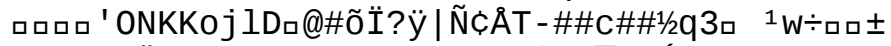

口

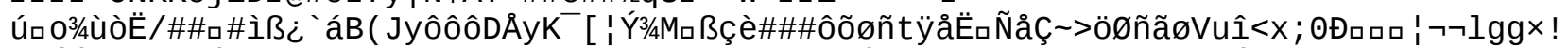

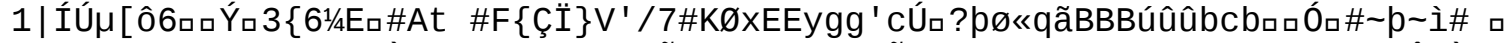

' '

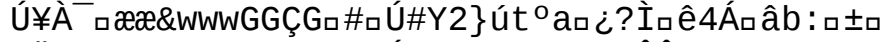

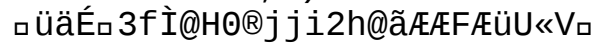

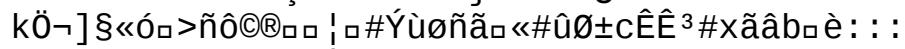

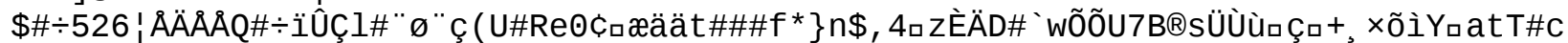

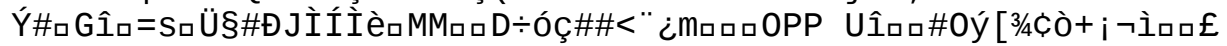

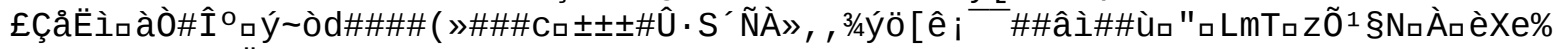

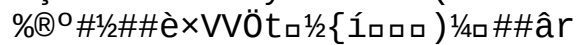

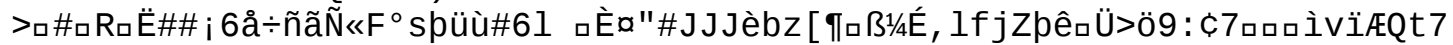

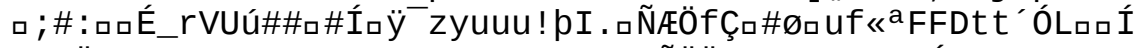

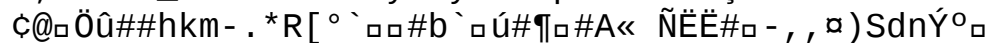

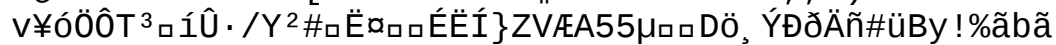






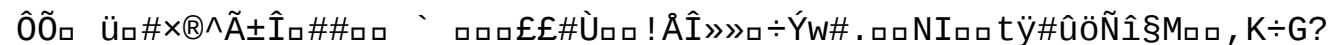

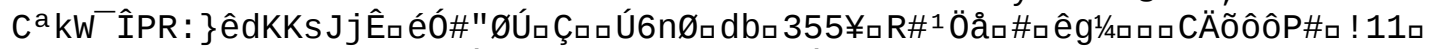

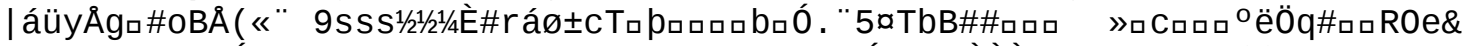

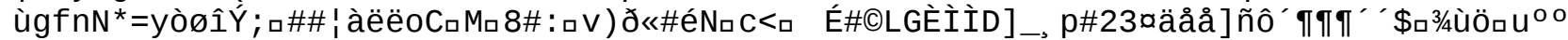

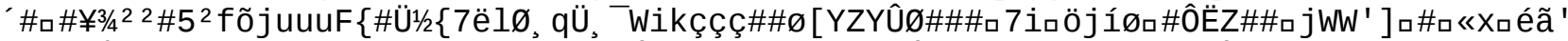

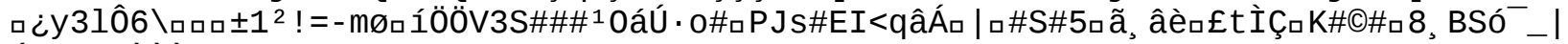
Át/ÆıロCİ̀̇İ, ᄀ(2 
¿ロ a\#1ÂÁjhÈo oúÑé\#@L!

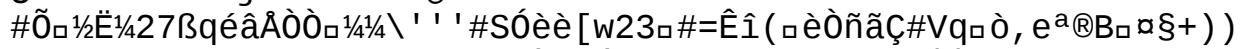

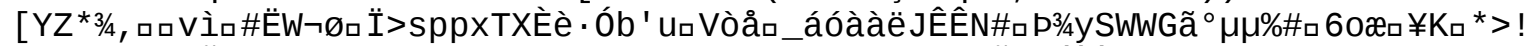

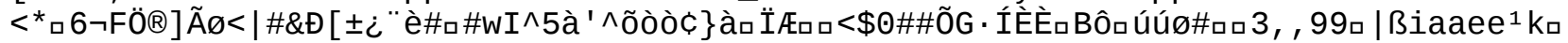

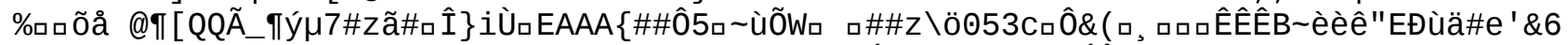

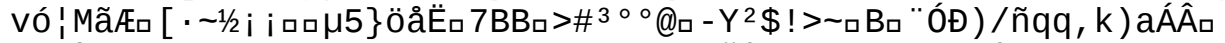

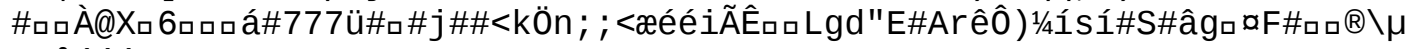

\#ャåááá6

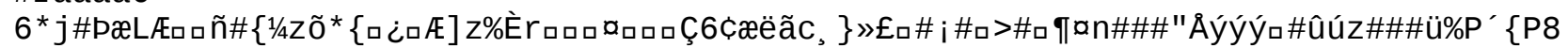

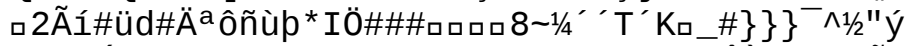

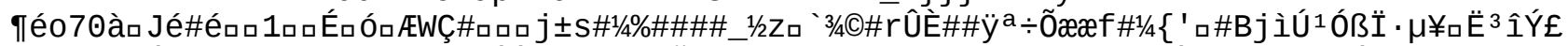

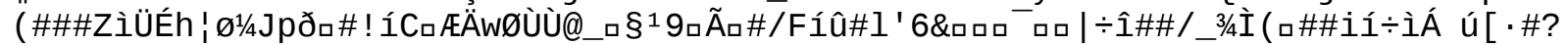

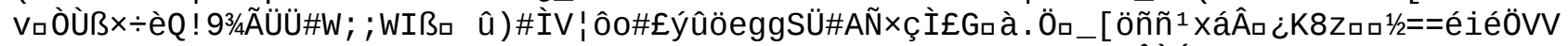

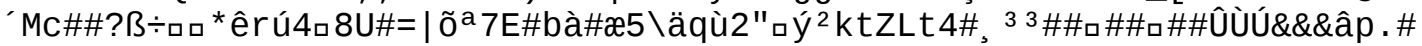


ðロ · , ÎaÏpÌø 


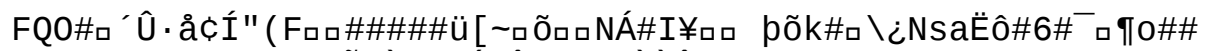

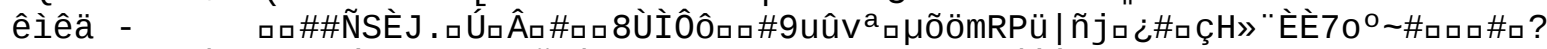

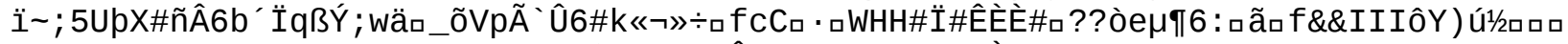

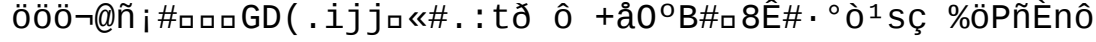




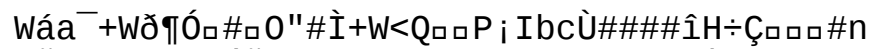

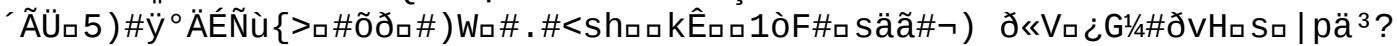

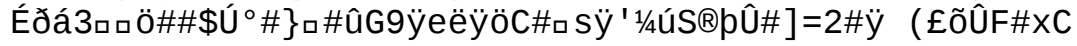

$\dddot{x}_{\mathrm{a}}$

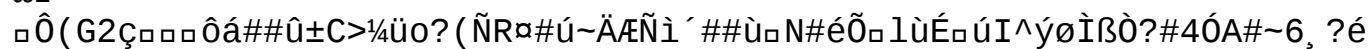

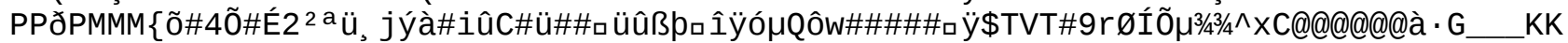

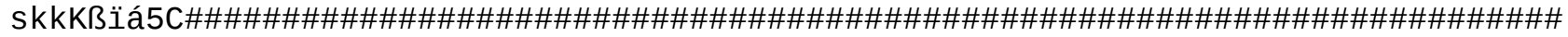

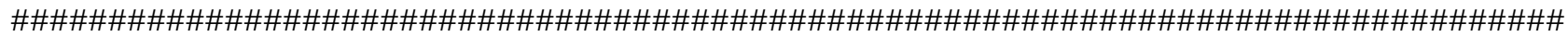

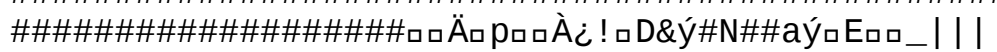

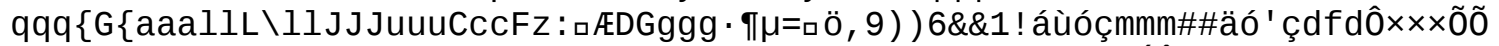

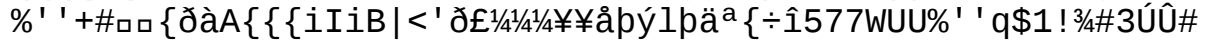

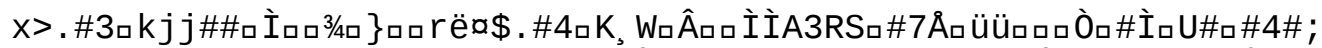

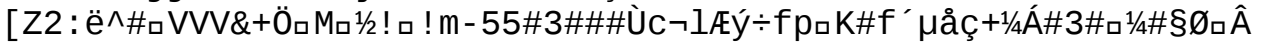




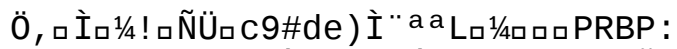

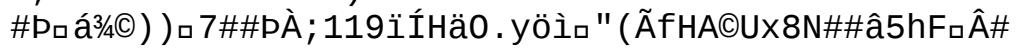

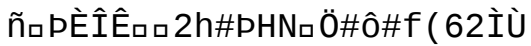




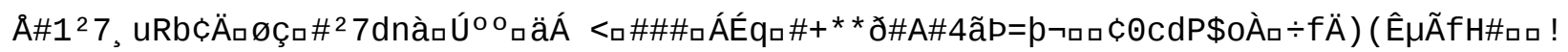
\#å\#0 


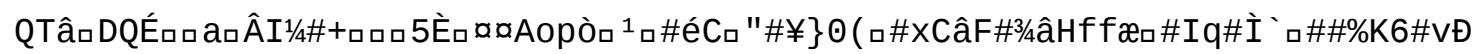


9 ( òÖ@\#VËf 


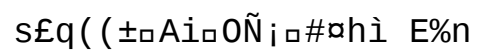




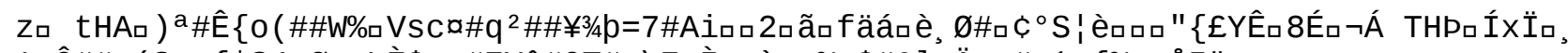

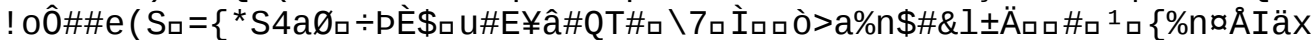

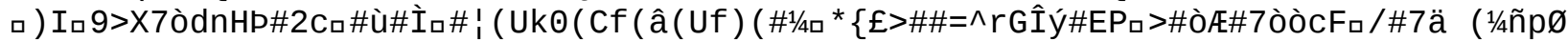




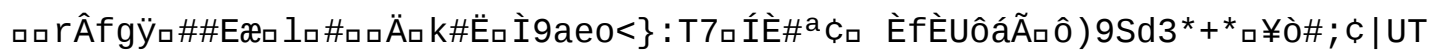




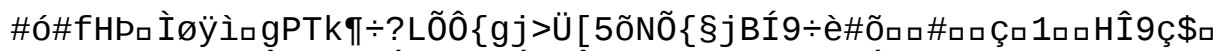

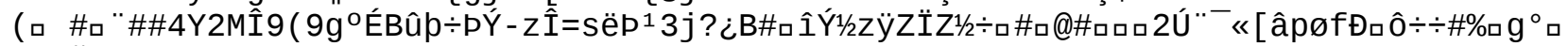

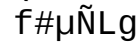

cFn\#S3/4\#mä1̂" \#XüÀ 


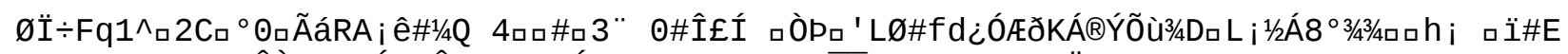

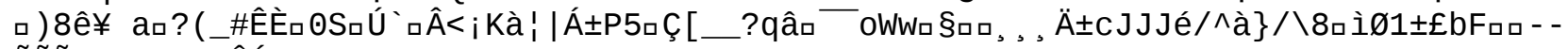

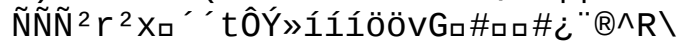




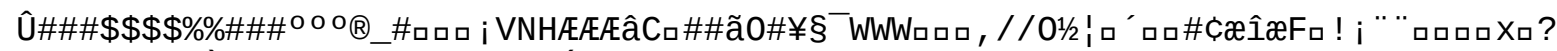

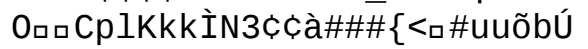




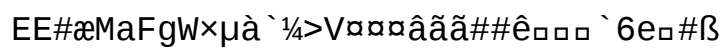


999Æ口ロ\#ロXîîNa!! \#oddd C八, Ànajjöööv?\&FNa: DFa1\#pxgFIq\#Â-ô\#, ììê 


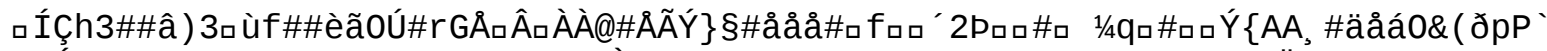

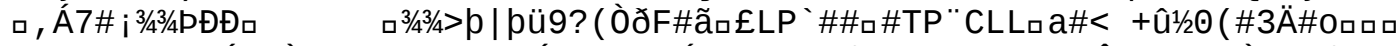

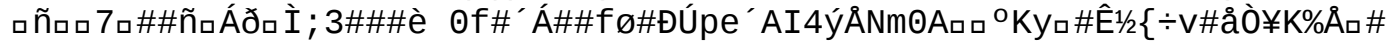

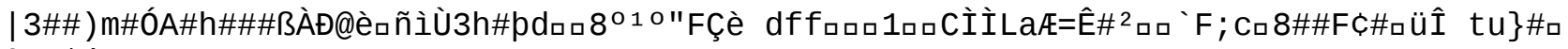
$\circ: \#{ }^{*}($ 6 


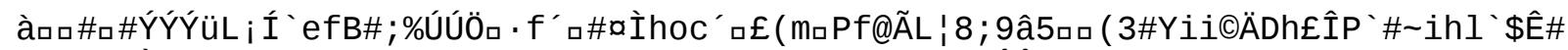

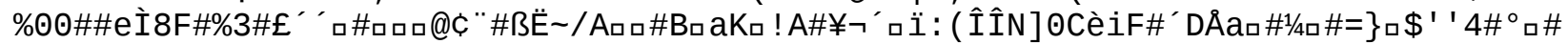

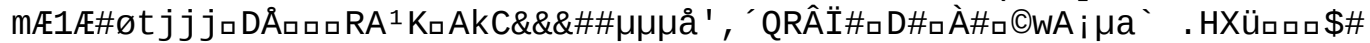

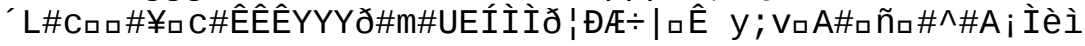




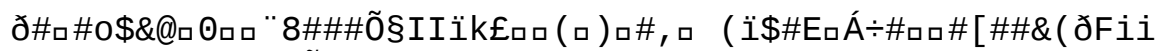
'\#Rx_\$\#>øロ ${ }^{\circ}$ ! FañøøôéSA 


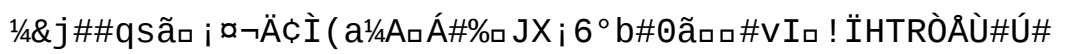




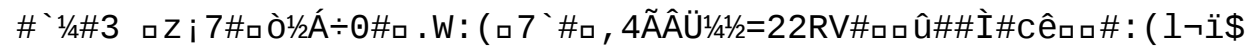

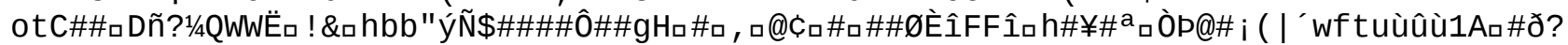

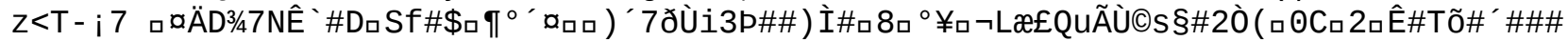




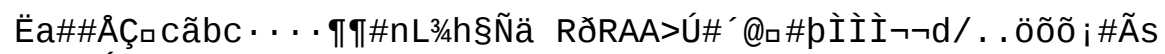

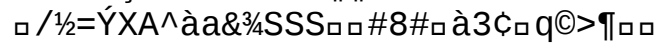

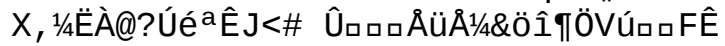




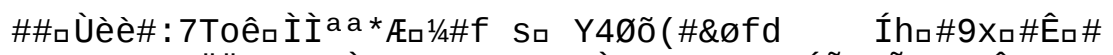

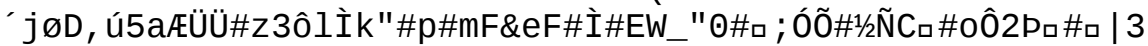

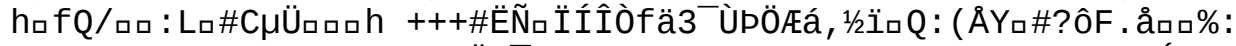

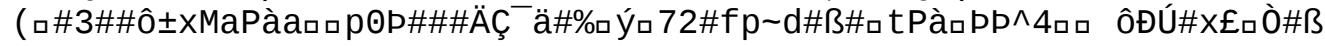




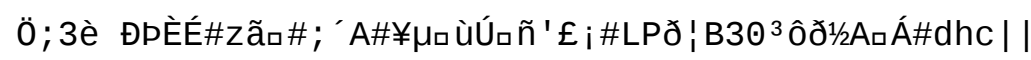


Aa $1 / 4$ ÑP_OKı

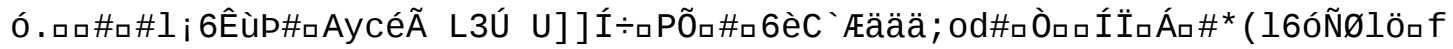
- ãı氏Fロ\#\#\#mPfde

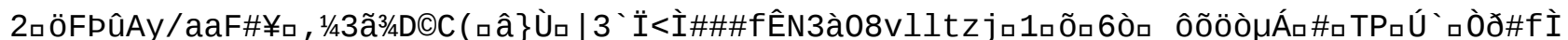

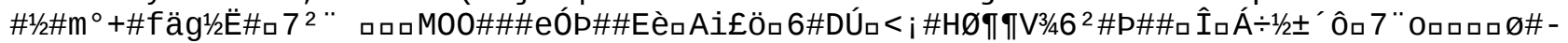

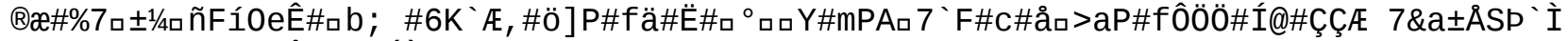

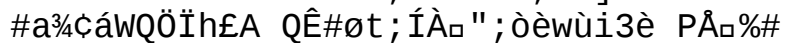

口

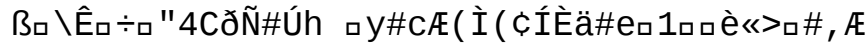


3/4 $\mathrm{D} \square[\square \mathrm{A}$ 


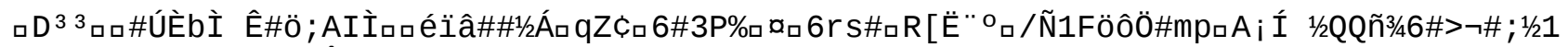
$\mathbb{E x \# \} H e \notin \{ \mathrm { P } @ P e ̀ 3 \# B \backsim A ̂}$ 


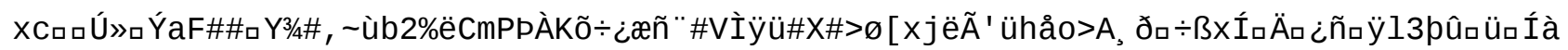
\}\#Pàȳ1/2' ñ?ם=їc\#

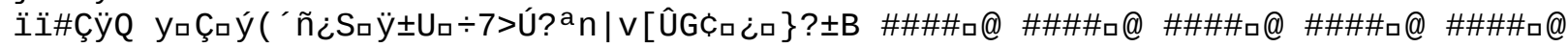

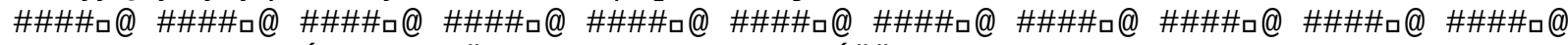

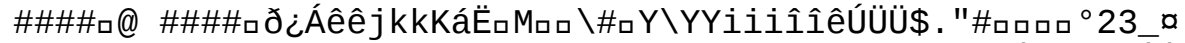

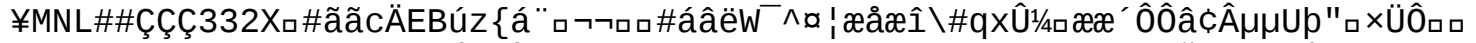

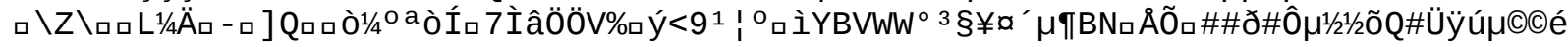

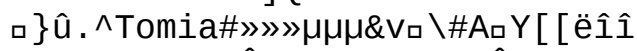

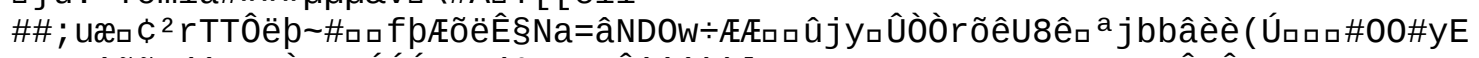

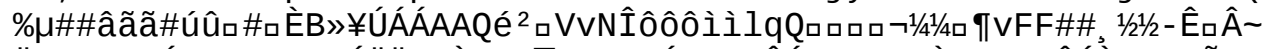

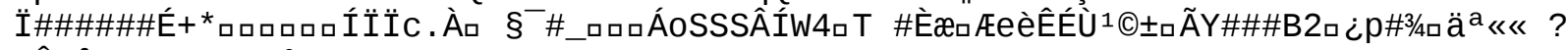
DÛঃÂWíííròò¿üå/¥ed

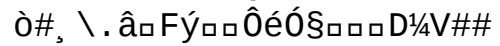




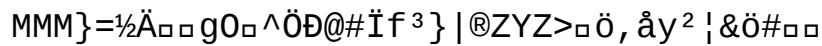

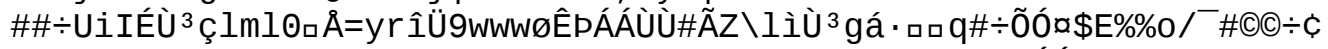

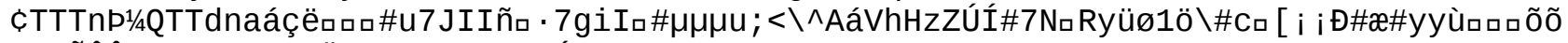

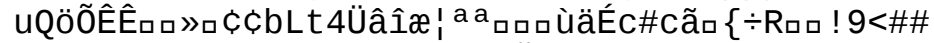

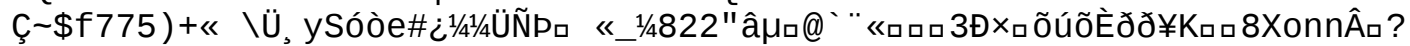

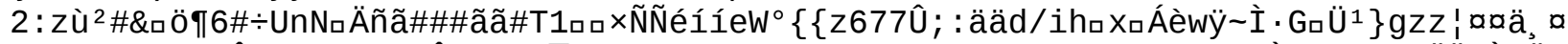

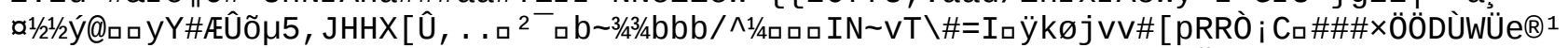
口\#”

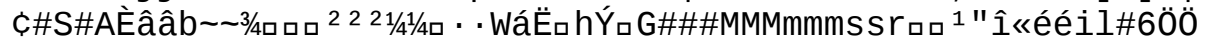

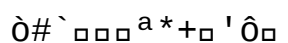

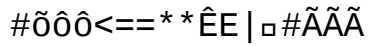

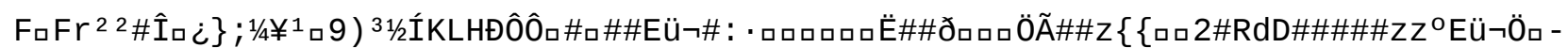
I! 


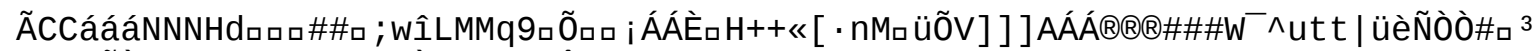

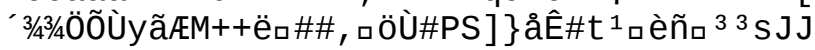

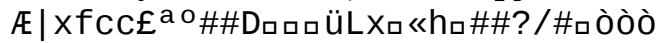

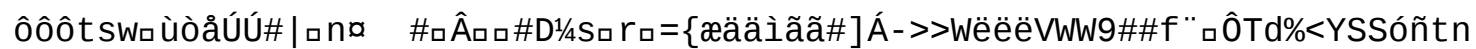

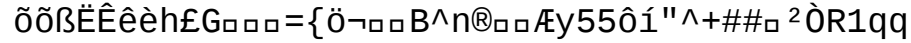




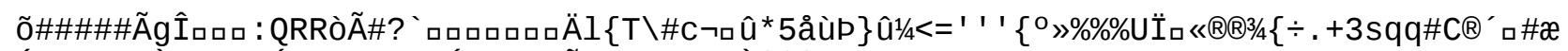

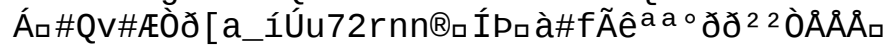

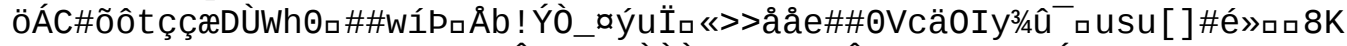

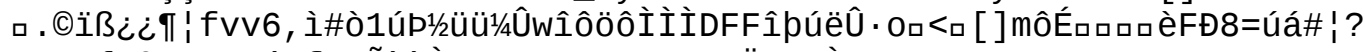

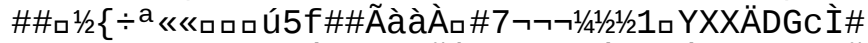

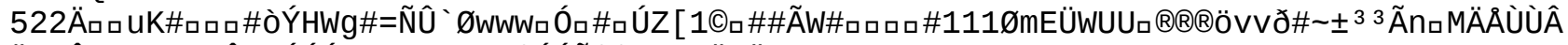

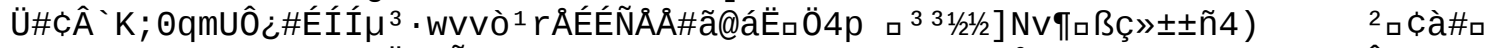

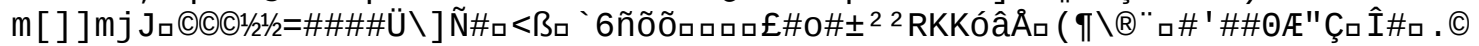

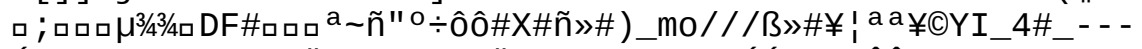

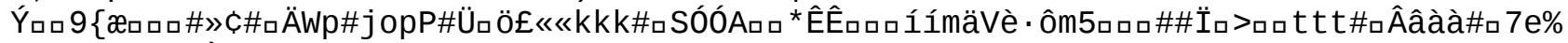

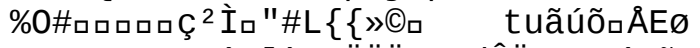

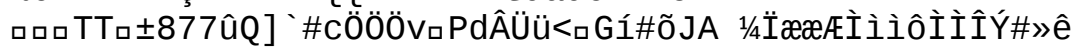


口ロÝ\$[Æ\#ÖÖ×à\#\#ロロ\#o\}\}mjz

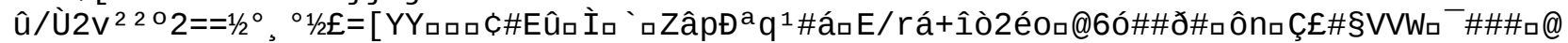

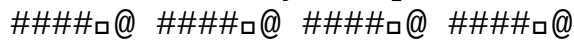

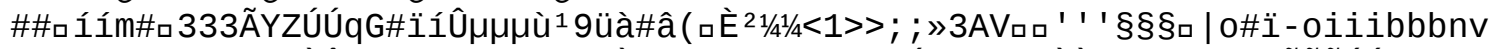

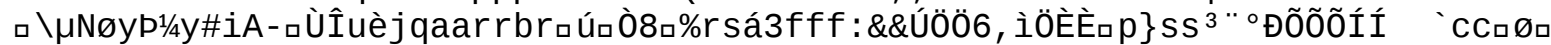

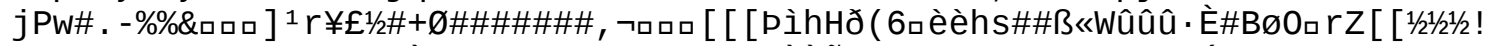

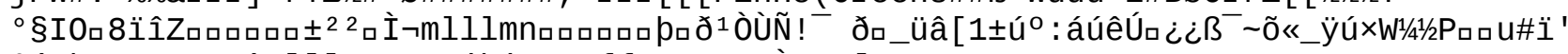

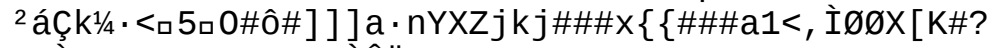

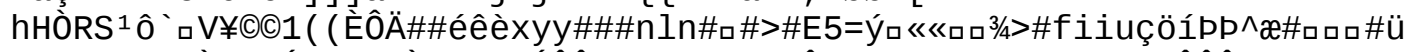

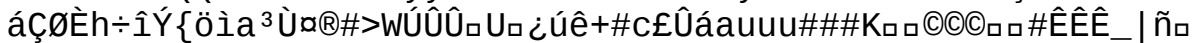

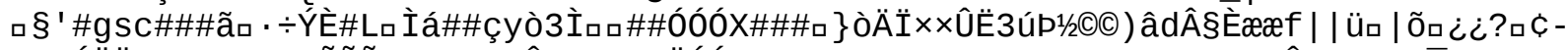

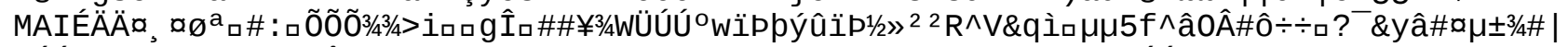

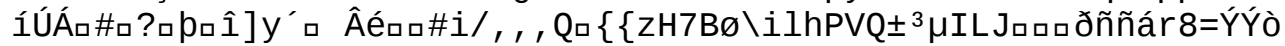

००:i/^ !ñðpoko0MIqqqÑóõ555A $\pm m j l \mid$

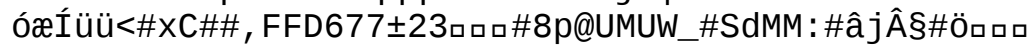

ccc' \# $7^{-}$-wrpÀp

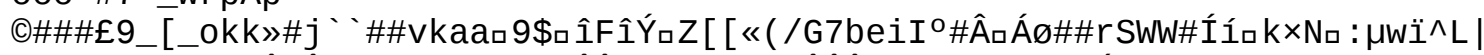

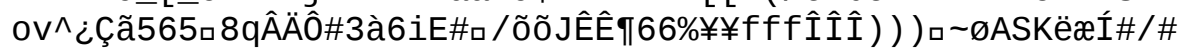

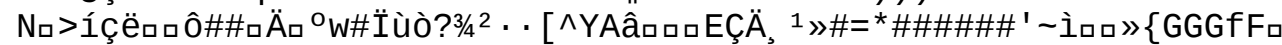

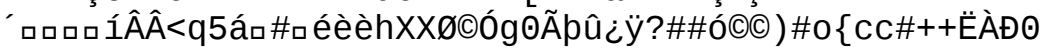

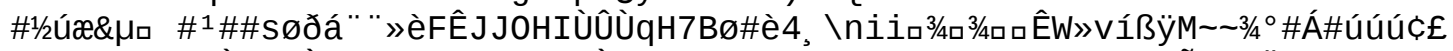

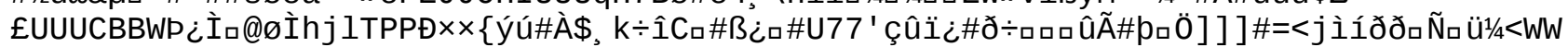
$W$

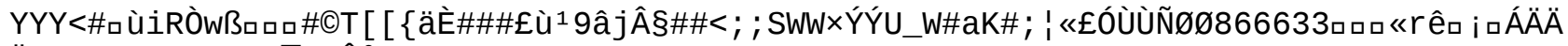
$\ddot{A}\left[{ }^{\circ} \#^{1}\left\{\div \hat{1} \square=\left\{{ }^{\circ}\right\urcorner^{-} \pm+\hat{E} \AA_{\square} \# 573 \# \wedge v E\right.\right.$

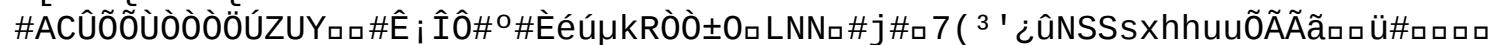

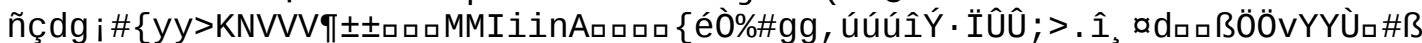

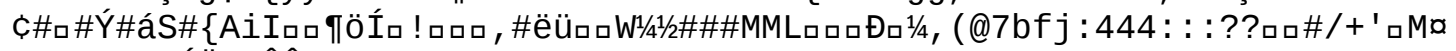

i i 1 ) $1 Q Q I E ́ \dot{I} \div$ êÊE

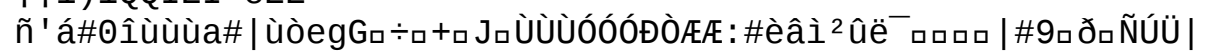

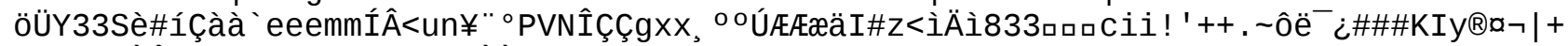

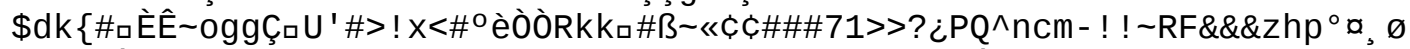

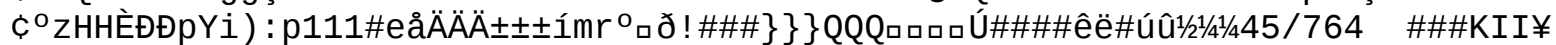

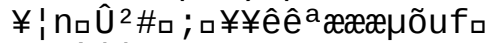

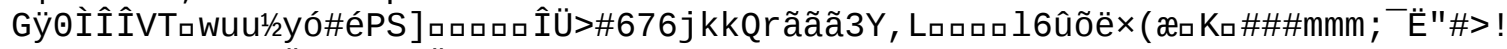

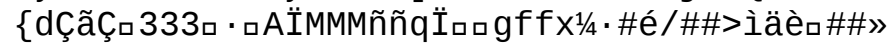




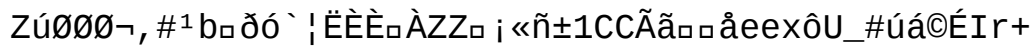

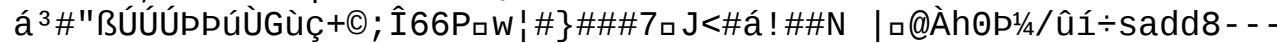

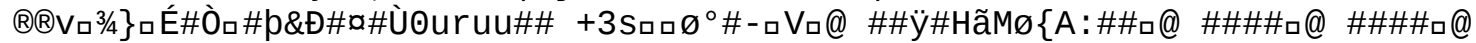

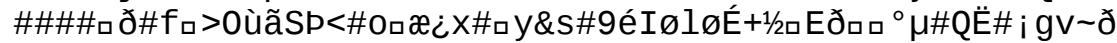

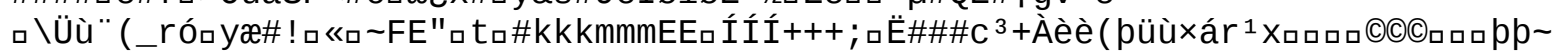

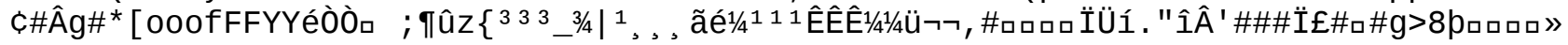

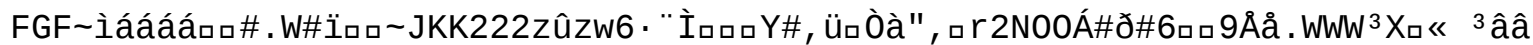

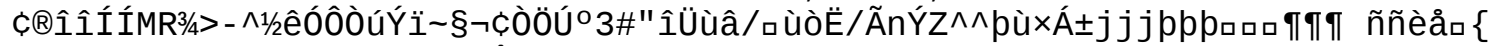

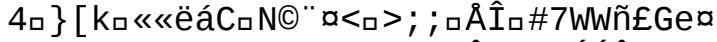

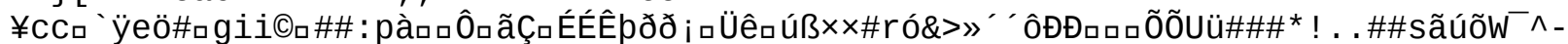

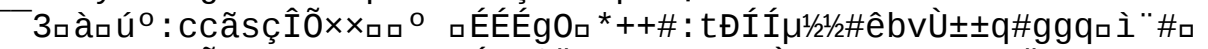

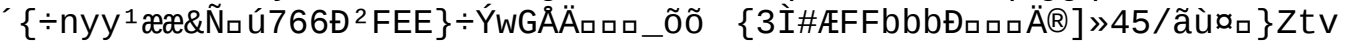

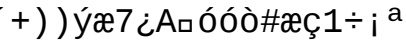




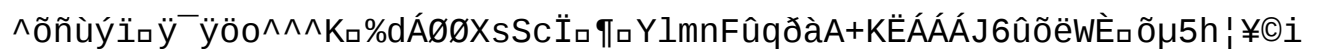

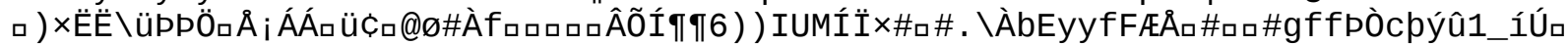

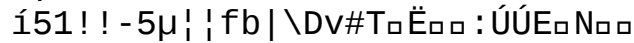





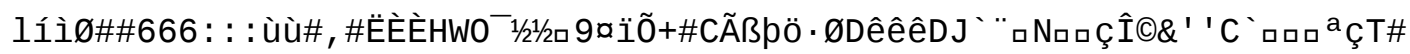

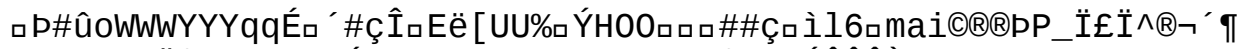

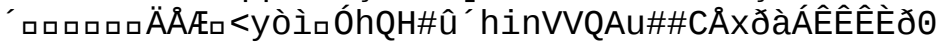

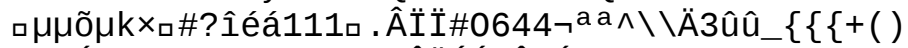

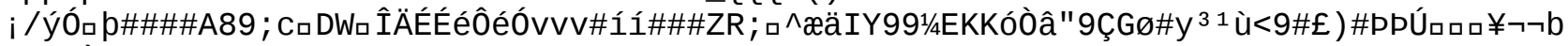
og $\div$ ìù3 


\section{pnnî́̇Mí/\#}

NHIC口; $7:$ : úı ÎF\#? üÇ?

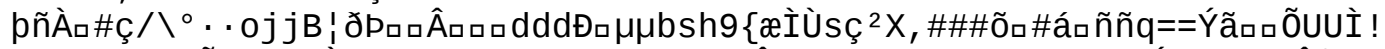

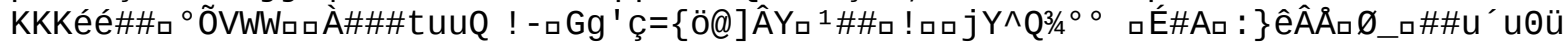

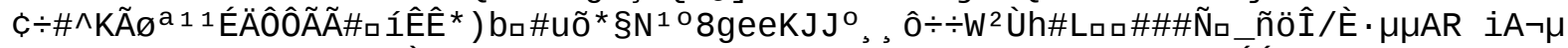

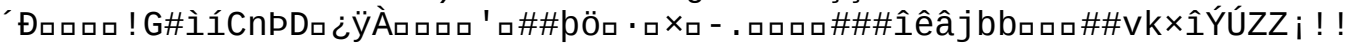

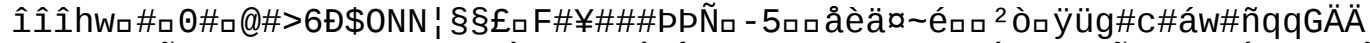

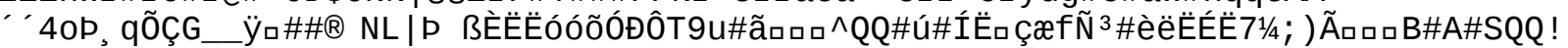

R\#[]]@1 \#\#

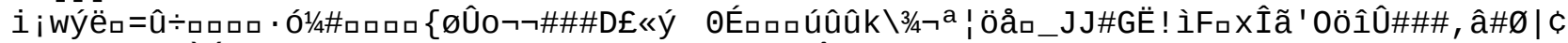

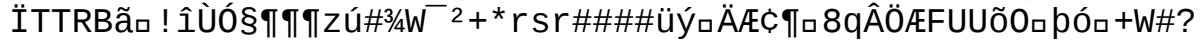

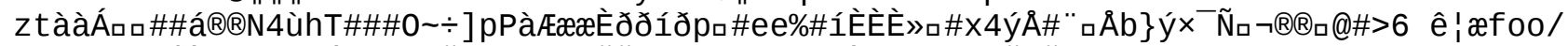

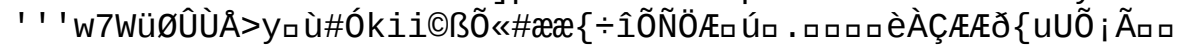




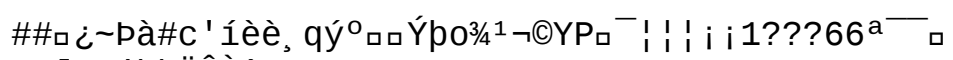
PW[\#<jbbüôİôäI\# 


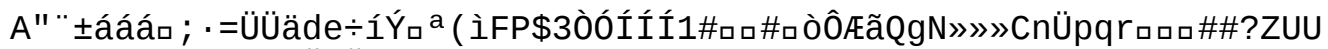

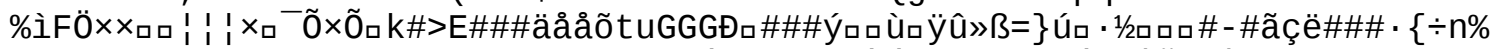

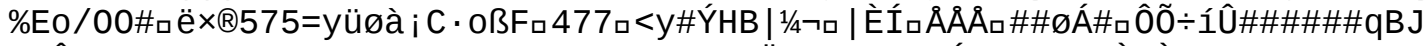

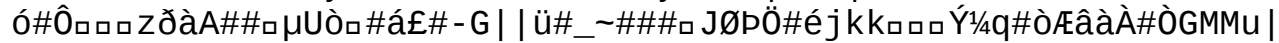
$b_{\square}{ }^{1} M \mu^{\circ a} \#\left\{q c c \# \# \hat{I} \otimes\left(\# \# \# \cdot{ }^{\circ} 0 \_a ̂ a A ̈ \bar{Y} " c £ £ i ! ! n ̃ q \partial\right.\right.$ 


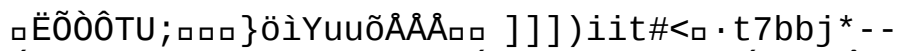

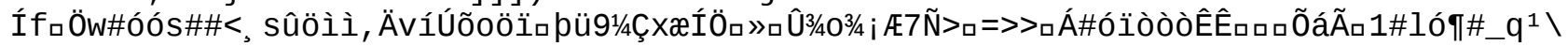




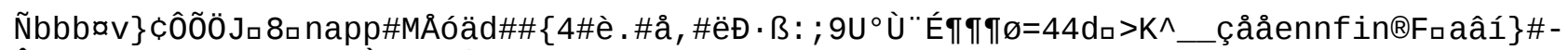

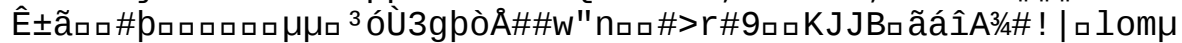

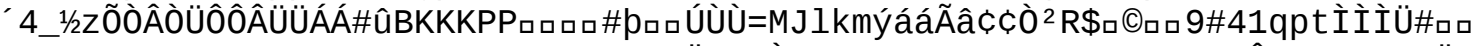

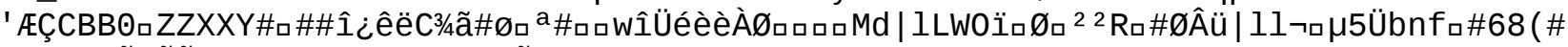

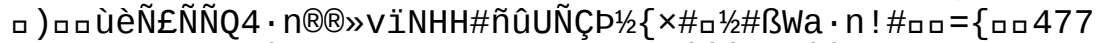

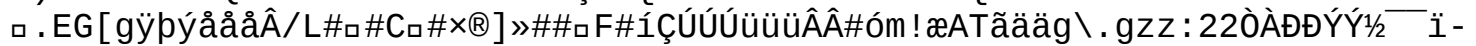

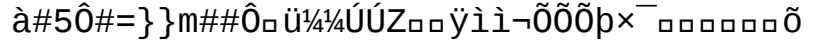




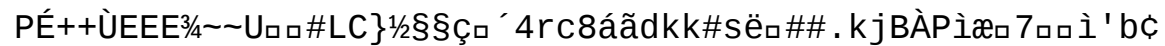

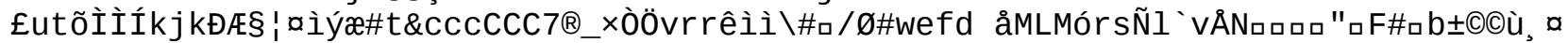

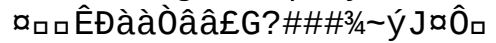

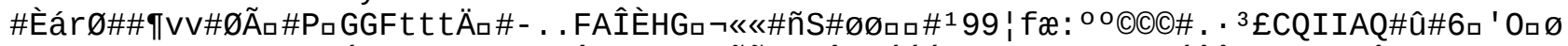

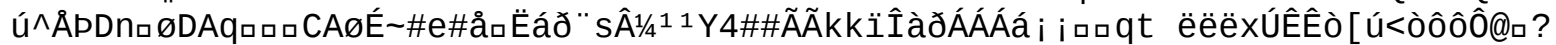

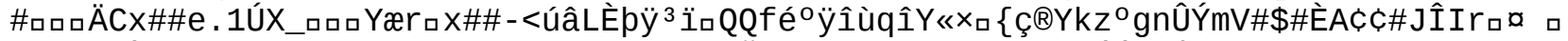

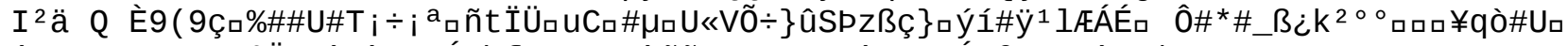

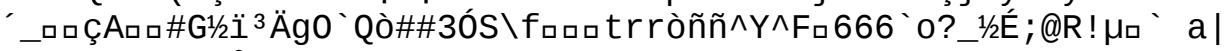

$E \# \wedge 1 / 4 \#^{2} \mu^{3}$ ëëíÅW\#9ロ\$口7VAÿC

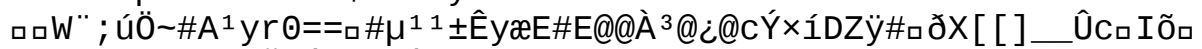

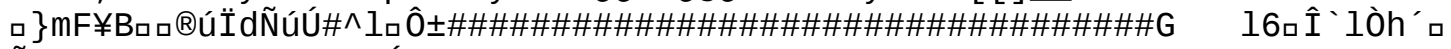

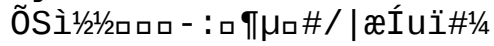




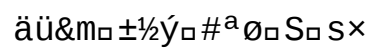




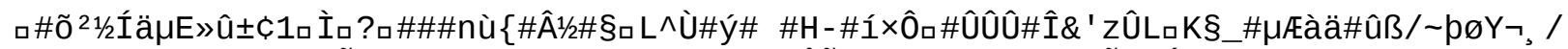

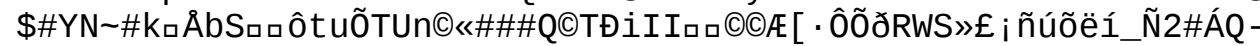

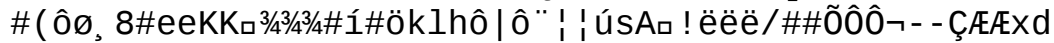

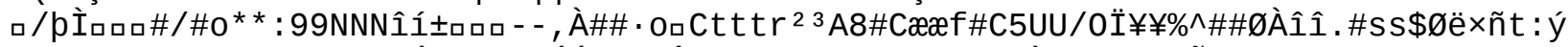

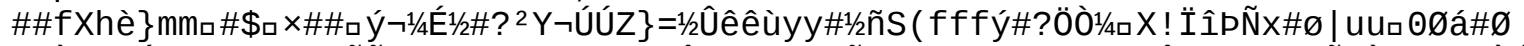

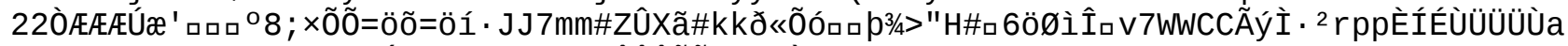

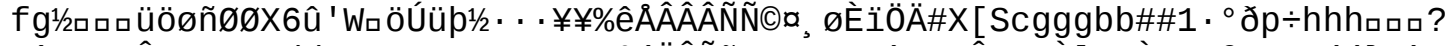

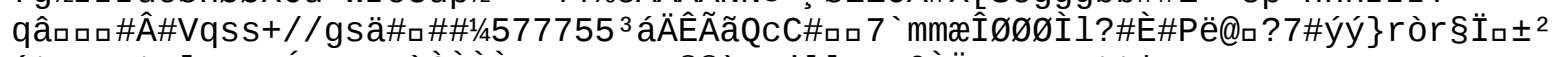

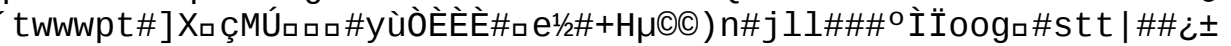

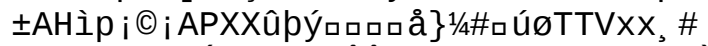

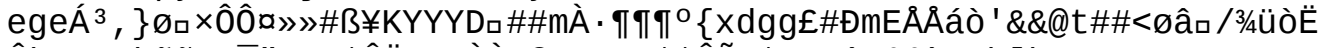

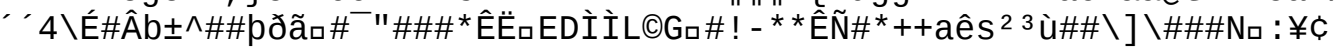

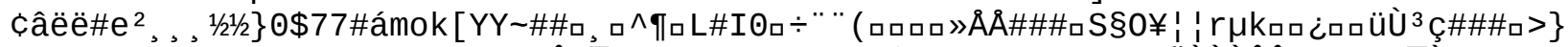

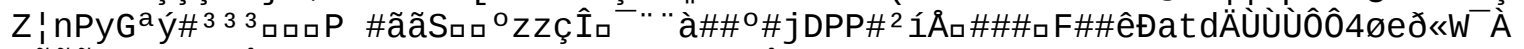

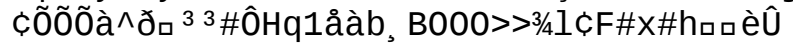




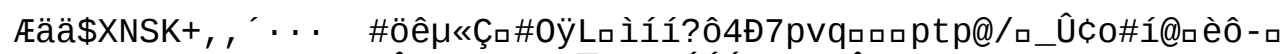

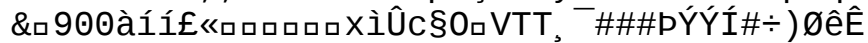

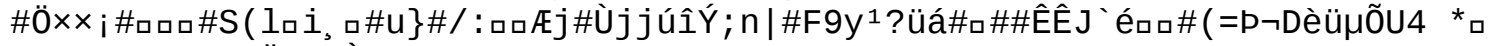
w ««kgg7ĐßÿöÜH\#aÈx_a-\#\#£®®\#é[SSっì" 


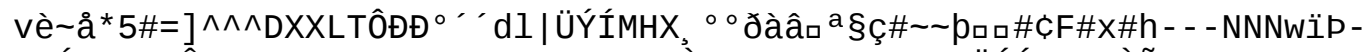

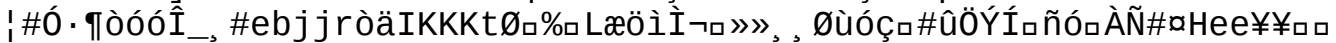

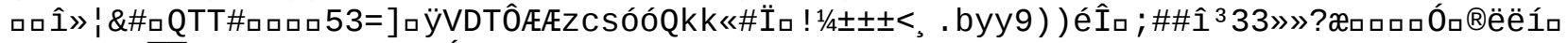
g466\#\#\# çeâE\#ロロ Ao\#\#\#Á\&\#\# 


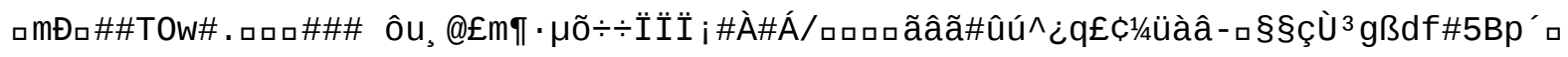




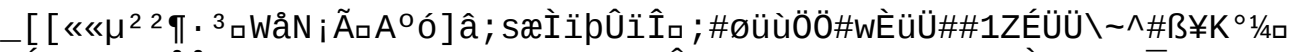

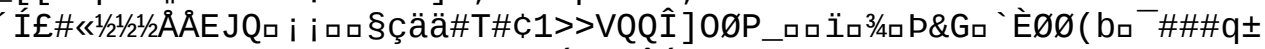

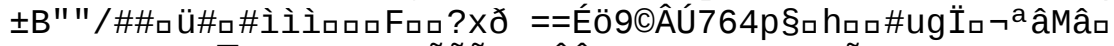

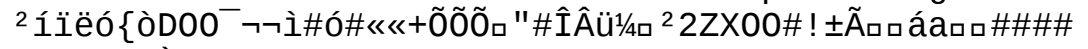

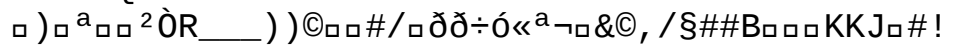

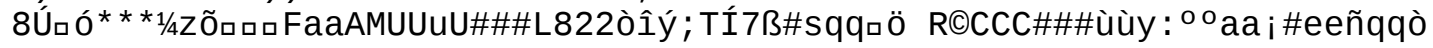

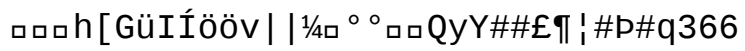

)/-ロZ\#)++ëãí5； ; ; 88øáÃ\# 


\section{$\tilde{\mathrm{N}} 3 / 4 \square ? *$ *}

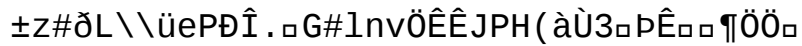

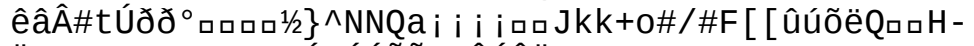

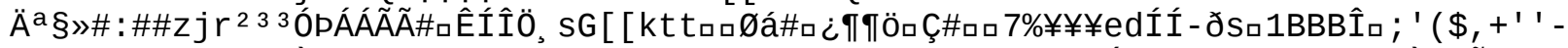

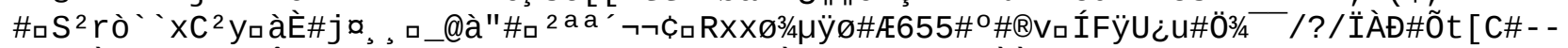

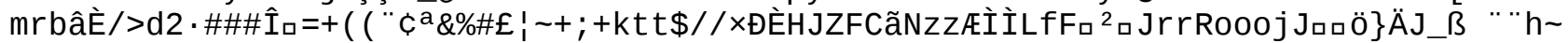

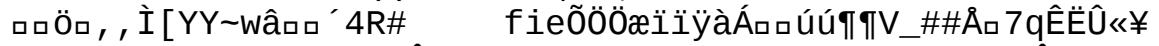

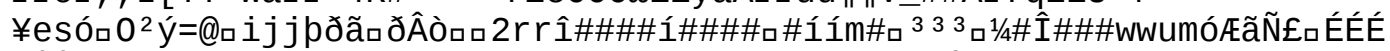

' ôôøèèøøØ: /FF

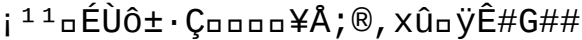




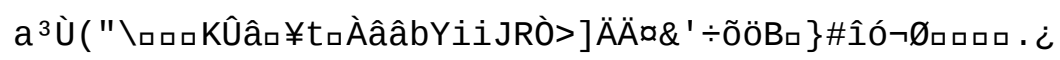




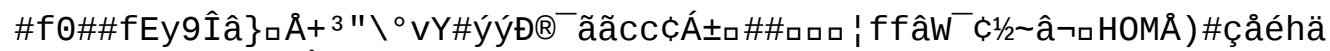

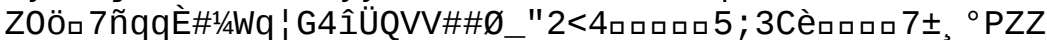

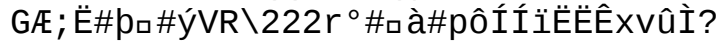

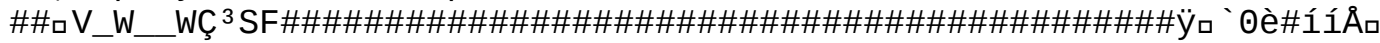

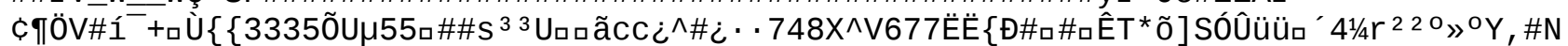

\# 


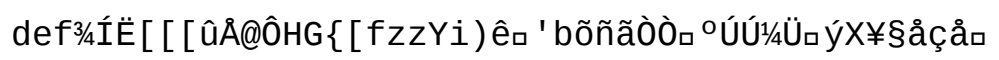


\#ï\}ü\# \#Ãdfdø"\# 
Æ/\#̂ิì̀̀464 VxgñØ\#_.̈̈×xKaKÒSS ${ }^{1} \#$

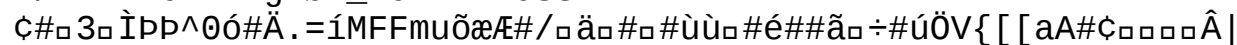

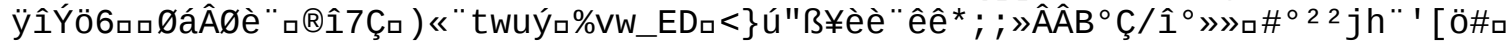




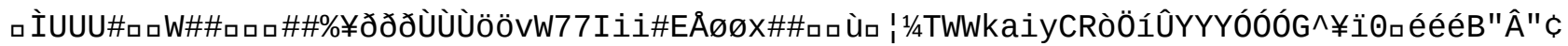
$c^{* * *}$

$a_{j}$ êè 


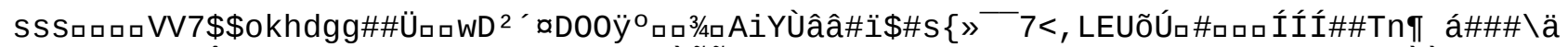

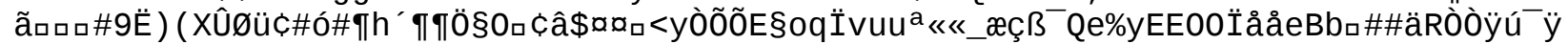

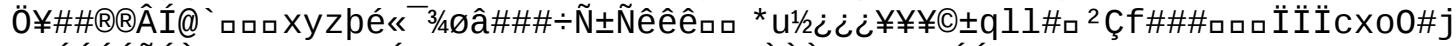

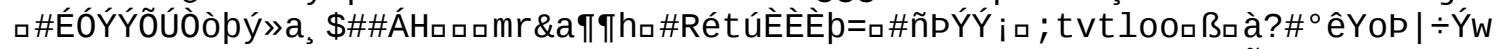

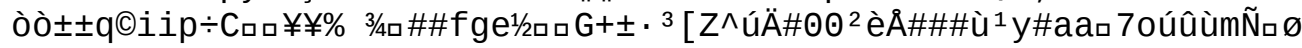




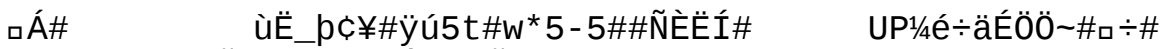

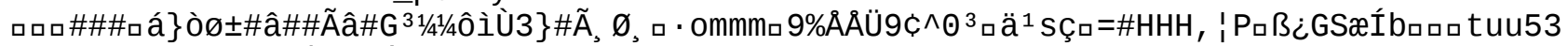

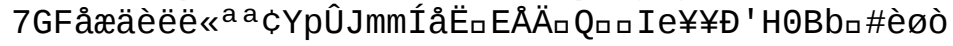

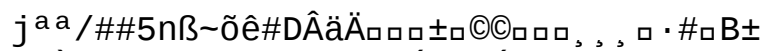

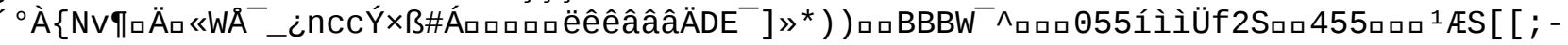
- $\left.\left.C^{1} / 4 \backslash\right]\right] M L L$ 


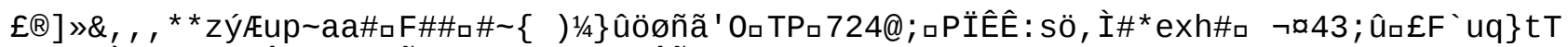

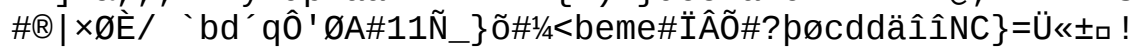




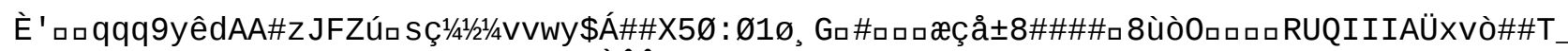

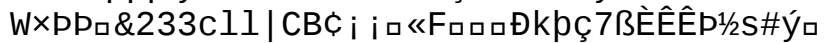

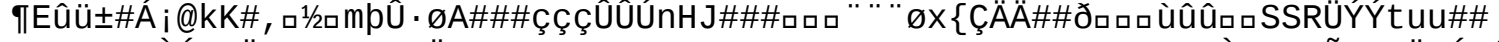

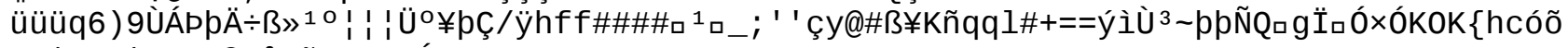
ฉ 


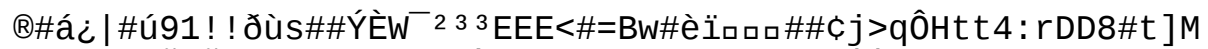

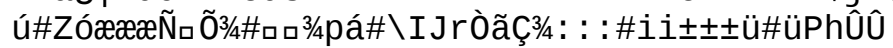




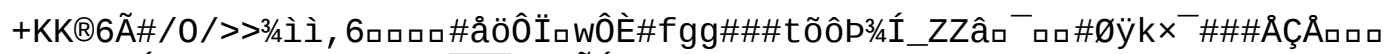

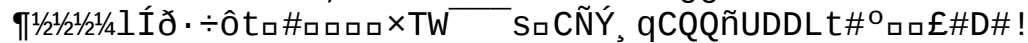

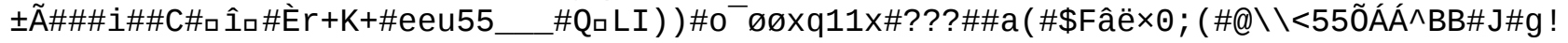
<@/àó1/2ロ\#\#

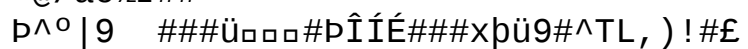

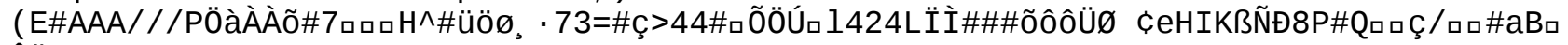
ÔÖ

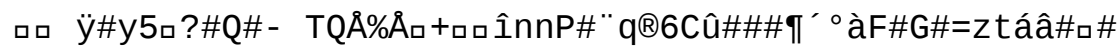




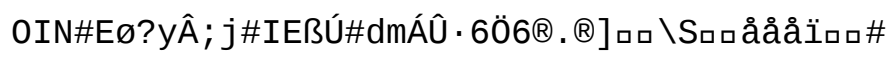




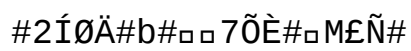




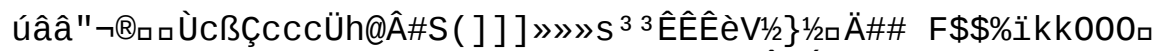

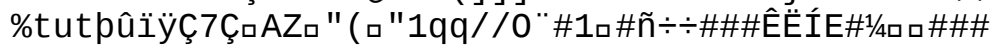

yñ"\#g1\#

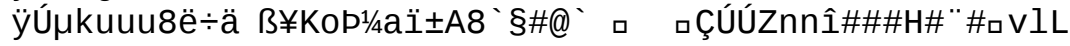




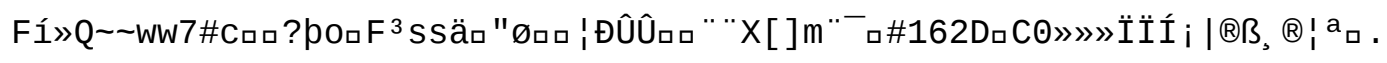




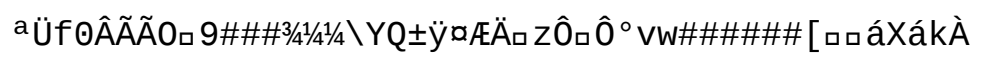


\#\#\#ロロQ\#.^ 


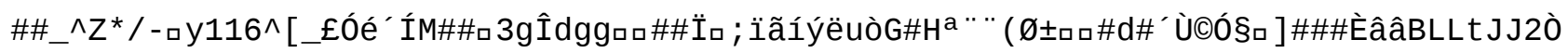


\#0̈ØØøâÅ॰0̃õ<a F\#\#dWDD\#

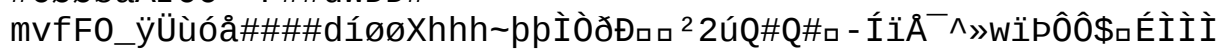

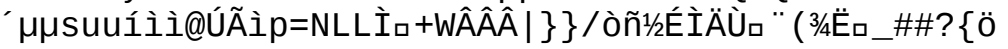




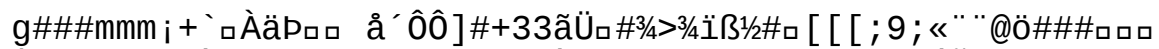

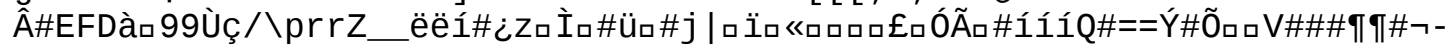

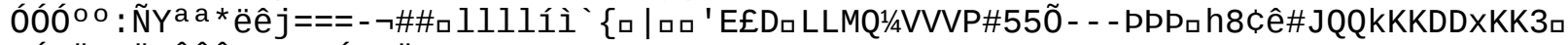

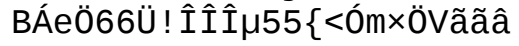


ฉ öÙĐÉÉÂÂA^^${ }^{-} \mu \mu \#$ 
$\mathrm{F}^{-}$££\#|\#\#\#REôpöåËロロロロ1/4@F Éロロ 


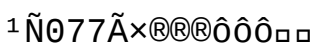




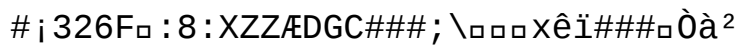

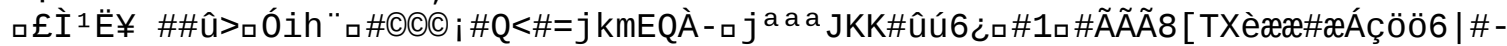

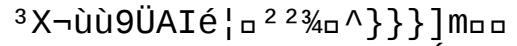

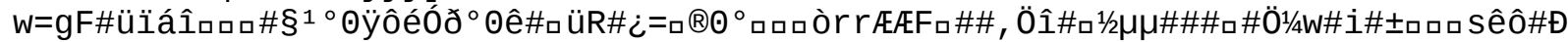

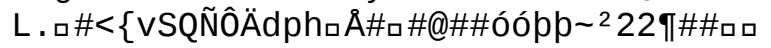




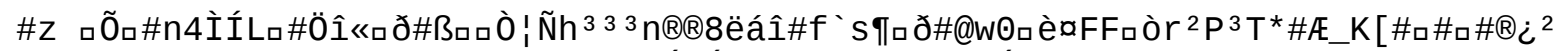

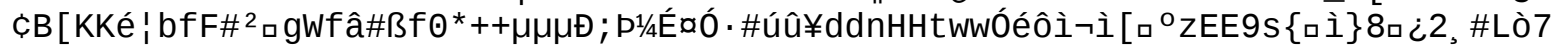

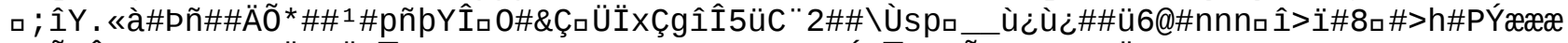

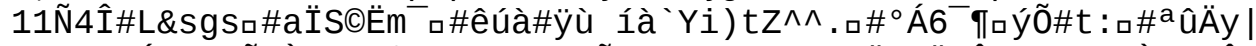

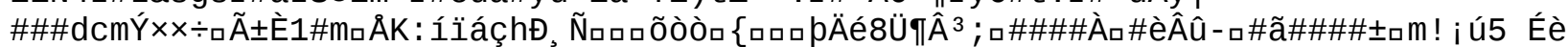


\#âcZê?\#ロÜ3/48Ùw\#\$ロ\#\#\#\#\#\#\#\#\#\#\#\#\#\#\#\#\#\#\#\#\#\#\#\#\#\#\#\#\#+öØìíí́

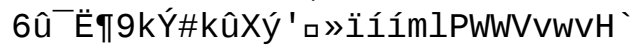

$$
\# ; \text {; Ö }(
$$

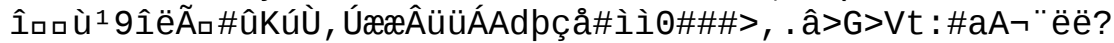

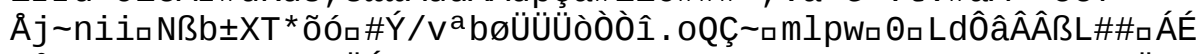

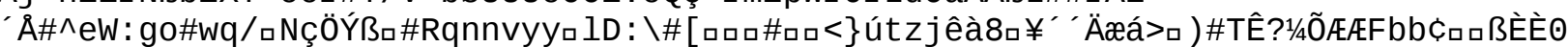

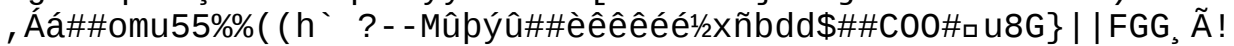

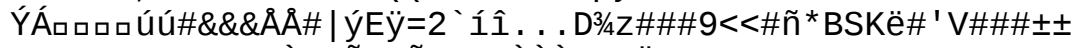

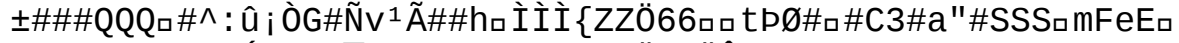

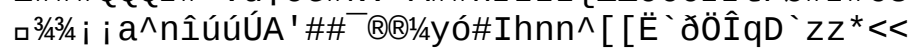


9\#5\#Wロㅁ

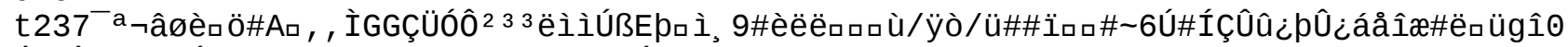
Á«\#Ò\} ̈̈ãGaÁç\} | bïíáTNNNDDÄäääPPG \ÆÝÝ

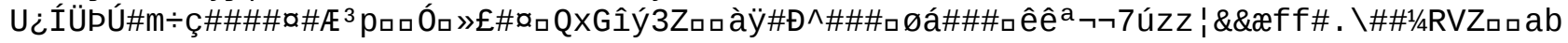




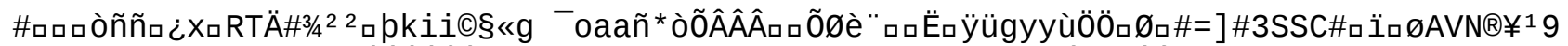

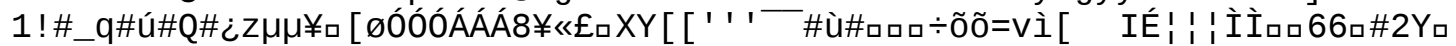

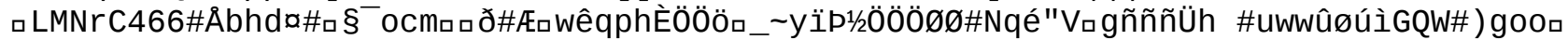

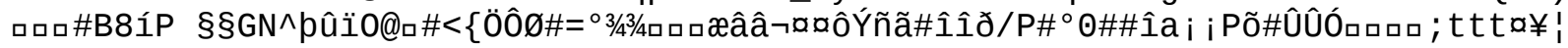




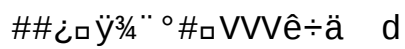




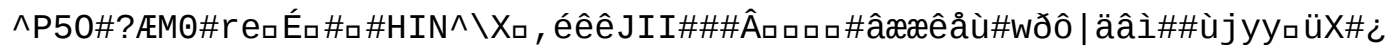
fff $\left.\backslash \backslash N_{a}<\right)$, , ÜÜÜ 


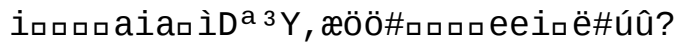

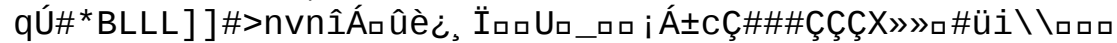

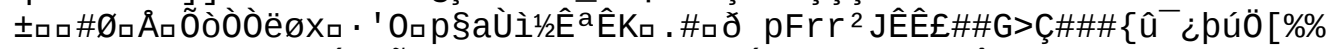

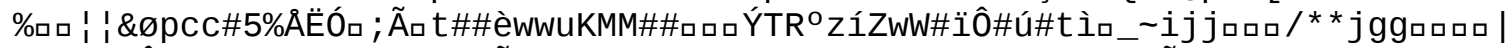

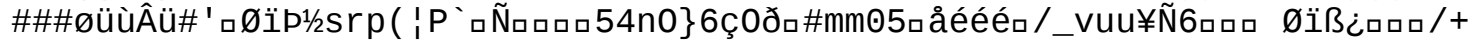

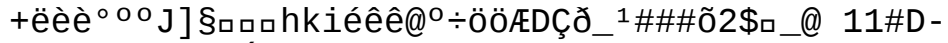

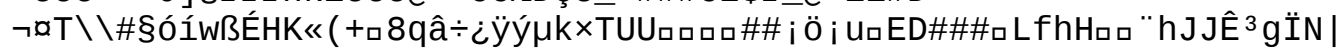

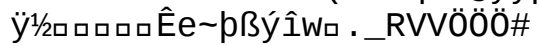

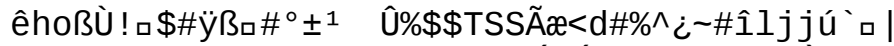

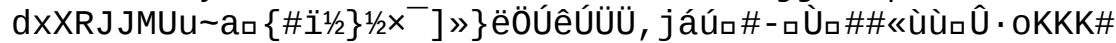





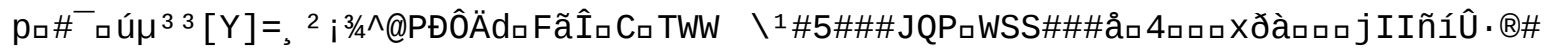

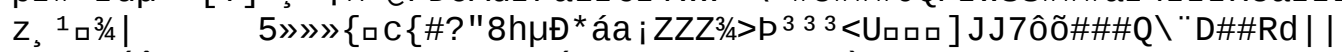

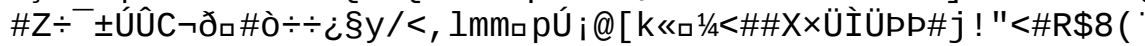


2 ÄpD\#j\#?

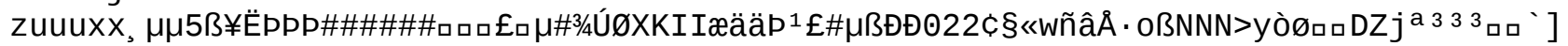

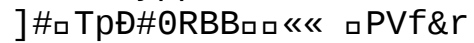




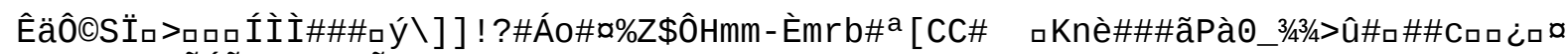

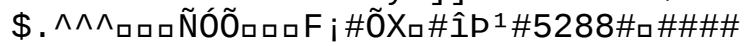





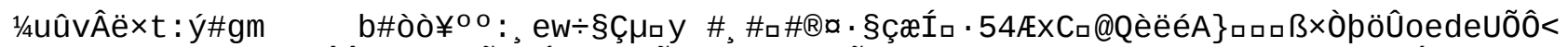

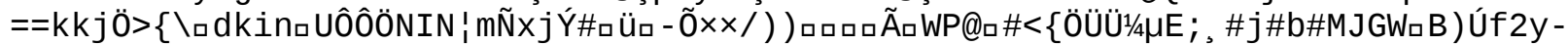

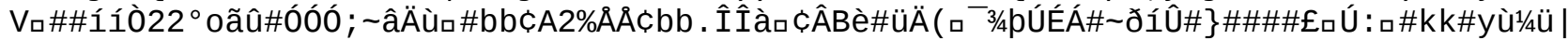
m - - MMMıロÚæ:

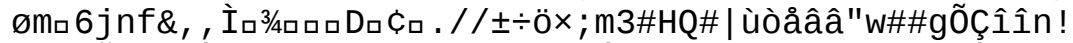

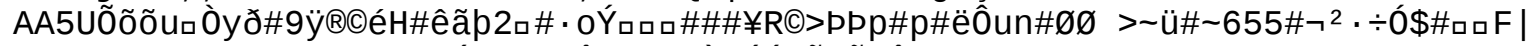

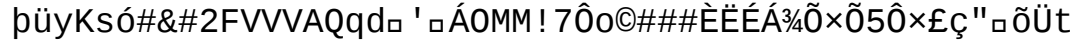

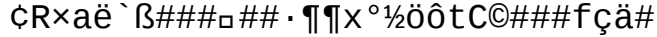

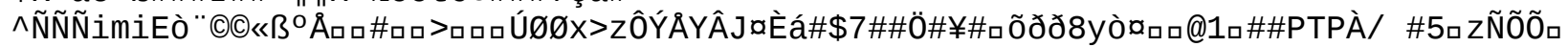
\#IJJvuq9uú4D 322 Wa 
!æo* *̂ễ̃̃ \#á

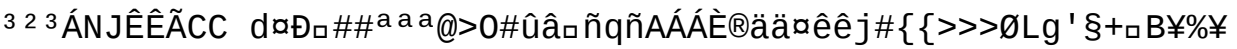


\#\#88\#Ӱロ ${ }^{\circ} Đ P,-J Q \tilde{N} E>>\# C F \# \sim+\square O ́$ 

口 h\# 


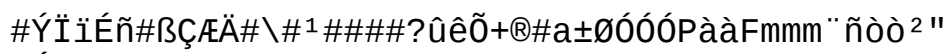

\#ÍEKK«§»ÛH\#

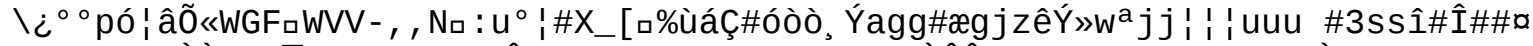
口WNN囚, Ø0̀òRWO ${ }^{-} \mid$i \#< ÂTWWoll

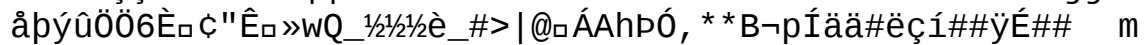

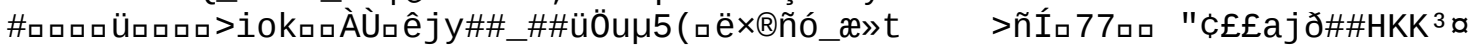

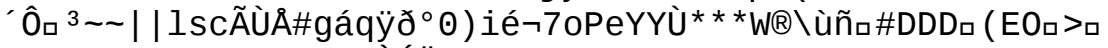

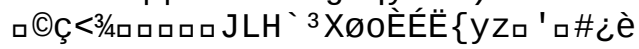




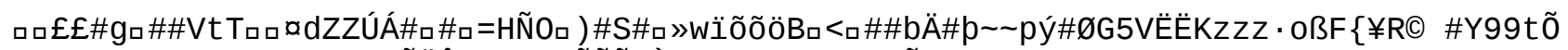

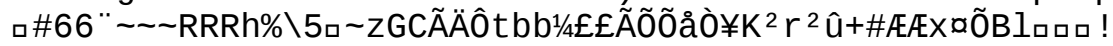

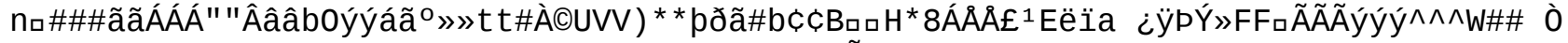

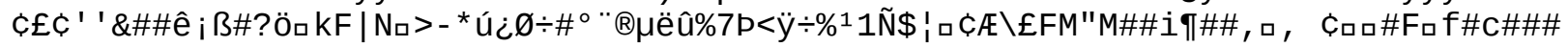


\#ロCôaT\#\#ロqta $\mathrm{i} \div \hat{\mathrm{I}}$ 


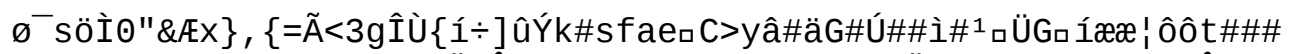

口@口

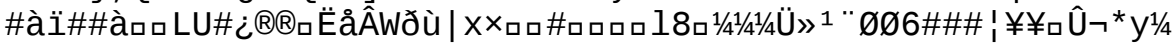

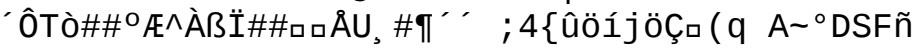


\#\#B·P]k+ó?Z\#ロquqJn\%quPUU

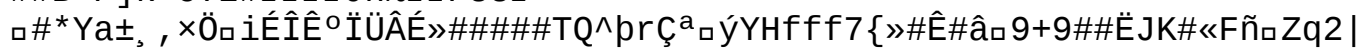

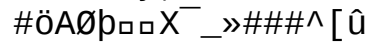

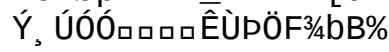



$1 / 4 Y \hat{I}$

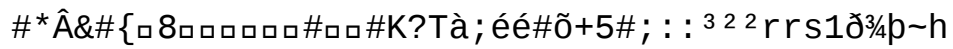




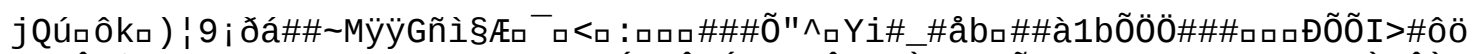

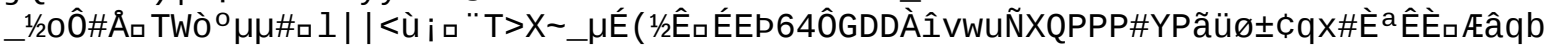

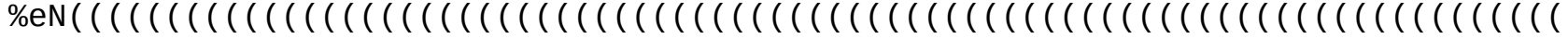

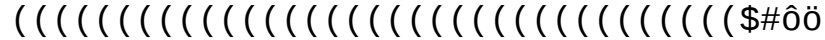

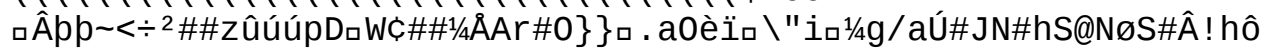

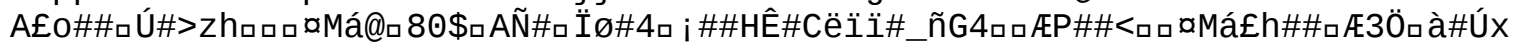




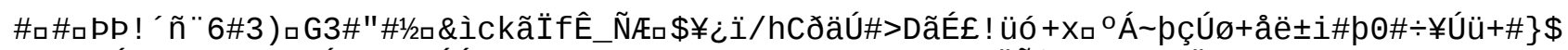

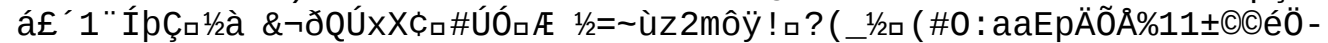

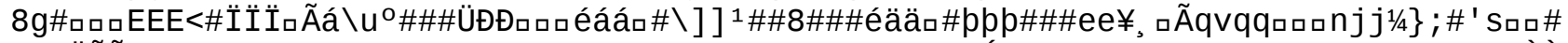

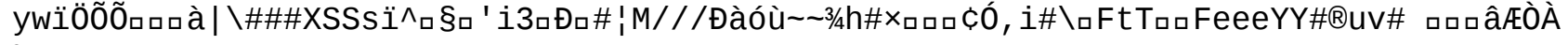
\%ууунн 


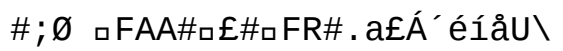


$\#<\square$ ??Â<, 


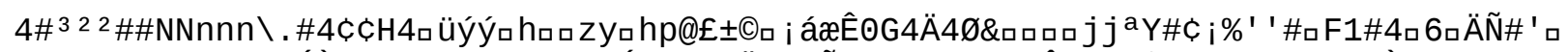

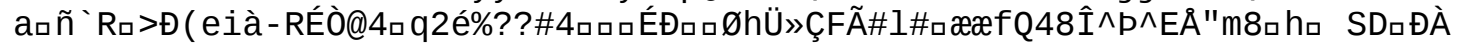

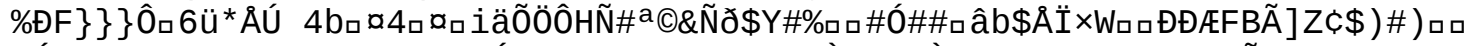

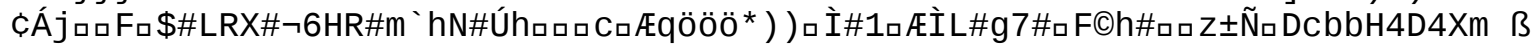

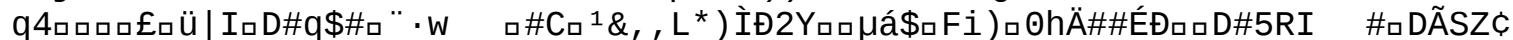

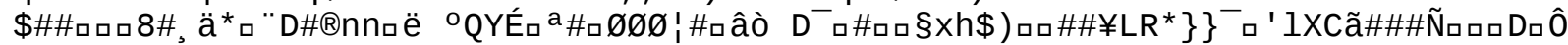

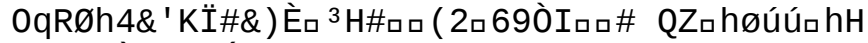

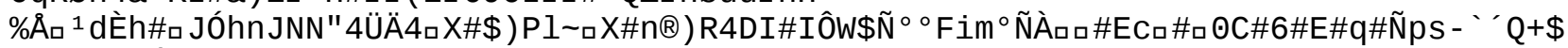
$\%_{-,}, Q_{\square} " 0 ́, \mathrm{~J}$

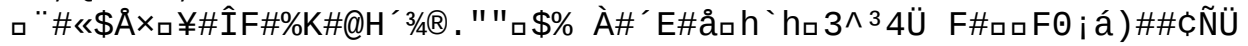




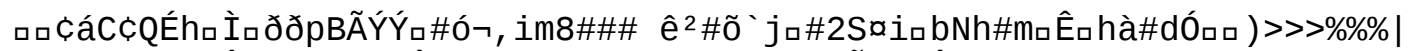

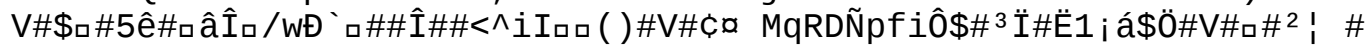




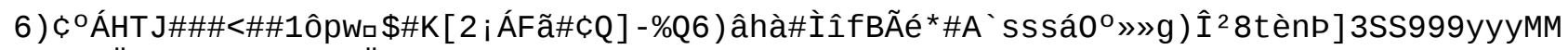

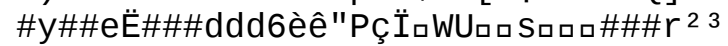

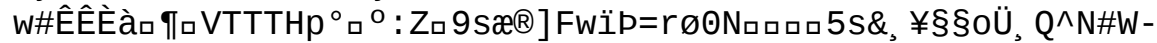

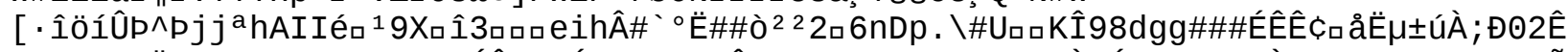

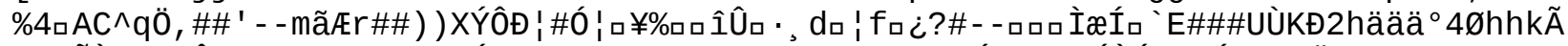

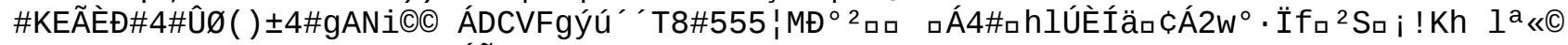

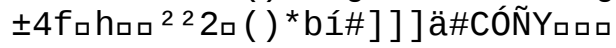

$\square^{2} 34 a ̈ a c a_{n} \cdot i i_{i} A a c \% I$

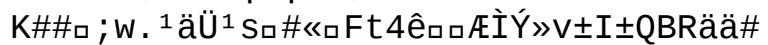

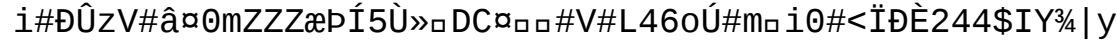




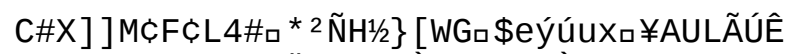

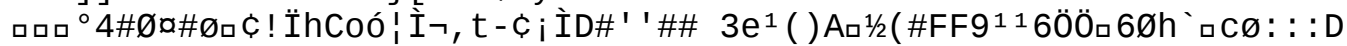

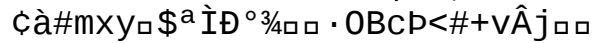




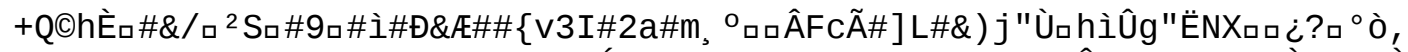

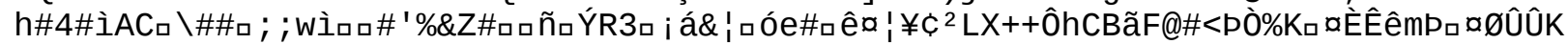

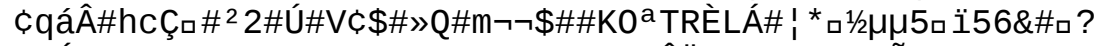

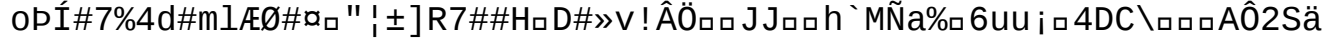

ㅁ 


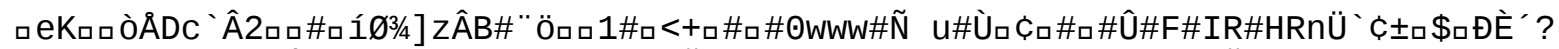

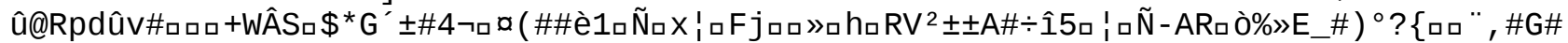

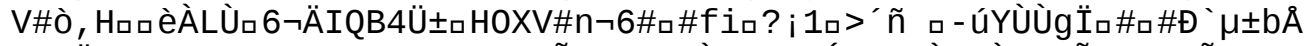

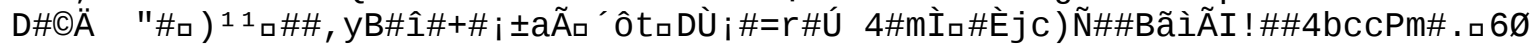

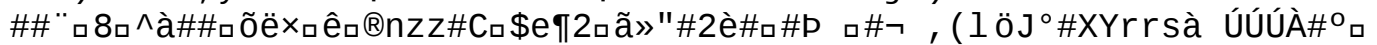

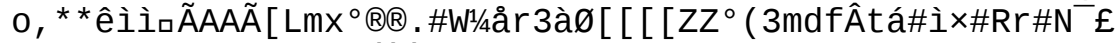

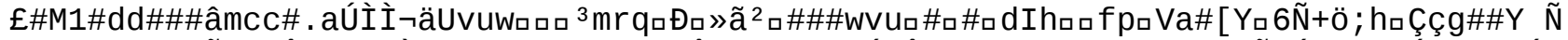

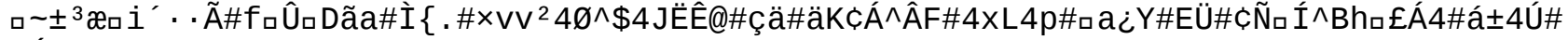
çÁĐèdi 


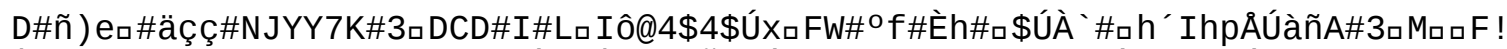

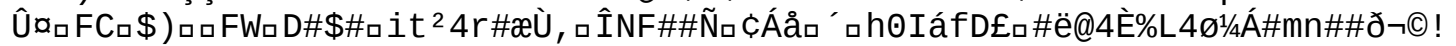

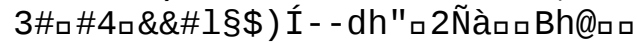




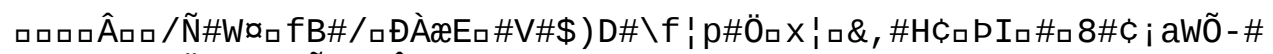

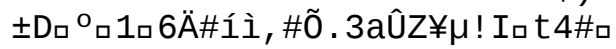


1ъÖロ V\#İa\%

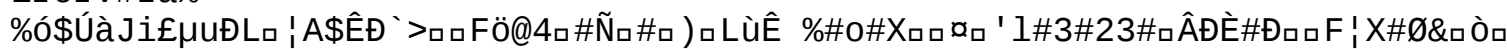

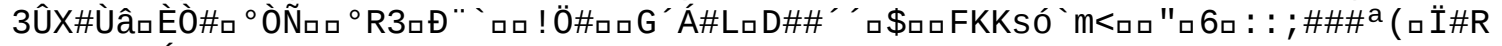

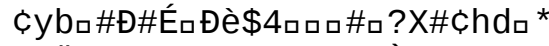

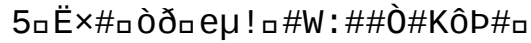




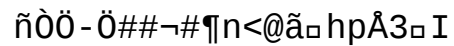

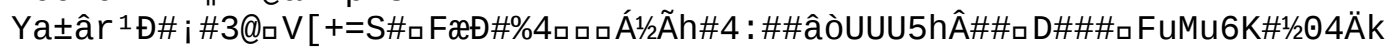

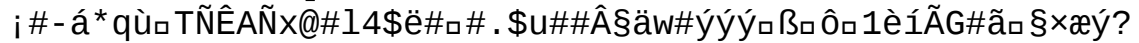

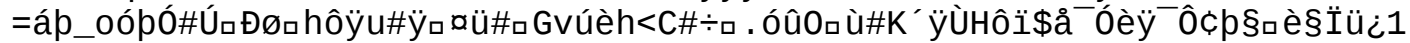

' ' ýS

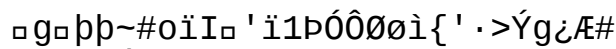

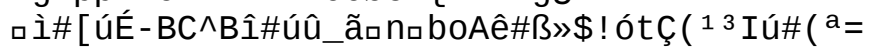

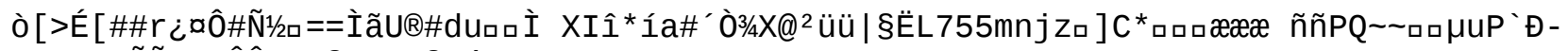

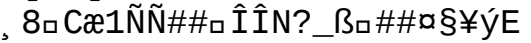

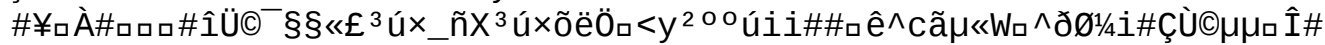

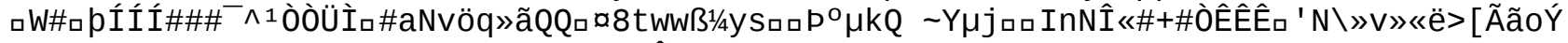

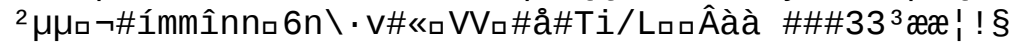




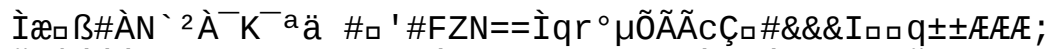

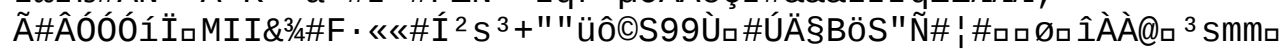

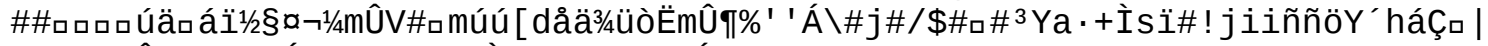

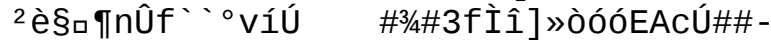

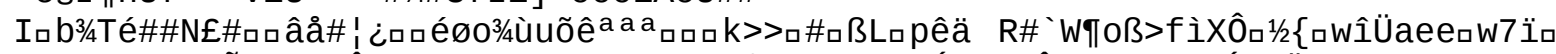

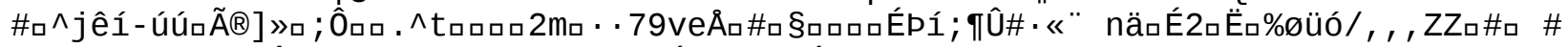

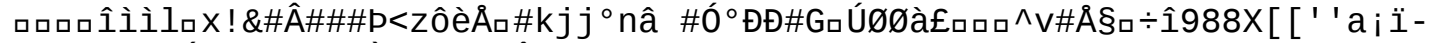

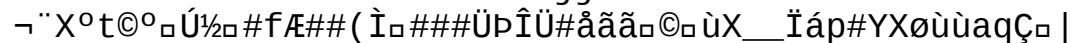

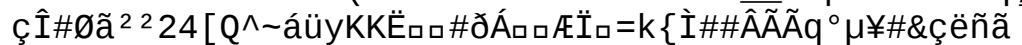

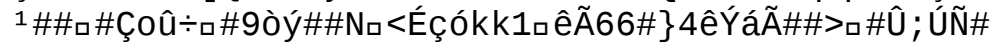


ù\#\# ' Na\#\#\#koog ( 2 231ðsç\#ÎáİÁÁÉÉ@ロ

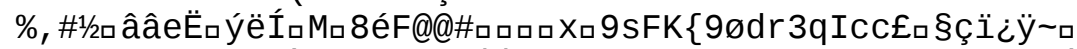

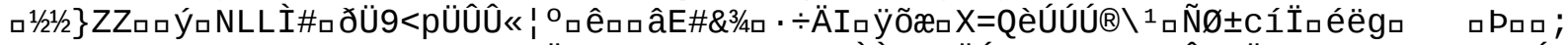

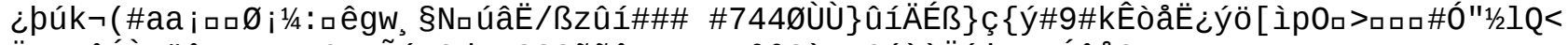

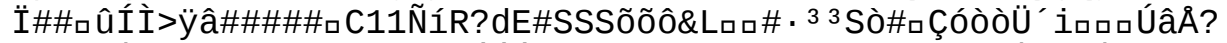

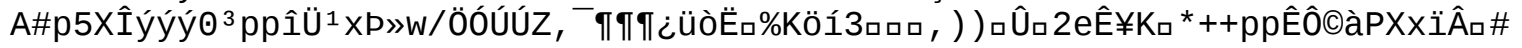

8\#Ëロé »;göì5kÖ, ${ }^{11}$ ÁçÀİİ $5 \ddot{E} \times x \cdot{ }^{\circ}{ }^{\circ} \# \# 0 ̂ e ̂ 0 ̃ \ll \tilde{N} \&$ 


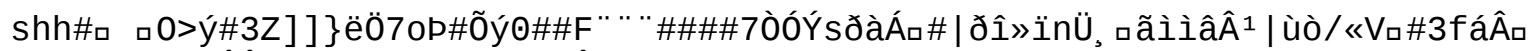

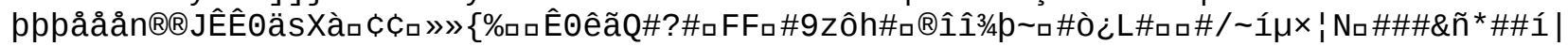
$\#^{-0}$ o

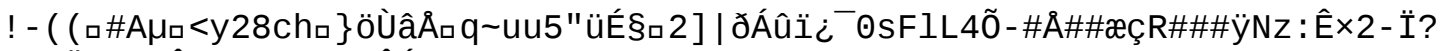

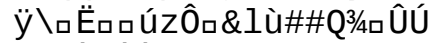

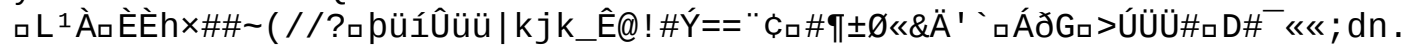

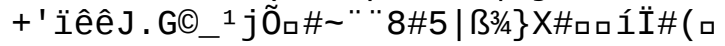


ロ\#ÉÉÉÛđmõõõ

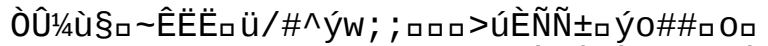

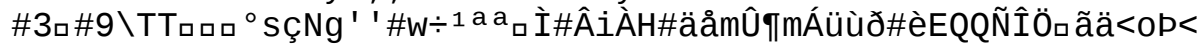

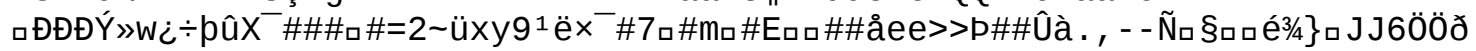




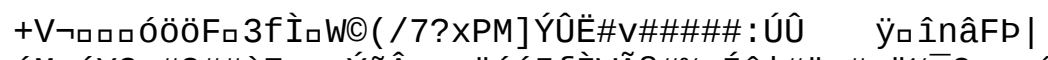

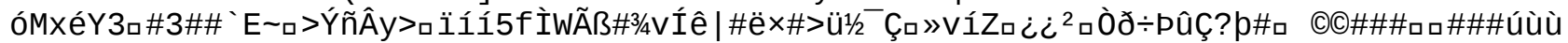

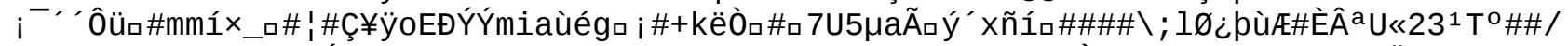

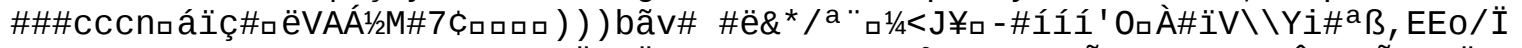

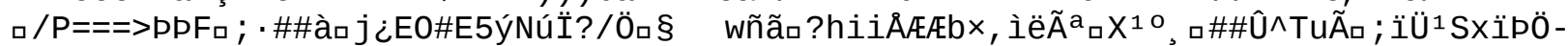

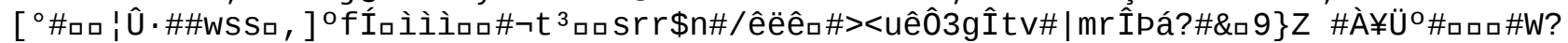

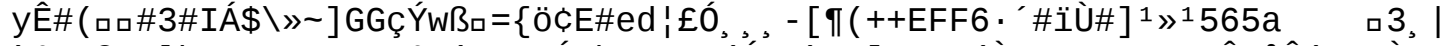

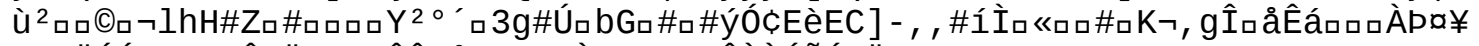

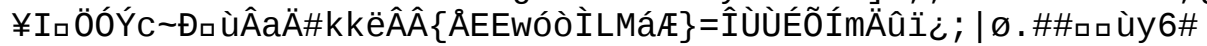

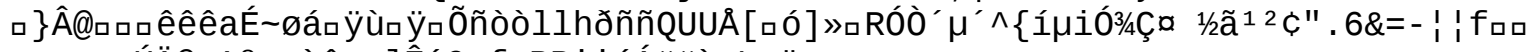

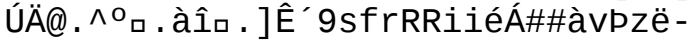




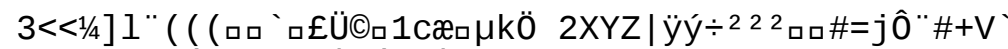

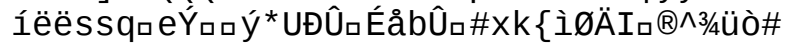

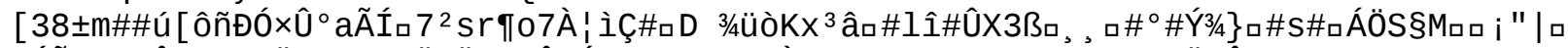

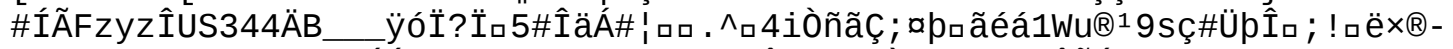

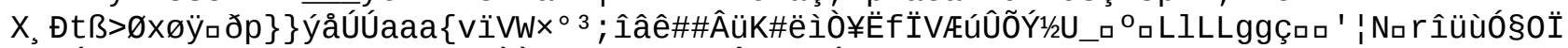

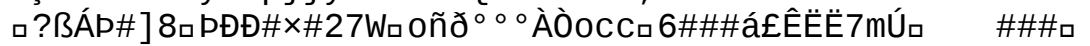

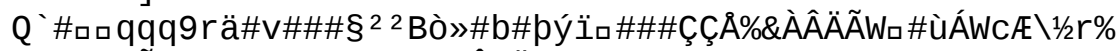

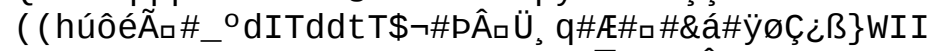

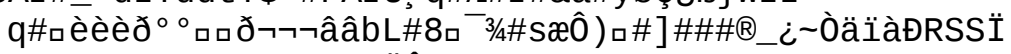

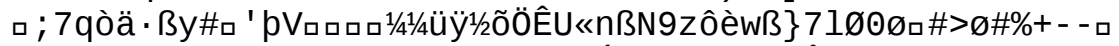

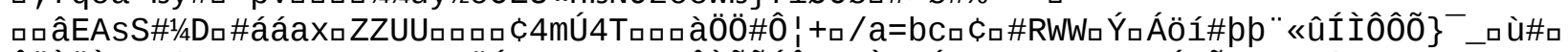

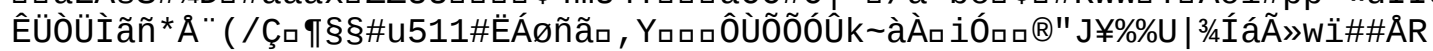

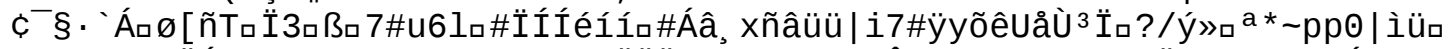

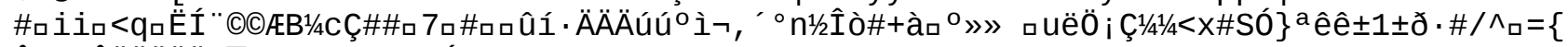

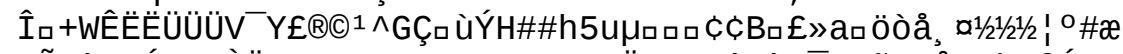

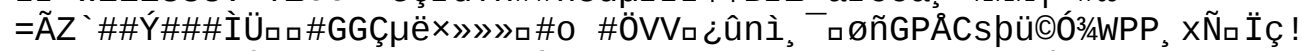

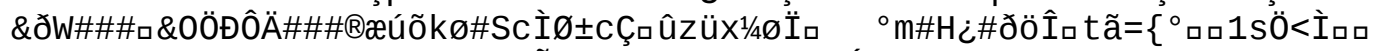

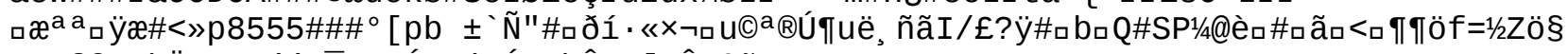

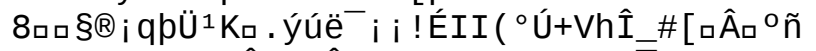

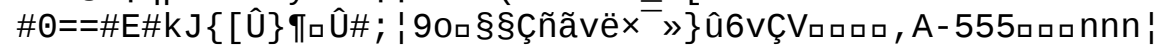

$\mid \square \#$ a

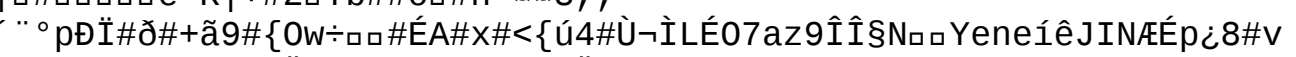

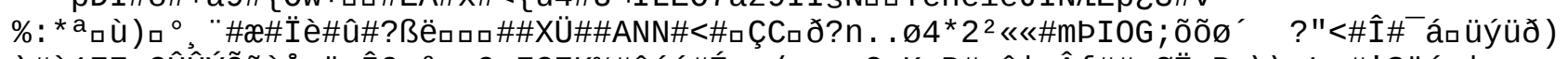

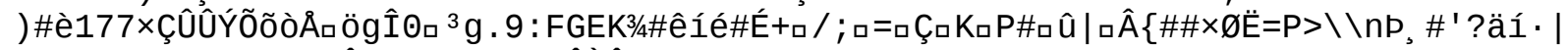

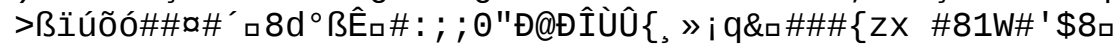




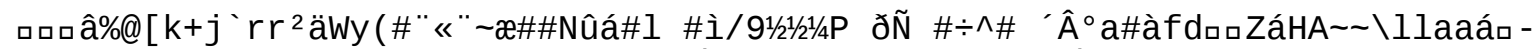

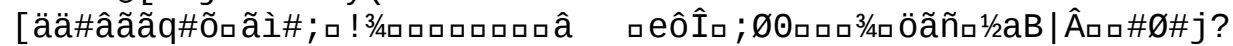

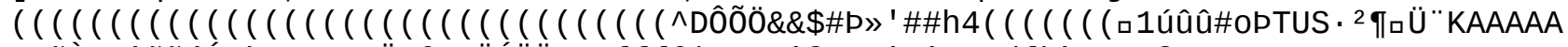

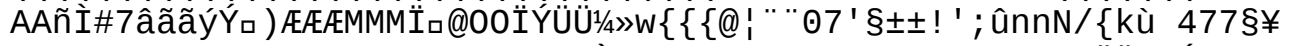

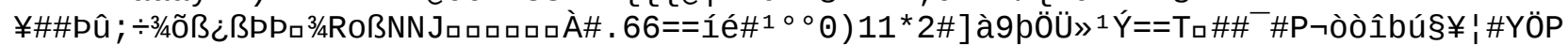

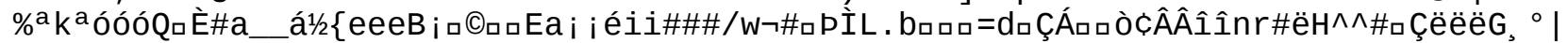

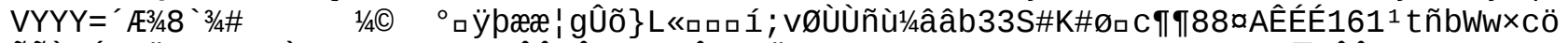

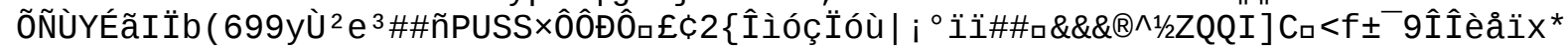

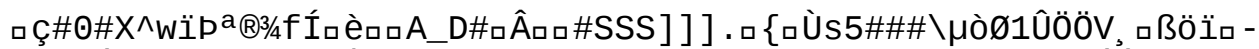

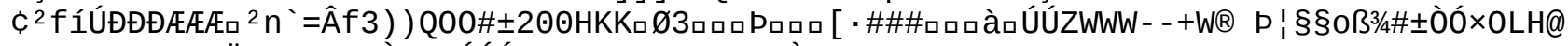

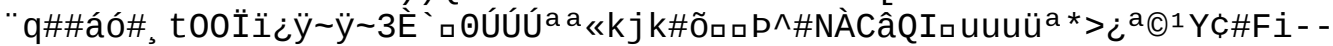

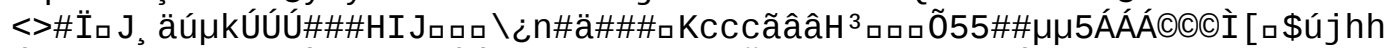

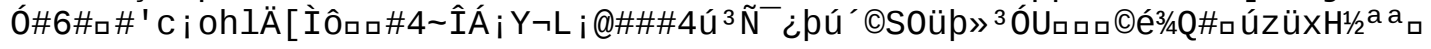




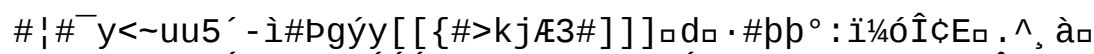

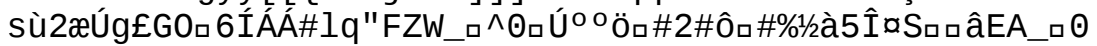

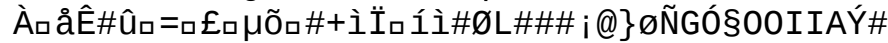




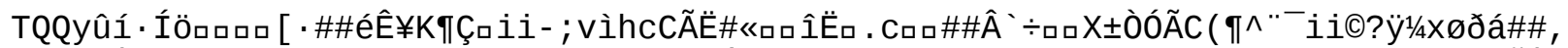

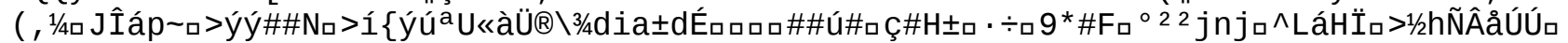

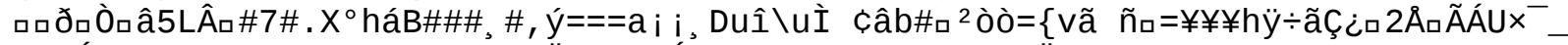

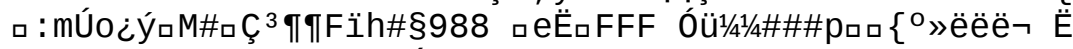

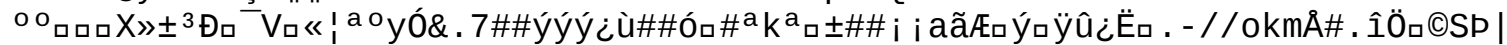

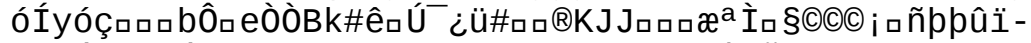

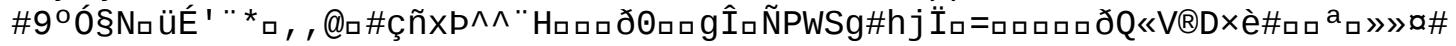

\section{$\square C^{1} / 2 \mathrm{I}$}

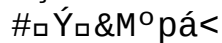

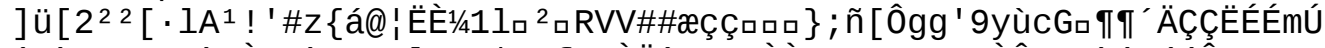

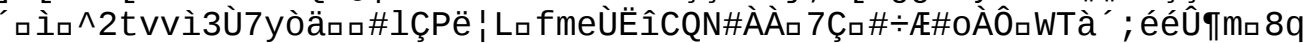

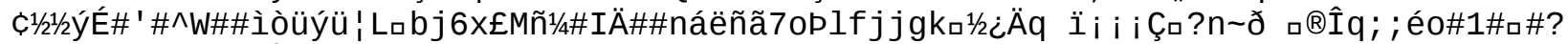
( 



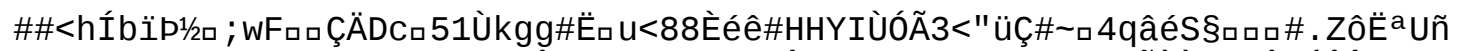

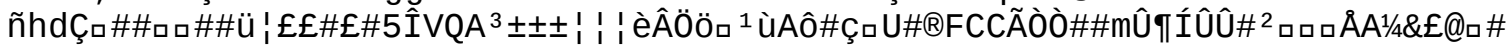

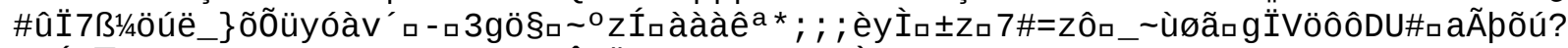

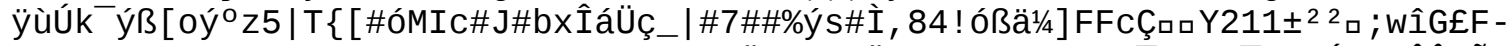

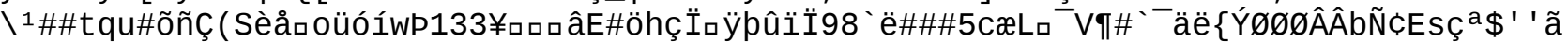

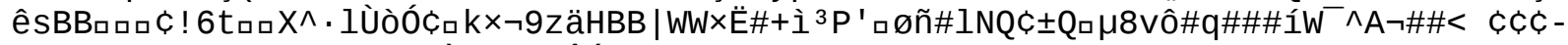

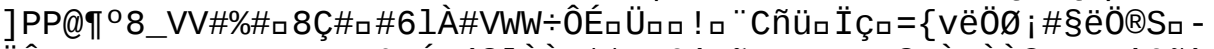

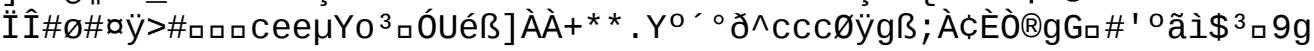
C口A2ロ\#F@ロ\#口\#\#\#1+Y\$

*\#\#ロ

$* * * 4$

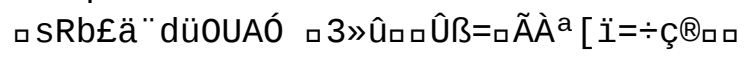





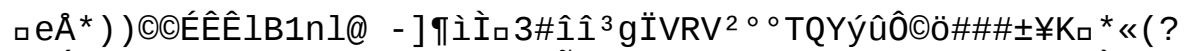

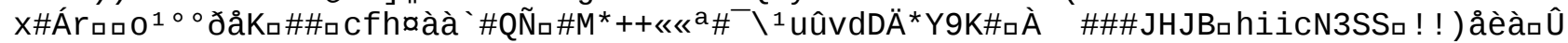

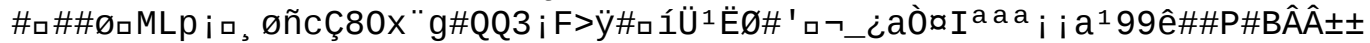

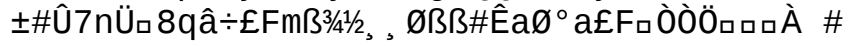




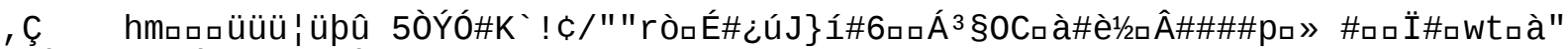

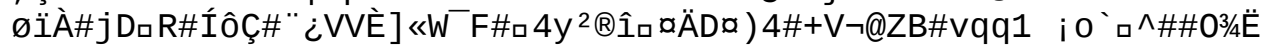

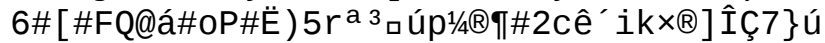




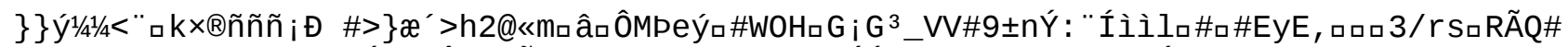

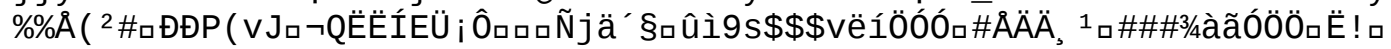

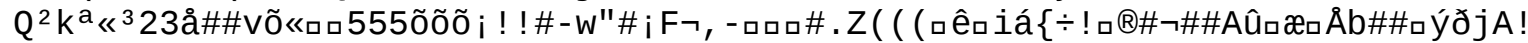

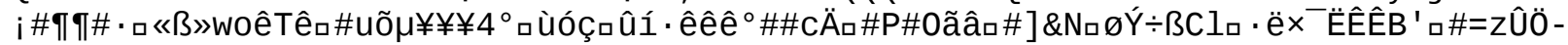

ĐTÅÅEèq@ ! :

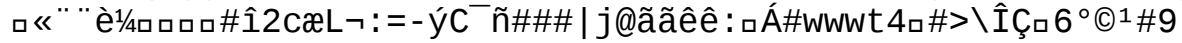

$\pm a^{1} / 4 \pm\{\div$ nd 
'E¿ý6fýúõ İø $>\AA ̊ 丿$ i èGÑN+V\#[ZYùx $\{/ \wedge 2$ är -

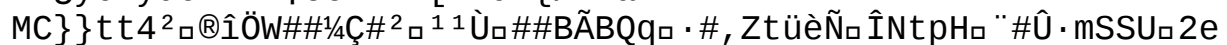

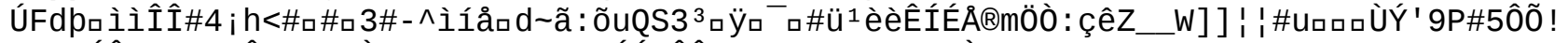

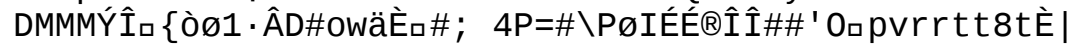

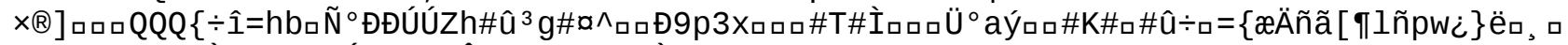

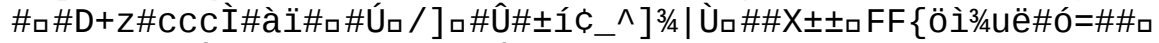

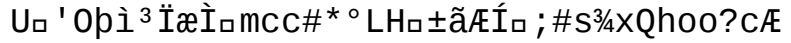




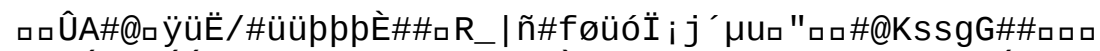

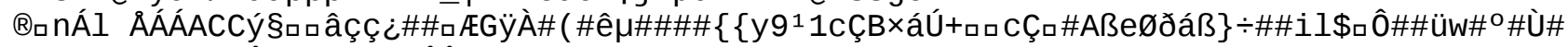

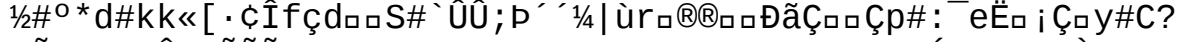

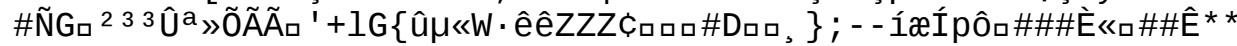

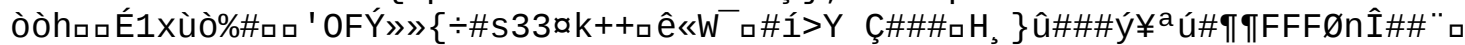

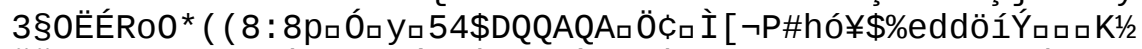

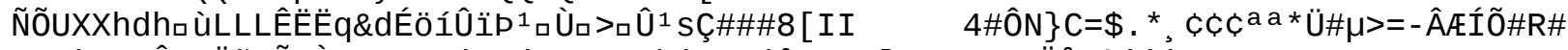

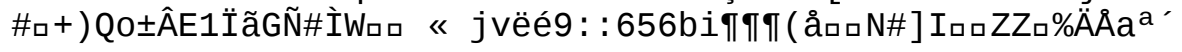


ü. ' ' \#\#\#ØLW. èeüý/+) *b|

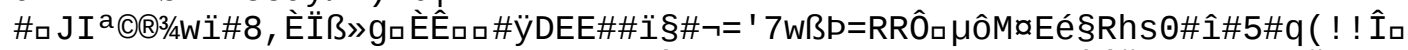
.\&口\#æPi \#\#IEY\#\#, ロ

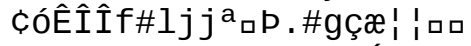

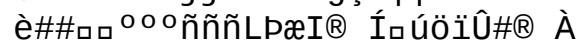

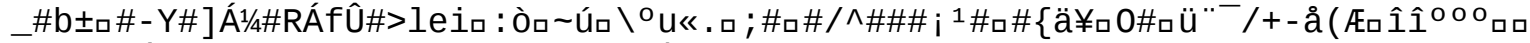

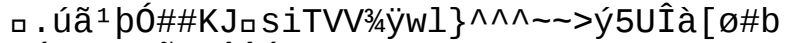

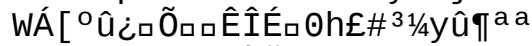

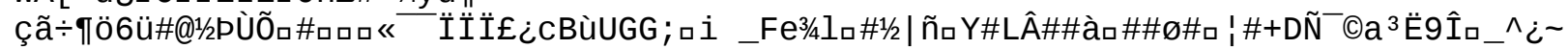

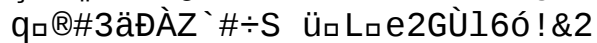




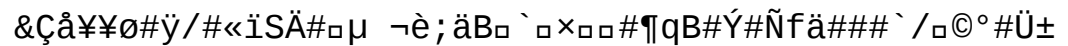

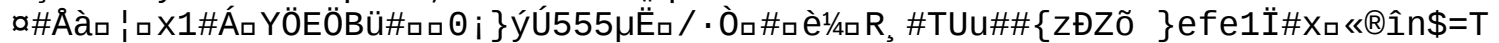

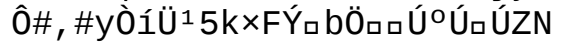

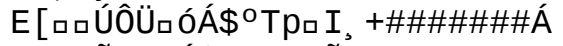

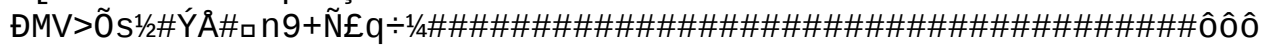

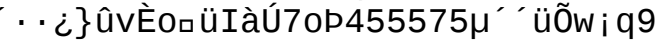




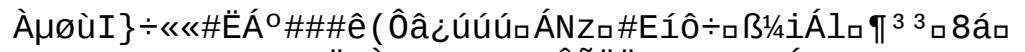

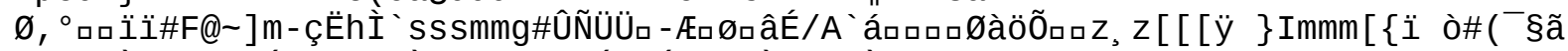

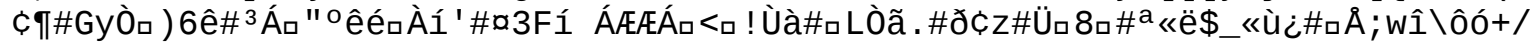

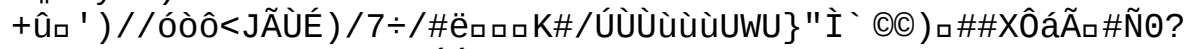

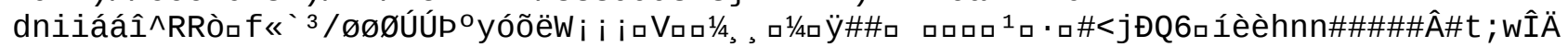

ÄäJ

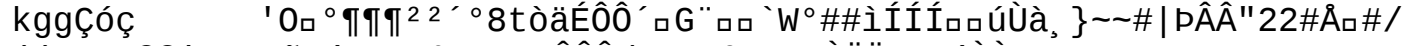

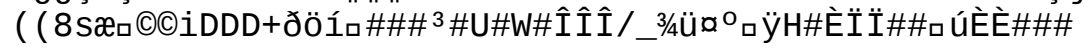




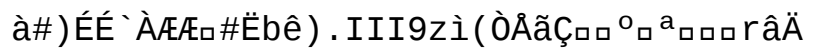

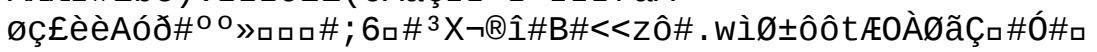




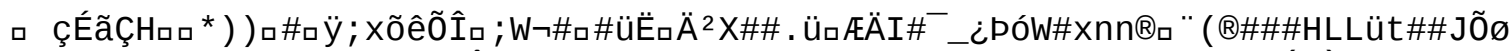

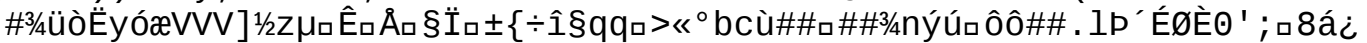

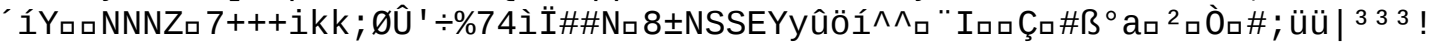

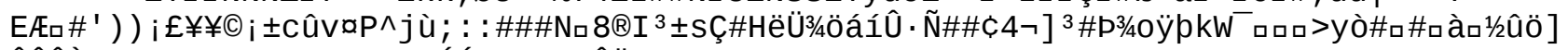

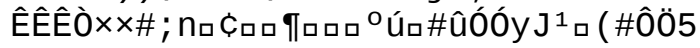




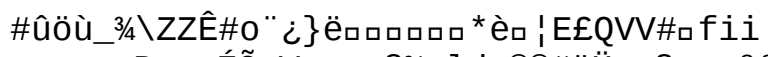

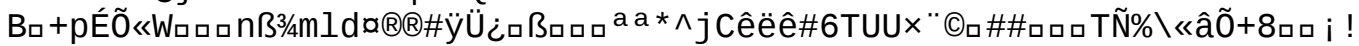

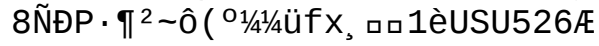




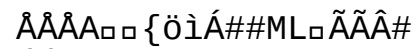
$\mathrm{f} \square \mathrm{O}$

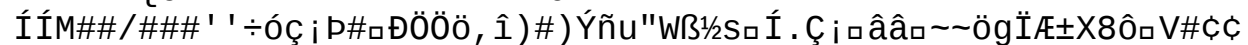

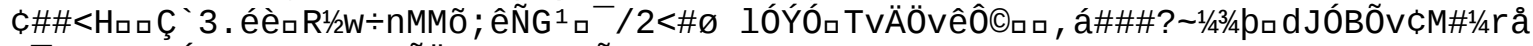

c'

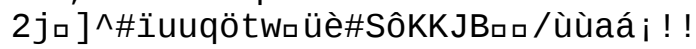

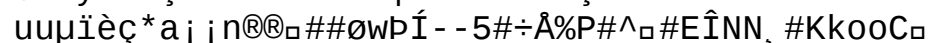

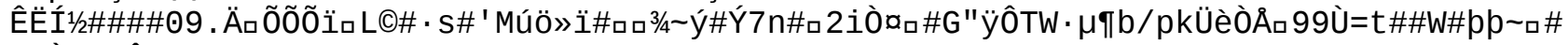
PDËÿP\#Â+V 


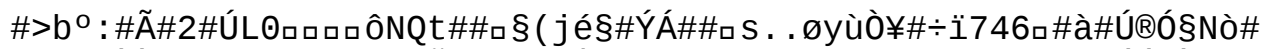

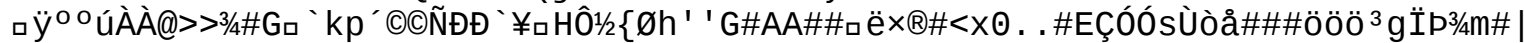

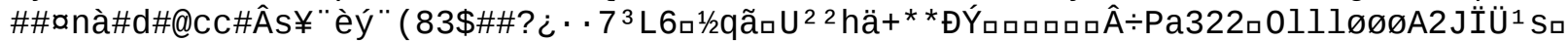




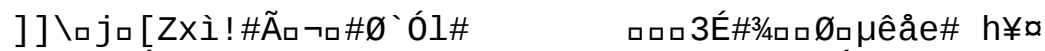

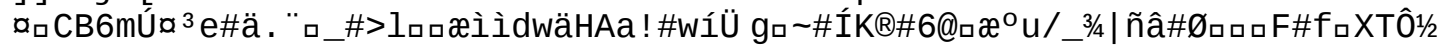

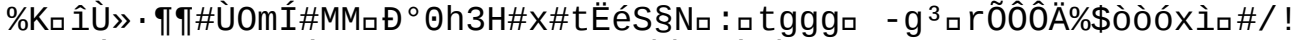

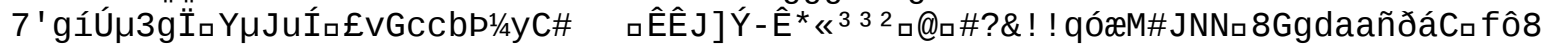

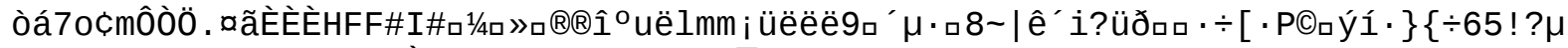

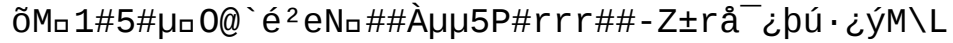


- -Ú.cc£yóçcu("+EVB9@]£ ¡ À 


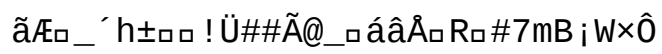




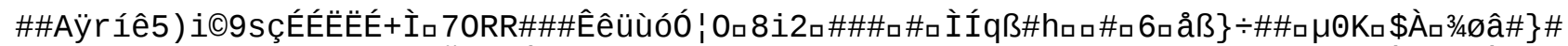

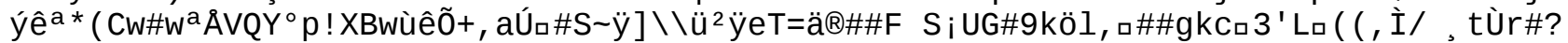

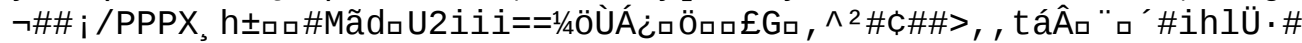
\#10̀öö

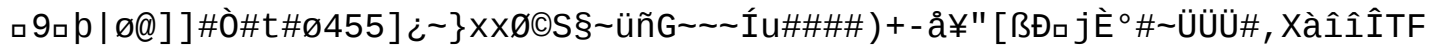

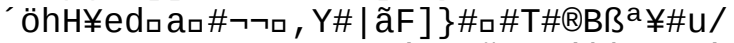

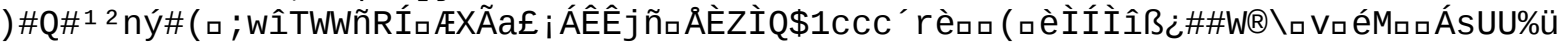

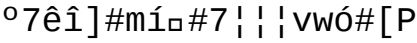

$\mathrm{X} \# \mathrm{ZZ}{ }^{*}$ 口

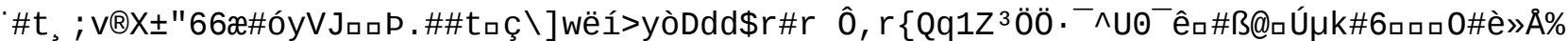

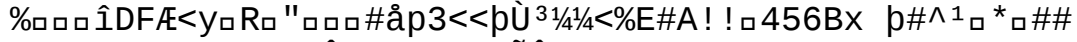

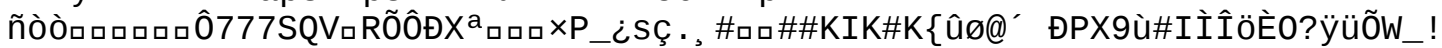

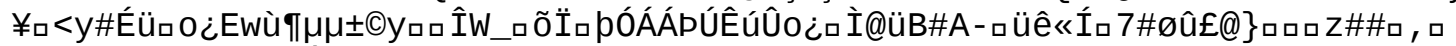

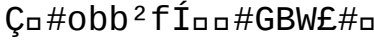




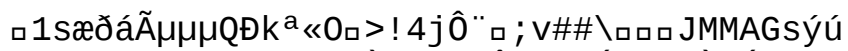

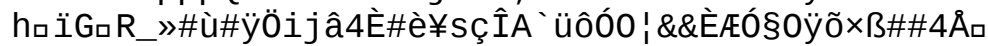

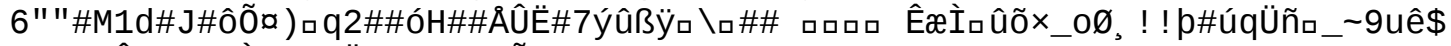

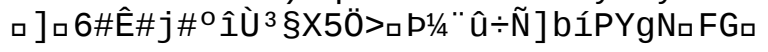




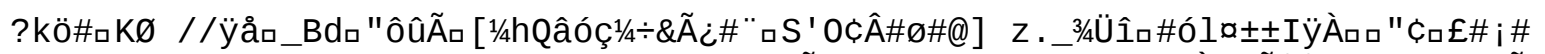

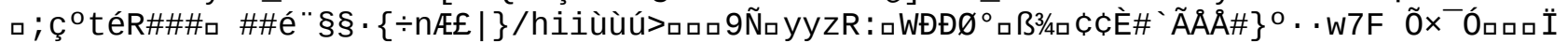

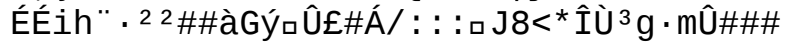




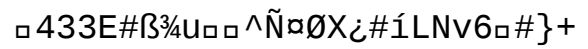

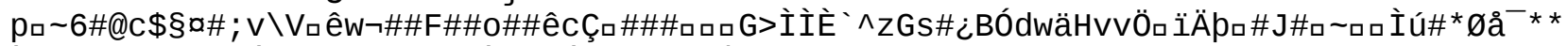

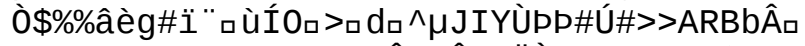

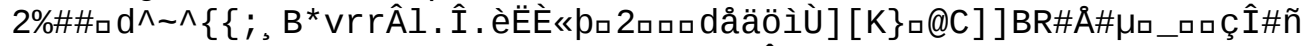
æææk54\#>Xıロ 


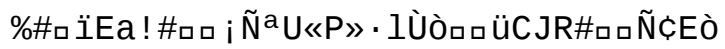

ò $\#^{0} 1 / 2 \ddot{I} \quad$ uV $3 / 4, * \square \backslash \# ?$

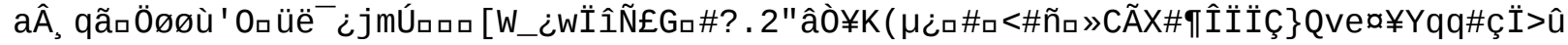


\#F\LLRR\#\%\#1/2êU Opîüù (Üærİ॰ * ; ; òr s i «eV -

Âi" "+5èõ"oøúúÂæ\#Fa662êa\#á1\# : iôhÿ\#ロ///ロ4iòd0\#ロ '0ロ , [ 


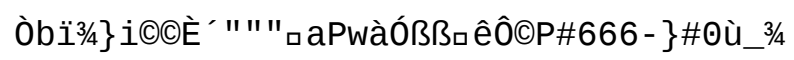




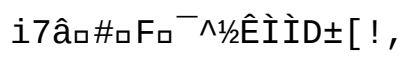




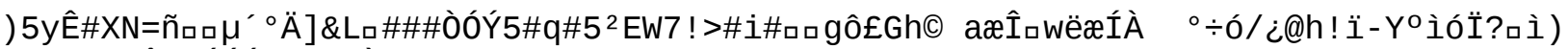

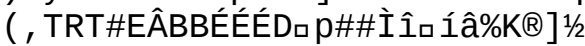


E¿?ðÙ口\#’

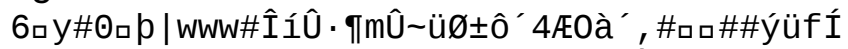

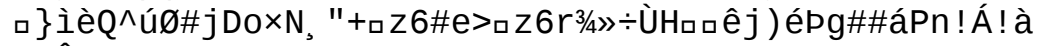

$1 / 2 \square \hat{I}_{\square}-n c ̧ \backslash \#$

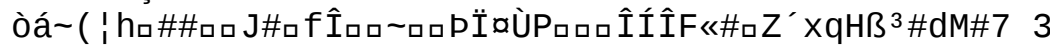

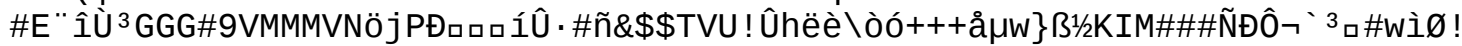

İõl\#R\#\#çÀn\#à\#İ\#4ロuÁí [ · \$Ä555ÃQ\{BCqínõëó\#

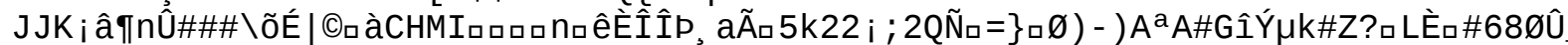

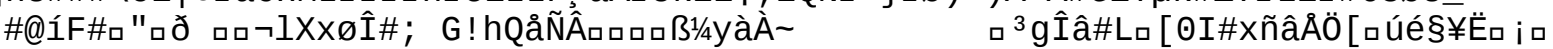

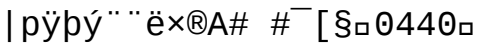




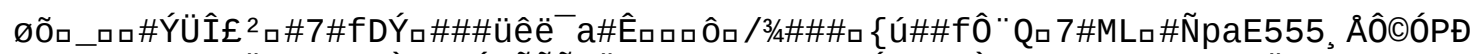

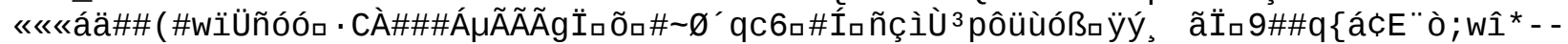

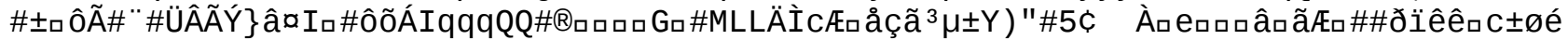

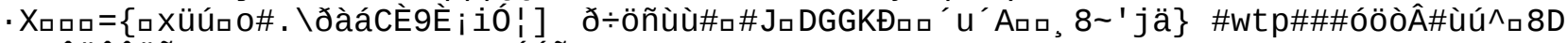

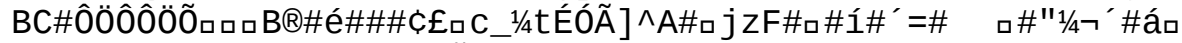

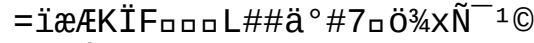
Ë/

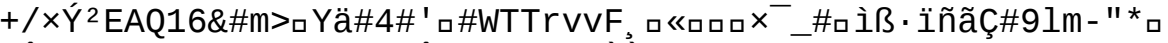

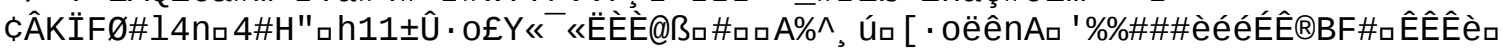
\#

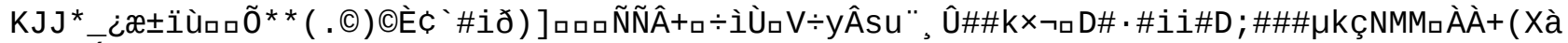
口םÇDÝâ1defBrÿ6f 


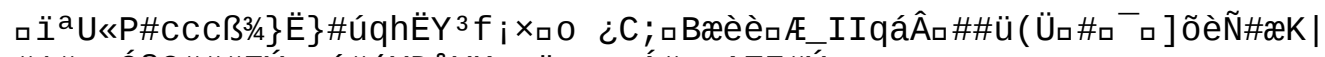

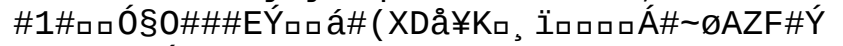

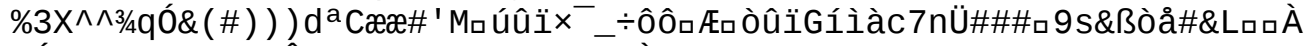

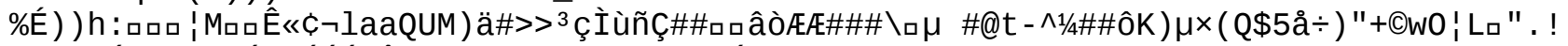

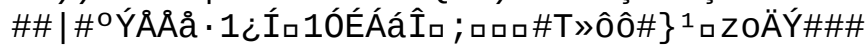


$\square=\{6 \# \# \ddot{A} \#$

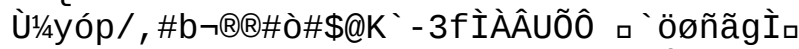

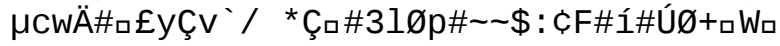

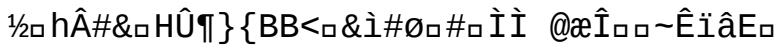




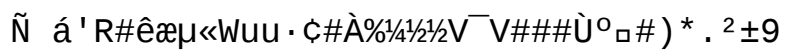




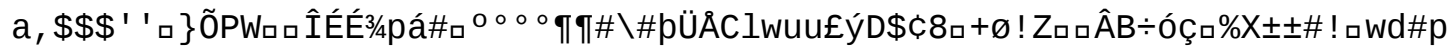

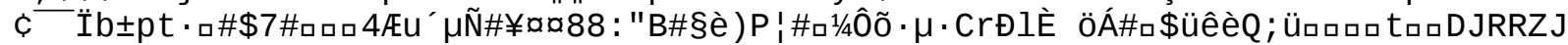

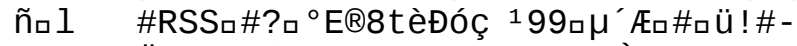

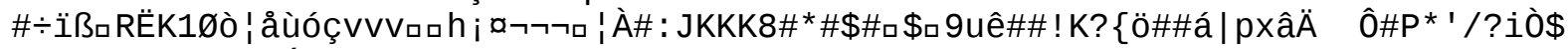

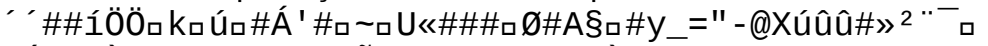

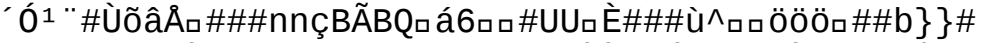

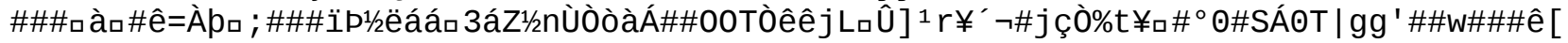


uu8ロ ò\#\#\#àêââää\#]॰\#ロG?ロ 



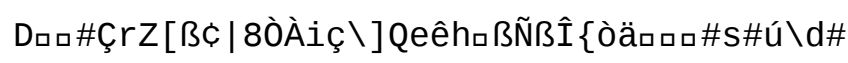




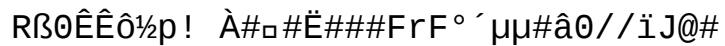

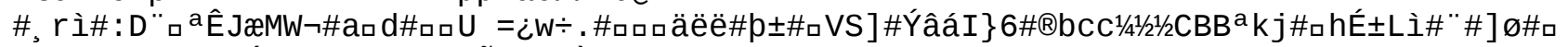

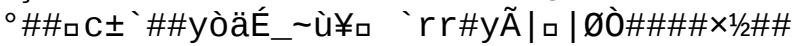




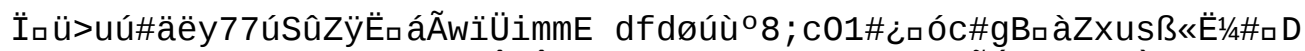

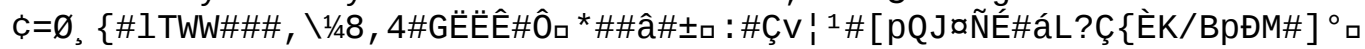




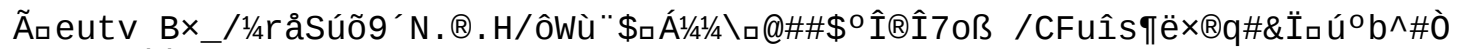

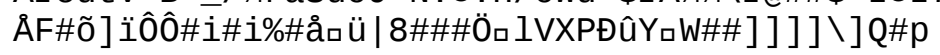

"722\#3ロ+ם 


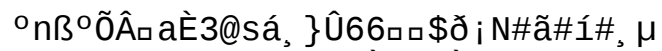

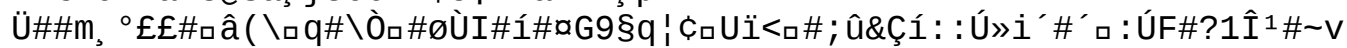

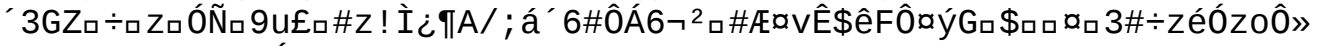

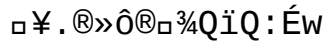




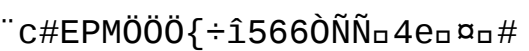

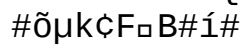


«LÖロ NOOg_òû\#\#̂̀àlî̀̀ıE\#uqv

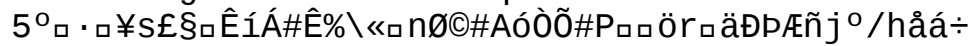




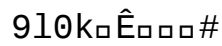

ᄀ|ýロñ"Æy, slß`+ãQ\#ロ\#\{s\#E`G_Nã1 


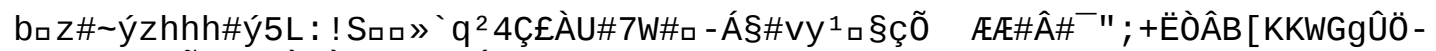

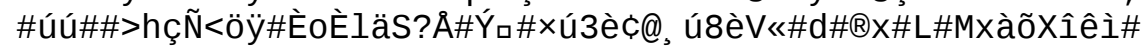

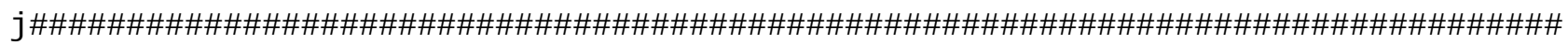

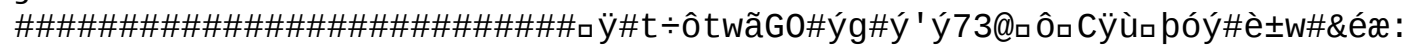

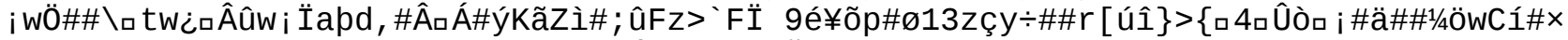

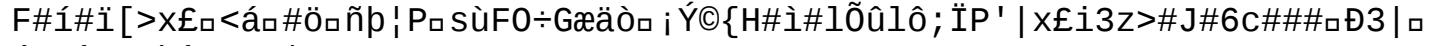
á\#\#ûçØøhôq»Bï! 


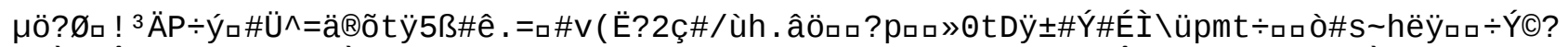

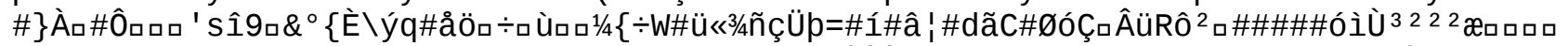

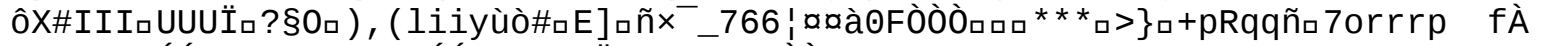

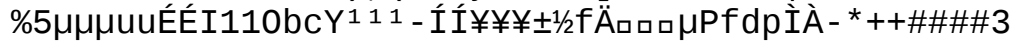

) 3^11/4àaı @À 


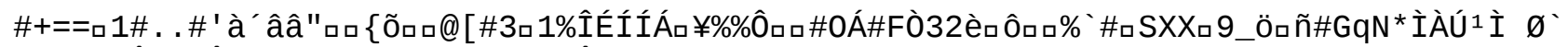

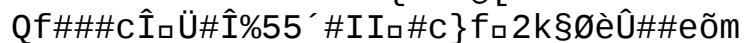

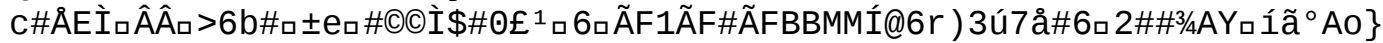




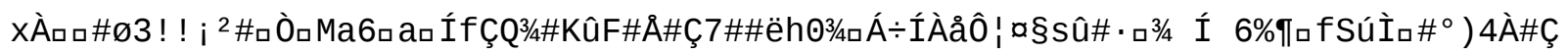




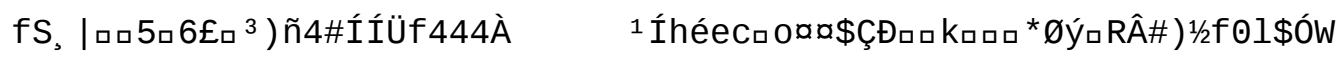


Ü\#Ê7`Fùûlôn

my! $\times$ i à $34 \hat{0}\}$ Töm

$5)$ ü

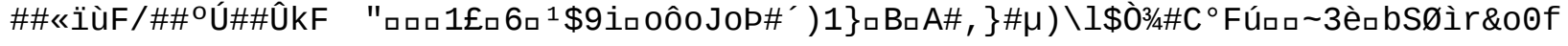




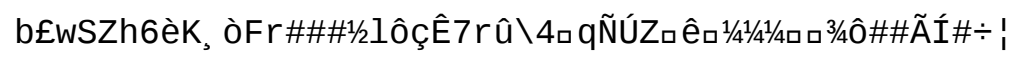




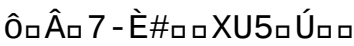

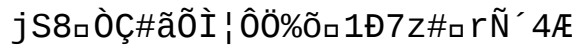




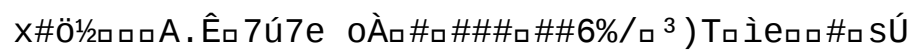


口, "Ç7h3

ØpMi\#的)E\#\#ロVQú 
ฉ Á 


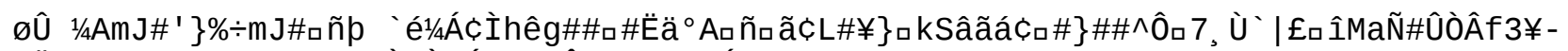

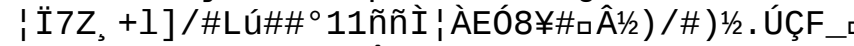

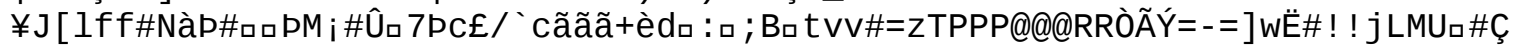

\#UTT\#\#à\#\#[ [ çäd\#?vLXø@pquq¿Võ\#! ]

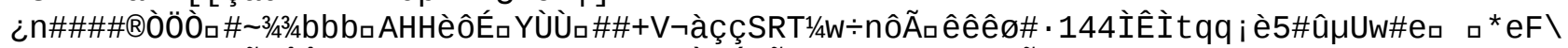

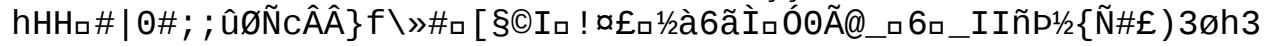





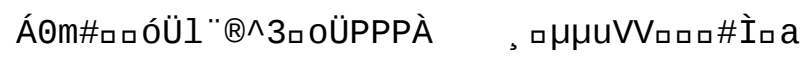

ò×i38lèèè\$3fıSf\#0́fdçpØàÇøîquèè>6\# 




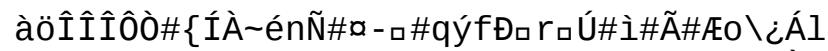

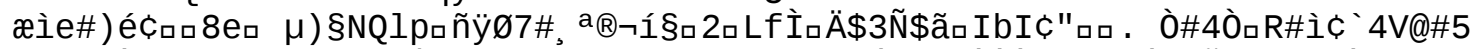

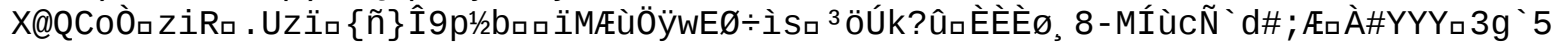


FSC\}5Je '̀̀

ฉQ\# . . Î (Úû

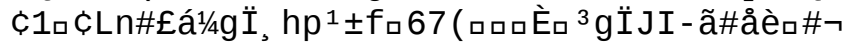


;@口\#dÄÆ\$\$Ä?È $\{\hat{1} 1 / 40 \ddot{U}$ i 
. \#9ロÆ口 


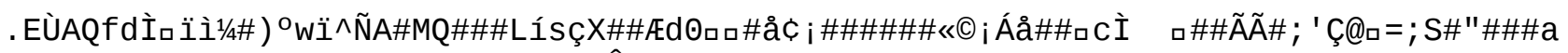

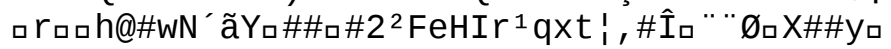




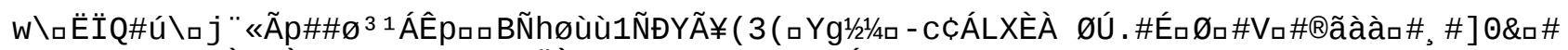

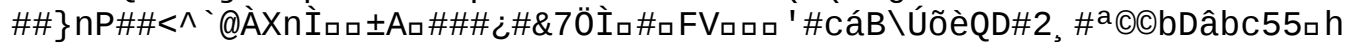

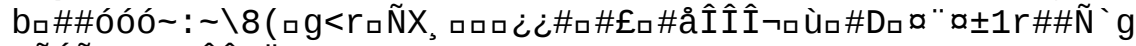

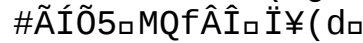

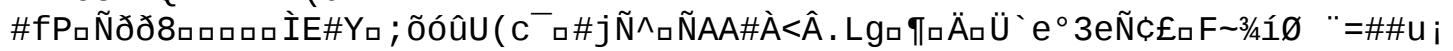




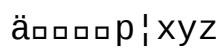

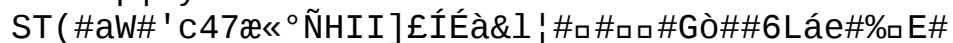

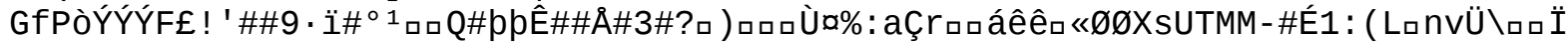


Ê\#N\#ロc9\#\#I . $740 ̂ q$ 


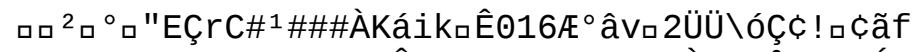

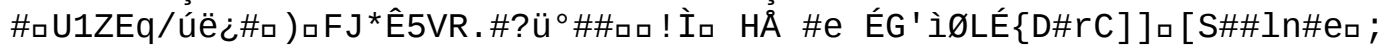

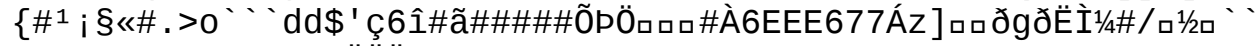

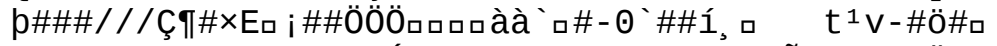

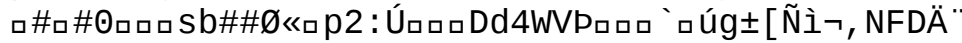


Ü; wÎ̃e\#\#\#22: ; :X\#ロ\#ロロkuᄀ 


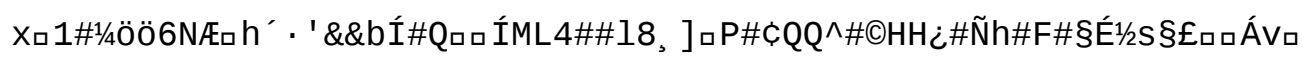




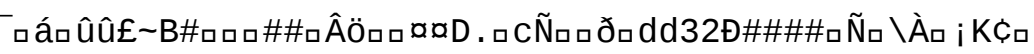




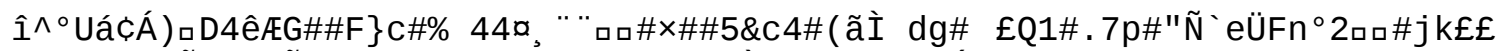

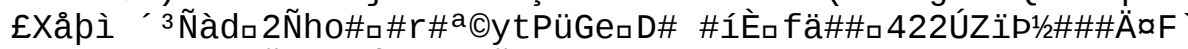

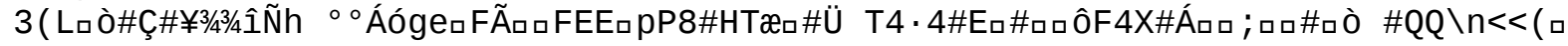




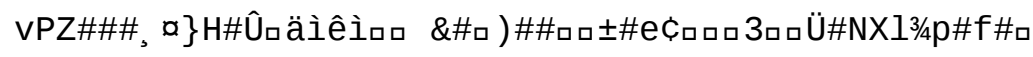




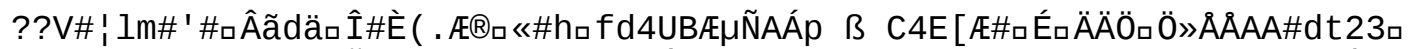

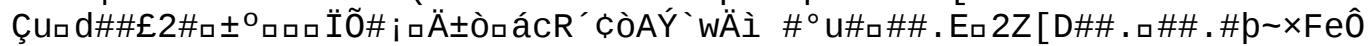

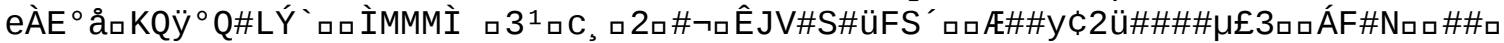

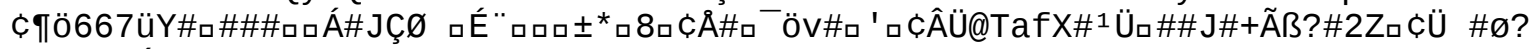
N\#D"bXÉFëFbç。 


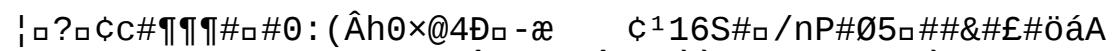

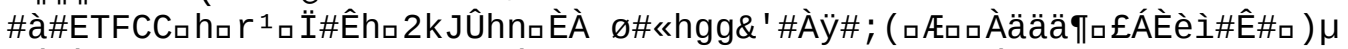

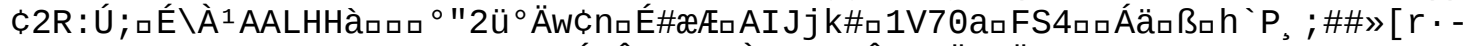

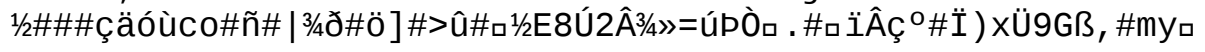




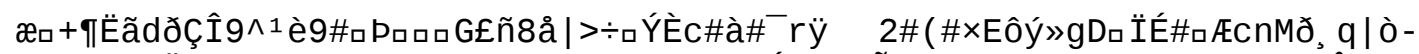

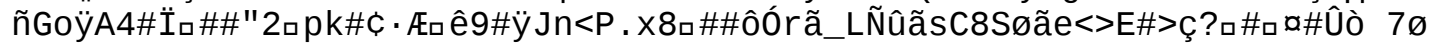

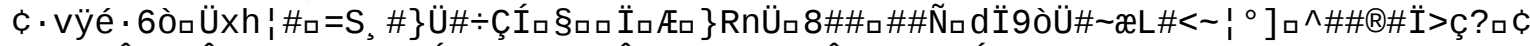

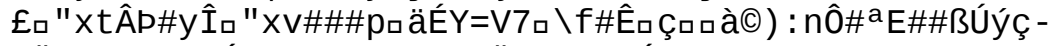

\#Ï\#\#psäÆ"NÁS\#Ü"

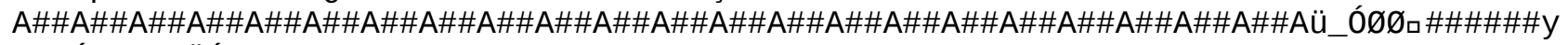
İ\#\#ÉII]]]ÏÓq`¿aäî1 $12\{a-\square$

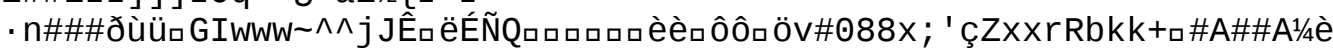




ฉष० :ujåêõr

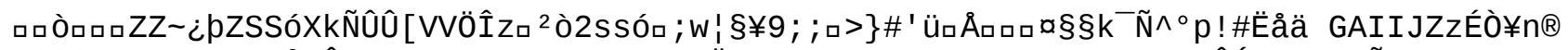

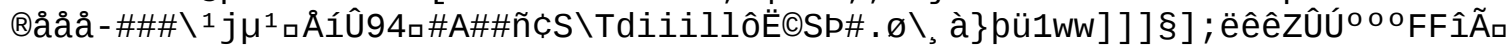

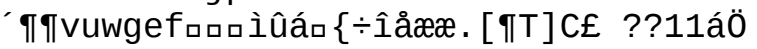




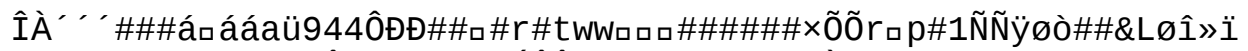

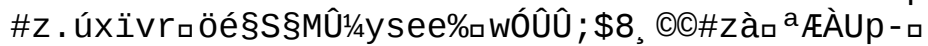

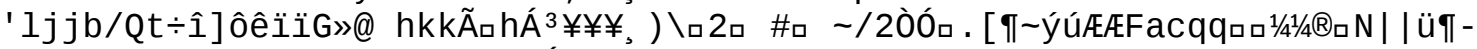

[=== \#\#\#ê·oßvì

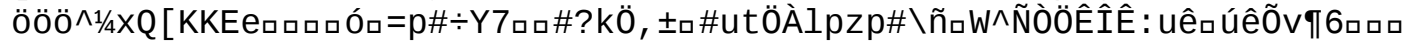

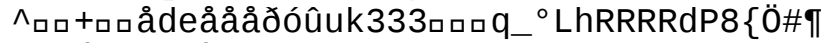

\#åĐÁq ${ }^{2}$ Ëı+ÈË3

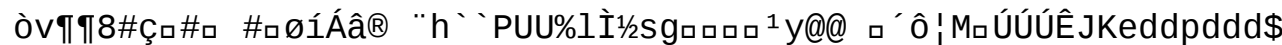

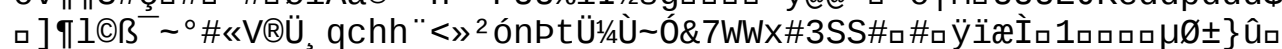

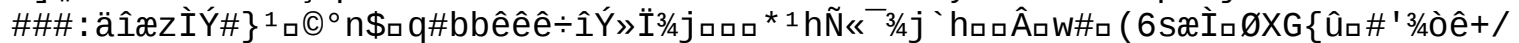

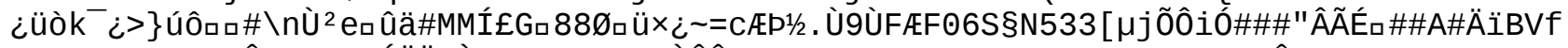

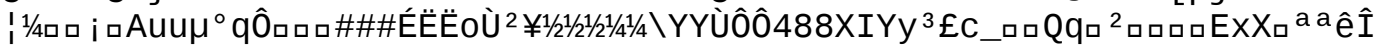

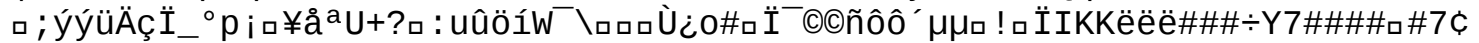

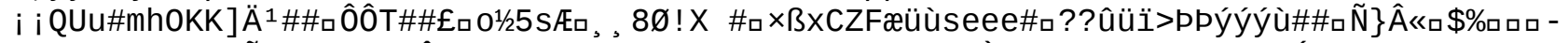

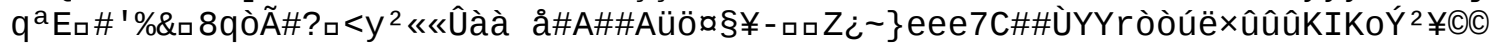

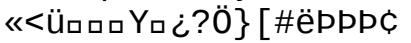

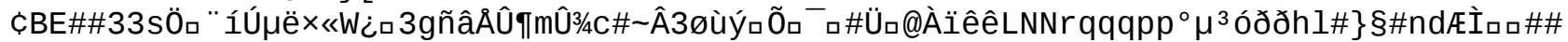

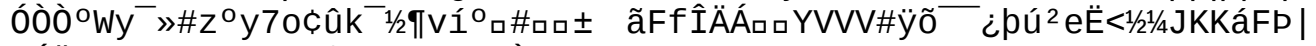

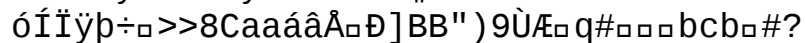

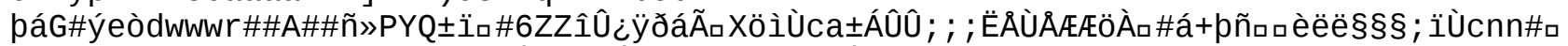

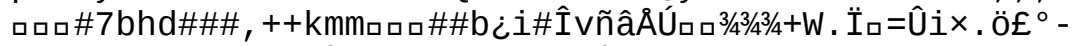

p, ®R®înn\#\#\#415ÅÙ

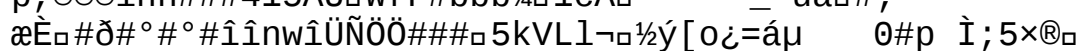

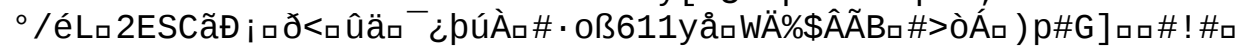

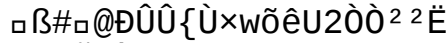

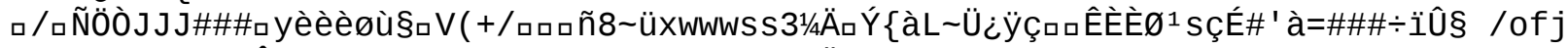

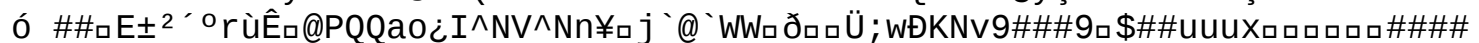

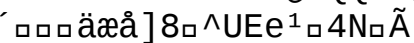




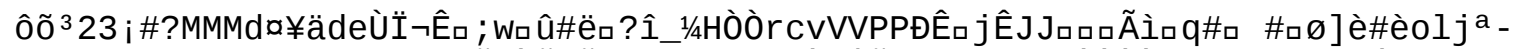

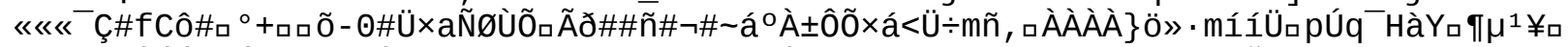

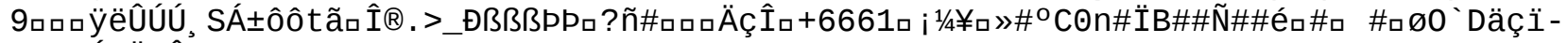

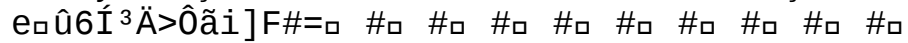

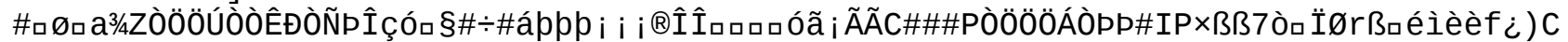

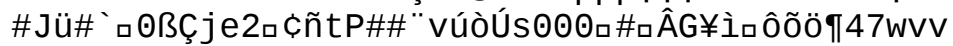

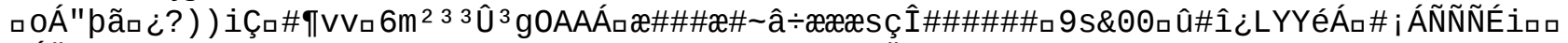

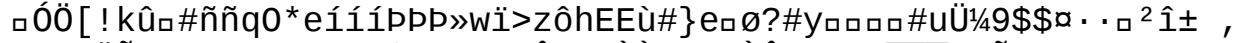

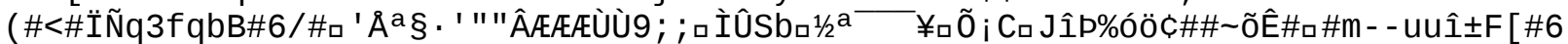

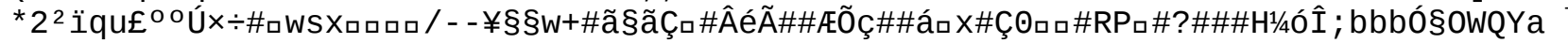
\#\#\#üa19VV! /6qâçם\}\#\#\#ロMë£+\#. -

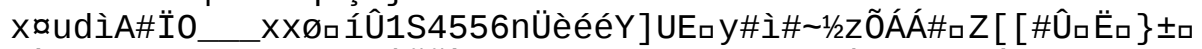

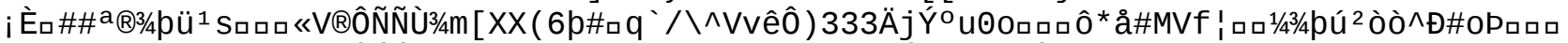

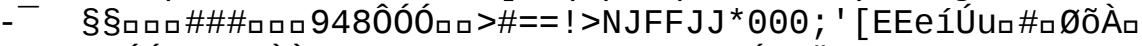

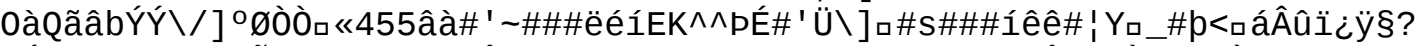

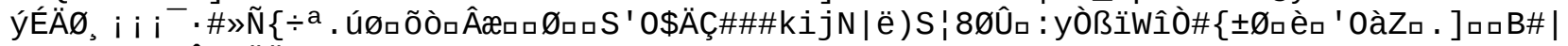
õ $\mu k \times<=<<=\hat{I} a ̀ q O ̈ E ̈ \# u ́ 9 m \# n ̃ T F \# \# \# \mu^{\prime}$ 'äää口

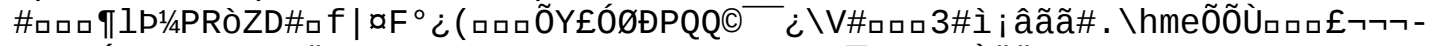

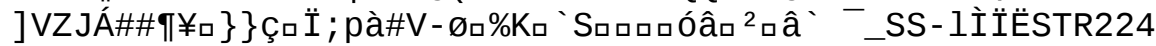




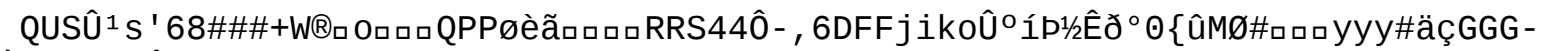

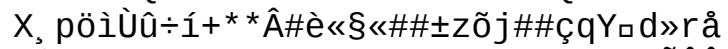

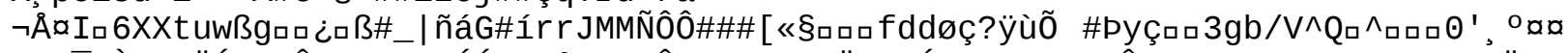

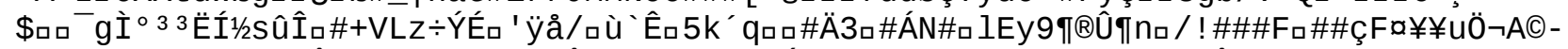

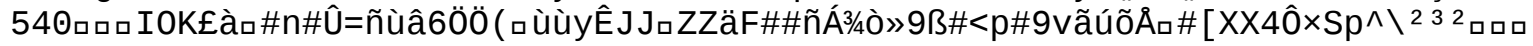

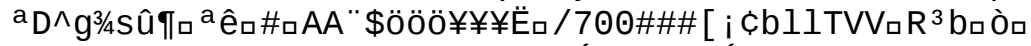

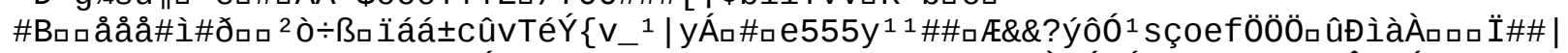

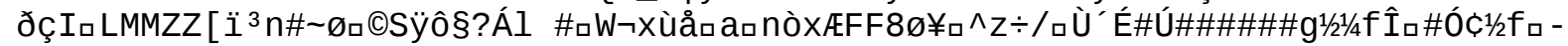

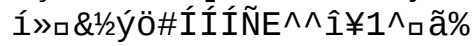

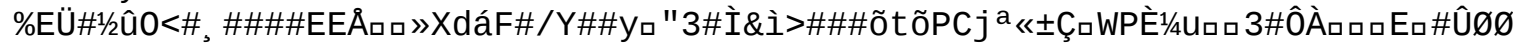






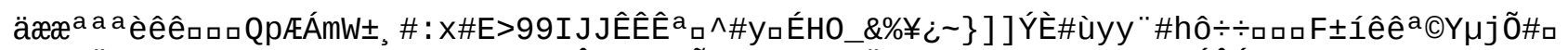

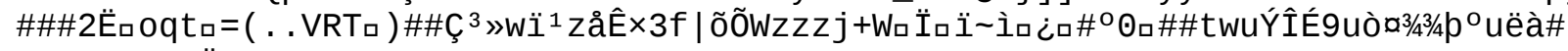
\#\#úú\#Zio@Äвロrם

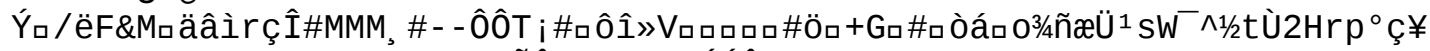

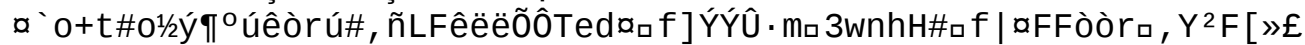

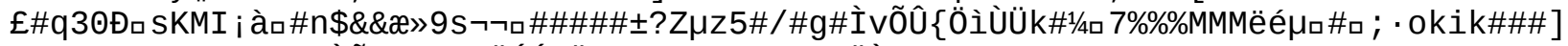

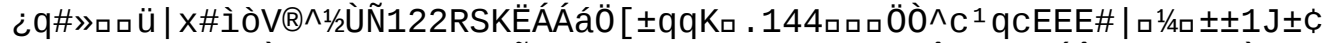

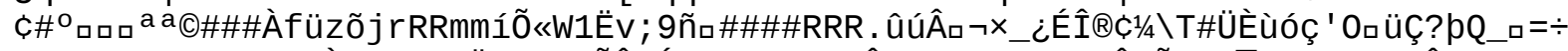

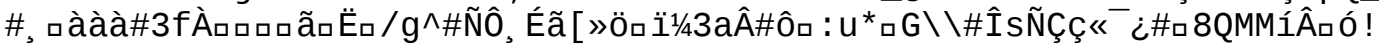

ÁÁ7@_Ç"ם_口- i i pöÛoã 


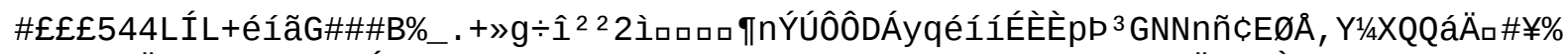

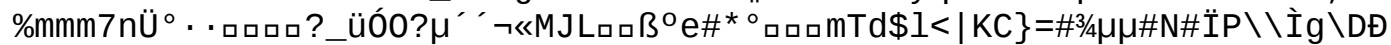

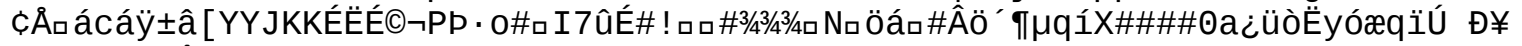

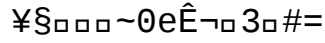

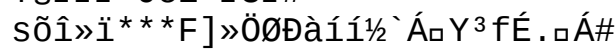

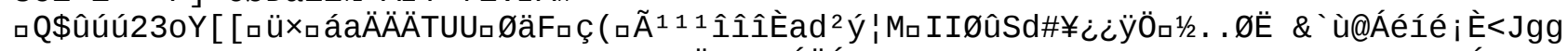

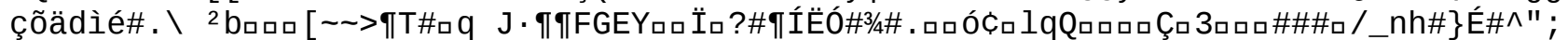

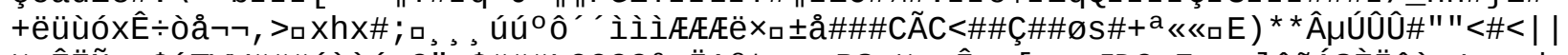

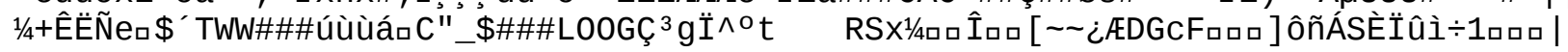

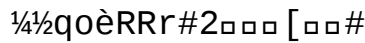

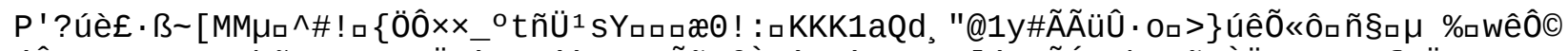

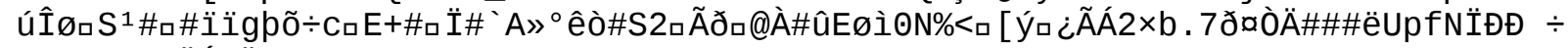

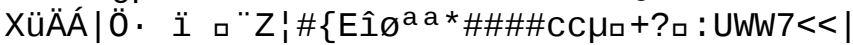

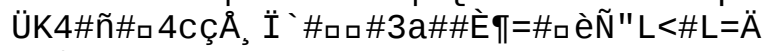

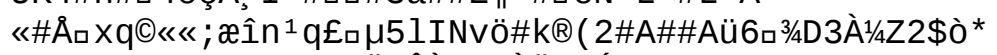

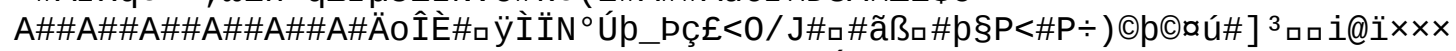

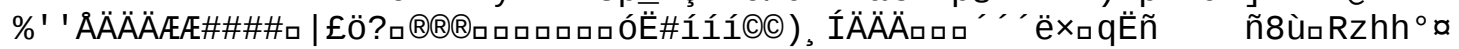

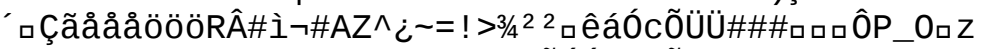

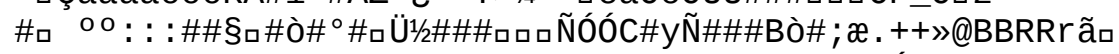

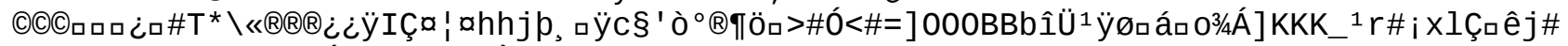

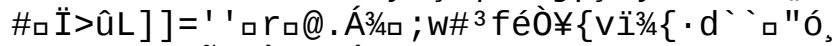

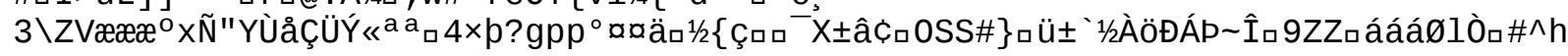
\#

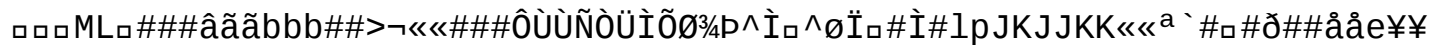

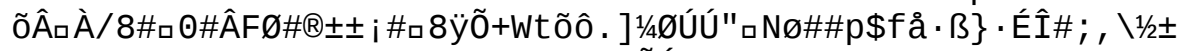

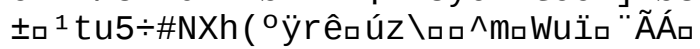




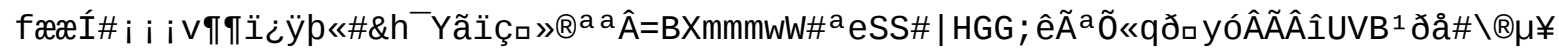

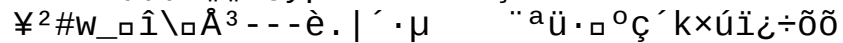

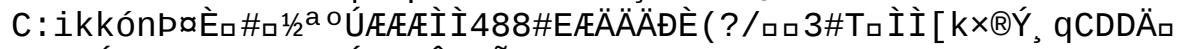

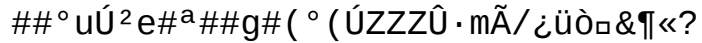

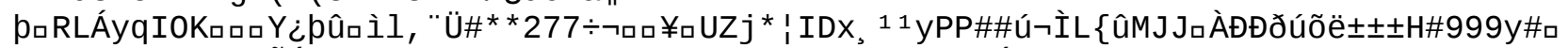

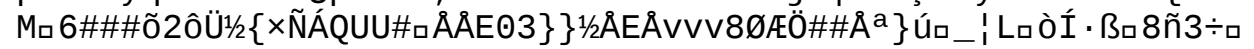




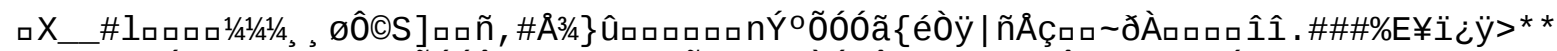

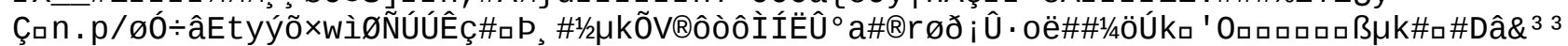

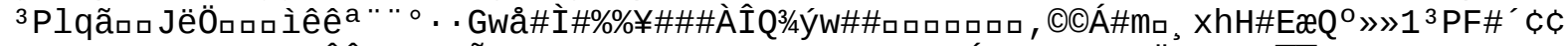

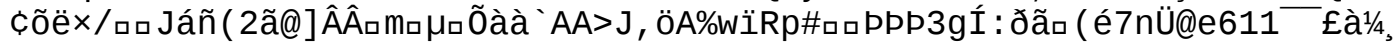

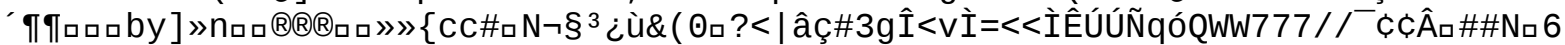

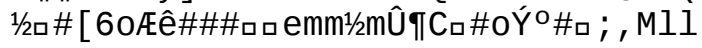




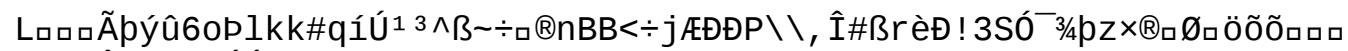

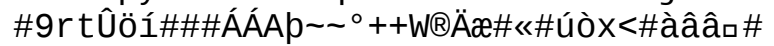

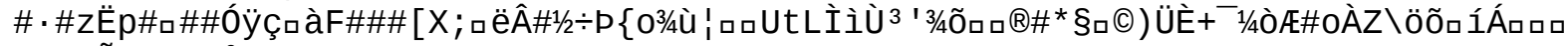
a $\left.\backslash \mu \mathrm{j} \tilde{0} \# \#\right|^{\circ} \mathrm{b} \AA$

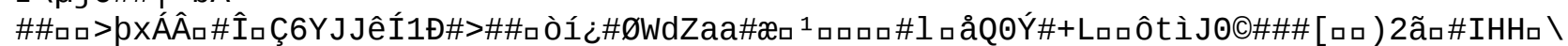

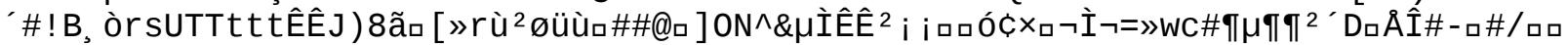

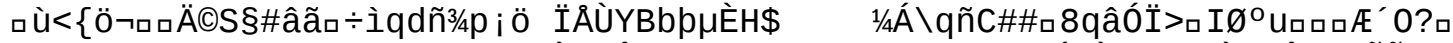

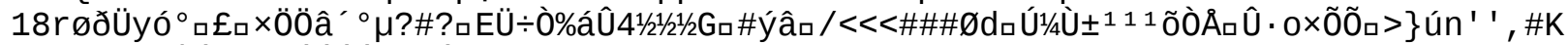

口 . = \}ú4®uÛÁ^, \& : ÚÉÉÉÜÜlú

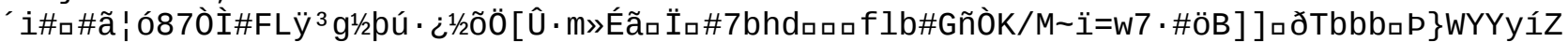

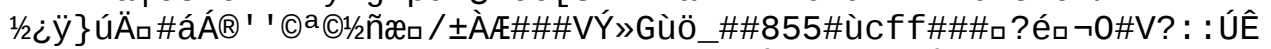

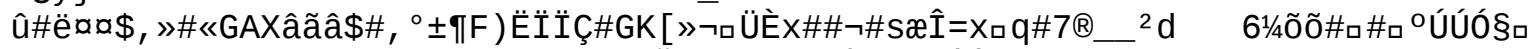

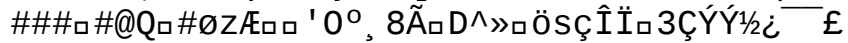

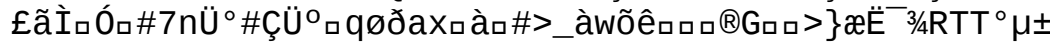

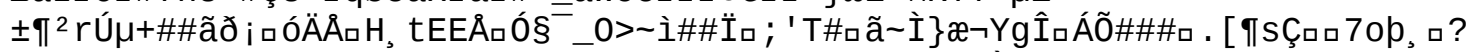

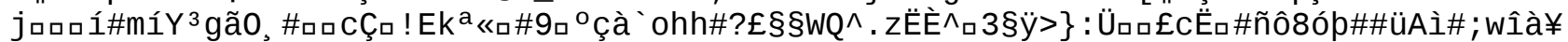

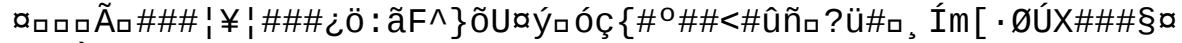

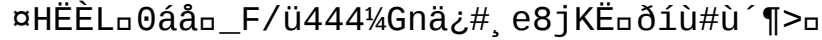

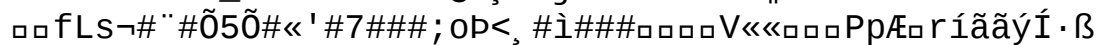

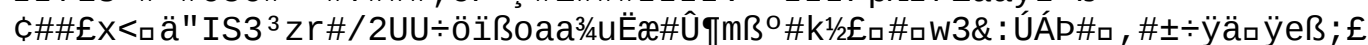

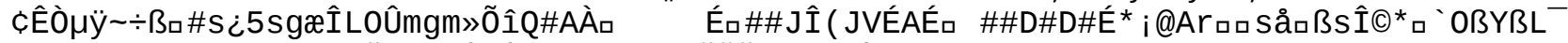

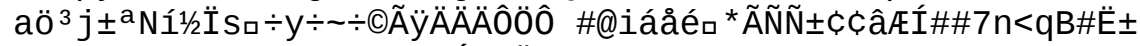

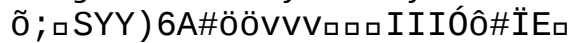

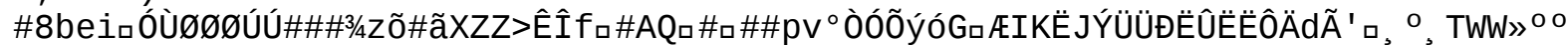

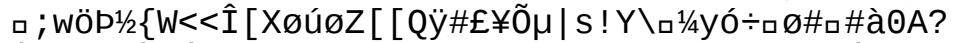

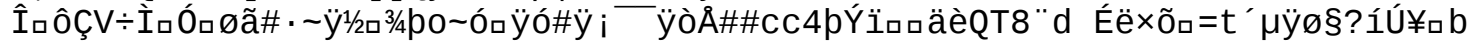

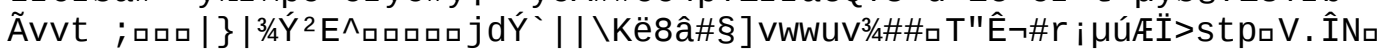

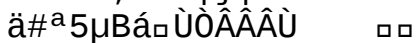

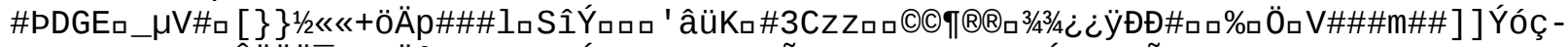

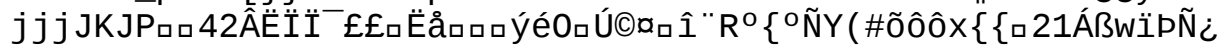

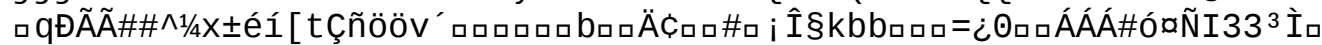




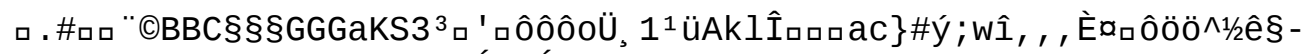

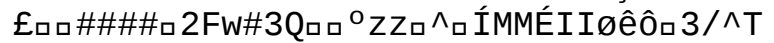

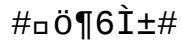

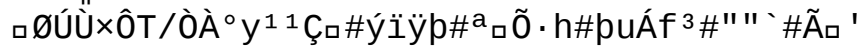


Ohk; », t'.\#eVcll 


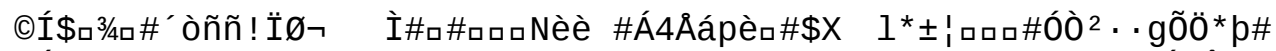

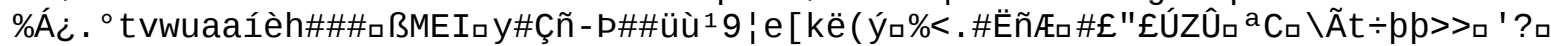

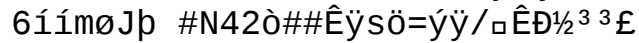

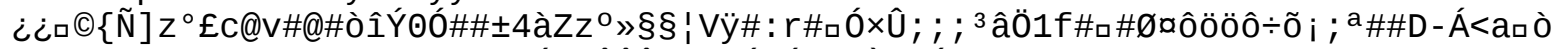

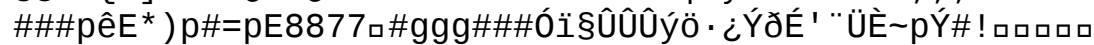

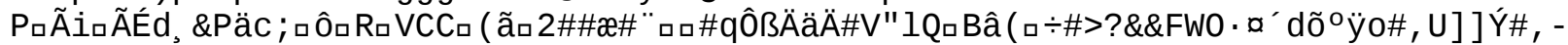

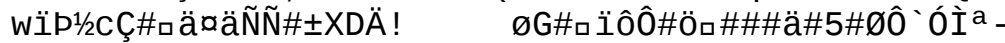

«ëì่̀ $0 \cdot \# \# \# \# \# \# \# \# \# \# \# \# \# \# \# \# \# \# \# \# \# \# \# \# \# \# \# \# \# \# \# \# \# \# \# \# \# \# \# \# \# \# \# \# \# \# \# \# \# \# \# \# \# \# \# \# \# \# \# \# \# \# \# \#$

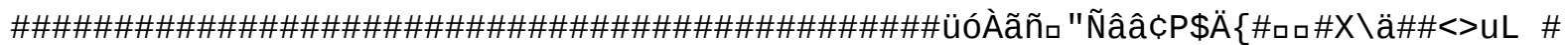
$c_{\square} X, \mathbb{E} \cdot \backslash \# \# / 1 / 4 \S^{\circ} \#$ é $\cdot h$

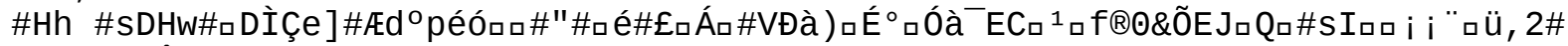

$\mathrm{c} \# \mathrm{j} \neg \mathrm{A} \_9 \hat{\mathrm{A} C ̧} \mathrm{Q}(\mathrm{C}+\square$

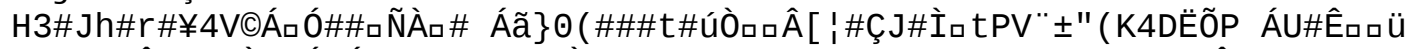

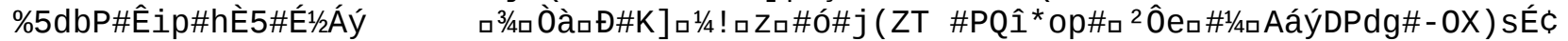

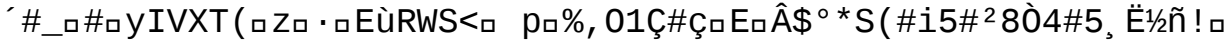

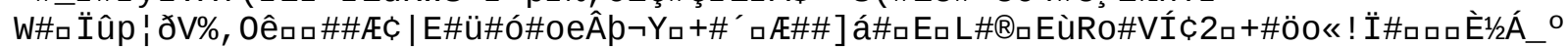

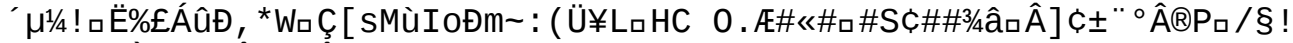

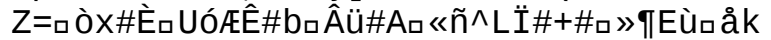

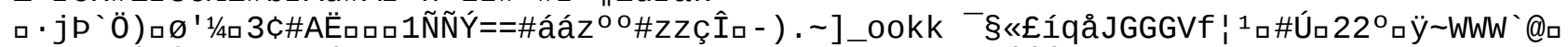

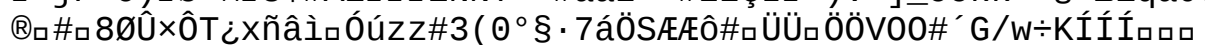

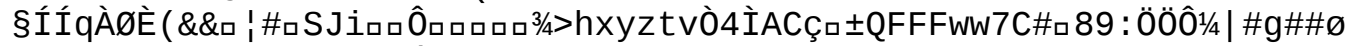

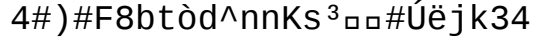

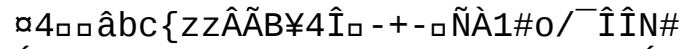

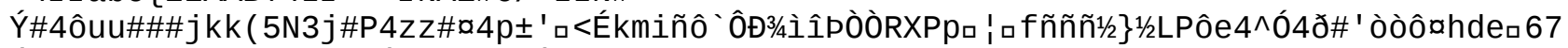

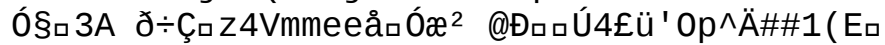

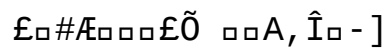

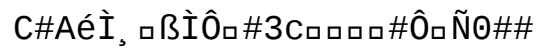




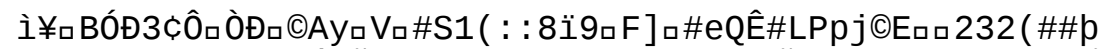

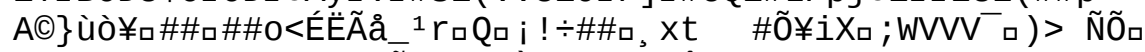

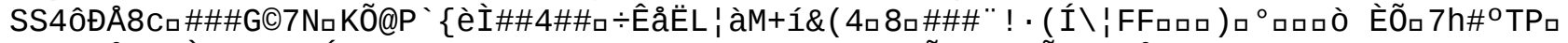

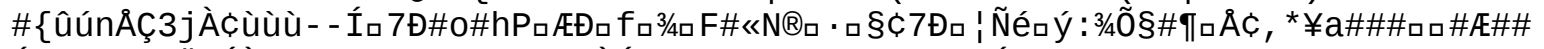

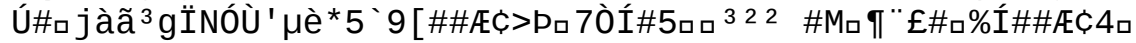




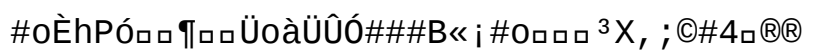


Æ\#T\#е4üý)æ

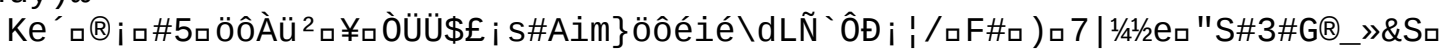

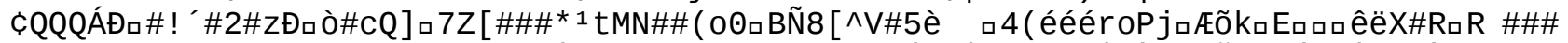

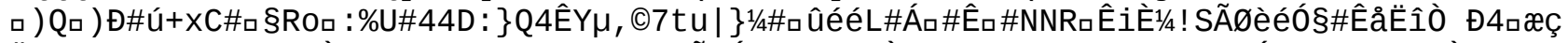

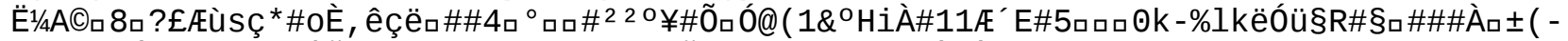

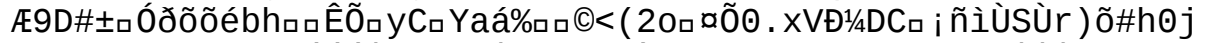

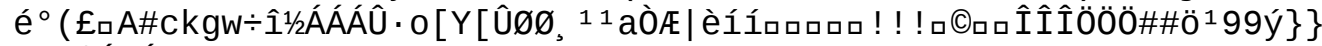

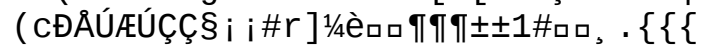


jggWôü9âa ¡ ĐÀÊÊ

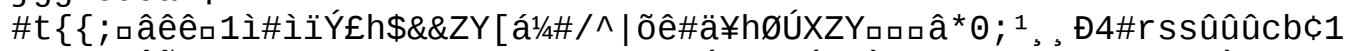

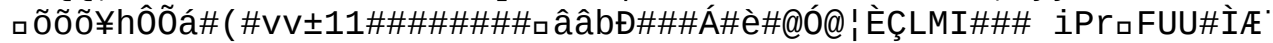

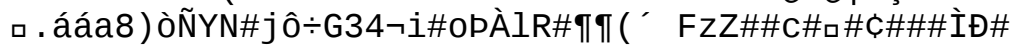





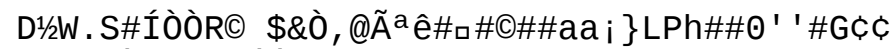

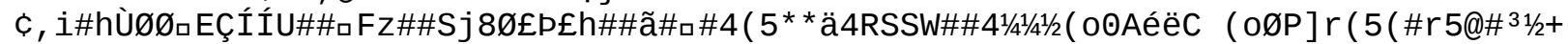

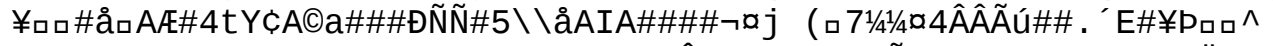

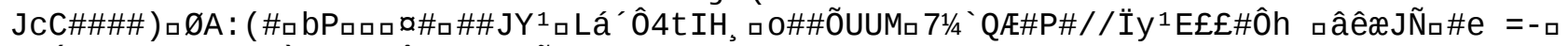

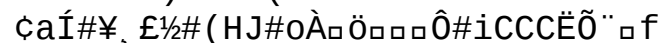

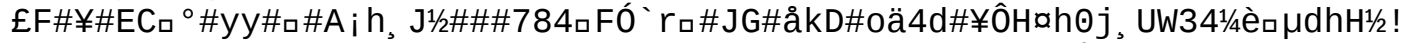

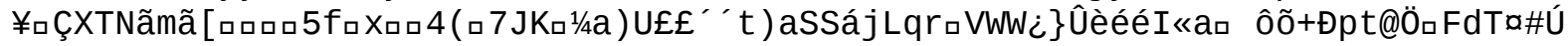
$\mathrm{C} \circledast \mathrm{R} \backsim 7 \mathrm{~h} 5 \# \mathrm{SSS} \square 1 / 4 \mathrm{~A}[\square$

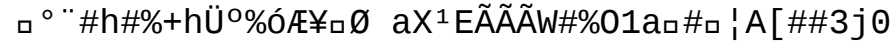


]C\#\#ロ\#ロRa a£Ü\#K\#\#*(4\#ロ i(f

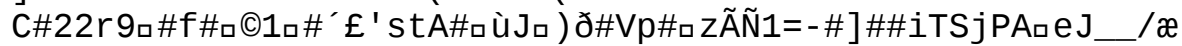

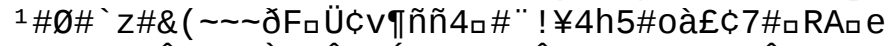

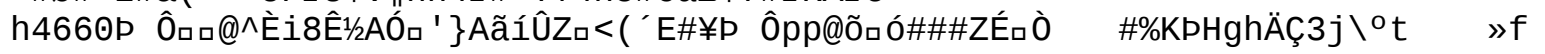




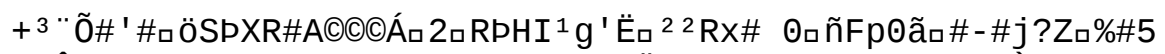

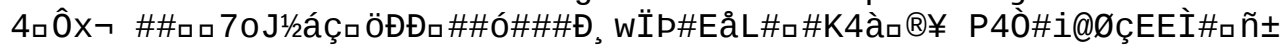

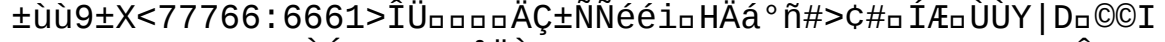

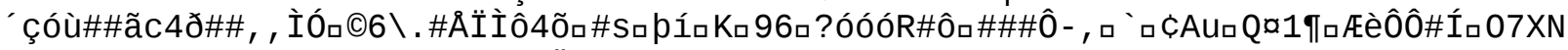

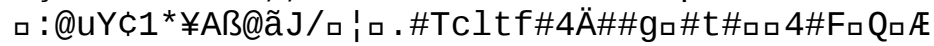




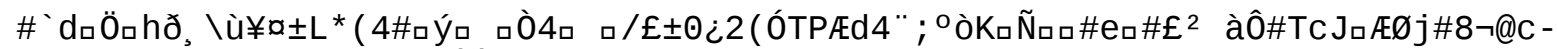

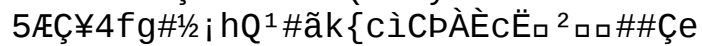

/P4Ä

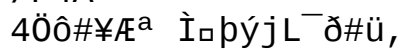

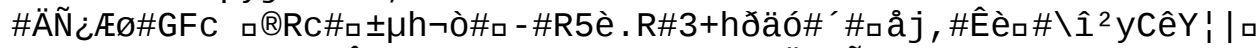

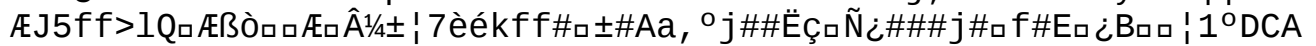

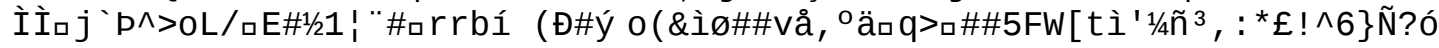

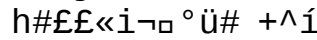

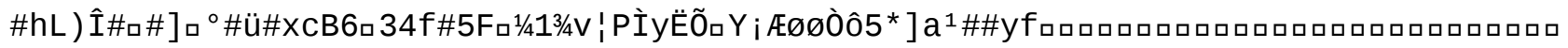

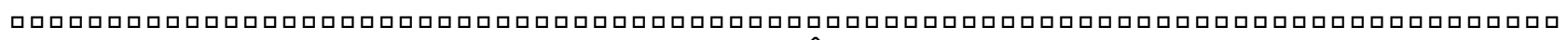

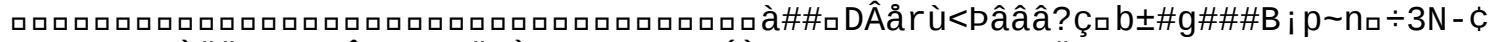

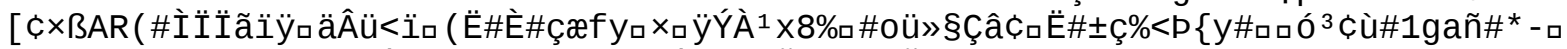

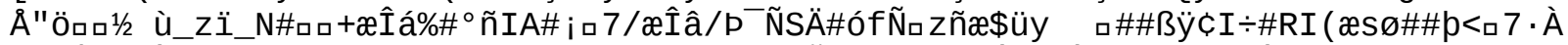

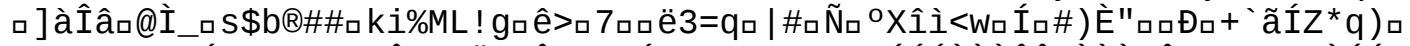

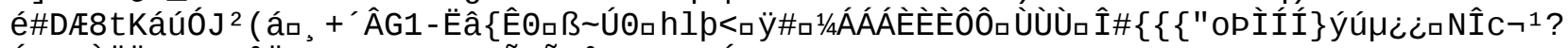

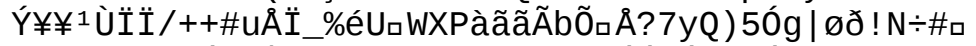

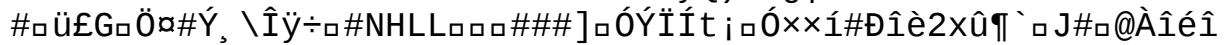

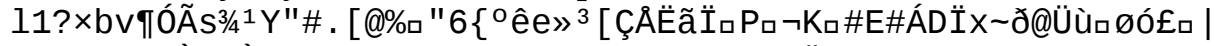

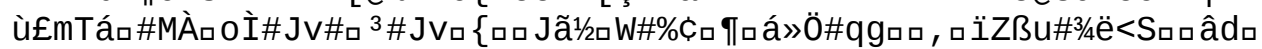

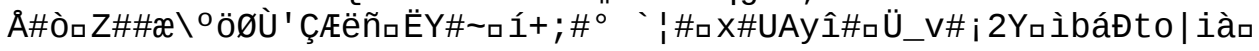




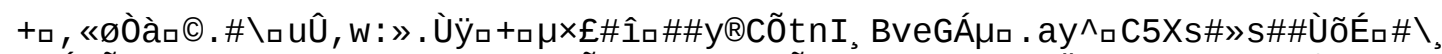

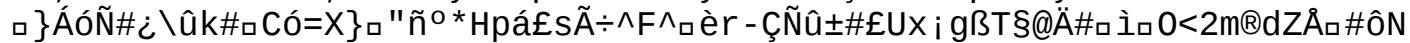

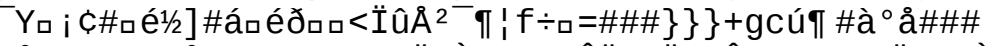

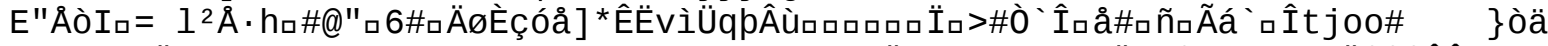

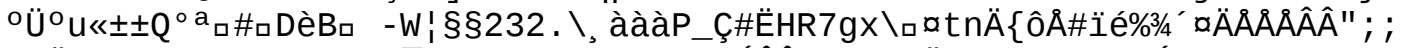

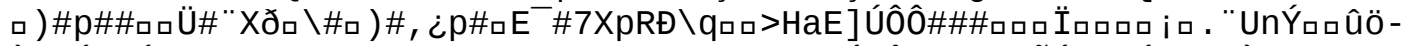

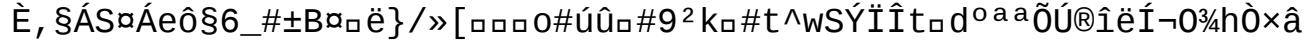





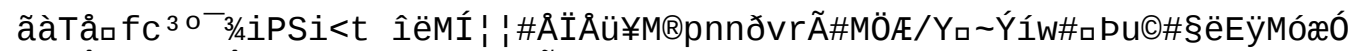

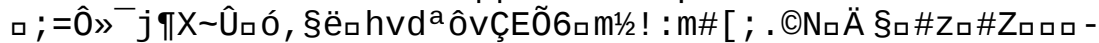

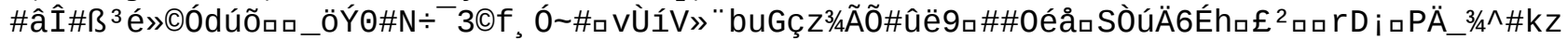

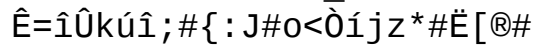




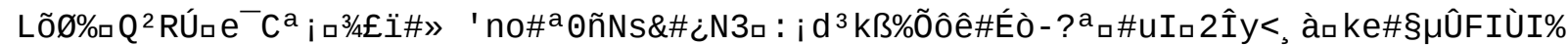

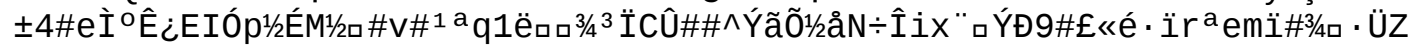

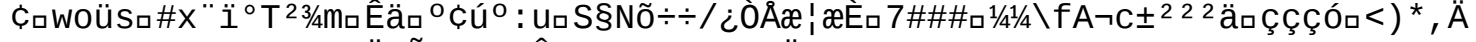

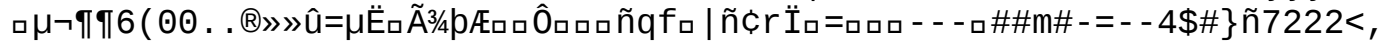




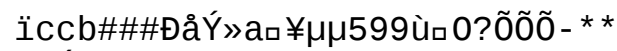

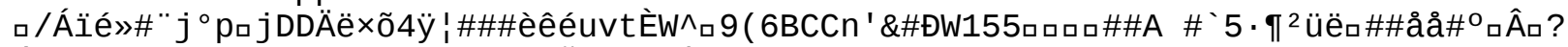

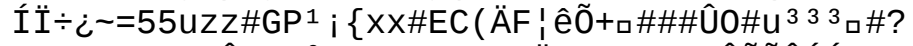

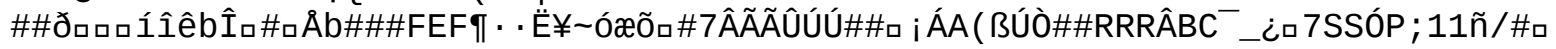




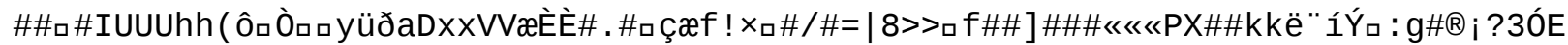

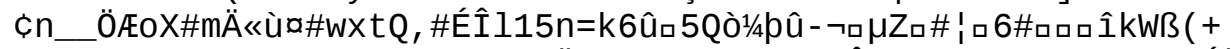

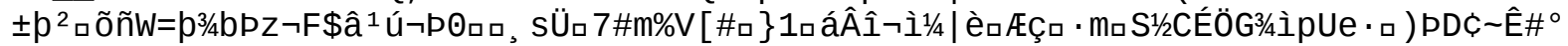

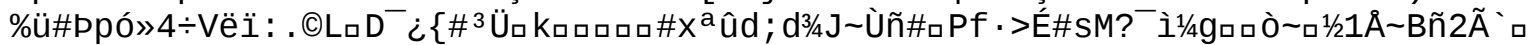

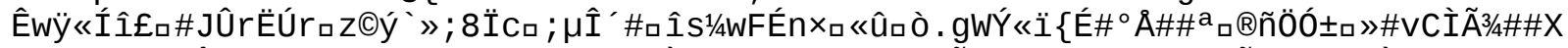

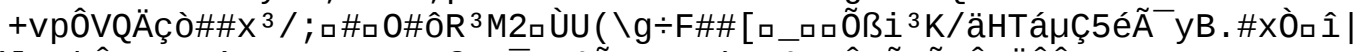

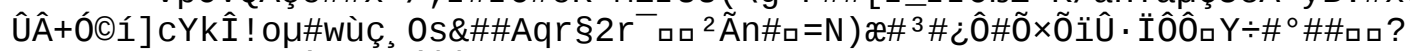

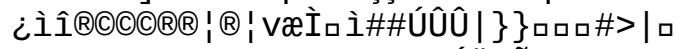

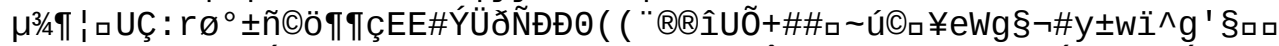

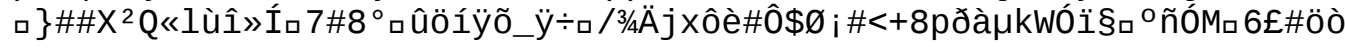




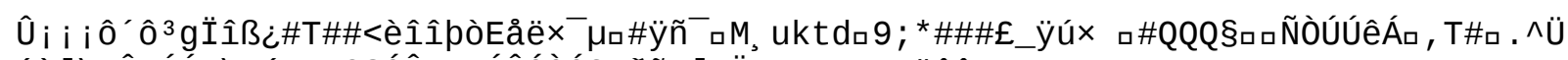

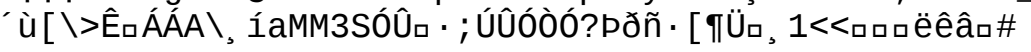





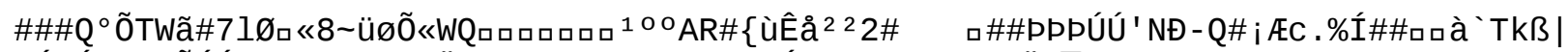

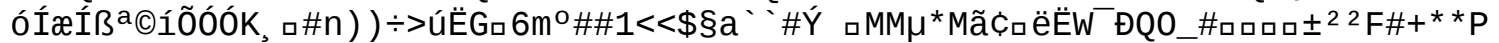

ฉロฉจ>\}ZóĐ! WWW\#6\#åÉaF\#\#êMMMëp

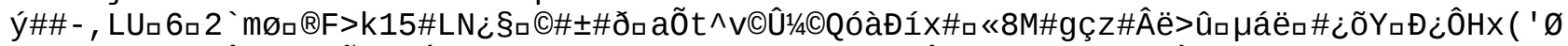

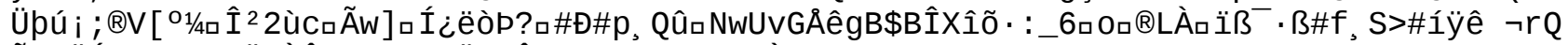

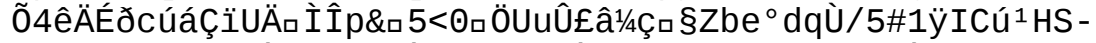

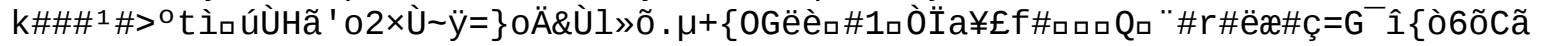




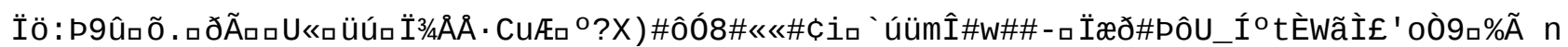

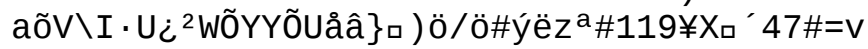


$\mathrm{kW}-\mathrm{a}$

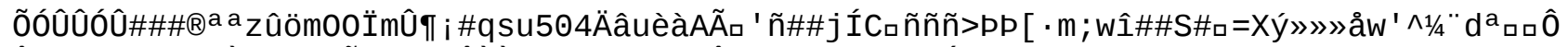

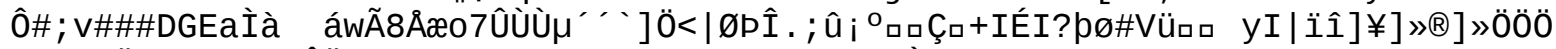

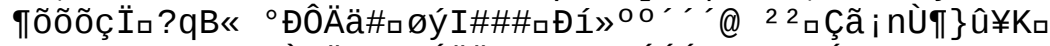

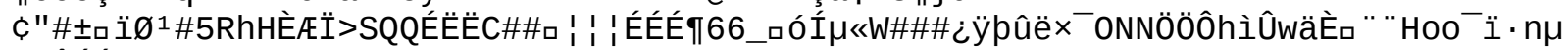

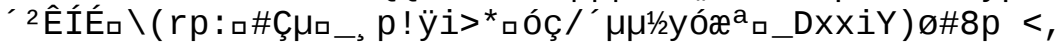




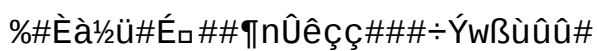




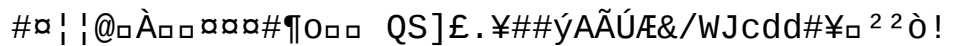

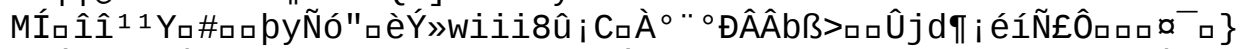

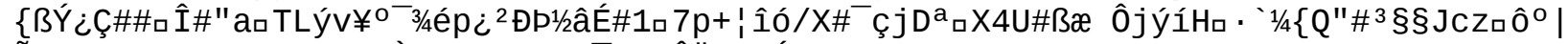

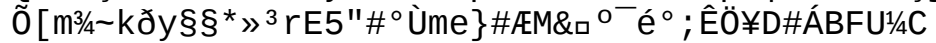

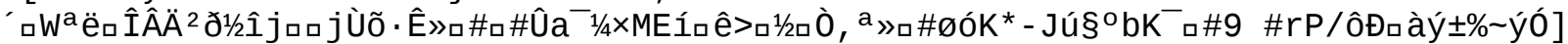

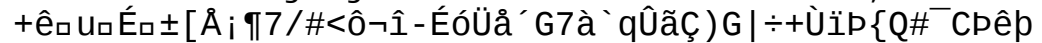

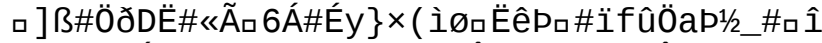

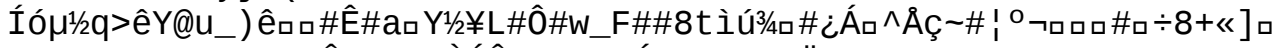

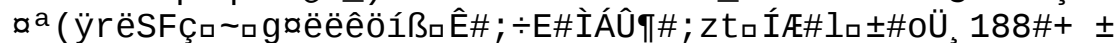

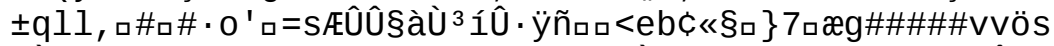

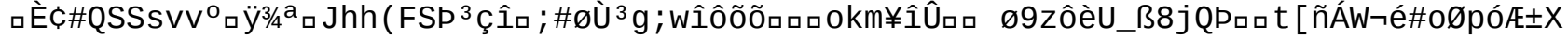




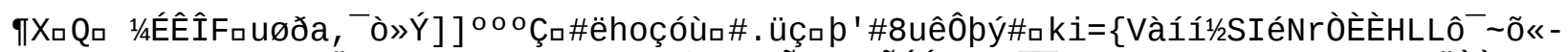

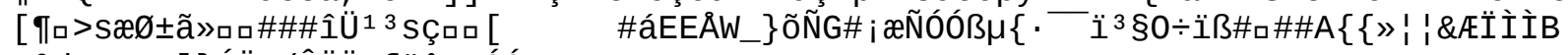

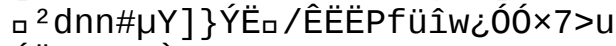

Ý̈̈ロ $\left.=; 88 \grave{E}^{\circ}\right\}$ ôè\#ロ 


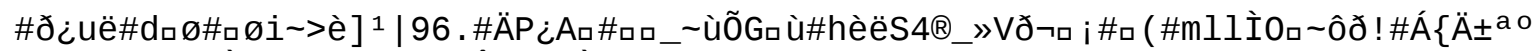

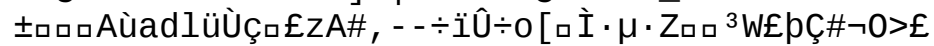

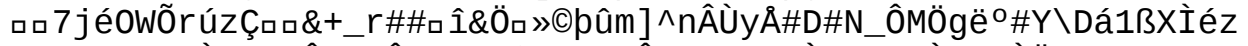

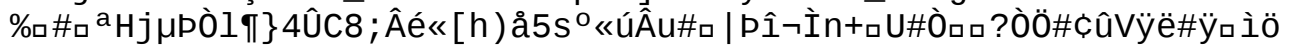
व1/2íô¥ व ZG\#

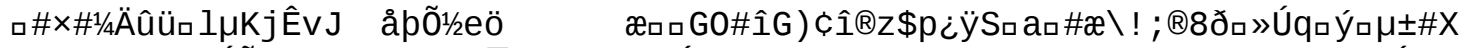

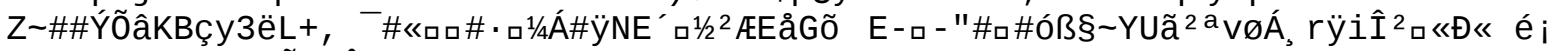

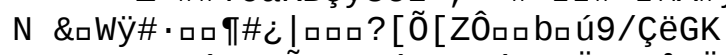

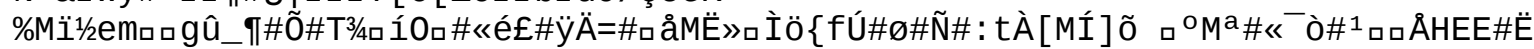

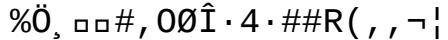

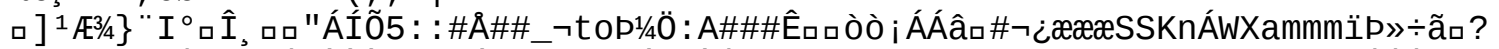

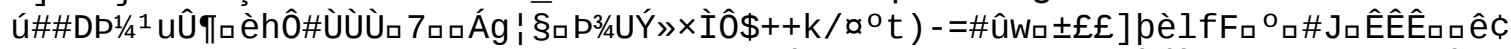

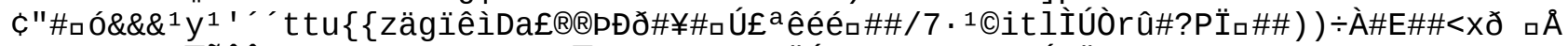

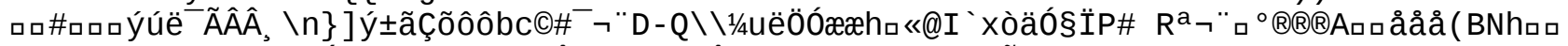

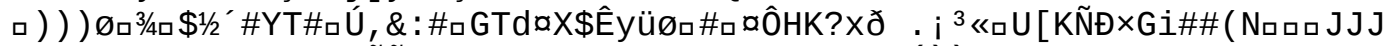

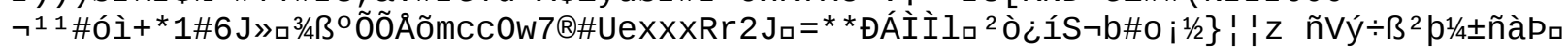

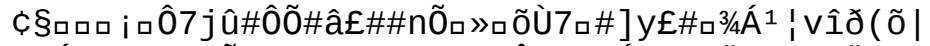

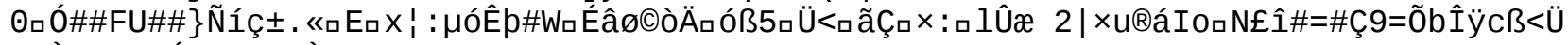

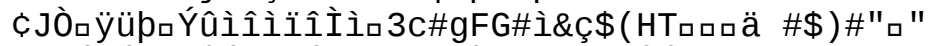

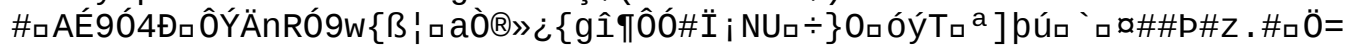

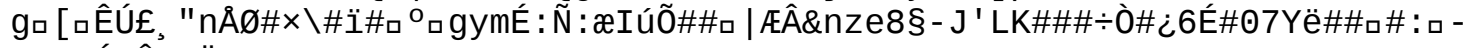

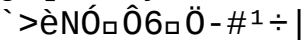

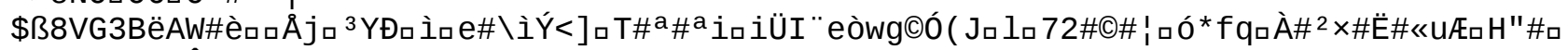
\#/Ç]4B5T\}Ôr [ם9BıRv ${ }^{\mathrm{a} 1 / 2}{ }^{1} \#$ rzKèõ 


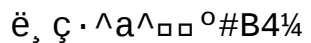

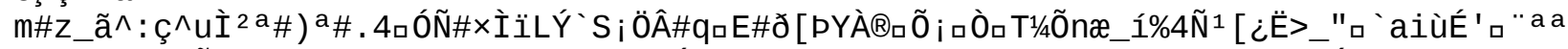

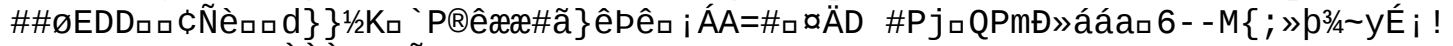

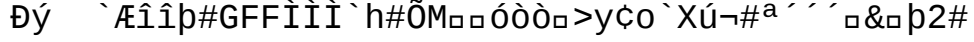


"םXÂÊ * Àßi ${ }^{1} 1$ ÙÂÂÂöí8

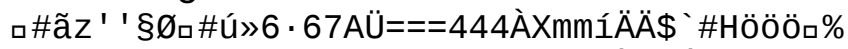

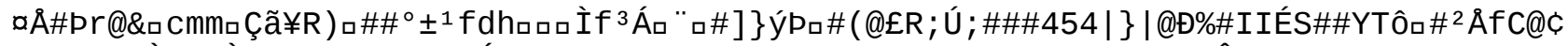

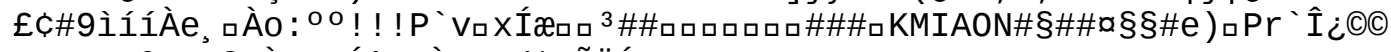

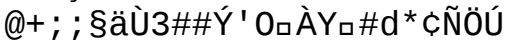

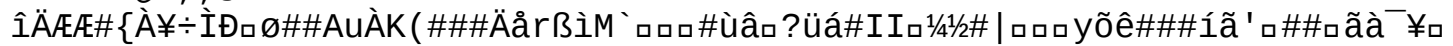

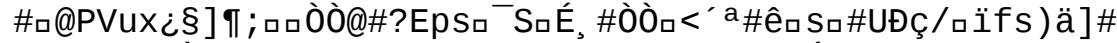

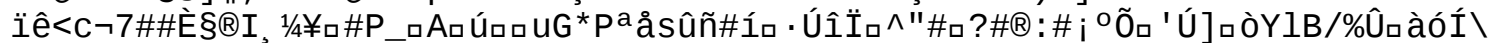

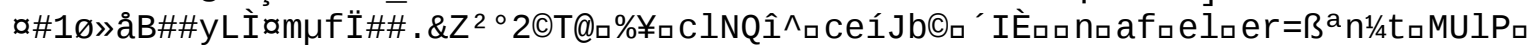

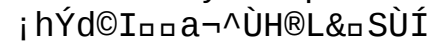

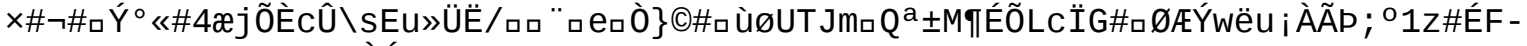

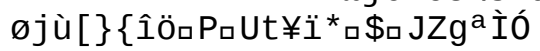




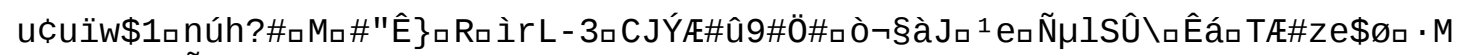

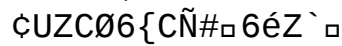




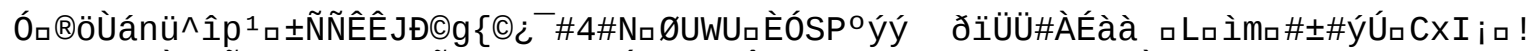

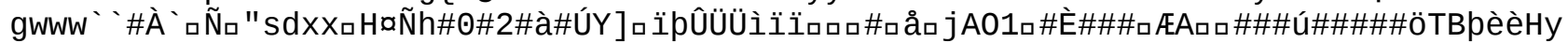

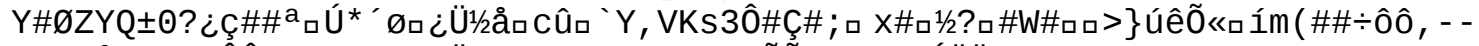

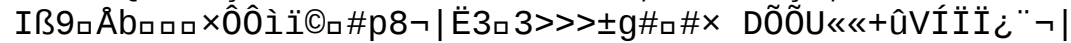

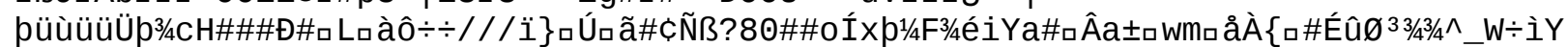
$\varnothing 066$

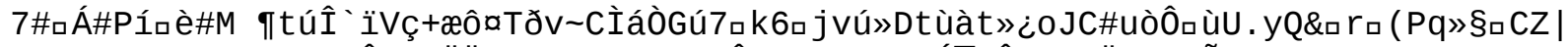

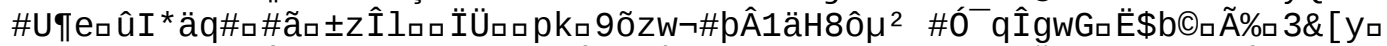

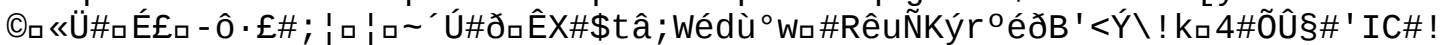

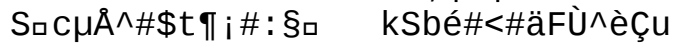




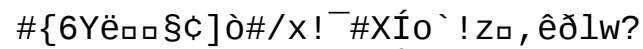

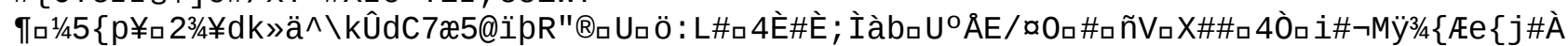

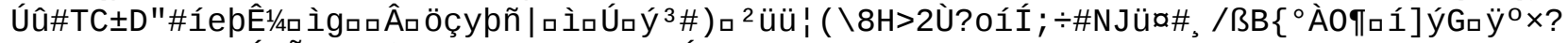

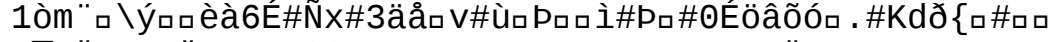

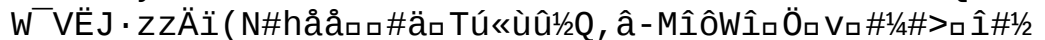

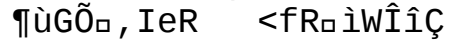

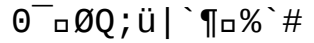

Ä\# ¿øfú_1 $\div$ foÈøbっÍðóNL3ロaß- 


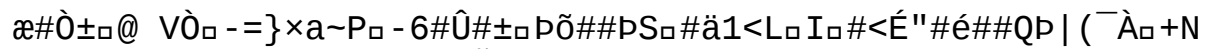

. b i L"zsøêûg i \#\#\#Iıb@èı\#ÕıûáıAıöb

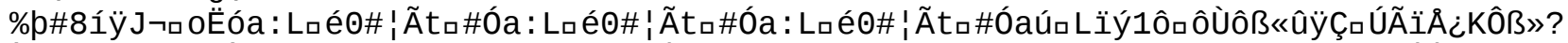

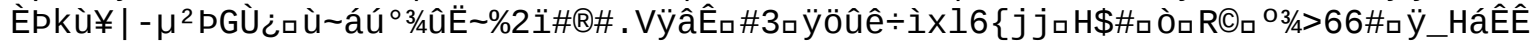

$Z$

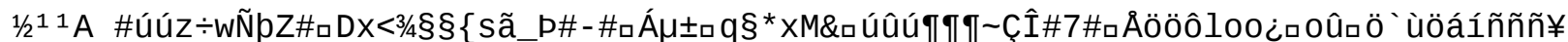

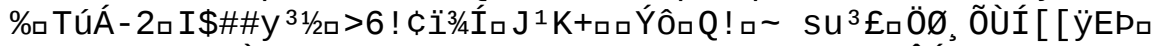

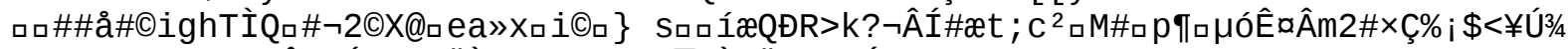

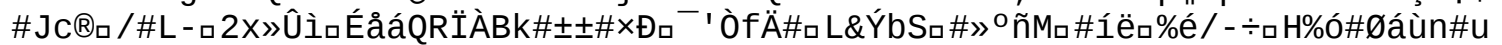

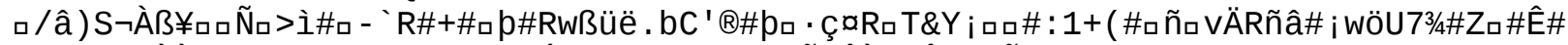

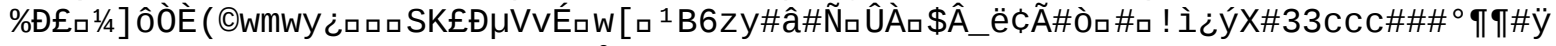

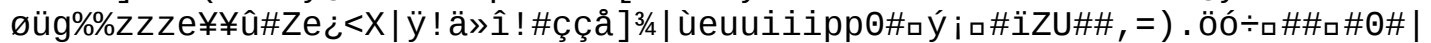

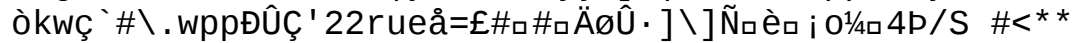

\#

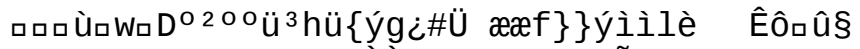

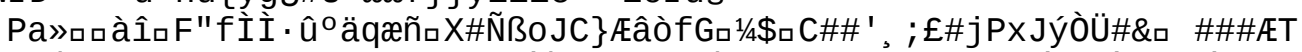

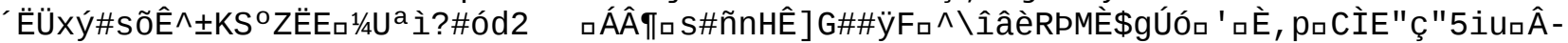

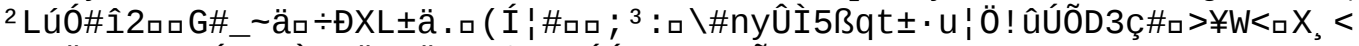

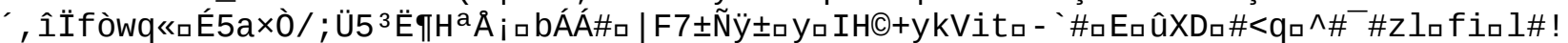

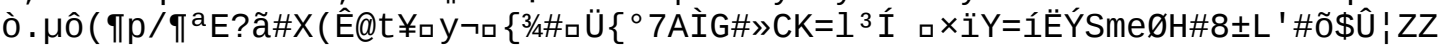

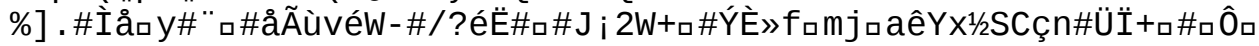

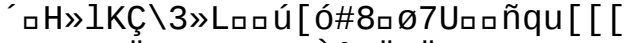

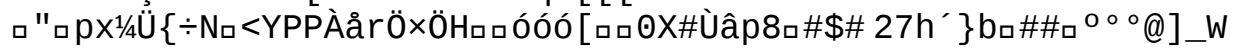




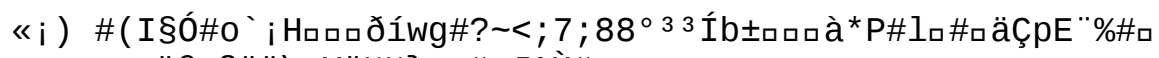

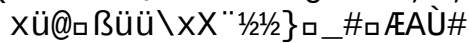

$\square$

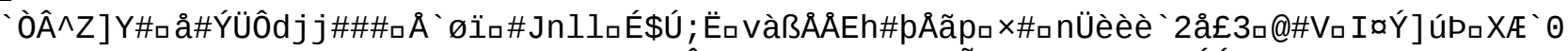

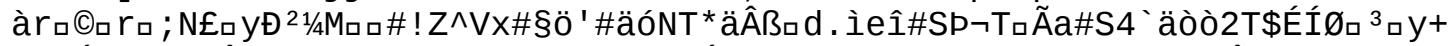

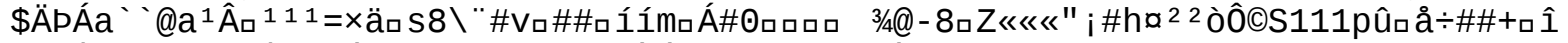

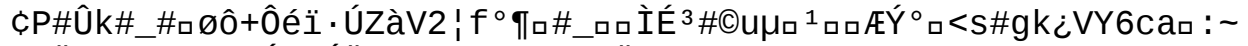

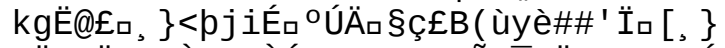

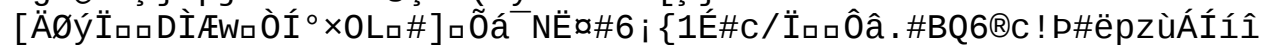

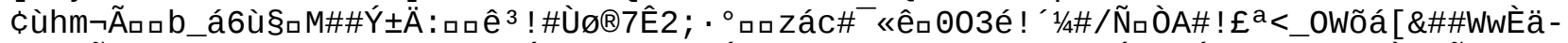

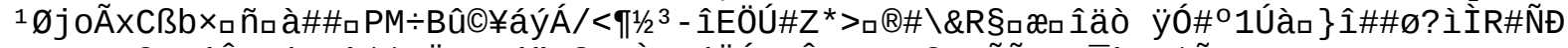

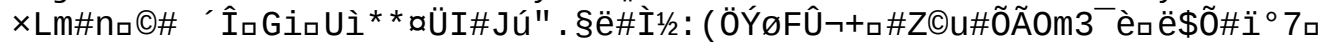

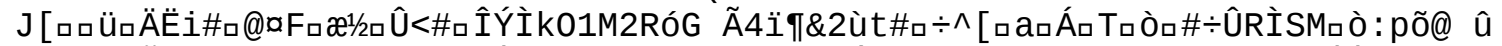

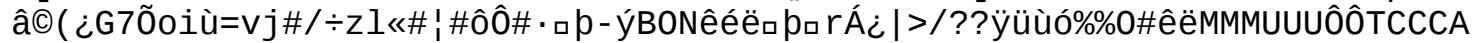

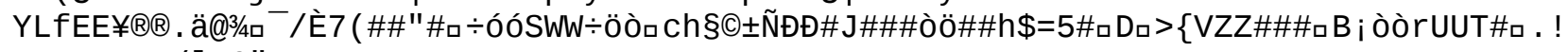
\#\#555ロロ\#/]RC"ם\#\#\#K\#

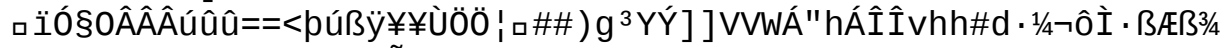

* $\neg P y(<>>f k g \# \square G E F \#) \tilde{N} w V J J J$ 

tut@ם\#»»\#\#\#Àf666 33 33 ButÉd

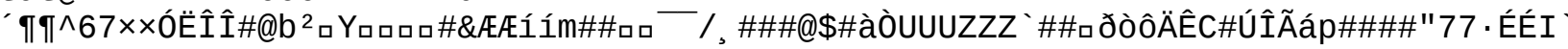




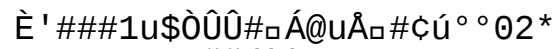

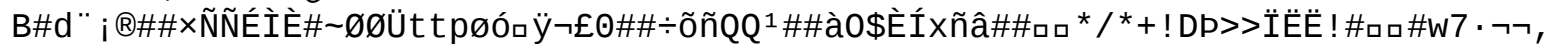

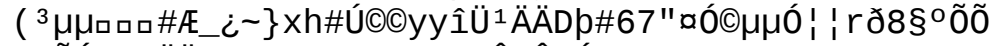

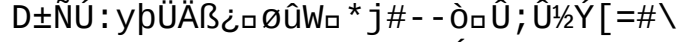

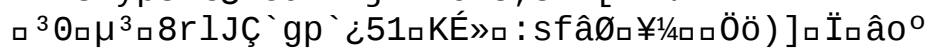

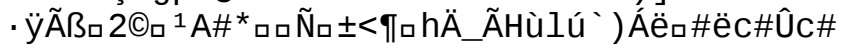

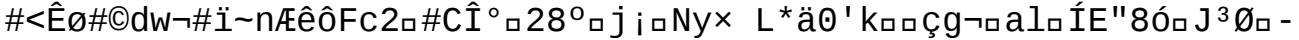

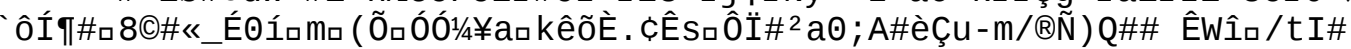




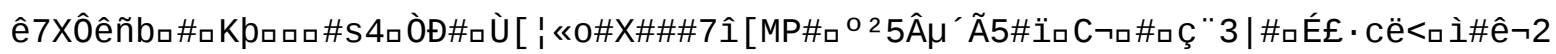




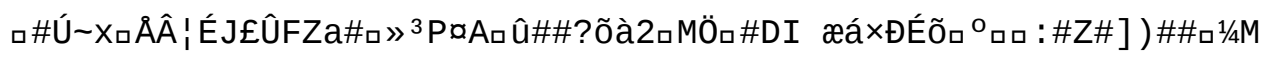




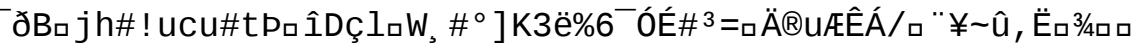

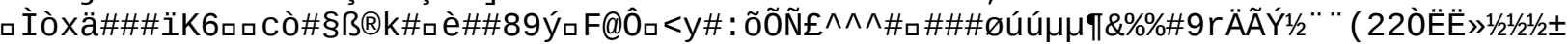

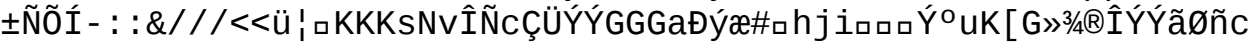

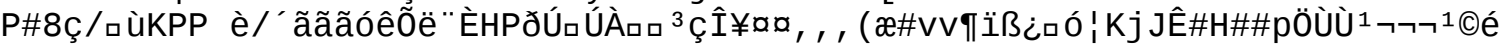

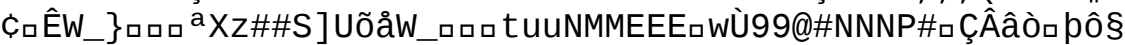

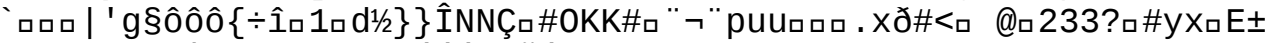

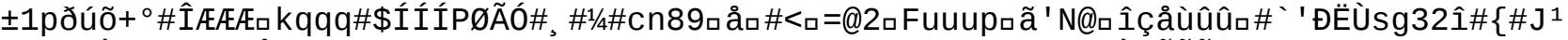

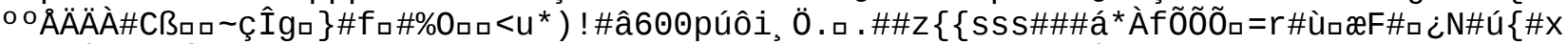

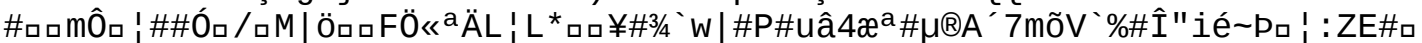

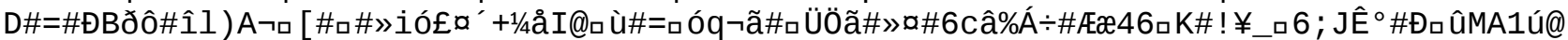

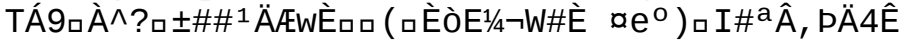

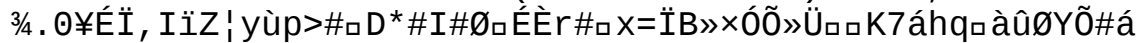

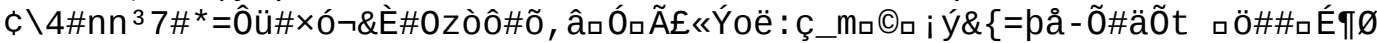

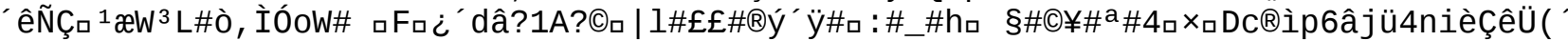

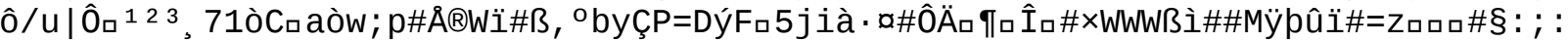
${ }^{3} ø q \tilde{n} \_\# ' r r \hat{1}$

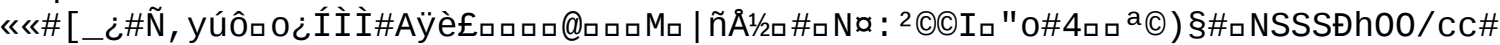
口\#qq9xhh 


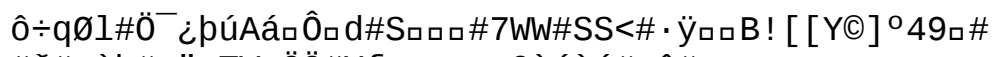

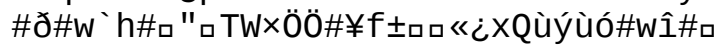


\#\#ロûp\{À\#o\#\#_ß̈̈>ÿÜøø,

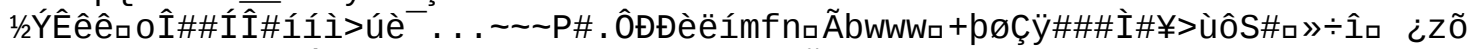

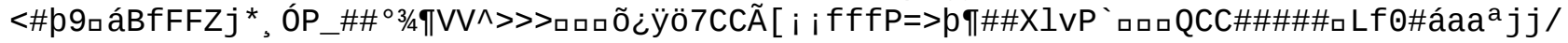

^11/4pwS353\#àñ 


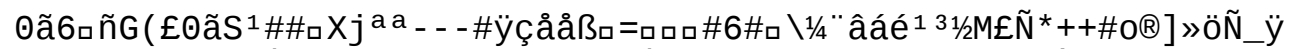

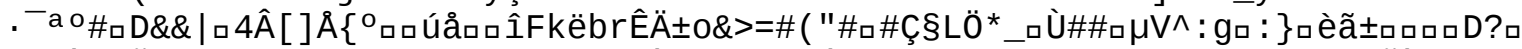

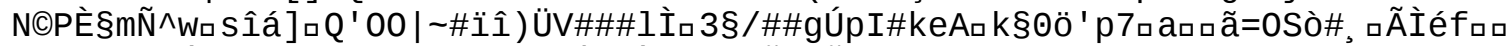

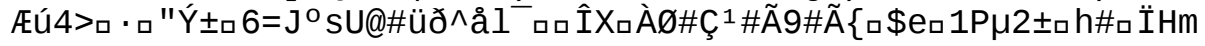




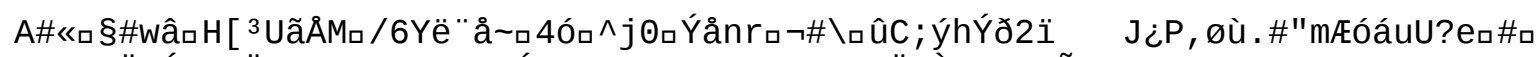

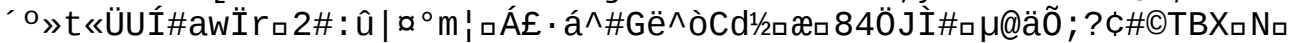

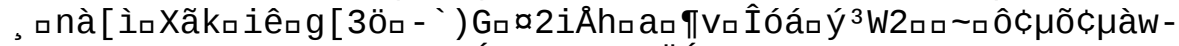

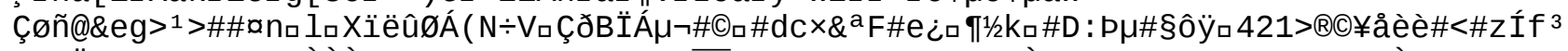

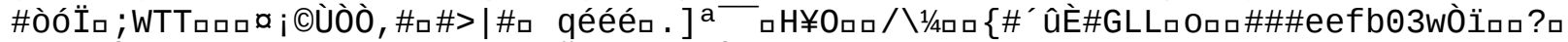

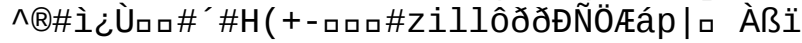

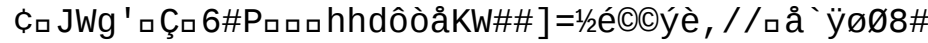




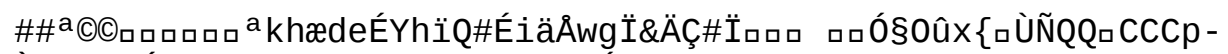

À\#*ロF§Óíìì3/4øòK§\#7\# \#\#\#bqX§\#×Á 


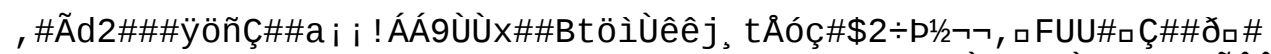

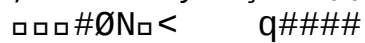

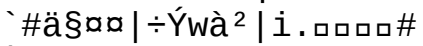

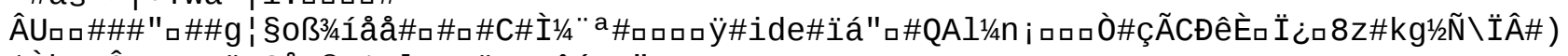

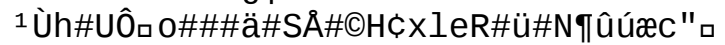

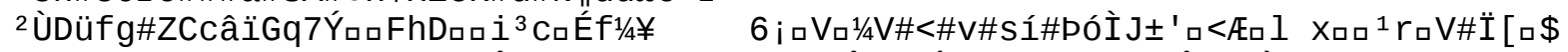

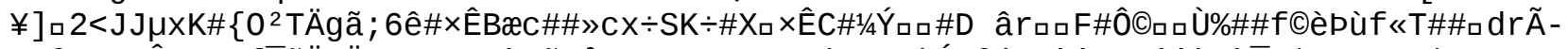

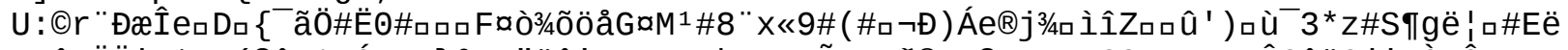

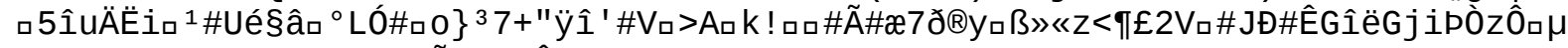

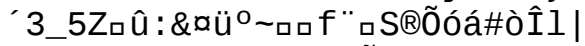

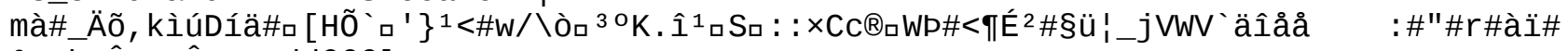

${ }^{2} \# \# \overline{\mathrm{h}} \# \mathbf{I}$ < <uÊÆæZTdd@@@IL 


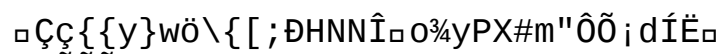

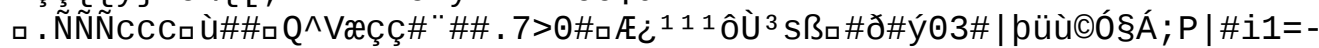

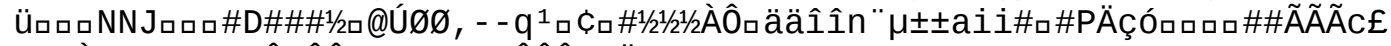

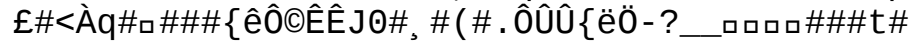




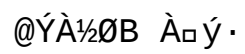

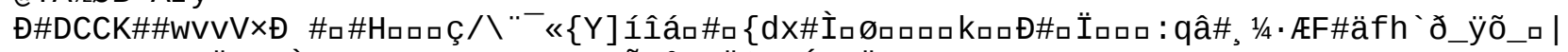

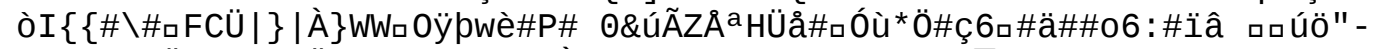

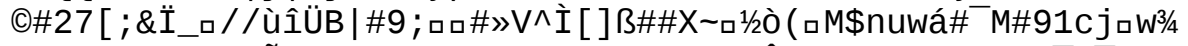

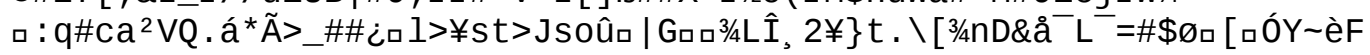




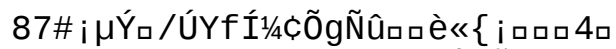

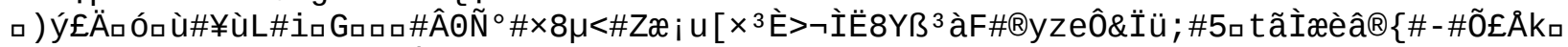
\%ü\# a äAEßEùMì\# ${ }^{2} C^{\prime}$ ó\} $\hat{U}^{3} \#$ j jyæÄuåYA

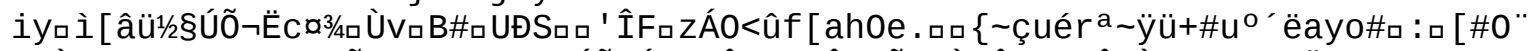

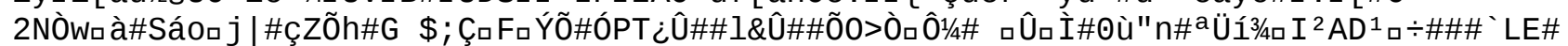

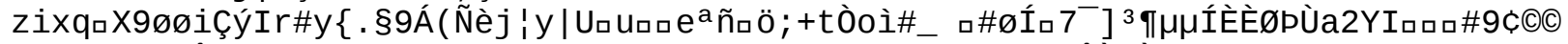

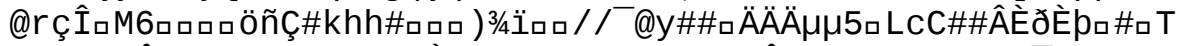

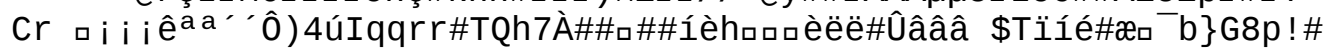

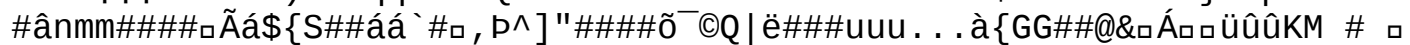

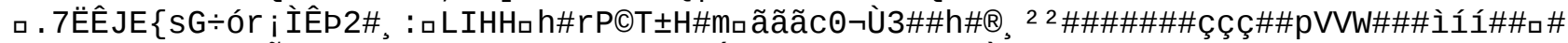

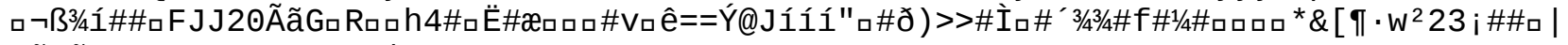

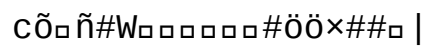

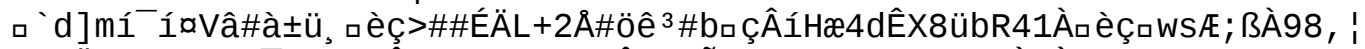

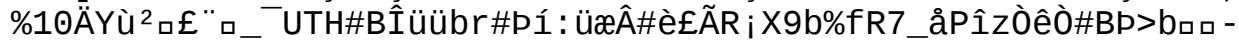

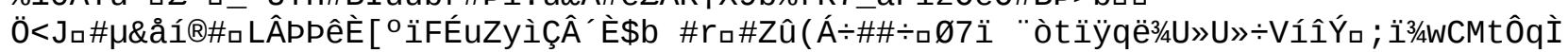

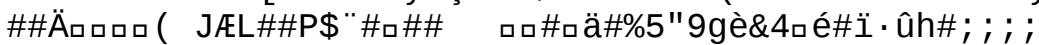

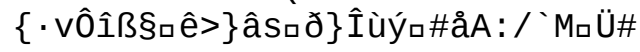




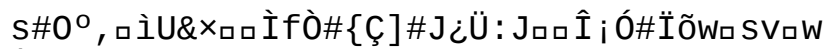

Îðn\#@C: ВÇ 7?

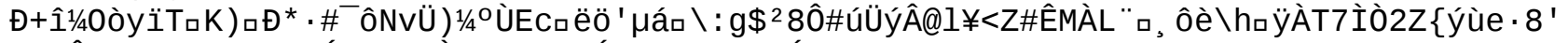

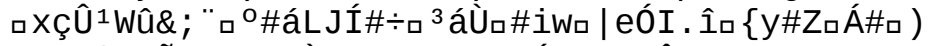

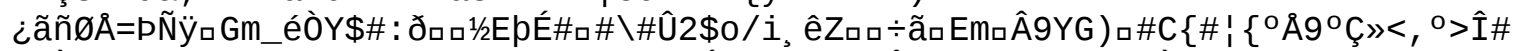

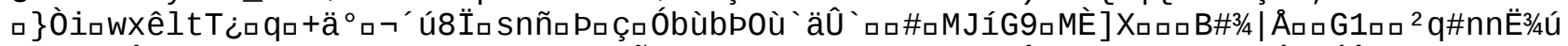

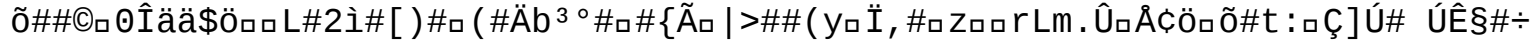


ô+\&Ï॰Å"æ 
0 ; $3 / 4 \mathrm{X}, \mathrm{A}>_{\square} . \ddot{\mathrm{A}} \mathrm{i}$ 


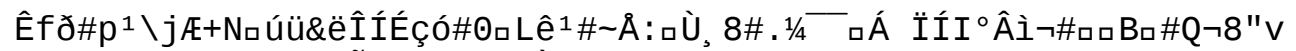

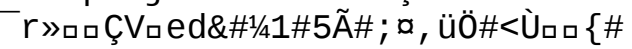




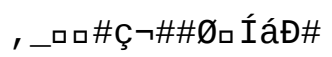




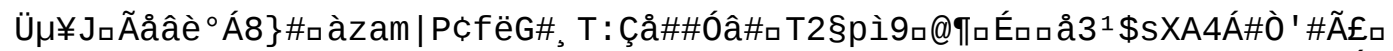

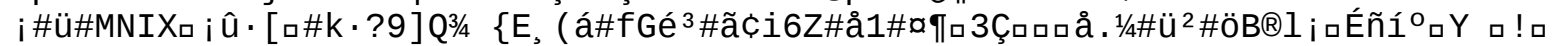

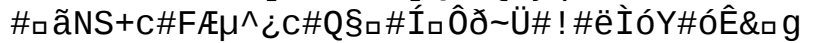

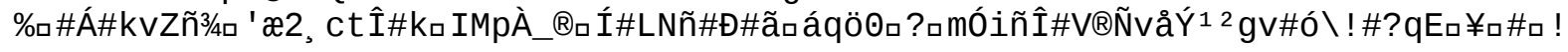




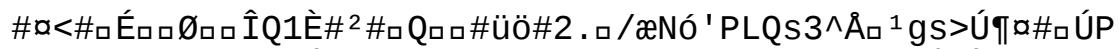

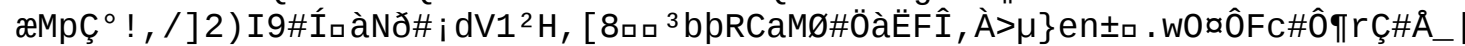

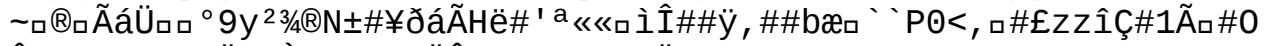

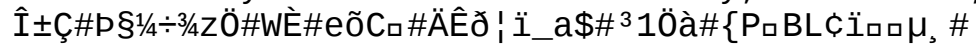

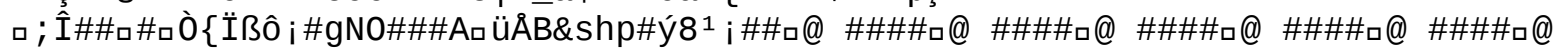

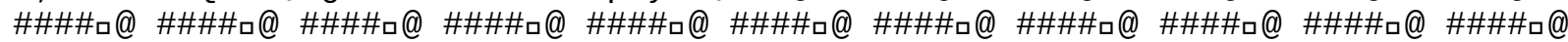

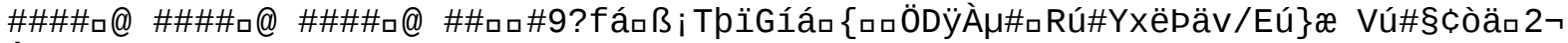
Ûpa ÄV?1;7ıwqáG\#à \{\#ャcccÅÅÅùùù 


\section{: ] \#\#ロロロロロ}

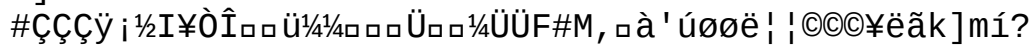

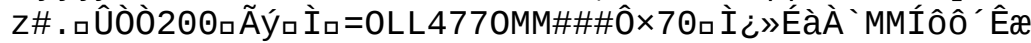

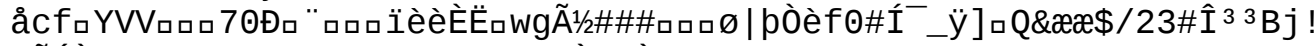

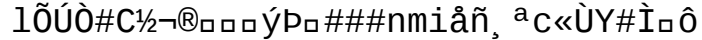

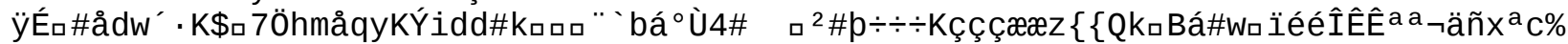

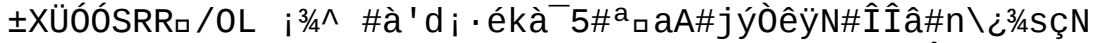

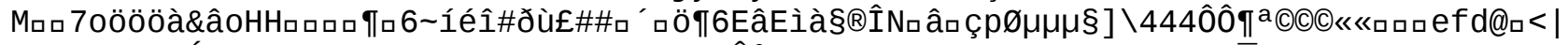

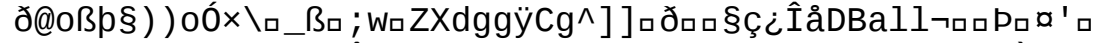

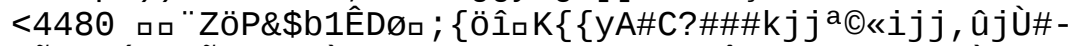

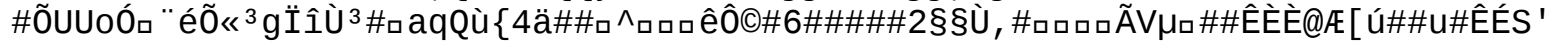



iaãW-\#\#\#èë ýõxz; vhií@q 

$\left.\# \square q^{*}+^{*}\right\urcorner$ 



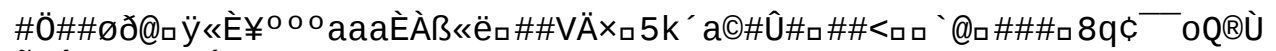

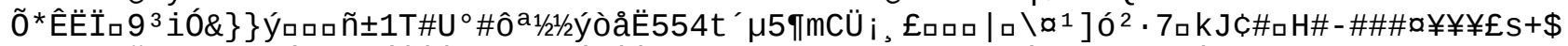

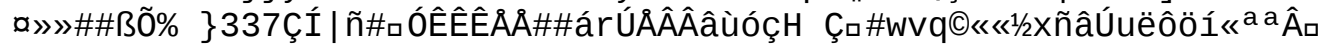




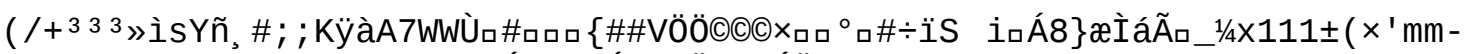

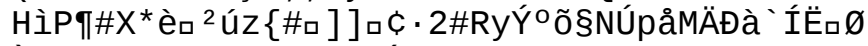

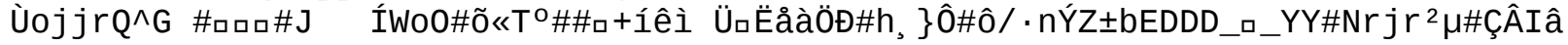




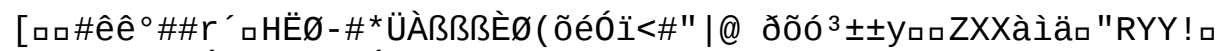

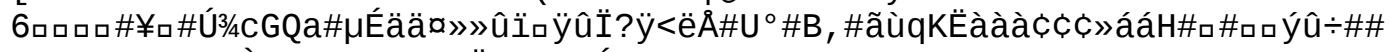

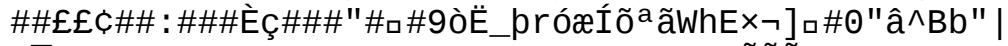

口 »§ûa -

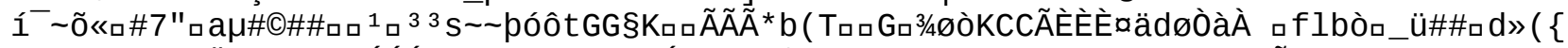

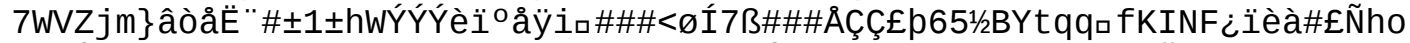

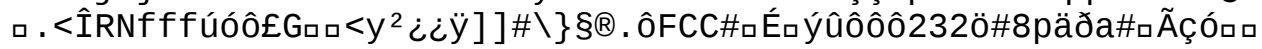




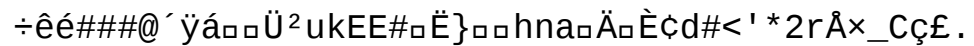

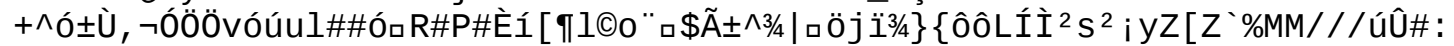

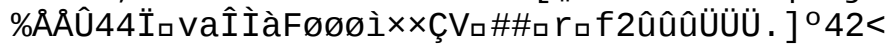




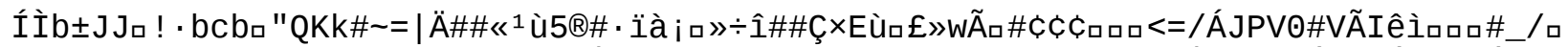

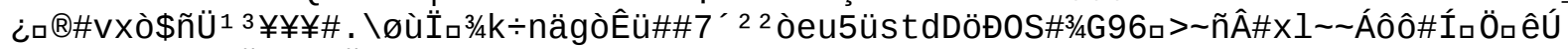
$1 / 4 \_d f \sim \# c \not c p I \tilde{A} » » » p \tilde{0}$ 


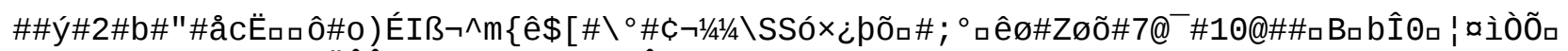

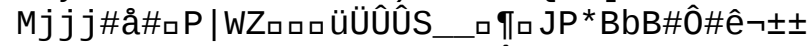

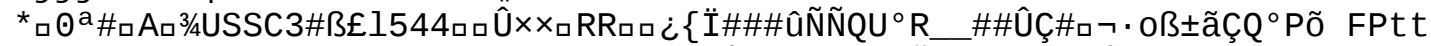

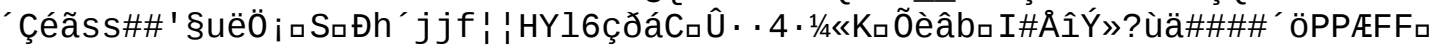

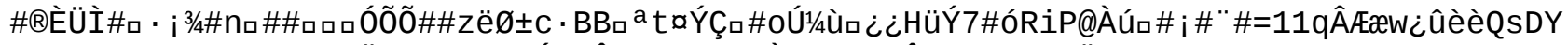

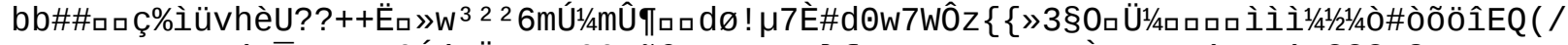

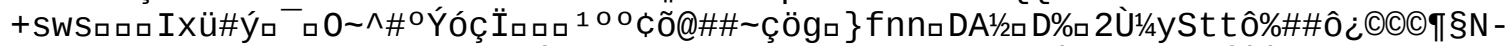

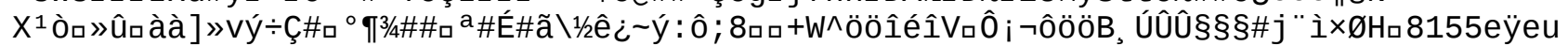

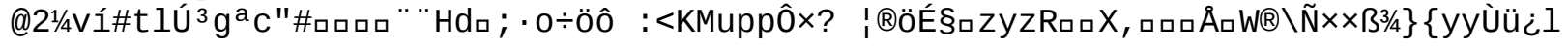




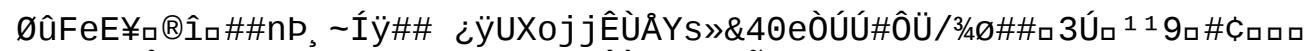

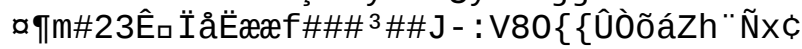




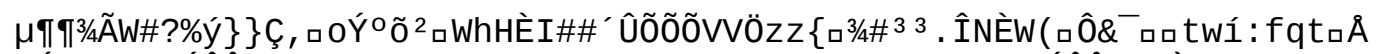

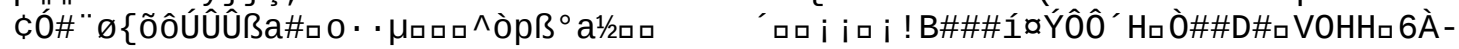

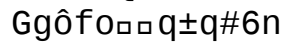




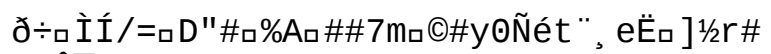

$\# \llbracket \hat{A}^{-}$

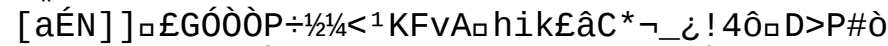

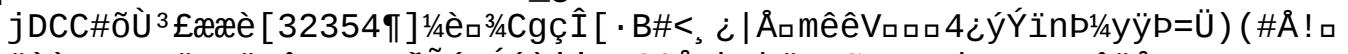

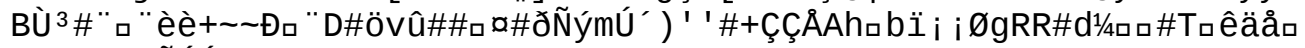

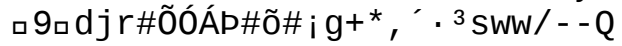


Úa`3XPP^\#6nHO\{!

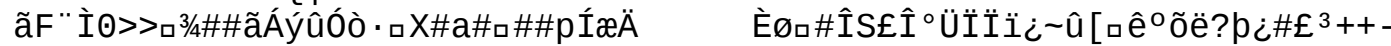

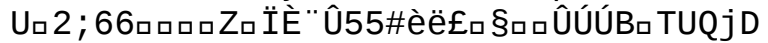

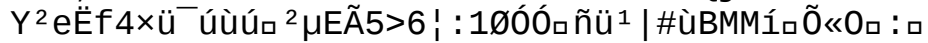

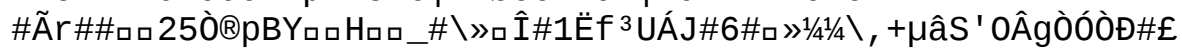

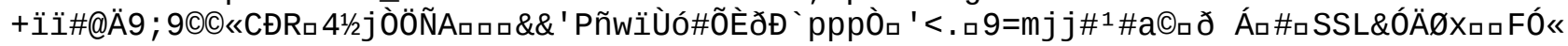
WhjםX

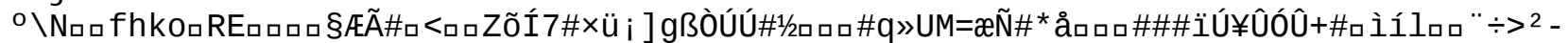

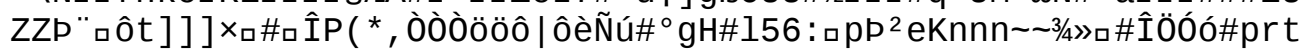



j | Õ\#ÍðÅロ_\#\#\#ロロÀn]\#\#nll 


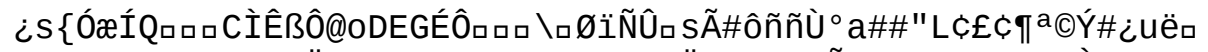

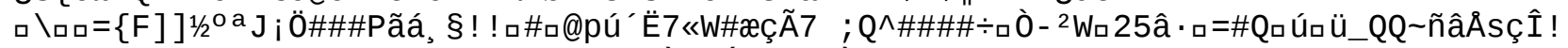

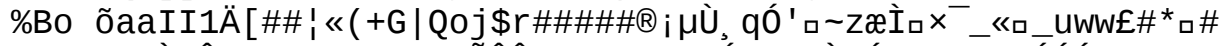

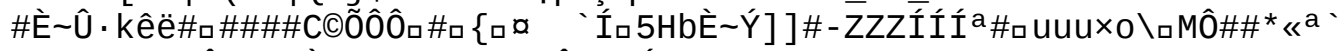

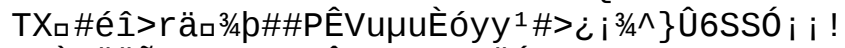

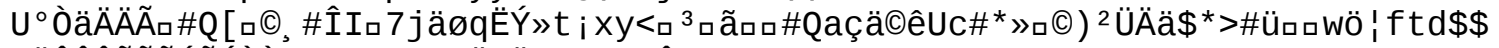

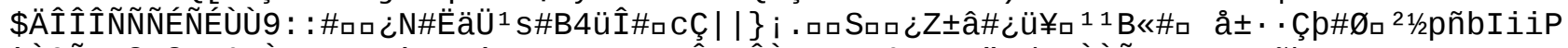

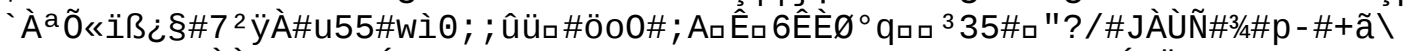

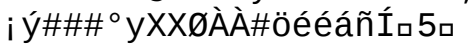

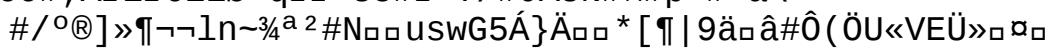

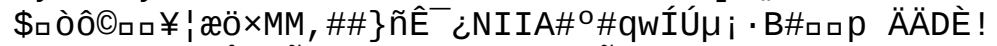

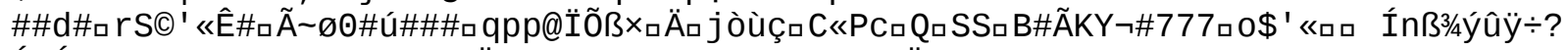

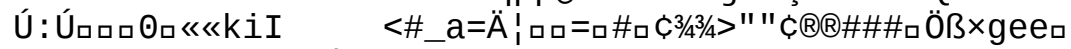

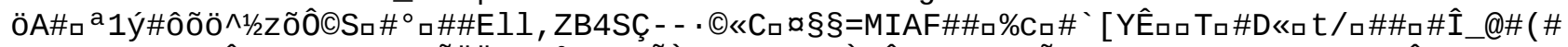

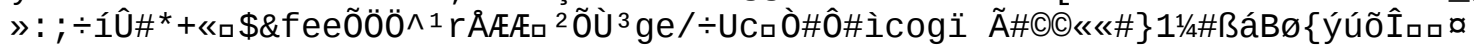

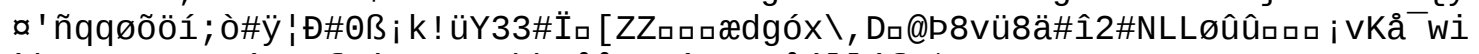

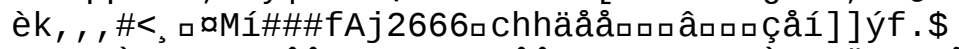

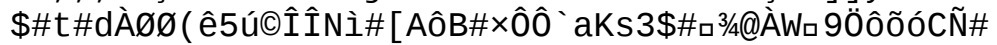

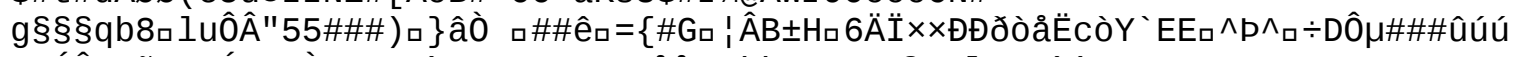

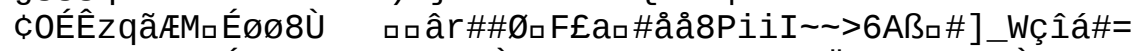

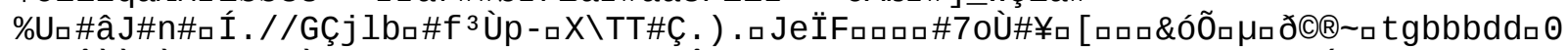

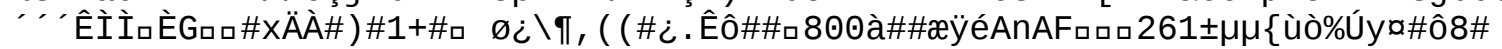

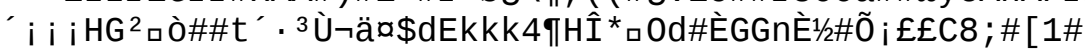




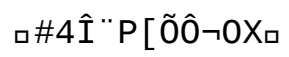




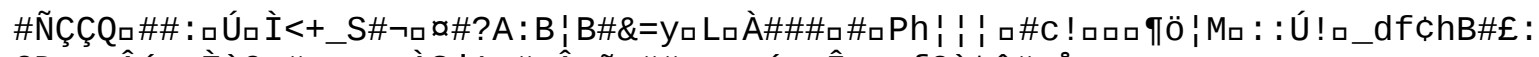

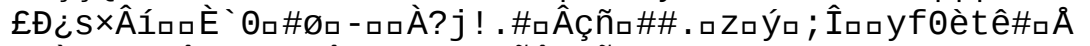

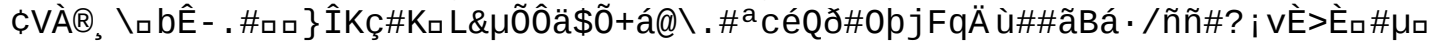
I, $\hat{\imath}$ 


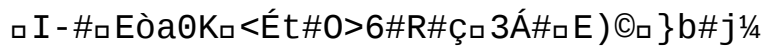

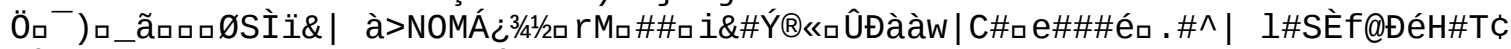

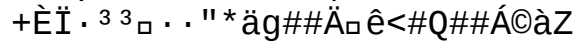

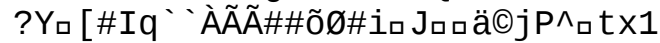

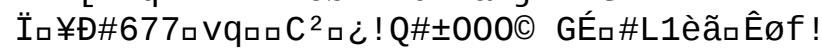

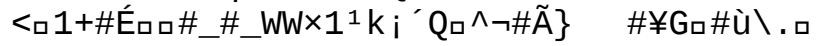




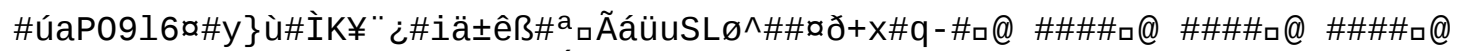

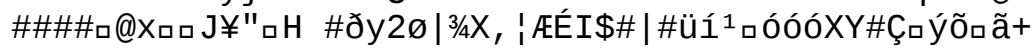




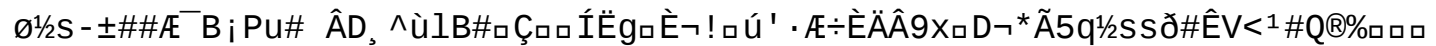

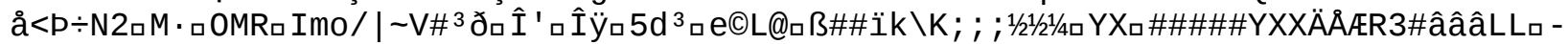

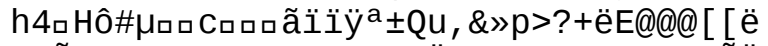

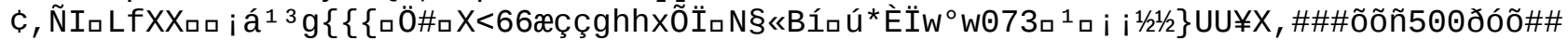

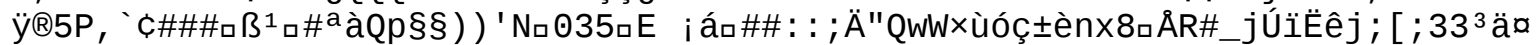

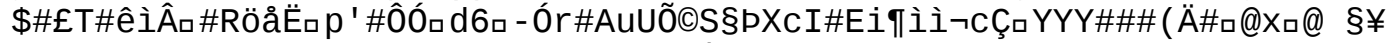

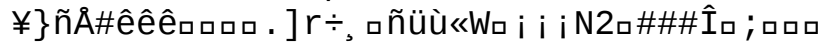




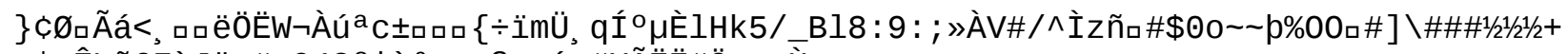

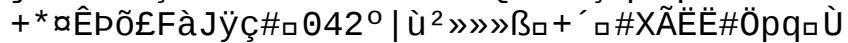

. $\div \# k @ E ̈ y x x \mid$ òÉ ' ûöínNV\#ロbĐéđVVaúón - -

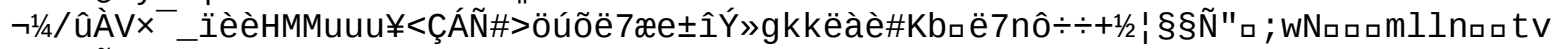

'ÇÇÇÃarkıÆò\#7n 




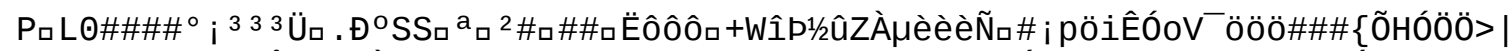

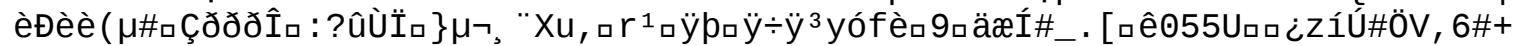
$£ e C æ \backslash \llbracket l \#$ ロ 


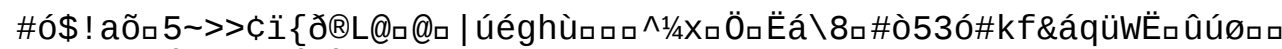

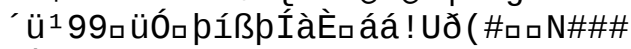

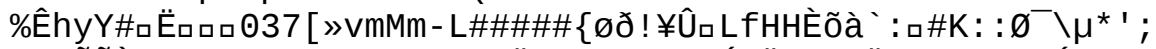

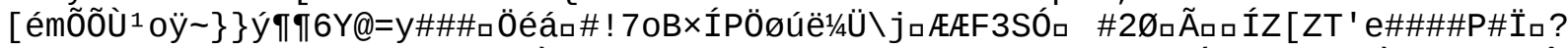

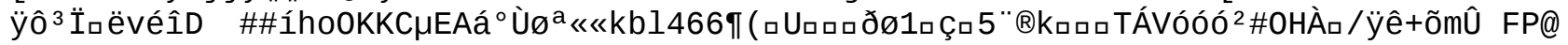
\#\#

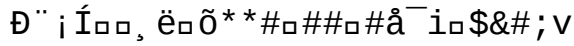




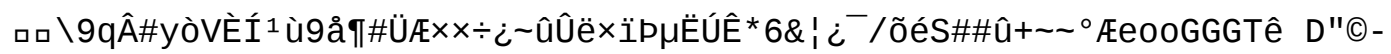
$1 / 2\}$ û ${ }^{1}$ a ù $q \sim j b l<<2 C$

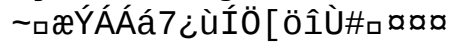




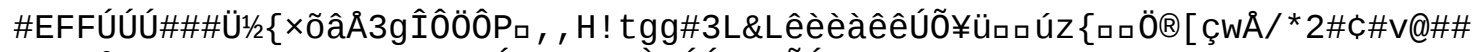

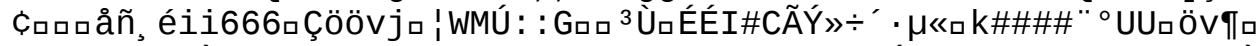

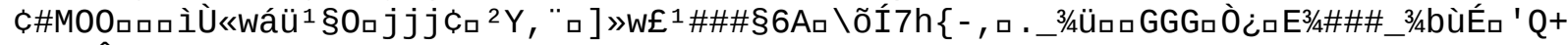

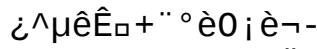

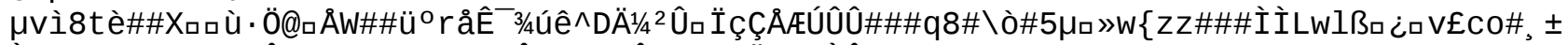

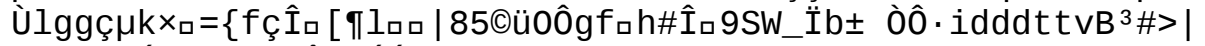

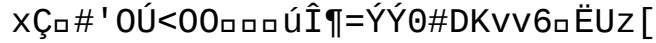




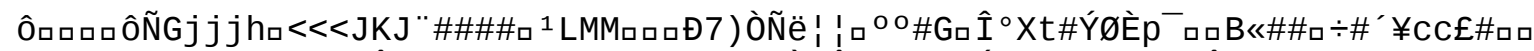

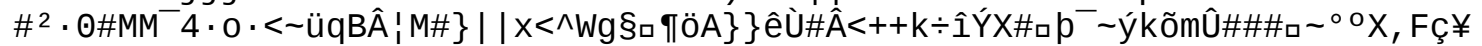

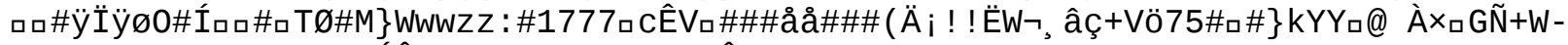

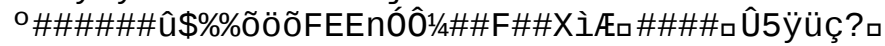

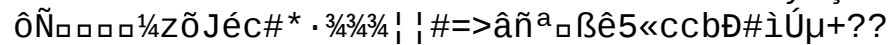

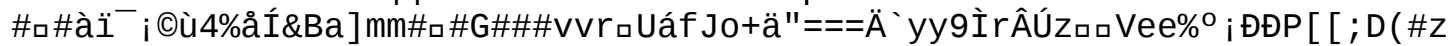

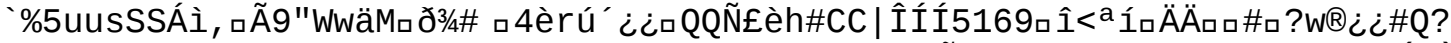

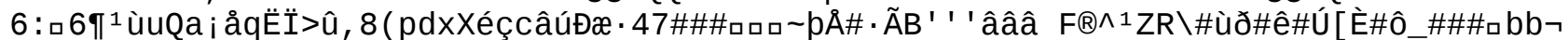

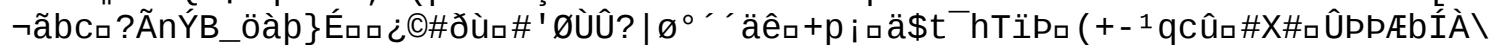

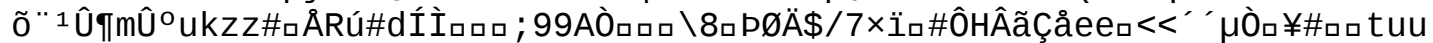

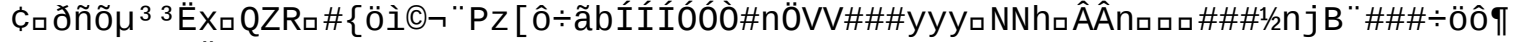

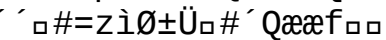

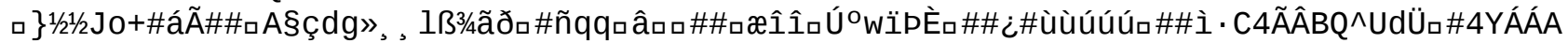

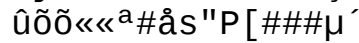





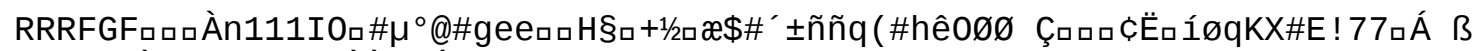

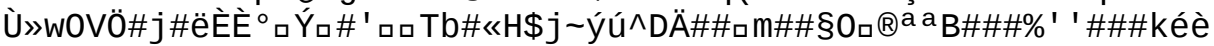

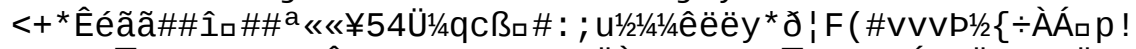

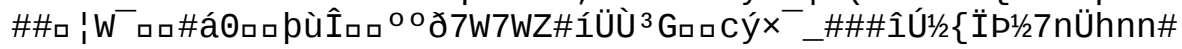
$\mathrm{gU}^{*} \mathrm{e \# \# \#}$

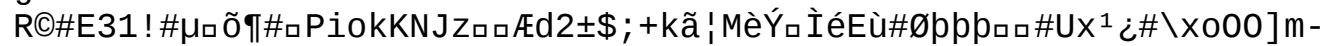

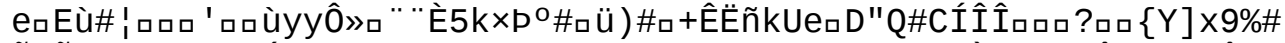

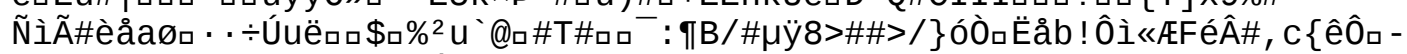

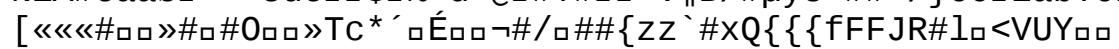




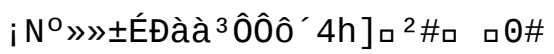


$\mathrm{T}\left[\mathrm{t} \#{ }^{*}\right.$ URÿw 


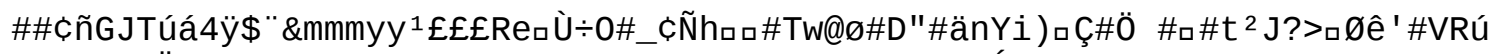

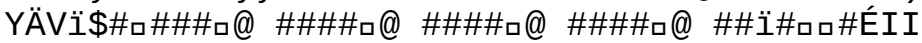

$\square$ 口 
口; Çgdx\$Y>-hhhð\#ÚıtaロÖĐ\#\#\#\#\#ó ( 6\&\&66ף

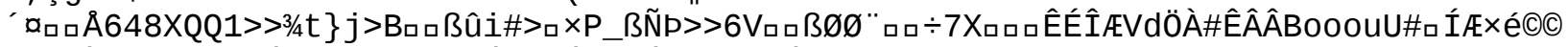

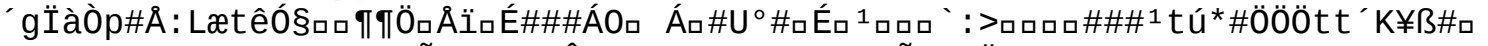

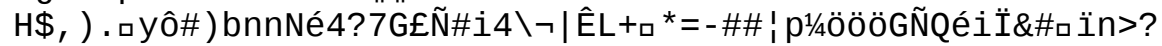

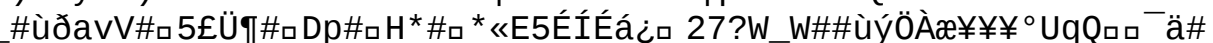

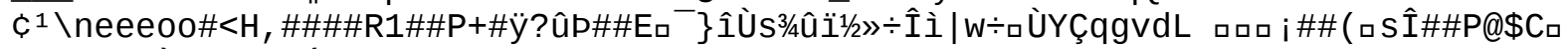
口J\#\#A\%*Ù@\#D2ロ\#ÉA

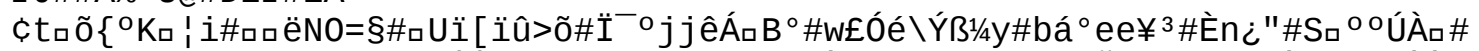

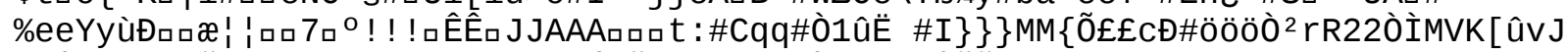

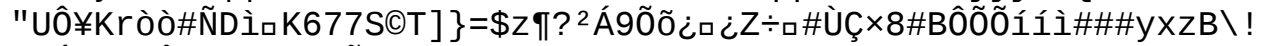

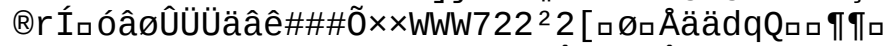

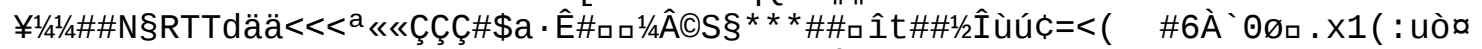

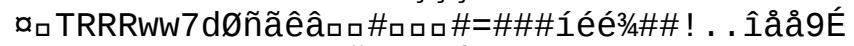

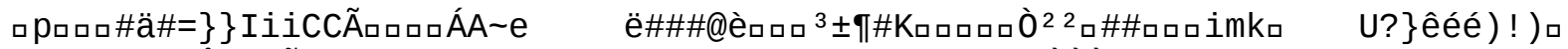

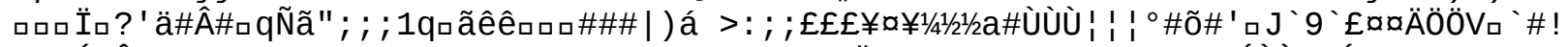

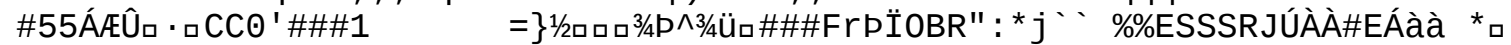

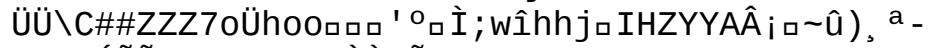

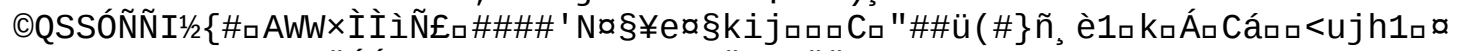

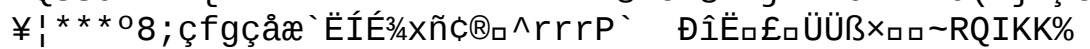

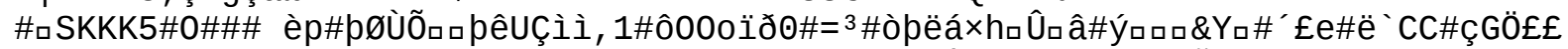

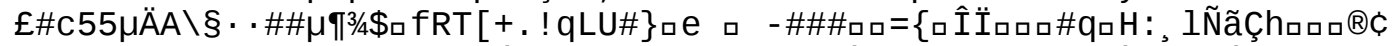

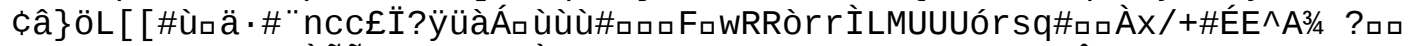

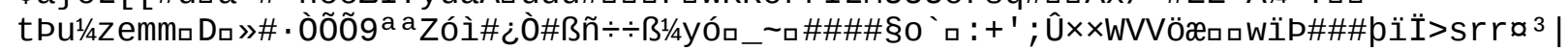

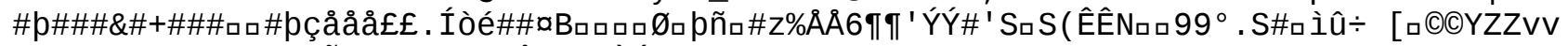

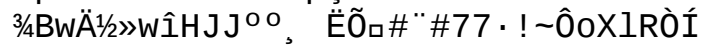

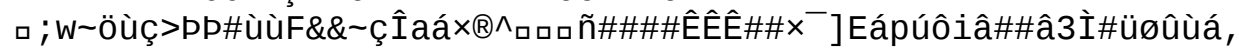




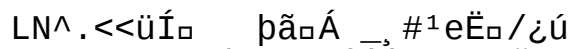

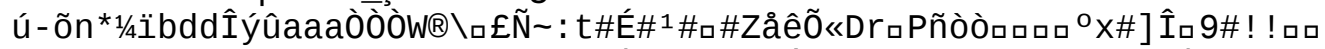

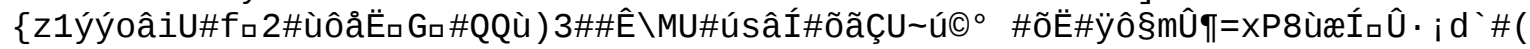


gfaÏF 


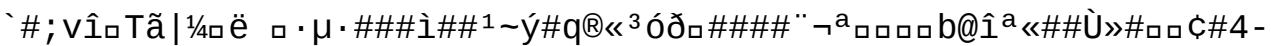

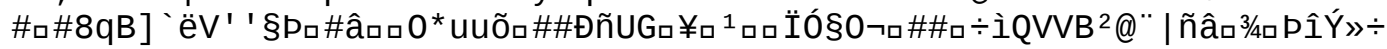

İロロ»ロ $t$ \# 
ddøòåË222RRロ

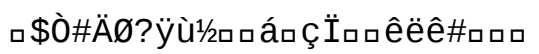




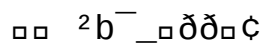

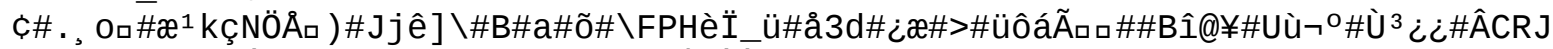

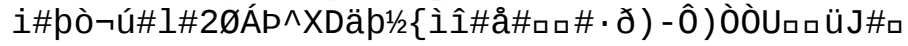

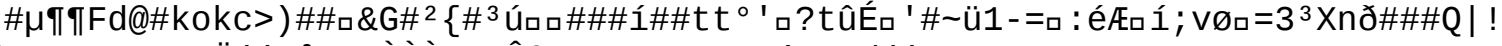

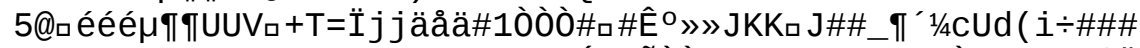

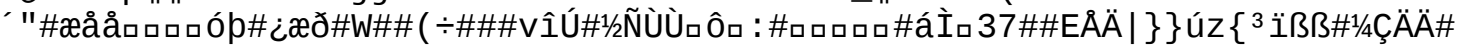




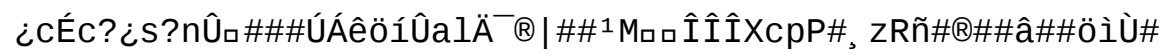
$5 \# \#, '\{7$

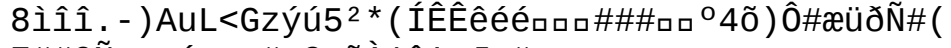

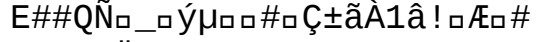

ฉםUUUÏ̄ $=K I I A R n n j \#$ 



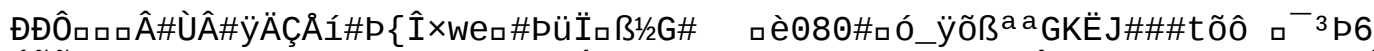
\#

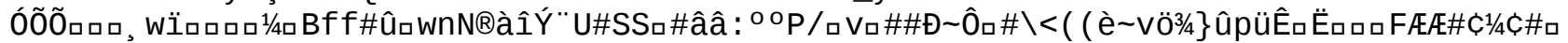

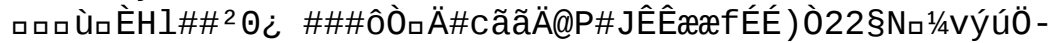

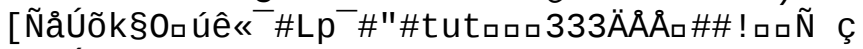

\#\#?Éý

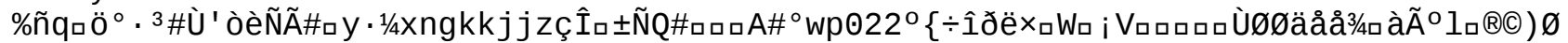

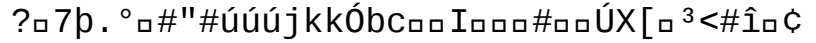




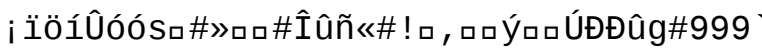

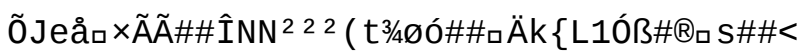

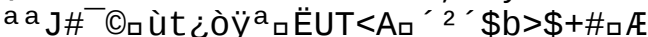

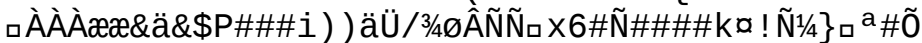

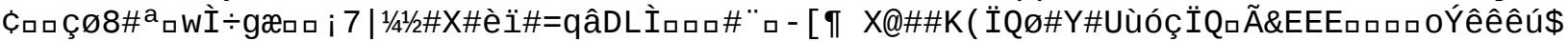

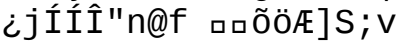


\#rTU\#Óロp\#\#\#0̃ 8 i

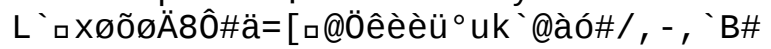

; Y 


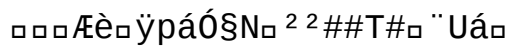

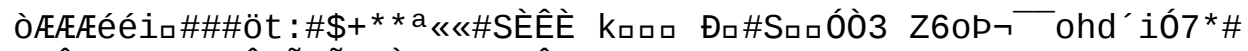

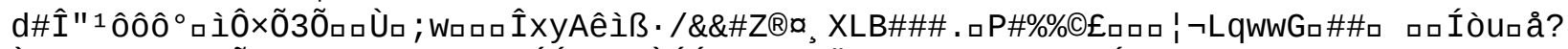

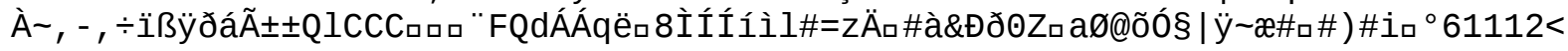




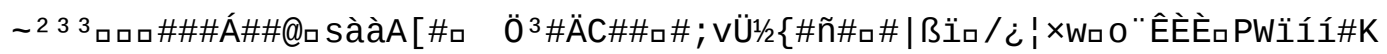


$f a j \# \# \#$ jEY

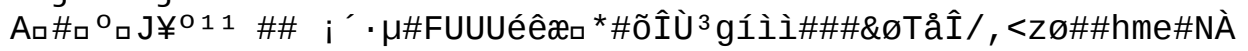




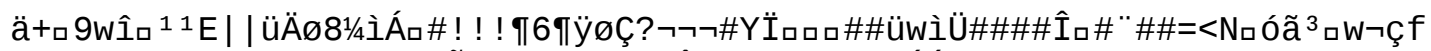
ñก̃, $\div 97=3 \# \#$, EREÑhccc $t z H p \odot O ̂ \square \| \square V 8 E ̈ 囚 ` 1)$ ÉÉ 


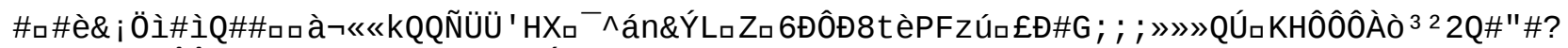

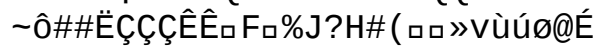


¿Çk\#d?? ¿Ü1/1/4/4à á á1/2"Wc®\#OS 0 : ; 1 


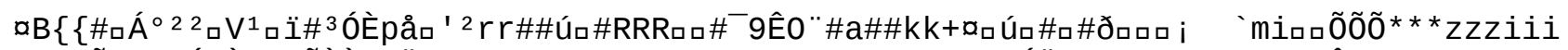

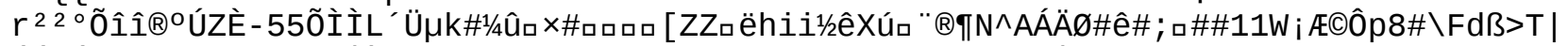

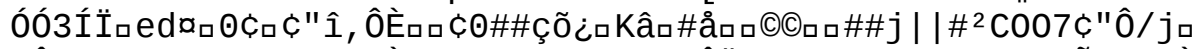

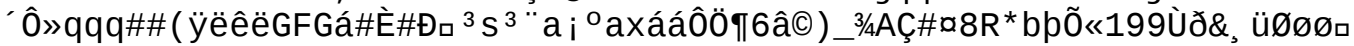

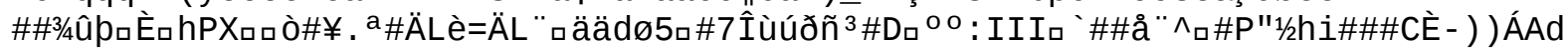

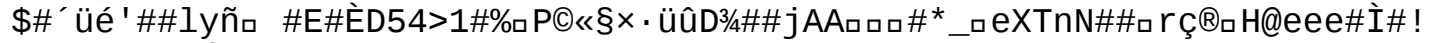

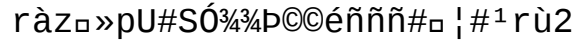

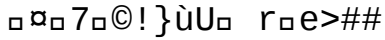

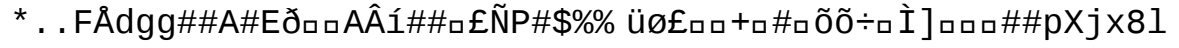




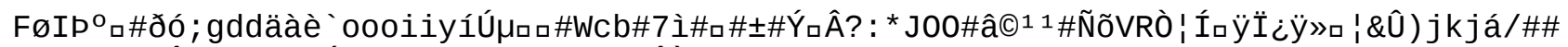

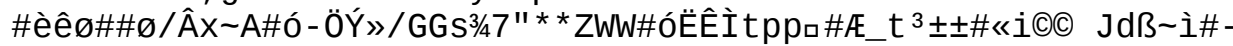

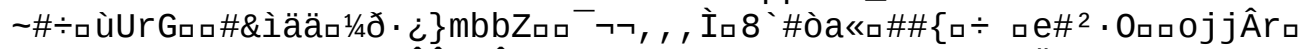

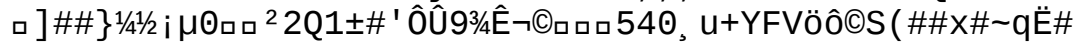

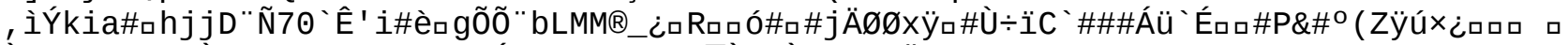

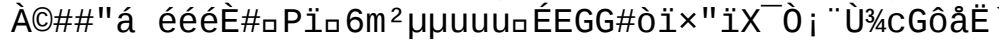




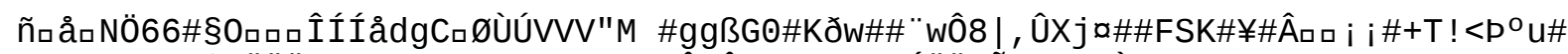

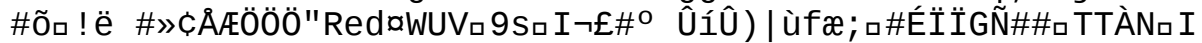

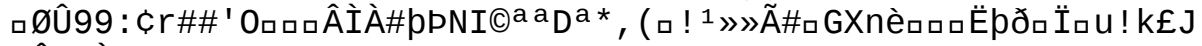

\#ÂB ¿Ùं $1 / 4 Y$ a

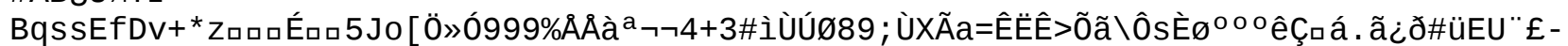

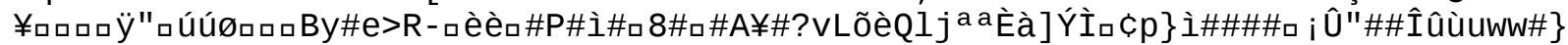

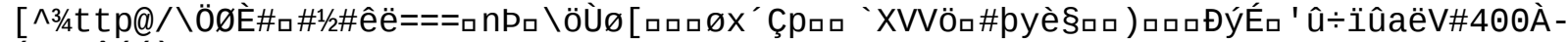
ÁAঃ\#Âóóòtt' $555 \# \# \tilde{n}_{-}(\# \mathrm{~L} \# 3 / 4$ ?

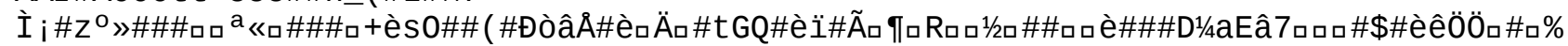

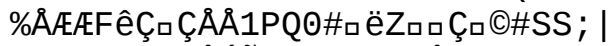

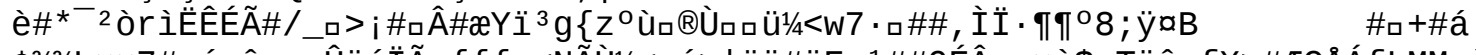

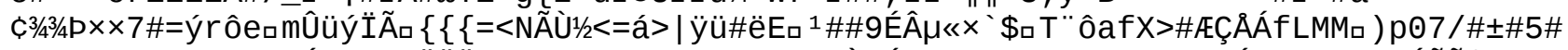

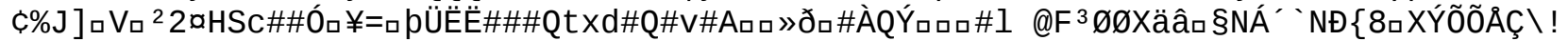

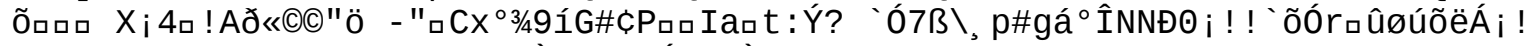

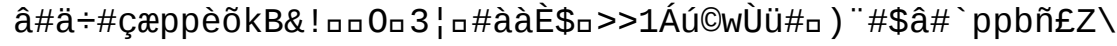

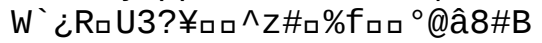

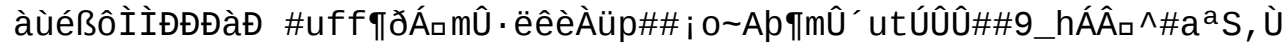




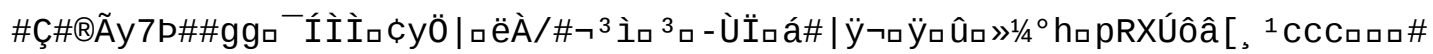




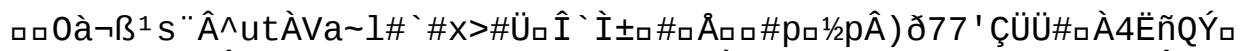

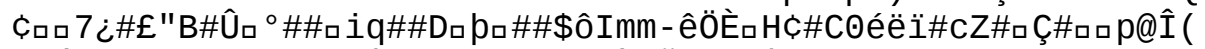

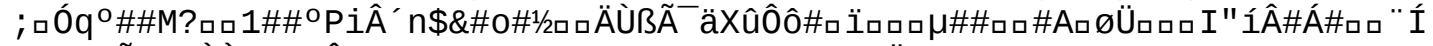

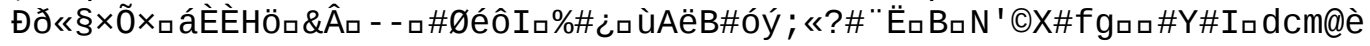




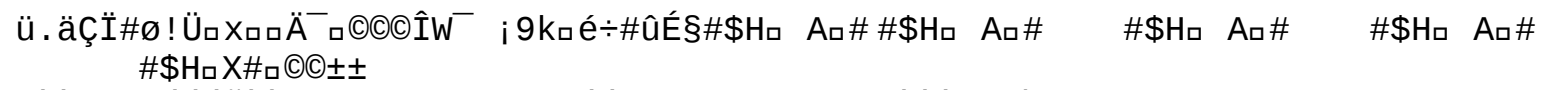

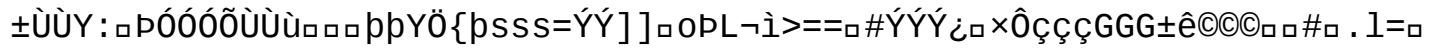


,,,$\square 0 \bigotimes 7$ 
B. , 


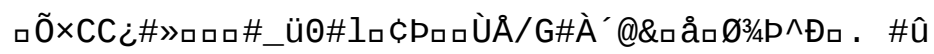




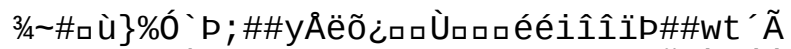

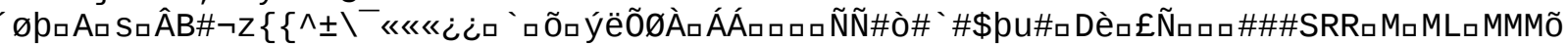




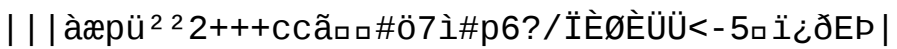

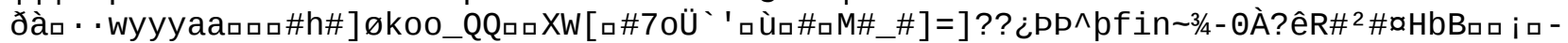

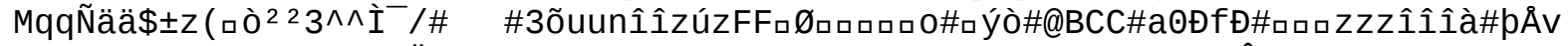

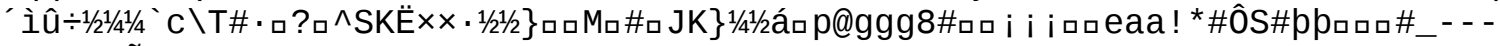

$3^{3} 31 » \#$ ÕMı \#§OkikGDD 


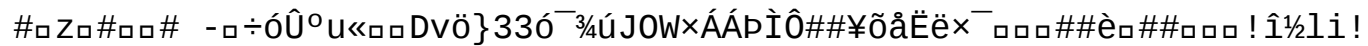

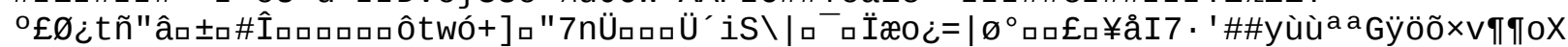

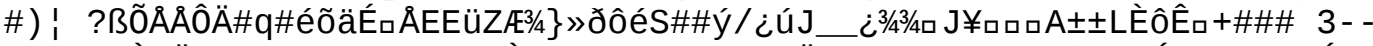

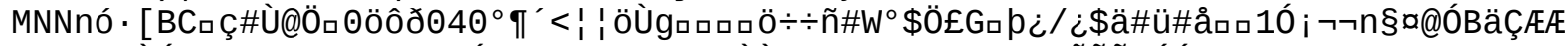

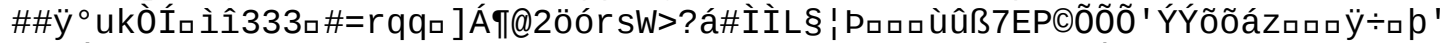

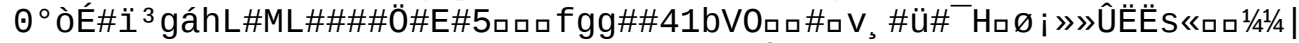

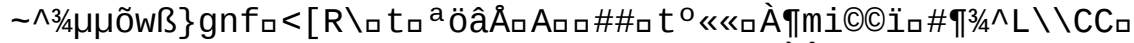

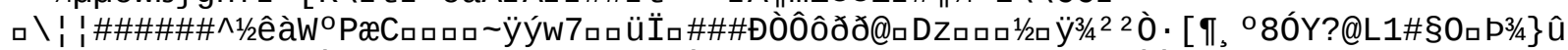

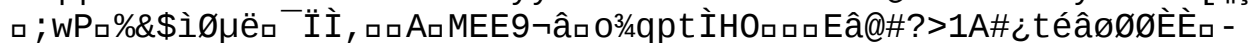

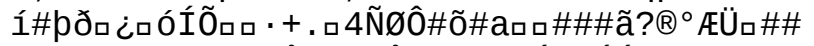

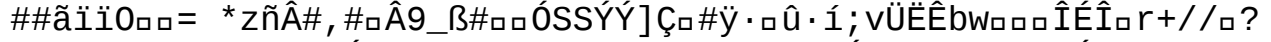

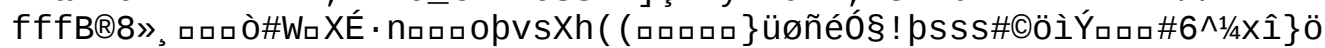




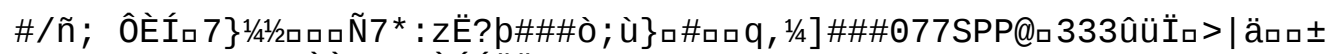

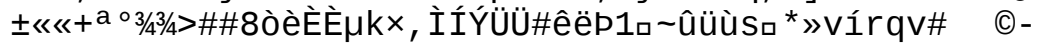

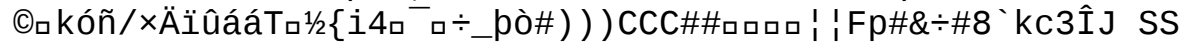




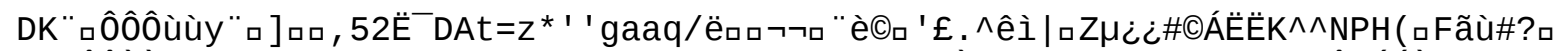

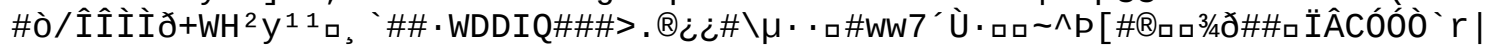

É\#ロ+66VPH\#å\#Ë` $D_{\square}=K \# \#$ PII

$\hat{\mathrm{E}} \%$ 


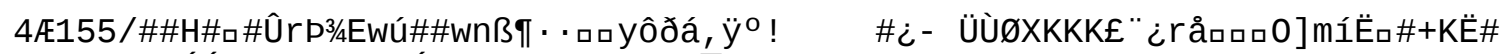

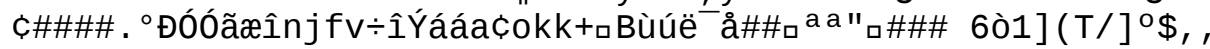




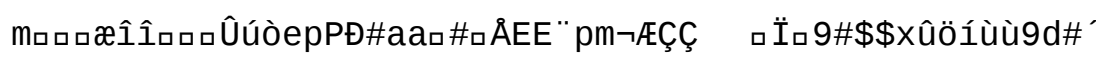




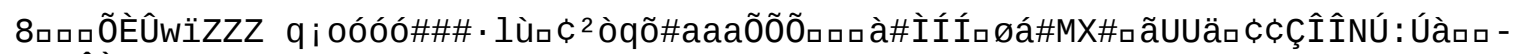
jáí̂̂Òロ\#ロüa\#ロĐ«P\#\#?

PTT tt t ${ }^{-} \wedge 1 / 2 U$ Úø

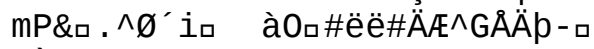

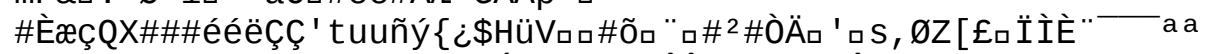

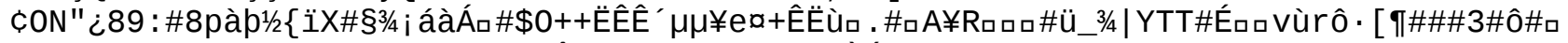

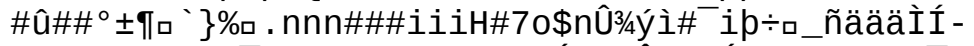

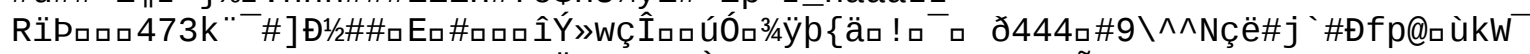

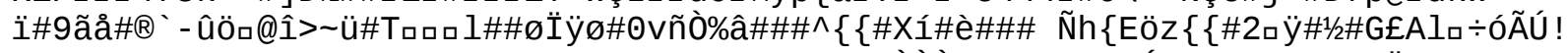

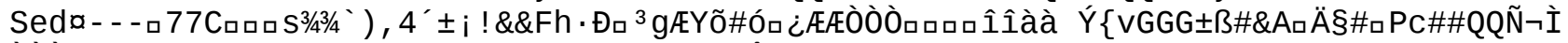

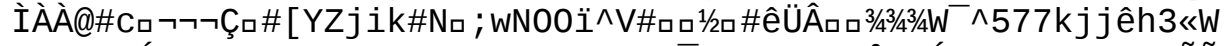

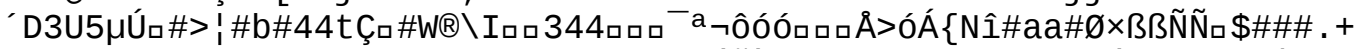

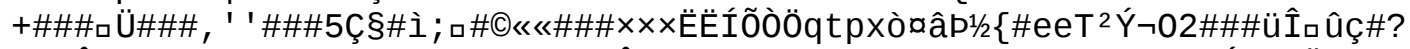

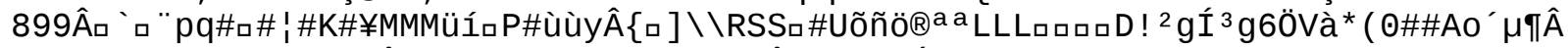

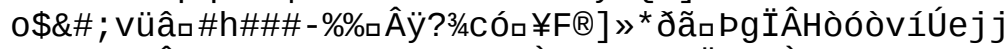

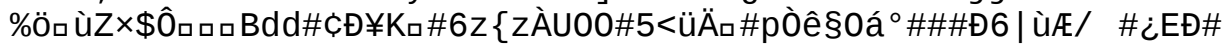




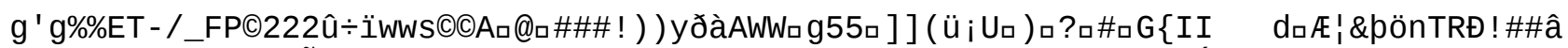

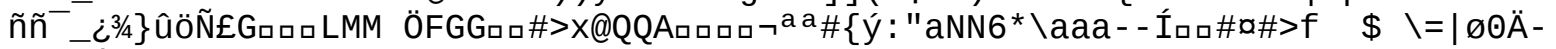

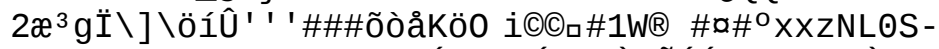

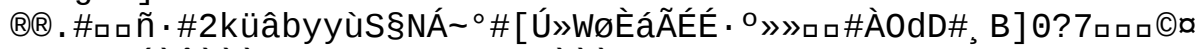

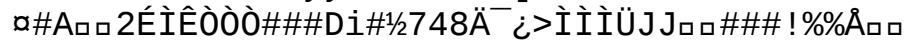

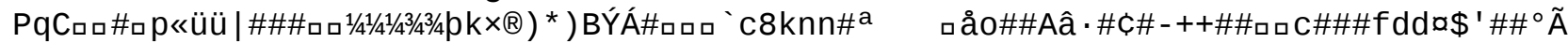

ÒnzZZJJ

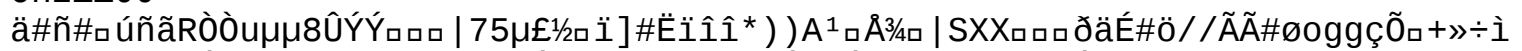

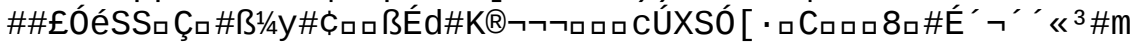

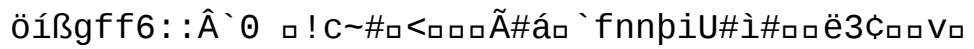


口ם\#3 3 3 $7 n \$$

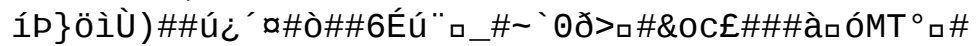

L6èn ${ }^{\circ}$ ฉðpA! i +

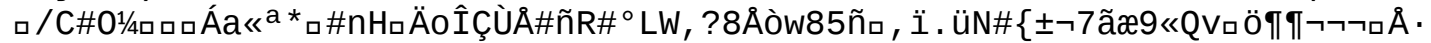

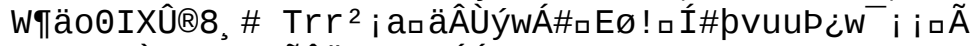

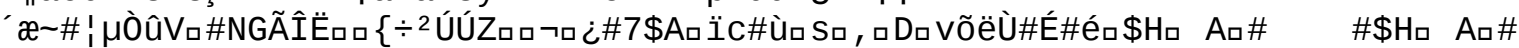

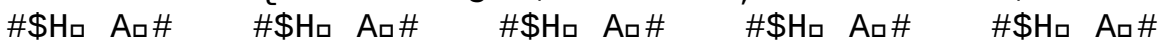

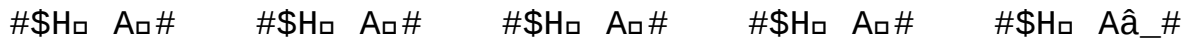

$\# \$ H_{\square} \quad A_{\square} \#$ 


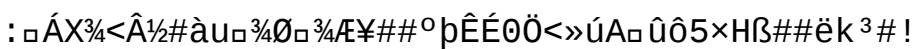




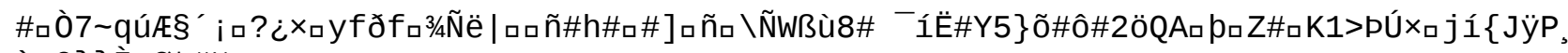
àr $\Theta\}\} I_{\square} \emptyset \mathrm{t} \# 1 / 4 \mathrm{n}$

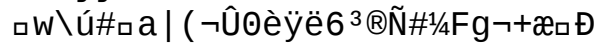




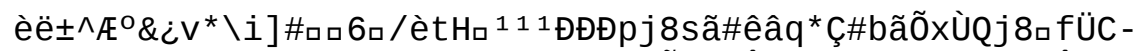

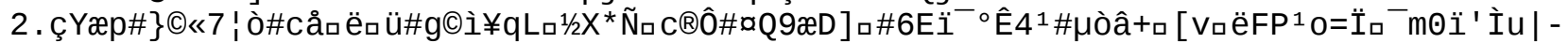

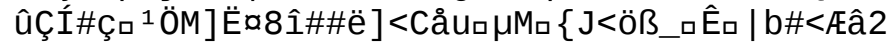




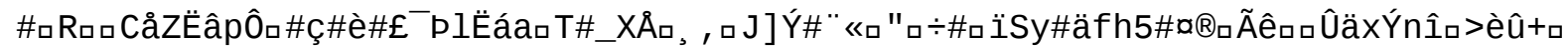

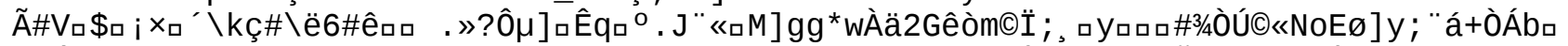

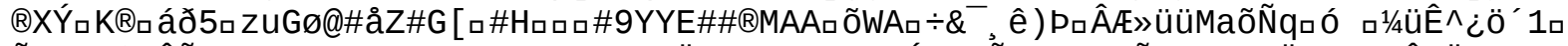

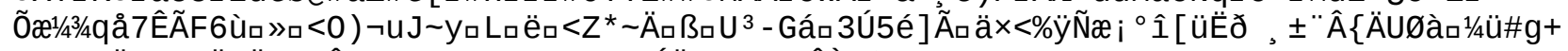

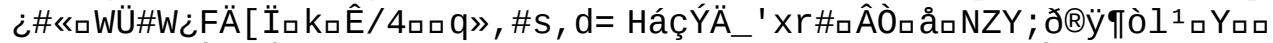

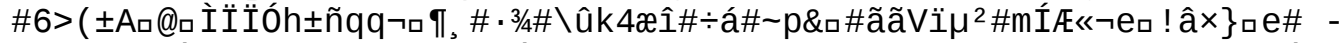

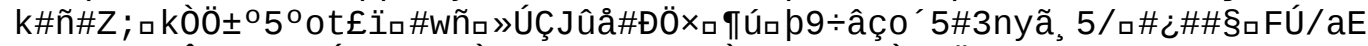

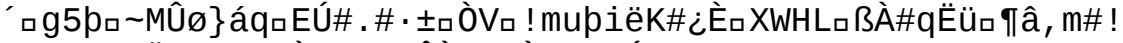

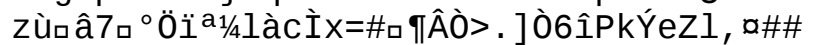

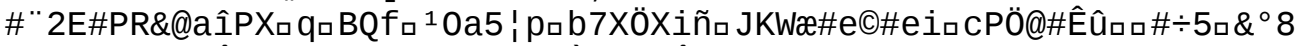

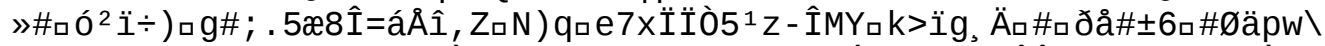

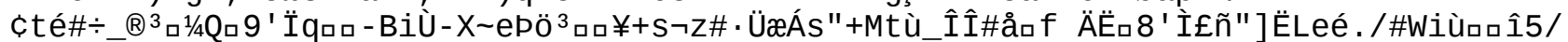

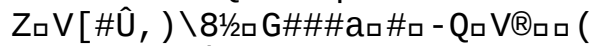

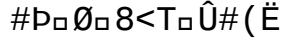




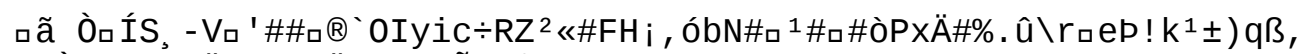

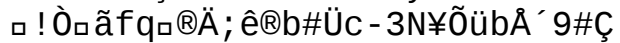

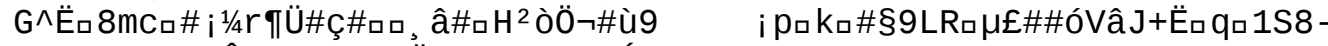

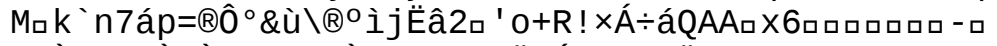

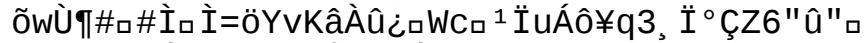

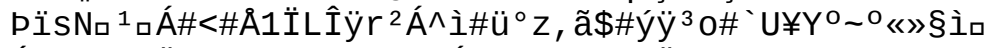

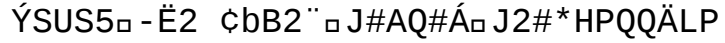




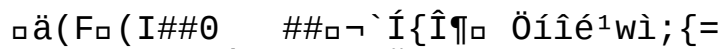

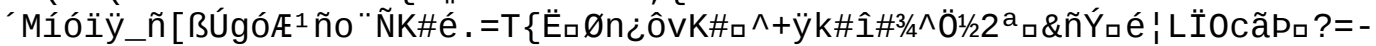

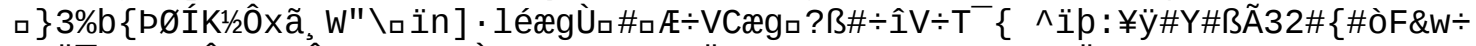

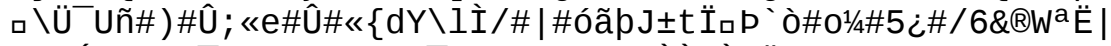

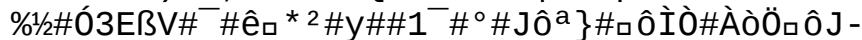

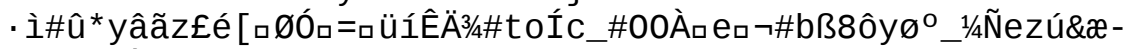

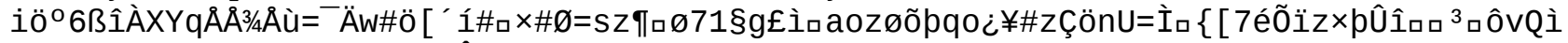

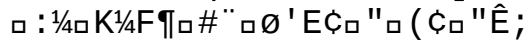

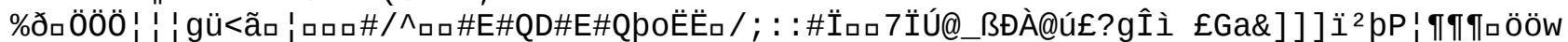

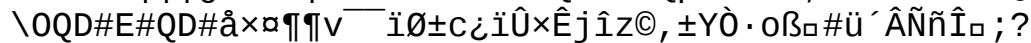

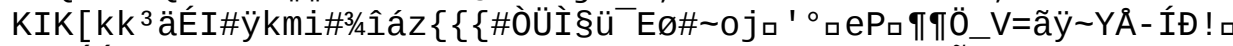

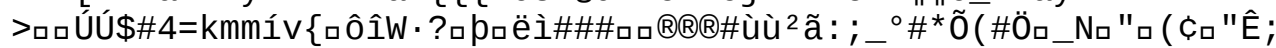

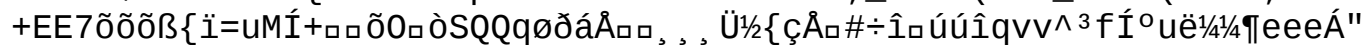

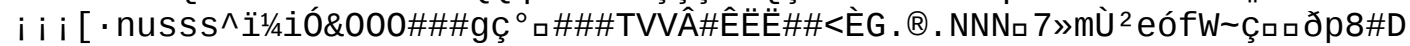

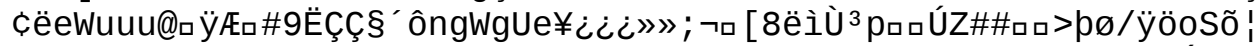

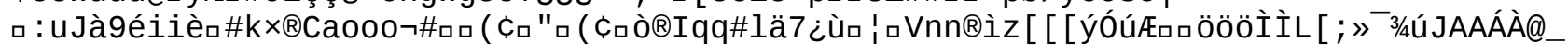
SSóëo3/4Ñ̃óKHH, \}ûTñ4£?ÿå/

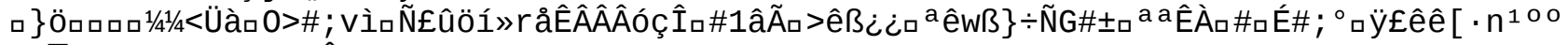

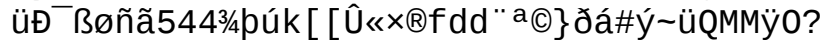

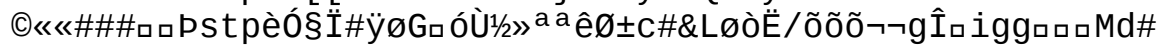

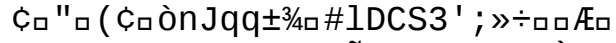

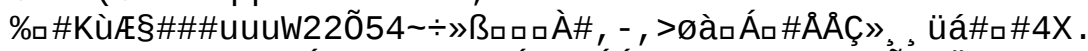

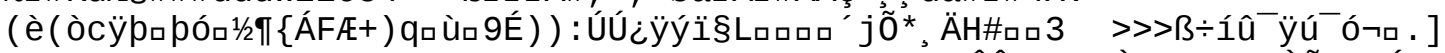

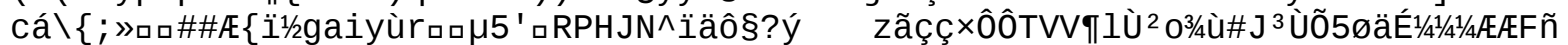

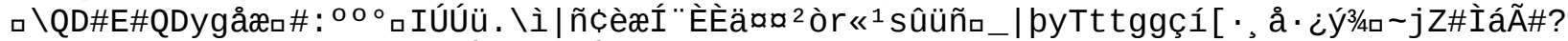

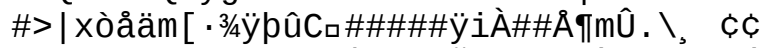

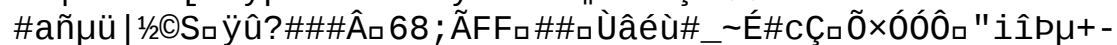

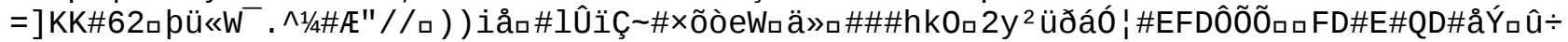

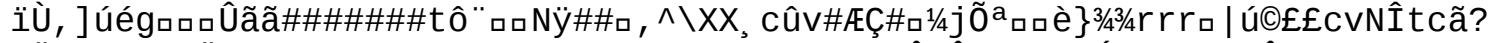

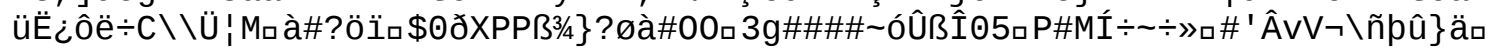




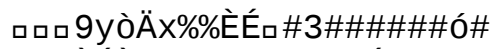

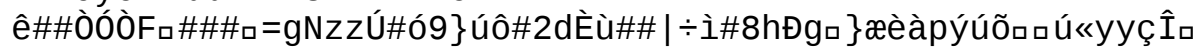

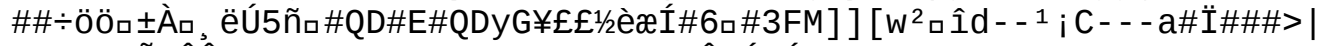

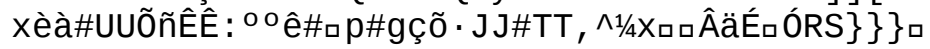




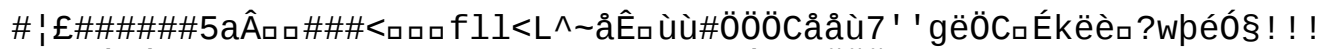

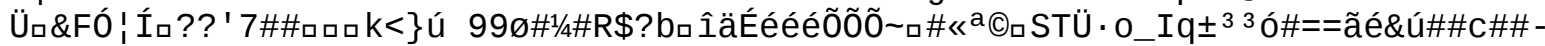

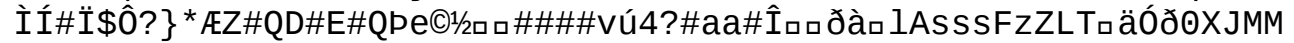

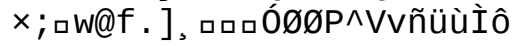




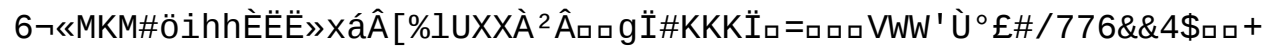

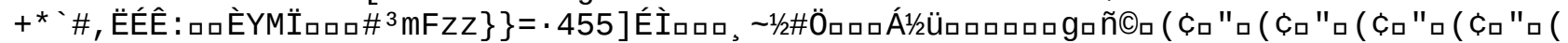

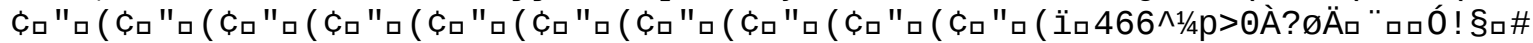
口

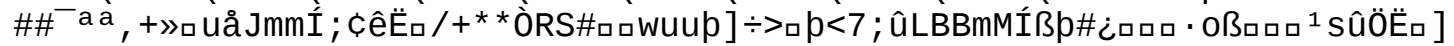

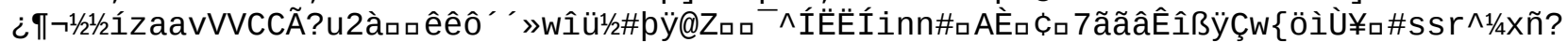

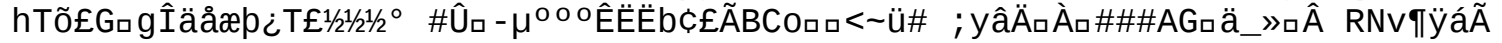

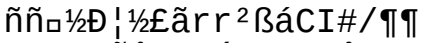

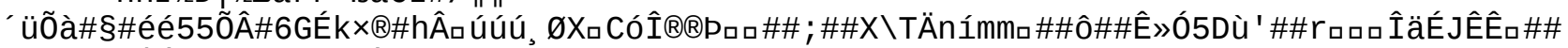

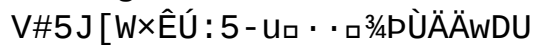

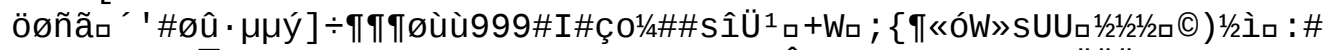

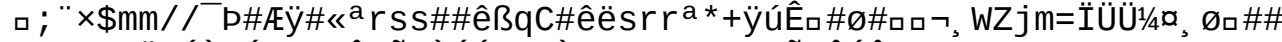

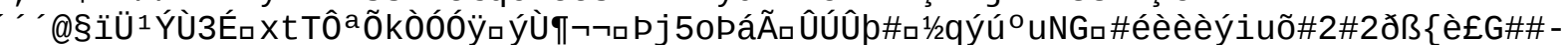

Z4cæİ_KıьÖ\#\#\# 
ฉ §úçCழ@]\#\#\#e\#\#Ó\#3LLLõ 


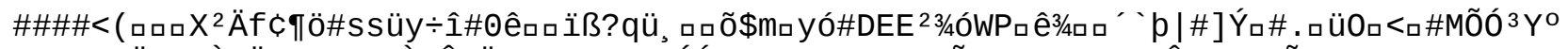

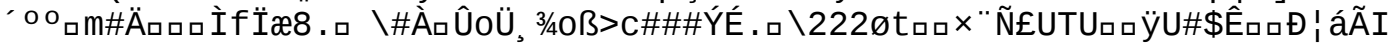

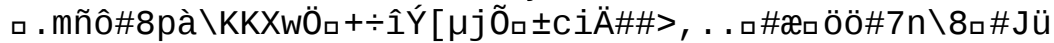

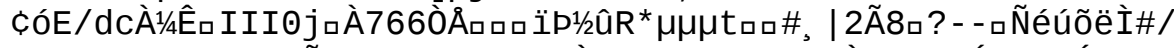

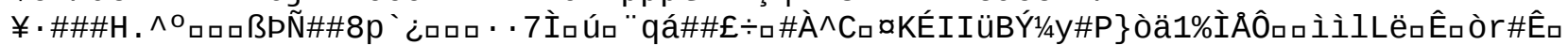




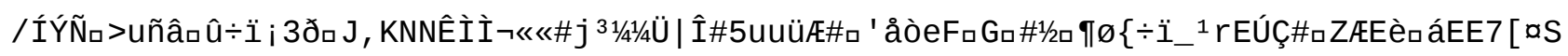




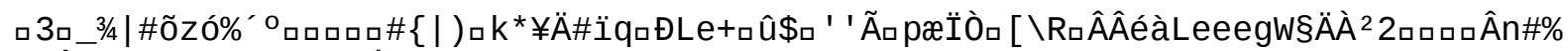
\$äôðáò $\left[\llbracket l a, \# p_{\square}>\right\} * 0_{\square}-$

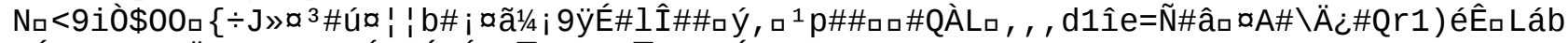

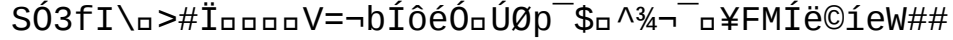




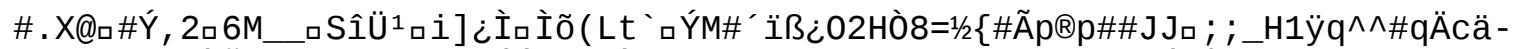

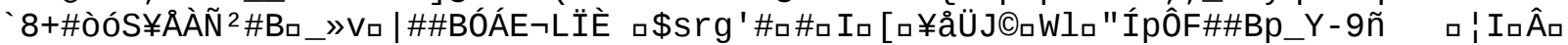

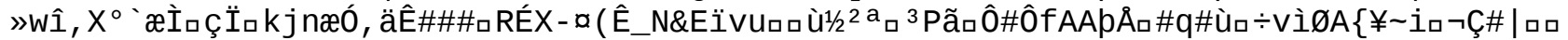

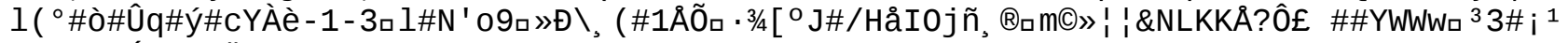

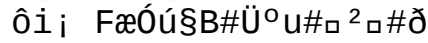


\$a YYÉïhHZò [ ] - *壬

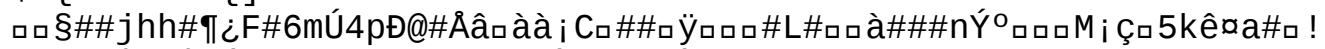

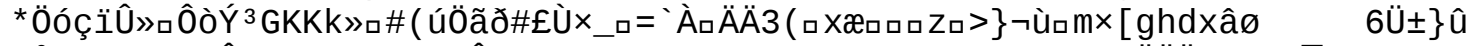

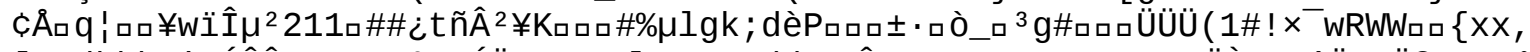

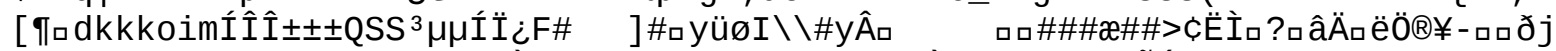

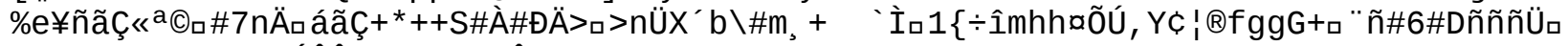

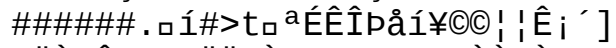

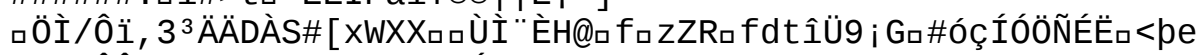

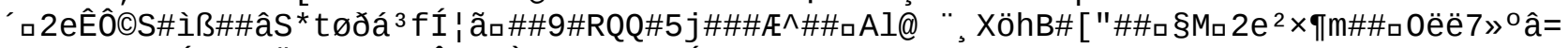

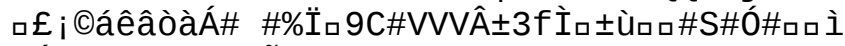

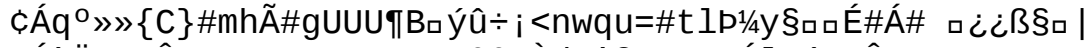

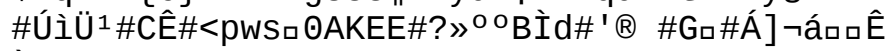

$\hat{A}_{\square} \#_{\square} 0_{\square} 2 \mathrm{u}$

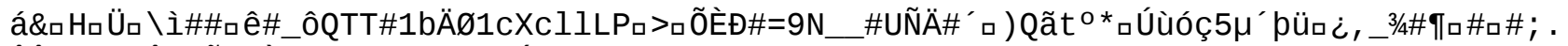

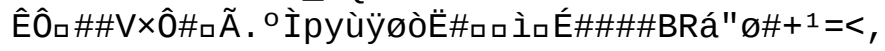




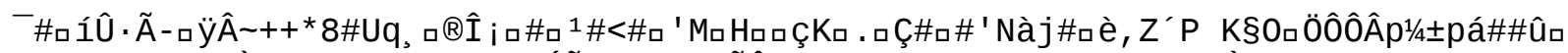

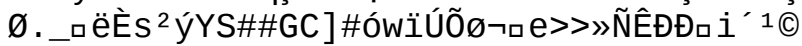
â॰7ĐGıôRVVPPÀ]

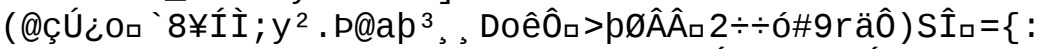

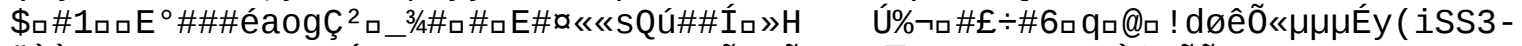

İÀ̀̀ $5 \# 7 n \mid \$ \#$

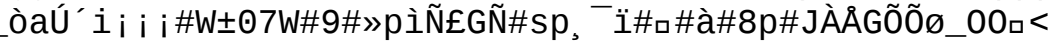




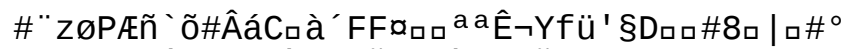

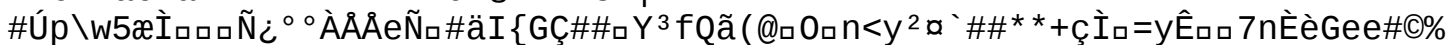

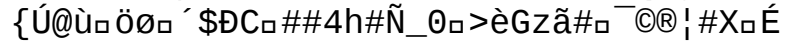


口 MRVロUロ\#\#Hø\#EA\}○khlÝoM`\#]\# 
$y$

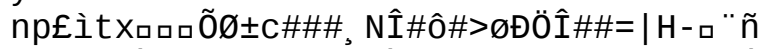

À\#ü3; +«æa ${ }^{-1}{ }^{-1}$ áÀ ${ }^{3}$ İ̈66quqqüP\} « ${ }^{1}$ sÉ^t£.

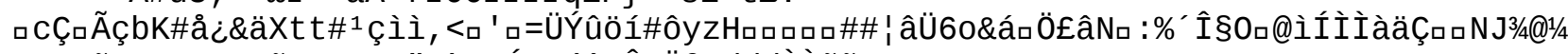

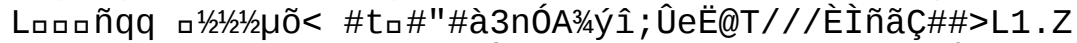

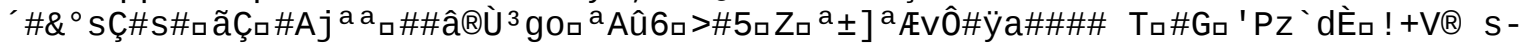

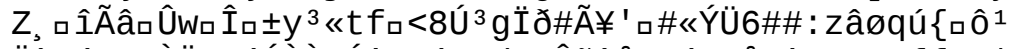

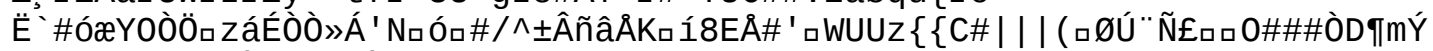

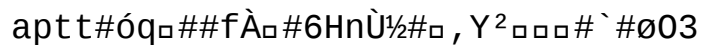

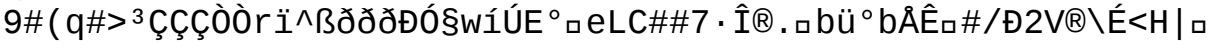


Å\#+cãâøT^^^ロ\&ëë»\#Ö\#0\#Æj 


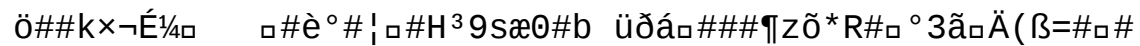

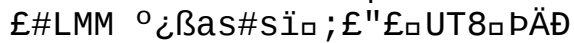

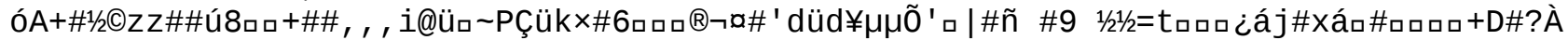

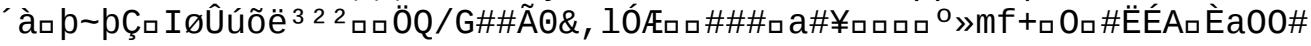





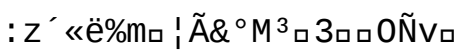




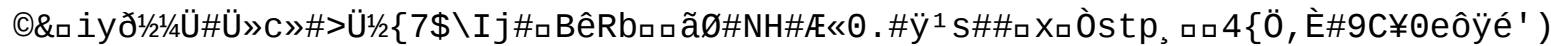

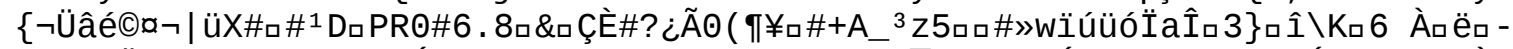

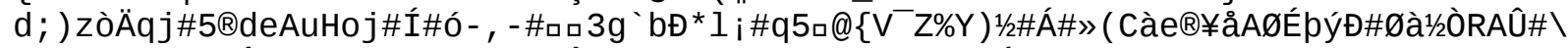

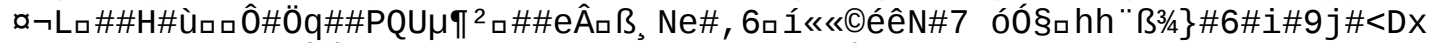

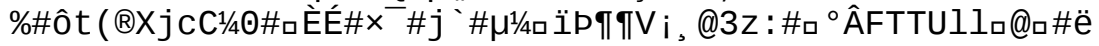




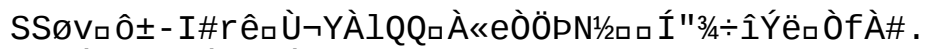

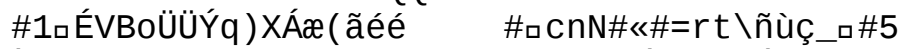

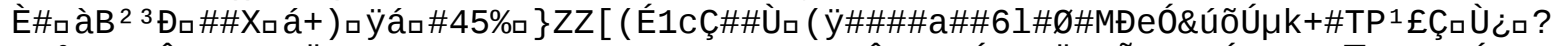

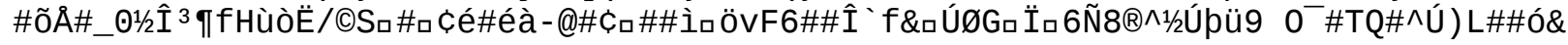

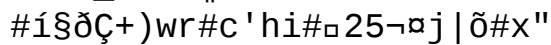




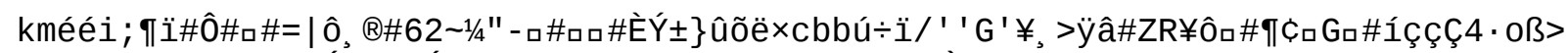

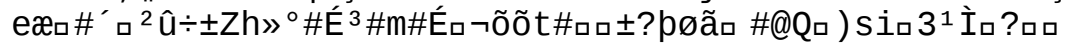

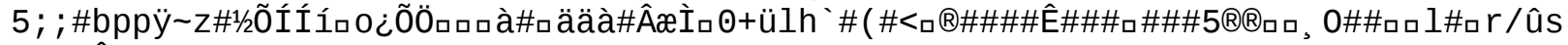

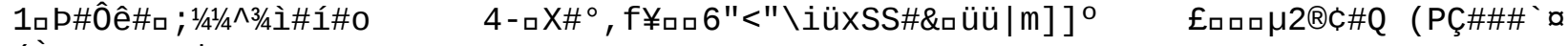
(À\#ロ\#\#Oロ1/4|

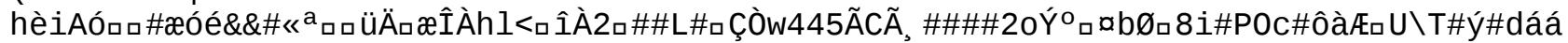

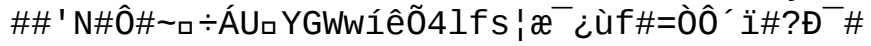




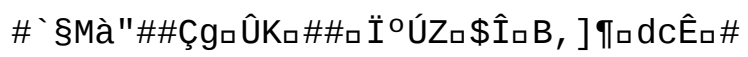


口\#İ̀̀\#u\#

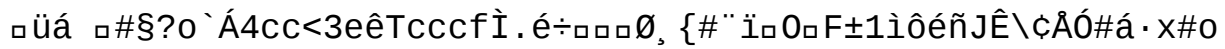




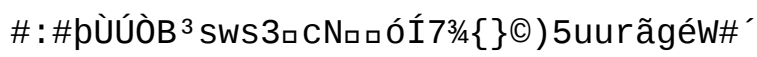




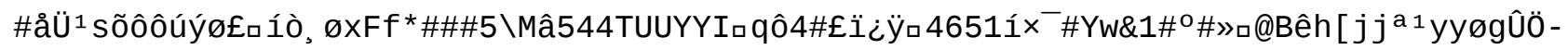
Z\#\&

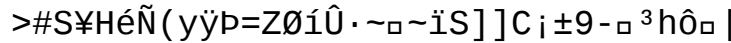

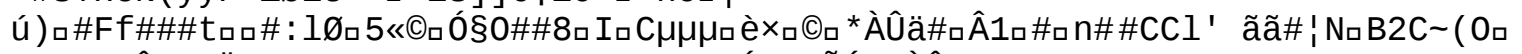

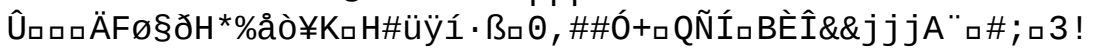




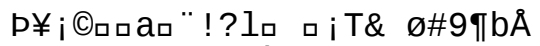

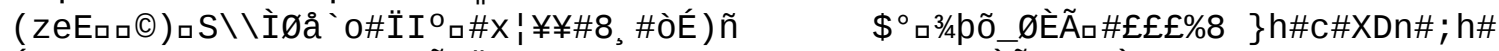

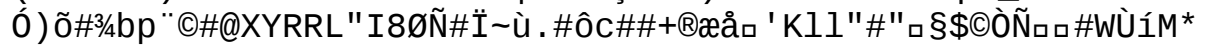

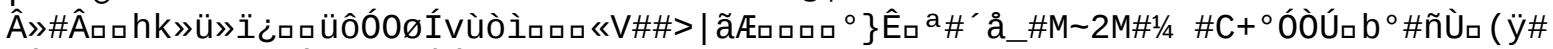

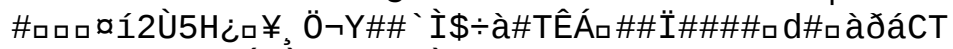

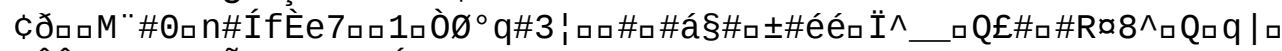

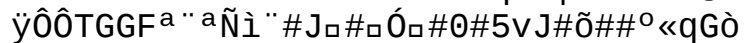

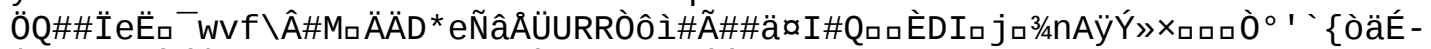

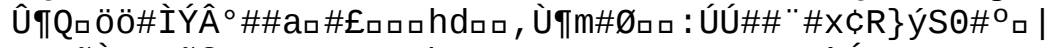

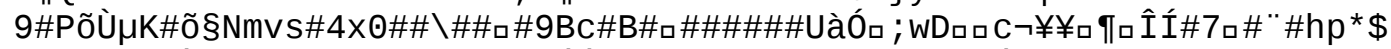

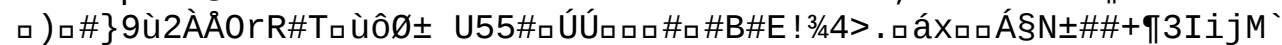

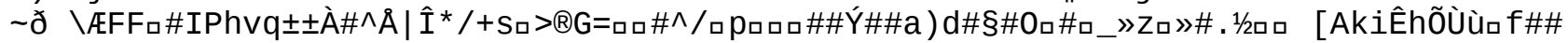

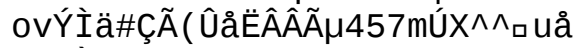

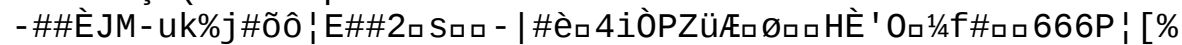

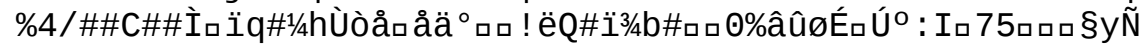

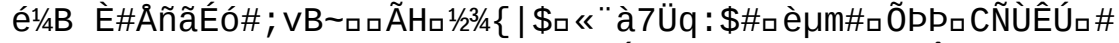

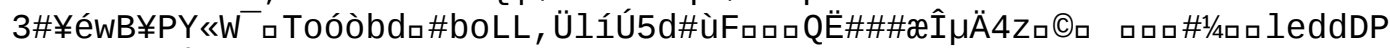

\#\#êêja\#ロÛôôt

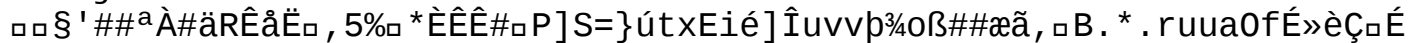

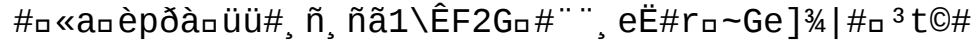




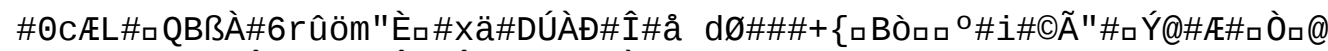

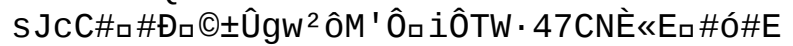

$\square^{\mathrm{a}} 19, \backslash^{\prime} \#$ 


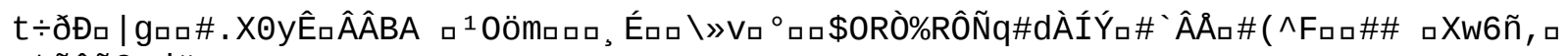
¿tñâãÇud\#u

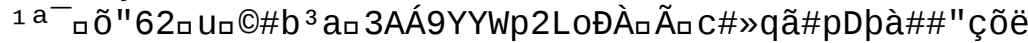

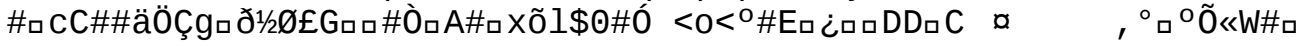




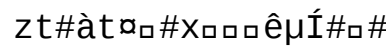

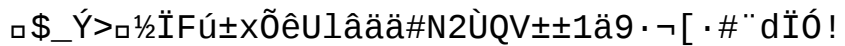

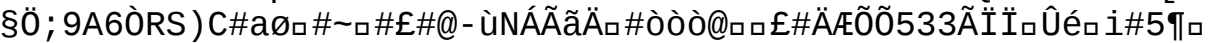

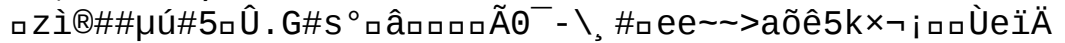

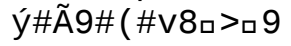




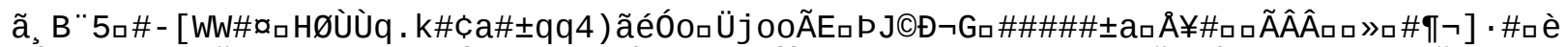

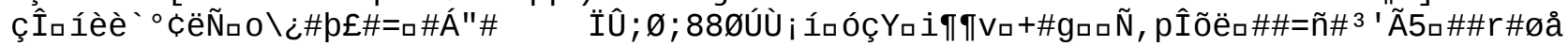
l§ãkhiA\{@ðgé\{ $119^{\circ}$, è\# 


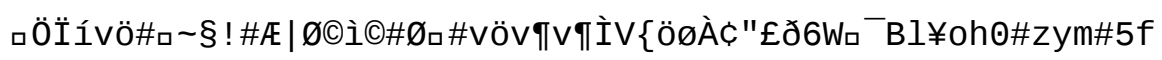




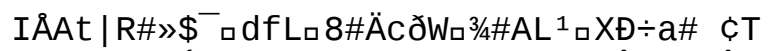

$\{$ ëyö?öïÏ\#

æïÝY»WZâ\#Ô< üøjI 


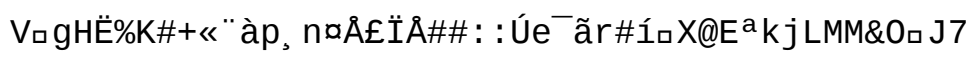




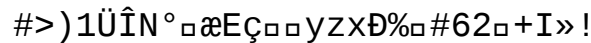

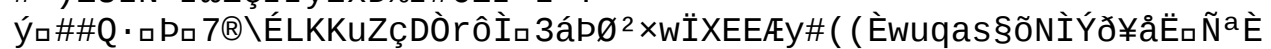
$\mathrm{a}+\mathrm{U}$ 


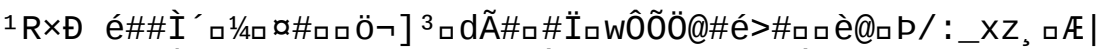

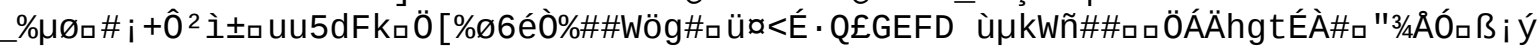


>, 3ééD\#WQ \, व i ÿèácZ@\#5YW7=\#\#Ý(\%\#[nkKQ\#\# 


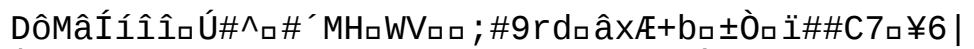

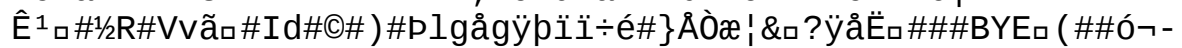

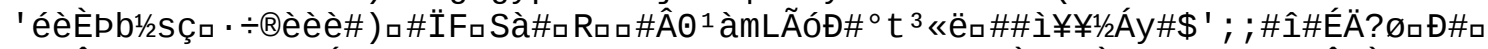

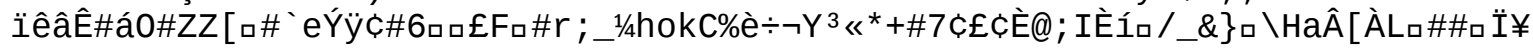
\%l»0ї3/4Gロ\#\#Vøロ

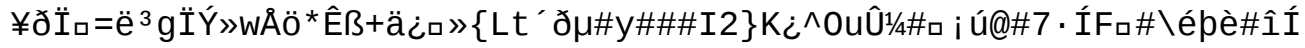




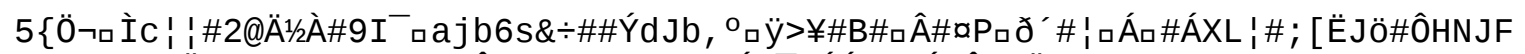

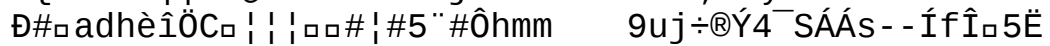




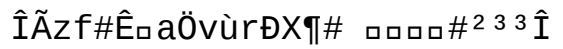

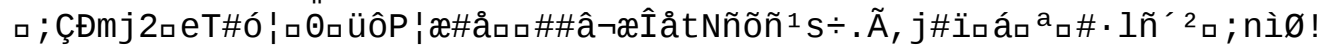

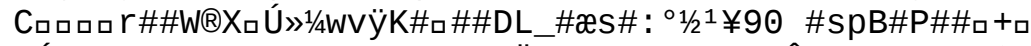

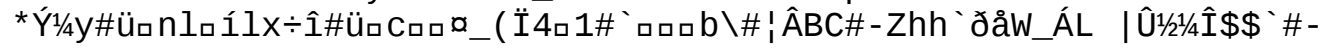




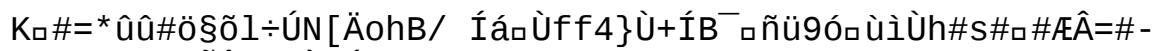

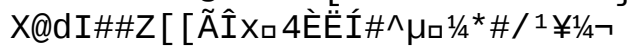




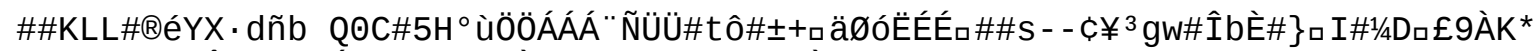

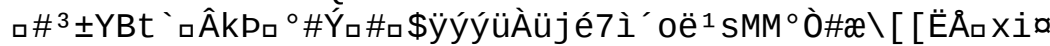

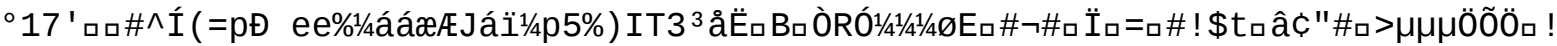

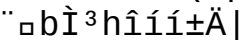

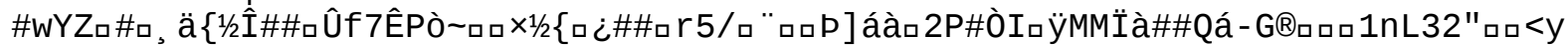




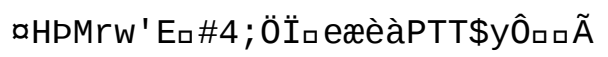


Âqঃòc 


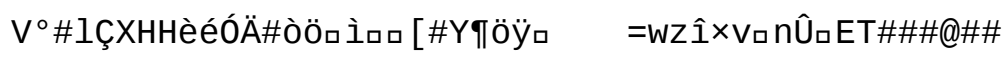




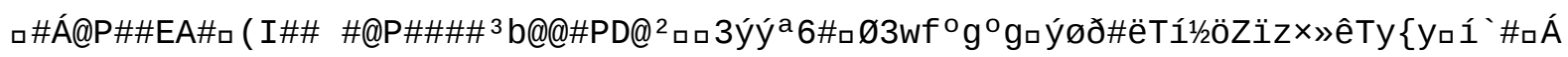




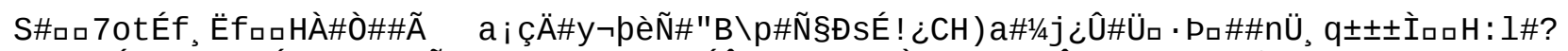

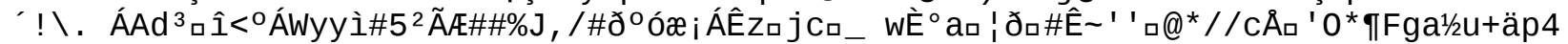

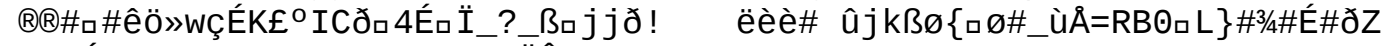

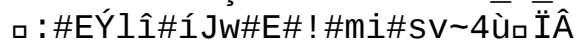




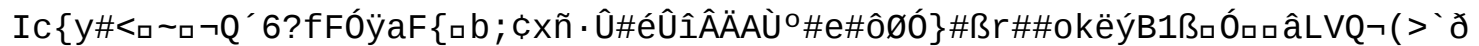

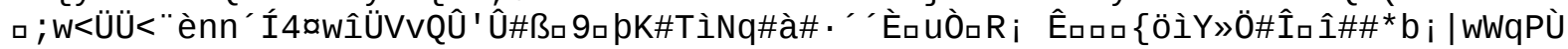


$\mathrm{a} \# £ \times_{\square}$

$\left.\# \#^{1} \mu ¥ ¥ a \tilde{a} w B \# W\right)$ 1ิå

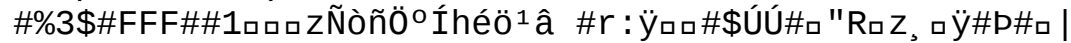

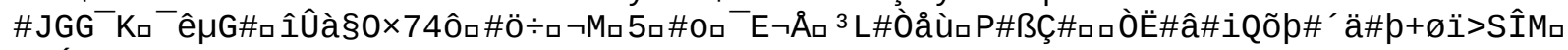

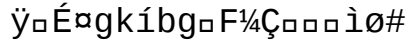

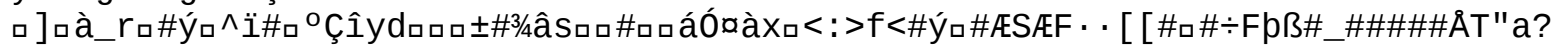
Íbrôz\#ロy\#KKa "Dabỗ̃x\#\#\#mÛfÜjõ

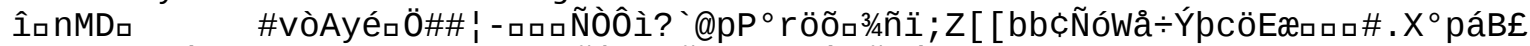

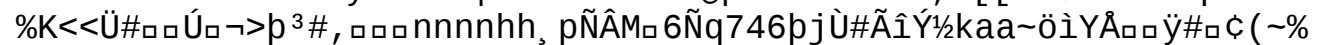

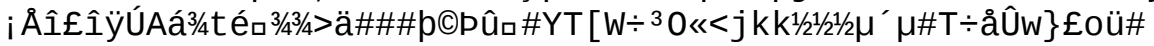




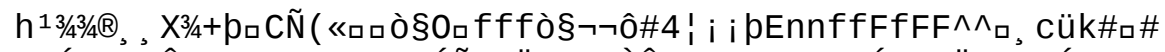

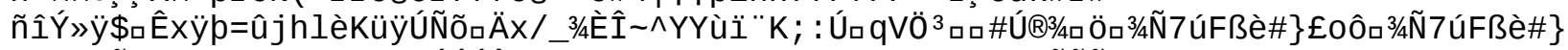

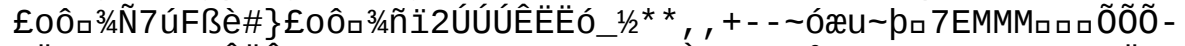

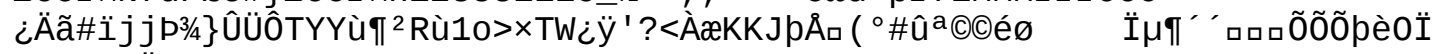

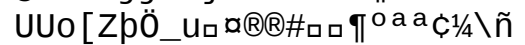

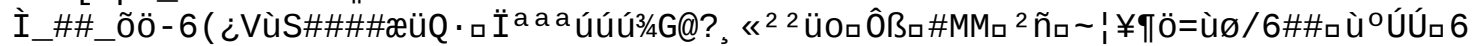

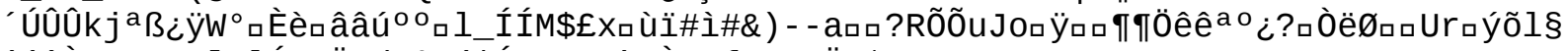

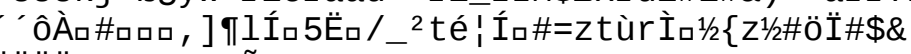

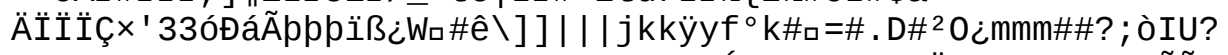

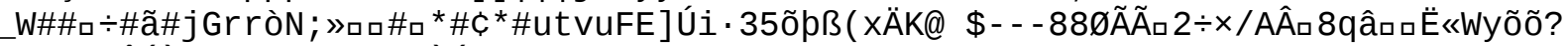

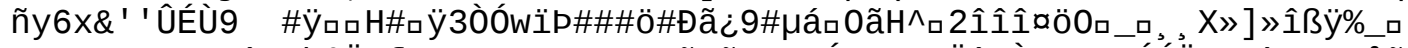

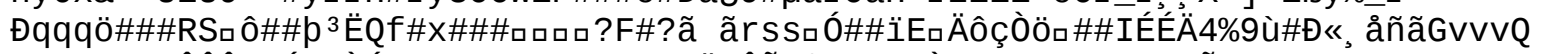

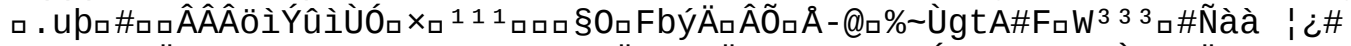

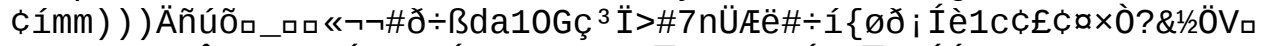

aÀÆ̃̃

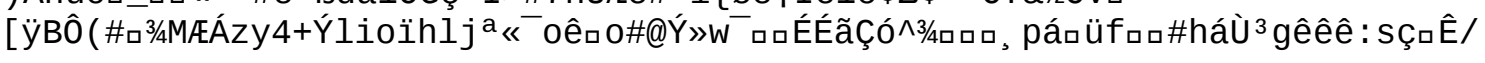

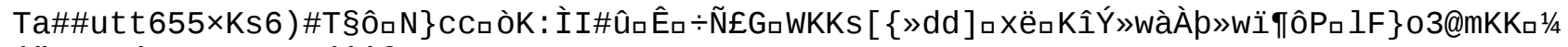

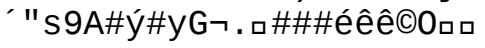




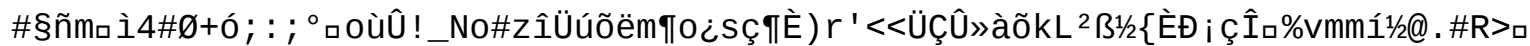

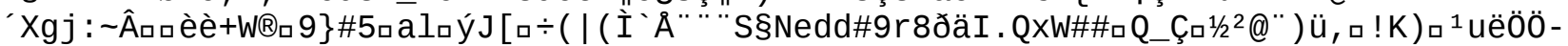

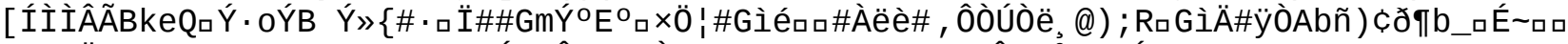

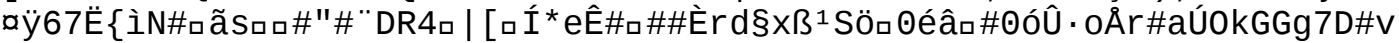

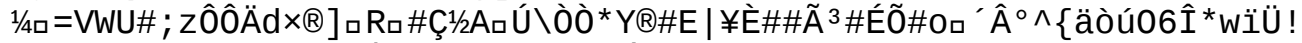

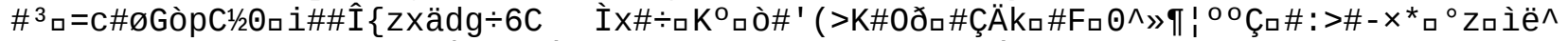

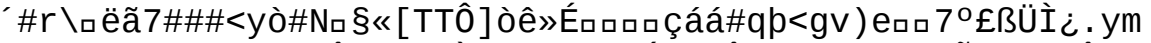

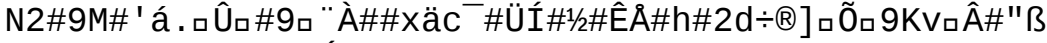

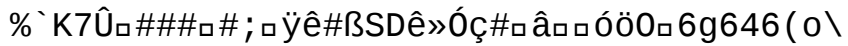




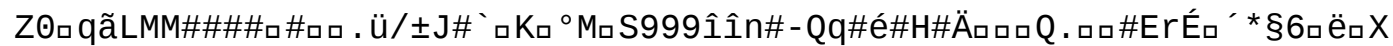

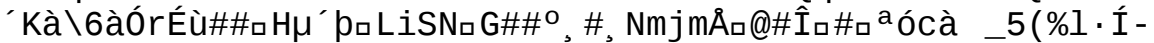

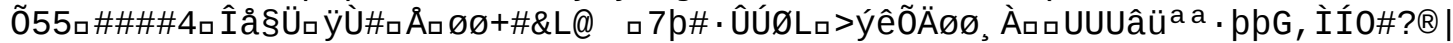

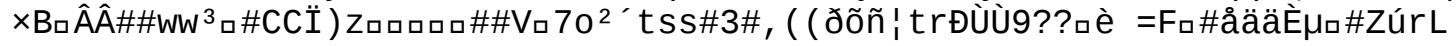




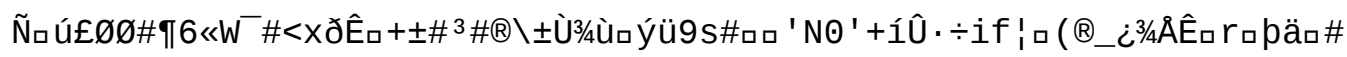




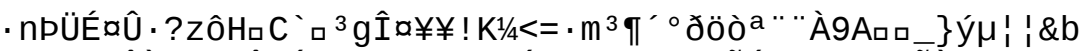

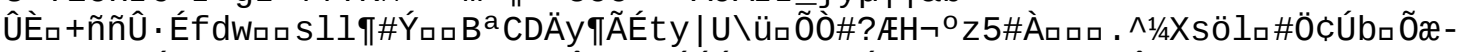

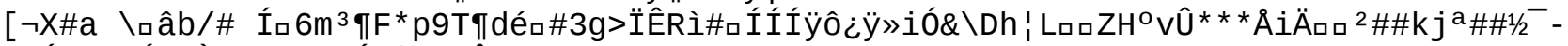

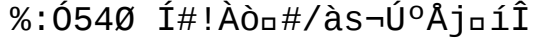

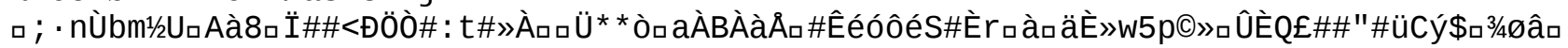

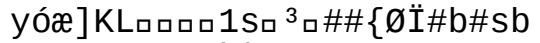

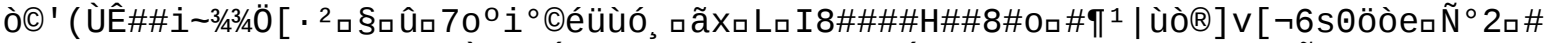

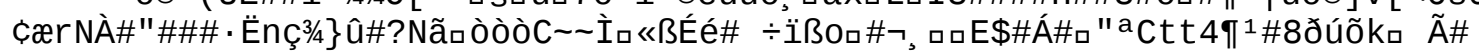




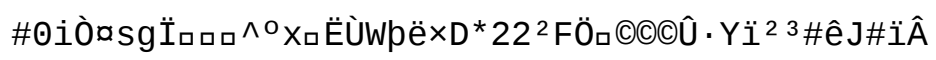




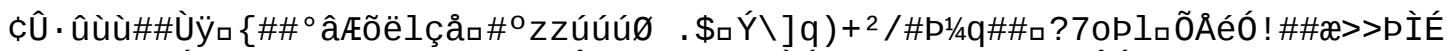

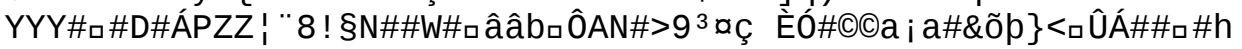


Üòíwß- ] ôöÍ [Â 


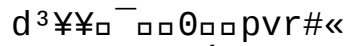

$W^{\wedge} 1 / 2 Z \AA ̊ \cdot W \# \#: d_{\square} \# 9 \# L ; \#: \odot::$

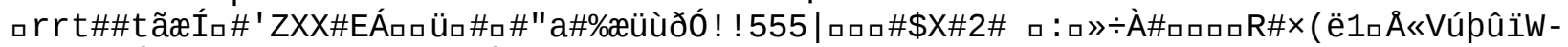

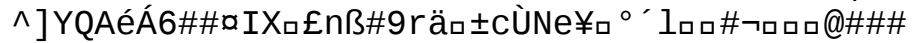


pロロ«\#.zôèáaýûùVFì6»ロ； \#pc\%\#PÈİHÇ\#ロ 


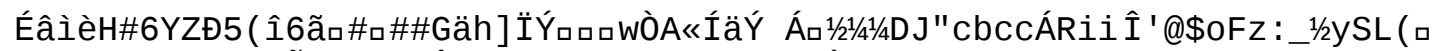

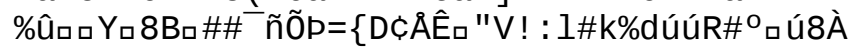




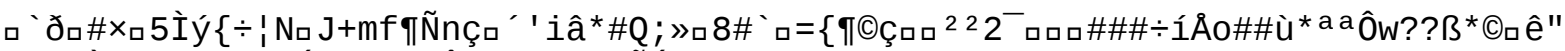

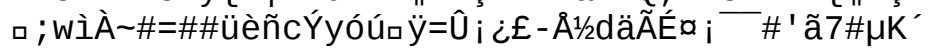




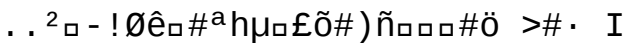

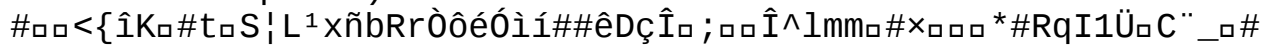

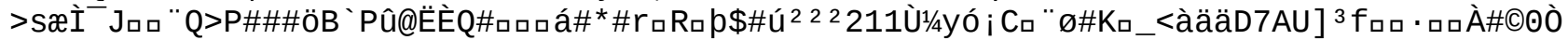

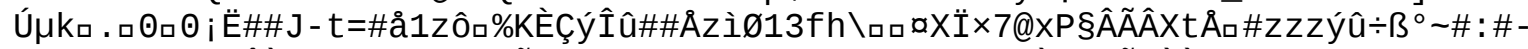

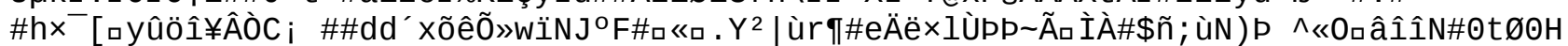

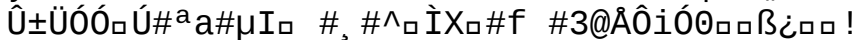

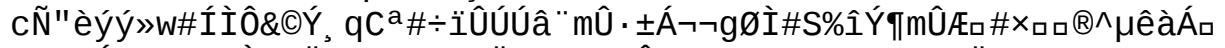

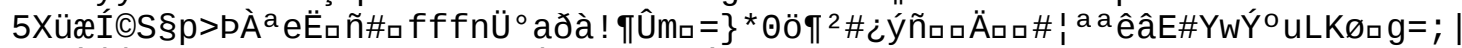

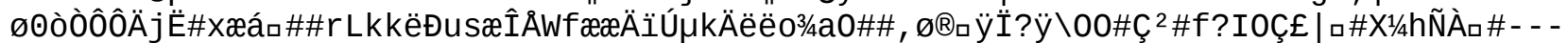

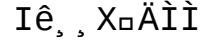




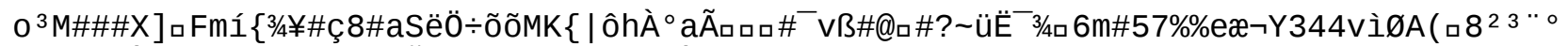

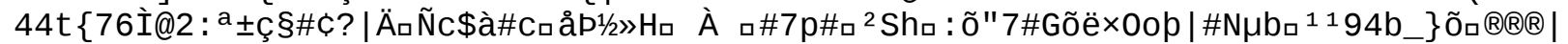

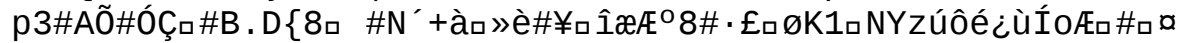




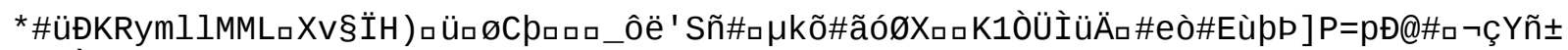
$\pm \mathrm{d} \otimes \mathrm{A} \# \%$ | I00' i\#\# 


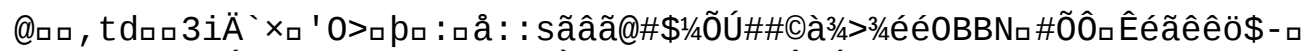

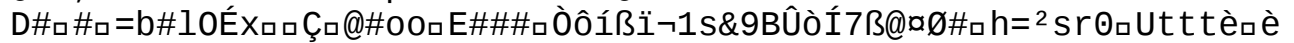
๑ÃZ6ロ\#\#\#áququopüçÉêê'\#

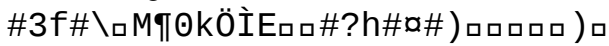


8_iFa\#\#'MC : 'ロÜäÎ̀̀\#+' ' '\#

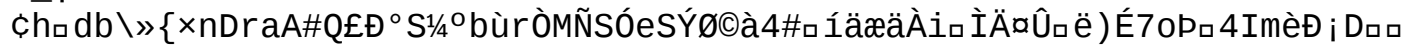

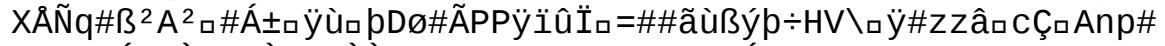

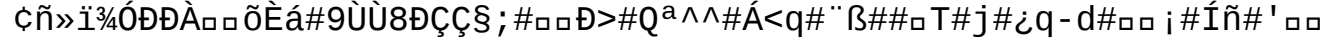

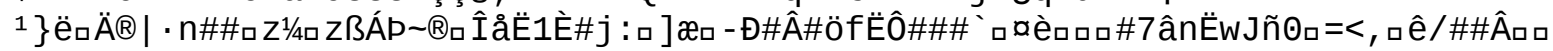


\#

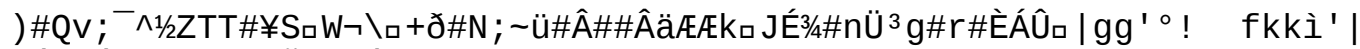

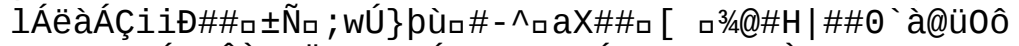

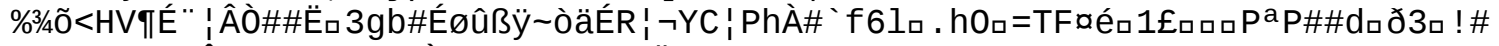

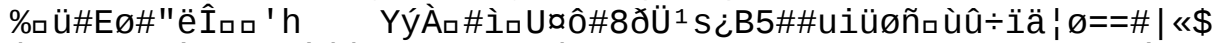

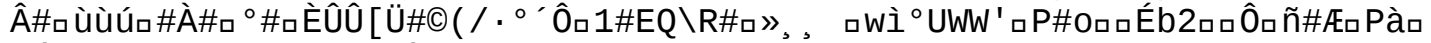

NÉ\#\#âââ\#Ea\#4ฉıZ_ß\#İ 'çhSıQ

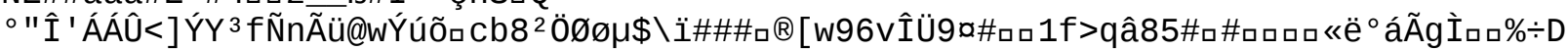




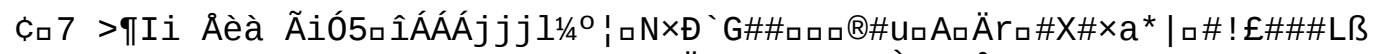

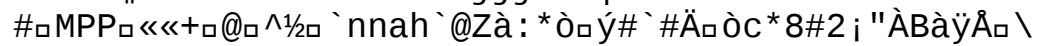

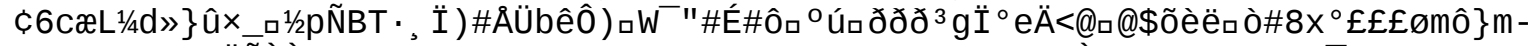

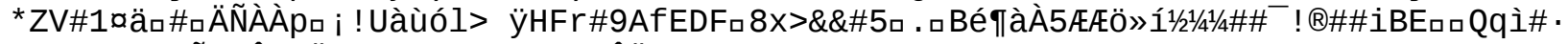

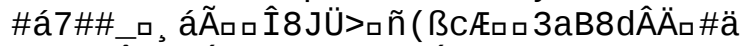

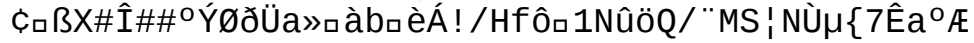


ØロÕ士ロ\#ロ\#\#ÀO@ロ

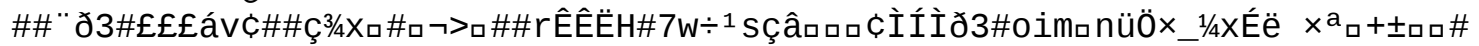

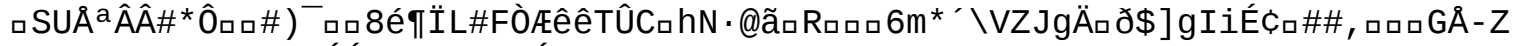

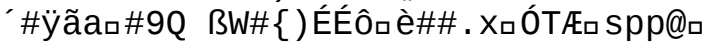

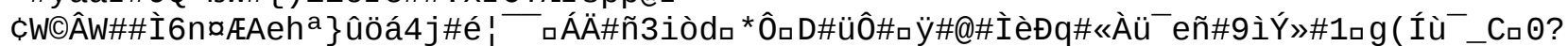

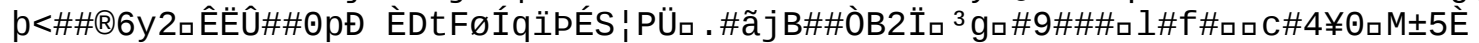

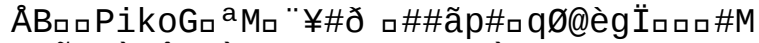

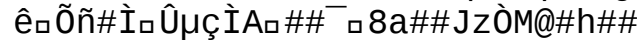




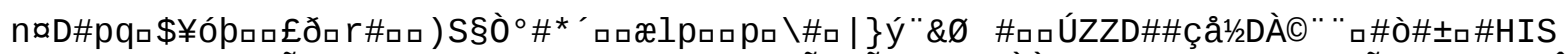

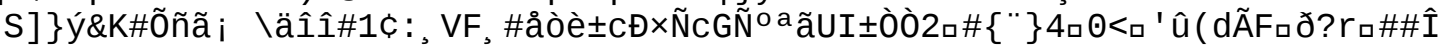

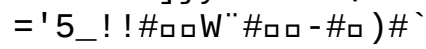




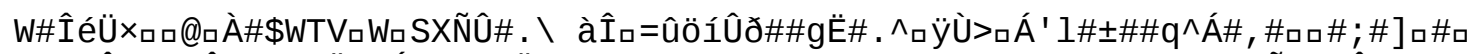

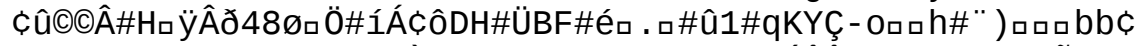
«ÕæMı $\hat{E}$

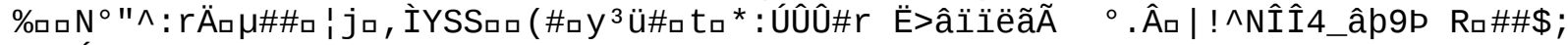

\% ôÁWì=: *

.

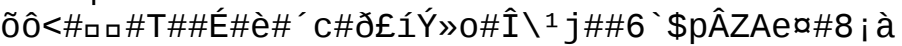

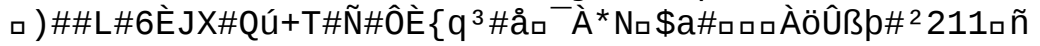





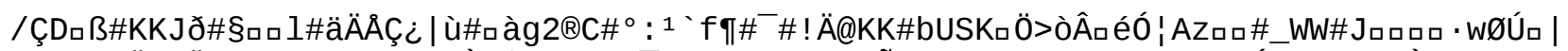

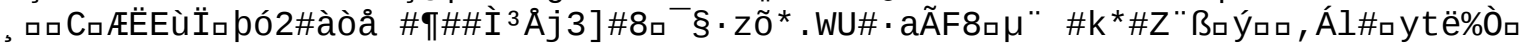

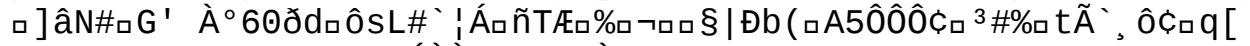

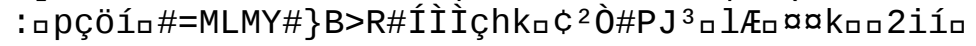




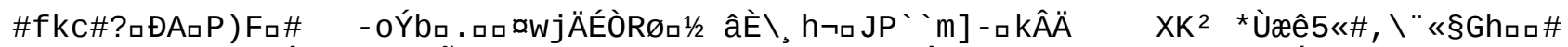

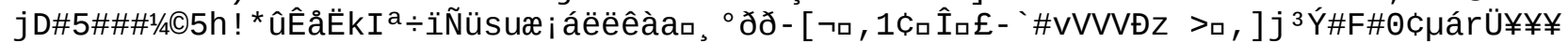

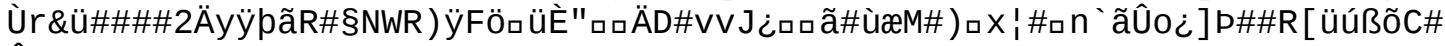
'

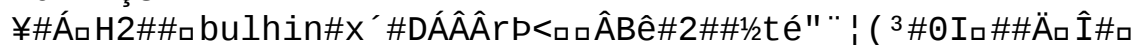

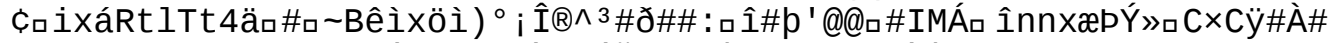

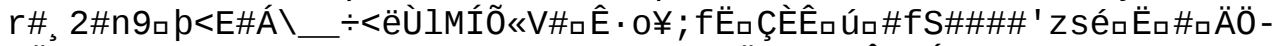

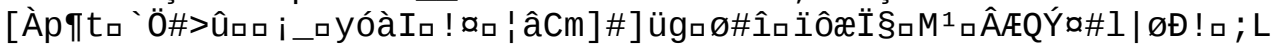

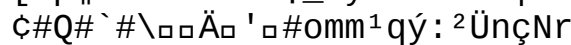

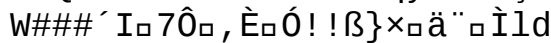

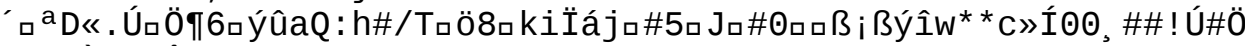

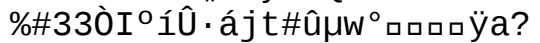

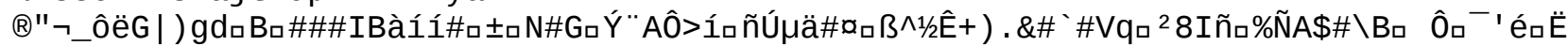

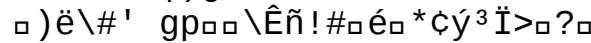

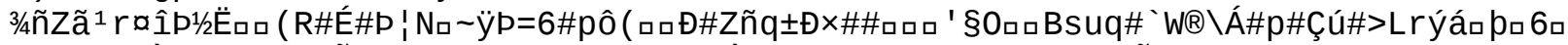

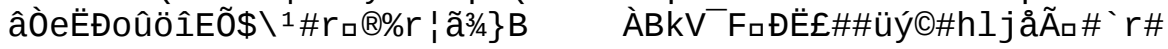




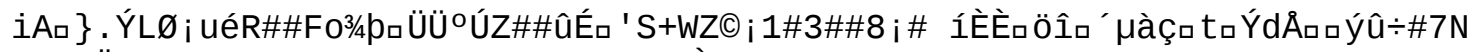

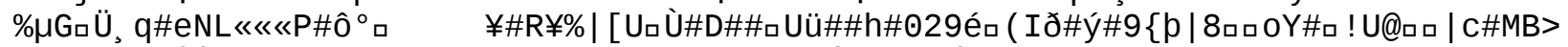

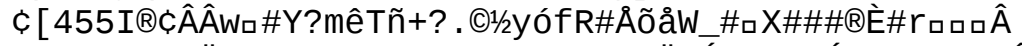

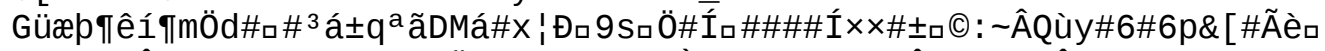

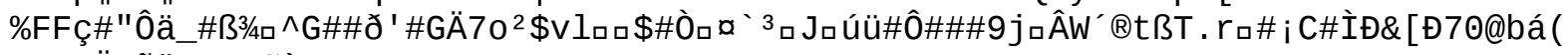
$\# \# P^{1 / 4 E ̈ P o ̃ o ̈-\# \square Đ \emptyset ~ J J ~}$

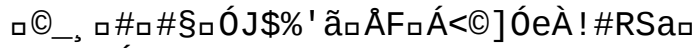

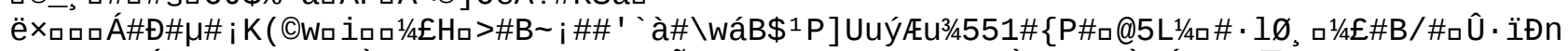

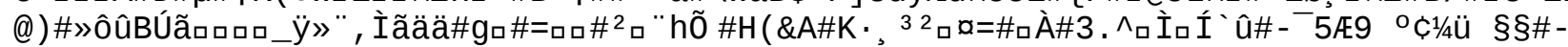

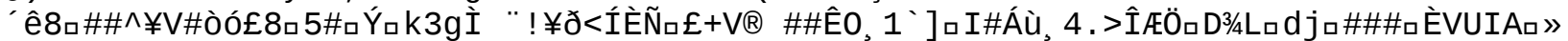

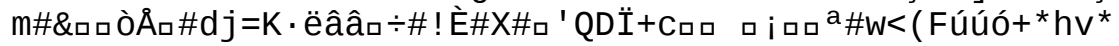




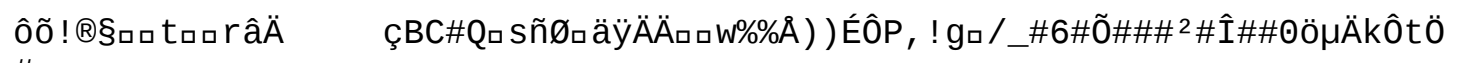

\#

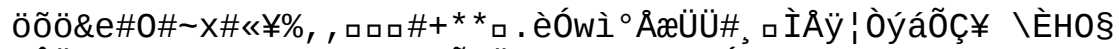

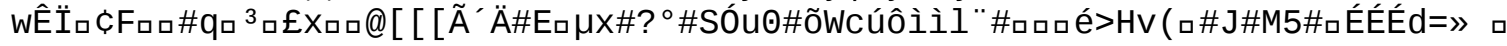




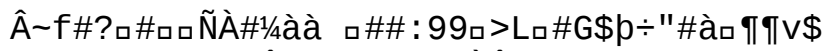

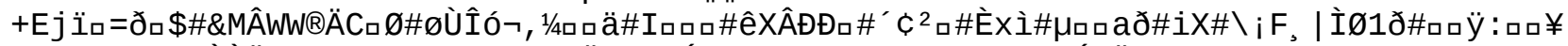

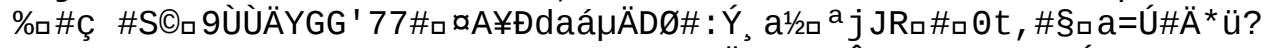

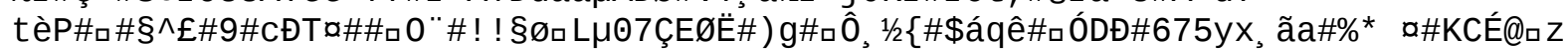

İKfÄÄ`\#ロ 
Ùı| õõı̇̀@gfg?ロ \}ûò̈E\#\#\#9r, 1/1/1/4 


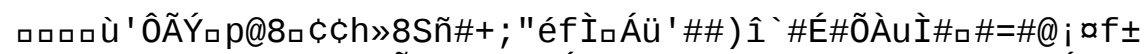

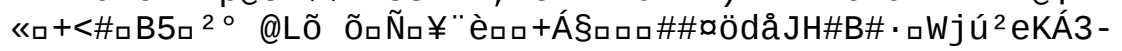




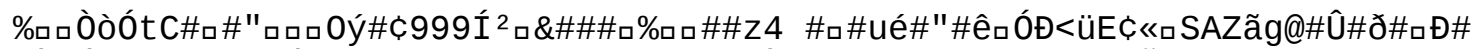

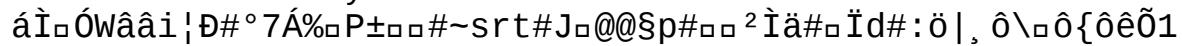

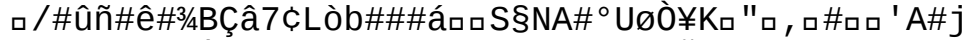

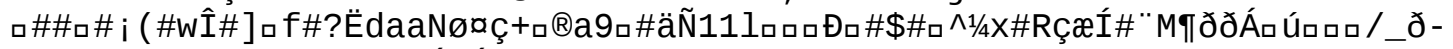

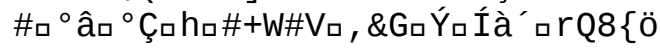


口\#8p\#1̂\# 


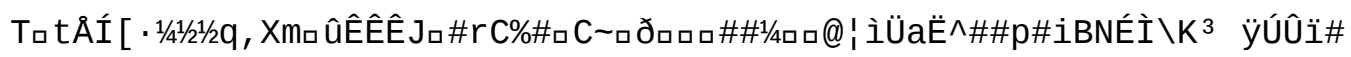




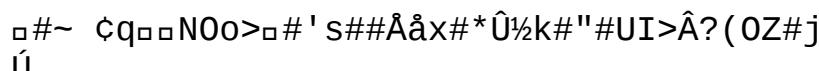




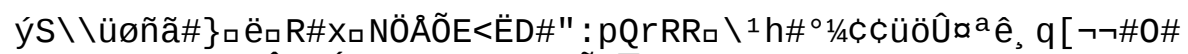

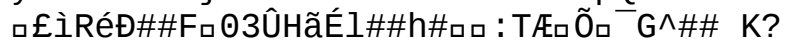




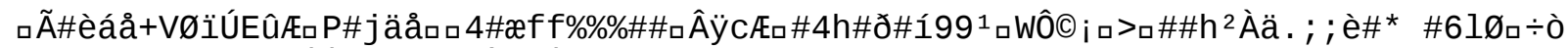

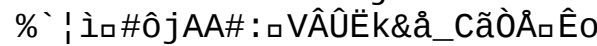

à==] à

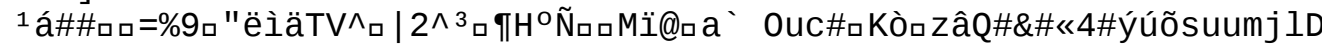

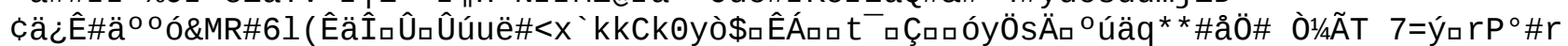
ac

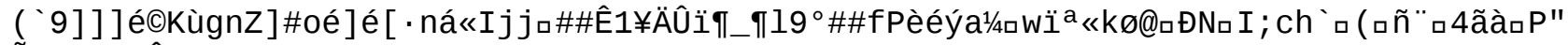
Õ $\square \# \square)$ SÔ' ORSı $\left[r^{\circ}\right.$ 


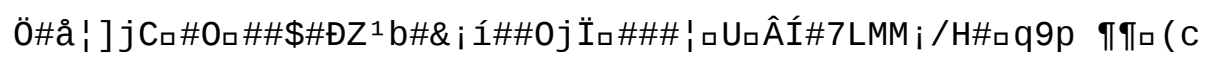




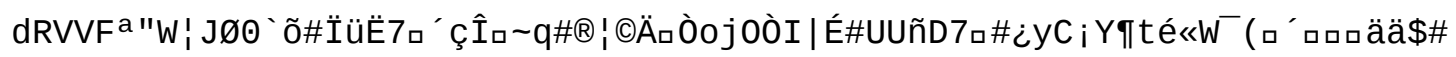


$\tilde{N} \# 9 r \$ U \hat{U} \# 0^{1} 9^{1}$ 口f . Õa ; wòa Ia\#1̂E ÛÛÛs

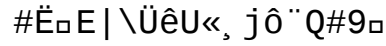




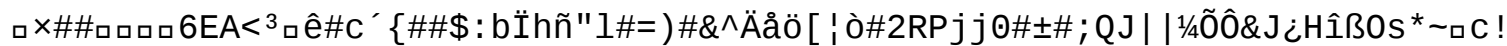

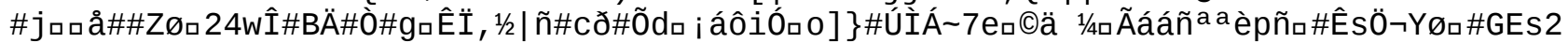

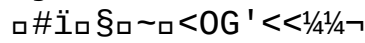




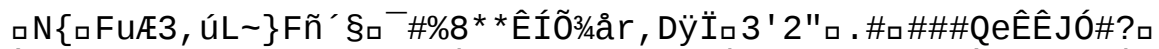

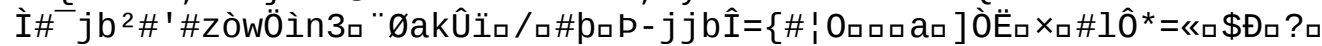

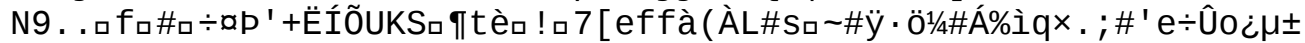

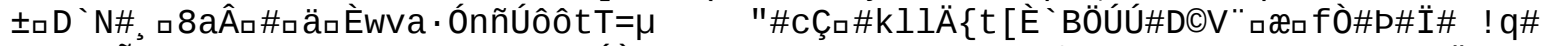

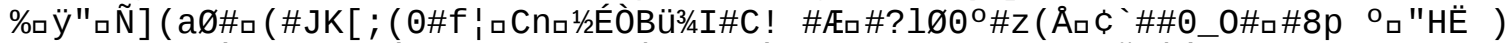

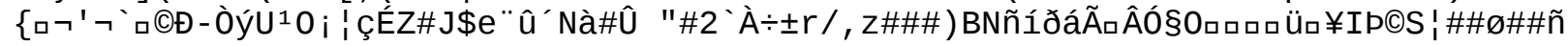


\#arÀ\#0b9\#ロロ०@\# 


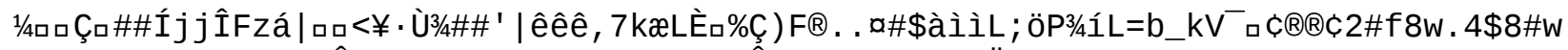

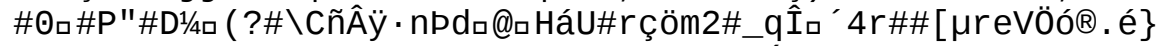

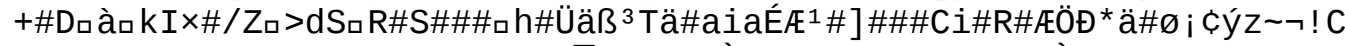

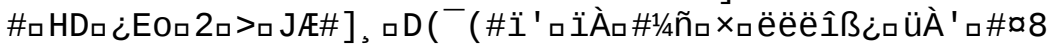

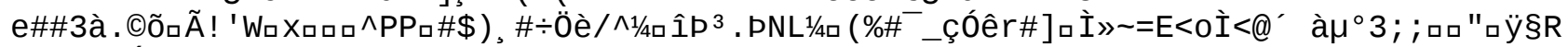
Đcıàı\#Ëä口Å\$)3@ø] J\#icıôsIY\#R

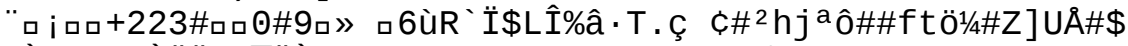

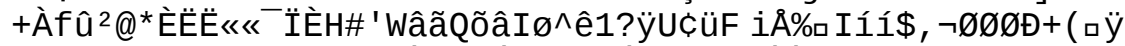

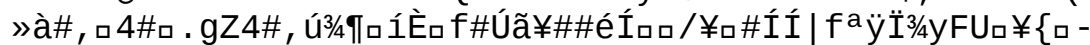

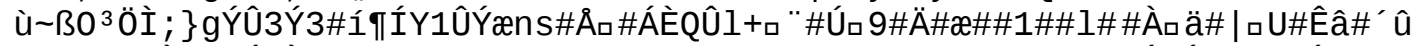

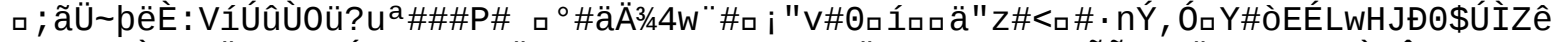

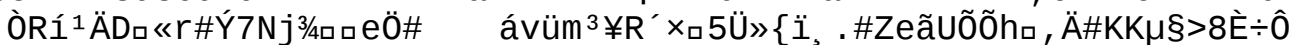

'Tê\#\#F»ãwú4ๆ0 


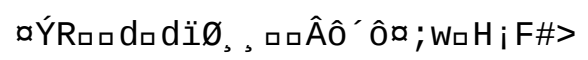




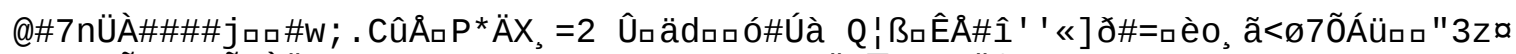

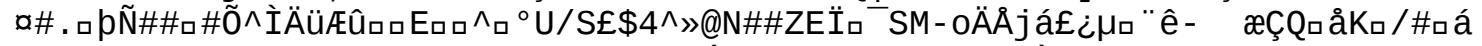

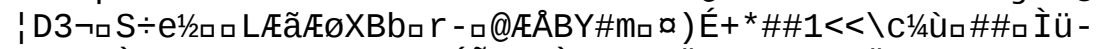

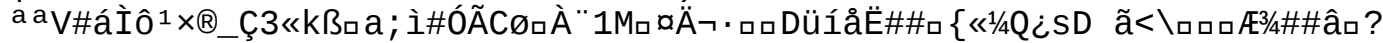

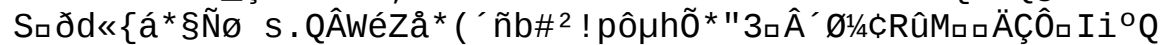

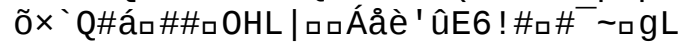




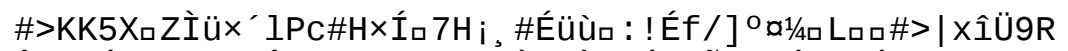

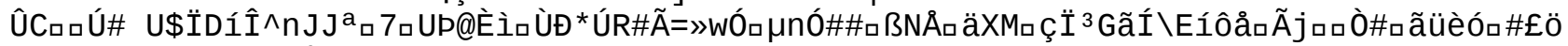

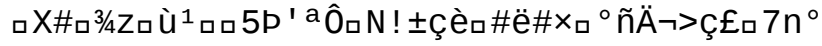

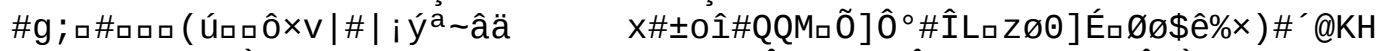

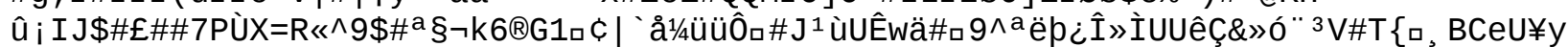

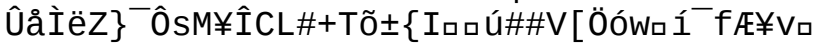

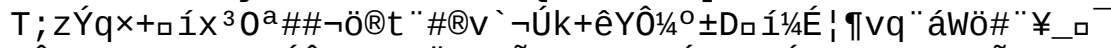

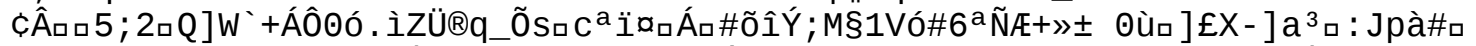

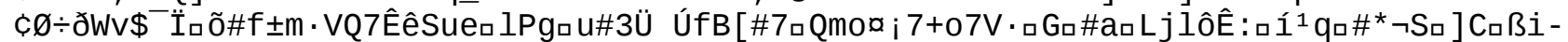

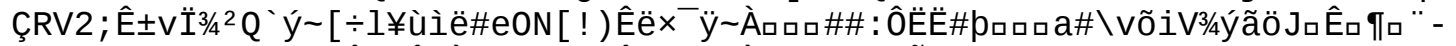

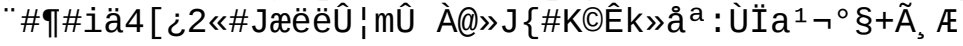




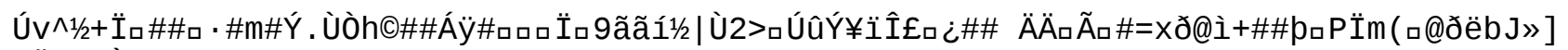
Eİîàvò¿ãaøı\$

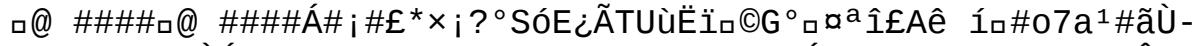

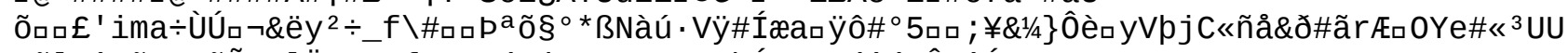

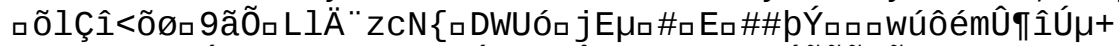

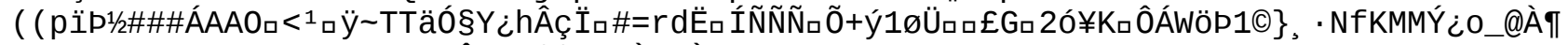

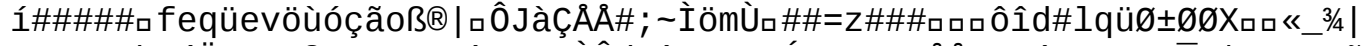

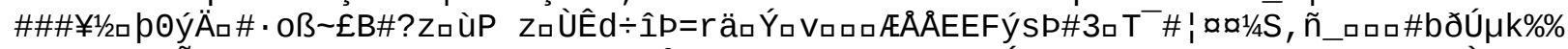

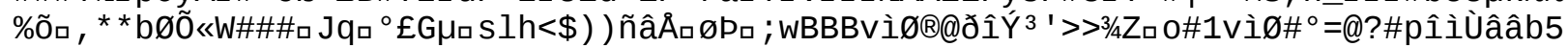
HJê\#> 


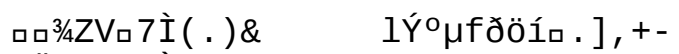

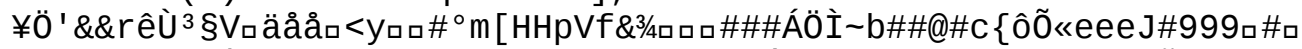

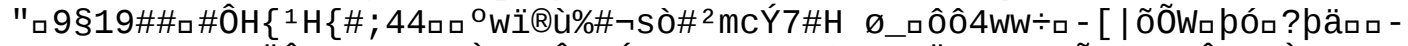

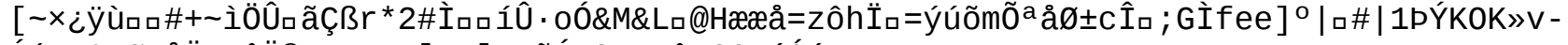

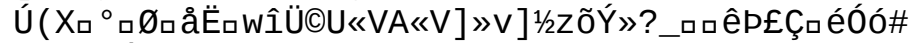

РேчcnÝòðð\#3f 
$¥^{1}$ ú\#] ]ロÈEiié C 
口 $\tilde{N}^{1 / 2 \#} i_{\square} 1 / 4$

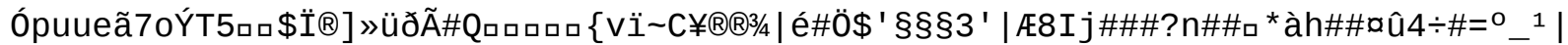

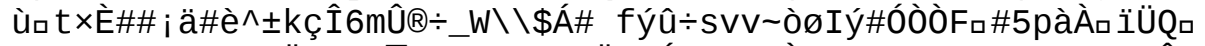

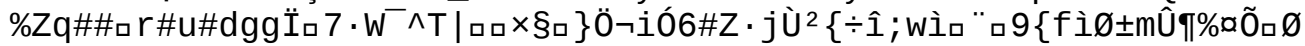

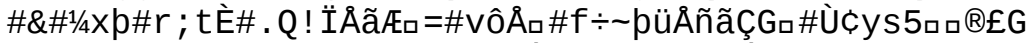

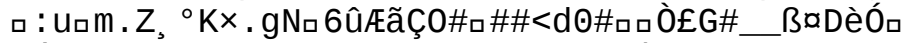

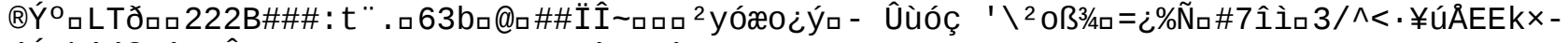

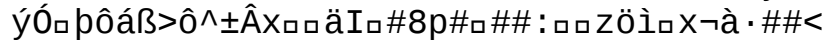

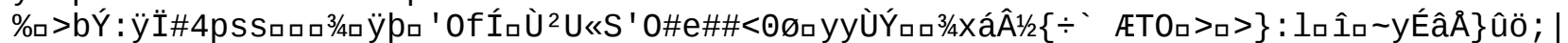

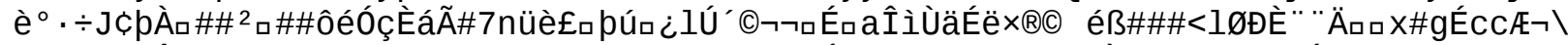

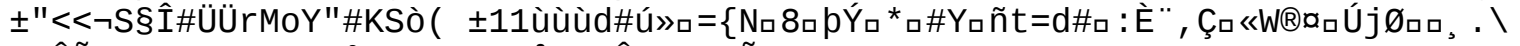

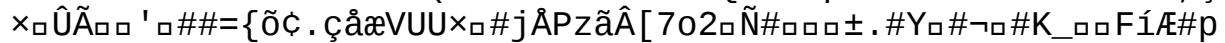

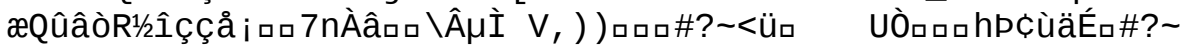




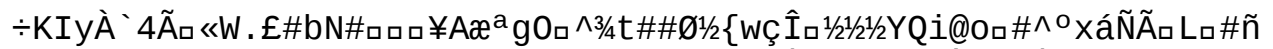

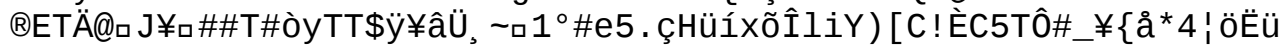




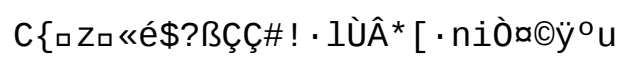




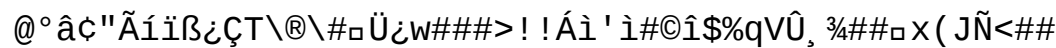

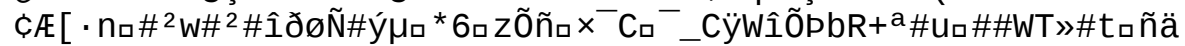

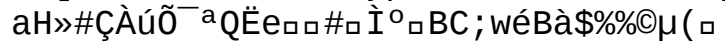

\#¿у£口mj», ロÿÆ $\mu \mathrm{kLÅ \% Vb?}$ 


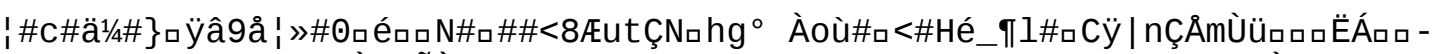

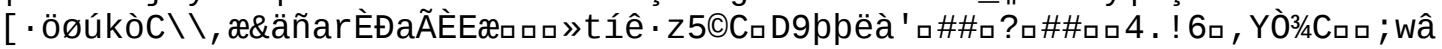




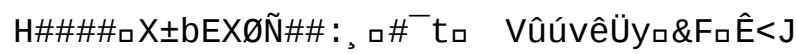




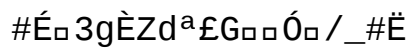
\#\#^ๆl)ロ\# 


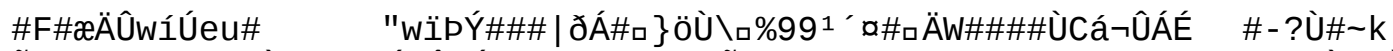

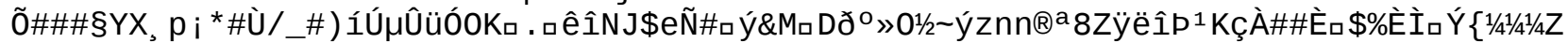

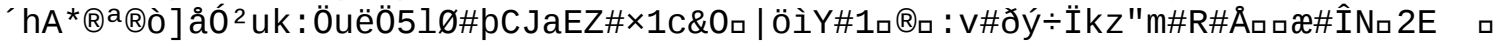




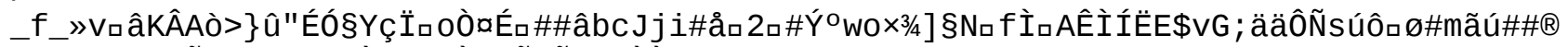

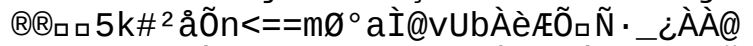

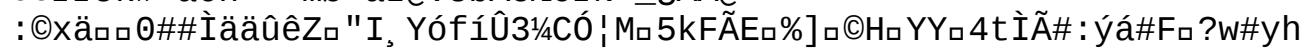




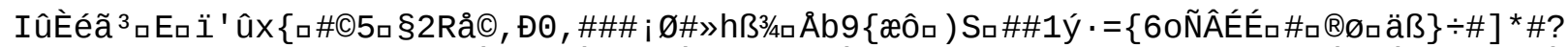

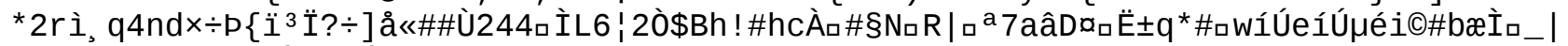

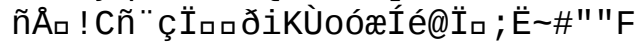




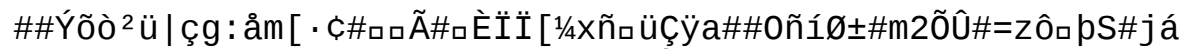

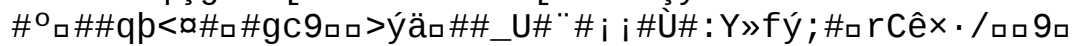

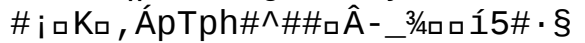




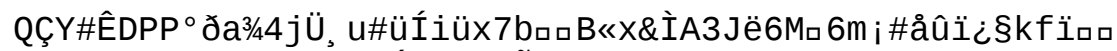

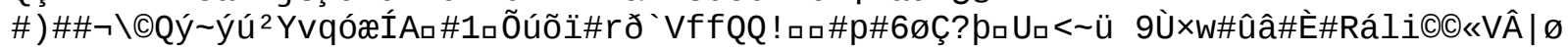


ๆÆ口Ø॰b॰Ä\# : 
Ä|ㄲzZõòäiß\#ロ 'ÁI 


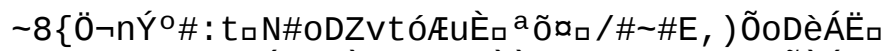

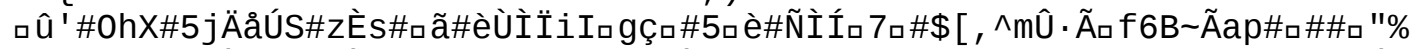

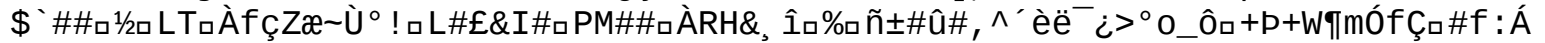




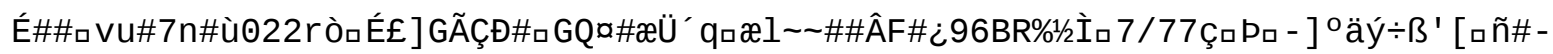
Z4zôèððp

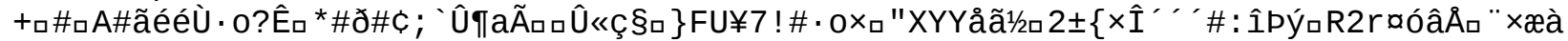

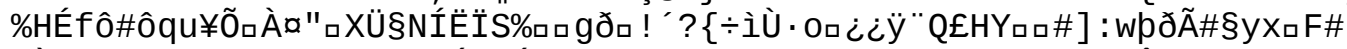

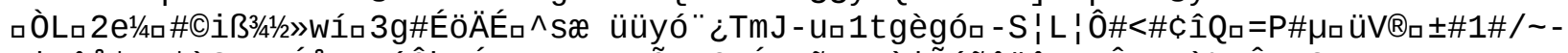

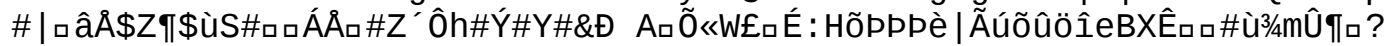

$p_{a}={ }_{a}$ Ùè . ÇaÕà $i \sim y ́ n a+$

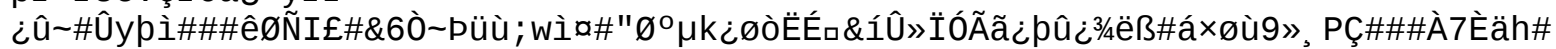

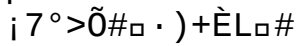

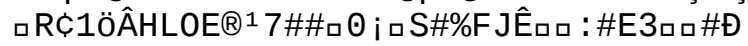




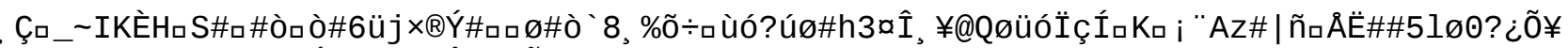

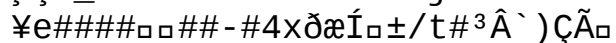

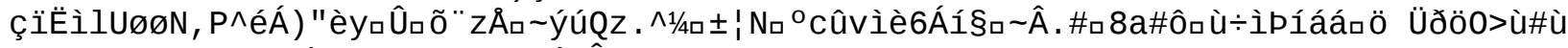

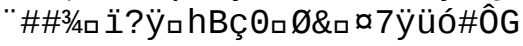




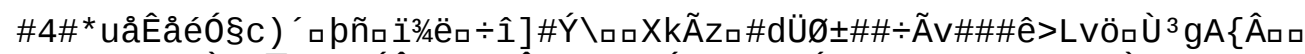

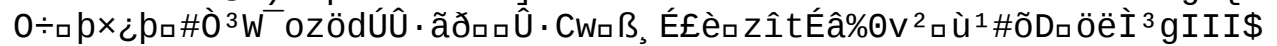
ØロßêṎ̈\# . $\hat{E} f$ 


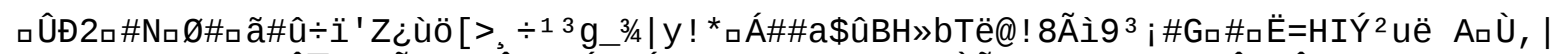

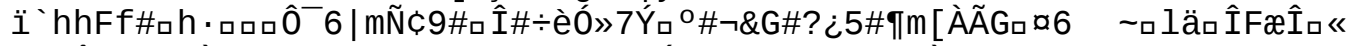

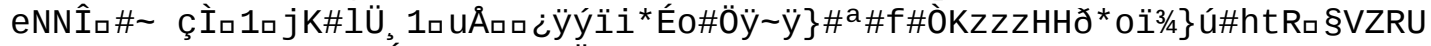

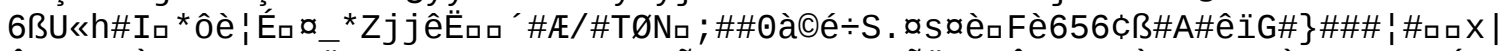

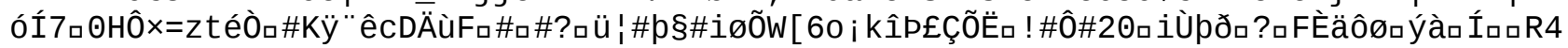




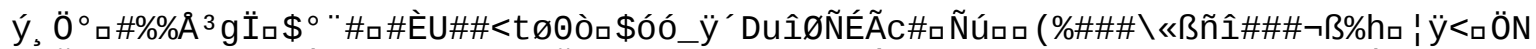

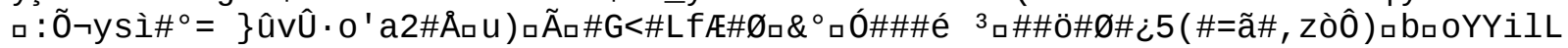




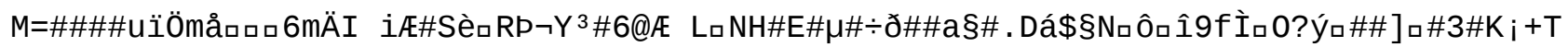
Eùê« âbcar

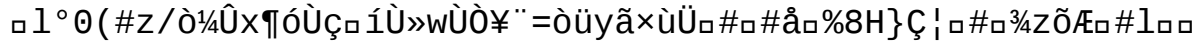

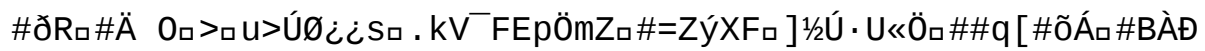




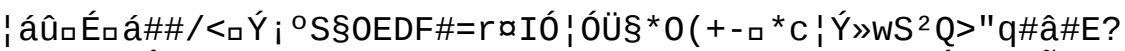

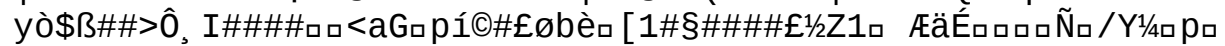

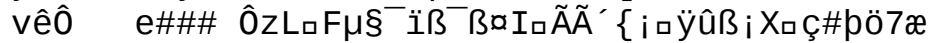




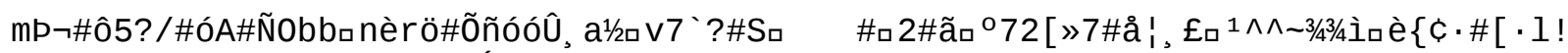
$h \div F L ? a^{*} \backslash_{\square}{ }^{a}$ 4RMhèÁ

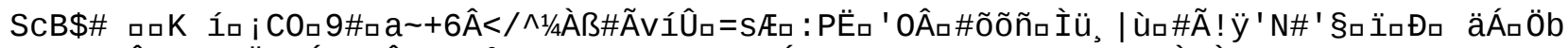

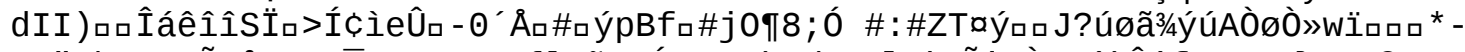

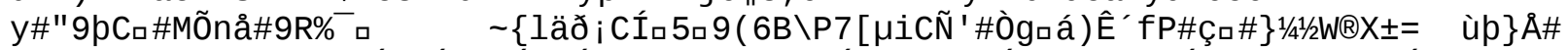

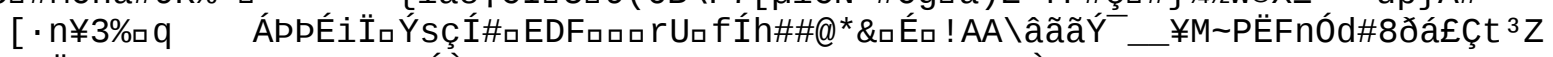

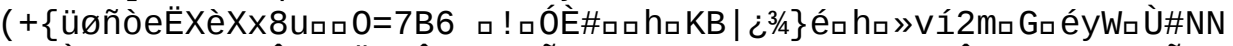

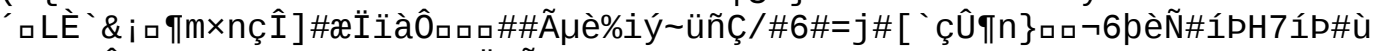

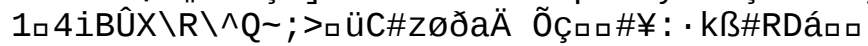

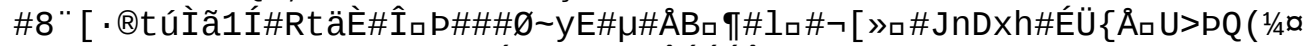

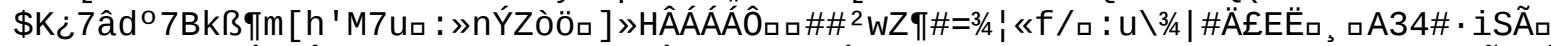

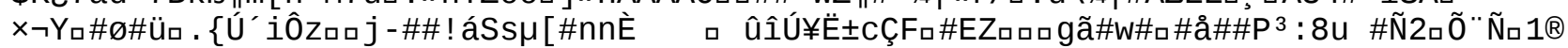

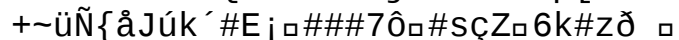

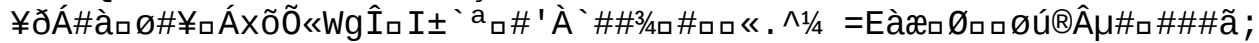

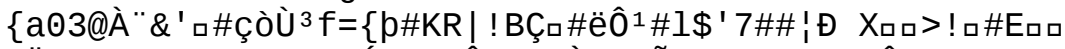

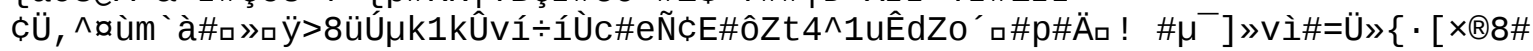




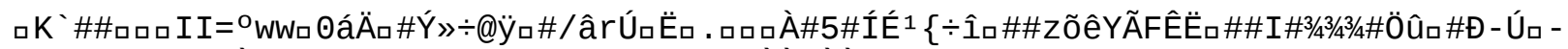

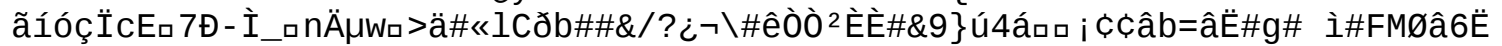

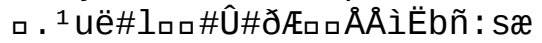




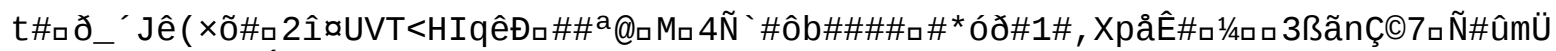

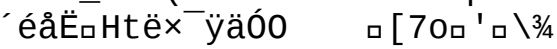




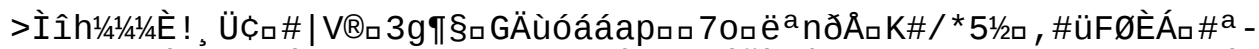

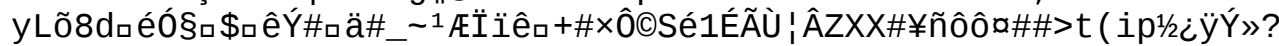

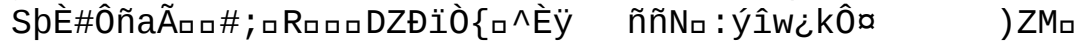




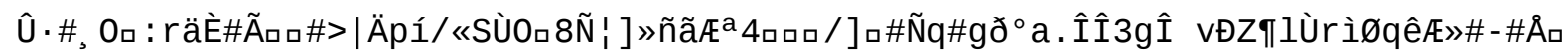
CFa\#; $0>$ 


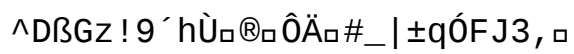

;

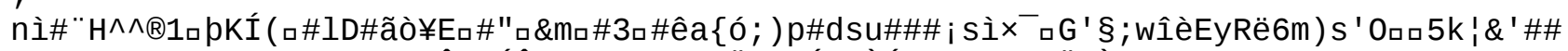

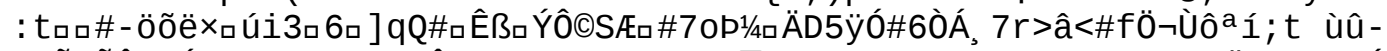

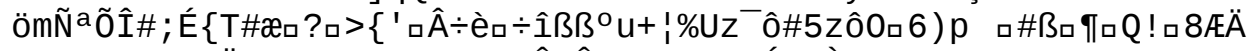

Ú/\#äj fP¹/4\%3\#ם

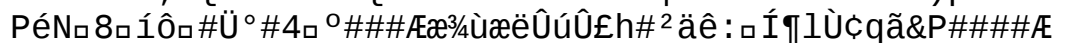

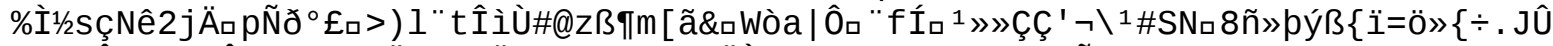

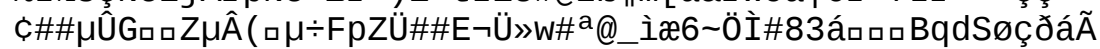

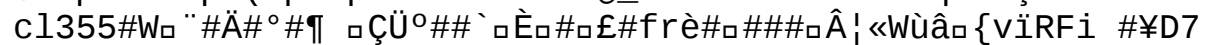

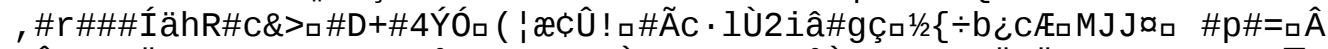

\#ÔaâñcÄSAっ fDø

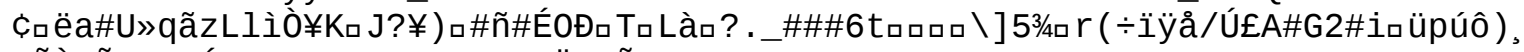

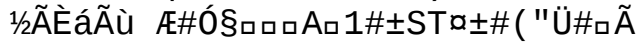

口 


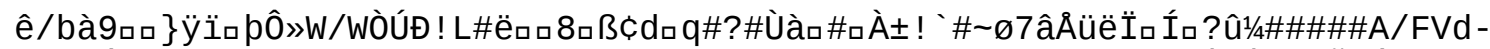

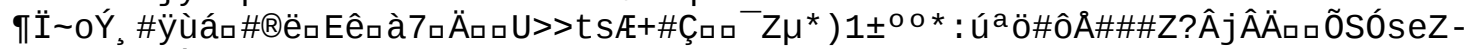

( / T5JOVİ॰\#; 


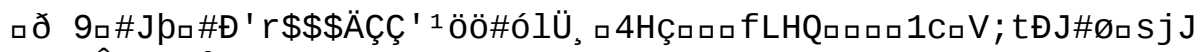

$9 ; 440 \hat{m}$ r\#ånq 


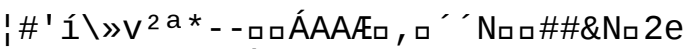

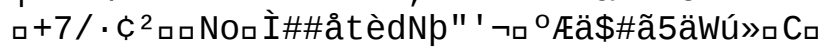


é"?ÅZU¥ÝaÏĐÅ@ZÄ QÃda t1üV \# \#

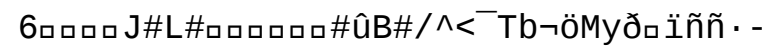

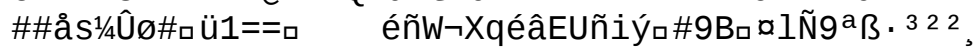

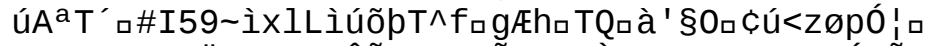

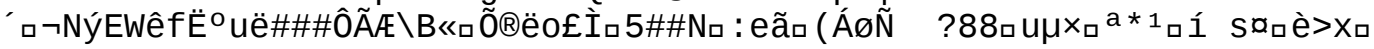




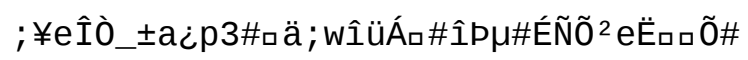

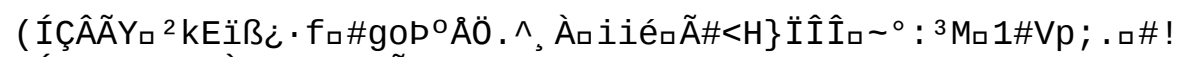

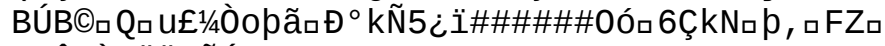

9\#ÛঃÒÇÄÜ* ÕÝ\#€\#

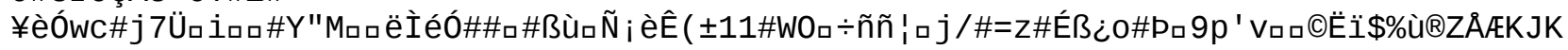

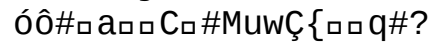


$\# \#$ व $<$ e

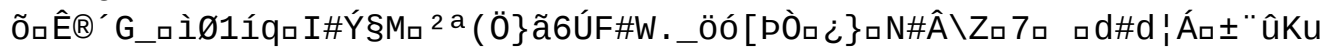




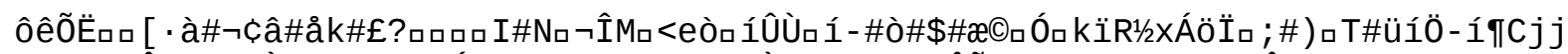

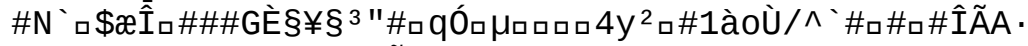

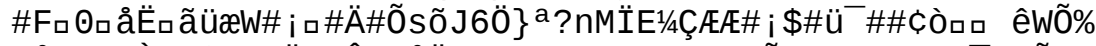

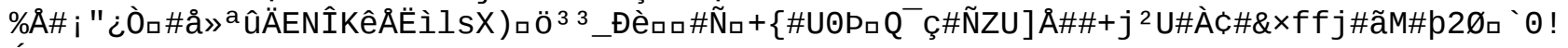
ó2 


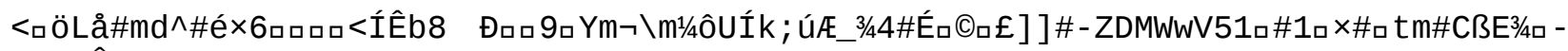
$Q^{\mathrm{a}} \oplus \square \hat{E}<J$ 


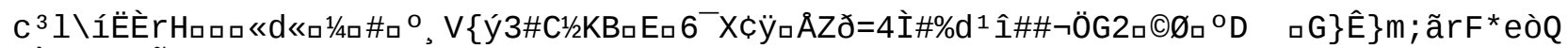

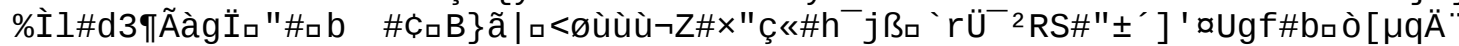

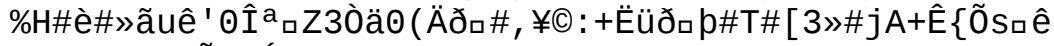

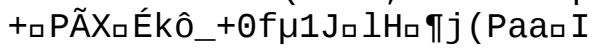




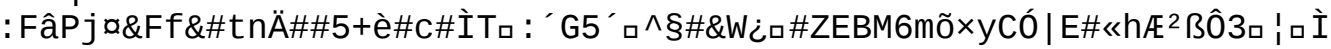




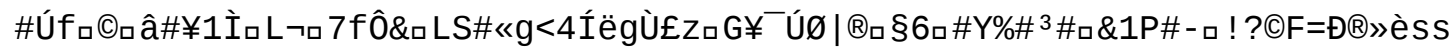

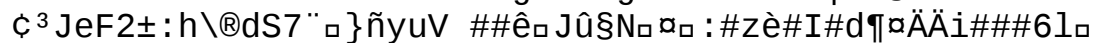

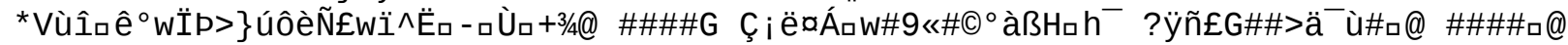

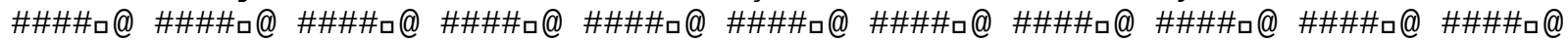

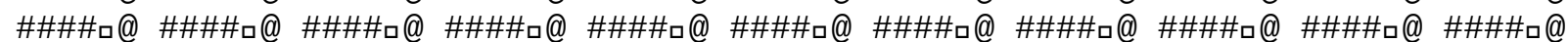

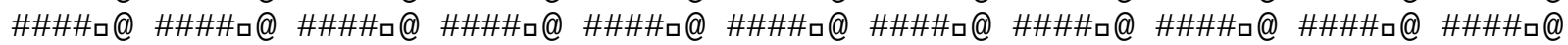

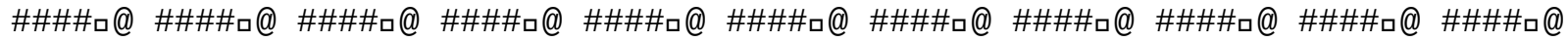

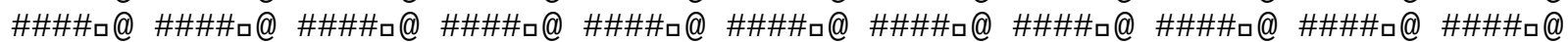

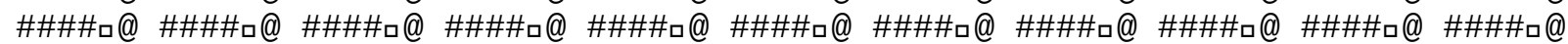

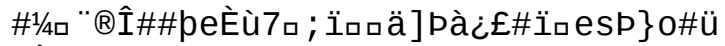

口Èûç . $\div$ व\#; då1/1/1/4 |

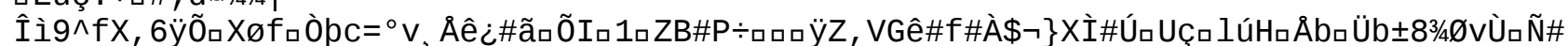

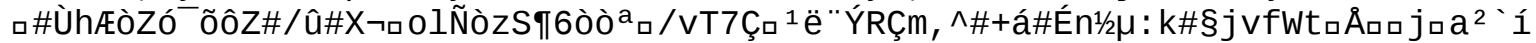

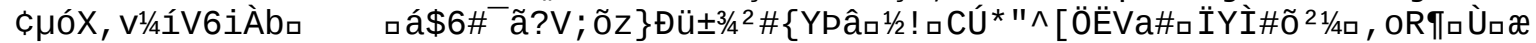

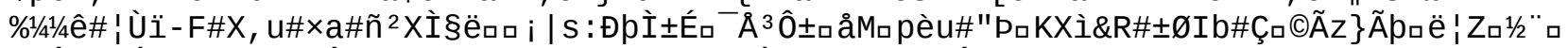

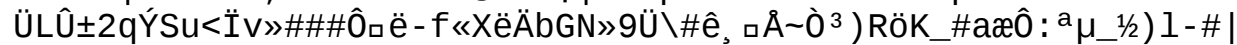

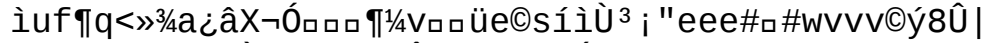

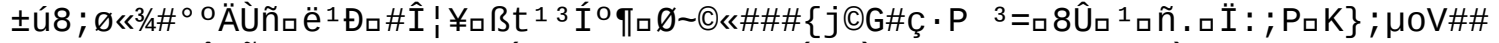

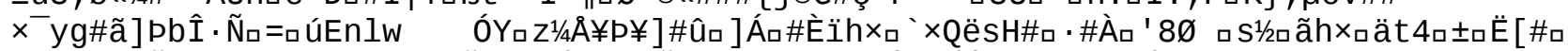

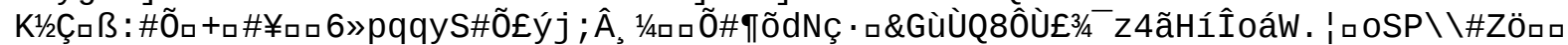

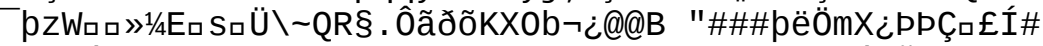

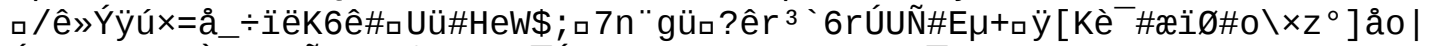

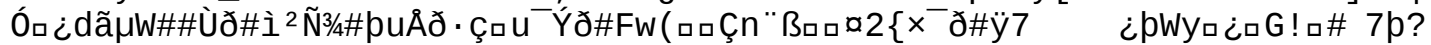

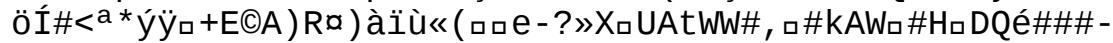

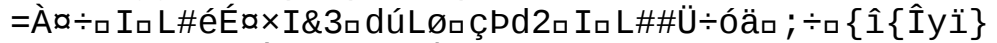

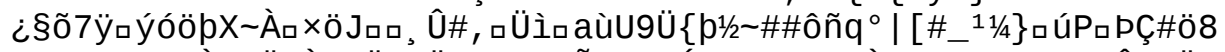

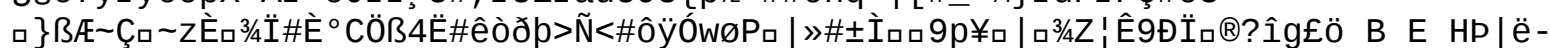

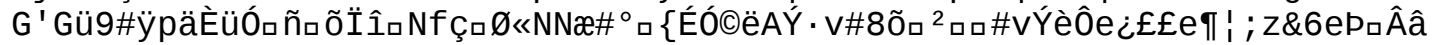

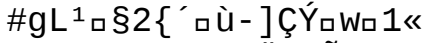

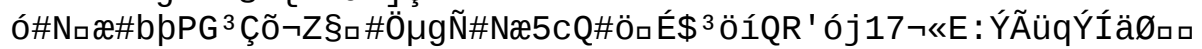

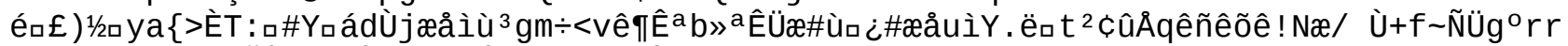

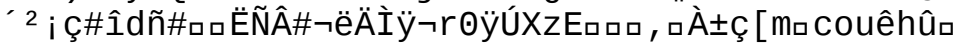

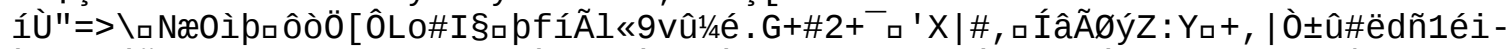

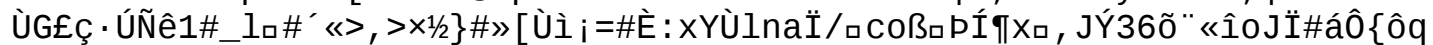

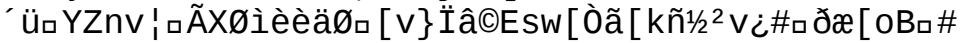




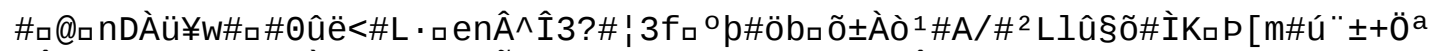

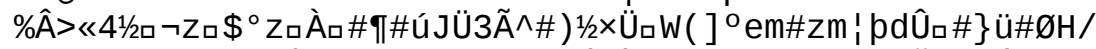

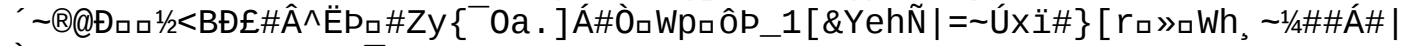
Òæ+l»BZsì^॰CĐOロ\#\#-ð

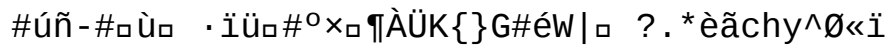

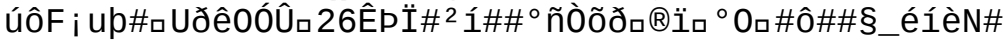

EhS\#A\#\#A\#\#A\#A\#\#A\#\#A\#\#\#\#A\#\#A\#\#A\#A\#\#A\#\#\#\#A\#\#A\#\#A\#A\#\#A\#\#A\#A\#\#A\#А\#\#A\#\#\#\#A\#\#А

\#\#A\#\#A\#\#A\#\#A\#\#A\#\#A\#\#A\#\#A\#\#A\#\#A\#\#A\#\#A\#\#AüロÓ\# 


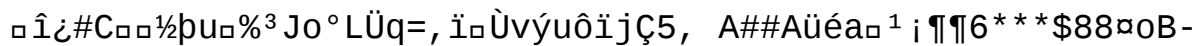

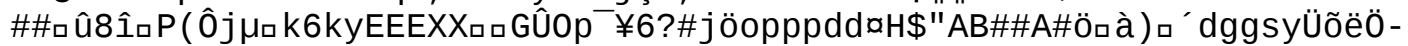

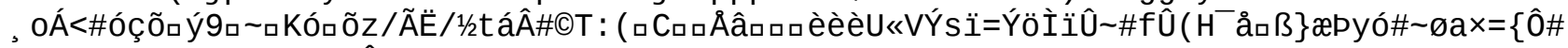

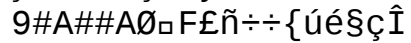

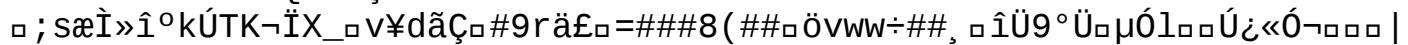

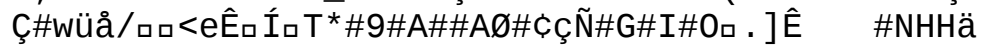

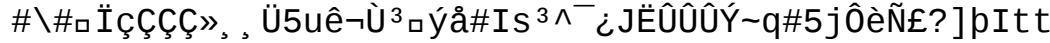




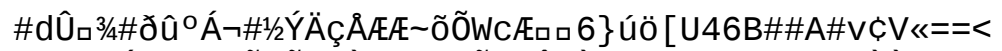

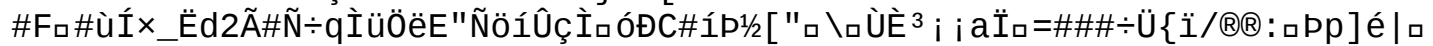

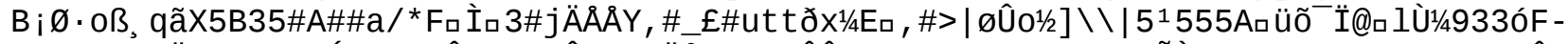

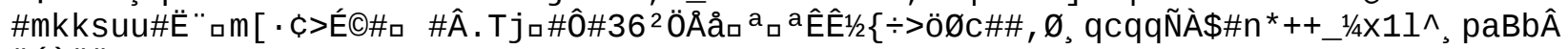
ÜÉÒÖÖ

52ロÔ\#A\#\#A 


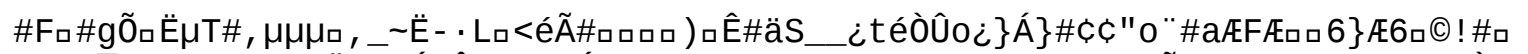

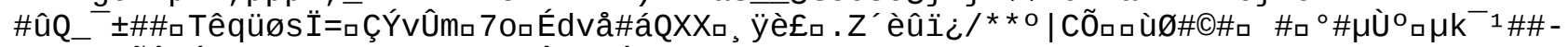

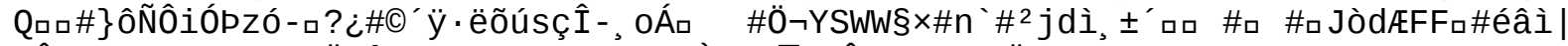

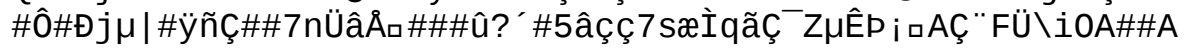




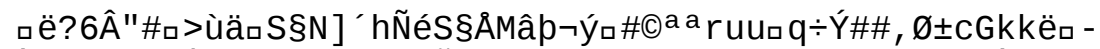

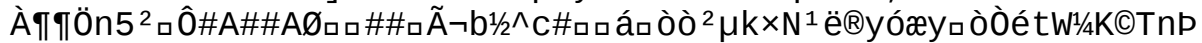

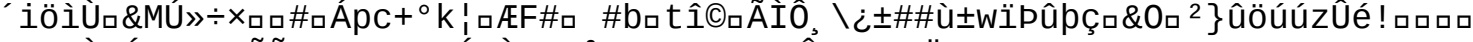

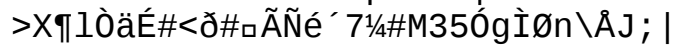

口 \#Â>LëF®Ï口\#S 


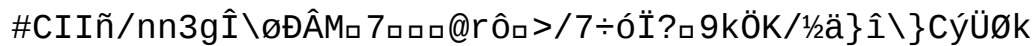

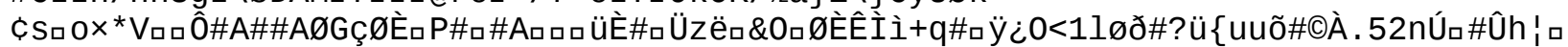

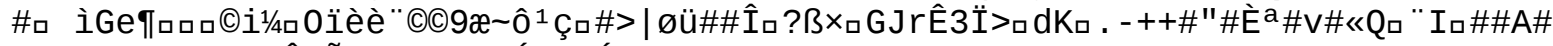

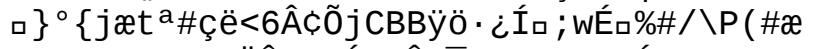

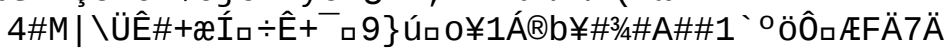




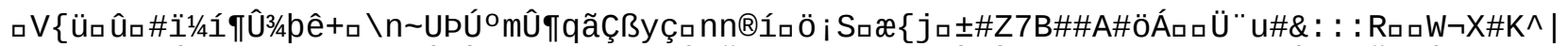

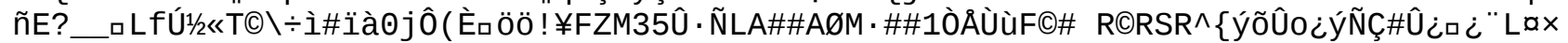
ëquËå._3/4|úôé ${ }^{-3 / 4 u ́ a}$ ¿¿ ? $\hat{I}$ 
ㅁ

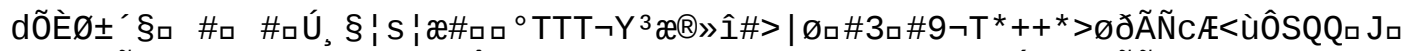

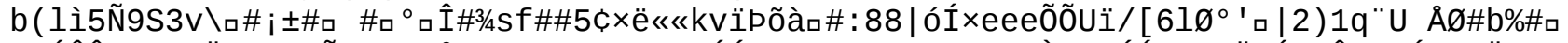

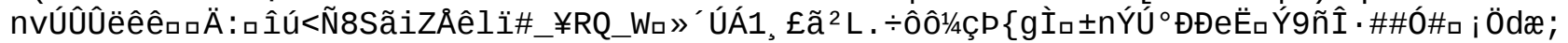

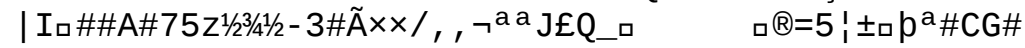




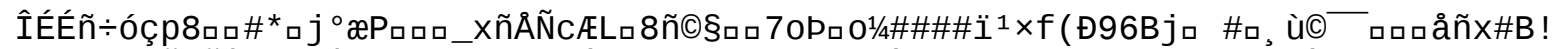

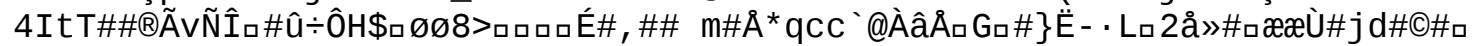

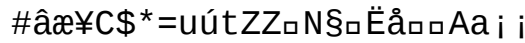

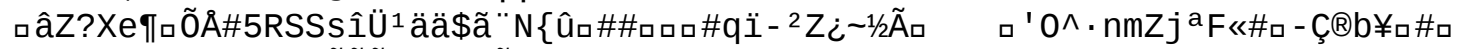

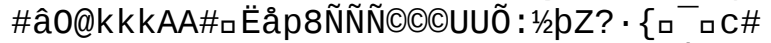

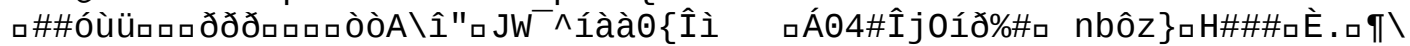

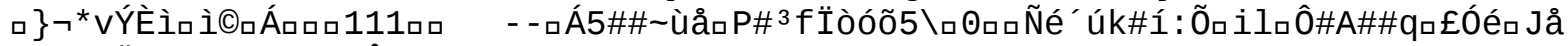

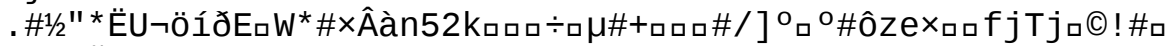

\#ûP@ÕfëFıíU\#x\#£\#ùâ\#Vıøúø 


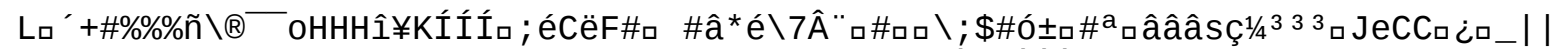

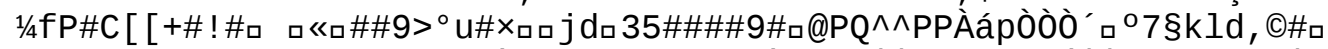

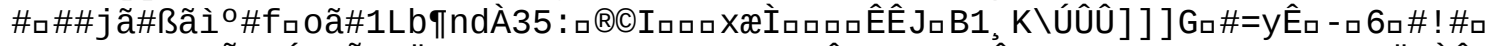

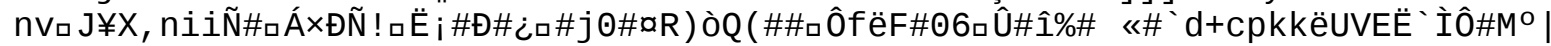

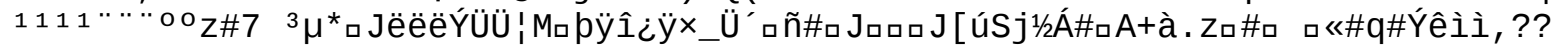

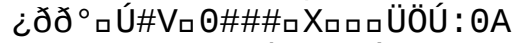

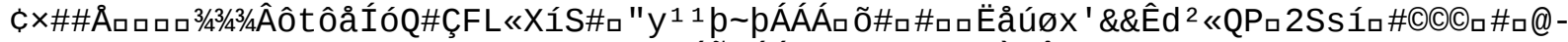

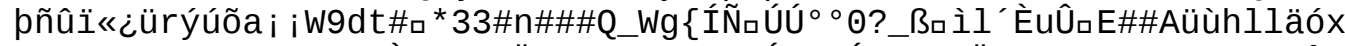

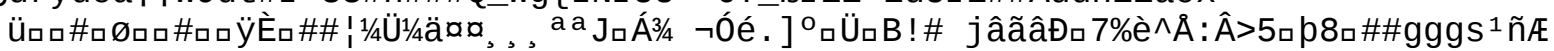

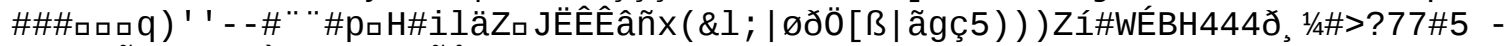

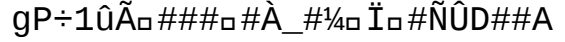




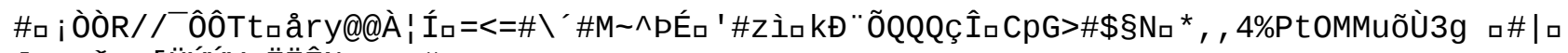

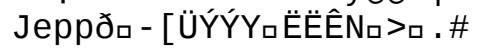




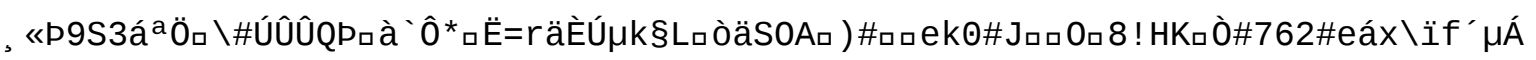




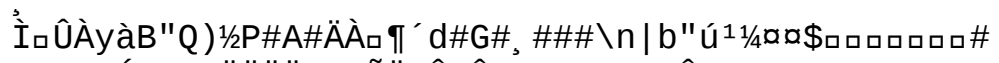

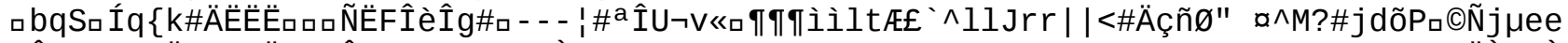

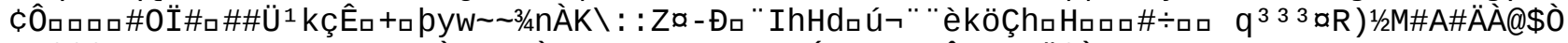

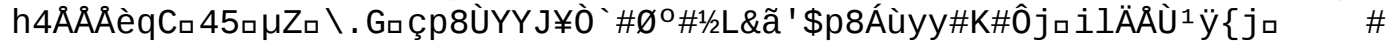

CCCbcc ak4aÖvôĐ9\#/Bó(\#

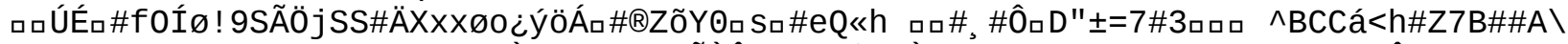

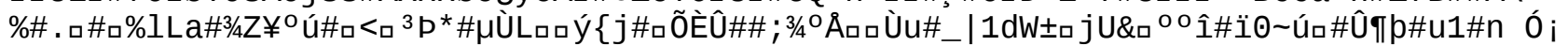

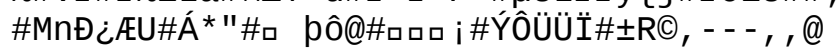




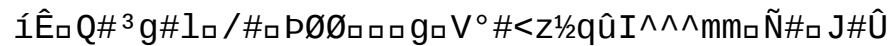

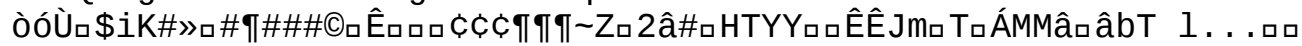

ú\#I>Øãêê : vìø๑ó | mýñGU\#+Na>

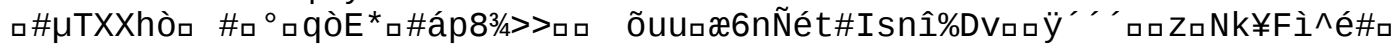

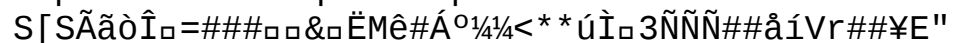

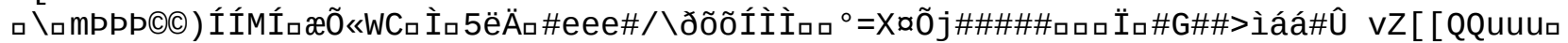

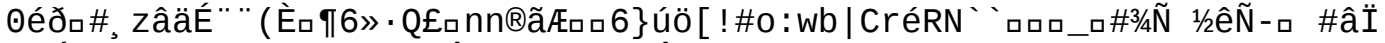

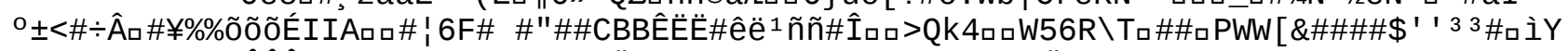

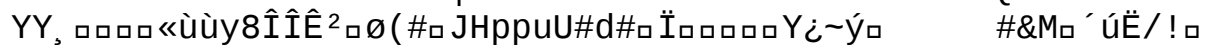

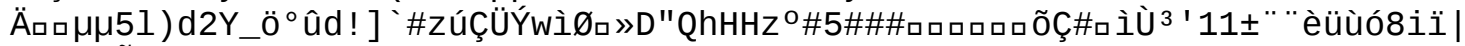

\#£FÜ ÕロææS\# ${ }^{1}$ 
õ\#\#\#\#\#WロロロO 


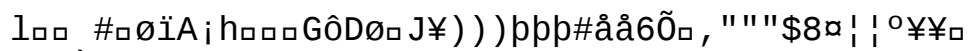

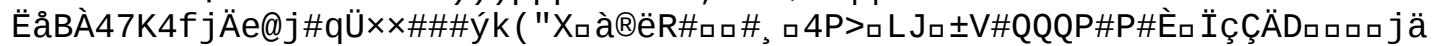

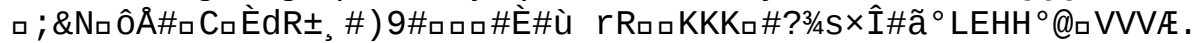


ÎËËÛ · O\#ÔH2ロ\#\#"\#

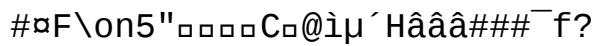

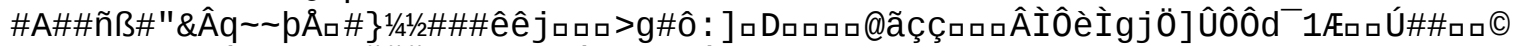

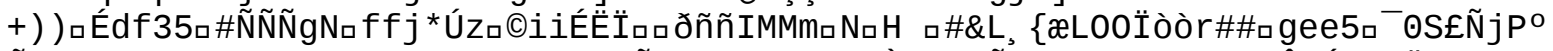

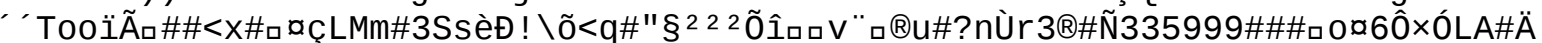

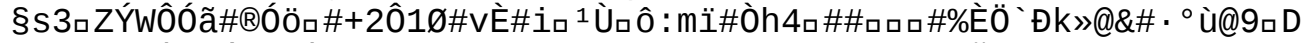

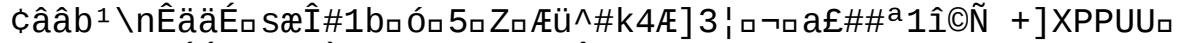

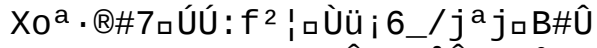

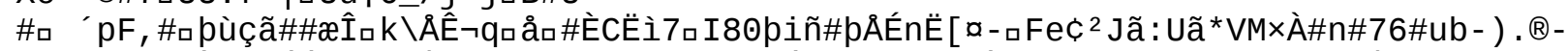

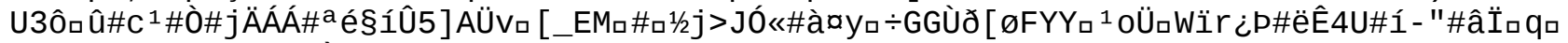

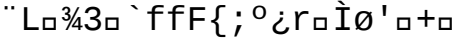

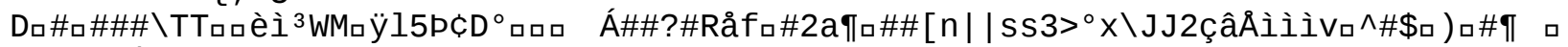
Bt $\ddot{\# \# " ~ " A ̂ \# ~}$

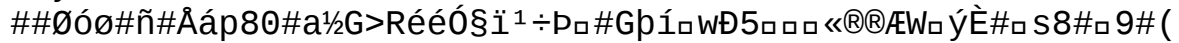

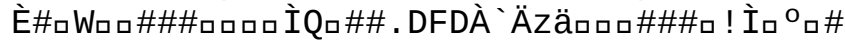




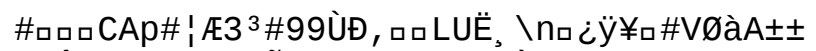

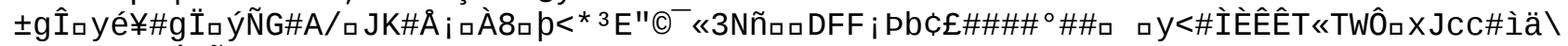
äüpûïロ $\mid$ òEễÕ $C C B \square$

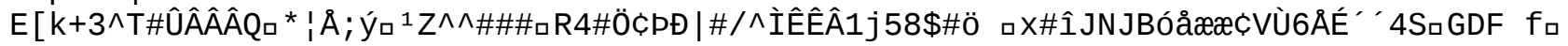
3

"ëéZì\# 


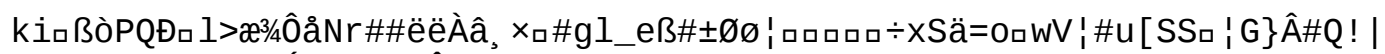
$>\# \mathrm{Px}{ }^{3} \mathrm{k}_{\square} \# 1 / 4 \#{ }^{*}{ }^{1} \mathrm{AÓ} \# \pm \mathrm{eP} \mu \# O \hat{\mathrm{m}}$; 
จ ${ }^{3} \bigvee ¥ W E \hat{E}:$ 


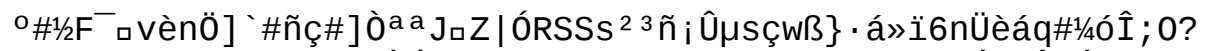

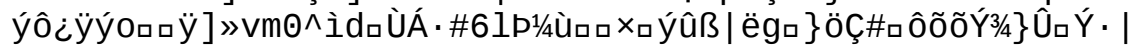

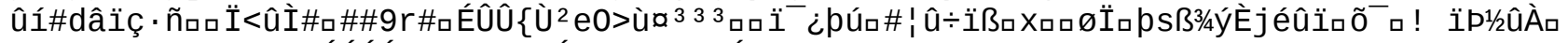

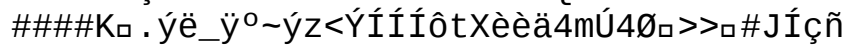

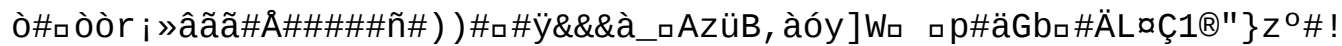

\#aÚX0̃<\#Â: \#ãRttTrrRJJ

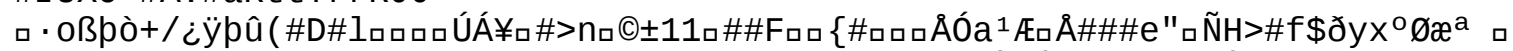

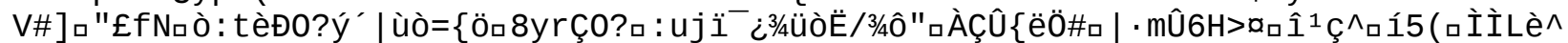

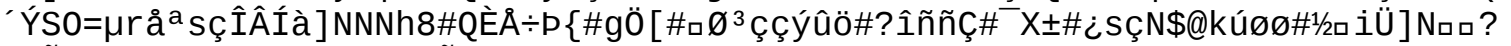

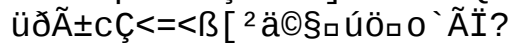

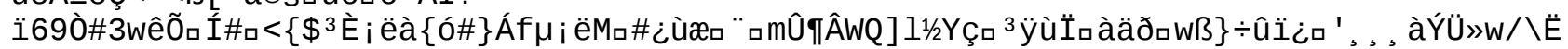

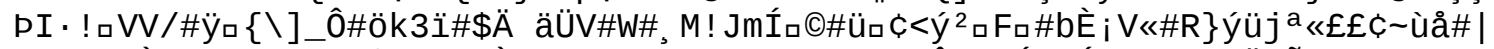

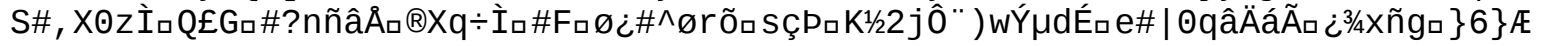




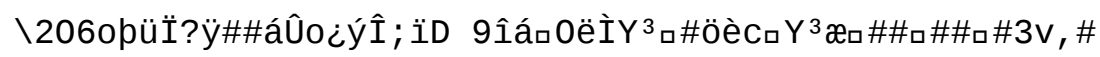




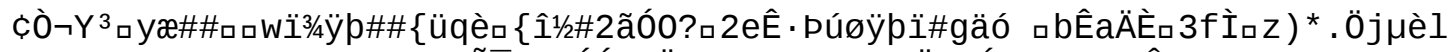

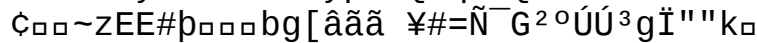

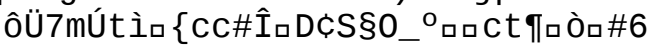

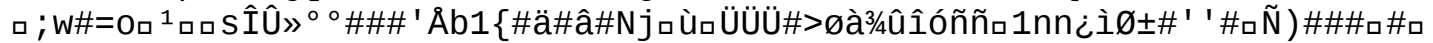

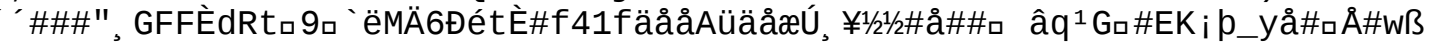

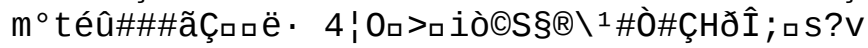




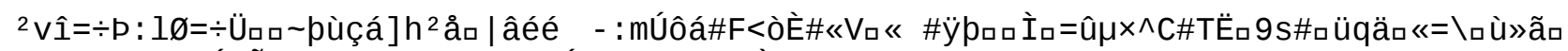

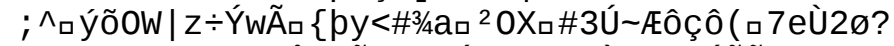

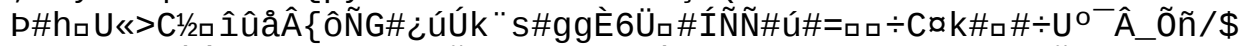

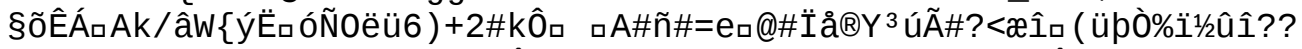

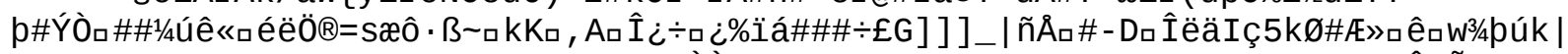

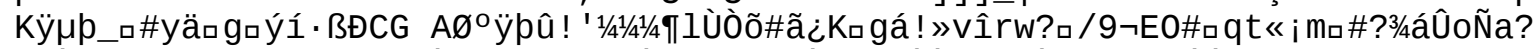

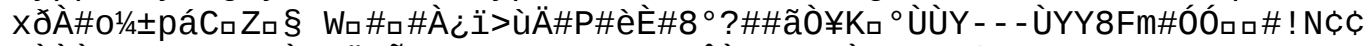

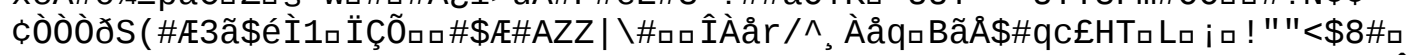

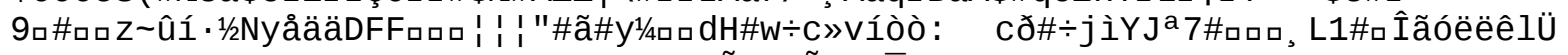

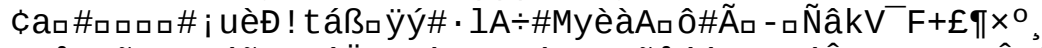

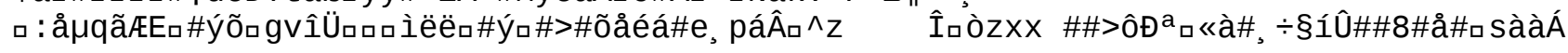
a; $\mathrm{V}$

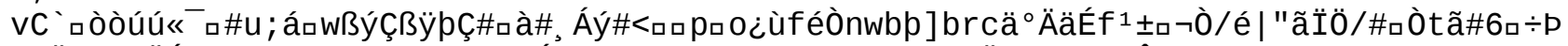

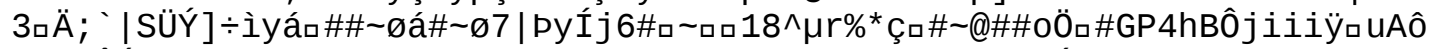

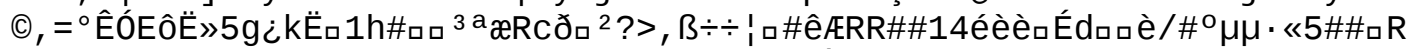

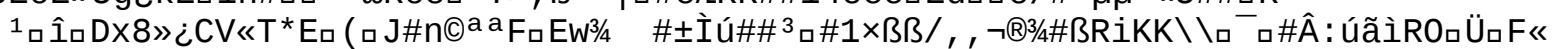

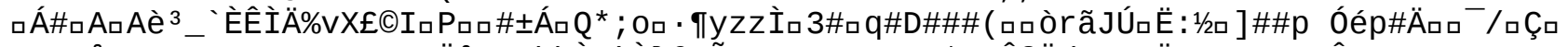

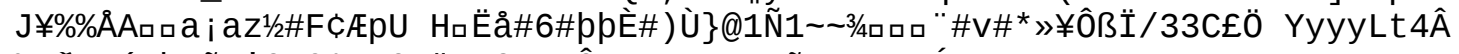

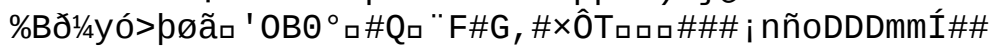




\section{À\#VM\#İ。}

R2

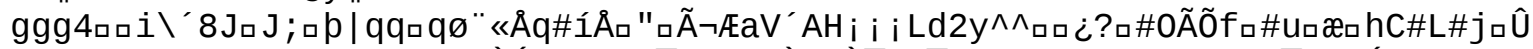

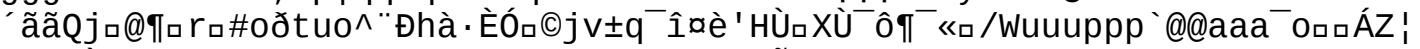

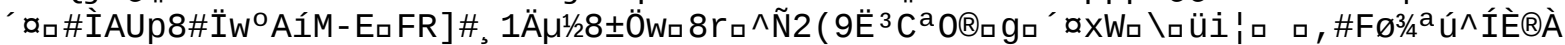

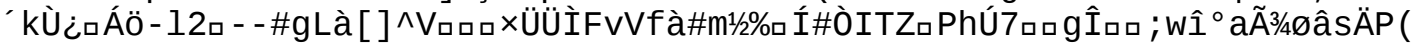

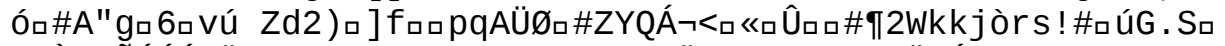

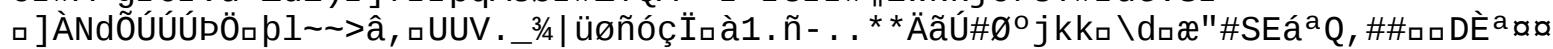

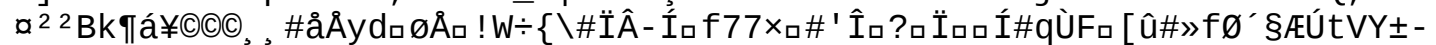

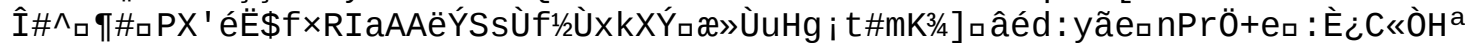




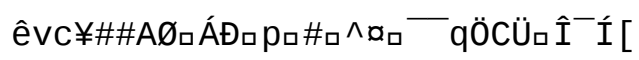




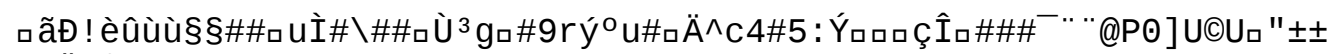

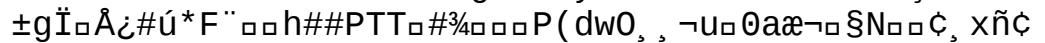

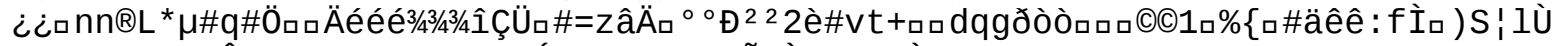

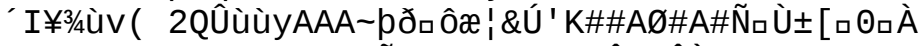

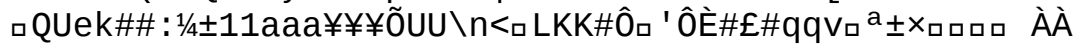




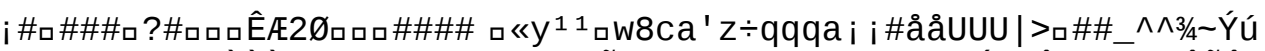

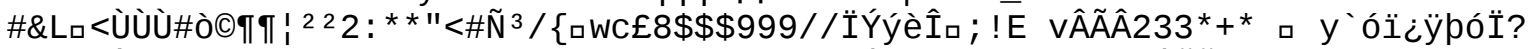

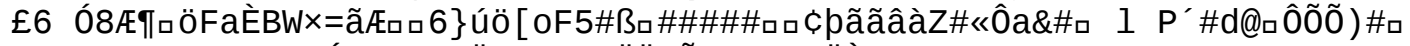

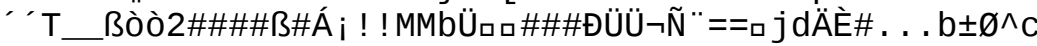

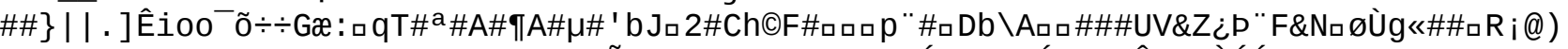

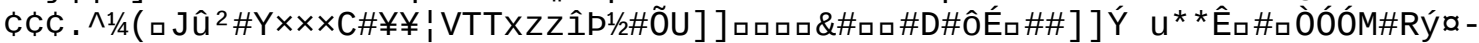

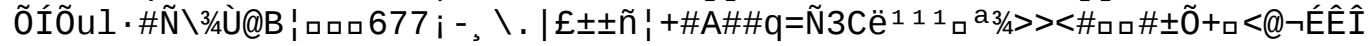




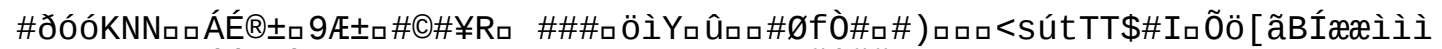

ooïan

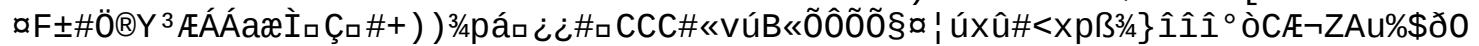

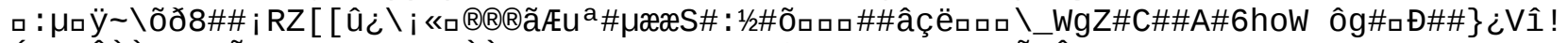

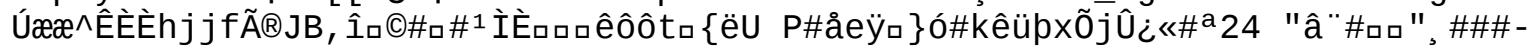

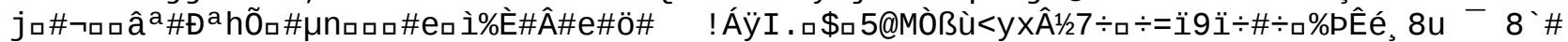

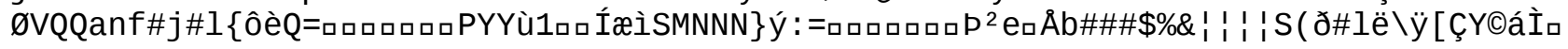




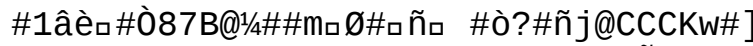

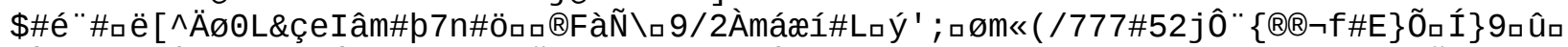

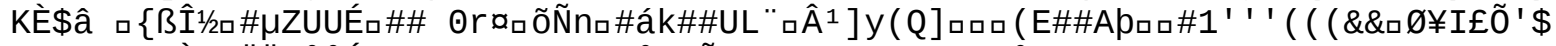

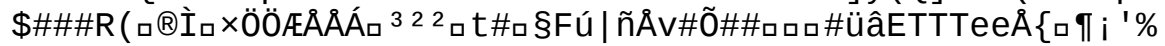

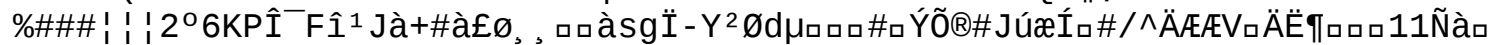

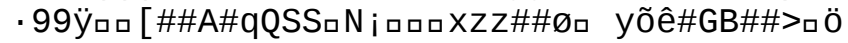


\#IJJrEE ${ }^{1}$ " ãVèı

\#^3/4|éëëëùôiHppJJ

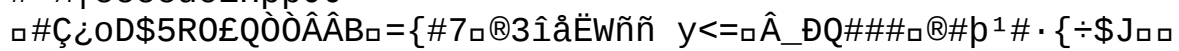

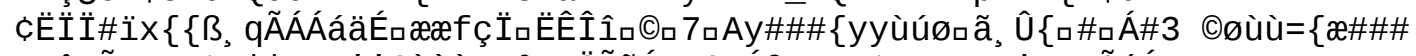

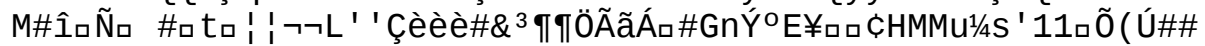


$\ddot{\mathrm{Y}}$ 口 $\{\mathrm{R} \mathrm{R} ¥$

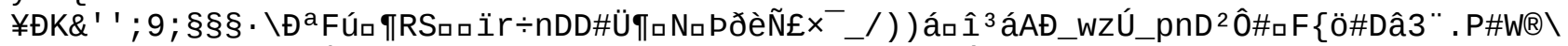

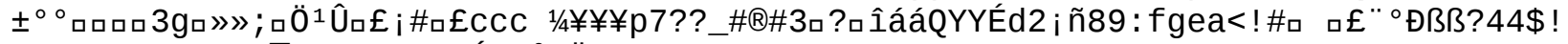

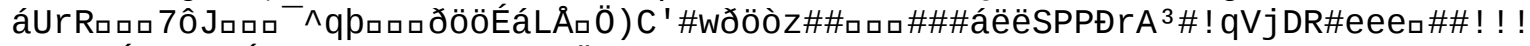

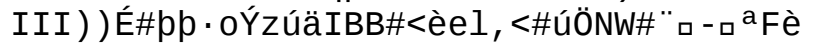


Fll1P` Èûnn\#\#\# \#uuu7mÚ\#\#Òé\# Ü

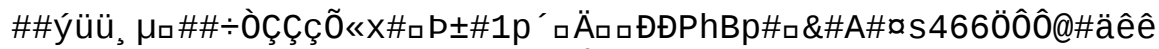

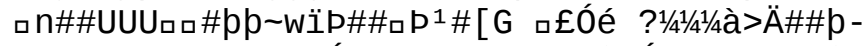

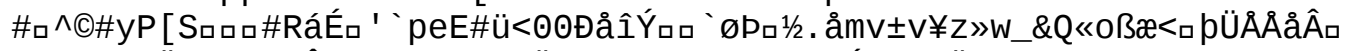

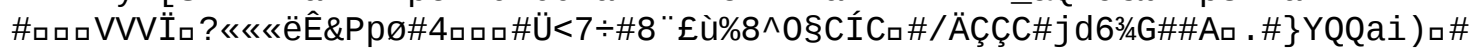




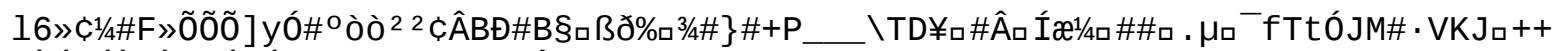
+ÙY : ÁòøĖøZÍĖÍ=súô (\#IuâÄóç Î1\#»A 
口ÁÅEqee¥, VãGıÑXZZ

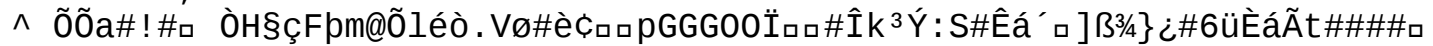

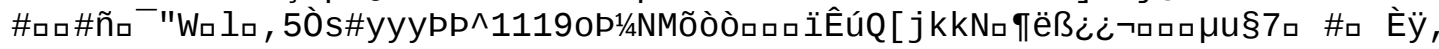




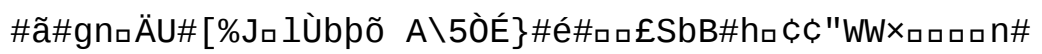


$\backslash 5$ rzÀı\#ঃ\# ¡ £\#A\#\#A\#\#á-

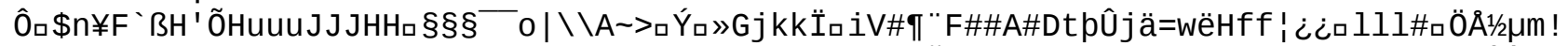

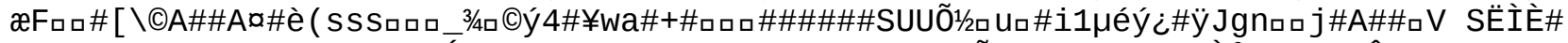

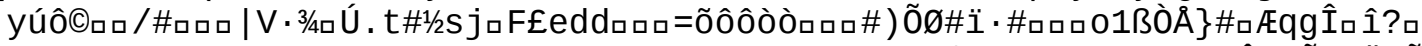

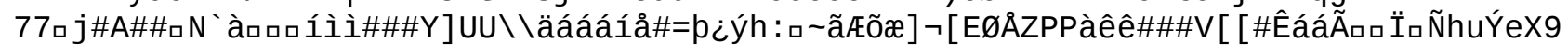

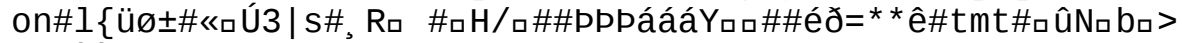

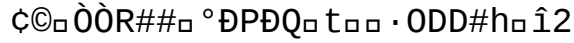




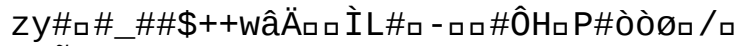

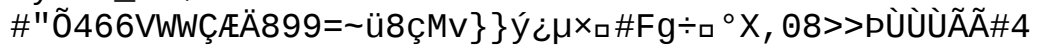

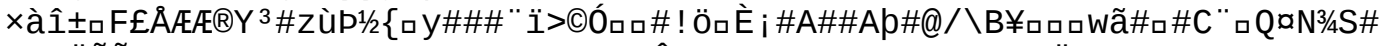

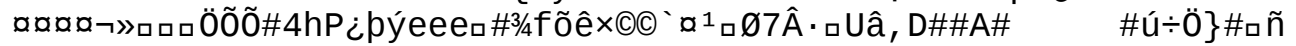

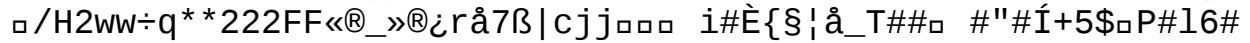




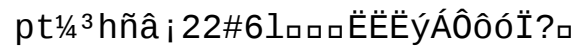

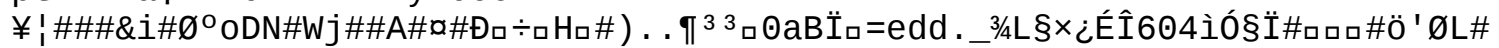

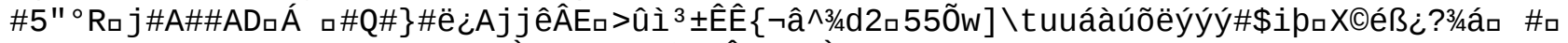
$\square \square X(\mathrm{C}) \# \mathrm{a} \pm \mathrm{X}$ 口

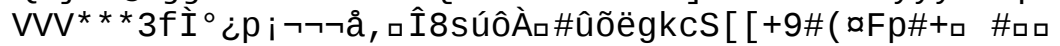

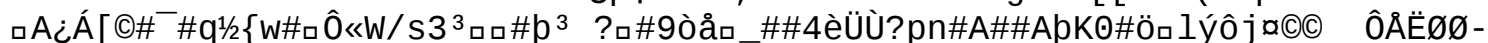

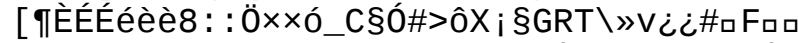

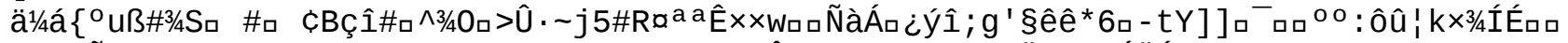

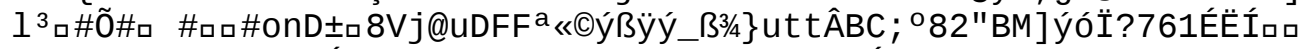

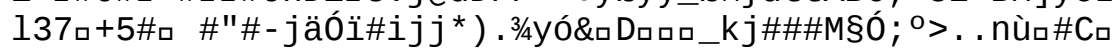




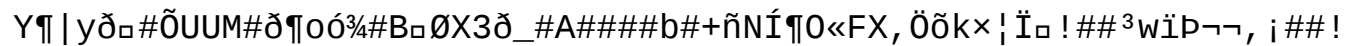

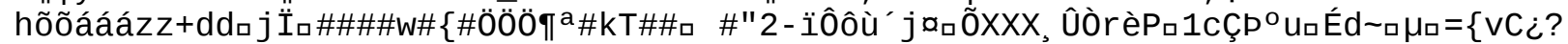

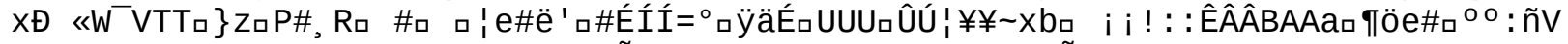

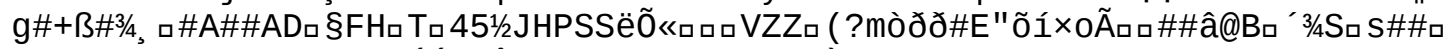

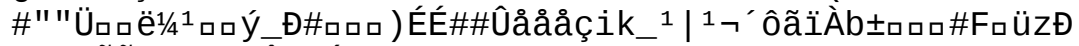

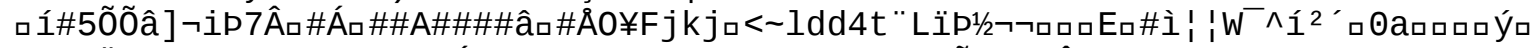

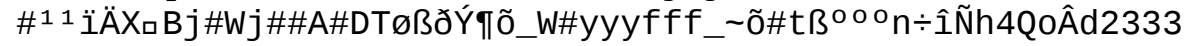


\#8uêÔव\#1NıðVj\#5r\#0̃\#ロ \#ロ

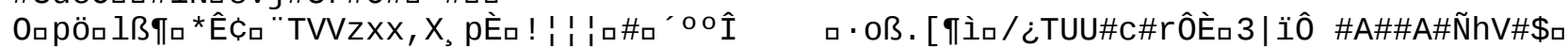

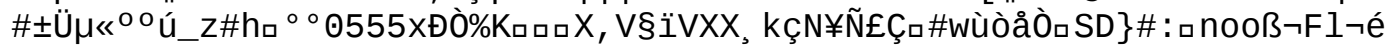


Ü7ロ \#ロ cÁQ\#7o( *) õéÓgÝ̛́

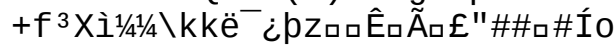




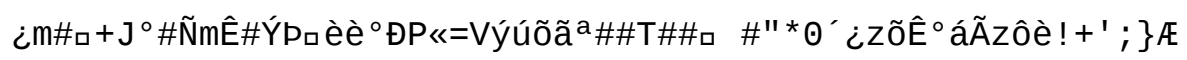




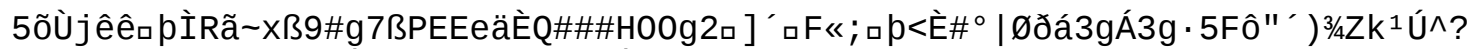

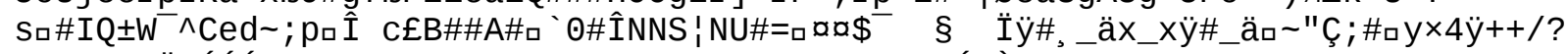

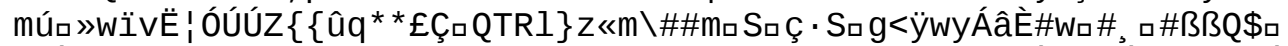

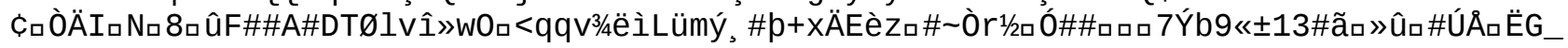
éñàA: :\#Õロ

A\#\#А\#\#А\#\#А\#\#\#\#А\#\#А\#\#А\#А\#\#А\#\#А\#\#\#\#А\#\#А\#\#\#\#А\#\#А\#\#А\#А\#\#А\#\#А\#А\#\#А\#\#А\#А\#\#А\#\#А\#

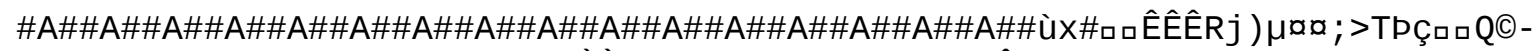

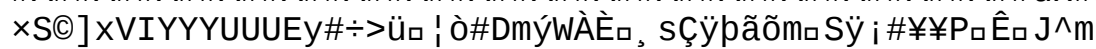

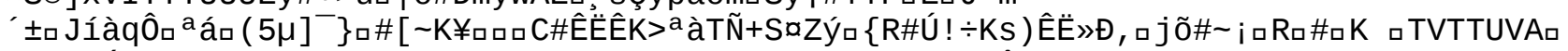

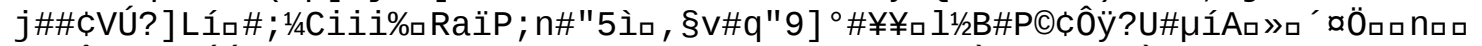

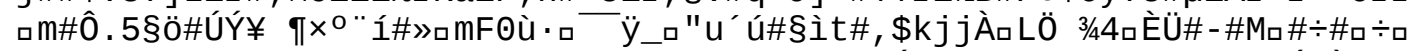

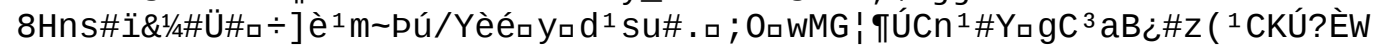
2 ÙÂ\#" $¥ \mathrm{X}^{\circ} @[\mathrm{G} \neg \neg \AA ̊ \AA \#$

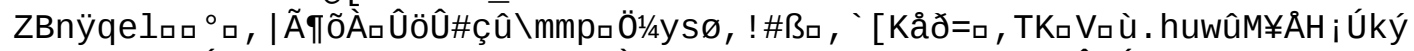

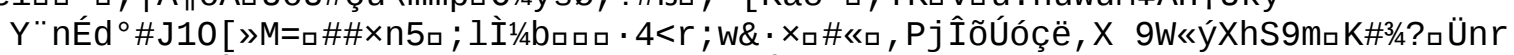

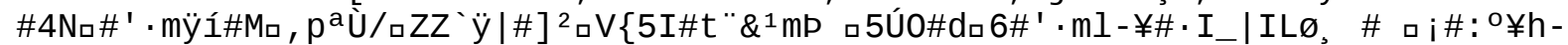

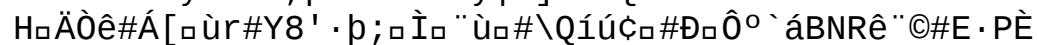

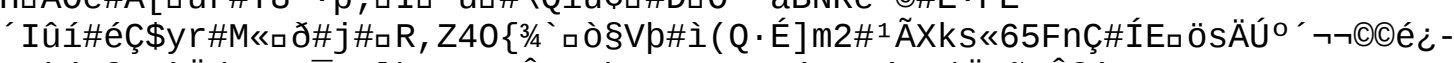

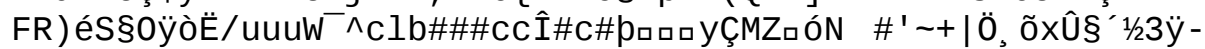

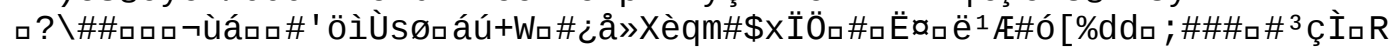


\#2ÄÀĐ@øçロ=ロNi [QFÆ\#gùıbôpo; ${ }^{2} Đ e ̀ ı E \odot \# n \# I ́ \wedge 6 \odot 4 T \#-$

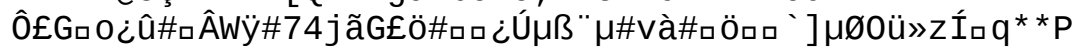

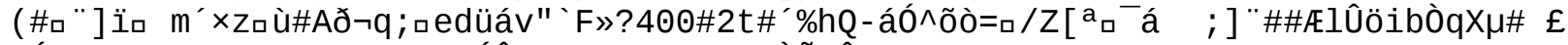

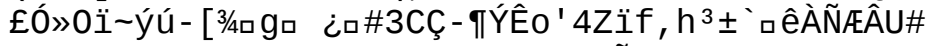

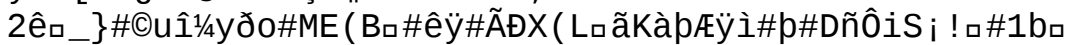

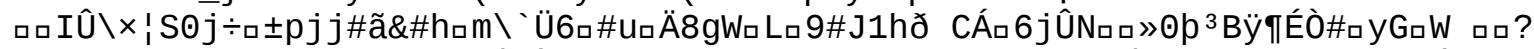

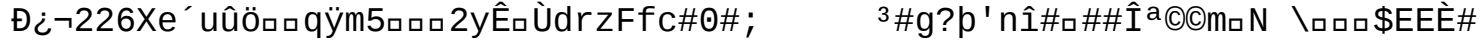




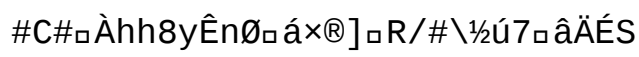




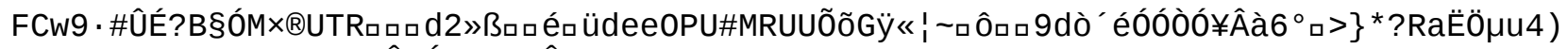

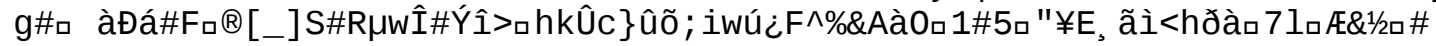
$\# \#^{*} \#^{3} \mathrm{~b} \AA$

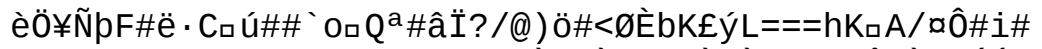

口DRSS«" "

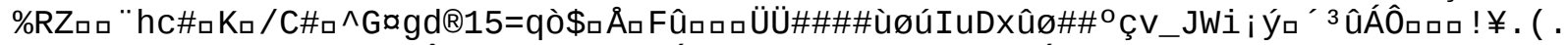

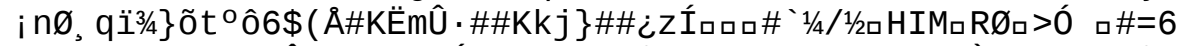

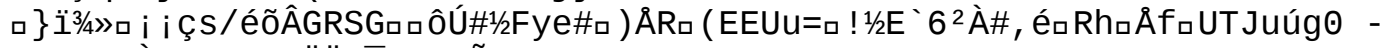

IÕ̃ · a\#ĖIRÿ\#\#' $\mu$ ÖÖI ${ }^{-}$ýlHUÕR:

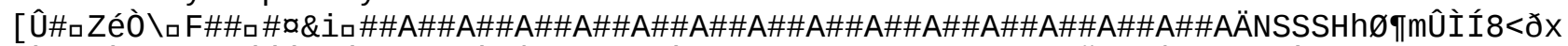

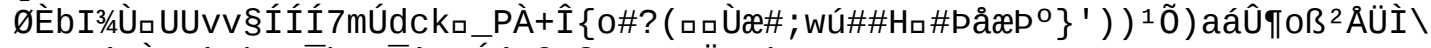

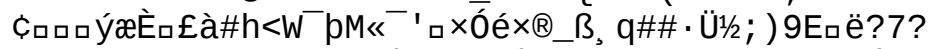

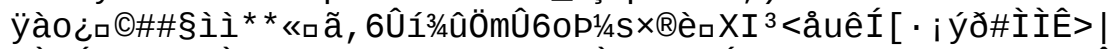

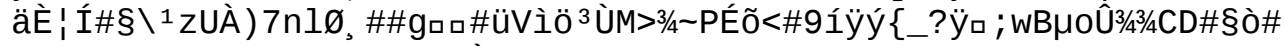

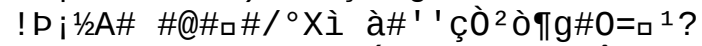

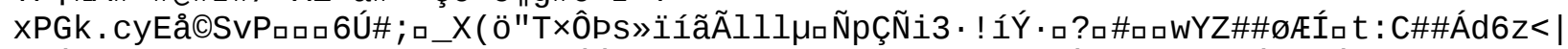

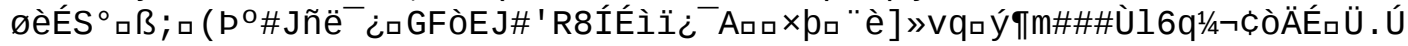





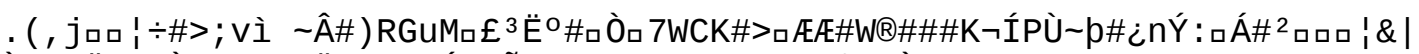

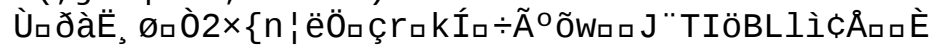




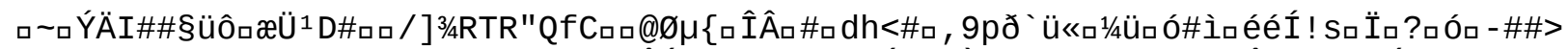

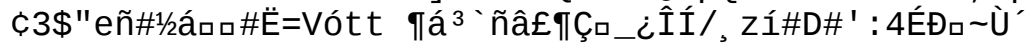

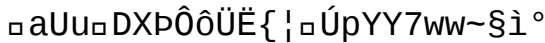

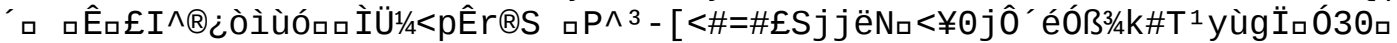

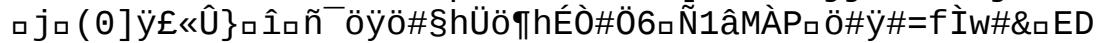

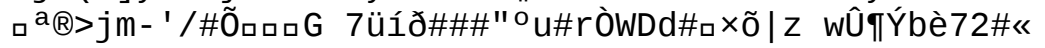




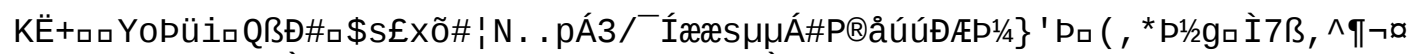

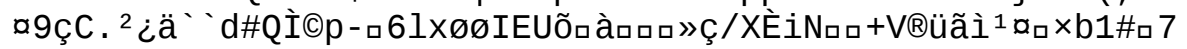




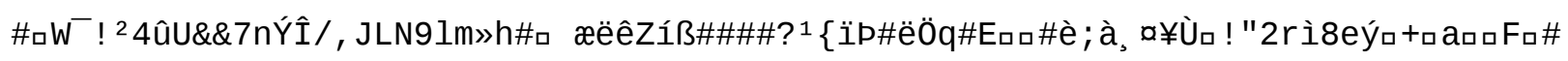




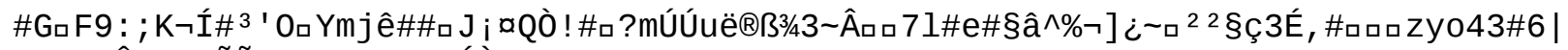

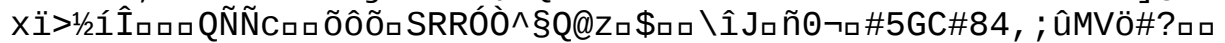

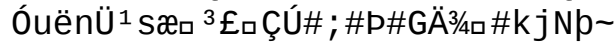

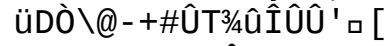

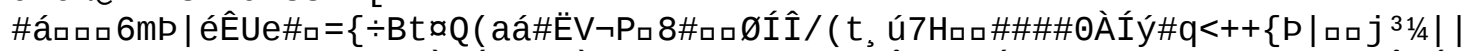

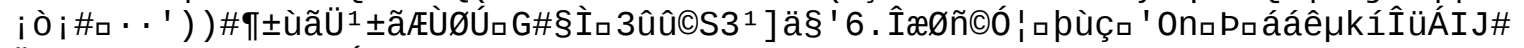

Äòn ¿ü\#q\}ãörõ?p8eú û\#\#ロ ;

YÝSSSTRZ: = \#ロÂb1 


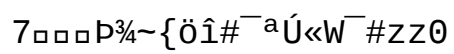




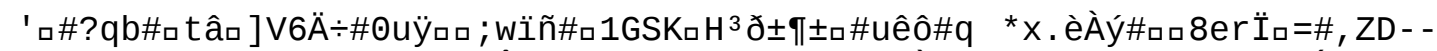

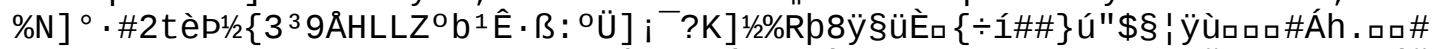

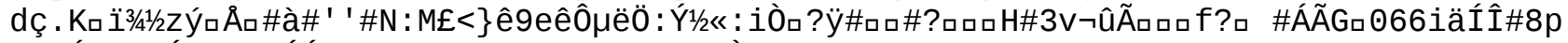

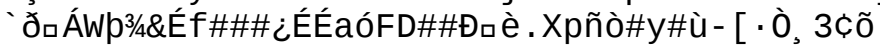


Æ/\#7 3|ØÃ $\square$ \$3

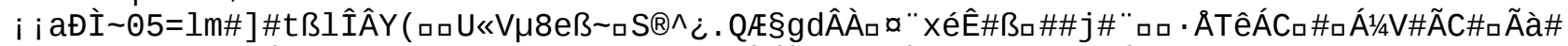

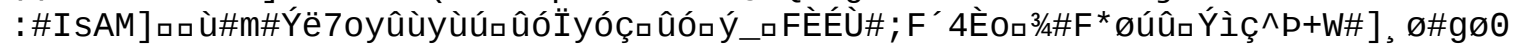
$\square$ \#.̈̈q

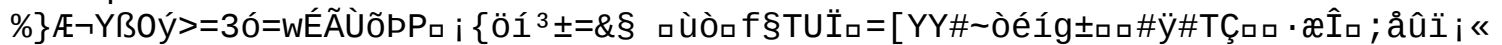

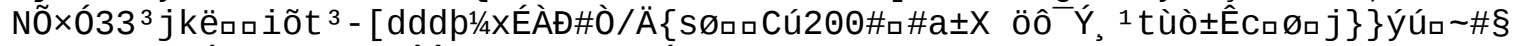

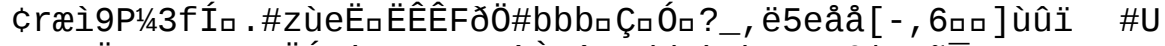

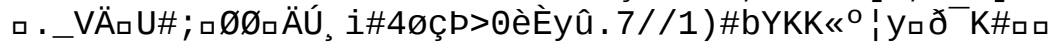




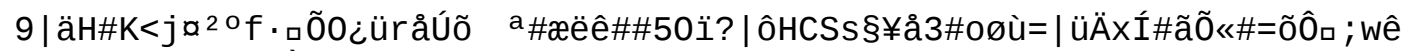

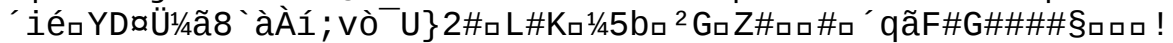

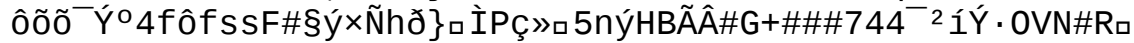

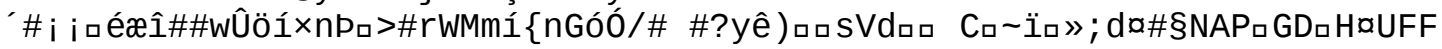




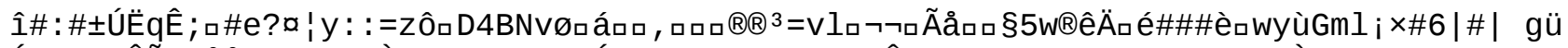

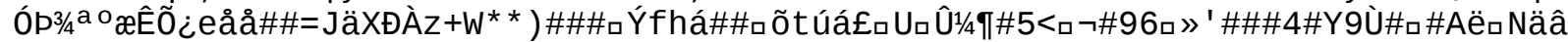

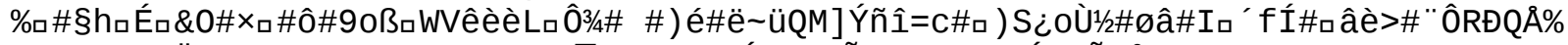

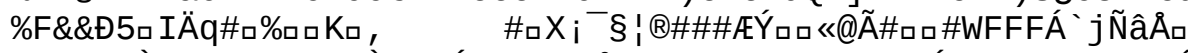

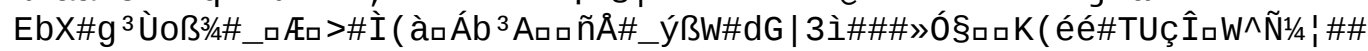




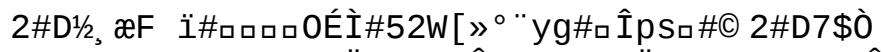

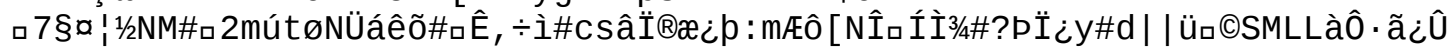

'Ù口q »ça |

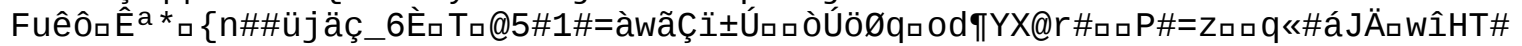

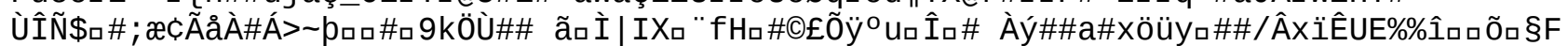

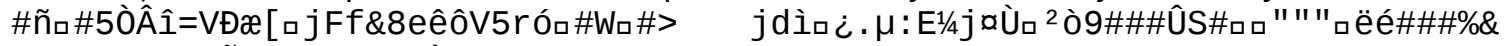

\%eåำ $]^{2}$ téxUÕa\#5\#ÄU\#? üÀda ${ }^{1}$ ûa $3 / 40$ a $P$

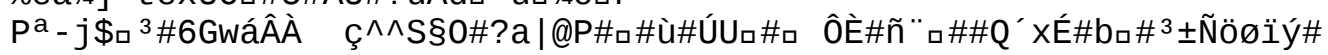




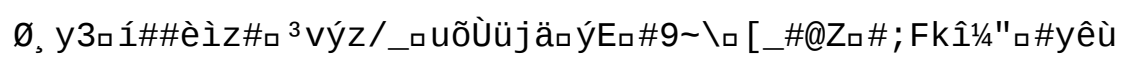




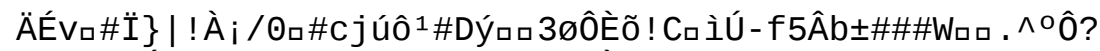

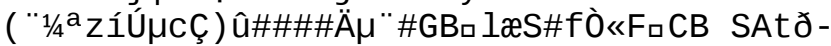

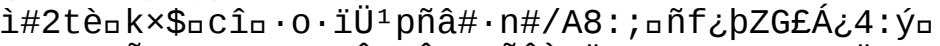

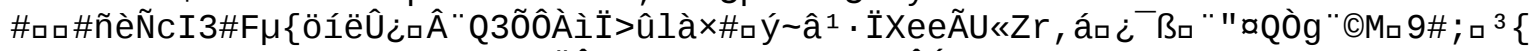

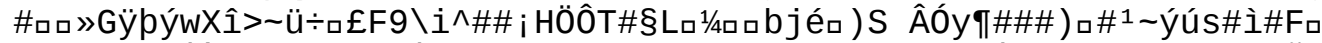

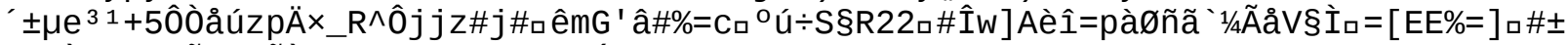

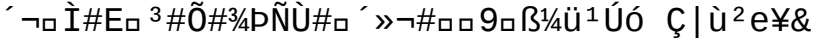




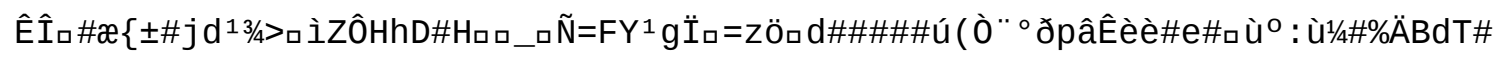




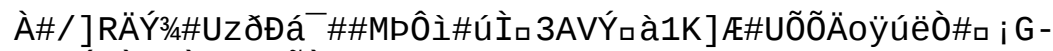

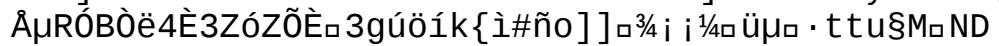

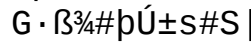

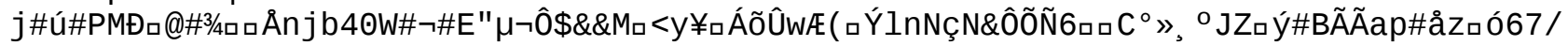

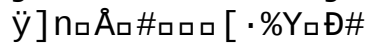




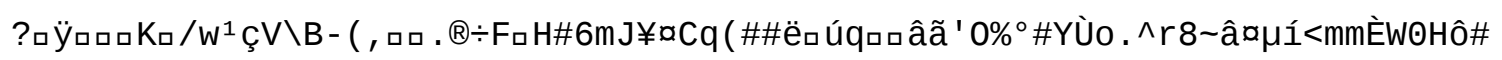





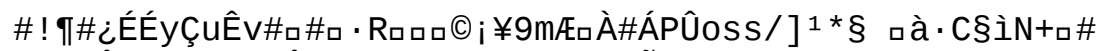

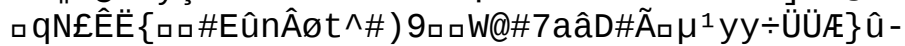




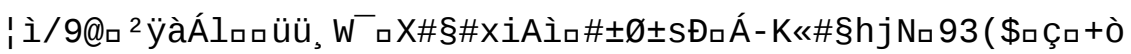

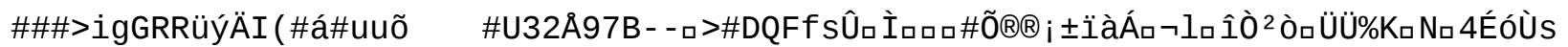

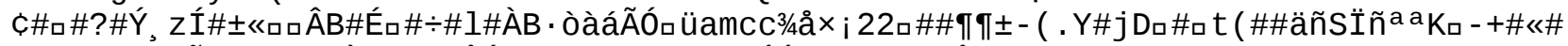

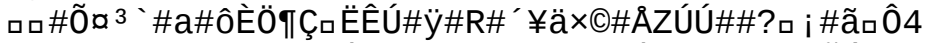

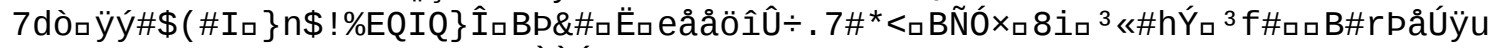

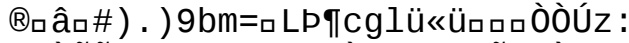

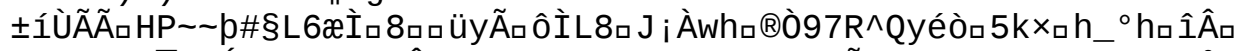

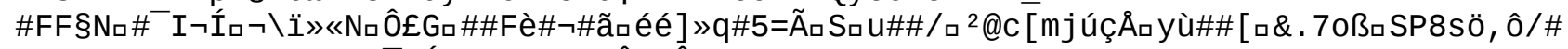

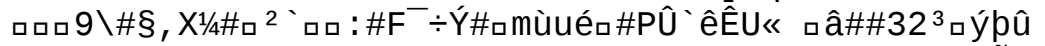

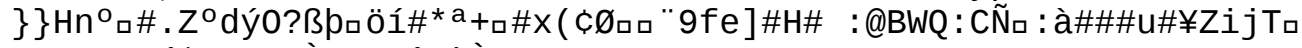

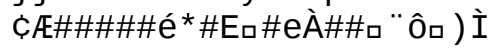




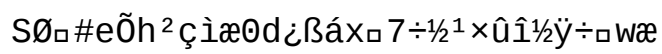




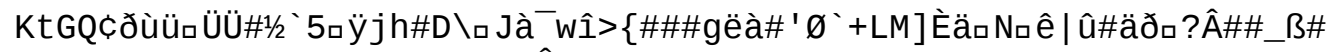

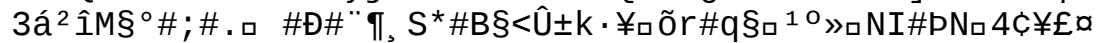

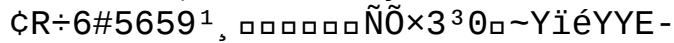

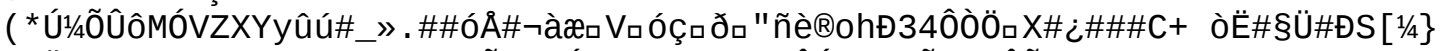

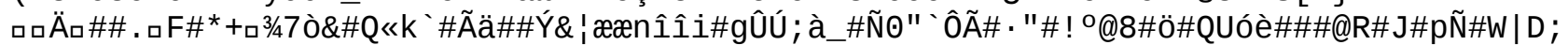

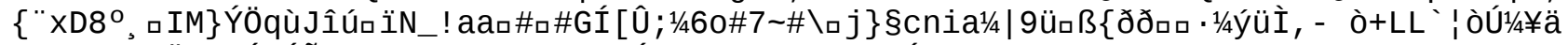

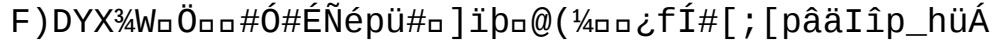




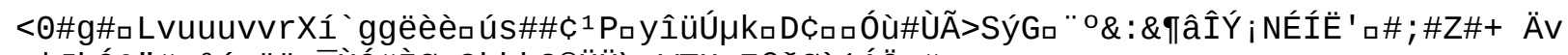

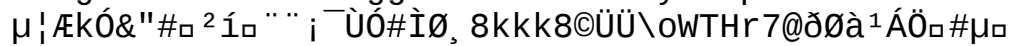

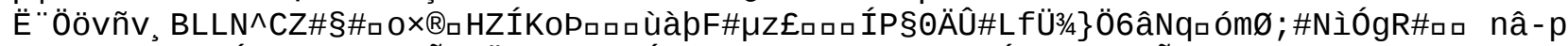

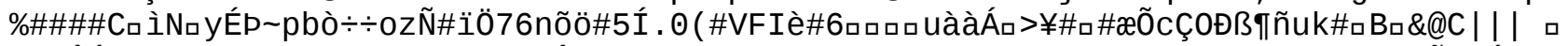

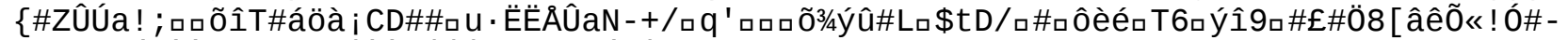

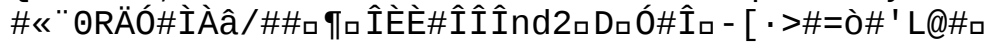

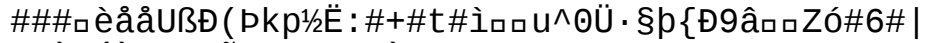

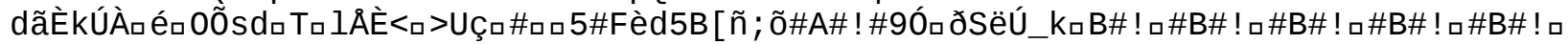

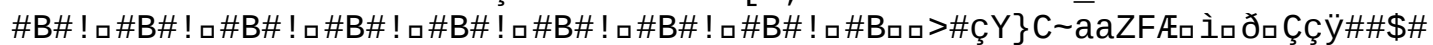
$\{$ é 


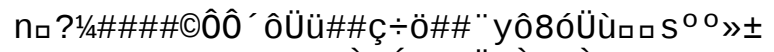

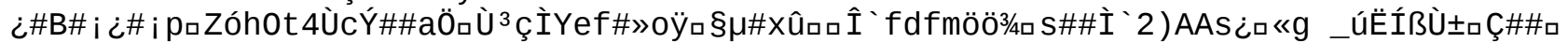

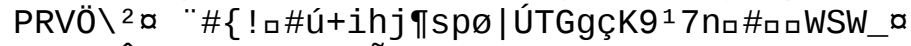

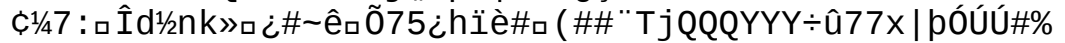

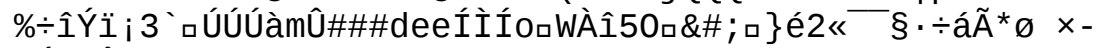

$\mathrm{mÍ} / Z \hat{E} O a W \times<e ̂ c s A ̈ \# \neg^{a} y$ tòLJFÖ98\#ロ $\AA^{\mathrm{a}} \neg \mid$

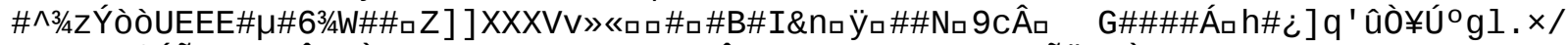

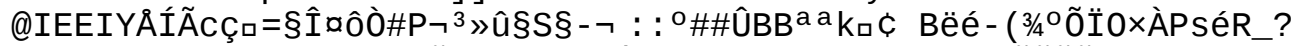

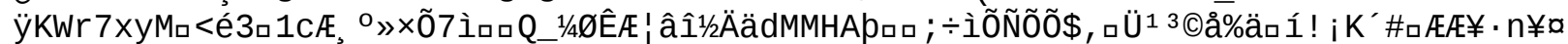
$¥ \ll " \sim o ́ \mu^{\text {a a } x æ i ́ 1 ~}\{c c o ̂$ 


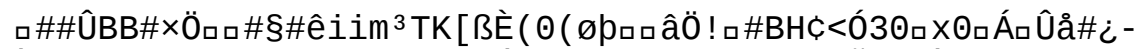

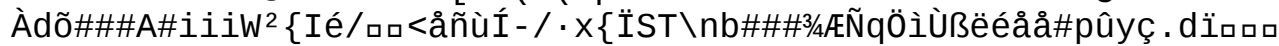

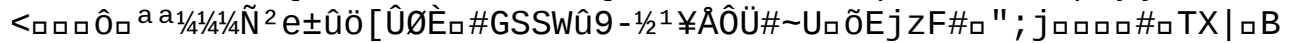

ü|

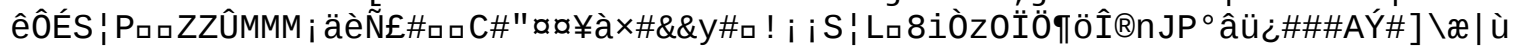

$\% A K+y ́ l: ø \hat{u}^{*} \# ! \square \# \square \# \div+\left.\#\right|^{a}$

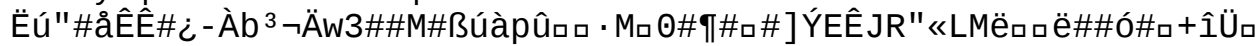

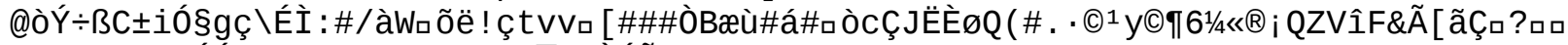

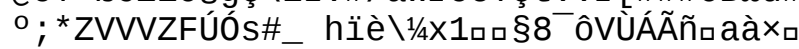

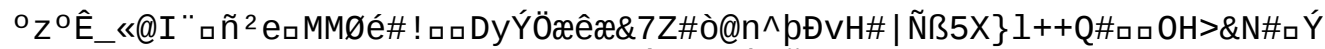

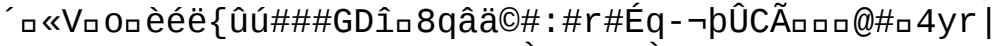

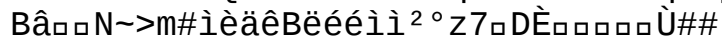

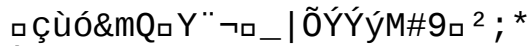

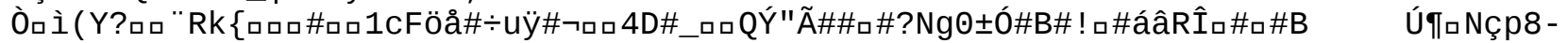

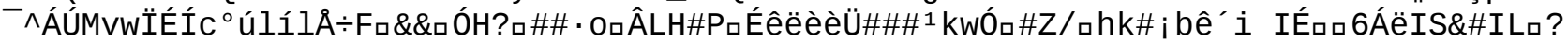

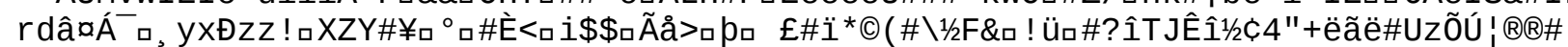

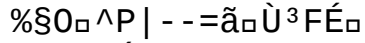

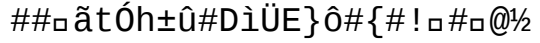


Ft $\backslash U ̈$ UU $\ll H N N[\} \mid$ |

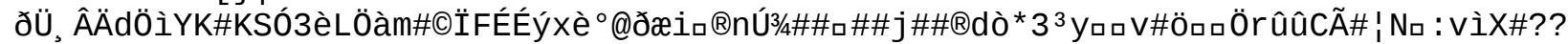

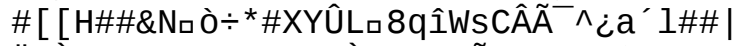

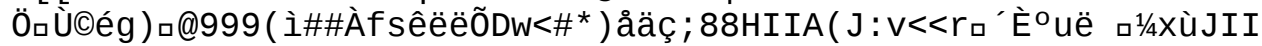

JBÔÉÉËg ${ }^{3}$ Ũ

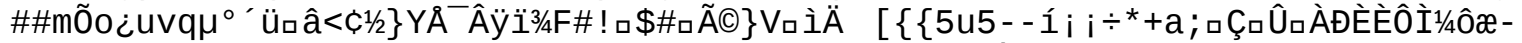

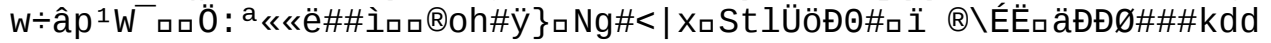




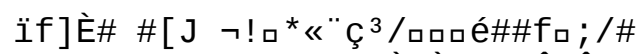

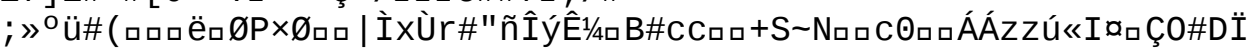

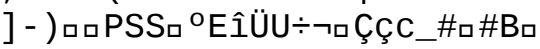




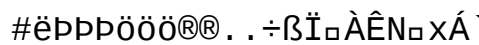

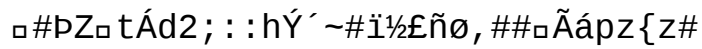




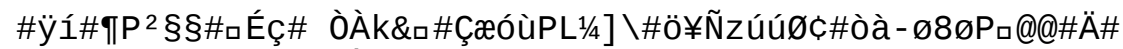

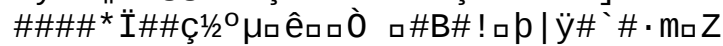

\section{endstream}

endobj

$15 \odot$ obj

</Length 1525

/Filter/FlateDecode

>stream

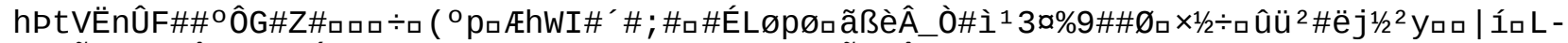

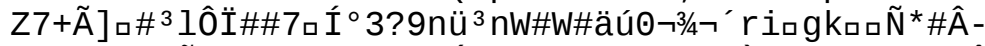

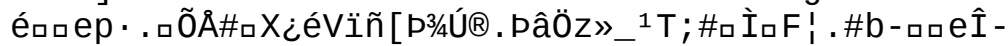

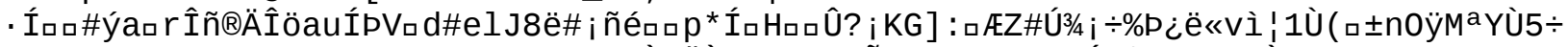

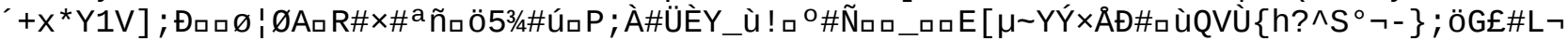

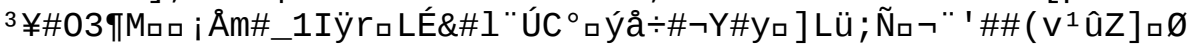


çiD\%Ùö̂ि\# $73 \mu \#$ a ${ }^{a}$

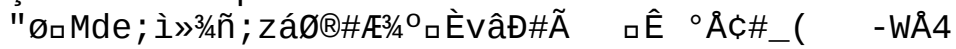




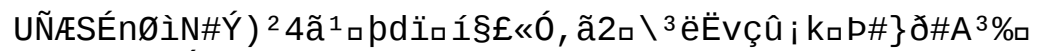

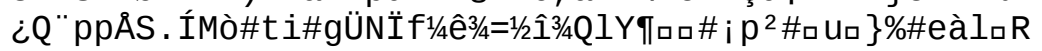


û9\#! ${ }^{-} \# 1$ b 
》)

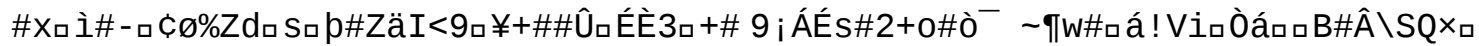

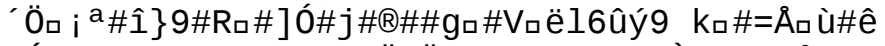

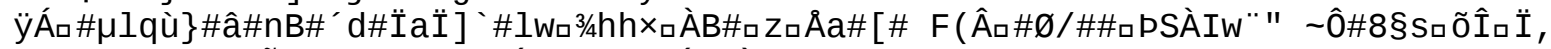

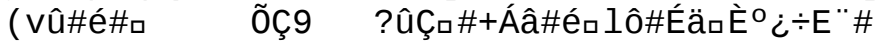

um\#Óquqd G\%Cksıq\#ロóà£ó\$jG7 


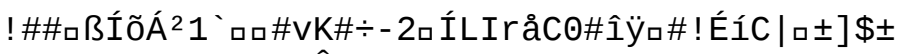
ฉロ\#\#6B\#ロ\#?Fö\#ÇםÔ 


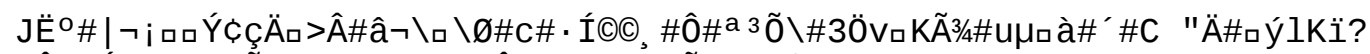

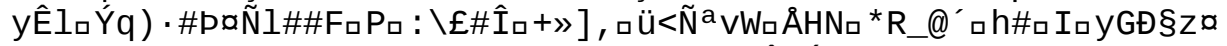

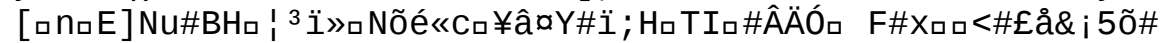

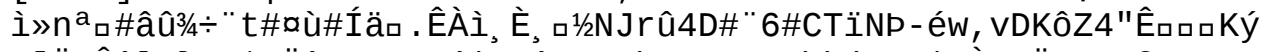

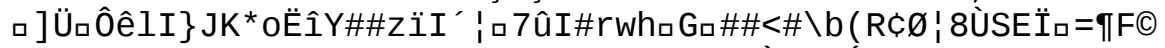

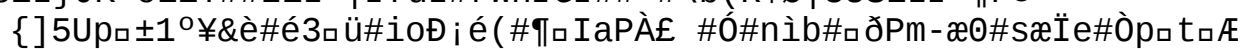

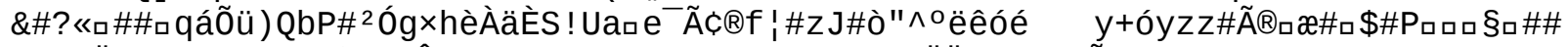

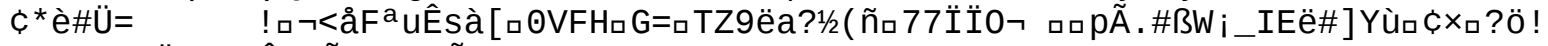

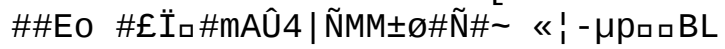




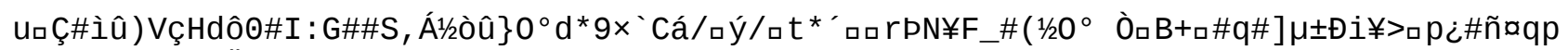

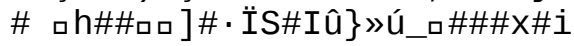

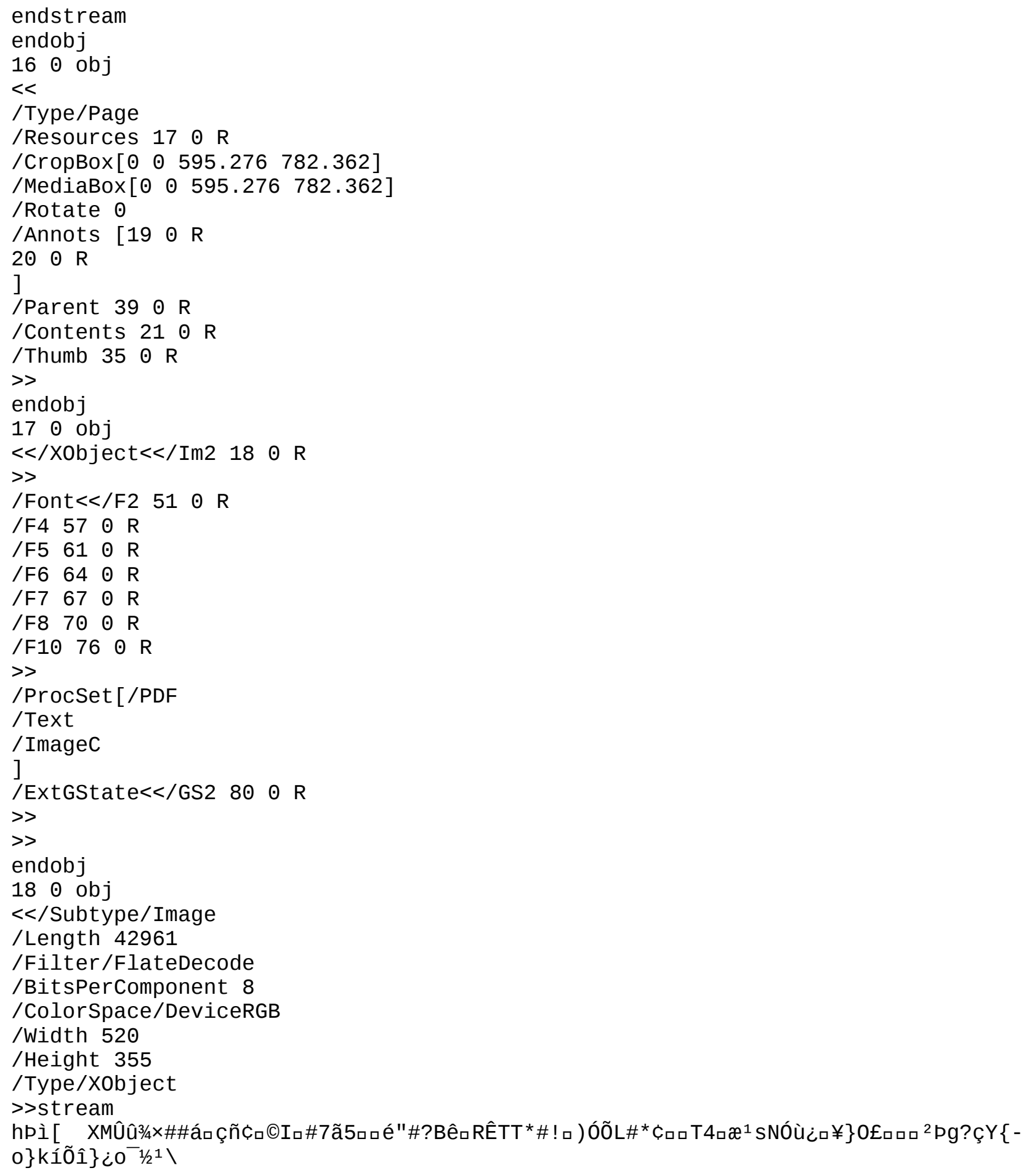

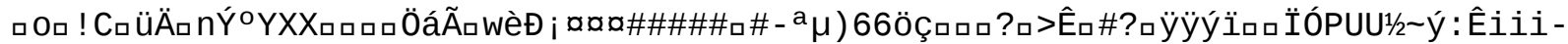
$[\cdot \square 6 \mathrm{mZ} \cap \AA \sim 丿 \tilde{A} a 9 s \& Y$

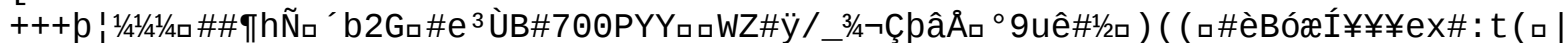

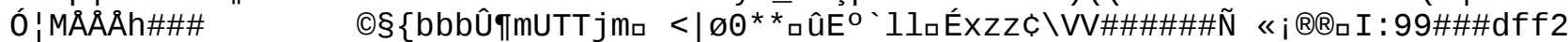

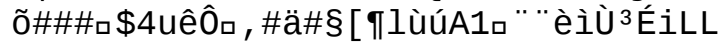




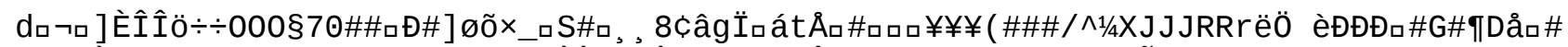

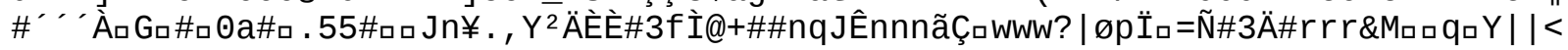




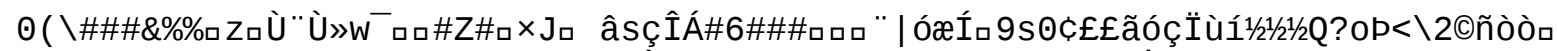

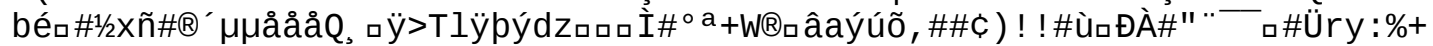

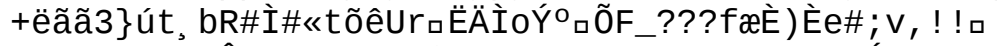

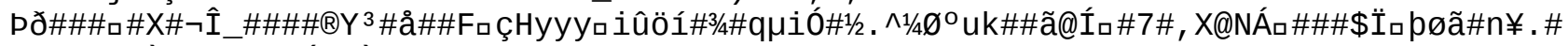

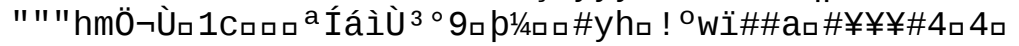




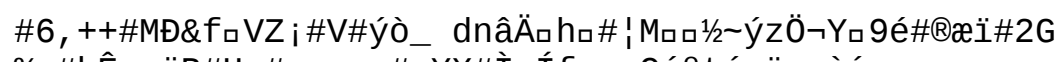

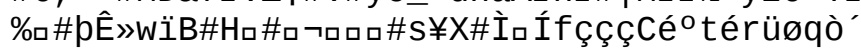

口 


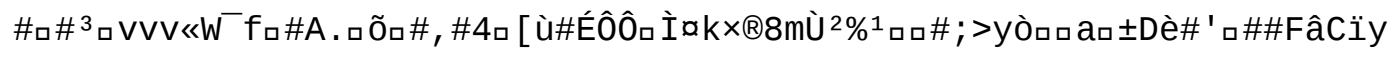

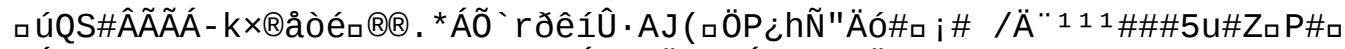

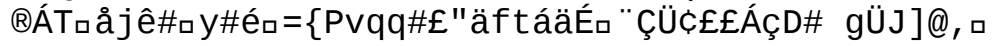


"\$\$aGa\# J 


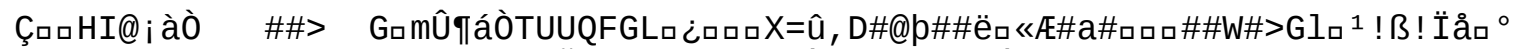

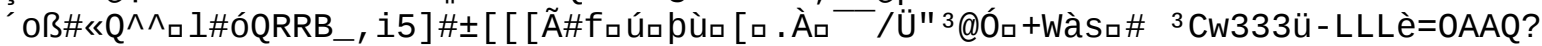

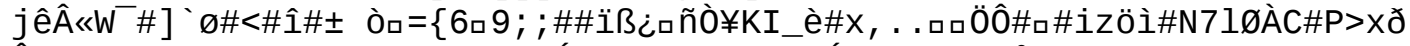

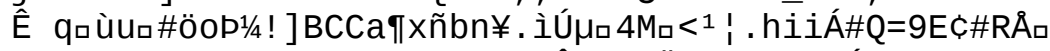

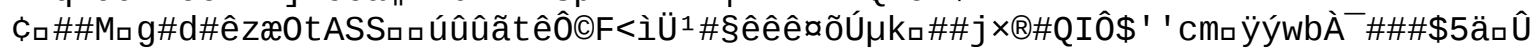

口;WP\#ロáÖ!\#\#\# 
ecccc\#111X| 


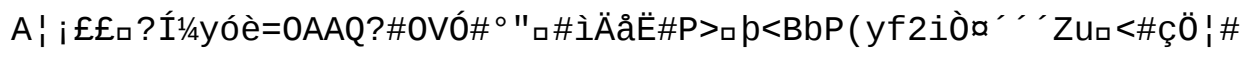




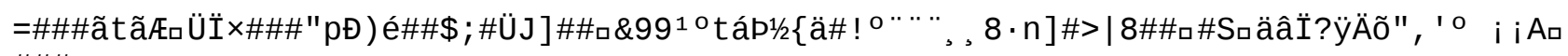
$\# \# \#$ p

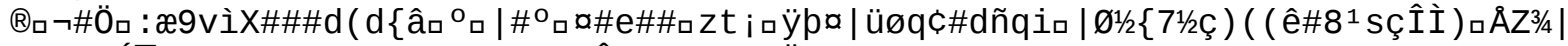

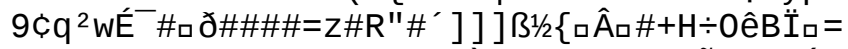

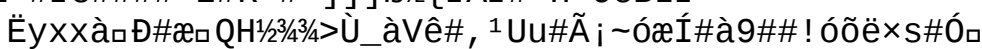

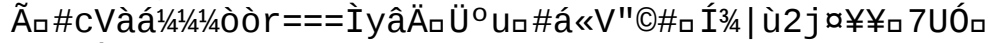

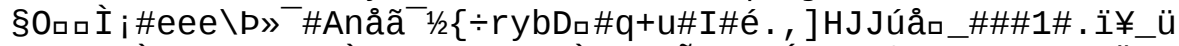

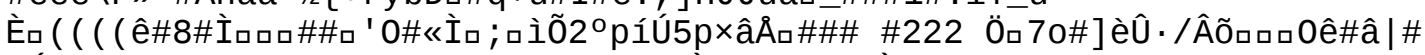

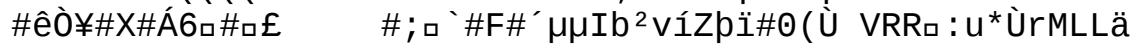

! \#àI a ' $\mathbf{0} q ¥ ?$

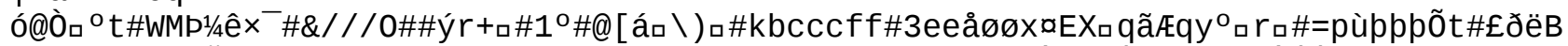

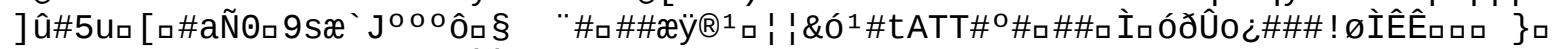

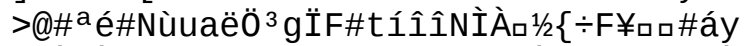

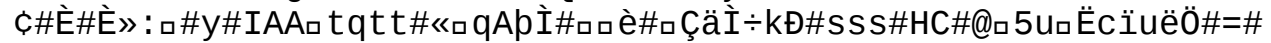

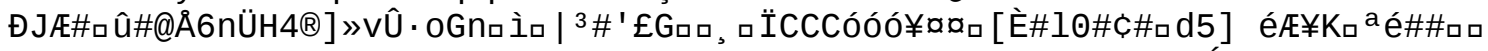

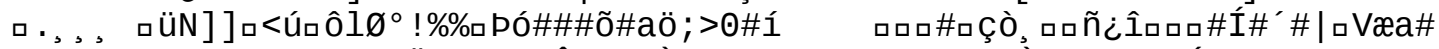

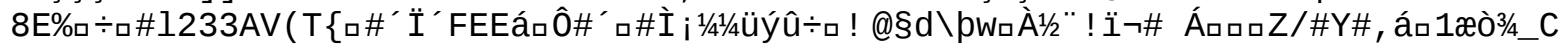

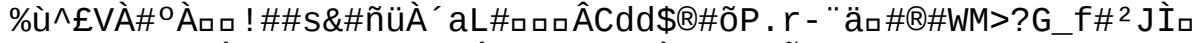

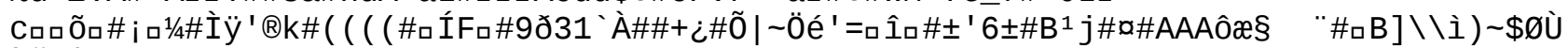

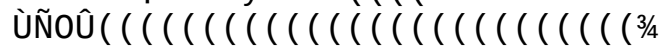




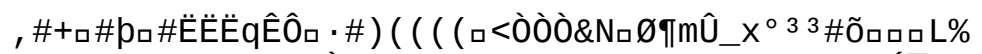

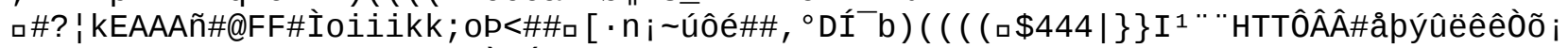

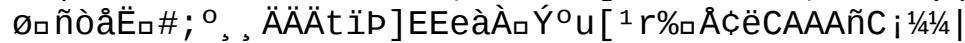

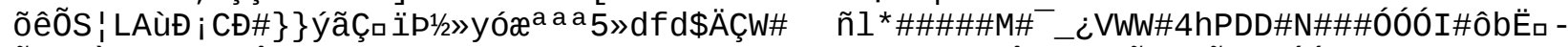

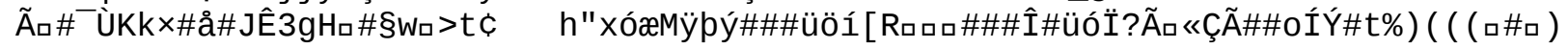




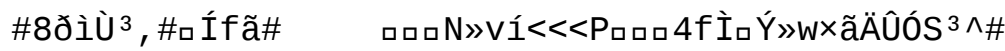

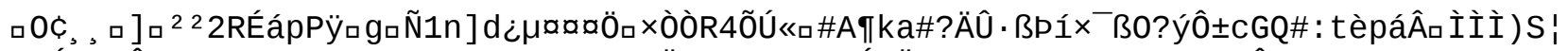

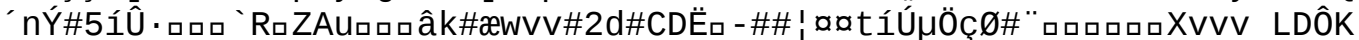

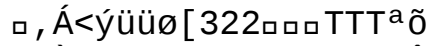

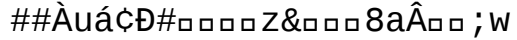

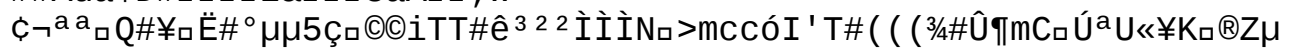

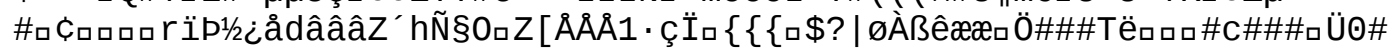

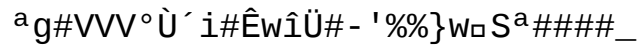




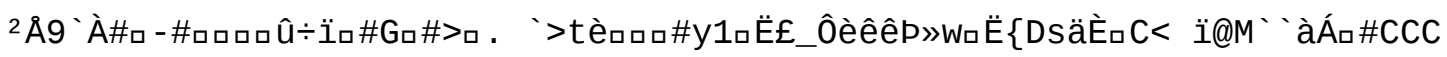





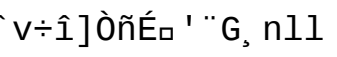




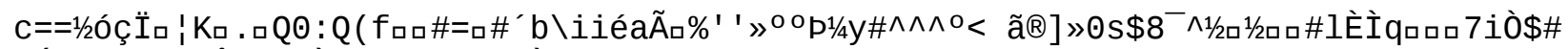

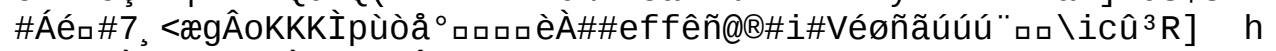
(ç\#äÒıs3Rò\#ôĖúq_Z\Ê 


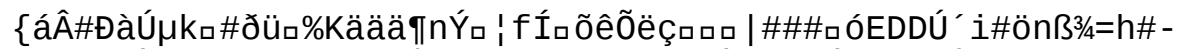

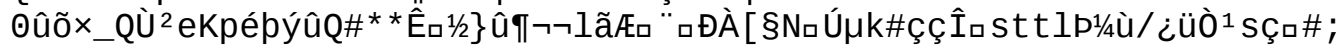

cõöíÛd2aqu"A+FiÛTmë0̈544\#ü\#í\#\#\#Æ\#ロ\#ê1

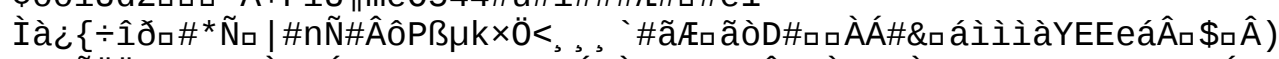

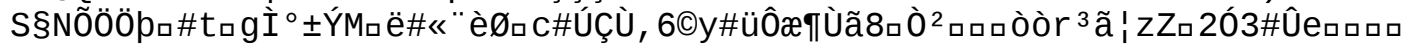

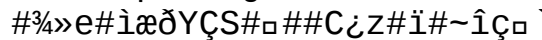

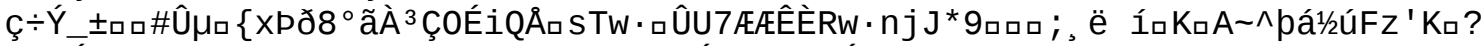

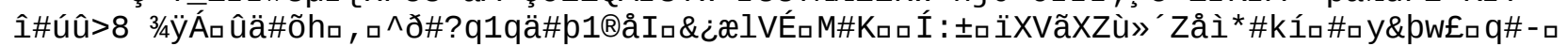

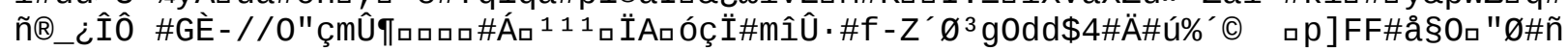

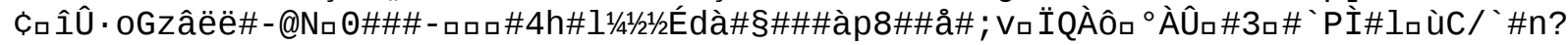

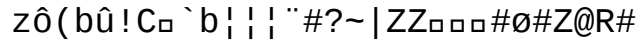




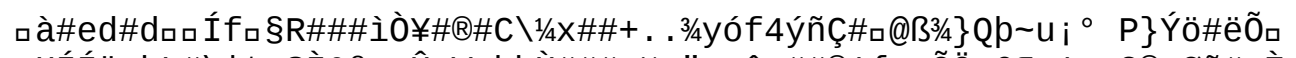

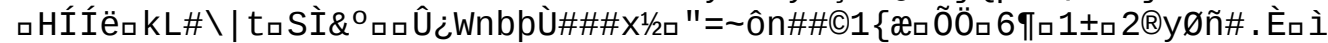

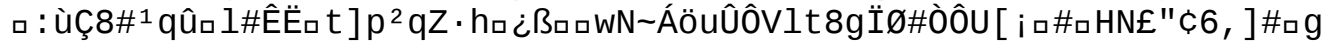




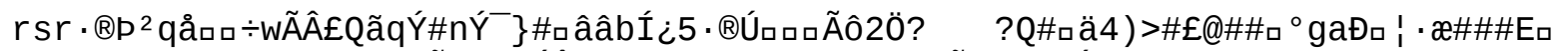

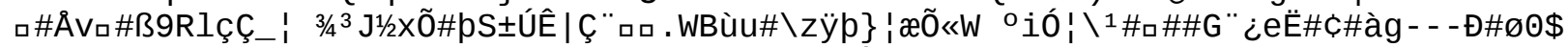

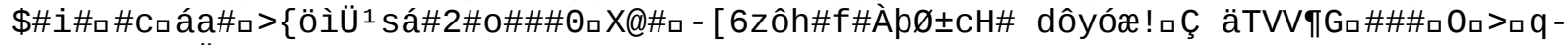
$\neg \neg ® \wedge 1 / 2 \square \square ` r u ̈ B \square$ 


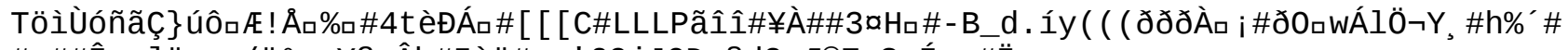

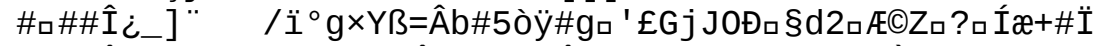

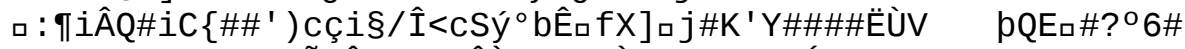

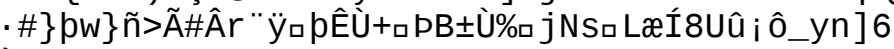

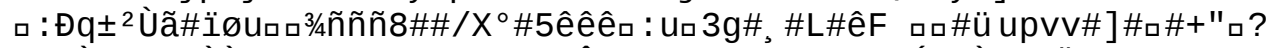

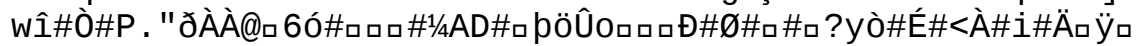

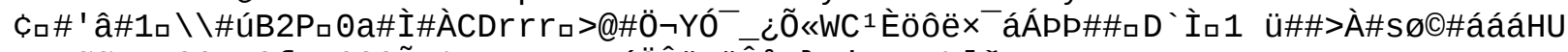

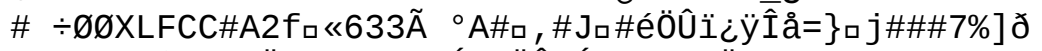

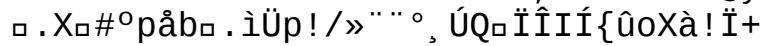

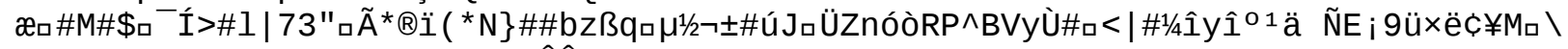

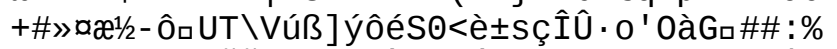

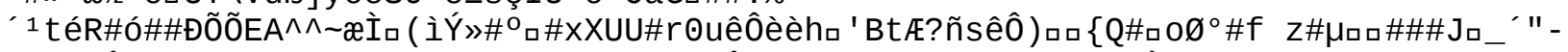

'i \#

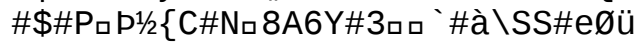

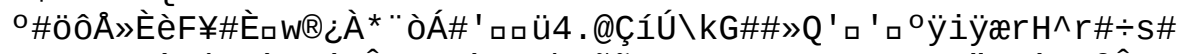

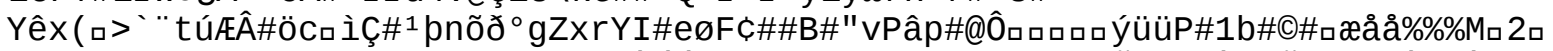

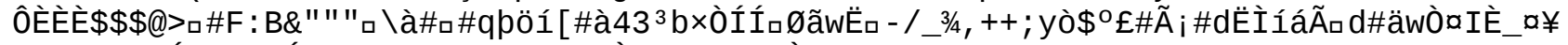

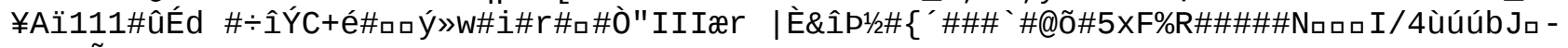
$\square \square \# \square \tilde{A}$ 


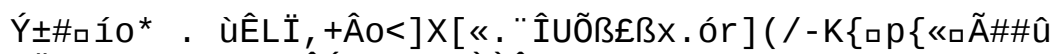

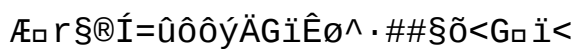

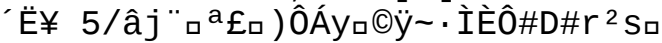

\#ロåÍëロ\#0̃\#

口० 


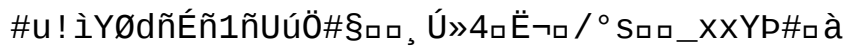

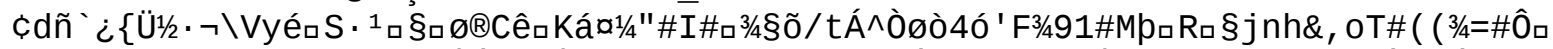

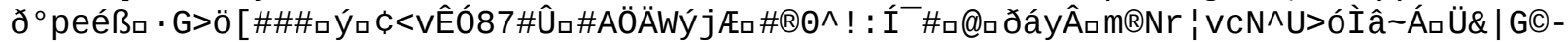
$W^{3} 6=+, 0 \square D \# N$

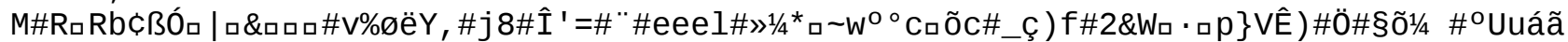

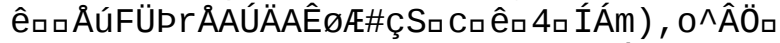

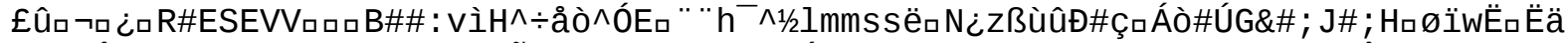

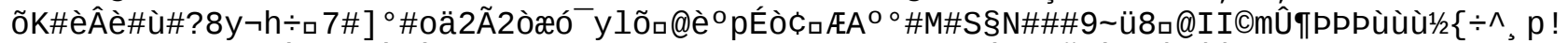

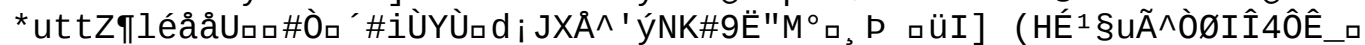

$\mathrm{K}$ \#Ý

$\emptyset, 1 U^{\prime}$ éÖ $[a$ 


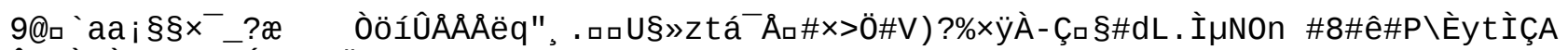

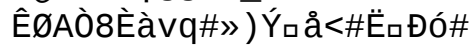

口\#\#ロ

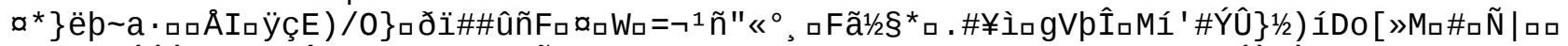

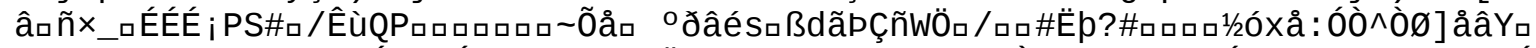

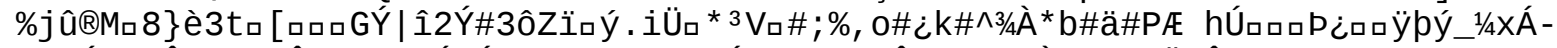

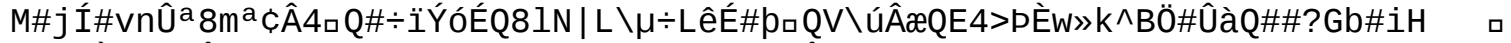

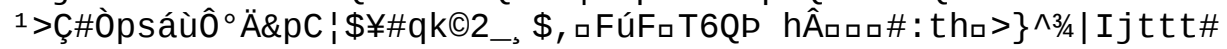




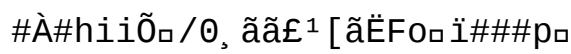




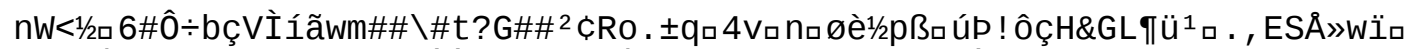

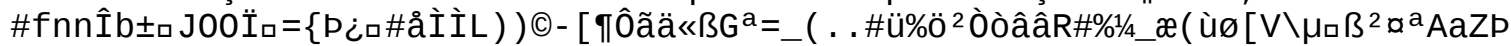

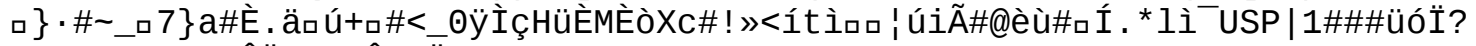

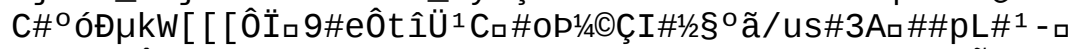

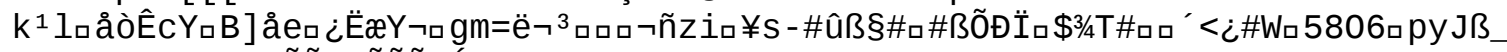
\%\# . PP4mXZZ\#ÇÃÑ£GÃÃÃIÓa; $W_{\square} \tilde{n} @ \# \square Z$

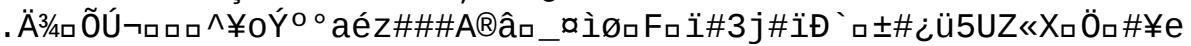

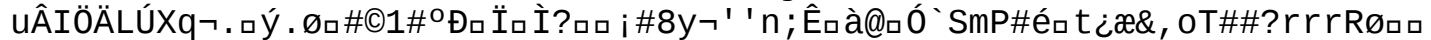

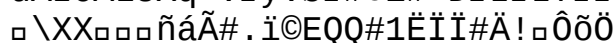

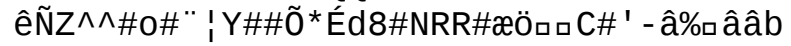




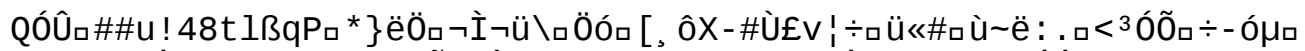

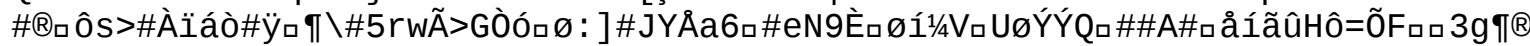

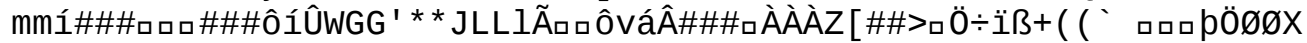

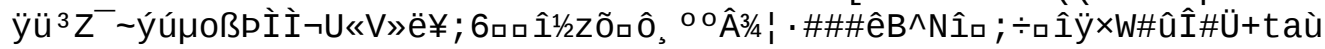




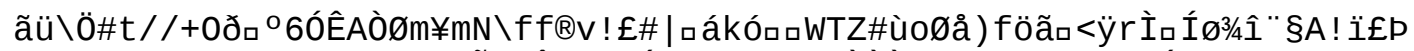

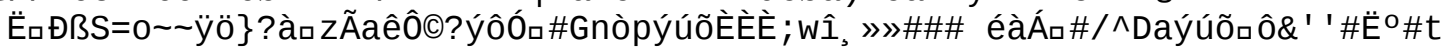




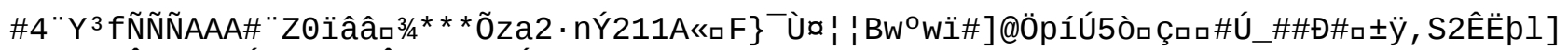

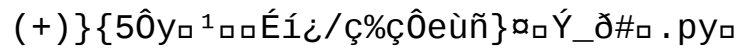




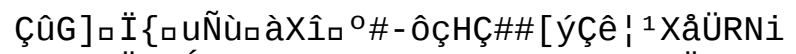

»ä\#\#İ«\#, WåååÅÅÃãããıロ\#\#\#kii!ฉロ

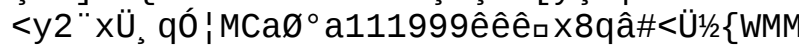

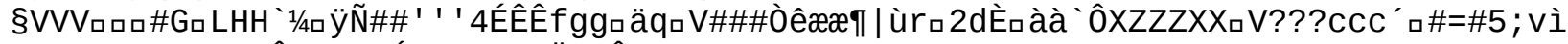

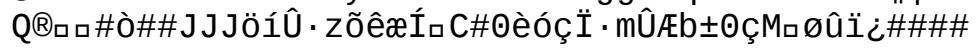

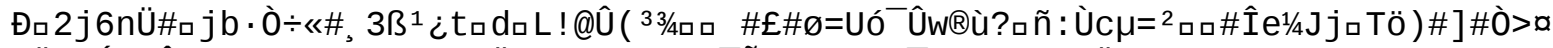

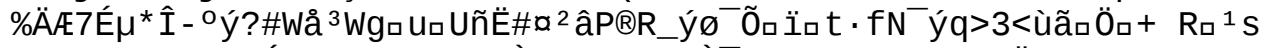

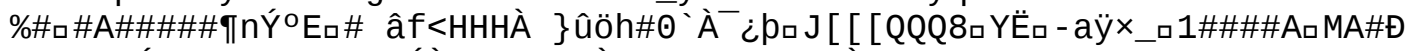

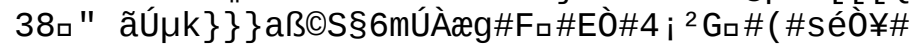

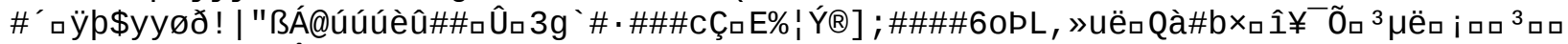

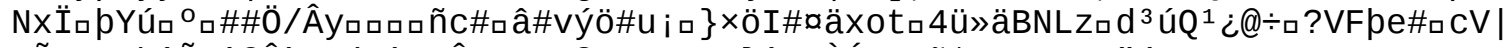

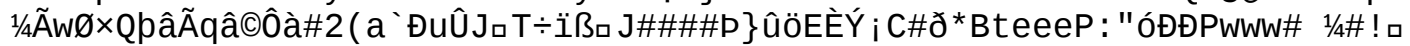

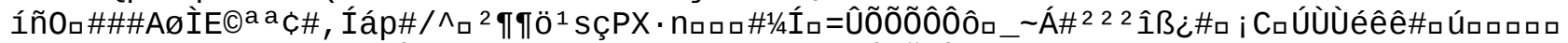

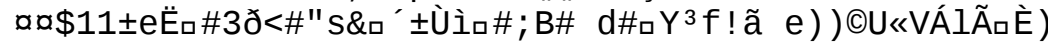

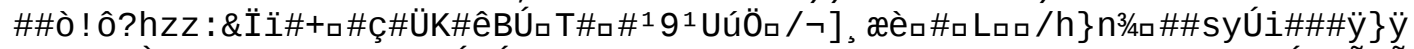

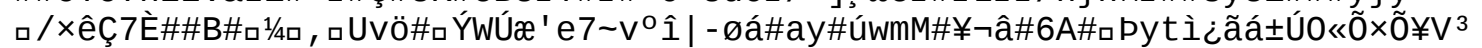

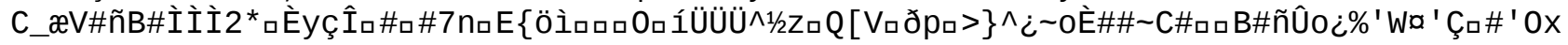

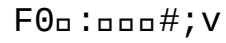




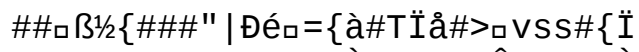

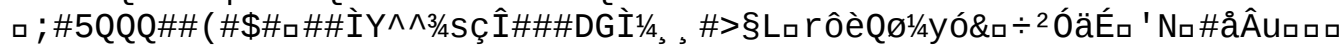

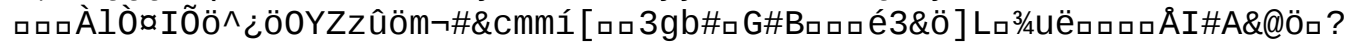

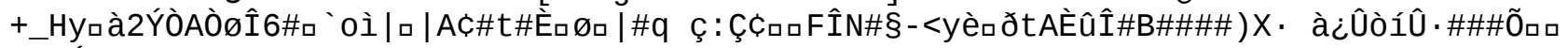

口 ‘Ó§O£\#ロ 


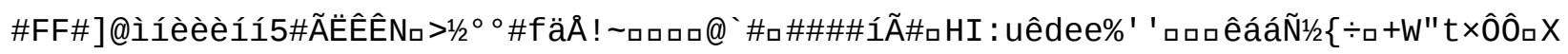




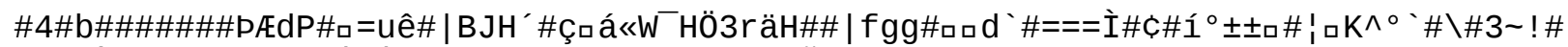

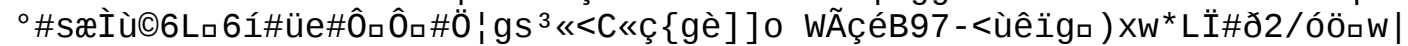

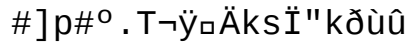

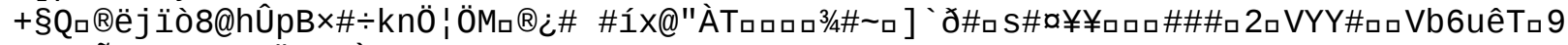

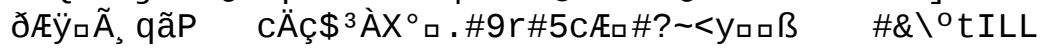




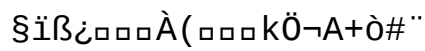




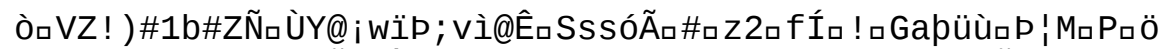

\}m ! ä1 \#ÆUTTTTPPÀ/\#ロ\$V

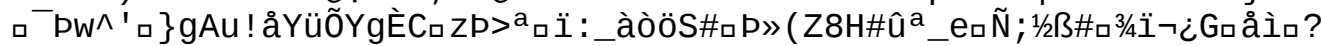

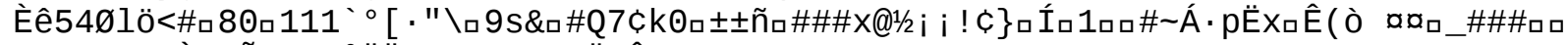

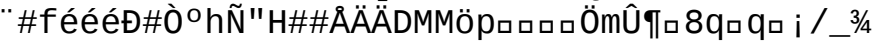




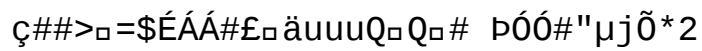

\#ロव 


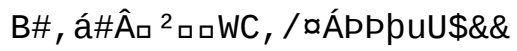

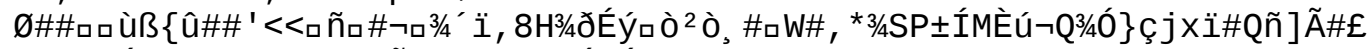

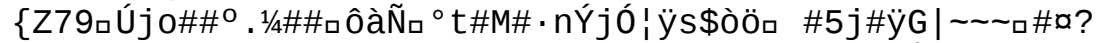

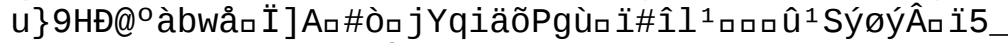

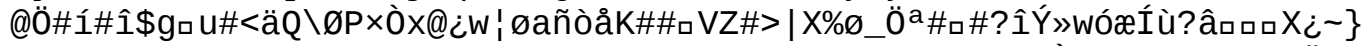

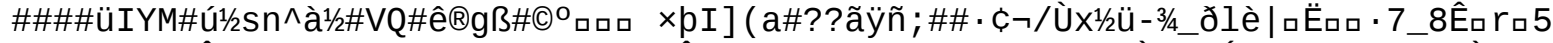

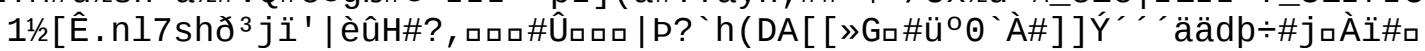

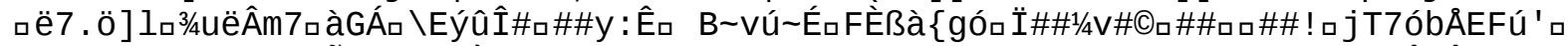

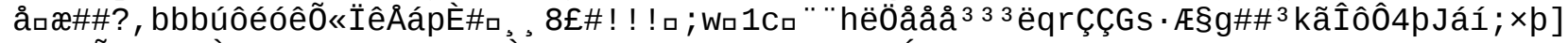

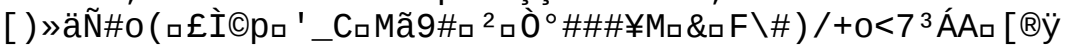

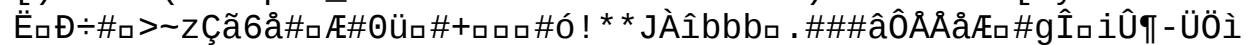

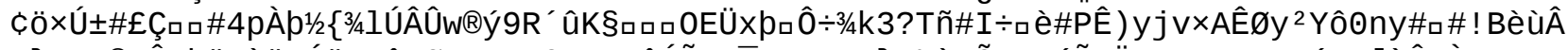

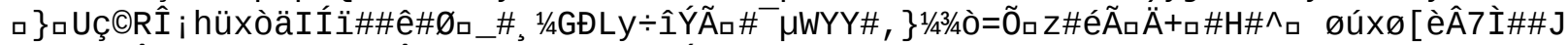

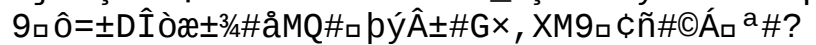

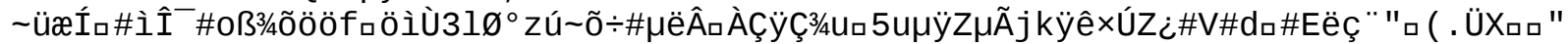

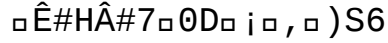

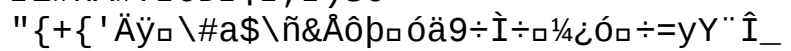




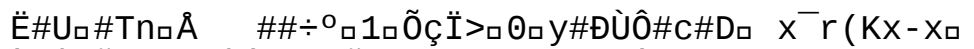

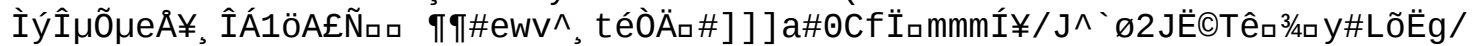

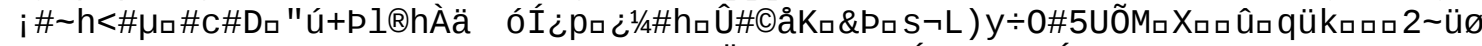

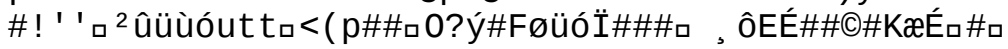




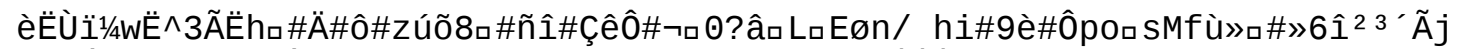

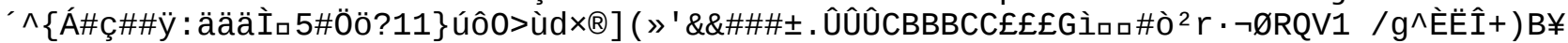
ÕÙy\#HáuH口x

$\{\#$ iRıÛ3: v

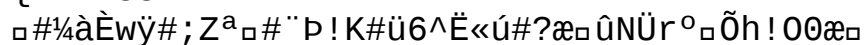

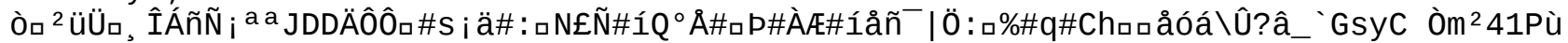

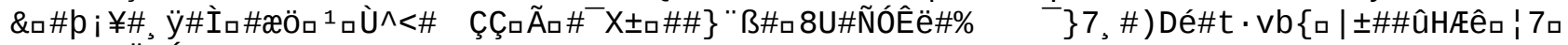

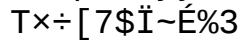

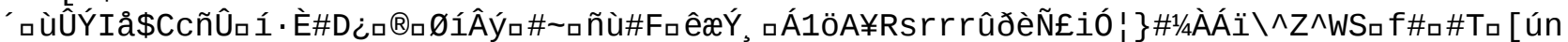

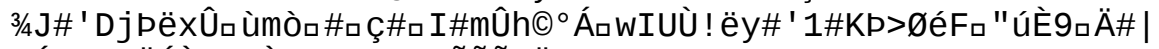

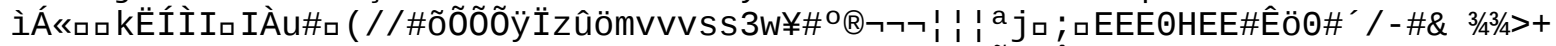

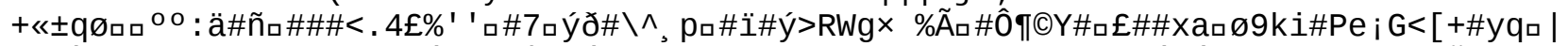

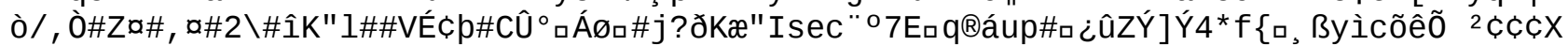

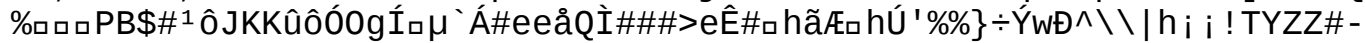

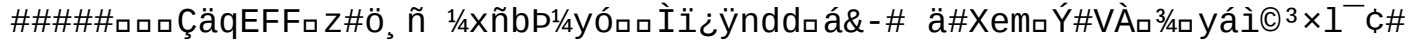

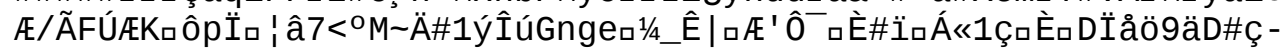

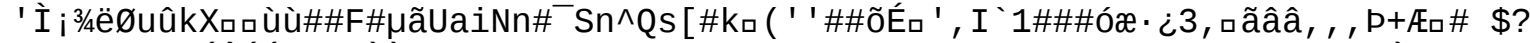

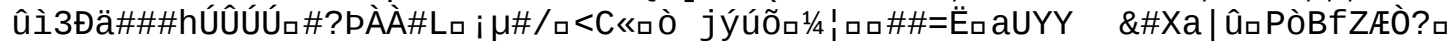

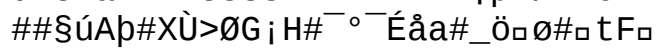




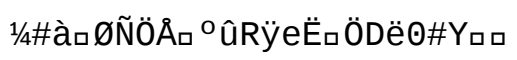




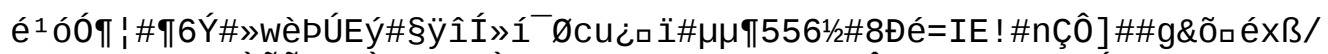

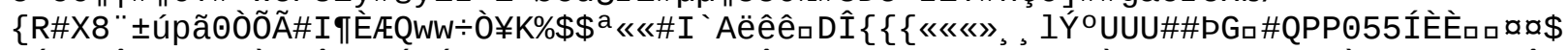

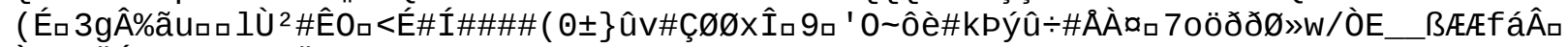

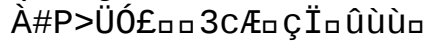




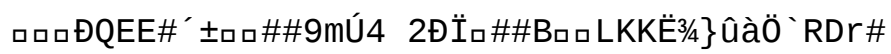




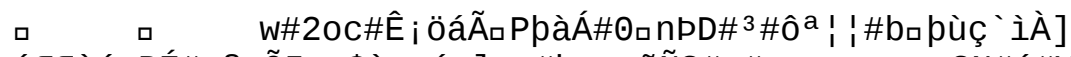

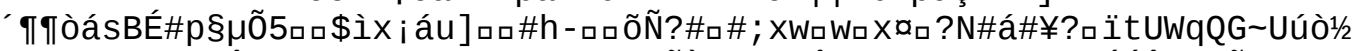

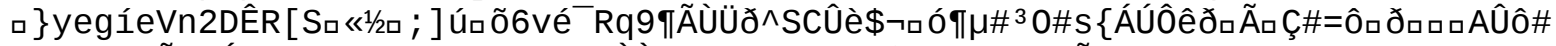

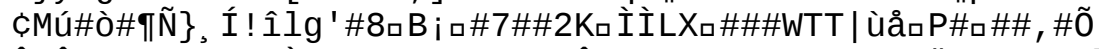

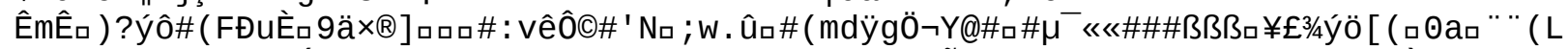

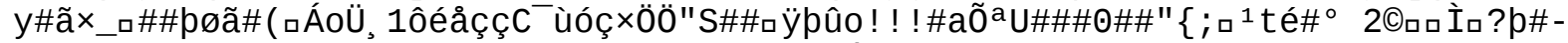

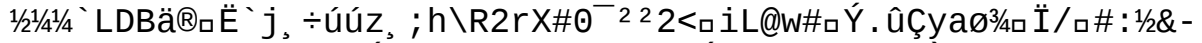

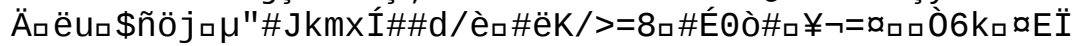




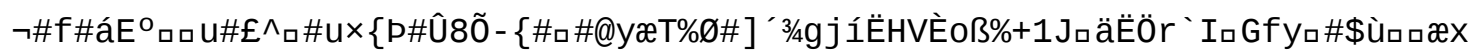
«â $\left[\cdot n_{\square} \hat{e ̂}\right.$ वK\#Xr\#

$1 \neg$ ר 
$\square ?$

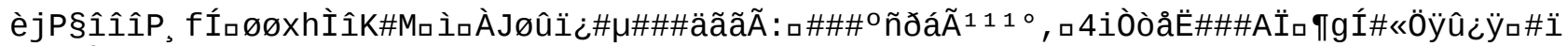
Ü13 1 Uे2P i वप्र E\#\#\#aL $\left\{\left\{\hat{u ̂}^{-3 / 4 u ́}\right.\right.$

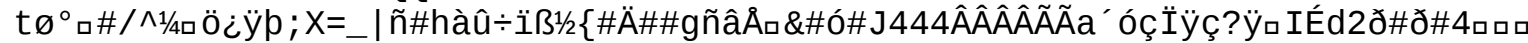

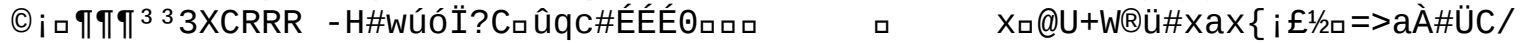

Ëõ] ̈̈в ฉ\#ó

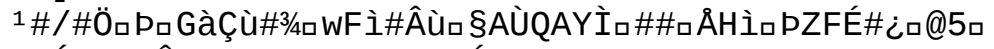

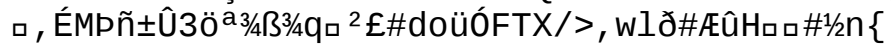

$\# / 1 / 4 \ddot{e}=\mathrm{dD} \# \#$ $h_{\square} / 9 U q \#$

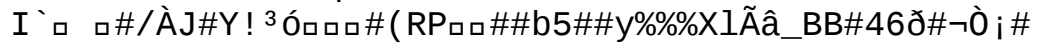




$$
\text { ㅁㅁ }
$$




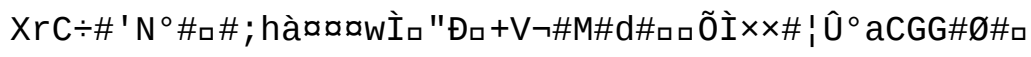




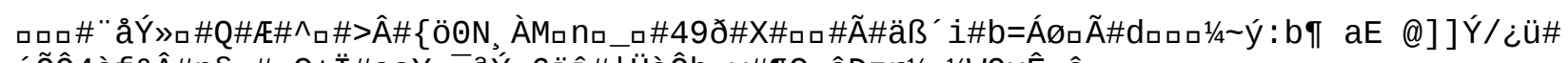

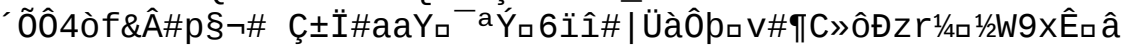




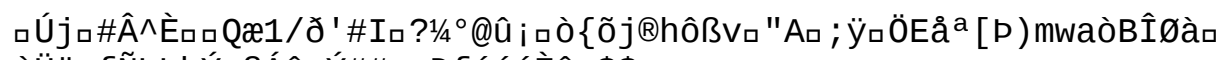

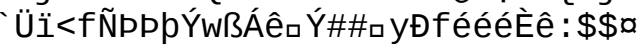




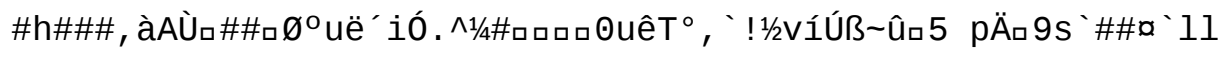




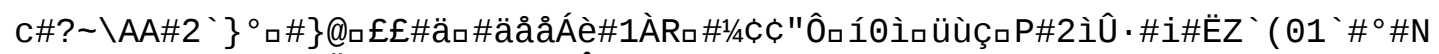

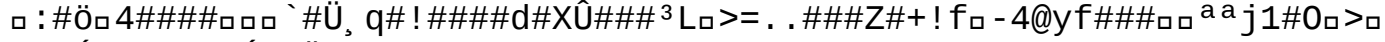
Ç, nÝ: $: 0 G]] \square Y ́ q \# \ddot{I}$

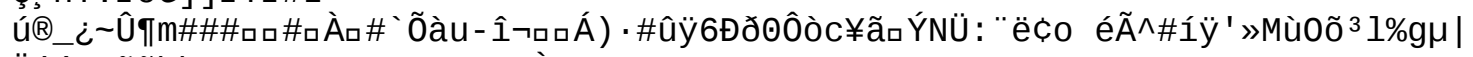

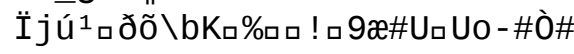




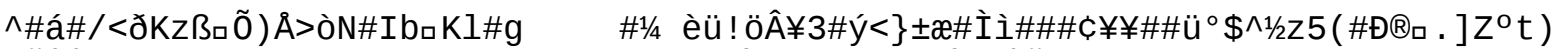

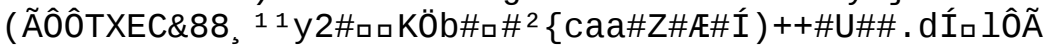

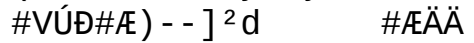




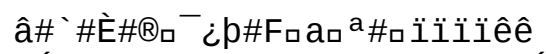

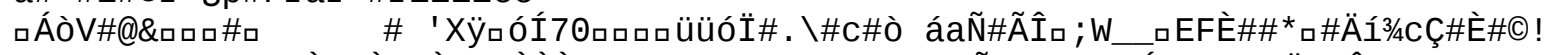

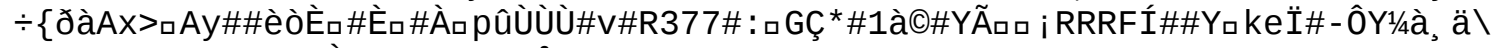

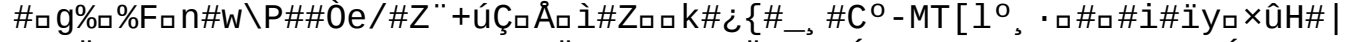

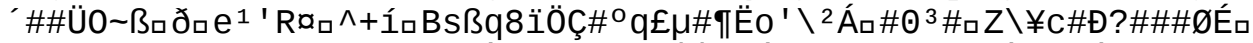

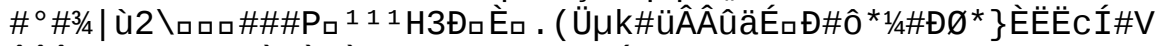

, \}口Y444ロðW² Yaa! «\#\#\#

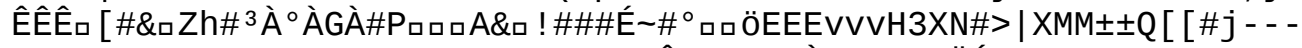

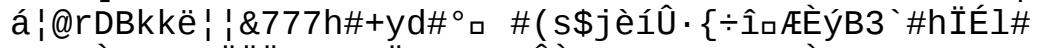

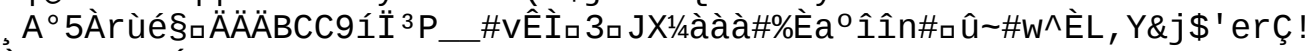

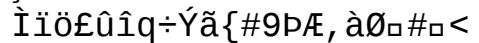




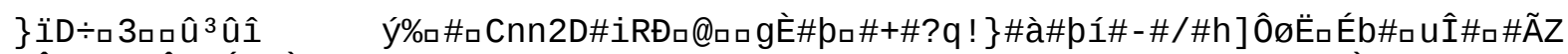

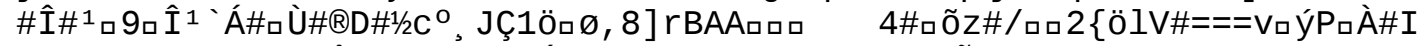

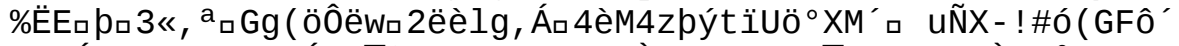

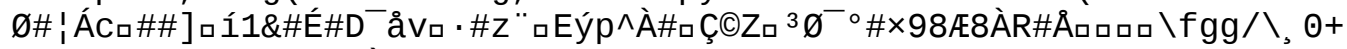

+\#ý\#\#\#, àÄ\#úúúóç̇̈çò7<, T_aãò

án

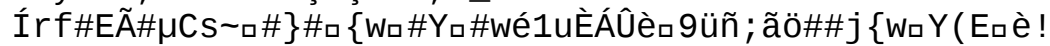

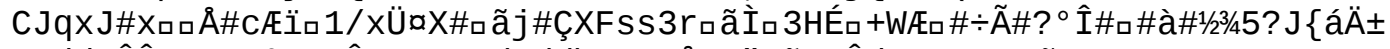

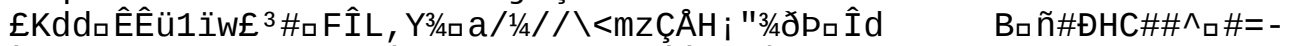

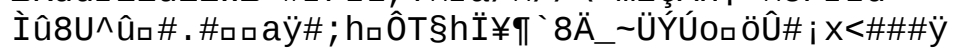




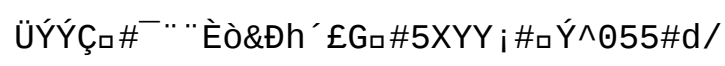




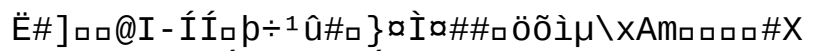

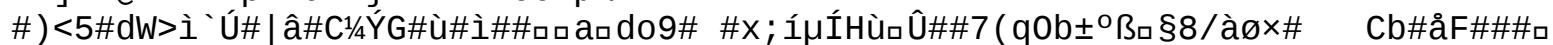

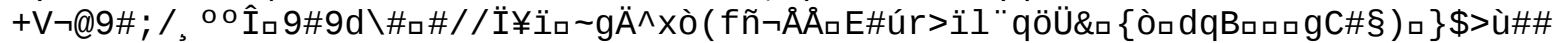

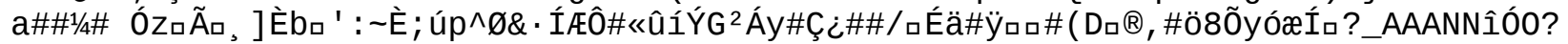

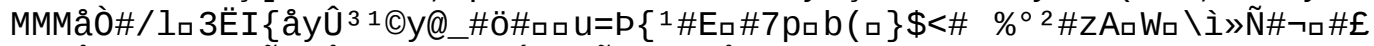

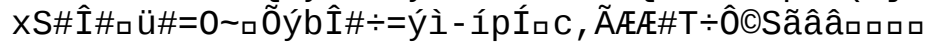




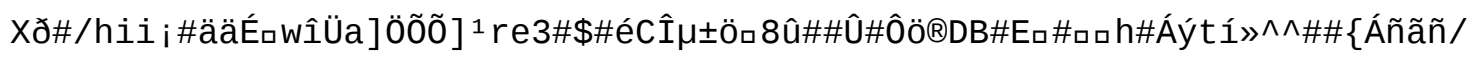




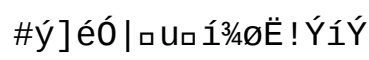




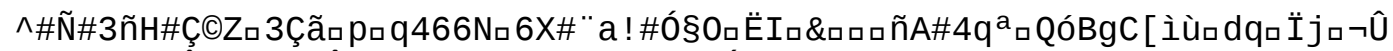

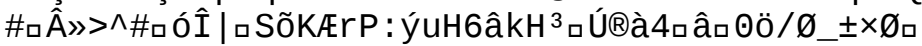

$x<8 \notin 8 \square \square \square V i ́ U ́ 5 n U ̈ 80 ̈ n ̃ p \# \#$

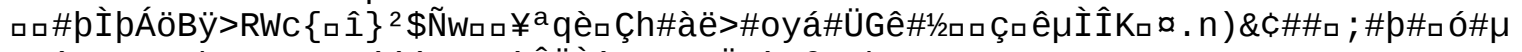

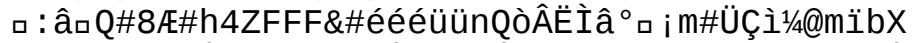

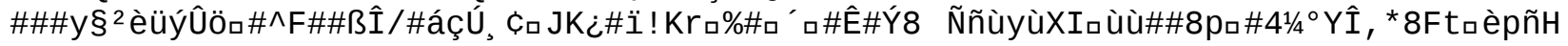




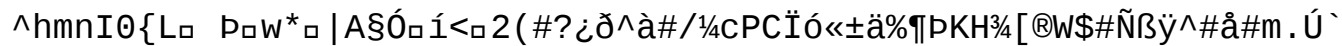

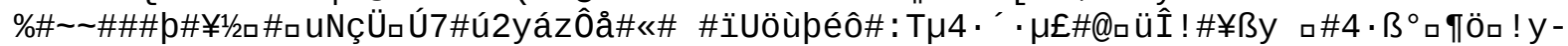

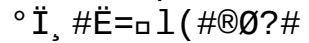




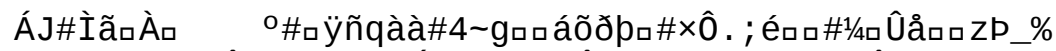

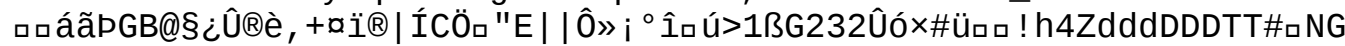

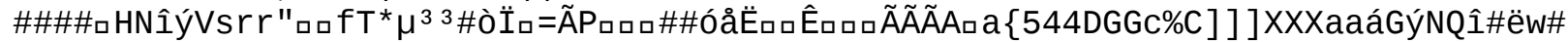

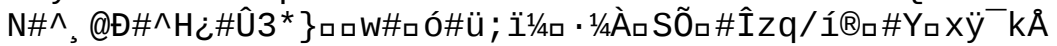

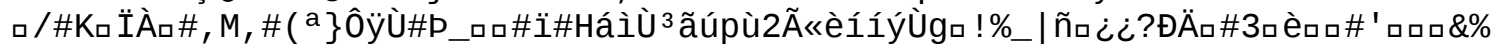

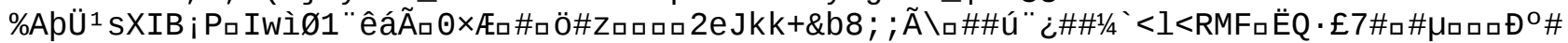

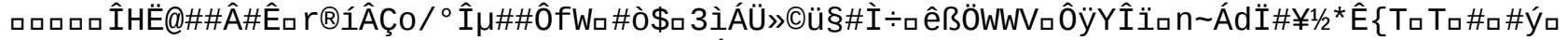

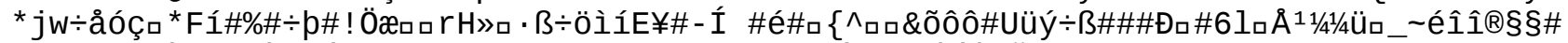

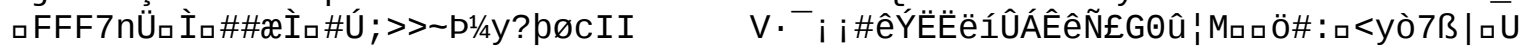

' cN\#PdH\#) Ra ãó\#ÝeÅ¥oëp ‘\%\#æ $1 \frac{1}{4}$

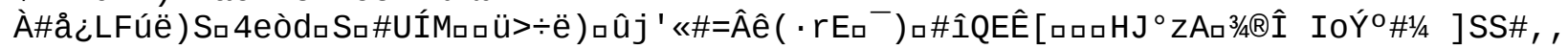




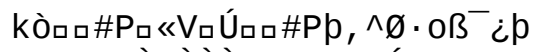

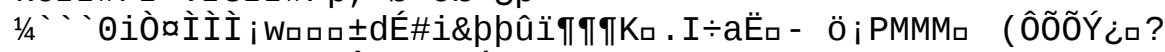

Ø\#_\}õUPPĐĐ10ß3/4=aÂn\#\#\#\#Ó], pAJJ

é" 


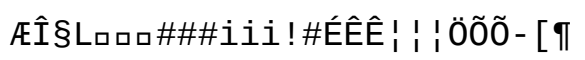




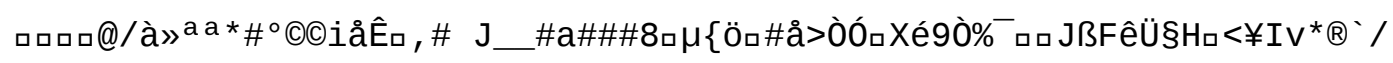




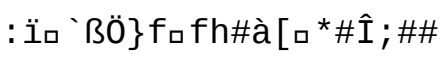




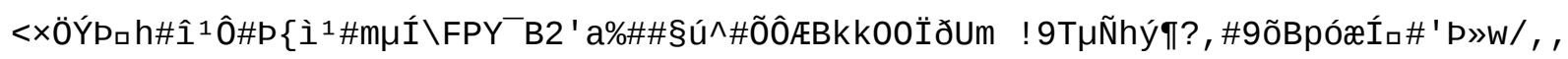


\#bûöíkö $\neg \#$

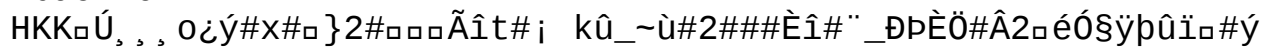




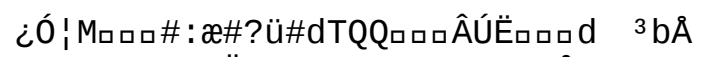

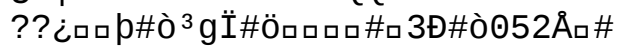


>\#6\#? <ÒwÏ̈ =Đ\#2ð\#\#\#\#Áúøúë- $\cdot$.

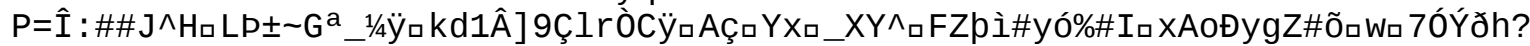

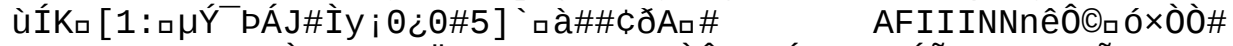

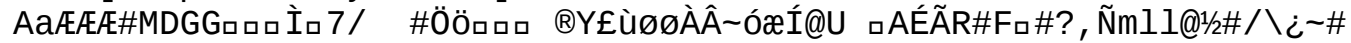

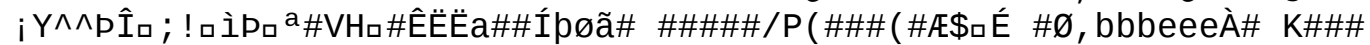

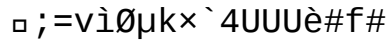




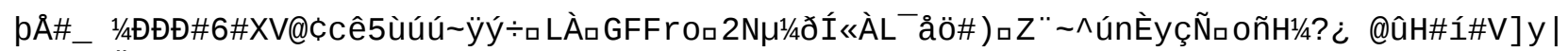
á1 $120 \tilde{0}$. ðÜ[Çםё口 


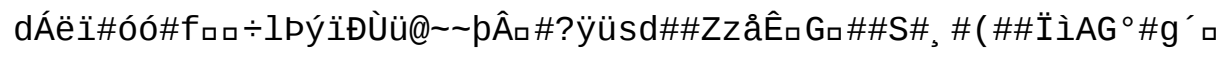




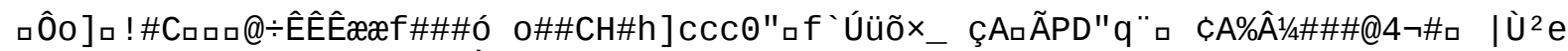

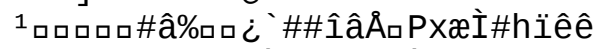

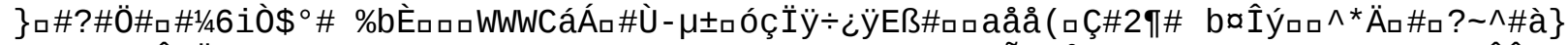

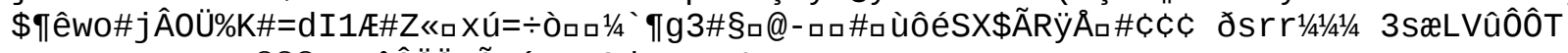

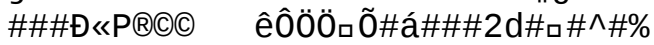




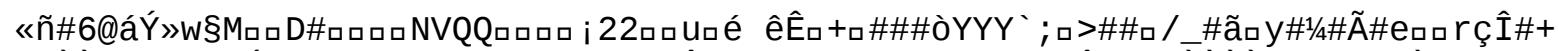

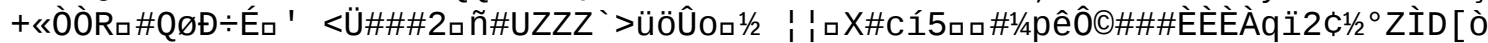

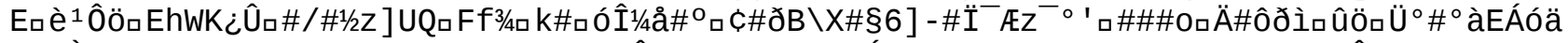

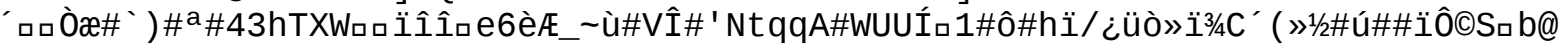

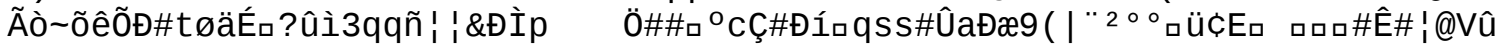




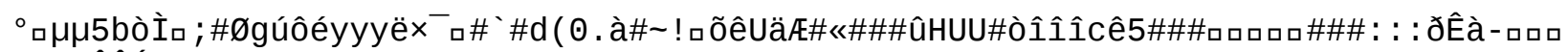
mI¥RÛUÚ =\# 
àÂ\#̈̈\#K

'

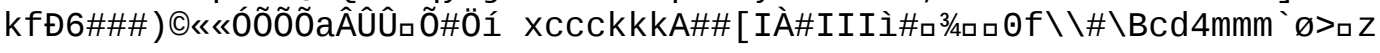


$\square \square \square A \square 1 / 2 Z 0 \widetilde{D}$

Ė\# 
$\#^{`} \#$ h\#Ö\#rÉ> hu(DF\#\#ロf !p

Đ\#ロH 


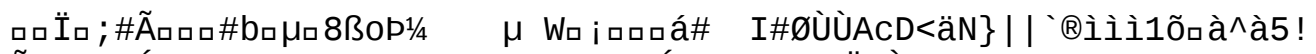

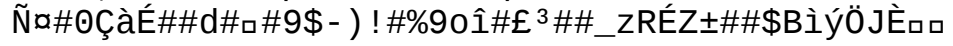




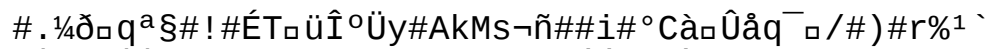

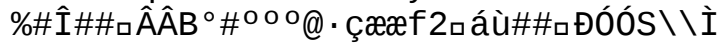

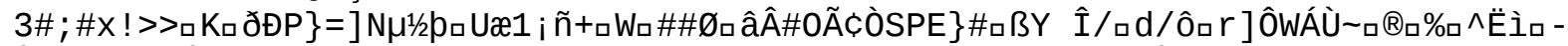

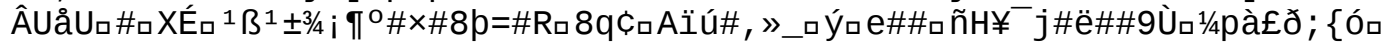

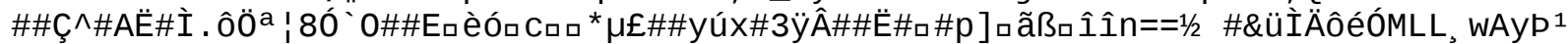

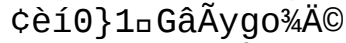

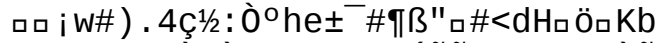

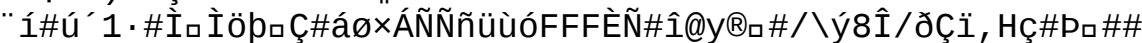

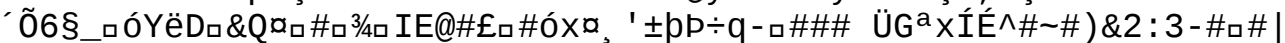

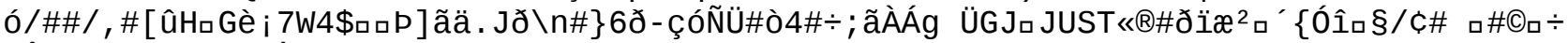
öÂaロ\#F\# [<Rî̀̀Foò@ôvc\#

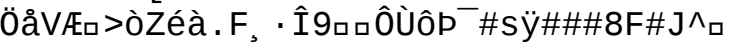

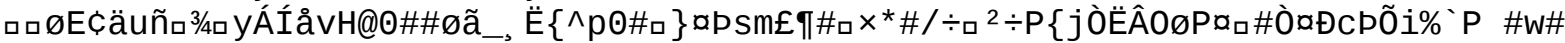

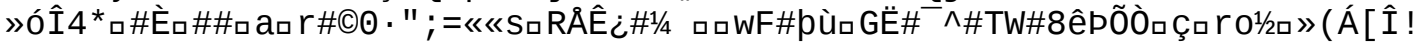

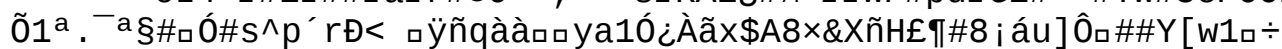

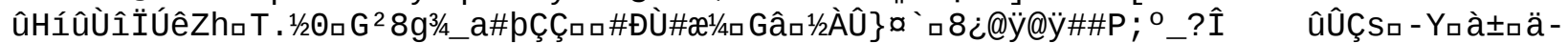

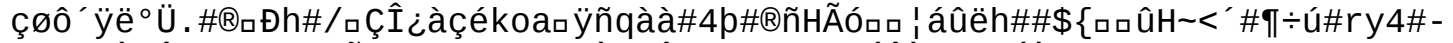

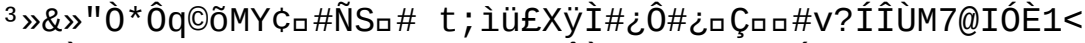

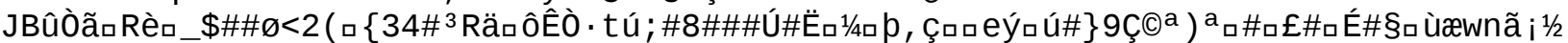

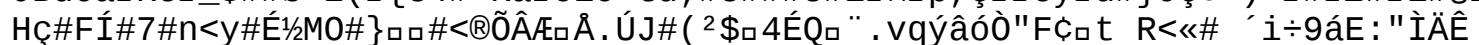

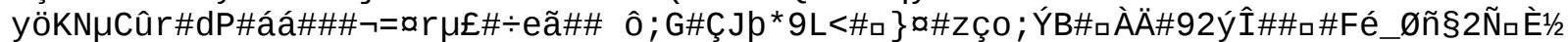

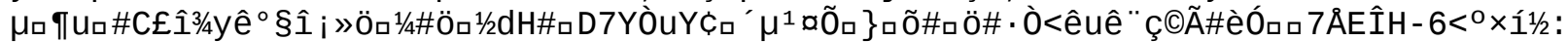

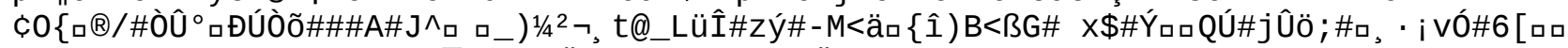

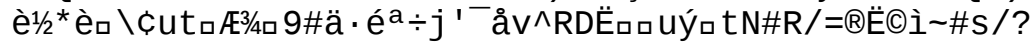

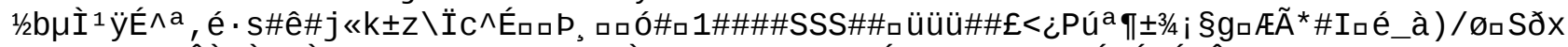

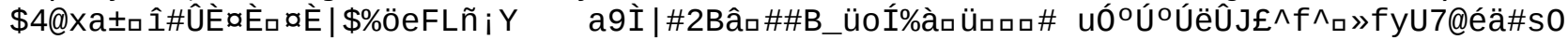

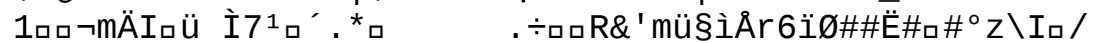

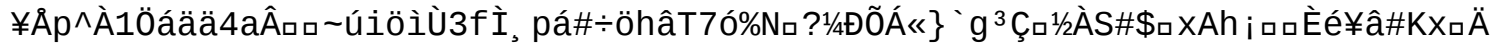




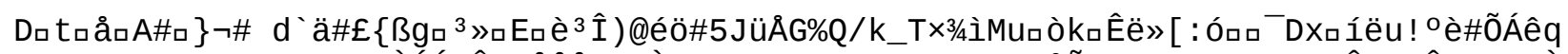

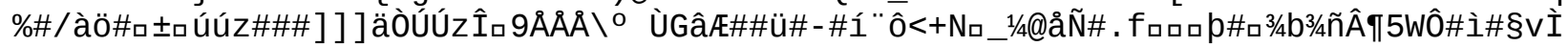


ãw?ฉ

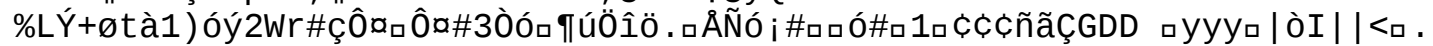

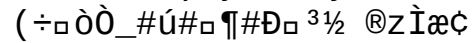

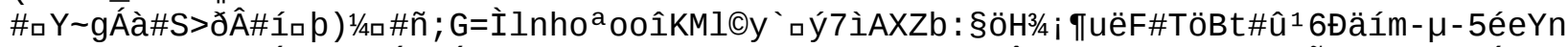

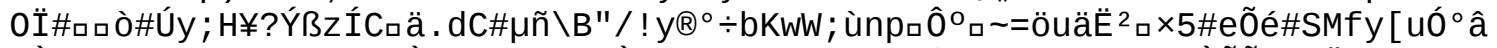

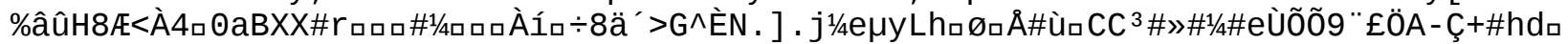

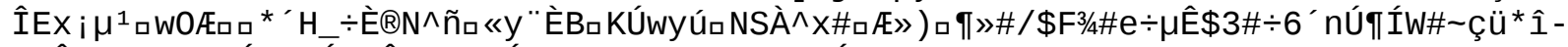

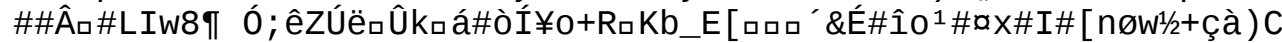

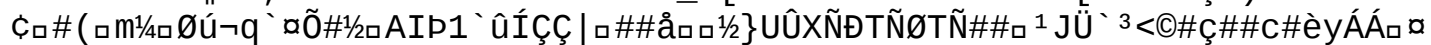

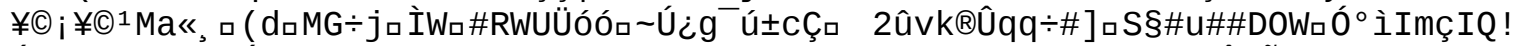

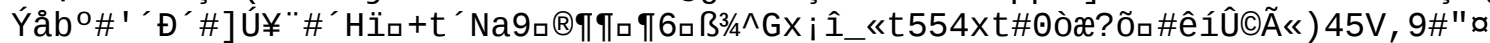

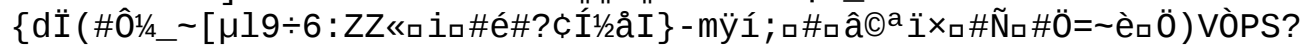

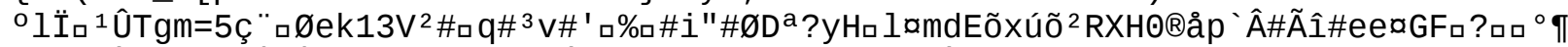

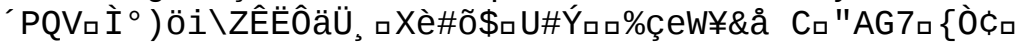

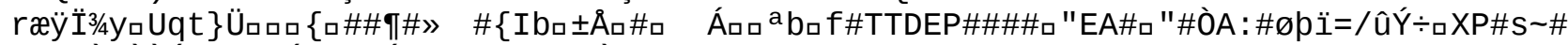

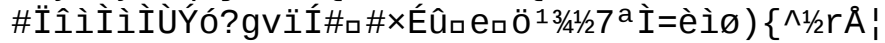

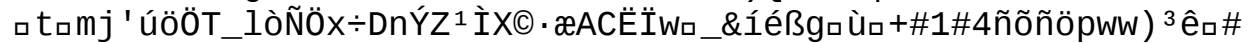

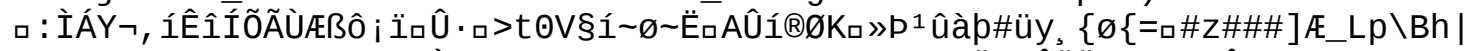

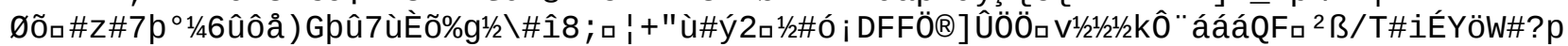

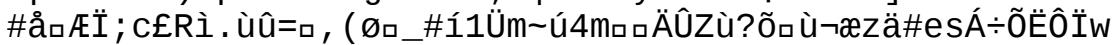

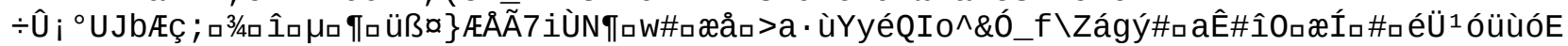

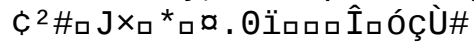




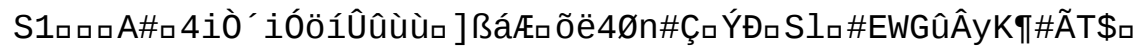

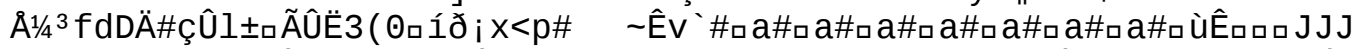

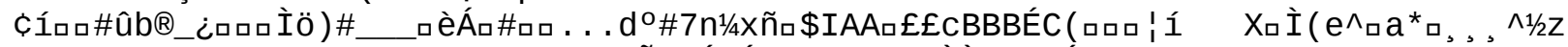

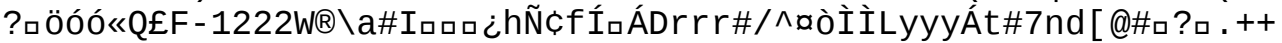

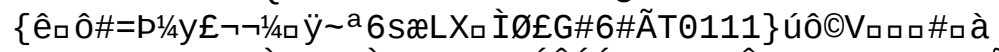

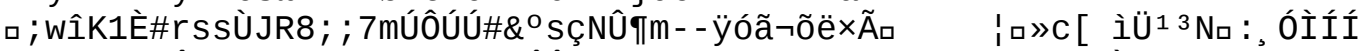

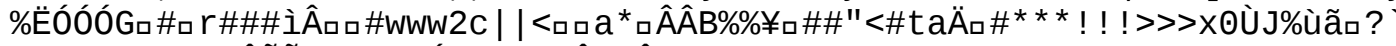

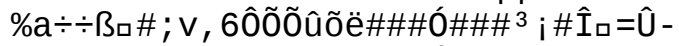

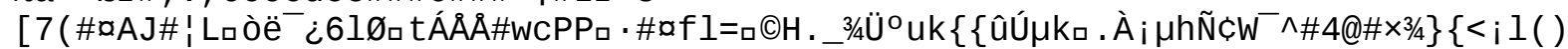

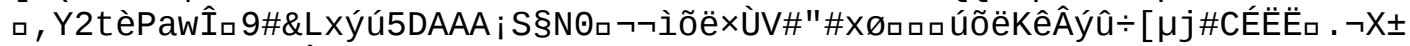

çZ̃ê.\#\#®\&FCC\#mÙロ 


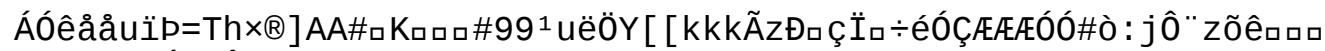

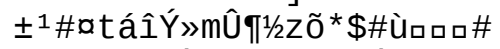

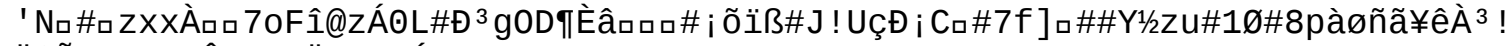
Üåõ\$I a taêô@ - [ ๆÄÿqãÆÁÿ +**\#\#\#J5A口

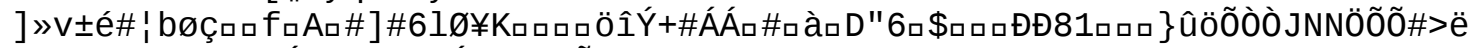
\%]xúô)ロK@J\#p§ía1\#wà'ió \#๑ããÃaMMMロ «

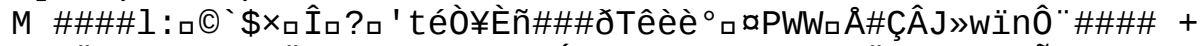

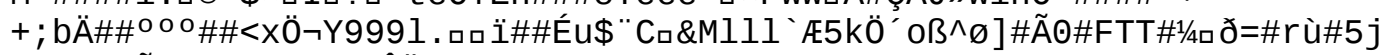

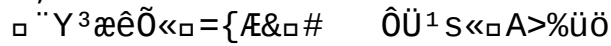

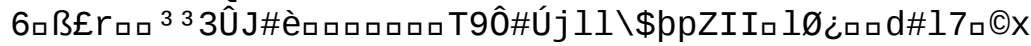

BCC口ß)Đ.xpü9\#çmdgggaฉÀJRÑ $l d d \$$

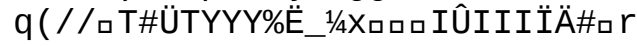




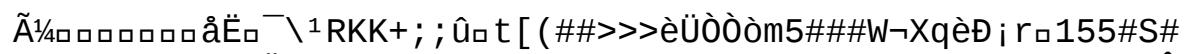

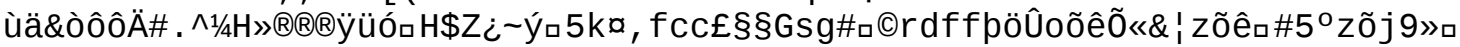

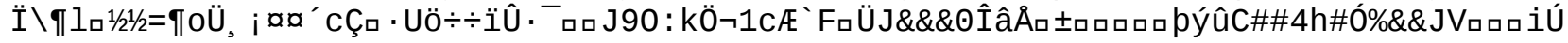
(ㅇ) ) ß] 


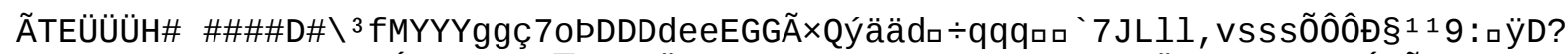

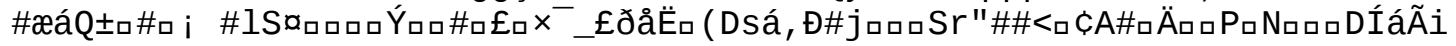
\#

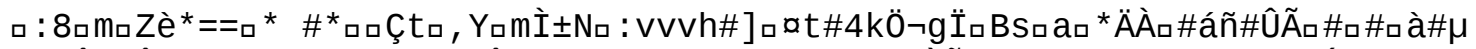

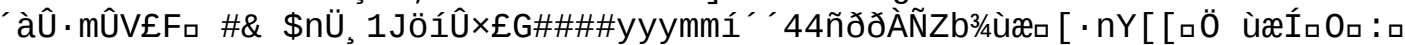

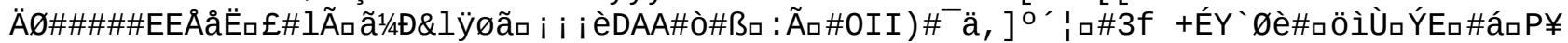

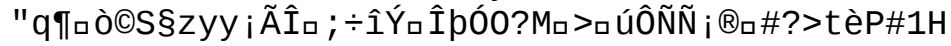

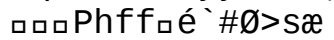


ê_${ }^{1} r ¥$ 
]ÀéPı\#À@ 


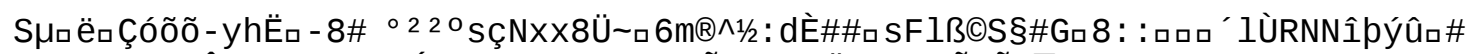

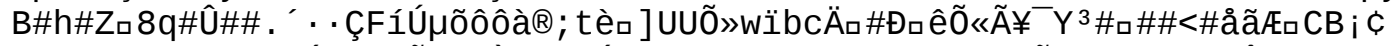

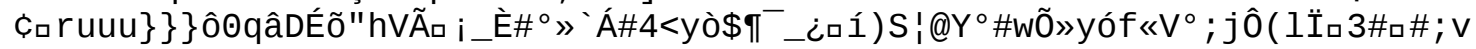




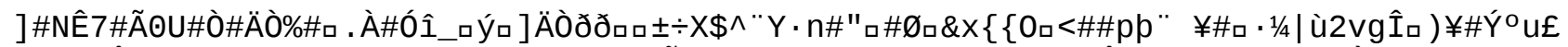

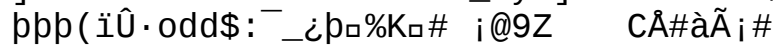

EeêÂ $\div$ ß OMàÿ $i \# / \wedge 1 / 4$ Äöq

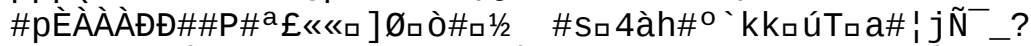

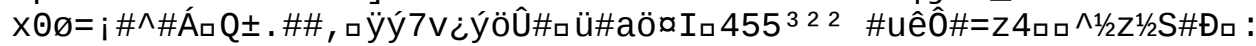

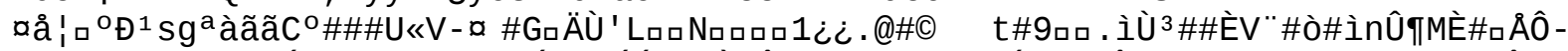

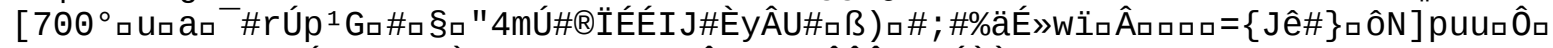

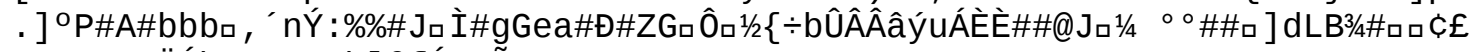

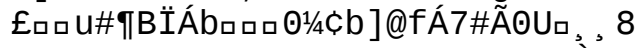

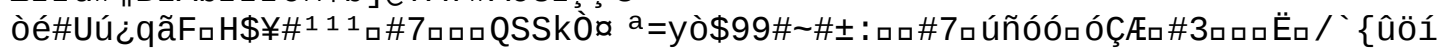

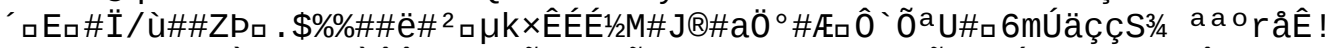

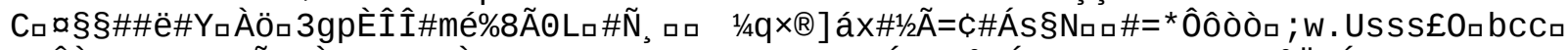

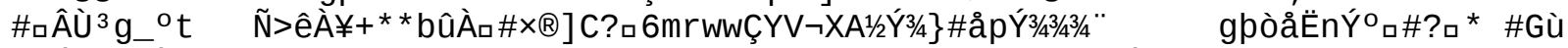

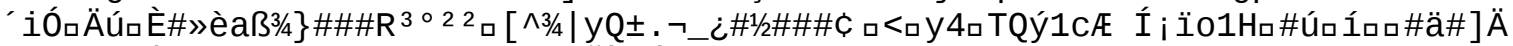

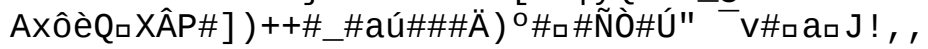


๖\#ÚTô¿ї\#*\#\#\#\#ä/ょêェã 


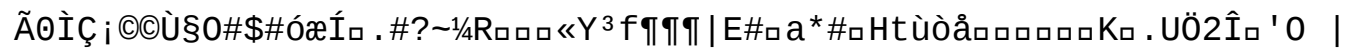

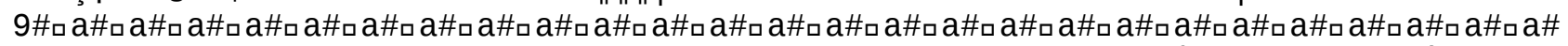

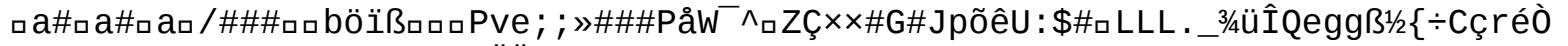

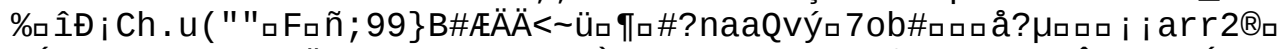

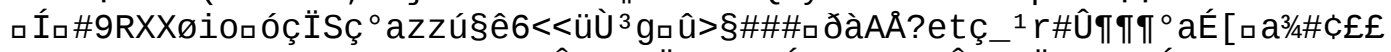

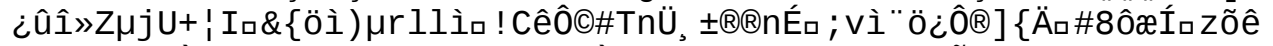

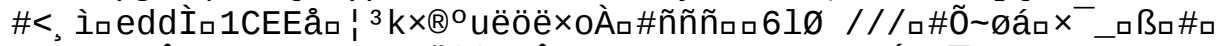

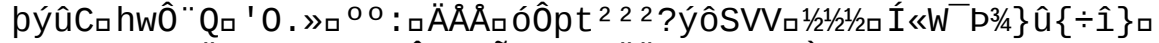

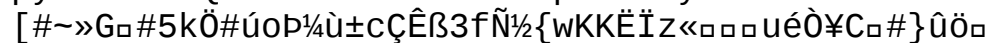

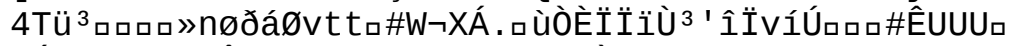

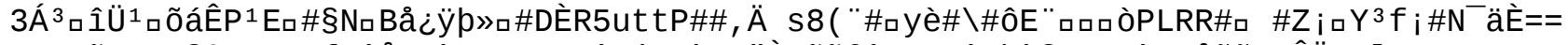

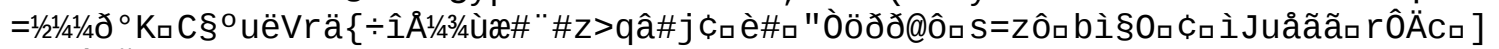

(ËæÍロ N\#\#zööö . ( (\#\#\#\# 



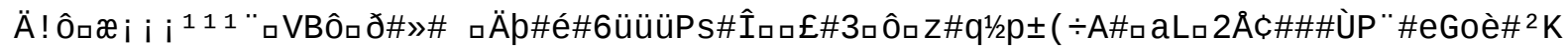

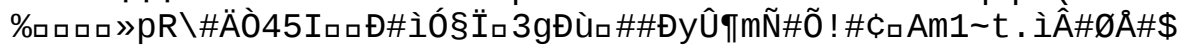

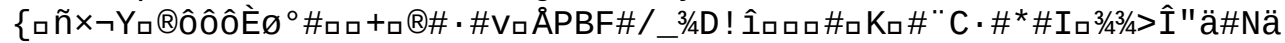

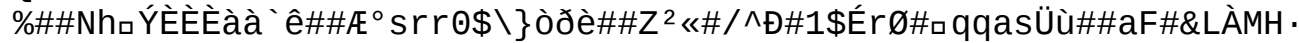

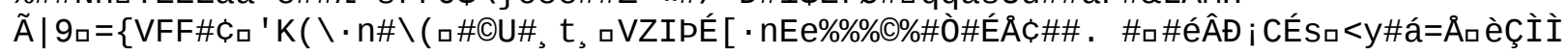




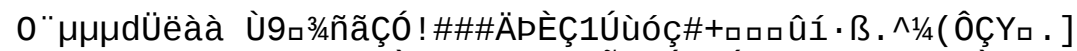

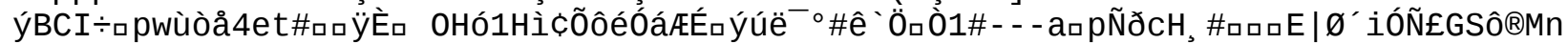

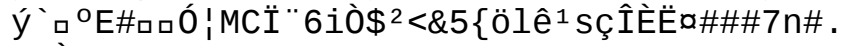

yZÒロ \#7

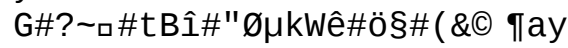


\#»\%£\#, D 


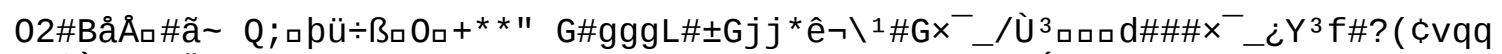

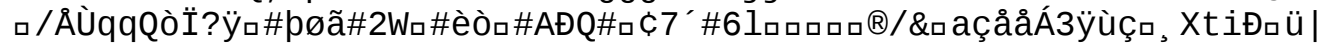

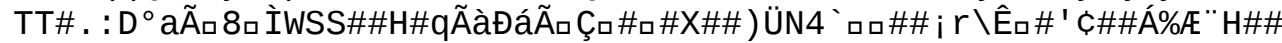

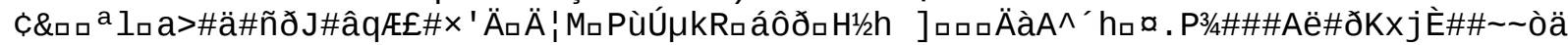

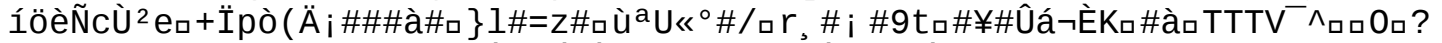

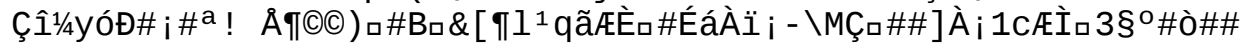




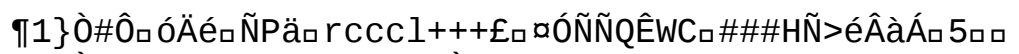

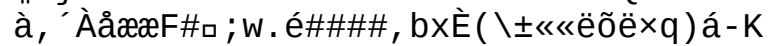

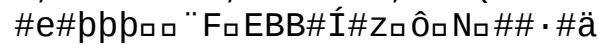




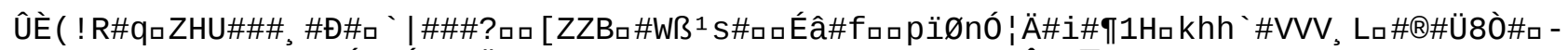

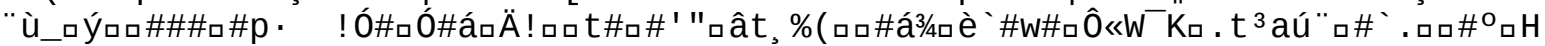

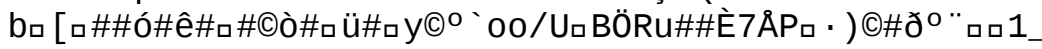




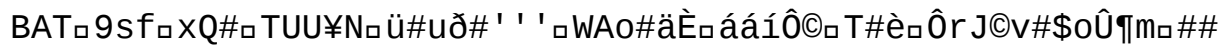

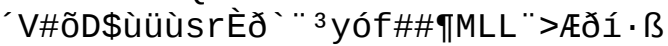

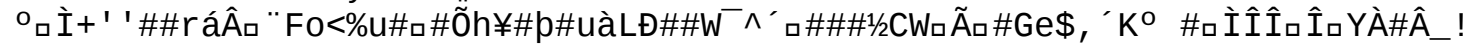

\#ı\#à ( $\left.{ }^{\circledR}\right) \# A^{a} p_{\square} \#$ ã : âda $\cdot K_{\square}$ è $\div ß_{\square} G y ́ R \# \# H$

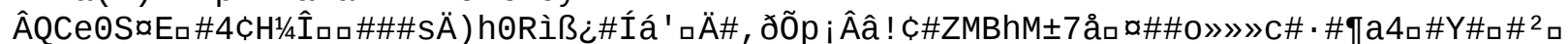

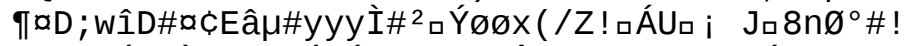

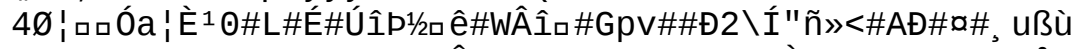

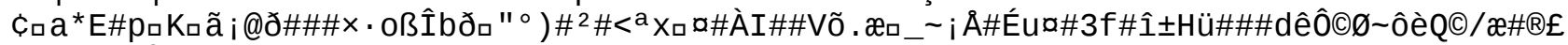

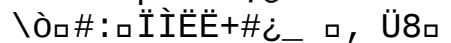

Na *wqq i 7\#d\#áû '

\#GMcÀaàá\#\#\#\%Ç0/ıi 


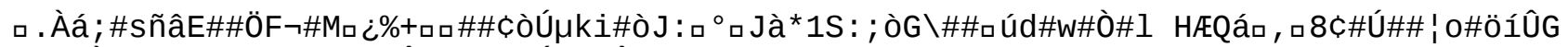

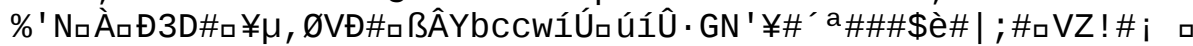

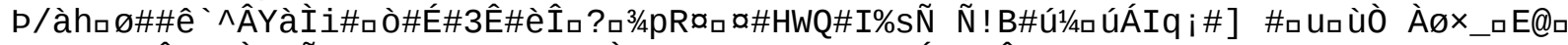

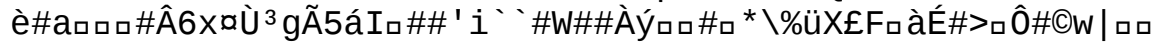

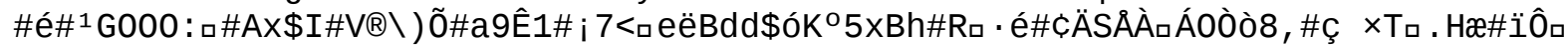

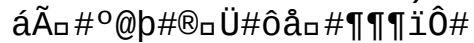




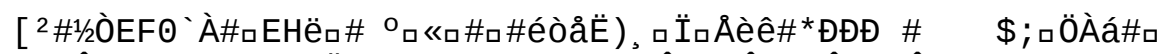

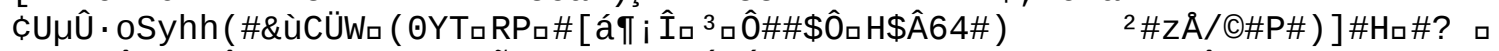

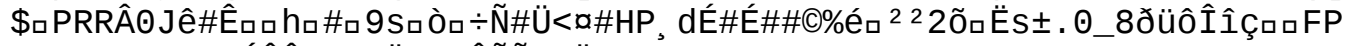

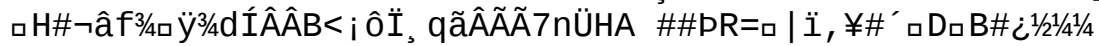

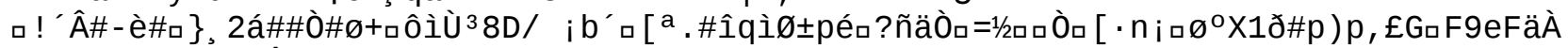

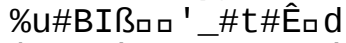

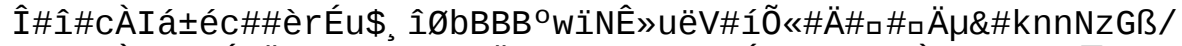

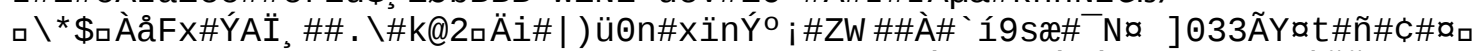

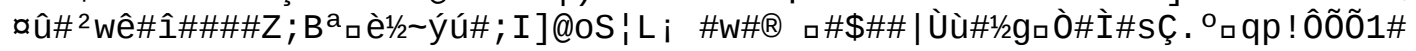




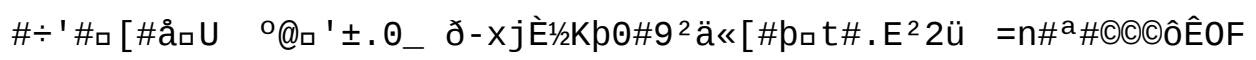




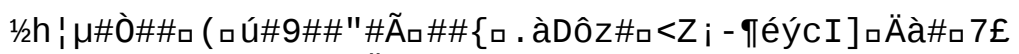

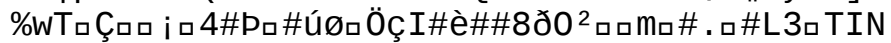

$\mathrm{N}$ 


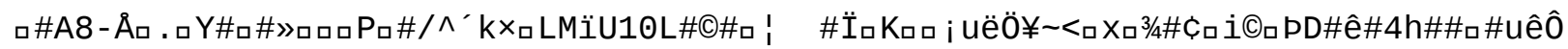

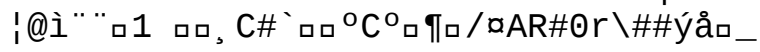

ô9é\#\}口TR\#

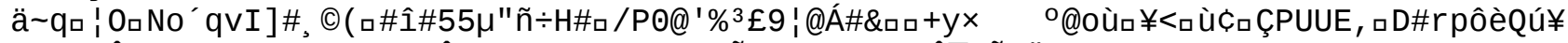

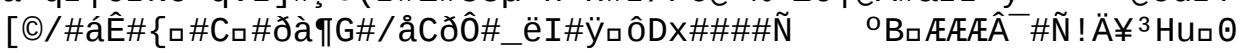




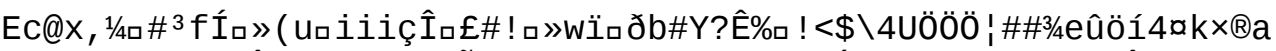

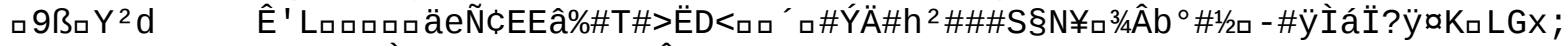

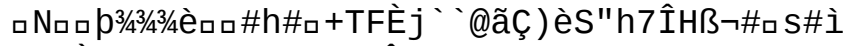

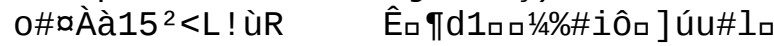

$\%$

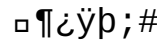




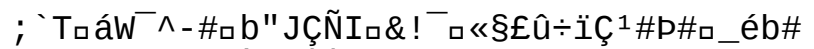

$H W \square \ll \ll+Y \# \S £_{\square} \grave{I}$ ÜÊ̂E

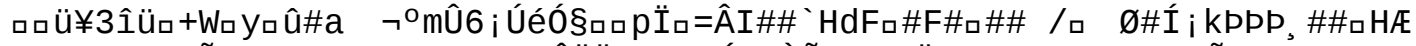

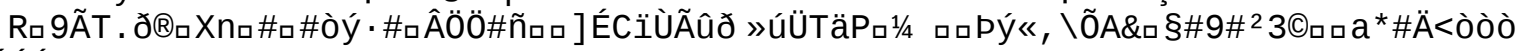
口* \#-1ÉÉÉ \pm$)^{a}$

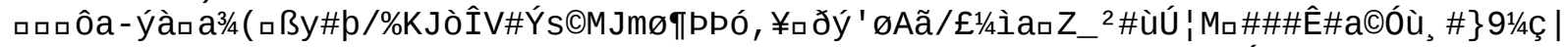

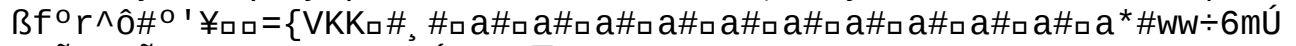

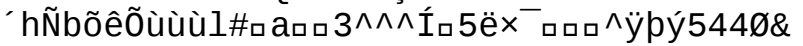




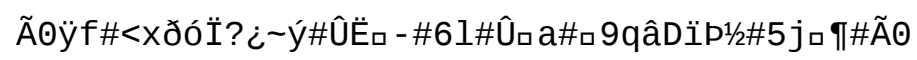




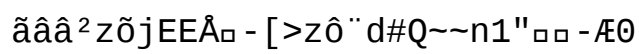




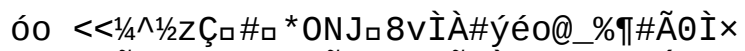

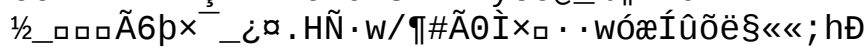

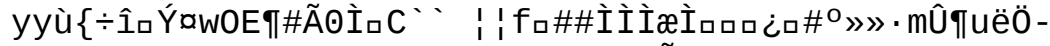

Zurvv gýp=óU`\#aùB ( ( (PVV®\&F_¿¿aÃa

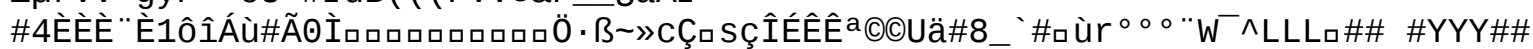


PQQ๑È1 ( $q^{3 / 4 A ̀ \Theta}$ 


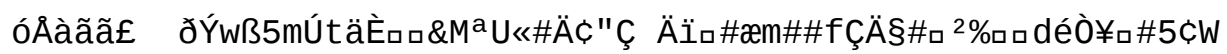


uêôA³/4PÁ?4ã —\# 


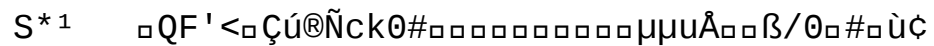

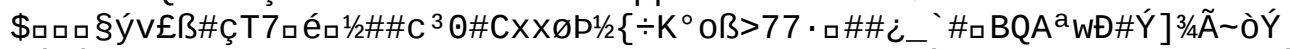

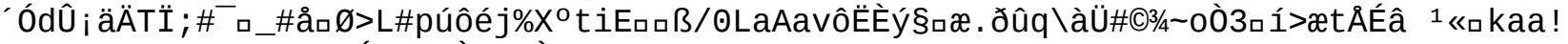

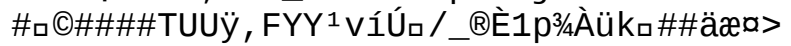

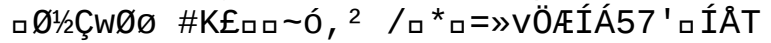

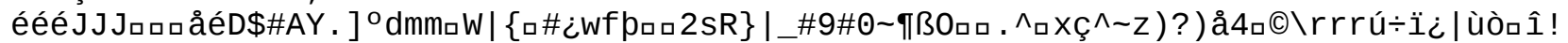

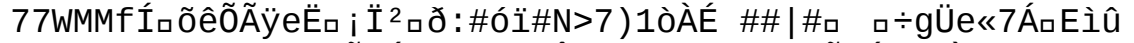

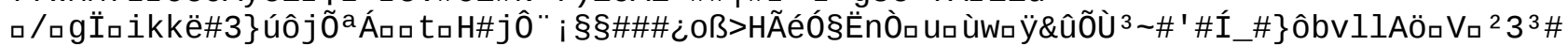

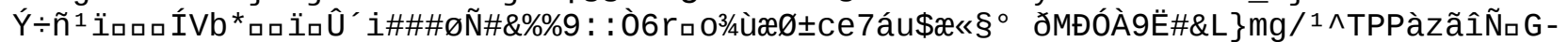

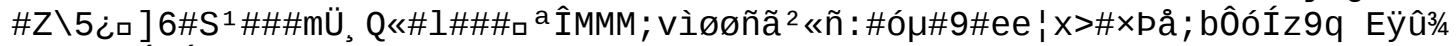

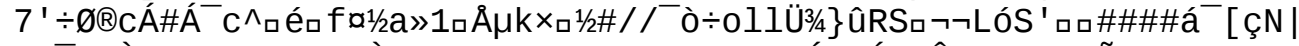

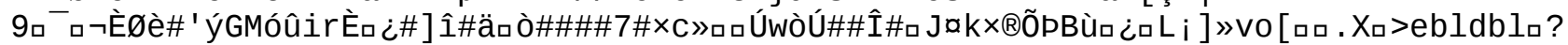

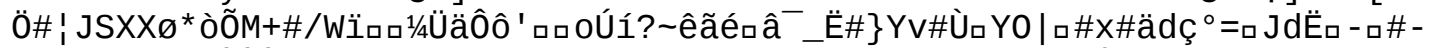

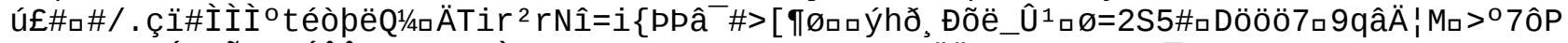

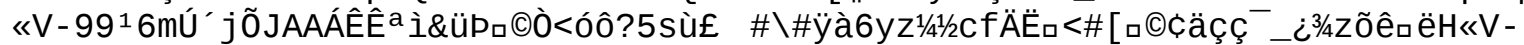

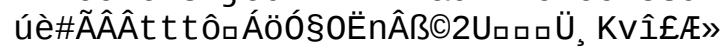




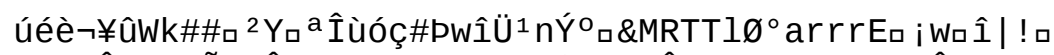

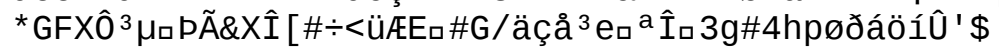

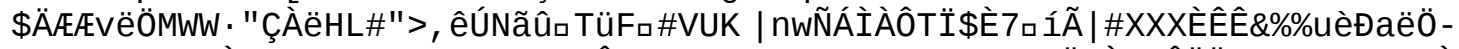

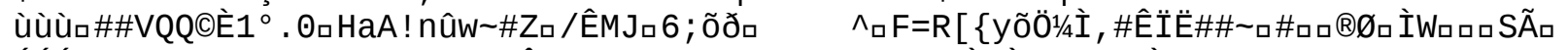

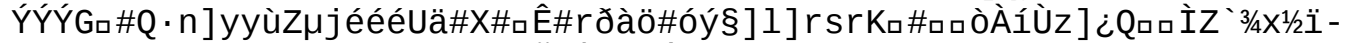

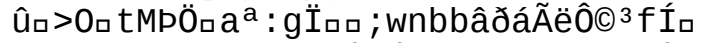

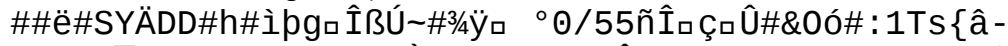

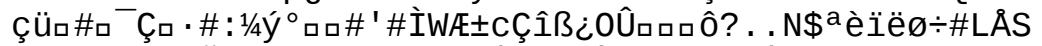

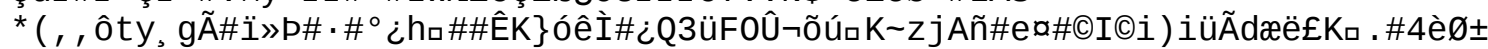

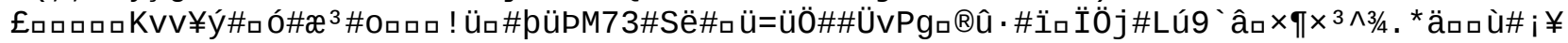
$¥ U C$ 


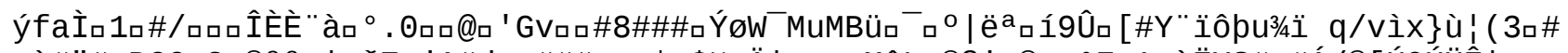

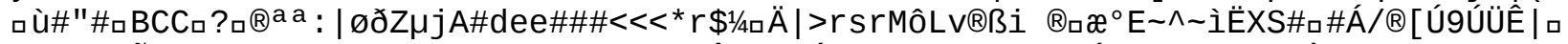

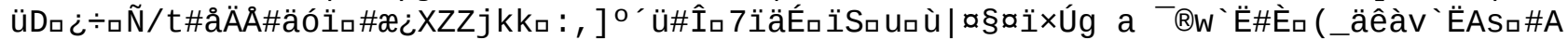

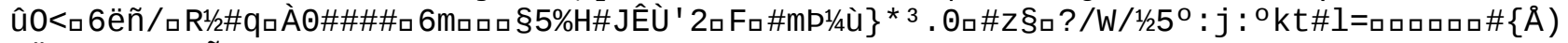

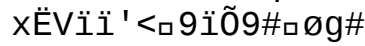




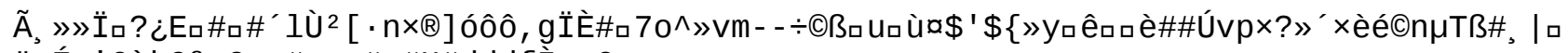

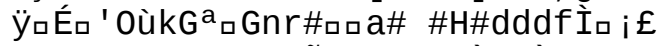

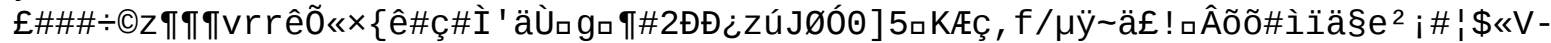

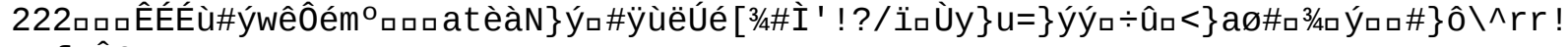
¿VfateE 


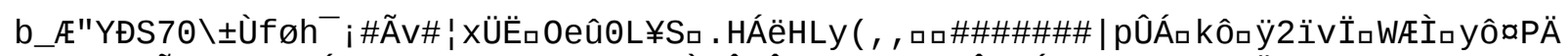

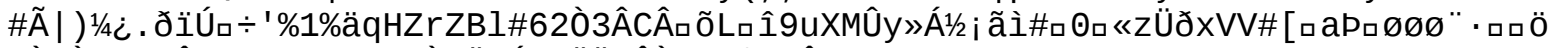

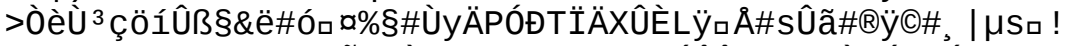

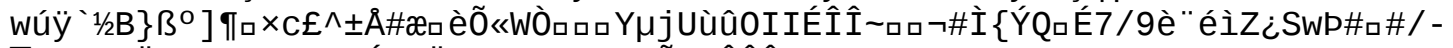

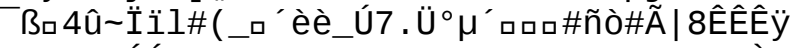

口\}ó\#ロêÚÚ

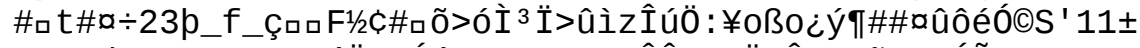

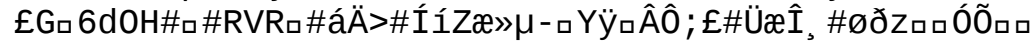




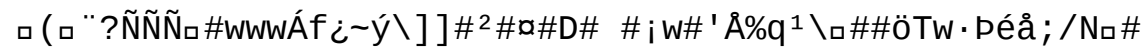

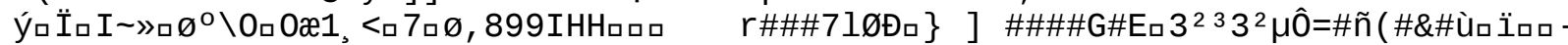

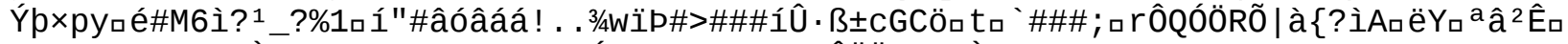
' 


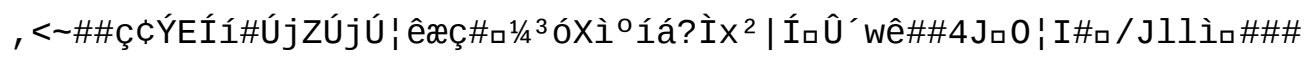




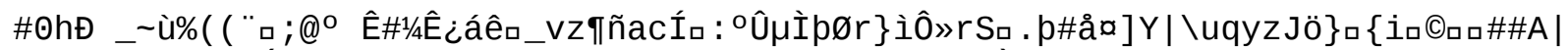

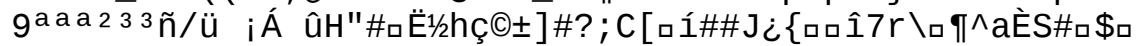

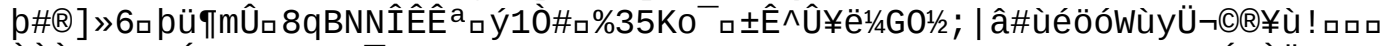

òòò

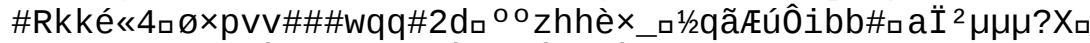

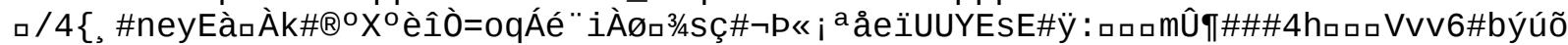

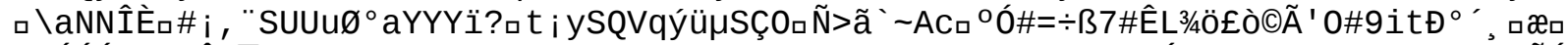

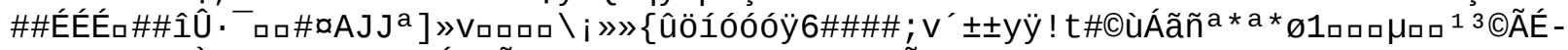

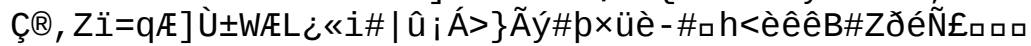

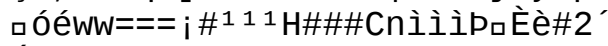

Í

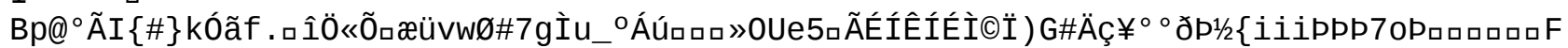

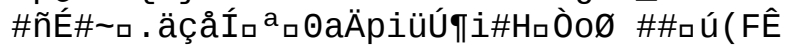




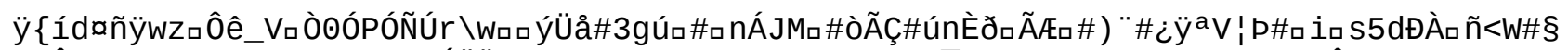

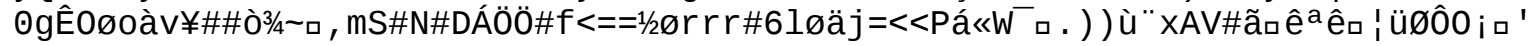
$\left.n g]^{1 / 4}\right]^{-} »[» u ́] y ́+y ́ E q \tilde{o}$

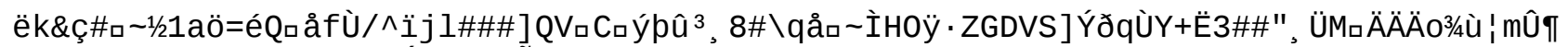

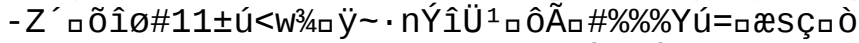

å@sıhçkkj ${ }^{1} \backslash n I a_{a} e ́ 1 S a ̃ \# F_{\square}{ }^{a} \#$, . xÙ_vÛßí3Y9xÜTï) ${ }^{3}$ ìç®òÝ|

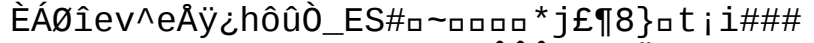

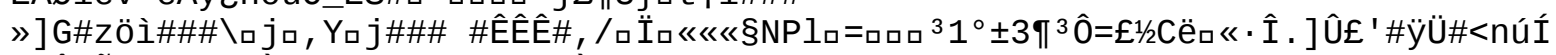

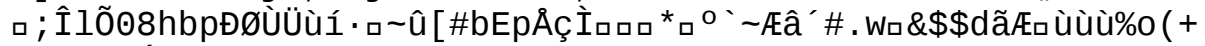

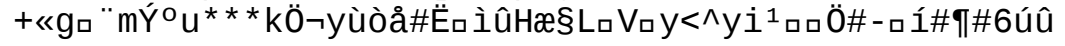


\#\#ロ ] ${ }^{\circ} o^{\prime} \mid$ ðò̃̃90â ${ }^{\mathrm{a}+\ll \square}$

$a^{++*}$ व

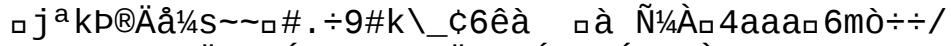

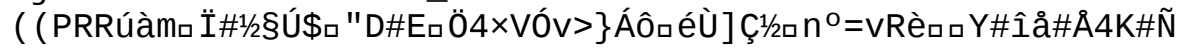




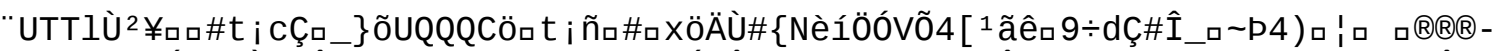

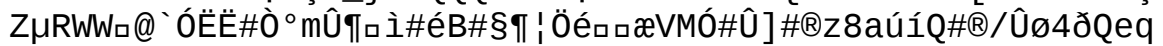

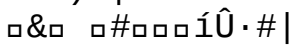
'PRR2j0̂ "ú1/4a Ô

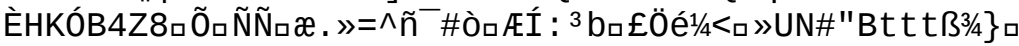




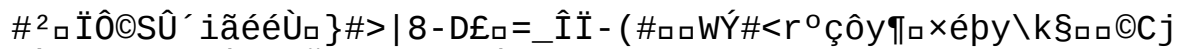

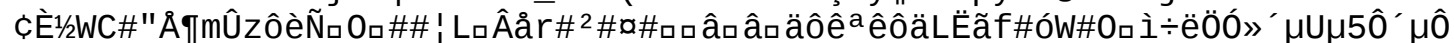

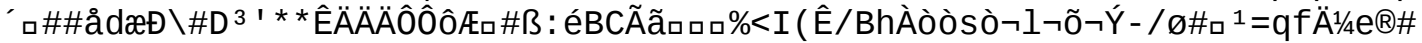

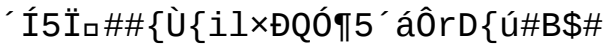

QWWÇÿåËロ\#3/4uÒı\#£ a çòA ${ }^{3} \# / 0^{3}-\mathrm{u}$

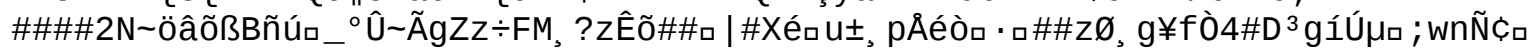

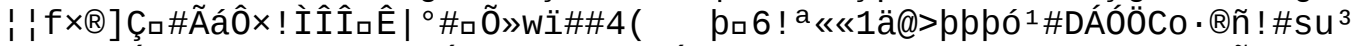

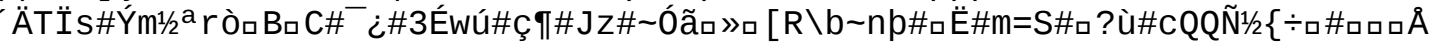




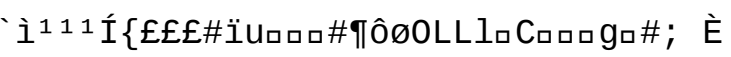




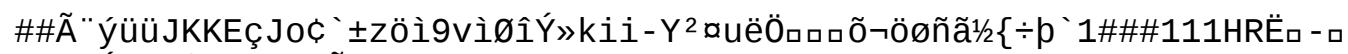

\#=*: Ó\#\#\#ÅFıáãÿêõ «

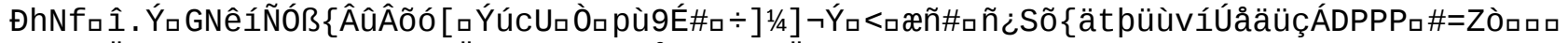

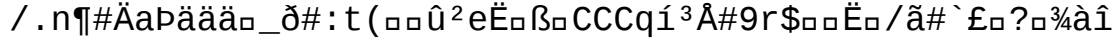

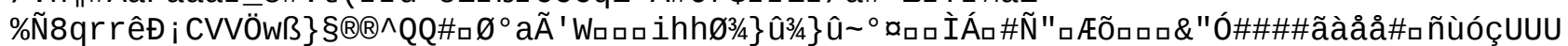

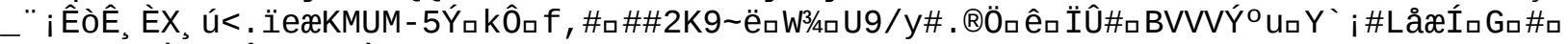
Iðöö口Créò¥f $1 \hat{\mathrm{E} \# \#<\square " A ̀ \# n U ̈, ~} Q \square)++\square \#$ $\left\{\wedge \wedge \wedge 3 \# \mathrm{rmmm}^{-} \wedge 1 / 2\right.$ aq

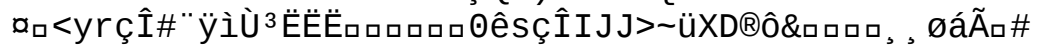




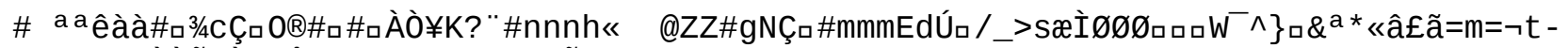

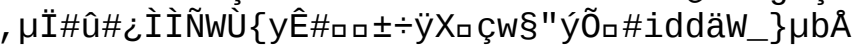

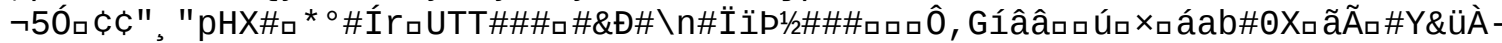

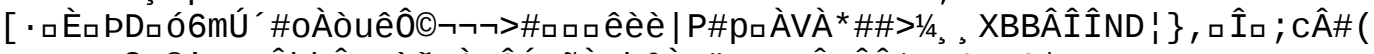

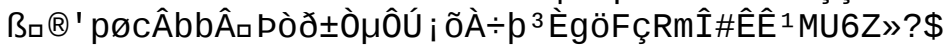

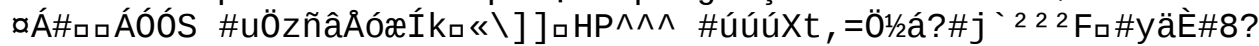

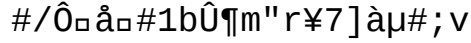




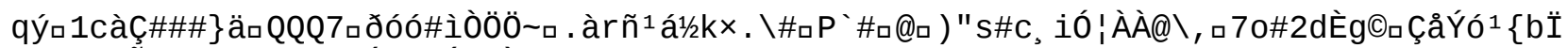

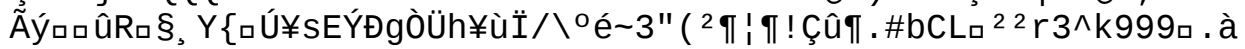




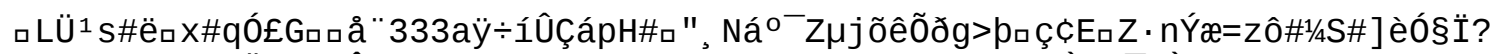

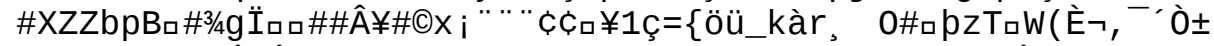

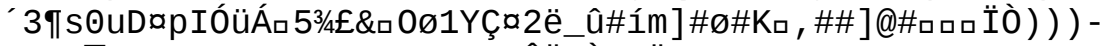

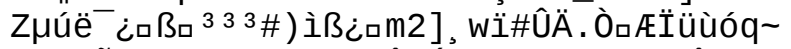

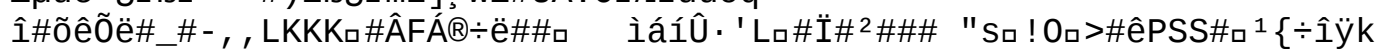

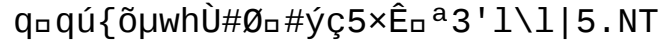

\#?\#a×DW \#j

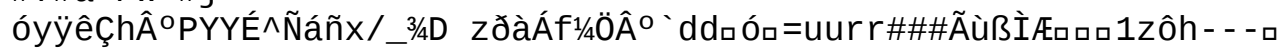

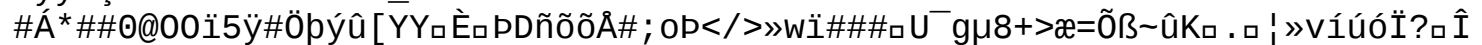

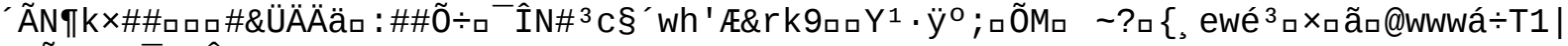

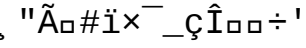




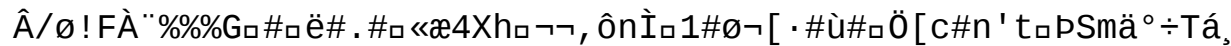

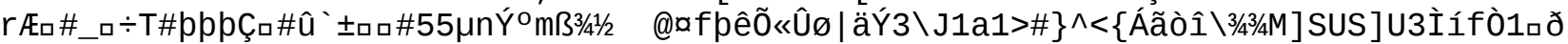

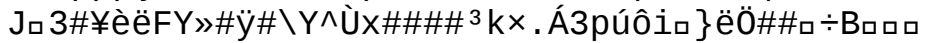

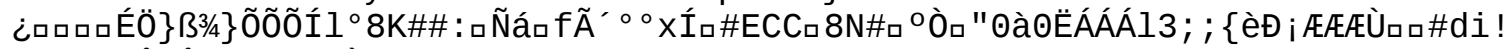

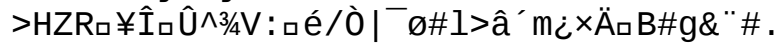

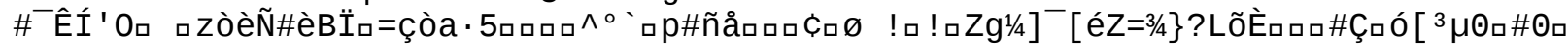

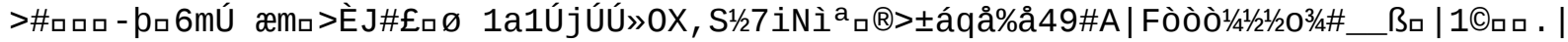




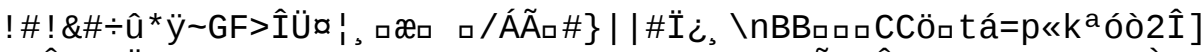

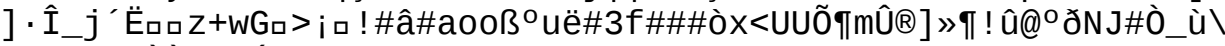

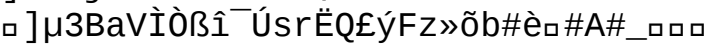


$99^{1} 6$ mÚHKKËËË̈ロロ $\div$ ìÙSðzRÃ@ ${ }^{\circ}$ 


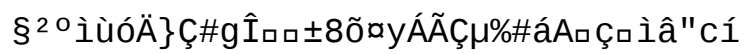




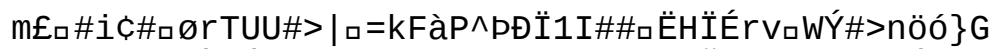

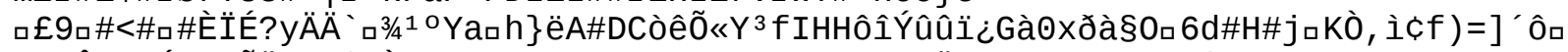

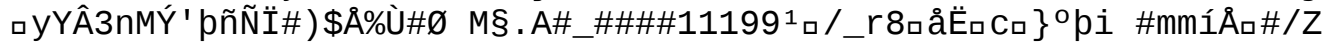

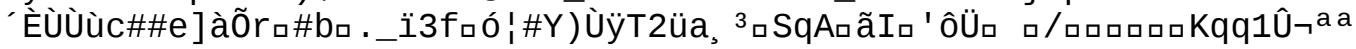

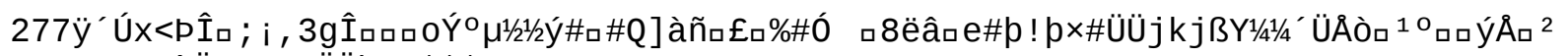

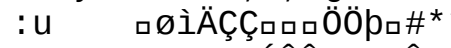

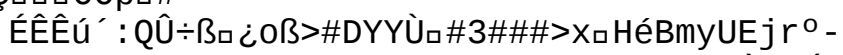

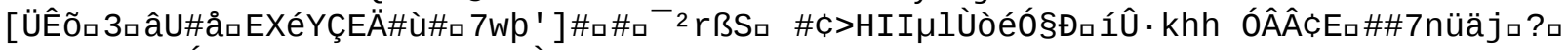

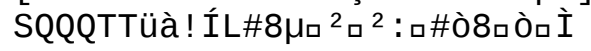




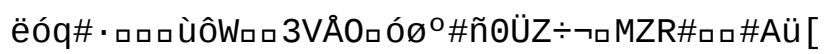




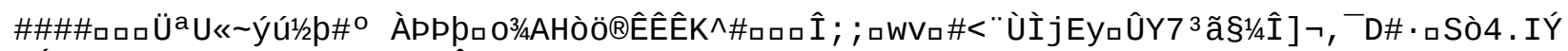

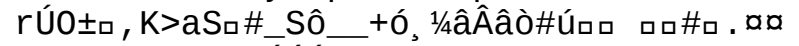

ם

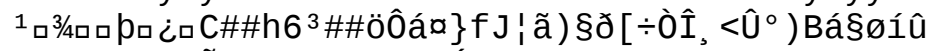

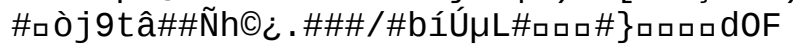




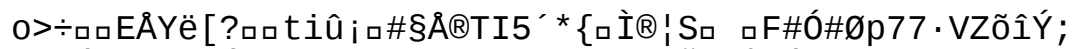

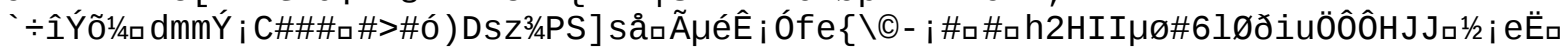

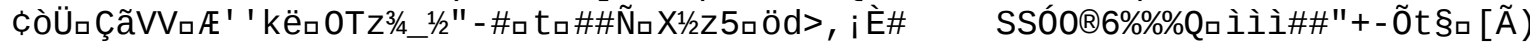

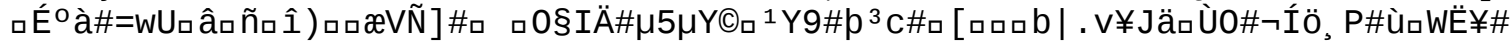
\%\#ロ ' MB\#îb 


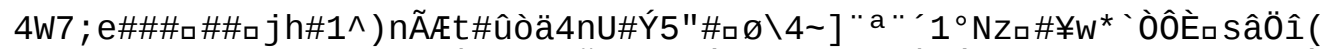

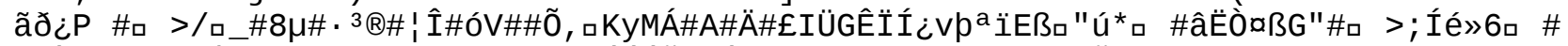

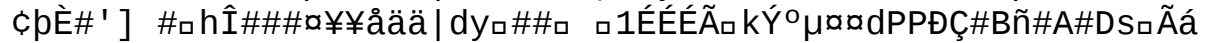




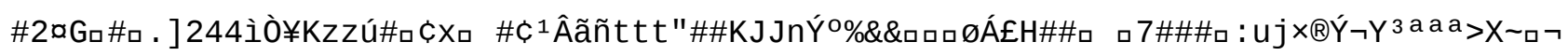




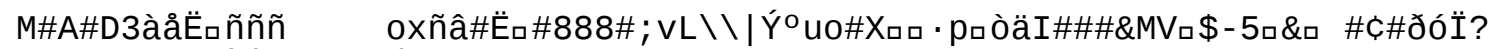

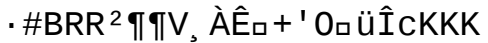

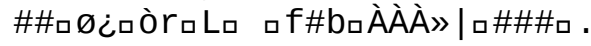


\#:\#ロ\#ÖVTTİ $=\left[A A \_\& \square \quad \# B d a ́ n ̃ x_{\square} 70 \# \# \# S V V F a a^{a} U+K K K_{\square} \# \square \quad \# Q \# ! \tilde{A}_{i} C_{\square} æ \hat{I}_{\square} \ll a\right.$

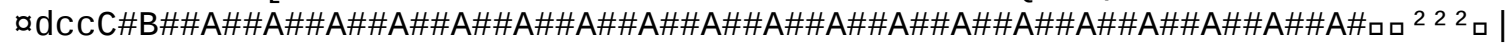

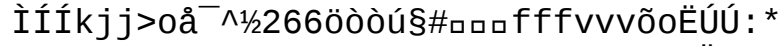

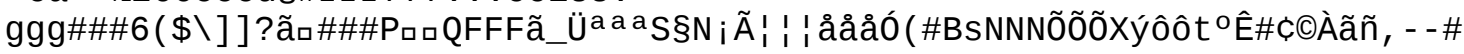




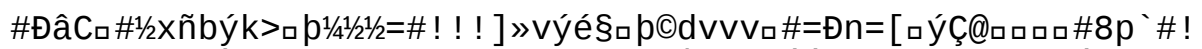

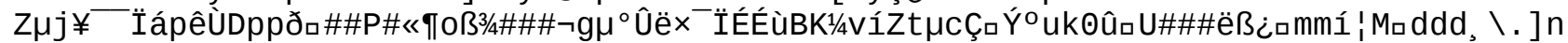

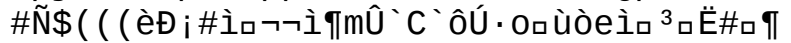

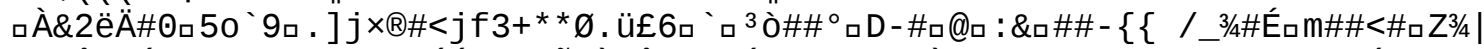

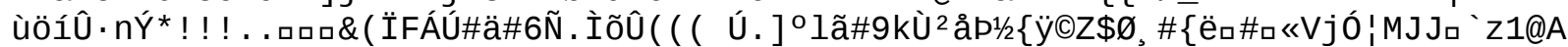

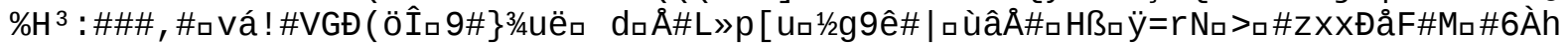




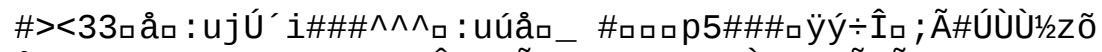

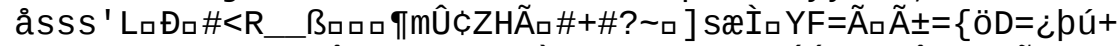

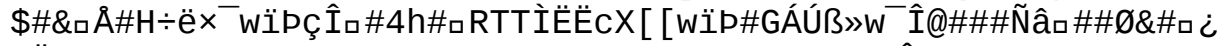

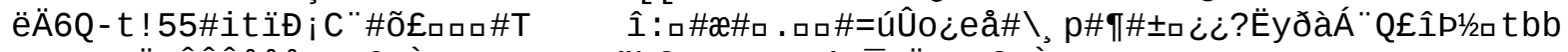

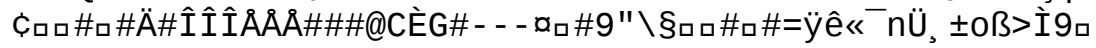




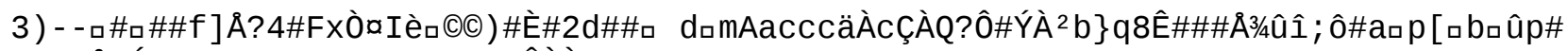

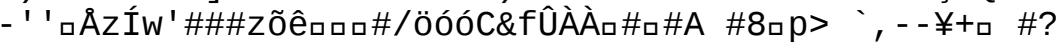

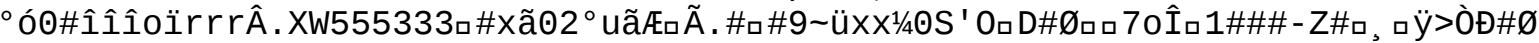

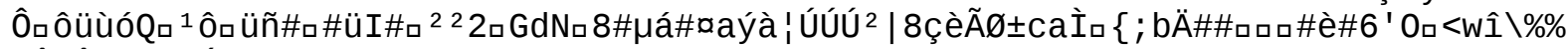

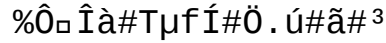




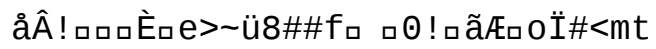




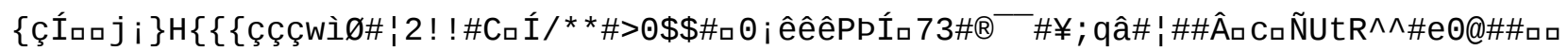

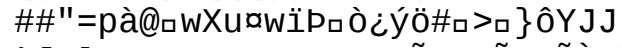

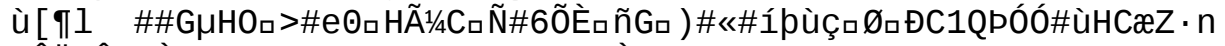

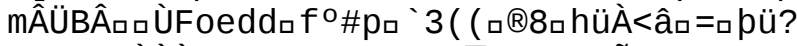

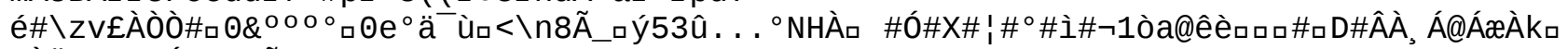

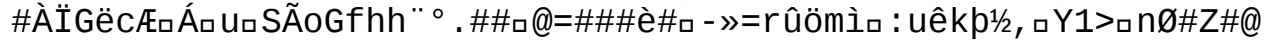




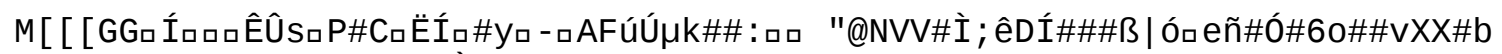

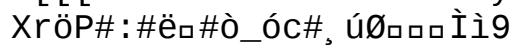


80̂\#\#Â $\pm g C ø n ̃ C ̧ \# \square ロ C ̧ \hat{I} \#(; D$

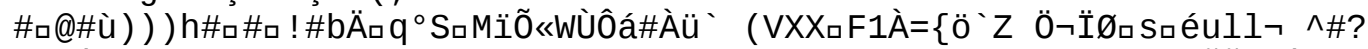

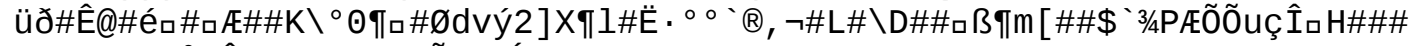
t॰Å\#ÂT"22òıtı9õè Ó\#X?

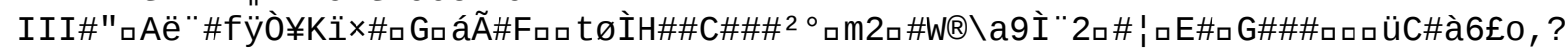

>>\#å1EløYløó: \#Äb\#\# 


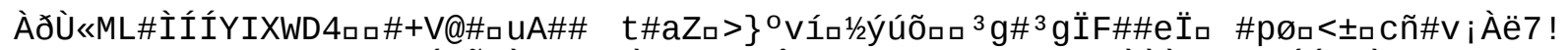

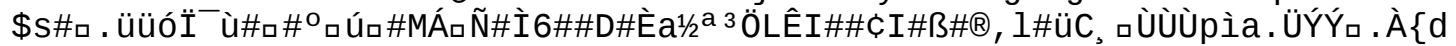

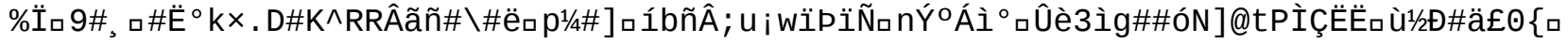
«ฉÜ) $S$ ! $1 / 4 æ$ ?

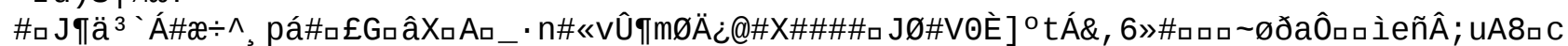

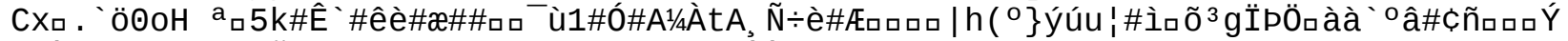

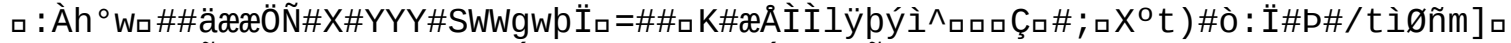

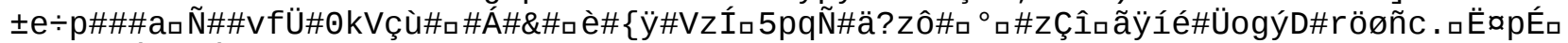

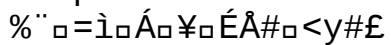

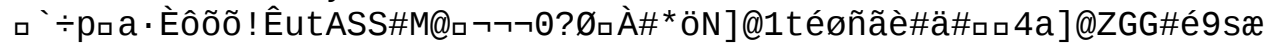




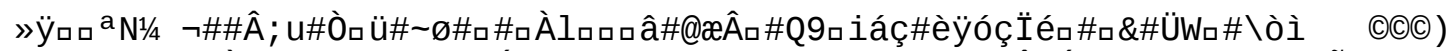

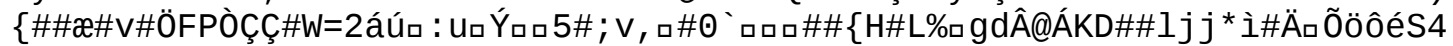

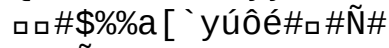

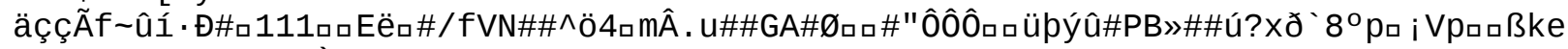

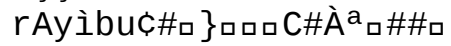




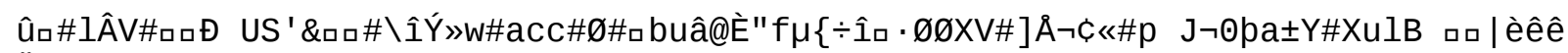
Ö\#口ロ\# 


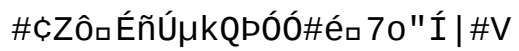




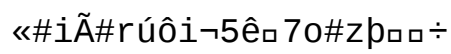




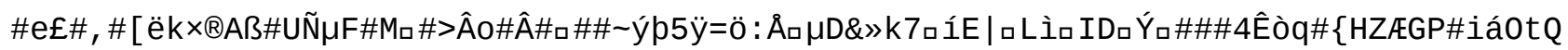




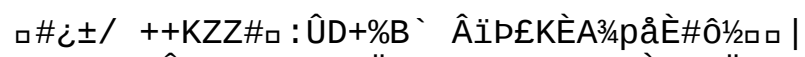

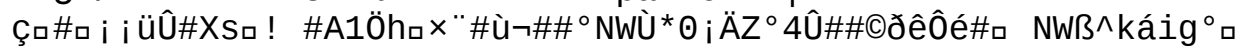

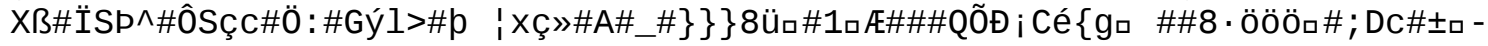
\{BM\#\#A\#\#ñ ¥ù?\#\#\#/\#i

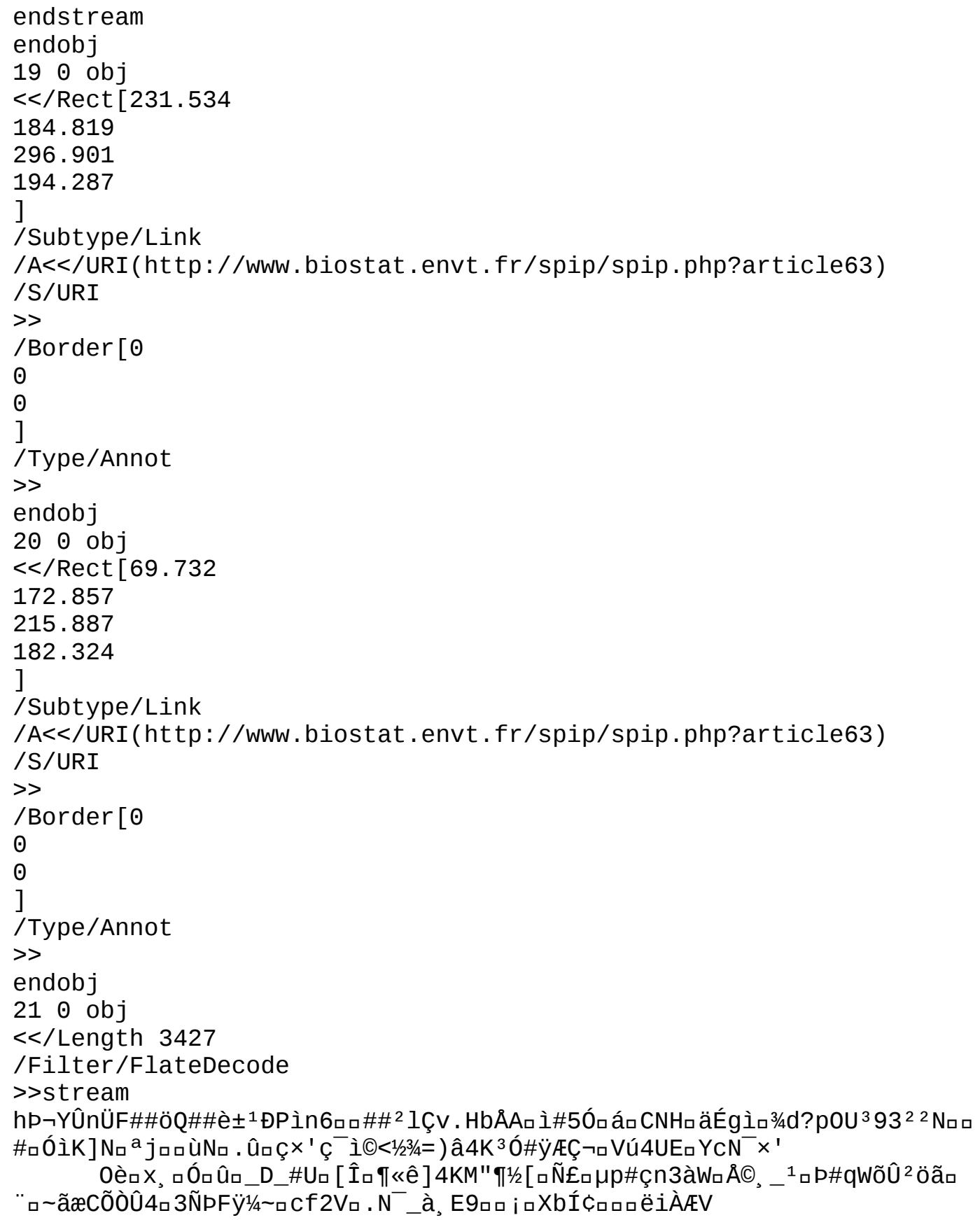




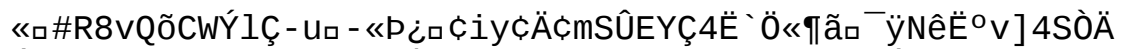

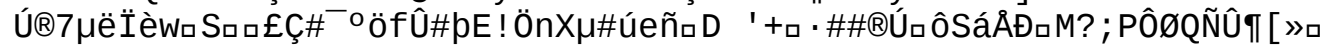

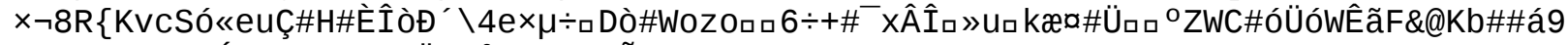

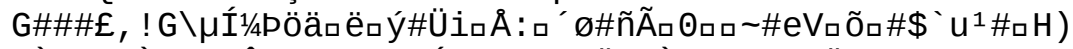

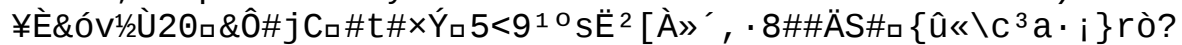

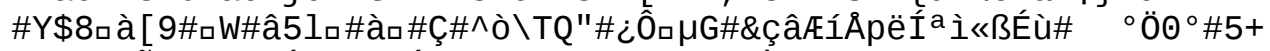

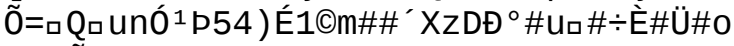

$\#$ \# *às ${ }^{3} \emptyset \# \# \tilde{A} \neg \ll$ ! ôq $£_{i}$ qe ; èr 


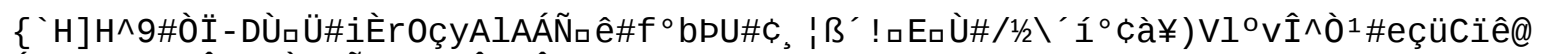

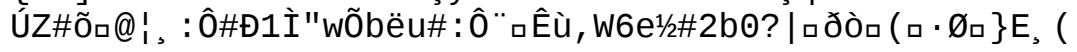




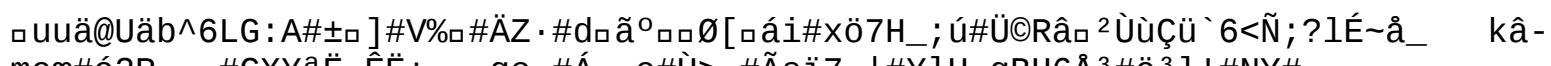

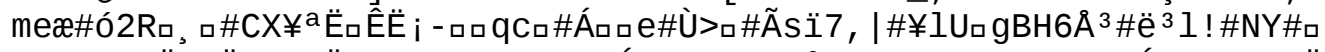

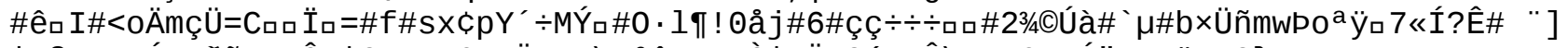

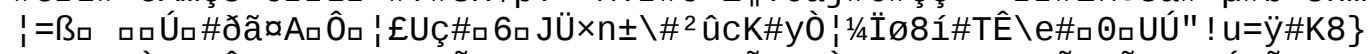

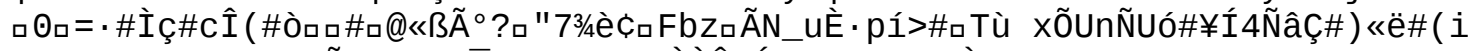

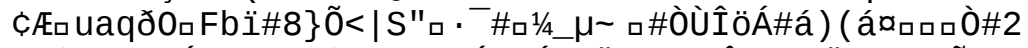

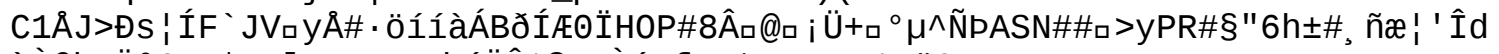

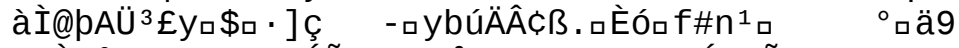

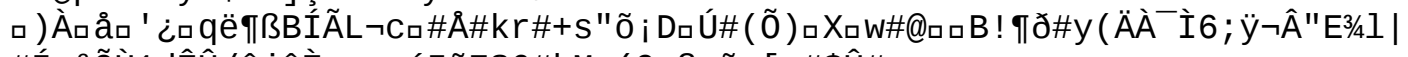

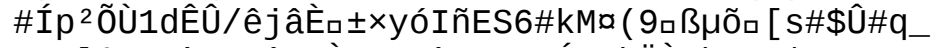

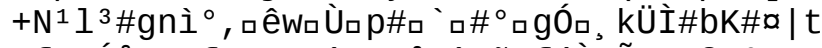

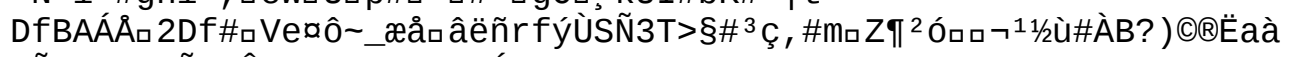

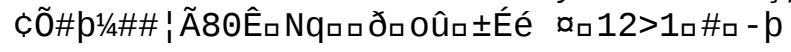


- iTÁ14aËÀ'

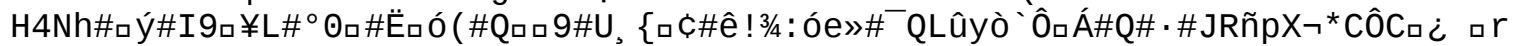

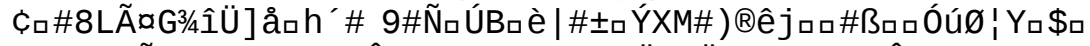

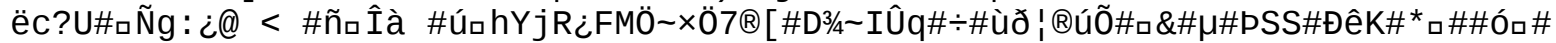

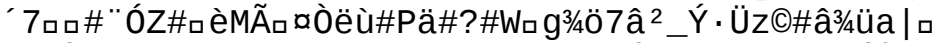

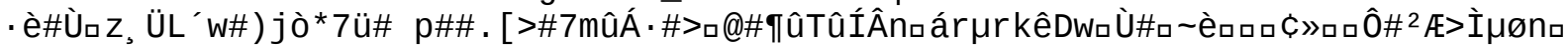

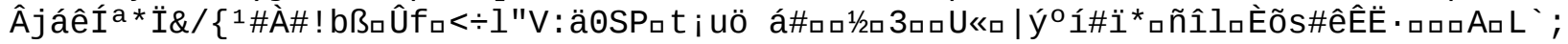




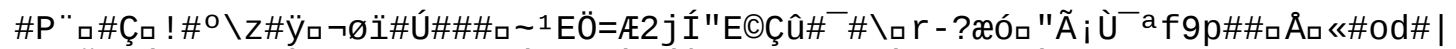

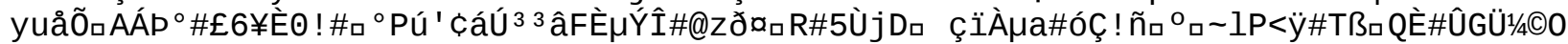

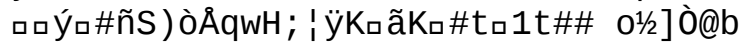

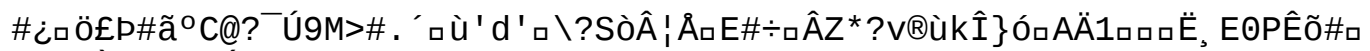

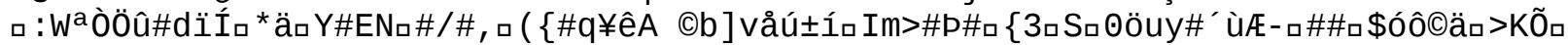

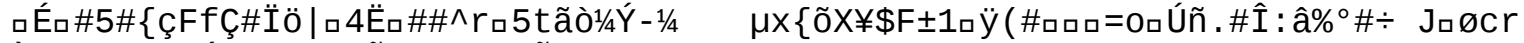

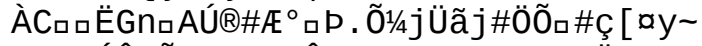

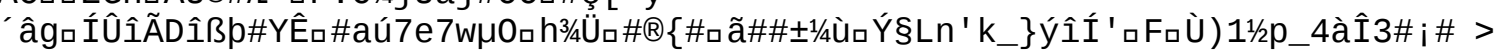




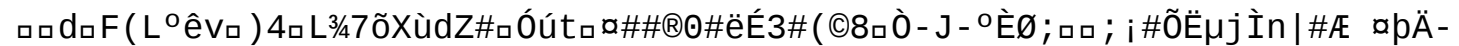

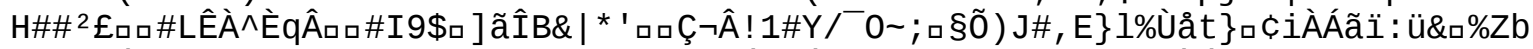

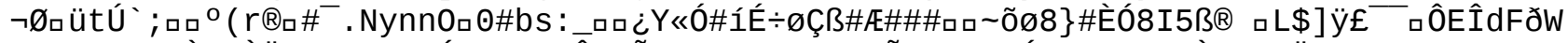

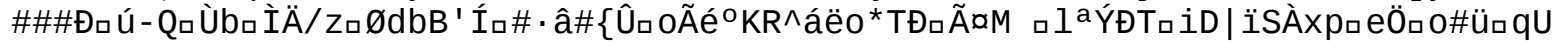




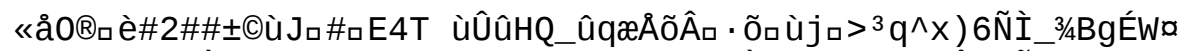

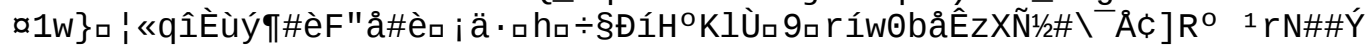

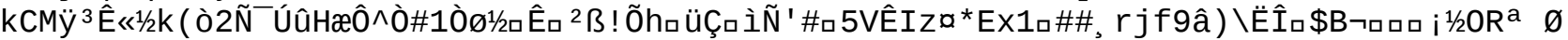
cã $\hat{I} \#^{\circ} \pm r(c ̧ \emptyset \boxminus @ »$ 
\#öÿ\#\#qnUv 


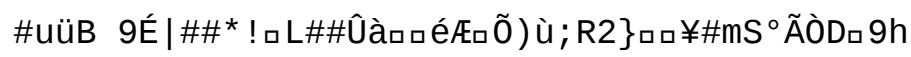




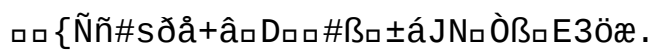




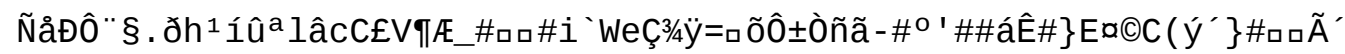
$W T \#<$ \& $2 \mathrm{2T} \cdot \mathrm{i}$

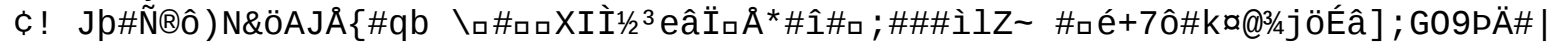

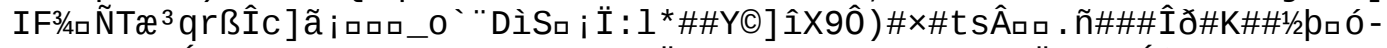

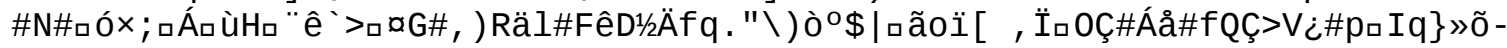

\$u口ôenÿ i \#Iõ¿\# 
\#N\#ĐC

\section{endstream}

endobj

$22 \odot$ obj

$<<$

/Type/Page

/Resources $23 \odot \mathrm{R}$

/CropBox [ $\left.\begin{array}{lllll}\odot & \odot & 595.276 & 782.362\end{array}\right]$

/MediaBox $\left[\begin{array}{llll}\odot & \odot & 595.276 & 782.362\end{array}\right]$

/Rotate 0

/Parent $39 \odot \mathrm{R}$

/Contents $24 \odot \mathrm{R}$

/Thumb $36 \odot \mathrm{R}$

$>>$

endobj

$23 \odot$ obj

$<</$ Font $<</ F 457 \odot \mathrm{R}$

/F6 $640 \odot \mathrm{R}$

/F7 67 ○ $\mathrm{R}$

/F8 $70 \quad 0 \mathrm{R}$

/F10 $76 \odot \mathrm{R}$

$>>$

/Procset [/PDF

/Text

]

/ExtGState $<</ G S 280 \odot \mathrm{R}$

$>>$

$>>$

endobj

$24 \odot$ obj

</Length 1243

/Filter/FlateDecode

>sstream

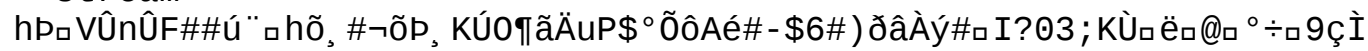


口?

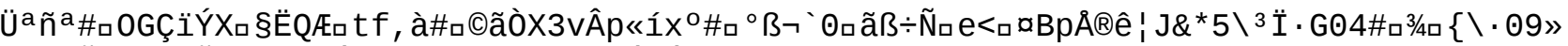

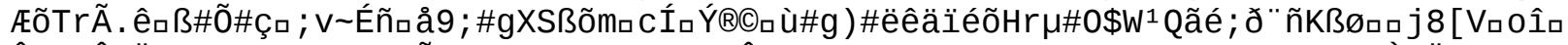

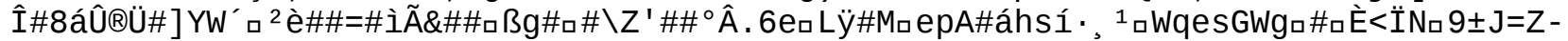

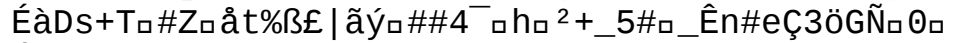

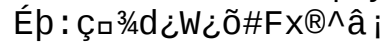




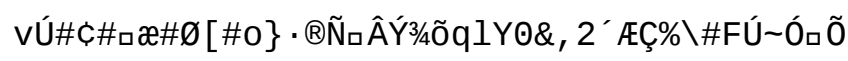
$\div$ 
Ø 


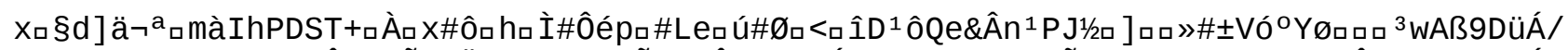

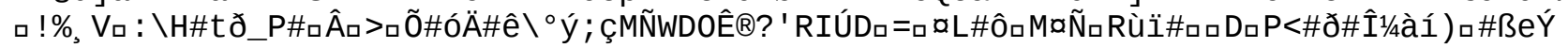




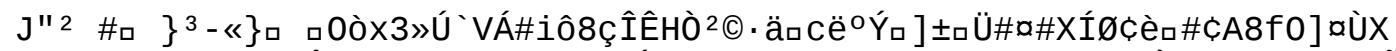

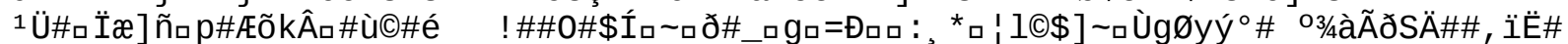

\%@ ’

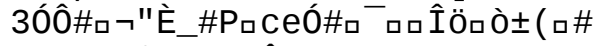

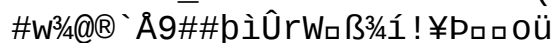

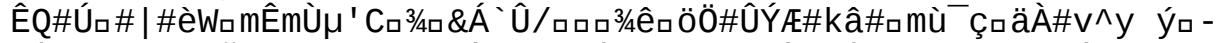

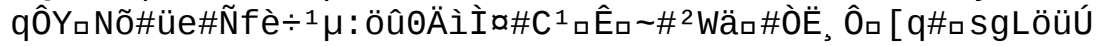


$5 \mathrm{M}$

$\div$ nã [9\#@ÒPıs\#*oølas

I

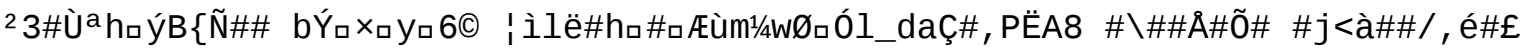


๑\#\# i zõ\#\#s\#3/4ì̇\#qhrÁsc\#/ロ<İèsÃ¥ö\#aA

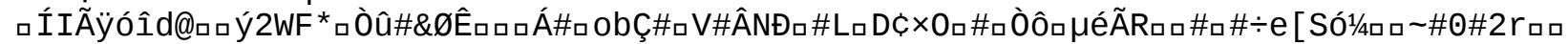

endstream
endobj

$25 \odot$ obj

$<</$ Subtype/Type1

/FontDescriptor $26 \odot \mathrm{R}$

/LastChar 111

/Widths [979

]

/BaseFont/KNMHHM+AdvEls-ent4

/FirstChar 111

/Encoding/WinAnsiEncoding

/Type/Font

$>$

endobj

$26 \odot$ obj

$<</$ StemV 0

/FontName/KNMHHM+AdvEls-ent4

/FontFile3 $27 \odot \mathrm{R}$

/Flags 32

/Descent - 324

/FontBBox $[0$

$-343$

1166

1218

]

/Ascent 926

/CapHeight 1224

/XHeight 716

/Type/FontDescriptor

ItalicAngle 0

/Charset(/o)

$>$

endobj

$27 \odot$ obj

$<$ </Subtype/Type1C

/Length 112

/Filter/FlateDecode

>sstream

hpbd`ad`dd\#ööóõððõvL)sÍ)ÖMÍ+1\#

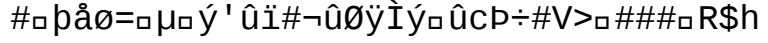

endstream

endobj

$28 \odot$ obj

$<</$ Subtype/Type1

/FontDescriptor $29 \odot \mathrm{R}$

/LastChar 109

/Widths [1000

$\odot$

$\odot$

$\odot$

$\odot$

0

$\odot$

0

$\odot$

0

0

○

$\odot$

$\odot$

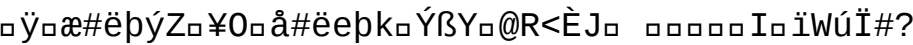




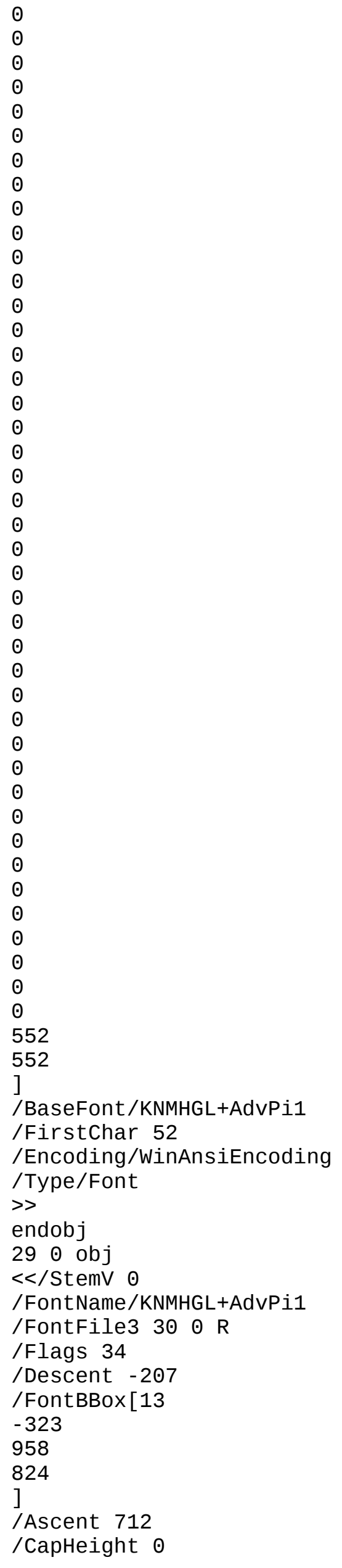


/XHeight 714

/Type/FontDescriptor

/ItalicAngle $\odot$

/CharSet (/l/four/m)

$>$

endobj

$30 \odot$ obj

$<</$ Subtype/Type1C

/Length 623

/Filter/FlateDecode

>sstream

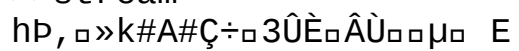

$\square \# C \quad C_{\square} \mathrm{a} \cdot \mathrm{Pb}$

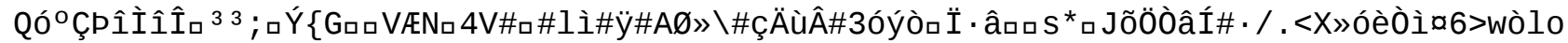

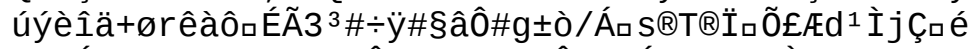

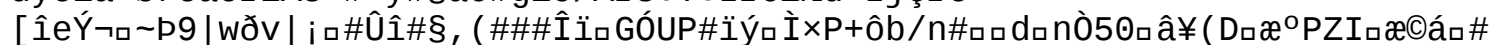

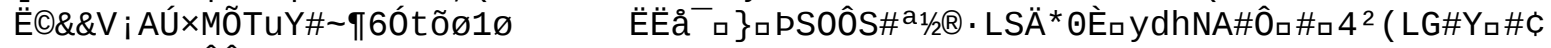

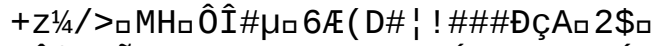

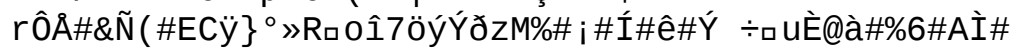

Cिf\#C

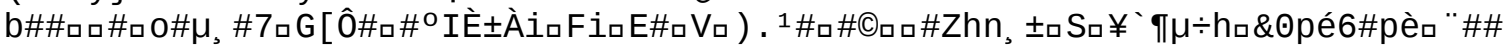

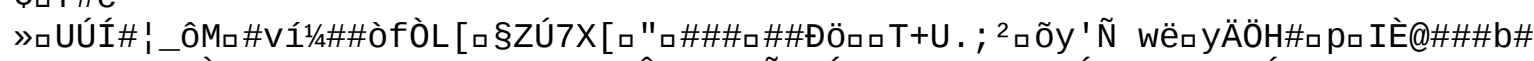
$\mathrm{Pý}+\mathrm{À} \#\{\# \mathrm{U} /$

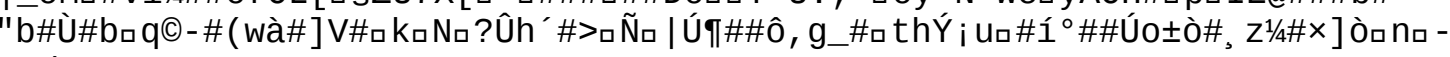

endstream

endobj

$31 \odot$ obj

$<</$ Length 415

/Filter [/ASCII85Decode

/FlateDecode

]

/BitsPerComponent 8

/Colorspace $37 \odot \mathrm{R}$

/Width 74

/Height 97

>stream

8;Z]!!?-S5\#_fhh"hDpIBdk3_Jog6X4Gknr@b5`hn"! IZ61]6Lo^"<8HjC1D8s5]

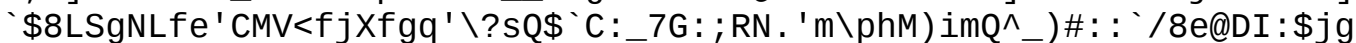
o [\$- lCJTs=) : HJ1\&YEYKFM 'XpjXA". A2RQkdbS<GMLKYq8r71\#2bo, _wbh@gG2E'

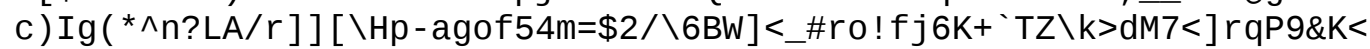
bY-@R/k; . Nd7L1XjM[ sm=) rNaXHFObS : 6VkeTeAunt0\$Ba, EcAm>Qk?Ta>0_qsU1. : . /K2dbQ^71\%DT=j=Vb\&Pd ! F, e+OBr3<]\%cn'q@f - q. I`3h<2@F15\&5*g`cDh7]j EiWj०`6YIO! . eD.M? >

endstream

endobj

$32 \odot$ obj

$<</$ Length 478

/Filter [/ASCII85Decode

/FlateDecode

]

/BitsPerComponent 8

/Colorspace $37 \odot \mathrm{R}$

/Width 74

/Height 97

>stream

$8 ; Z]$ "\$PJ ( 7\$q.Z+" 'p5p^JXT*\#R\%B, l *SnTf^\&tQ8G\%L)BmDA(^/=;A?\Cp9EQlfk : ! 50k1Njj1GEGRj9 ' $a^{*}\left[F^{*} 9\right.$ )6FgBnipE(RXtQmXE, =Xha6s)aQ_^RZ, ';7i+f;.G 8k\$_Ll_cTj<=\&g5B" ; ( j NaJ : = ○oNOo, \%sfqh8*MZ\%A\%="coTr\#fKR\%bDbFnAl1. v

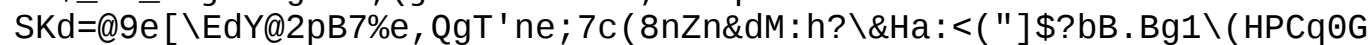
-cl\&c : 8 (\&<9Y\$OnI\%4gRS_e \Z\#80S,Prt\&/6:mqN; J : 1 [Vc3> ! quku* !^bi\$i4N, <

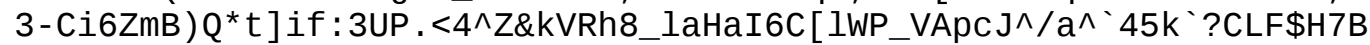
$7 \%<F 38 P=Y 1 \backslash H T \&$ ! e . ' ?h/+I . , A\#7U\$ ${ }^{\top} M m t W E N t I \_h . \bar{V}: X V F .7 p=U 7 \wedge Q \% I \# \_N C 1 p ? \wedge$ $\operatorname{IC} 5\left[d^{\wedge} B^{\prime \prime}>? g^{*} 1 X \sim>\right.$ 


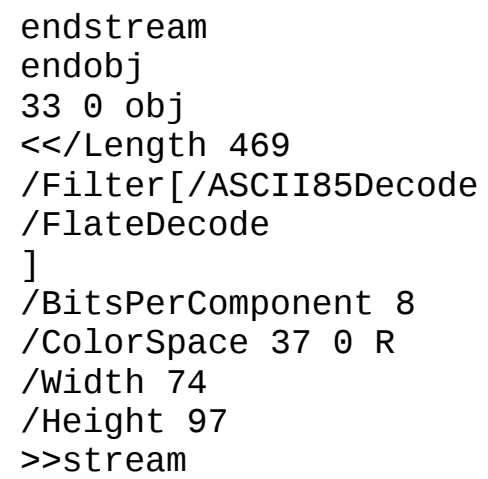
39@kt<dWAnTjhEJ/5V@N\%SlEVJ6/jm.o)h?+]D8\#J]=//, >) O+_TYc ! cH3\#F?\$kPt M) 6k ! - Nd; >1[Pi ! FKYQliuFf] ]N?\#8e0]0G^GrjTec47DS8 . e*Q27ud_f*1YSU0T.

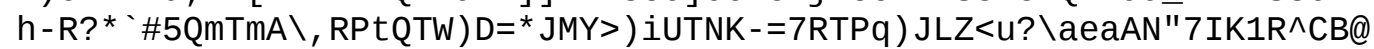
7E!1J >

endstream

endobj

340 obj

$<$ /Length 753

/Filter [/ASCII85Decode

/FlateDecode

]

/BitsPerComponent 8

/Colorspace $37 \odot \mathrm{R}$

/Width 74

/Height 97

>sstream

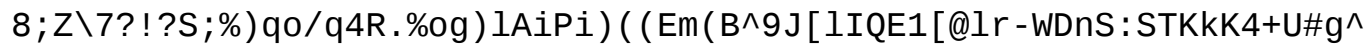
rr>Z2idBFX`C"fR! *s@LVO7rZOK* J\WAV, "9`\&KbgH=tUij\$M1b-oVT]"M1)1. [L2 CMXq2ZQtAgFgeo11u/7J UED]XHRIWZ)+\%U9 [bgoVm"8R\#u3R\$! 8b [53-63aY5a- )

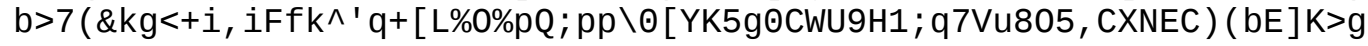
$<$, . NHX] pE $>+\backslash=K r j N r d t H>4 c I Q Z B a Y J k d " L N d 4$ ? . RR ' qLRJeRKRCO`^g1dGs-pgf< ! \#S<5LtY3G \ZV\&9g/pVg-uH1g ! VH\&Xf= : / :\%TZnJ8g ! \$716I\%r ! (\#J4<T=f<pbqY> $<e^{\prime}$ UYZ8T<6m4 *W3qXsM\&bKj : ?MoAW] If6c \8 ' bn247T9U_i : hZo\&lmWKCa?p9B\%s( e_Zk*) : \&i ' G\%dqmJaSc0FU[h7"H"dY-kN6; M\Gmh7o2APFLXg ! N2)nEQq5( S ( ०3Mf jRKG \5, <Vn\#JYZtfHET! . F2pJI4jYgQB\#IW/ Ba+)A\&IWM._hjnZ<K\&rYccG7JG[\& qCFJ\&639 ' [ 1^! 1lcUrmqZr3U?2Mmj jZd>bY9J7tqA [+K>a2QWGP<4q\$9pA०Cq"\$kI 3. Kr51T\$be, :03(FX. ]bERJLqXEV\%K+8nFGg10opJ '3nWo6[q/_\$X[-Y7At_-"Zlm Ih"u7=]0tQb8 $\$$; ; ; up\&T5! m) >

endstream

endobj

$35 \odot$ obj

$<$ /Length 552

/Filter [/ASCII85Decode

/Flatedecode

]

/BitsPerComponent 8

/Colorspace $37 \odot \mathrm{R}$

/Width 74

/Height 97

>stream

8;Z\7a `W7j\$ro@qLjH! FS8nq6P`V4934Y2/i-\&Q`Mm4jMH\%D@iIthSug : eh\&c ]Rd * ! +> q\$mq ‘QKPoiNTN/) p<, 8, kU*Q@34[U)g_sfB55=C`,TR+Q\#W68QN3tG' ; (p_Jk $\wedge^{\prime}$ ? ) 47( \$FnoIe^C: : \="rc] H^8(fZ/Mo+7lc ! /XnW[D3u04HK8BY\&0>\#Q^k. JL] $) \mathrm{m}$ ( f?hmES \hSrh!+Z<\$co >g=b-kfg^KHaC7;X\%QEhrXIdkhH3+@0o2/LWoSA $\$$ ! *6dYR 27? ! 3XoP>Aqe28j ! I \t:9(i1dVp ! p8F ( (m\%(Cf\#1J3i\&6DW $\backslash R t m L, g, ? p b R q Y \# 8 R G$

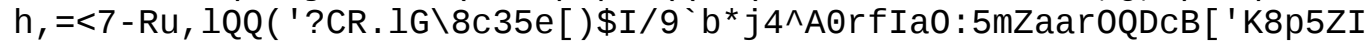
3h/>JAS"BVY>Cd+3u0J\%Fc_iXQXibnC2klgk_'G! JsPG-4 ' 7iDq\%, 9; ○ 'u\%q ! ej9I

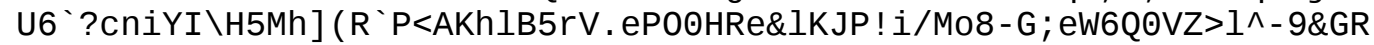




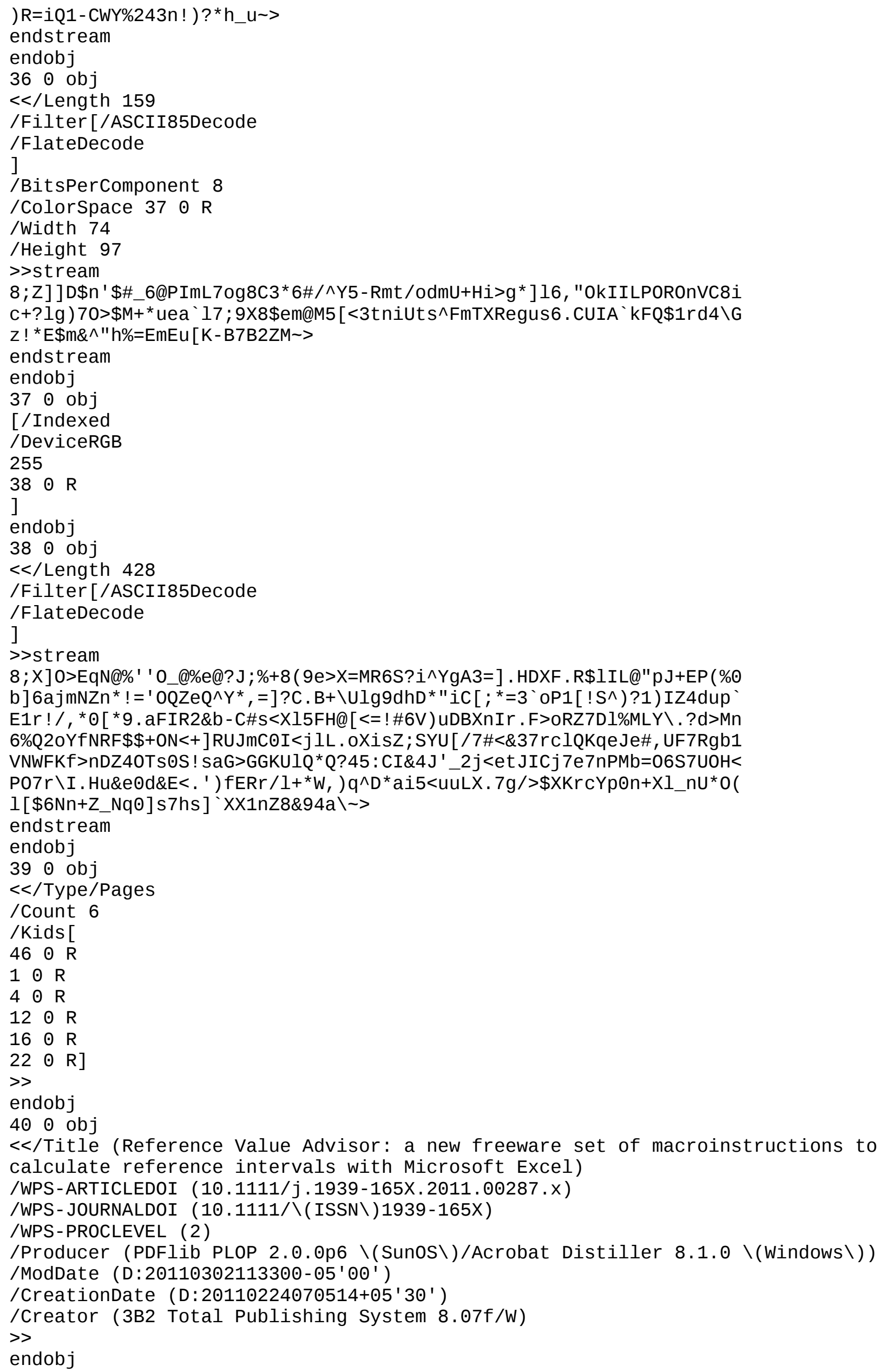




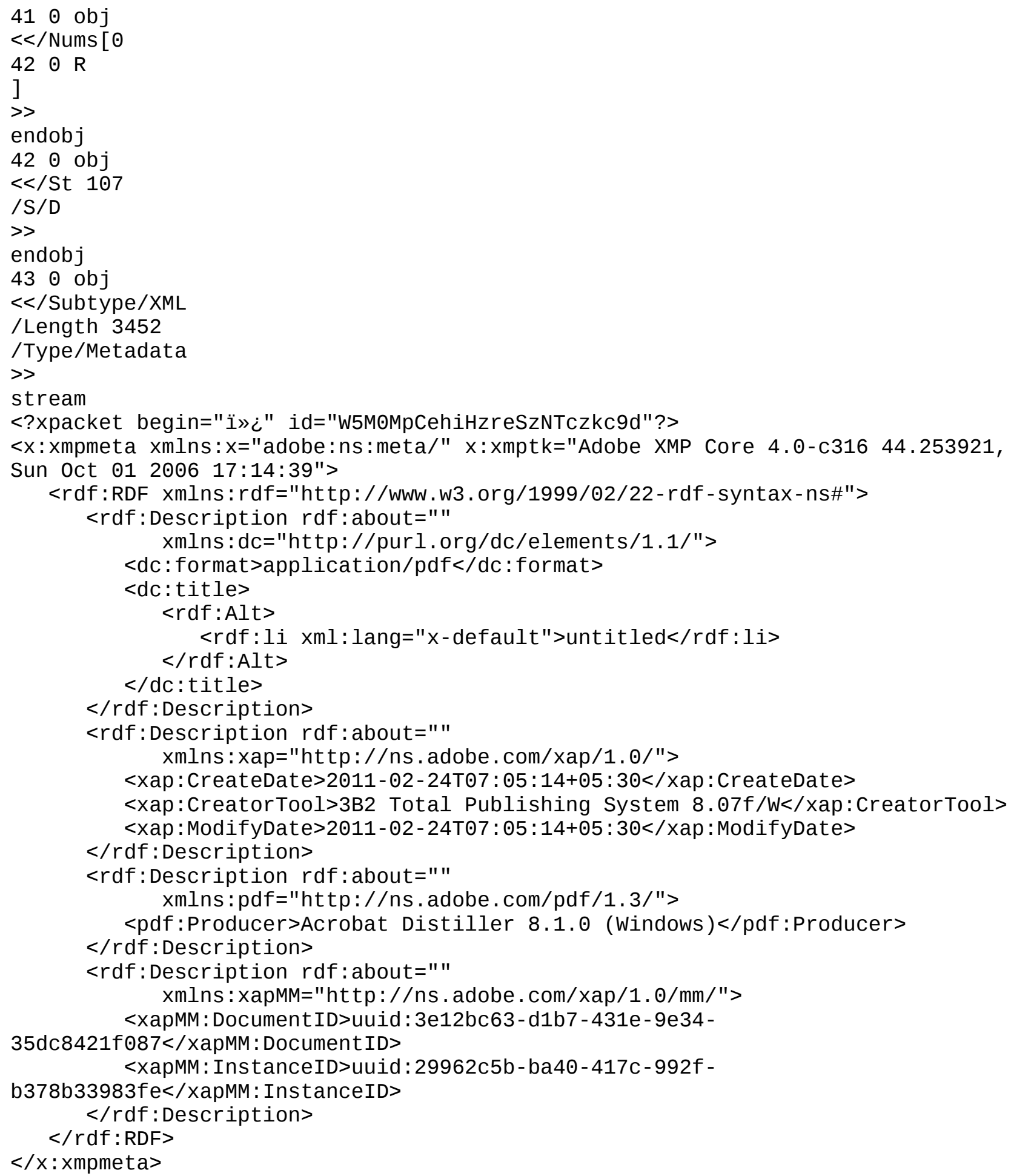




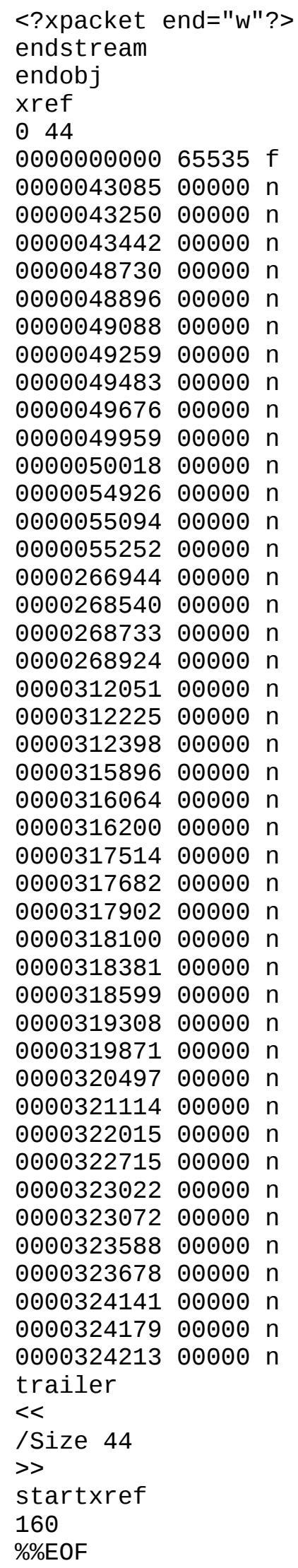

